\title{
Komplexität im Unternehmensplanspiel
}

\author{
Ein integrierter, systemtheoretischer \\ und kognitionstheoretischer Ansatz \\ unter Bezugnahme auf den
}

Gegenstand Unternehmensgründung

\begin{abstract}
Dissertation
zur Erlangung des wirtschaftswissenschaftlichen Doktorgrades des Fachbereichs Wirtschaftswissenschaften der Georg-August-Universität Göttingen
\end{abstract}

vorgelegt von

Regina Ahlbrecht

aus Hameln

Göttingen 2002 
Erstgutachter:

Zweitgutachter:

Tag der mündlichen Prüfung:
Prof. Dr. Dr. h.c. Jürgen Bloech

Prof. Dr. Jörg Biethahn

01. Juli 2002 


\section{Vorwort}

Der Umgang von Unternehmensplanspielern mit Unternehmensplanspielen bedeutet die Auseinandersetzung mit dem komplexen System der Unternehmung in simulierter Form. Dabei sieht sich der Unternehmensplanspieler zumeist einer hohen Komplexität gegenüber, die zum einen zum gegenwärtigen Zeitpunkt nur unzureichend definiert und zum anderen vom Unternehmensplanspieler lediglich eingeschränkt bewältigt werden kann. Hier will die vorliegende Arbeit einen Beitrag leisten und beschäftigt sich mit den Dimensionen der Komplexität im Unternehmensplanspiel. Dabei nähert sie sich der Problemstellung in zweifacher Weise an. Einerseits bezogen auf die Funktion des Unternehmensplanspiels als Modell über das komplexe System der Unternehmung sowie andererseits hinsichtlich dessen Einsatz als betriebswirtschaftliches Lehr-Lern-Instrument.

Die vorliegende Arbeit wurde am 01. Juli 2002 vom Fachbereich Wirtschaftswissenschaften der Georg-August-Universität Göttingen als Dissertation angenommen.

Allen, die mich bei der Erstellung meiner Dissertation unterstützt haben, spreche ich an dieser Stelle meinen Dank aus. Im Besonderen danke ich Herrn Prof. Dr. Dr. h.c. Jürgen Bloech, der die Arbeit betreute, für das entgegengebrachte Vertrauen sowie Herrn Prof. Dr. Jörg Biethahn für die Übernahme des Korreferats. Weiterhin auch Herrn Prof. Dr. Günther Gabisch für die Übernahme des volkswirtschaftlichen Prüfungsteils.

Ein herzlicher Dank gilt außerdem Frau Dipl.-Psychologin Ingeborg Nowack. In dem von ihr initiierten und geleiteten Arbeitskreis für Doktorandinnen zur wissenschaftlichen Qualifizierung speziell von Frauen an der Georg-August-Universität Göttingen, an dem ich teilnahm und zeitweilig mitarbeitete, erfuhr ich in konstruktiven Diskussionen vielfältige Anregungen.

Für die sorgfältige Durchsicht des Manuskripts danke ich Frau M.A. Astrid Krause in Göttingen sowie Herrn Marketing-Kommunikationswirt Gerald Groß in Düsseldorf. Ferner danke ich den Mitgliedern des Arbeitskreises für Doktorandin- 
nen sowie allen Kollegen und Freunden, die mich während der Erstellung der Arbeit methodisch, technisch und moralisch unterstützt haben, insbesondere Frau Studienrätin Kerstin Cummins in Hamburg sowie Frau M.A. Sandra Konold in Münster.

Nicht zuletzt danke ich ganz herzlich meiner Familie, insbesondere meinen Eltern, für die volle Unterstützung. Meinen Eltern Wilhelm \& Sigrid Ahlbrecht ist diese Arbeit gewidmet.

Regina Ahlbrecht 


\section{Inhaltsverzeichnis}

Abbildungsverzeichnis

VII

Abkürzungsverzeichnis

$1 \quad$ Einleitung

1.1 Thematischer Zusammenhang 1

1.2 Gang der Untersuchung 3

$\begin{array}{lll}2 & \text { Theoretische Grundlagen } & 7\end{array}$

$\begin{array}{lll}2.1 & \text { Grundlagen zu Unternehmensplanspielen } & 7\end{array}$

2.1.1 Historische Entwicklung der Planspielmethode 7

2.1.2 Simulationstechnik beim Unternehmensplanspiel 8

2.1.2.1 Das Unternehmensplanspiel:

ein Simulationsmodell 8

2.1.2.2 Das Unternehmensplanspiel:

ein Entscheidungsspiel

2.1.2.3 Interpretation der Simulationstechnik beim Planspiel

2.2 Einsatz von Unternehmensplanspielen

in der Betriebswirtschaftslehre

2.2.1 Das Unternehmensplanspiel: Eine Praxissimulation 20

2.2.1.1 Einsatzgebiete von Unternehmensplanspielen 20

2.2.1.2 Einsatz von Unternehmensplanspielen in der betriebswirtschaftlichen

Aus- und Weiterbildung

2.2.2 Zur Relevanz von Komplexität

im Unternehmensplanspiel

2.2.2.1 Der Komplexitätsbegriff

2.2.2.2 Mehrdimensionalität von Komplexität im Unternehmensplanspiel 
2.2.2.3 Zum Zusammenhang von Komplexität, Lernzielen und Lernerfolg im Unternehmensplanspiel 28

2.2.2.3.1 Zum Zusammenhang von Komplexität und Lernzielen

2.2.2.3.2 Zum Zusammenhang von Komplexität und Lernerfolg

2.3 Denkpsychologie

2.3.1 Komplexes Problemlösen - ein Aufriß 38

2.3.2 Einfache und komplexe Probleme

2.3.3 Einsatz von Simulationsmodellen im Forschungsfeld des Komplexen Problemlösens

3 Das S-H-E-L-L - Modell: Ein Mensch-Maschine-System

3.1 Das System der Unternehmung 50

3.2 Arten und Ablauf von Unternehmensplanspielen 51

3.2.1 Arten von Unternehmensplanspielen 51

3.2.2 Ablauf von Unternehmensplanspielen 56

3.3 Umgang von Personen mit komplexen Systemen 59

3.3.1 Gegenstand der Expertiseforschung 59

3.3.2 Kontrastive Methode der Experten-Novizen-Vergleiche

3.3.3 Untersuchungsdesigns zum Umgang von Personen mit komplexen betriebswirtschaftlichen Systemen $\quad 62$

3.4 Das S-H-E-L-L - Modell 70

3.4.1 Die Natur menschlichen Fehlverhaltens 70

3.4.2 Das S-H-E-L-L - Modell als eine Möglichkeit zur Beschreibung der Natur menschlichen Fehlverhaltens 71

3.4.3 Liveware 76

3.4.3.1 Der Einfluß von Personenmerkmalen auf den Umgang mit komplexen Systemen $\quad 76$

3.4.3.2 Der Spieler als Handlungssubjekt im Unternehmensplanspiel

3.4.3.2.1 Das epistemologische Subjektmodell 
3.4.3.2.2 Fehlverhalten in Zusammenhang mit komplexen Systemen 82

3.4.4 Liveware-Liveware

3.4.4.1 Umgang mit Unternehmensplanspielen in Gruppen

3.4.4.2 Mögliches Fehlverhalten in der Mensch-Mensch-Interaktion 88

3.4.5 Liveware-Software 90

3.4.5.1 Darstellung des Unternehmensplanspiels EpUS 90

3.4.5.2 Darstellung der Entscheidungssituation im Planspiel EpUS

3.4.5.3 Fehlverhalten in Entscheidungssituationen 95

3.4.6 Liveware-Hardware 97

3.4.6.1 Instrumente zur Entscheidungsvorbereitung 97

3.4.6.1.1 Allgemeiner Überblick über das Unternehmensplanspiel MARKUS

3.4.6.1.2 Entscheidungsvorbereitung im Planspiel MARKUS

3.4.7 Liveware-Environment

3.4.7.1 Darstellung des

Unternehmensplanspiels PENTA

$4 \quad$ Unternehmensplanspiele als Modelle über einen Gegenstandsbereich 107

$\begin{array}{lll}4.1 & \text { Die Allgemeine Systemtheorie } & 110\end{array}$

4.2 Systemdifferenzierung und Systemkomplexität 114

4.2.1 Differenzierung als konstitutive

Eigenschaft von Systemen

4.2.2 Komplexität als attributive

Eigenschaft von Systemen

4.3 Das Unternehmensplanspiel als Modell über das komplexe System der Unternehmung

4.3.1 Differenzierung und Komplexität im Unternehmensplanspiel 
4.3.1.1 Differenzierung im Unternehmensplanspiel 123

4.3.1.2 Komplexität im Unternehmensplanspiel

125

4.3.2 Entwicklung eines Komplexitätsmaßes für das offene System der Unternehmung 131

4.4 Grundlagen der Unternehmensgründung 135

4.4.1 Chronologische Entwicklung der Unternehmensgründungsforschung 135

4.4.2 Terminologische Abgrenzungen 142

4.4.2.1 Der Begriff der Unternehmensgründung 142

4.4.2.2 Der Prozeß der Unternehmensgründung 143

4.4.2.3 Formen der Unternehmensgründung 145

4.5 Insolvenz als zentraler Faktor

des Scheiterns junger Unternehmen 147

5 Das Unternehmensplanspiel als Lehr-Lern-Instrument 152

5.1 Das Unternehmensplanspiel:

Ein komplexes Lehr-Lern-Arrangement 152

5.2 Komplexes Problemlösen und Wissen 157

5.3 Mentale Repräsentationen von Wissen 162

5.3.1 Gegenstand der Wissenspsychologie 162

5.3.2 Propositionale, analoge und regelbasierte

Ansätze der Wissensrepräsentation 164

5.3.2.1 Propositionale Repräsentationssysteme 164

5.3.2.2 Analoge Repräsentationssysteme 166

5.3.2.3 Regelbasierte Repräsentationssysteme 168

5.4 Mentale Modelle als analoges

Repräsentationssystem komplexer Systeme 169

5.5 Produktionssysteme als eine Form

regelbasierter Repräsentationssysteme 172

5.5.1 Dörner's Theorie der Absichtsbehandlung 172

5.5.1.1 Elemente der Theorie

der Absichtsbehandlung $\quad 172$

5.5.1.2 Prozesse des Wissenserwerbs 174

5.5.2 Newell's SOAR-Theorie 176

5.5.2.1 Die Architektur von SOAR 176 
5.5.2.2 Wissenserwerb durch den

CHUNKING-Mechanismus 179

5.5.3 Anderson's ACT*-Theorie 181

5.5.3.1 Die Architektur von $\mathrm{ACT}^{*}$ 181

5.5.3.2 Prozeß des Wissenserwerbs in $\mathrm{ACT}^{*}$ 185

5.6 Diskussion und Gegenüberstellung der Produktionssysteme

6 Entscheidungshandeln in Unternehmensplanspielen

6.1 Der Entscheidungsansatz in der Betriebswirtschaftslehre

6.1.1 Der Gegenstand der normativen und deskriptiven Entscheidungstheorie

6.1.2 Zur Relevanz von normativer und deskriptiver Entscheidungstheorie in der Betriebswirtschaftslehre

6.1.3 Die operative Differenz zwischen normativer und deskriptiver betriebswirtschaftlicher Entscheidungstheorie als Ausgangspunkt der Analyse

6.2 Der Erfahrungshintergrund eines Individuums

6.2.1 Merkmale des Erfahrungshintergrundes eines Individuums

6.2.2 Vorwissen: Spiegel des Erfahrungshintergrundes eines Individuums

6.2.2.1 Methoden zur Erhebung von Vorwissen

6.2.2.2 Methoden zur Erhebung des Wissenserwerbs 210

6.3 Die unternehmerische Handlungskompetenz

6.4 Komplexität als attributive Eigenschaft von Entscheidungen

6.4.1 Die objektive Komplexität einer Entscheidungssituation

6.4.2 Die subjektive Komplexität einer Entscheidungssituation

6.5 Lernprozessbezogene Komplexität im Unternehmensplanspiel

6.5.1 Dimensionen der Kontrollkomplexität im Unternehmensplanspiel

6.5.2 Die Systemschwierigkeit: Ausdruck der Problemkomplexität in Wissensgrößen 
6.6 Betriebswirtschaftliche Lernprozesse

im Unternehmensplanspiel

230

6.6.1 Zur Relevanz der ACT*-Theorie

in der Betriebswirtschaftslehre

230

6.6.1.1 Interpretation kognitiver

betriebswirtschaftlicher Lernprozesse

durch die $\mathrm{ACT}^{*}$-Theorie 230

6.6.1.2 Kognition, Emotion und Motivation 232

6.6.1.3 Metakognition: Die Steuerung

des eigenen Denkens

236

6.6.2 Kognitive Grenzen: Der Klassifikationsansatz menschlicher Fehler von Reason

6.6.3 Darstellung von Expertenwissen in Expertensystemen 246

6.6.4 Entscheidungsunterstützung

im Unternehmensplanspiel

6.6.4.1 System, Handlung und Zeit 249

6.6.4.2 Entscheidungsunterstützungssysteme 254

6.7 Entrepreneurship Education 257

6.7.1 Unternehmensgründung als Gegenstand der Lehre 257

6.7.2 Der Begriff des Unternehmensgründers 258

6.8 Liquiditätsplanung einer Unternehmensgründung 259

6.8.1 Der Geschäftsplan als Instrument der Gründungsplanung 259

6.8.2 Dekomposition einer Planungsaufgabe 262

6.8.3 Dekomposition einer Finanzmittelplanung im Hinblick auf den Aspekt der Beteiligungsfinanzierung $\quad 265$

$7 \quad$ Schlußbetrachtung und Ausblick

$\begin{array}{ll}\text { Abkürzungsverzeichnis für Zeitschriftentitel } & 279\end{array}$

$\begin{array}{ll}\text { Literaturverzeichnis } & 280\end{array}$ 


\section{Abbildungsverzeichnis}

$\begin{array}{lll}\text { Abbildung 2-1: } & \text { Modellbildung bei Planspielen }\end{array}$

Abbildung 2-2: $\quad$ Arbeitsablauf am Unternehmensplanspiel 15

Abbildung 2-3: $\quad$ Phasen einer Spielrunde im Planspiel 16

Abbildung 2-4: $\quad$ Aktions- und Reaktionsbereich beim Simulationsmodell 19

Abbildung 2-5: $\quad$ Systematisierung von Lernzielen im Hinblick

auf die Entwicklung eines Unternehmensplanspiels

Abbildung 2-6: Ablauf der Untersuchung 35

Abbildung 2-7: Klassifikation von Barrieretypen 44

Abbildung 3-1: $\quad$ Arten von Unternehmensplanspielen 56

Abbildung 3-2: $\quad$ The traditional sequence in system design in the domain aircraft

Abbildung 3-3: The dynamic HELS system, showing the interactions between Hardware, Software and Liveware components, and between each of these and the Environment

Abbildung 3-4: The dynamic HELLS system, showing the interactions between Hardware, Software, Liveware and Liveware components, and between each of these and the Environment

Abbildung 3-5: Aufnahme und Austausch der Informationen in einer Gruppe während der Situationsanalyse

Abbildung 3-6: $\quad$ Schema des Kommunikationsvorganges 88

Abbildung 3-7: Modellentscheidungen im Planspiel EpUS 93

Abbildung 3-8: $\quad$ Entscheidungssituation im Planspiel EpUS mit 94

Abbildung 3-9: $\quad$ Entsorgungs- und Abfallwirtschaft im Planspiel PENTA 104

Abbildung 4-1: $\quad$ Offenes und geschlossenes System

Abbildung 4-2: $\quad$ Allgemeine Systemeigenschaften in Abgrenzung zu einer bloßen Menge von Elementen

Abbildung 4-3: $\quad$ Statische Dimensionen systemtheoretischer 
Abbildung 4-4: Dynamische Dimensionen systemtheoretischer

Komplexität und Kompliziertheit

Abbildung 4-5: Horizontale Differenzierung im Sinne

einer bottom-up-Betrachtung

Abbildung 4-6: Vertikale Differenzierung im Sinne

einer top-down-Betrachtung

Abbildung 4-7: Das Konzept der Iso-Komplexitätskurven

Abbildung 4-8: $\quad$ Top-Down-Zerlegung eines offenen Systems mit

Abbildung 4-9: $\quad$ Dimensionen der Abbildungskomplexität eines

offenen Systems mit

Abbildung 4-10: Gründungsformen

Abbildung 4-11: Zerlegung des Elementes Finanzierung im

offenen System der Unternehmung

Abbildung 5-1: $\quad$ Überblick über die SOAR-Architektur

Abbildung 5-2: $\quad$ Ein allgemeiner Rahmen für das ACT-Produktionssystem

Abbildung 5-3: $\quad$ Kognitive Strukturierungen nach

DÖRNER, NEWELL \& ANDERSON

Abbildung 6-1: Variablen zur Errechnung der

Spiel-Performanz eines Teilnehmers

Abbildung 6-2: Die Kontrollkomplexität eines offenen Systems

und ihr Zusammenhang mit der Kognitiven

Komplexität eines Planspielteilnehmers mit

Abbildung 6-3: Rahmenkonzeption zum Begriff der Systemschwierigkeit

Abbildung 6-4: Die Kontrollkomplexität eines offenen Systems

und ihr Zusammenhang mit dem Vorwissen

eines Planspielteilnehmers mit

Abbildung 6-5: $\quad$ Struktur des psychophysischen Systems

im Person-Umwelt -Bezug mit

Abbildung 6-6: $\quad$ Ebenen der Kontroll- und Steuerungstätigkeit

Abbildung 6-7:

Architektur eines Expertensystems

Abbildung 6-8:

Beteiligungsfinanzierung in den Lebensphasen

eines Unternehmens 
Abbildung 6-9: $\quad$ Möglichkeiten der Beteiligungsfinanzierung in der Phase des Seed Financing

Abbildung 6-10: Möglichkeiten zur Beteiligungsfinanzierung in der Phase des Start Up Financing

Abbildung 6-11: Möglichkeiten zur Beteiligungsfinanzierung in der Phase des First Stage Financing

Abbildung 6-12: Möglichkeiten zur Beteiligungsfinanzierung im Zeitverlauf 


\section{Abkürzungsverzeichnis}

\begin{tabular}{ll} 
Abb. & Abbildung \\
akt. & aktualisierte \\
Aufl. & Auflage \\
BALPA & British Air Line Pilots Association \\
Bd. & Band \\
bearb. & bearbeitete \\
BMFT & Bundesminister für Forschung und Technologie \\
BMWi & Bundesministerium für Wirtschaft und Technologie \\
bzw. & beziehungsweise \\
ders. & derselbe \\
dies. & dieselbe(n) \\
DGfE & Deutsche Gesellschaft für Erziehungswissenschaft \\
DGfP & Deutsche Gesellschaft für Psychologie \\
DGOR & Deutsche Gesellschaft für Operations Research \\
Dr. & Doktor \\
ed. & edition \\
Ed. & Editor \\
Eds. & Editors \\
erw. & erweiterte \\
et al. & et alii \\
e.V. & eingetragener Verein \\
f. & folgende \\
ff. & fortfolgende \\
H. & Heft \\
Hrsg. & Herausgeber \\
hrsg. & herausgegeben \\
insbes. & insbesondere \\
Jg. & Jahrgang \\
N.F. & Neue Folge \\
N.J. & New Jersey \\
No. & Number \\
\hline
\end{tabular}




$\begin{array}{ll}\text { Nr. } & \text { Nummer } \\ \text { Prof. } & \text { Professor } \\ \text { S. } & \text { Seite } \\ \text { sog. } & \text { sogenannte(n) } \\ \text { Sp. } & \text { Spalte } \\ \text { Suppl. } & \text { Supplement } \\ \text { u. } & \text { und } \\ \text { u.a. } & \text { und andere } \\ \text { überarb. } & \text { überarbeitete } \\ \text { unveränd. } & \text { unveränderter } \\ \text { v. } & \text { von } \\ \text { v. Chr. } & \text { vor Christi } \\ \text { verb. } & \text { verbesserte } \\ \text { vgl. } & \text { vergleiche } \\ \text { Vol. } & \text { Volume } \\ \text { vollst. } & \text { vollständig } \\ \text { z.B. } & \text { zum Beispiel }\end{array}$



UND GERADE DADURCH, DASS

MAN SICH IRRT, FINDET MAN

MANCHMAL DEN WEG

-Vincent van Gogh-

\subsection{Thematischer Zusammenhang}

Die Überlegungen zu dieser Arbeit gehen vom Gegenstandsbereich der Unternehmensgründung aus. Die Initiierung durch das Unternehmertum wie auch die Entstehung eines Unternehmens als betriebswirtschaftliches Erkenntnisobjekt sind in der Vergangenheit wenig theoretisch thematisiert worden. Auch heute kann festgestellt werden, daß ein hoher Anteil der wissenschaftstheoretischen Diskussionsbeiträge deskriptiv bleibt. Weiterhin weisen die wenigen modellgestützten Untersuchungen Lücken auf, wodurch sie der Komplexität der Problemstellung, verursacht durch die Vielzahl von Entscheidungen, welche die Gründung einer Unternehmung hervorruft, nicht gerecht werden. Der Untersuchungsraum wird demnach eingeschränkt. In diesem Zusammenhang wurde durch das von Dietz, ${ }^{1}$ unter Berücksichtigung chaostheoretischer Aspekte, entwickelte Modell eine modelltheoretische Auseinandersetzung mit dem Bereich der Unternehmensgründung angeregt, wozu die Möglichkeit der Modellbildung durch Unternehmensplanspielmodelle ausgewählt wurde.

So verweist bereits Szyperski zum Ende der 70er Jahre auf die Eignung der Planspielmethode für den Gegenstand der Unternehmensgründung. Er führt aus, daß ein in Zusammenhang mit der Unternehmensgründung zu entwickelndes Modell zum einen in der Lage sein muß, Ergebnisse des Systemverhaltens zu reproduzieren, zum anderen aber auch die Wechselbeziehungen aufzuzeigen, die zu einem bestimmten Ergebnis geführt haben. Demnach muß ein entsprechendes Modell dynamischer Natur sein, um Zustands- und Verhaltensänderungen faßbar zu ma- 
chen und aufzuzeigen. Szyperski gelangt zu dem Schluß, daß die Simulation diesem Anspruch in hohem Maße Rechnung trägt. ${ }^{2}$

Dabei ist zunächst davon auszugehen, daß das Planspiel als spezifische Form einer Simulation zwei elementare Funktionen besitzt. Das ist zum einen die Funktion als Modell, die sich durch die Möglichkeit bedingt, einen realen, meist sehr komplexen Gegenstand durch ein Planspielmodell abzubilden. Zum anderen ist es die Funktion des Planspiels als Lehr-Lern-Instrument. In diesem Zusammenhang wird sowohl durch die Funktion des Planspiels als Modell als auch als Lehr-LernInstrument die Berücksichtigung von Komplexität impliziert. Weiterhin wird durch die Funktion des Planspiels als Modell die Berücksichtigung der Systemtheorie impliziert sowie durch die Funktion des Planspiels als Lehr-LernInstrument mit dem Charakter eines Entscheidungsspiels die Berücksichtigung des Menschen. Schließlich wird durch die Berücksichtigung des Menschen die Notwendigkeit einer Integration der Psychologie impliziert. Zum einen die Integration der Denkpsychologie, die im Bereich der Problemlöseforschung, einem Teilbereich der Denkpsychologie, den Umgang von Personen mit komplexen Systemen untersucht sowie zum anderen die Integration der Wissenspsychologie, da Problemlösen als Anwendung und Erwerb von Wissen angenommen wird.

Weiterhin ist davon auszugehen, daß Komplexität sowohl im Bereich der Unternehmensgründung als auch im Unternehmensplanspiel eine Schlüsselfunktion hat. Um in diesem Zusammenhang zu Aussagen über Komplexitätsmerkmale zu gelangen, ist Komplexität in Zusammenhang mit Unternehmensplanspielmodellen zu betrachten. Dabei ist zunächst festzuhalten, daß das Merkmal Komplexität im Unternehmensplanspiel offensichtlich mehrdimensional ist. Durch eine grundlegende Betrachtung des Komplexitätsbegriffes kann jedoch weiterhin festgehalten werden, daß Komplexität unter kontextabhängigen und kontextunabhängigen Gesichtspunkten zu beleuchten ist. In Zusammenhang mit der Modellbildung ist Komplexität dabei als attributive Eigenschaft von Systemen anzusehen. In Zu-

2 Vgl. Szyperski, N.; Kirschbaum, G., (GRÜMOD 1979), S. 14 f. Diese Sichtweise wird auch in der anglo-amerikanischen Forschung vertreten. So verweisen Ulrich \& Cole darauf, daß eine der Methoden, ,which are best suited to an entrepreneurial learning style“, die der „management simulation“ darstellt. Vgl. Ulrich, T.A.; Cole, G.S., (Entrepreneurs 1987), S. $36 \mathrm{ff}$. 
sammenhang mit der Funktion als Lehr-Lern-Instrument als attributive Eigenschaft von Entscheidungen.

Durch diesen Zusammenhang erwächst zum einen die Möglichkeit einer modelltheoretischen sowie zum anderen die einer lernprozeßbezogenen Betrachtung von Komplexität im Unternehmensplanspiel, wobei, durch die Betrachtung von Unternehmensplanspielen in bezug auf ihren Einsatz in der Lehre, eine Integration der Wirtschaftspädagogik impliziert wird. Die modelltheoretische Betrachtung kann nun durch die Integration der Systemtheorie unter systemtheoretischen Gesichtspunkten erfolgen. Die lernprozeßbezogene Betrachtung kann unter Berücksichtigung sowohl psychologischer als auch entscheidungstheoretischer Erkenntnisse erfolgen. Die modelltheoretischen und die lernprozeßbezogenen Betrachtungen können schließlich bezüglich modelltheoretischer Implikationen auf die in $\mathrm{Zu}$ sammenhang mit dem Lernprozeß gewonnenen Erkenntnisse sowie entscheidungsunterstützender Maßnahmen untersucht werden, was als letzten Aspekt eine Integration der Wirtschaftsinformatik impliziert.

In Zusammenhang mit der Unternehmensgründung ergeben sich dadurch letztlich Anknüpfungspunkte zu einer Betrachtung der gewonnenen Erkenntnisse am konkreten Beispiel zweier kritischer Faktoren im Gründungsgeschehen.

\subsection{Gang der Untersuchung}

Der erste Teil der Einleitung diente dazu, den thematischen Zusammenhang der Arbeit darzustellen. Hier wird nun der Aufbau der Arbeit im einzelnen überblickartig dargestellt.

In Kapitel zwei wird zunächst der theoretische Bezugsrahmen ausgearbeitet. Nach grundlegenden Ausführungen zum historischen Hintergrund der Planspielmethode wird die spezifische Simulationstechnik beim Planspiel erläutert. Aus dieser Betrachtung läßt sich zum einen die Funktion des Planspiels als Simulationsmodell, zum anderen dessen Funktion als Entscheidungsspiel ableiten, wobei die Faktoren insgesamt als Mensch-Maschine-Simulation interpretiert werden können. Im weiteren werden mögliche Einsatzgebiete von Planspielen beleuchtet um dann bezug zu nehmen auf den in dieser Arbeit relevanten Bereich des Einsatzes von Plan- 
spielen in der Ausbildung. Daran anschließend wird die Relevanz von Komplexität im Unternehmensplanspiel aufgezeigt und schließlich Grundlagen der Problemlöseforschung als einem Teilgebiet der Denkpsychologie ausgeführt.

In dem sich anschließenden Kapitel drei werden Erkenntnisse der betriebswirtschaftlichen sowie der psychologischen Forschung in einen gemeinsamen Bezugsrahmen gestellt. Dies kann zum einen unter Berücksichtigung der in Kapitel zwei ausgeführten Diskussion bezüglich einer wünschenswerten Zusammenarbeit zwischen der Betriebswirtschaftslehre und der Psychologie gesehen werden; geschieht jedoch vordergründig im Hinblick auf den in Kapitel sechs ausgeführten Aspekt, daß die Teilnehmer an einem Unternehmensplanspiel, gemäß einer Untersuchung von Bloech, Kauer \& Orth, ${ }^{3} \mathrm{ihr}$ in Seminaren und Vorlesungen erworbenes Sachwissen nur unzureichend oder gar nicht anwenden. So zielt die in Kapitel drei vorgenommene Betrachtung zum einen auf eine Spezifikation des Unternehmensplanspielsystems, zum anderen auf die möglichen Ursachen für die unzureichende Anwendung des Sachwissens im Unternehmensplanspiel ab. Als problematisch wird in diesem Zusammenhang angesehen, daß sich aus der grundlegenden Simulationstechnik beim Planspiel unterschiedliche Modellkonstruktionen entwickelt haben sowie weiterhin die psychologischen Untersuchungen an unterschiedlichen Versuchsanordnungen durchgeführt werden. In diesem Zusammenhang wird das in der Luft- und Raumfahrttechnik entwickelte S-H-E-L-L - Modell ${ }^{4}$ herangezogen und betriebswirtschaftlich und kognitionspsychologisch interpretiert.

In Kapitel vier wird den weiteren Ausführungen zunächst eine kurze Zusammenfassung der Vorgehensweise und Erkenntnisse aus Kapitel drei vorangestellt, um danach den Systemgedanken wieder aufzugreifen. In diesem Zusammenhang wird zunächst aufgezeigt, unter welchen Gesichtspunkten die Allgemeine Systemtheorie zu ihrem universellen Anspruch gelangt. Da diese Gesichtspunkte gleichsam den Hintergrund für die durch Luhmann vollzogenen Annahmen einer Theorie der Systemdifferenzierung sowie einer Theorie der Systemkomplexität darstellen, ${ }^{5}$

Vgl. Bloech, J.; Kauer, G.; Orth, C., (Unternehmensplanspiele 1996), S. 38.

Vgl. Edwards, E., (Machine 1972); Hawkins, F.H., (Human 1984).

Vgl. Luhmann, N., (Systeme 1996), S. 41. 
wird Komplexität im folgenden als attributive Eigenschaft von Systemen, Differenzierung als konstitutive Eigenschaft von Systemen verstanden wobei auf eine Anwendbarkeit auf das Unternehmensplanspielmodell abgezielt wird. Die weiteren Betrachtungen widmen sich dann einer chronologischen Entwicklung des Forschungsstandes im Bereich der Unternehmensgründungsforschung. Daran schlieBen sich wiederum Betrachtungen zum Begriff der Unternehmensgründung, Formen der Unternehmensgründung sowie weiterhin des zeitlichen ausgedehnten Prozesses der Unternehmensgründung an. Zum Schluß erfolgt dann die Identifikation des Elementes Finanzierung als problematische Variable im Gründungsgeschehen sowie eine Berücksichtigung der in diesem Kapitel gewonnenen Erkenntnisse in Zusammenhang mit dieser Variable.

Die zweite Funktion eines Unternehmensplanspiels, die als Lehr-Lern-Instrument, bildet die Grundlage der folgenden zwei Kapitel. In Kapitel fünf werden zunächst wirtschaftspädagogische Hintergründe beleuchtet, um im Anschluß daran, unter dem Gesichtspunkt, daß Lernhandeln und Problemlösen sich gegenseitig bedingen, eine Betrachtung bezüglich des Zusammenhangs von Problemlösen und Wissen vorzunehmen. Da zwischen einer angemessenen Wissensrepräsentation und dem erfolgreichen Umgang mit einem komplexen System eine positive Korrelation festgestellt werden kann und Problemlösen weiterhin als Anwendung und Erwerb von Wissen angenommen wird, wird durch die gewonnenen Erkenntnisse die Relevanz der Wissenspsychologie impliziert. Diese nimmt bezüglich der Repräsentation von Wissen im menschlichen Gedächtnis unterschiedliche Repräsentationsformate an, die zunächst unter grundlegenden Gesichtspunkten beleuchtet werden. Daran anschließend wird das Konzept mentaler Modelle als analoges Repräsentationssystem komplexer Systeme erläutert sowie weiterhin die Theorie der Absichtsbehandlung von Dörner, ${ }^{6}$ die SOAR-Theorie von Newell 7 und die ACT*-Theorie von Anderson $^{8}$ als regelbasierte Konzeptionen dargestellt. Die Betrachtungen werden dann mit einer Diskussion und Gegenüberstellung der regelbasierten Konzeptionen abgeschlossen. Dabei zielt die Betrachtung auf eine mögliche Anwendbarkeit der dargestellten Konzeptionen auf den betriebswirtschaftlichen Lernprozeß. 
Im Kapitel sechs dient die Identifikation einer operativen Differenz zwischen normativer und deskriptiver Entscheidungstheorie als Ausgangspunkt der Analyse. In diesem Zusammenhang wird Komplexität als attributive Eigenschaft von Entscheidungen angenommen und eine potentielle Differenz zwischen objektiver und subjektiver Komplexität angenommen, die im weiteren Verlauf als potentielle Problemkomplexität identifiziert wird. Weiterhin wird eine Gegenüberstellung weiterer Komplexitätsdimensionen, die in den Ausführungen abgeleitet werden, mit den in Kapitel vier analysierten Komplexitätsgraden und Komplexitätsdimensionen vorgenommen. In den weiteren Betrachtungen wird die Interpretation des betriebswirtschaftlichen Lernprozesses, unter Berücksichtigung der in Kapitel fünf ausgeführten $\mathrm{ACT}^{*}$-Theorie von Anderson, ${ }^{9}$ durch Orth,${ }^{10}$ ausgeführt und die Relevanz von emotionalen, motivationalen und metakognitiven Aspekten im Unternehmensplanspiel aufgezeigt.

Im weiteren Verlauf wird dann auf die Entscheidungsunterstützung im Unternehmensplanspiel bezug genommen sowie weiterhin an die Betrachtungen in Kapitel drei angeknüpft; und ein Klassifikationsschema menschlicher Fehler von Reason ${ }^{11}$ erläutert. Schließlich wird an den Aspekt angeknüpft, daß Informationsdefizite die zweithäufigste Ursache des Scheiterns von Unternehmensgründungen darstellen und eine kurze Betrachtung der gewonnen Erkenntnisse am konkreten Beispiel vorgenommen.

Kapitel sieben hat Schlußbetrachtung und Ausblick zum Inhalt. Hier werden die Ergebnisse dieser Arbeit zusammenfassend dargestellt und ein Ausblick auf mögliche, anknüpfende Forschungsaktivitäten gegeben.

9 Vgl. Anderson, J.R., (Architecture 1983).

10 Vgl. Orth, C., (Unternehmensplanspiele 1999), S. 49 ff.

11 Vgl. Reason, J., (System 1987). 


\section{Grundlagen zu Unternehmensplanspielen}

\subsection{1}

\section{Historische Entwicklung der Planspielmethode}

Der Stammbaum des Planspiels weist eine bis weit in die Vergangenheit reichende Historie auf. An dessen Anfang standen um 1000 v. Chr. die Kampfspiele. Die Entwicklung ging weiter über das Kriegs-Schach und das Karten-Manöver bis hin zu den Kriegsspielen als unmittelbare Vorläufer von Simulationen im militärischen Bereich. ${ }^{12}$ Die Kriegsspiele dienten seit dem Anfang des 17. Jahrhunderts sowohl zur Darstellung militärischer Operationen als auch zum Training und zur Ausbildung von Offizieren. Mit Hilfe von Landkarten und Sandkästen wurden Gebietsausschnitte sowie Truppenkonstellationen mit Stecknadeln oder Figuren modelliert.

Im 19. Jahrhundert wurde das durch den Artillerie-Leutnant von Reisswitz entwikkelte strenge Kriegsspiel offiziell in die Preußische Armee eingeführt. Hier gelang es zum ersten Mal, auch den Faktor Zeit zu simulieren, indem im Spielverlauf neue Fakten hinzugefügt wurden. Auf dem strengen Kriegsspiel bauten schließlich die neuesten Weiterentwicklungen, angefangen beim freien Kriegsspiel, auf. Waren in strengen Kriegsspielen die Manöver bspw. noch durch umfangreiche Regeln bestimmt, so wurde in freien Kriegsspielen der immer komplizierter werdenden Kriegstechnik entsprochen und das umfangreiche Regelwerk vermehrt durch den Einsatz von Schiedsrichtern abgelöst, die den Spielverlauf regelten. Das freie Kriegsspiel wurde später ausschließlich der militärischen Ausbildung zu Grunde gelegt und löste damit das starre Kriegsspiel gänzlich ab. ${ }^{13}$

Simulationen im militärischen Bereich wurden dann zu Beginn des 20. Jahrhunderts entwickelt und dienten später als Vorläufer wirtschaftlicher Planspiele. Als quasi-praktische Aus- und Weiterbildung wurden dort Ernstfall-Szenarien einge-

12 Vgl. den Stammbaum des Planspiels bei Rohn, W.E., (Führungsentscheidungen 1964), S. 25 .

13 Eine umfangreiche Darstellung zur Historie des Planspiels findet sich bei Rohn, W.E., (Führungsentscheidungen 1964), S. 19 ff.; Bleicher, K., (Unternehmungsspiele 1962), S. $31 \mathrm{ff}$. 
setzt. ${ }^{14}$ Unmittelbarer Vorläufer wirtschaftlicher Planspiele war schließlich das 1955 von der Rand Corporation nach den Prinzipien des strengen Kriegsspiels entwickelte Planspiel MONOPOLOS. Entwickelt für logistische Fragestellungen der US-Luftwaffe, wurden dort vorwiegend betriebswirtschaftlich-organisatorische Elemente aufgegriffen. Die Anstrengung, ein spezifisches Unternehmungsspiel zu entwickeln, gelang im Jahre 1956 als erstes der AMERICAN MANAGEMENT ASSOCIATION. ${ }^{15}$ Im Jahre 1959 begann dann auch in Deutschland die Auseinandersetzung mit Unternehmensplanspielen. ${ }^{16}$ So entstand das erste deutsche Unternehmensplanspiel kurze Zeit darauf. Es wurde im Jahre 1960 von IBM vorgestellt. ${ }^{17}$ Inzwischen finden Unternehmensplanspiele, zumeist als Planspiele in computergestützter Form, eine weite Verbreitung. ${ }^{18}$

\subsubsection{Simulationstechnik beim Unternehmensplanspiel}

\subsubsection{Das Unternehmensplanspiel: ein Simulationsmodell}

Planspiele besitzen einen breiten Geltungsbereich. Sie können neben dem wirtschaftlichen Gegenstandsbereich unterschiedlichste Realitätsbereiche abbilden. ${ }^{19}$ Demgemäß kann der Planspielbegriff als sehr allgemeingültig aufgefaßt werden. Wird ein Unternehmen oder werden Unternehmensbereiche durch ein Planspiel abgebildet, so kann der Planspielbegriff, nach Rohn, als eine Anwendung der Planspiel-Idee auf wirtschaftliche Vorgänge verstanden werden. ${ }^{20}$ Begriffe wie Management Game, Business Game, Decision Exercise, Executive Game, Mana-

Vgl. Faßbender, S., (Management 1973), S. 82; Bleicher, K., (Unternehmungsspiel 1960), S. 36 .

Vgl. Arbeitskreis Gamer,(Unternehmungsspiele 1963), S 149.

Vgl. Rohn, W.E., (Führungsentscheidungen 1964), S. 30.

Albach, H., (Unternehmensspiele 1974), S. 29.

Vgl. bspw. die Übersicht existierender Planspiele bei Rohn, W.E., (Planspiel-Übersicht 1988), S. 1 ff. Rohn unterscheidet die Planspiel-Datenbank Synopsen sowie die PlanspielDatenbank Texte, die weit über 300 Planspiele beschreiben. Vgl. zur Datenbank Synopsen ders., (Planspiel-Übersicht 1988), S. 1 ff. Zur Datenbank Texte vgl. ders., (Planspiel-Übersicht 1988), S. $168 \mathrm{ff}$. 
gement Decision Game, Simulation Exercise usw. werden in diesem Sinne oft synonym verwendet. ${ }^{21}$

Einem Planspielmodell kommt die Aufgabe zu, komplexe Systeme und komplizierte Prozesse, bei denen es auf menschliche Entscheidung und Verhaltensweisen ankommt, durchschaubar zu machen. Sie werden eingesetzt, um Entscheidungsfindung und Kommunikation im betriebswirtschaftlichen Bereich zu trainieren, sie ermöglichen es, Folgen von Entscheidungen zu prüfen, Lösungen $\mathrm{zu}$ finden und Handlungsalternativen heraus zu arbeiten. ${ }^{22}$ Dies gelingt durch Simulation, das heißt, mit Hilfe eines Modells werden Situationen geschaffen, welche die Probleme, Spannungen und Vorgänge unserer Arbeitsumgebung nachahmen. ${ }^{23}$

Ebenso wie der Planspielbegriff kann auch der Simulationsbegriff als sehr allgemeingültig aufgefaßt werden. Er findet in den unterschiedlichsten Anwendungsbereichen Verwendung, und eine Definition dazu, was unter Simulation verstanden werden soll, liegt nicht vor. ${ }^{24}$ Kern führt jedoch aus, daß ein Charakteristikum aller Simulationen das „Nachahmen“ ist. ${ }^{25}$ Mit dem Begriff des „Nachahmens“ ist sprachlich der Begriff des „Vortäuschens“ verbunden, womit das „Nachahmen“ als auch das „Vortäuschen“ im Begriff Simulation miteinander verbunden sind. ${ }^{26}$

Um etwas „nachzuahmen“, ist es zunächst erforderlich, daß ein Gegenstand existiert, der „,nachgeahmt“" werden kann. ${ }^{27}$ Ein solcher Gegenstand wird allgemein als System bezeichnet. Ein System, auf das die Methode der Simulation angewendet wird, kann als Simulationssystem bezeichnet werden. Entscheidend ist, daß die Simulation des Systems nicht direkt erfolgt, sondern über ein gedankliches Modell des Systems, welches somit auch als Simulationsmodell bezeichnet werden kann. Um ein System in einem Modell abzubilden, müssen das System sowie dessen Prozesse beschrieben werden. Dabei sollen - der zugrundeliegenden Fra-

\footnotetext{
21 Vgl. Rohn, W.E., (Führungsentscheidungen 1964), S. 11. Bleicher subsumiert die Planspielidee unter der Bezeichnung „Management Games“. Vgl. Bleicher, K., (Entscheidungsprozesse 1965), S. 11.

22 Vgl. Heidack, C., (Planspiel-Praxis 1980), S. 35

23 Vgl. zum Wesen des Planspiels Bleicher, K., (Unternehmungsspiele 1962), S. 14 ff.; Koller, H., (Planspieltechnik 1969), S. 65 ff.

24 Vgl. Zayer, H., (Prinzipien 1975), S. 18.

25 Vgl. Kern, W., (Research 1987), S. 115.

26 Vgl. Bleicher, K., (Unternehmungsspiele 1962), S. 15.

27 Vgl. Petzing, F., (Untersuchung 1993), S. 6.
} 
gestellung entsprechend - meist nur bestimmte Aspekte betrachtet werden. Wichtig ist in diesem Zusammenhang, daß bei der Abstraktion kein wesentlicher Aspekt verlorengeht. ${ }^{28}$ Systeme existieren dabei nicht von vornherein in der Umwelt, sondern stellen eine gedankliche Strukturierung der Umwelt im Sinne einer abgrenzbaren Einheit dar. ${ }^{29}$

Systeme sind Gegenstand der Systemtheorie. Seit ihrer Entstehung haben sich unterschiedliche Zweige entwickelt. Deshalb sollte die Verwendung des Begriffes Systemtheorie in unterschiedlichen Disziplinen auch nicht darüber hinwegtäuschen, daß es sich hier um eine Vielzahl unterschiedlicher Denk- und Sichtweisen handelt. ${ }^{30}$ Gemeinsam ist den Systemtheorien jedoch, daß sie versuchen, Systeme abzubilden und zu verstehen. Dabei werden die Geschehnisse in der Welt als ein Wirkungsgefüge verstanden. Das bedeutet, daß hier nicht nur einfache Ursache-Wirkungs-Ketten untersucht werden, sondern diese als systemische Gebilde höchst komplexe Netzwerke darstellen. ${ }^{31}$ Auch werden in einer ersten Annäherung an den Systembegriff stets zwei Bereiche angesprochen: Zum einen werden materielle Objekte behandelt, zum anderen Aussagenkomplexe über diese Systeme in Form von Modellen und Theorien verfaßt. ${ }^{32}$ „In der Literatur sind viele von unterschiedlichen Intentionen abhängige Systemdefinitionen $\mathrm{zu}$ finden.“"33 Systeme lassen sich zunächst jedoch sehr allgemeingültig definieren. ${ }^{34}$ So umfaßt ein System eine Gesamtheit von Elementen. Diese Elemente weisen Eigenschaften auf und sind durch Beziehungen untereinander verknüpft, wobei die Beziehungen den Systembegriff festlegen. Ohne Beziehungen handelte es sich um eine Menge. ${ }^{35}$

Vgl. Bossel, H., (Simulation 1992), S. 12 ff.

Vgl. z.B. Petzing, F., (Untersuchung 1993), S. 6 f.; Orth, C., (Unternehmensplanspiele 1999), S. 7.

Vgl. Strohner, H., (Systeme 1995), S. 27.

Vgl. Strohner, H., (Systeme 1995), S. 27.

Vgl. Fuchs, H., (Systemtheorie 1973), S. 34; Grochla, E., (Systemtheorie 1972), S. 127.

Fuchs, H., (Systemtheorie 1969), Sp. 1620.

Vgl. bspw. Fuchs, H., (Systemtheorie 1973), S. 35; Kosiol, E.; Szyperski, N.; Chmielwicz, K., (Standort 1972), S. 67.

Vgl. Flechtner, H.-J., (Grundbegriffe 1969), S. 12. Die Beziehungen zwischen den Systemelementen werden in der Literatur unterschiedlich bezeichnet. Ackoff \& Emery sprechen bspw. auch von Beziehungen. Vgl. Ackoff, R.L.; Emery, F.E., (Systeme 1975), S. 18. Luhmann spricht von Interdependenzen. Vgl. Luhmann, N., (Funktionen 1964). Aber auch von Relationen. Vgl. Luhmann, N., (Komplexität 1980). Beer spricht schließlich von der Konnektivität des Systems und meint damit ,[...] jede Ansammlung miteinander in Beziehung stehender Teile“. Beer, S., (Kybernetik 1962), S. 24. 
Aus einer solch allgemeingültigen Definition erwächst der Vorteil, daß der Systembegriff in seiner Anwendung nicht nur eingeschränkte Gültigkeit erlangt, sondern quasi auf jeden Gegenstand, logischer oder materieller Natur, angewendet werden kann. Kontextspezifisch kann der System-Begriff dann, im Hinblick auf den Anwendungsbereich, weiter spezifiziert werden. Diesem formalen Systembegriff folgt die Allgemeine Systemtheorie, deren zentrales Anliegen es ist, Struktur, Beziehungen und Verhalten von Systemen darzustellen und deren Gemeinsamkeiten $\mathrm{zu}$ erforschen. ${ }^{36}$ Der Ursprung der Allgemeinen Systemtheorie liegt, historisch gesehen, in Denkansätzen der Ganzheitslehre sowie in den organismischen Auffassungen der Biologie. ${ }^{37}$ Die Denkansätze der Ganzheitslehre sind eine Sichtweise der Wissenschaftsgeschichte. Diese und die atomistischmechanistische Sichtweise wechselten einander ab. Während die systemische Perspektive eine ganzheitliche Betrachtung verfolgt, wird durch die atomistische Perspektive der klassischen Naturwissenschaft eine elementare Betrachtung angestrebt. Diese Gegenpole drücken sich in Reduktionismus und Holismus aus. Während der Reduktionismus den Versuch unternimmt, Ganzheiten auf ihre Teile zu reduzieren, fokussiert der Holismus die Ganzheit und sieht deren Teile als sekundär an. ${ }^{38}$ Heute kann man jedoch nicht mehr von einem Wechsel dieser Perspektiven sprechen. Es vollzieht sich ein Wandel, der beide Perspektiven beinhaltet. Dies ist der Übergang zu elementaren und gleichwohl ganzheitlichen Denkweisen, wodurch Fakten und Ereignisse in den Rahmen von Ganzheiten eingeordnet werden und integrierte Gebilde mit eigenen Eigenschaften und Beziehungen entstehen. ${ }^{39}$ Dieser Sichtweise ist die Systemtheorie zuzuordnen, die ,[...] versucht, eine Mittelposition einzunehmen und das Wechselspiel zwischen Teil und Ganzem in ausgewogener Weise zu berücksichtigen“40.

$\mathrm{Zu}$ zentralem Anliegen und Zielen der Allgemeinen Systemtheorie vgl. bspw. Bertalanffy, L. v., (System 1988), S. 36 ff.; Boulding, K.E., (Systems 1956), S. 197 ff.; Fuchs, H., (Systemtheorie 1973), S. 24 ff.; Schanz, G., (Wissenschaftspraxis 1975), S. 5 ff. der Allgemeinen Systemtheorie bspw. Grochla, E., (Systemtheorie 1970), S. 4 ff.; Ulrich, H., (Unternehmung 1970), S. 102 ff. 
Begründet wurde die Allgemeine Systemtheorie durch den Biologen Bertalanffy, der die Idee zu einer Allgemeinen Systemtheorie ${ }^{41}$ das erste Mal in einem philosophischen Seminar an der Universität Chicago präsentierte und später in Zusammenarbeit mit dem Biomathematiker Rapoport und dem Ökonomen Boulding weiterentwickelte. ${ }^{42}$ Bertalanffy's Überlegungen gehen von seiner Forderung nach einer Systemauffassung des Organismus aus. Er konkretisiert und erweitert seine Ausgangsüberlegungen im Rahmen der organismischen Biologie durch die Entwicklung eines speziellen Beschreibungs- und Erklärungsmodells, das er als „Offenes System“ bezeichnet. Mit Hilfe dieses Modells können biologische Organisationsprobleme wie Wachstum, Anpassung, Regulation und Gleichgewichtsfragen dargestellt und erklärt werden. Weiterhin geht Bertalanffy von der Annahme aus, daß die Strukturen biologischer Organisationsprobleme, die durch das Modell des „Offenen Systems“ beschrieben und erklärt werden können, häufig formal isomorph zu den Problemstrukturen anderer Disziplinen sind. Daraufhin verallgemeinert und erweitert er die spezifisch biologische Systemauffassung und das „Offene-System-Modell“ zu einer „Allgemeinen Systemtheorie““43

Die Ausführungen haben gezeigt, daß der Systembegriff der Allgemeinen Systemtheorie einen breiten Geltungsbereich besitzt. So ist er auf jeden Gegenstand materieller Natur, ja selbst auf den Menschen anwendbar. Bei der Modellbildung muß ein Systemmodell kein isomorphes Abbild des Realsystems sein. Dafür sind zwei Gründe anzuführen: Zum einen kann der Umgang mit einem Modell nur dann sinnvoll sein, wenn dies eine Abstraktion des Realsystems darstellt, zum anderen kann es nur selten gelingen, Realsysteme in allen ihren Ausprägungen

41 Bertalanffy verwendet den Begriff Allgemeine Systemtheorie, aber auch die Begriffe Allgemeine Systemlehre, General System Theory und General Theory of Organization. Vgl. Bertalanffy, L. v., (Systemtheorie 1957), S. 8 ff.; Bertalanffy, L. v., (Systemlehre 1949), S. 114; Bertalanffy, L. v., (General 1962); Bertalanffy, L. v., (System 1988), S. 30 ff. Vgl. bspw. Bertalanffy, L. v., (System 1988), S. 89 f.; Kappel, R.; Schwarz, I.A., (Systemforschung 1981), S. 99. Bertalanffy war in dem Versuch, eine interdisziplinäre Systemtheorie $\mathrm{zu}$ entwickeln, nicht der erste. Bereits zu Beginn des 20. Jahrhunderts entwickelte Bodganov seine Theorie der Tektologie. Die Überlegungen Bodganov's basieren auf drei Ausprägungen von Komplexen. So ist seiner Auffassung zufolge ein organisierter Komplex derjenige, in dem das Ganze mehr ist als die Summe seiner Teile. In einem disorganisierten Komplex ist das Ganze weniger als die Summe seiner Teile. In einem neutralen Komplex ist das Ganze schließlich gleich der Summe seiner Teile. Vgl. Gorelik, G., (Bodganov's 1987), S. 159. Gorelik nimmt auch einen Vergleich mit der Allgemeinen Systemtheorie Bertalanffy's vor. Vgl. ders., (Bodganov's 1987), S. 164 ff. und die dort angegebene Literatur. 
vollständig zu erfassen, so daß Modelle notwendigerweise unvollständig sein müssen. Ausreichend ist es, Homomorphie zu fordern, womit auch der Systemansatz Modelle nicht von der ceteris-paribus-Klausel befreit. ${ }^{44}$ Es erscheint jedoch wesentlich, den geeignetsten Detaillierungsgrad sorgfältig zu bestimmen. Wird eine Detaillierung zu oberflächlich vorgenommen, so führt dies zu wenig aussagekräftigen und nicht voll verwendbaren Ergebnissen. Bei einer zu hoch gewählten Detaillierung hingegen ist ein steigender Zeitbedarf notwendig, um das Modell zu erstellen. Außerdem wächst auch der Rechenzeitaufwand für die einzelnen Experimente, wodurch weniger Szenarien betrachtet werden können. ${ }^{45}$

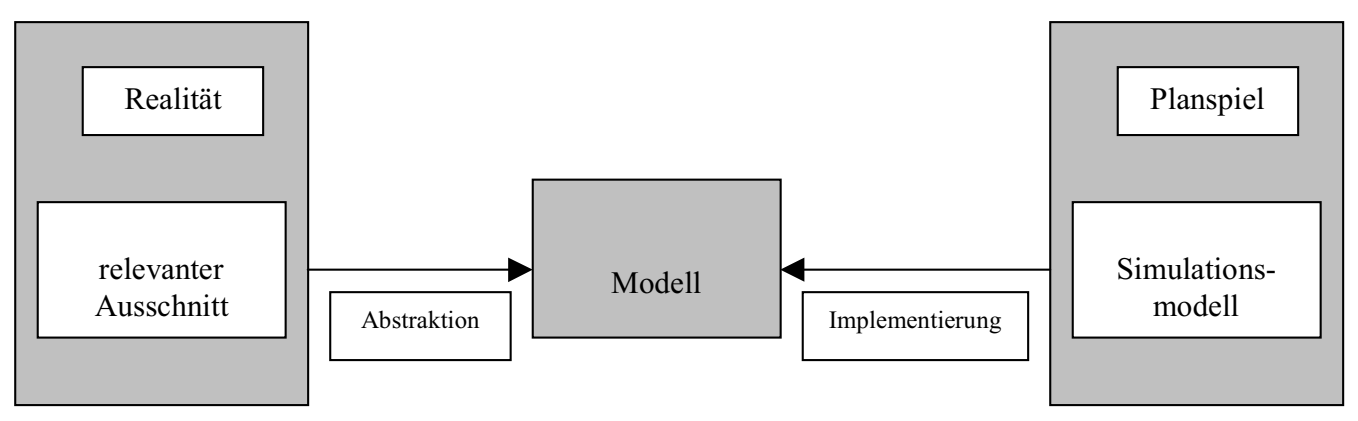

\section{Abbildung 2-1: Modellbildung bei Planspielen ${ }^{46}$}

Wird die Simulation auf das Systemmodell angewendet, so ist in diesem Sinne auch das „Nachahmen“ zu verstehen. Es entsteht dadurch ein Simulationsmodell, welches die Realität in den - im Hinblick auf die zu Grunde liegende Fragestellung - als wichtig erachteten Aspekten abbildet. Da es die Realität jedoch nur in ausgewählten Aspekten erfaßt, wird lediglich „,vorgetäuscht“, daß die Abbildung der Wirklichkeit entspricht. ${ }^{47}$ Diese Aspekte sind gleichsam Prinzip jeder Modellbildung. So kann eine allgemeine Definition bspw. wie folgt lauten: „Auf der Basis von Funktions-, Struktur- oder Verhaltensähnlichkeiten bzw. -analogien zu einem Original werden Modelle zum Zwecke speziell solcher Problemlösungen benutzt, deren Durchführung am Original nicht möglich oder zu aufwendig wäre. "48 [Vgl. Abbildung 2-1].

\footnotetext{
44 Vgl. Wolf, M., (Leistungsfähigkeit 1979), S. 25.

45 Vgl. Rabe, M., (Einführung 1998), S. 3.

46 Klotzbücher, R., (Planspielentwicklung 1996), S. 28.

47 Vgl. Bleicher, K., (Unternehmungsspiele 1962), S. 15.

48 Sellien, R., Sellien, H. (Hrsg.),(Wirtschaftslexikon 1988), Sp. 433.
} 
Dadurch wird jedoch wenig über die spezifische Simulationstechnik beim Planspiel ausgesagt. ${ }^{49}$ Daher soll im folgenden auf die zweite wesentliche Komponente des Planspiels, das Entscheidungsspiel eingegangen werden.

\subsubsection{Das Unternehmensplanspiel: ein Entscheidungsspiel}

Rohn führt aus, daß ein Planspiel immer aus zwei Teilen besteht. Das ist neben dem Modell das Spiel. Das Modell ist zunächst in gewissem Sinne eine Momentaufnahme und damit seinem Wesen nach statisch. Es ,[...] bildet das Untersuchungsobjekt als formalisiertes System ab, aus dem mit Hilfe von exakten Rechenregeln oder Algorithmen die Ergebnisse abgeleitet werden“".50

Im Spiel wird dieses Modell dann der Gegenstand der vorgesehenen Geschäftsmaßnahmen. ${ }^{51}$ So dienen Planspiele vorwiegend zur Simulation von Entscheidungen. Die meisten Modelle sind so konstruiert, daß sie eine systematische Durchführung des Entscheidungsprozesses, seiner Teile, Schritte und Phasen ermöglichen. Die Entscheidungen wirken nun ihrerseits auf das Modell, auf die in ihm gespeicherten Daten und Zusammenhänge ein und verändern das Modell. Durch die mathematische Verknüpfung mit den Modell-Daten werden die Ergebnisse der getroffenen Entscheidungen ausgelöst. ${ }^{52}$ Dabei setzt sich der Simulationsprozeß $\beta$ selbst aus mehreren aufeinander aufbauenden Entscheidungsphasen zusammen. ${ }^{53}$ [Vgl. den formalisierten Prozeß in Abbildung 2-2].

Im Gegensatz zum Simulationsmodell, stellt das Spiel demgemäß ein dynamisches Funktionsgeschehen dar. Hier steht das Verhalten, das im Rahmen und in den gegebenen Begrenzungen des ökonomischen Spielmodells abläuft, im Vordergrund. Der Simulationsprozeß erzeugt eine Wechselwirkung zwischen Modell und dem Spielverhalten. ${ }^{54}$ Das Spielverhalten wird dabei durch das Entscheidungsverhalten des Spielers hervorgerufen. Dieses setzt die dynamische Entwicklung in Gang. Die dynamische Darstellung von Entscheidungen stellt einen

Vgl. Bleicher, K., (Unternehmungsspiele 1962), S. 15.

Koller, H., (Planspieltechnik 1974), S. 6.

Vgl. Rohn, W.E., (Führungsentscheidungen 1964), S. 11.

Vgl. Rohn, W.E., (Führungsentscheidungen 1964), S. 11.

Vgl. Prehm, H.-J.; Ehlken, J.,(ISOS 1995), S. 21. 
entscheidenden Vorteil von Planspielen dar. Sie ermöglicht es, Entscheidungen mehrfach durchzuführen, unterschiedliche Alternativen in Betracht zu ziehen und die Entscheidungen im Hinblick auf ihre Güte zu kategorisieren. Wenn Fehlentscheidungen rechtzeitig erkannt wurden, können korrigierende Maßnahmen abgeleitet werden. ${ }^{55}$

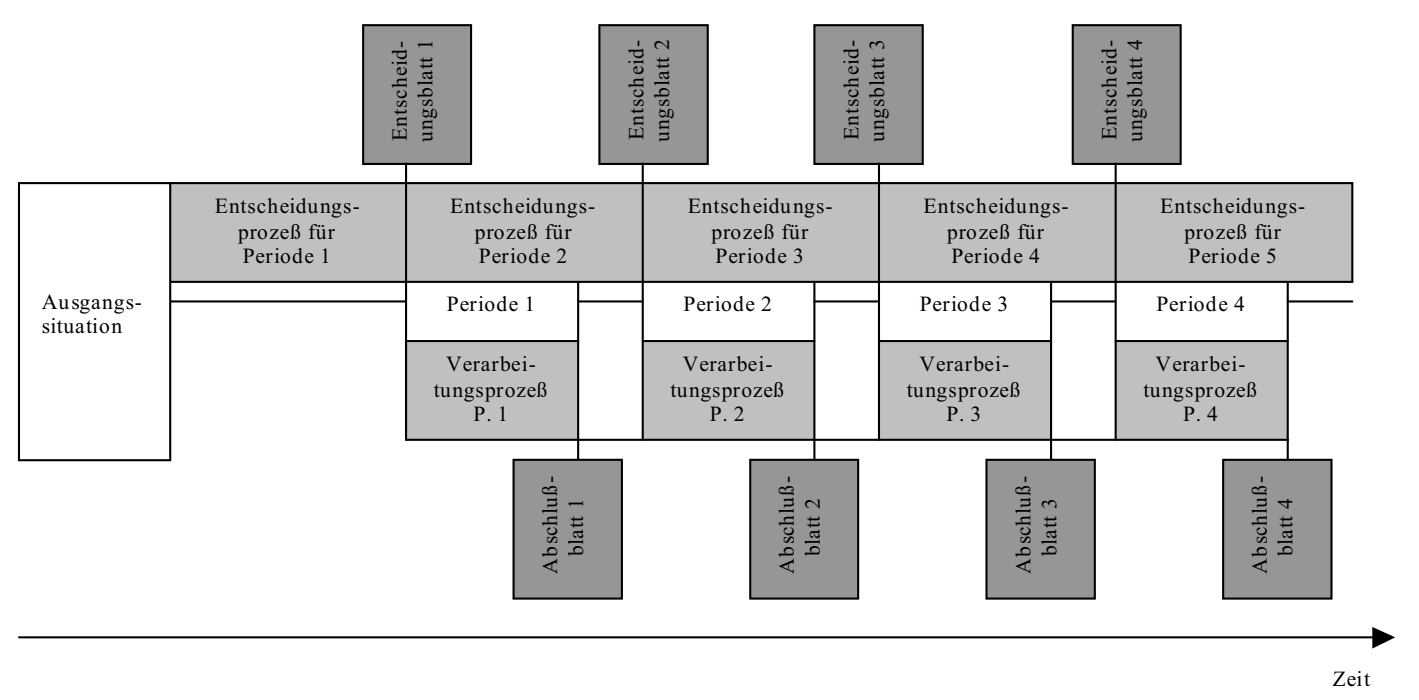

\section{Abbildung 2-2: $\quad$ Arbeitsablauf am Unternehmensplanspiel ${ }^{56}$}

Ein Phasenablauf findet sich bei Koller. Danach ist es Aufgabe eines Spielers, in einer Kette von Spielzügen die Entscheidungen im Hinblick auf eine vorgegebene, oder von ihm selbst definierte Zielgröße zu fällen. Durch die Analyse der Entscheidungssituation bildet der Spieler gewissermaßen ein internes Modell über das Spielmodell aus ${ }^{57}$ und entwickelt dabei eine Hypothese über die vermuteten Zusammenhänge im Spielmodell. [Vgl. Abbildung 2-3].

Diese bildet die Grundlage seiner Entscheidung. Nach Berechnung der Ergebnisse durch das Modell erhält der Spieler Rückmeldung über die von ihm erzielten Ergebnisse in Form von Berichten. Der Spieler kann nun den Grad der Zielerreichung überprüfen und erhält damit Informationen über die Richtigkeit der von ihm angenommenen Zusammenhänge im Modell. Wird die Hypothese bestätigt, so kann diese weiter verfeinert werden und als Grundlage weiterer Entschei-

55 Vgl. bspw. Graf, J., (Prinzip 1992), S. 17; Bleicher, K., (Entscheidungsprozesse 1965), S. 16. 
dungen dienen. Bei großen Abweichungen der Ergebnisse vom angestrebten Zielzustand können die Ursachen analysiert und die Hypothese über das Spielmodell modifiziert werden. Dies geschieht im Hinblick auf eine weitere Spielrunde, die sich daran anschließt und den Spielablauf von neuem beginnen läßt. ${ }^{58}$ Der Lernende muß im Planspiel also Lösungsansätze entwickeln, diese beurteilen und sich für eine Alternative entscheiden. Er wird gleichzeitig als Planer und Entscheider sowie als Spieler und Beobachter gefordert. ${ }^{59}$

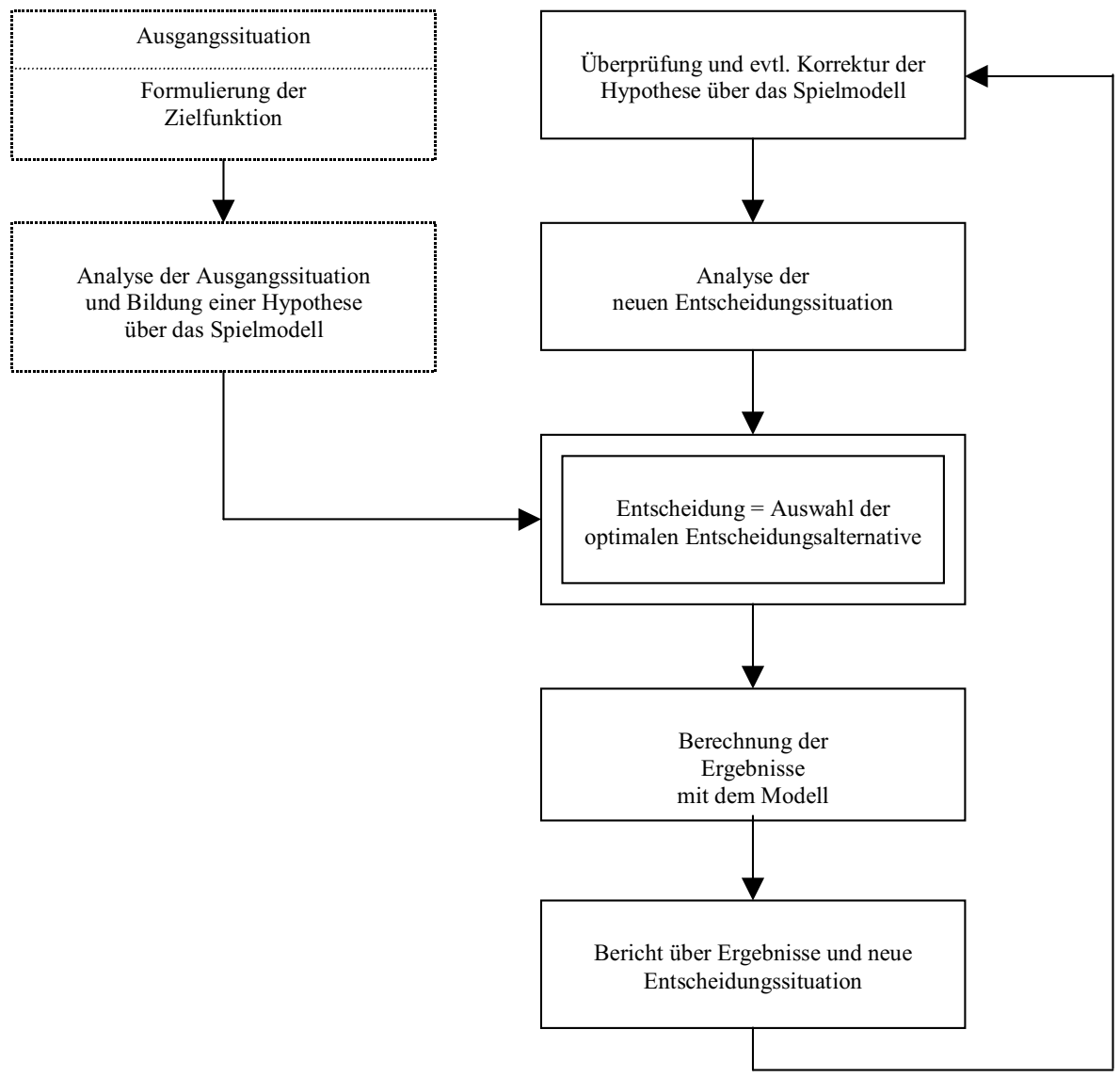

\section{Abbildung 2-3: $\quad$ Phasen einer Spielrunde im Planspiel ${ }^{60}$}

\subsubsection{Interpretation der Simulationstechnik beim Planspiel}

Es wurde gezeigt, daß sich die Simulationstechnik beim Unternehmensplanspiel aus zwei Bereichen zusammensetzt - dem Simulationsmodell und dem Entschei-

60 Koller, H., (Planspieltechnik 1974), S. 8. 
dungsspiel. ${ }^{61}$ Dabei ermöglicht das Modell die Simulation von Entscheidungsprozessen. Planspiele stellen somit Simulationsprozesse dar, wie sie im Bereich des Operations Research vielfach entwickelt wurden. ${ }^{62}$ Dieser Simulationsprozeß beim Planspiel kann unterschiedlich interpretiert werden.

Zum einen kann der Simulationsprozeß beim Planspiel als Mensch-MaschineSimulation aufgefaßt werden, wie dies die RAND CORPORATION getan hat. ${ }^{63}$ In der Mensch-Maschine-Simulation erhält der Aktionsbereich eine hohe Bedeutung. Durch die Beteiligung mehrerer Personen werden hier menschliche Verhaltensweisen im Rahmen von Handlungs- und Entscheidungsprozessen im Modell berücksichtigt. So ergibt sich ein interaktives Verhältnis zwischen Mensch und Maschine. ${ }^{64}$

Zum anderen kann das Planspiel auch kybernetisch interpretiert werden. So kann das Planspiel nach Bleicher als kybernetischer Regelkreis aufgefaßt werden. Hier stellt das Simulationsmodell die Regelstrecke dar, die von den Spielgruppen (Regler) durch die Entscheidungen (Stellgrößen) geregelt wird. ${ }^{65}$

Die Kybernetik wurde durch Wiener, etwa zur gleichen Zeit wie die Allgemeine Systemtheorie, begründet. ${ }^{66}$ Die Gedanken und spezifischen Probleme der Kybernetik gehen bereits bis ins Altertum zurück. Als ausschlaggebend für die Entstehung der Kybernetik sieht Flechtner jedoch die zur Zeit ihrer Entstehung existierende allgemeine wissenschaftliche Situation und als konkreten Anstoß den Ausbruch des zweiten Weltkrieges an. Die wissenschaftliche Situation stellte sich so dar, daß es den spezifischen Disziplinen zum einen nicht möglich war, die Wirklichkeit vollständig abzudecken, wodurch sich „weiße Gebiete“ auf der wissenschaftlichen Landkarte ergaben. Zum anderen förderten diese die Ausbildung von

61 Vgl. Abschnitt 2.1.2.1: Das Unternehmensplanspiel: Ein Simulationsmodell sowie Abschnitt 2.1.2.2: Das Unternehmensplanspiel: Ein Entscheidungsspiel. wird die Simulationstechnik beim Planspiel als Planspieltechnik bezeichnet. Vgl. bspw. Koller, H., (Planspieltechnik 1974), S. 6 f. Vgl. Geisler, M.A., (Simulation 1962), S. 242.

Vgl. Böhret, C.; Wordelmann, P.,(Planspiel 1975), S. 27 ff.

Bleicher, K., (Entscheidungsprozesse 1974), S. 14 ff.

66 Vgl. Wiener, N., (Kybernetik 1963). Nicht immer wird die Kybernetik als eine eigenständige Wissenschaftsrichtung angesehen. So subsumiert bspw. Vogt die Kybernetik als einen systemorientierten Ansatz unter den Theorien der Systemwissenschaften. Vgl. Vogt, R., (Systemwissenschaften 1983), S. 32. 
Spezialistentum. Probleme weisen jedoch zumeist einen ganzheitlichen Charakter auf. Durch die Teilung der Wissenschaft in spezifische Disziplinen sowie die Spezialisierung wird demgemäß deren Klärung erschwert. ${ }^{67}$ Der Eintritt der USamerikanischen Streitkräfte in den Krieg gab dann den konkreten Anstoß zu einer Entwicklung der Kybernetik. Es war erforderlich, eine verbesserte Flugzeugabwehr zu entwickeln. Die Schwierigkeit bestand dabei darin, feindliche Kampfflugzeuge abschießen zu können, ohne ihre genaue Flugrichtung zu kennen, da der Pilot diese willkürlich verändert, um den feindlichen Geschossen auszuweichen. Wiener und seine Mitarbeiter entdeckten in diesen Zusammenhang, daß auch das Verhalten von Menschen dem Prinzip der Rückkoppelung unterliegt und damit vorauszusehen ist. ${ }^{68}$ Die überragende Bedeutung der Kybernetik liegt demgemäß darin, vom reinen Spezialistentum hin zu ganzheitlichen Sichtweisen zu gelangen. Dazu verbindet sie vorher getrennte Wissensgebiete und erforscht diese unter Verwendung gleichartiger Denkmodelle und mit Hilfe gleichartiger Methoden. ${ }^{69}$ So spricht Wiener von „der neuen Wissenschaft der Kybernetik [...] oder der Theorie der Kommunikation und der Steuerungs- und Regelungsvorgänge bei Maschinen und lebenden Organismen“. ${ }^{70}$ Darüber hinaus bezieht sie im Zeitablauf sich veränderndes Verhalten in ihr Kalkül mit ein. Sie kann daher auch definiert werden als ,[...] die allgemeine, formale Wissenschaft von der Struktur, den Relationen und dem Verhalten dynamischer Systeme“.71 Sowie unter dem Aspekt der Zielorientierung eines dynamischen Systems ,[...] als die Wissenschaft von der Struktur, den Relationen und dem Verhalten zielorientierter, dynamischer Systeme“ .72

Der Zusammenhang von Kybernetik und Entscheidungssimulation kann demnach so aufgefaßt werden, daß die Entscheidungssimulation - wie die Kybernetik Menschen und Sachverhalte zusammenbringt. Durch das Entscheiden erlangen die ausgelösten menschlichen Reaktionen Relevanz, und das Ziel der Entscheidungssimulation ist es, bessere Lösungen der gestellten Sachaufgaben zu erlan-

67 Vgl. Wiener, N., (Kybernetik 1963), S. 26 ff.

68 Vgl. Wiener, N., (Kybernetik 1963), S. 30 f. sowie die Darstellung bei Flechtner, H.-J., (Grundbegriffe 1969), S. 3 ff. und die dort angegebene Literatur.

69 Vgl. Hartmann, R., (Betrieb 1967), S. 65.

70 Wiener, N., (Mathematik 1962), S. 233.

71 Vgl. bspw. Flechtner, H.-J., (Grundbegriffe 1969), S. 10.

72 Vogt, R., (Systemwissenschaften 1983), S. 82. 
gen, indem sie das menschliche Entscheidungsvermögen bewußt macht und systematisiert. ${ }^{73}$

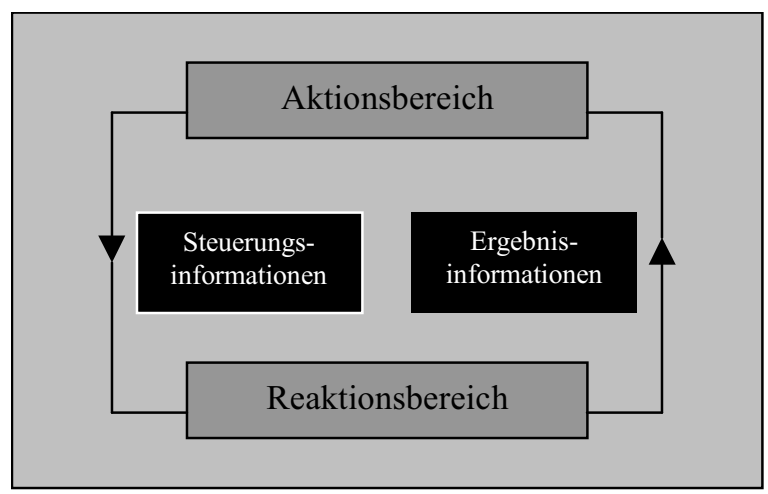

\section{Abbildung 2-4: $\quad$ Aktions- und Reaktionsbereich beim Simulationsmodell ${ }^{74}$}

Bei Bleicher findet sich weiterhin die Interpretation der Simulationstechnik beim Planspiel als Systementwurf. Dieser Systementwurf besteht dabei aus zwei Teilbereichen. So umfaßt er zum einen den Reaktionsbereich und zum anderen den Aktionsbereich. Die im Aktionsbereich gefällten menschlichen oder vom Menschen schematisierten maschinellen Entscheidungen [Aktionen] werden im Reaktionsbereich zu Ergebnissen [Reaktionen] verarbeitet, ${ }^{75}$ wobei sich durch die Annahme solch eines Regelkreises die dynamische Periodenstruktur bei Planspielen ergibt. ${ }^{76}$ [Vgl. Abbildung 2-4].

Hier wird im weiteren vom Konzept einer Mensch-Maschine-Simulation ausgegangen, die einerseits einen Reaktionsbereich zum anderen einen Aktionsbereich aufweist.

Vgl. Rohn, W.E., (Führungsentscheidungen 1964), S. 57. Rohn führt weiter aus, daß eine Zusammenarbeit mit der Kybernetik nicht in dem Sinne verstanden werden kann, daß naturwissenschaftliche Einsichten, Verfahren und Methoden auf geistig-seelische Prozesse übertragen werden, um sie zu ersetzen. Es besteht jedoch eine Wechselwirkung mit der hformationstheorie, die eine Grundlage der Kybernetik darstellt. Die methodische Durchbildung und Einbeziehung des Informationsprozesses würde eine Systematisierung des Entscheidungsprozesses vom Theoretischen her erleichtern. Vgl. ders., (Führungsentscheidungen 1964), S. 57.

Orth, C., (Unternehmensplanspiele 1999), S. 9 in Anlehnung an Bleicher, K., (Unternehmungsspiele 1962), S. 20.

75 Vgl. Bleicher, K., (Unternehmungsspiele 1962), S. 15; An anderer Stelle unterscheidet Bleicher auch nach Aktions- und Reaktionsbereich, verwendet aber auch die Begriffe Steuerungs- und Simulationsmodell. Vgl. Bleicher, K., (Unternehmungsspiele 1969), Sp. $1686 \mathrm{f}$.

76 Vgl. Karczewski, S., (Entwicklung 1991), S. 15. Karczewski unterscheidet in gleichem Sinne nach Regel- und Gestaltungssystem. Dabei gibt das Regelsystem den Rahmen eines 
Einsatz von Unternehmensplanspielen in der Betriebswirtschaftslehre

\subsection{1}

\section{Das Unternehmensplanspiel: Eine Praxissimulation}

\subsubsection{Einsatzgebiete von Unternehmensplanspielen}

Planspiele können zu unterschiedlichen Zwecken eingesetzt werden. Neben dem Einsatz in den Bereichen Forschung und Diagnostik, werden Planspiele im Bereich Ausbildung und Training eingesetzt. ${ }^{77}$

Der Einsatz von Planspielen im Bereich von Forschung und Diagnostik kann dabei in unterschiedlichen Ausprägungen erfolgen, die nicht immer unumstritten sind. Böhret \& Wordelmann unterscheiden die Simulation zur Theoriebildung, die als Labor- oder Quasi- Experiment, durchgeführt werden kann sowie die Prognosesimulation. ${ }^{78}$ Erfolgt der Einsatz einer Simulation bspw. im Laborexperiment, so wird damit das Ziel verfolgt menschliches Verhalten in simulierten Realitäten zu beobachten und zu analysieren. Der Mensch wird dabei Teil eines zumeist sehr einfachen Simulationsmodells, an dem, unter variierenden und kontrollierten Bedingungen, Aufgaben und Probleme gelöst werden müssen. Als problematisch erweist sich in diesem Zusammenhang der Rückschluß auf menschliches Verhalten in der Realität, da hier aufgrund der stark vereinfachten Bedingungen nicht immer zweifelsfrei auf eine Übereinstimmung geschlossen werden kann. In jedem Fall sind die Erkenntnisse jedoch hilfreich im Hinblick auf die Formulierung von Hypothesen. ${ }^{79}$

Im Bereich von Lehre und Fortbildung erfährt das Planspiel einen umfangreichen Einsatz; und ist hier, im Gegensatz zum Einsatz des Planspiels in Forschung und Diagnostik, auch unumstritten. Der entscheidende Vorteil des Planspiels ist hier die Vermittlung von Lernzielen in einer „Quasi-Realität“, die risikofreie Ent-

Spiels vor, während im Gestaltungssystem die Rollen der Teilnehmer definiert werden. Vgl. ders., (Entwicklung 1991), S. 14 f.

77 Vgl. Dörner, D.; Schaub, H.,(Spiel 1986), S. 64 ff.

78 Vgl. Böhret, C.; Wordelmann, P.,(Planspiel 1975), S. 32 ff.

79 Vgl. Böhret, C.; Wordelmann, P.,(Planspiel 1975), S. 34. 
scheidungen ermöglicht. ${ }^{80}$ Dabei erfolgt der Einsatz von Planspielen an Schulen, an Fachhochschulen und Berufsakademien sowie an Universitäten. ${ }^{81}$

In diesem Zusammenhang führt Högsdal weiterhin den Einsatz von Planspielen als Fernplanspiele an. Sie können dabei als offene Fernplanspiele und firmeninterne Fernplanspiele durchgeführt werden. Öffentliche Institutionen und Bildungsträger wie auch Firmen regen durch Ausschreibungen weltweit Menschen zur Teilnahme an offenen Fernplanspielen an. Unternehmen bieten Fernplanspiele als Trainingsmaßnahme, oder zur Vorbereitung auf ein Planspielseminar für ihre Mitarbeiter an. Da die Teilnehmer bei Fernplanspielen nicht an einem Ort zusammentreffen besteht auch die Möglichkeit diese in Form von Telelearning durchzuführen. Hier erfolgt die Datenverbindung über Euro-ISDN, so daß eine Kommunikation zwischen Teilnehmern an verschiedenen Orten sowie weiterhin dem Planspielleiter mit Sitz in einer Zentrale möglich wird. ${ }^{82}$

Das Planspiel kommt schließlich auch in Assessment-Centern zum Einsatz. Hier erfolgt der Einsatz zur Personalauswahl. Die Bewerber werden in Gruppen mit der Planspielaufgabe konfrontiert und in der Bewältigung der an sie gestellten Anforderung durch Psychologen beobachtet und bewertet. ${ }^{83}$

\subsubsection{Einsatz von Unternehmensplanspielen in der betriebswirt- schaftlichen Aus- und Weiterbildung}

Schon seit längerem wird in erster Linie in der universitären Lehre hervorgehoben, daß es notwendig ist, die Ausbildung an den Anforderungen der Wirtschaftspraxis $\mathrm{zu}$ orientieren. Hier bietet das Wirtschaftsleben täglich komplexe Situationen, in denen Entscheidungen getroffen werden müssen. Unternehmensplanspiele werden dabei als geeignete Methode angesehen, um Studierende auf diese Situationen vorzubereiten. ${ }^{84}$

\footnotetext{
80 Vgl. Böhret, C.; Wordelmann, P.,(Planspiel 1975), S. 42 f.

81 Vgl. Högsdal, B., (Planspiele 1996), S. 191 ff.

82 Vgl. Högsdal, B., (Planspiele 1996), S. 186 ff.

83 Vgl. Högsdal, B., (Planspiele 1996), S. 107.

84 Vgl. Friedrich, H.; Tschersig; R.,(Planspiel 1978), S. 11.
} 
Damit erfährt das Unternehmensplanspiel seinen vordergründigen Einsatz im Bereich von Ausbildung und Training, ${ }^{85}$ da unternehmerisches Handeln nicht nur durch die Praxis zu erlernen ist. So ist es einer Person nicht möglich, Entscheidungen, die auf höherer Führungsebene getroffen werden, zu fällen, so lange sie nicht die entsprechende Entscheidungsbefugnis auf einer entsprechenden Ebene erhält. Dadurch entfällt die Möglichkeit, solche Entscheidungen zu trainieren. Werden dann später solche Entscheidungen vorgenommen, können falsche Entscheidungen den Betrieb unter Umständen teuer zu stehen kommen. Daher wird es als besserer Weg angesehen, bei Führungskräften planmäßig Entscheidungssituationen zu trainieren. Dadurch wird es Personen möglich, unternehmerische Handlungsfähigkeiten - gewissermaßen im „Schonraum“ - zu entwickeln, ${ }^{86}$ welcher Umstand bereits als entscheidender Vorteil der Planspielmethode herausgestellt wurde 87 .

Dem Planspiel liegt demgemäß die Idee einer praktischen Erprobung des theoretisch erlernten Wissens zugrunde. Diese Idee fand nicht erst mit der Entwicklung von Planspielen Eingang in die Betriebswirtschaft. Schon sehr viel früher, bereits um die Wende des 19. zum 20. Jahrhunderts, war man sich der Wichtigkeit dieses Aspektes bewußt. Er wurde durch die Einrichtung von Übungs- und Musterkontoren umgesetzt. ${ }^{88} \mathrm{Im}$ weiteren Verlauf hat sich dann aus dem Musterkontor die Scheinfirma entwickelt. Dies diente der Anstrengung, die Realität in noch stärkerem Umfang als es der Musterkontor zuließ zu simulieren. ${ }^{89}$ Die Scheinfirma etablierte sich sowohl in der pädagogischen als auch in der betrieblichen Lehre. Neben Berliner Wirtschaftsoberschulen beteiligten sich auch Industrieunternehmen, Versicherungsgesellschaften, Banken und Kaufhäuser an der Methode der Scheinfirmenarbeit. ${ }^{90}$

Vgl. Bleicher, K., (Unternehmungsspiele 1962). Es findet aber auch Anwendung als Experimentiermodell für Forschungszwecke, zur Antizipation betriebswirtschaftlicher Aufgabenstellungen, wie auch zur Bewerberauswahl in Assessment-Center-Veranstaltungen. Vgl. Orth, C., (Unternehmensplanspiele 1999), S. 12 ff. und die dort angegebene Literatur. Vgl. Bleicher, K., (Entscheidungsprozesse 1965), S. 18.

89 Vgl. zur Methode der Scheinfirmenarbeit Kabuschat, H., (Scheinfirmenarbeit 1958), S. 19 ff. 
Das Planspiel ist somit eine aktivere Lehrmethode als klassische Lehrmethoden wie z.B. die Vorlesung. Im Planspielunterricht wird der Lernende stärker in das Geschehen eingebunden und kann durch Übernahme der Geschäftsführung eines Planspielunternehmens, möglicherweise erstmalig, aktiv am Entscheidungsprozeß teilhaben. ${ }^{91}$ Darüber hinaus weist die lernpsychologische Forschung Ergebnisse nach, die der Planspielmethode eine besonders hohe Bedeutung als Lehrmethode zuschreiben. ${ }^{92}$ Im Gegensatz zu anderen Lehrmethoden verdeutlicht der Umgang mit einem Spiel den Studenten stärker was sie tun, sehen und hören. Spiele geben den Studenten eine schnelle Rückmeldung über die Qualität ihrer Entscheidungen. Außerdem erhöhen Spiele das Interesse und die Motivation der Studenten. ${ }^{93}$ In diesem Sinne stellen Planspiele in Modellen betriebswirtschaftliche Wirklichkeiten dar, um die Wirkungen aufzuzeigen, die sich aus den von den Teilnehmern getroffenen unternehmerischen Entscheidungen ergeben. ${ }^{94}$ Heidack spricht in diesem Zusammenhang von Rückkoppelungsprozessen. Durch diese stellt ein Planspiel einen vielfältigen kybernetischen Lernprozeß dar, der für die Teilnehmer eine hohe Wirksamkeit besitzt, da sich durch die Rückkoppelungsprozesse die folgenden Effizienzkriterien feststellen lassen: 95

TRANSPARENZ:

VERGLEICHBARKEIT:

VERIFIZIERBARKEIT:
Im Planspiel ist es einem Teilnehmer möglich, die Wirkungen seiner Entscheidungen wahrzunehmen. Im Planspiel ist es sehr gut möglich, Entscheidungsalternativen heraus $\mathrm{zu}$ arbeiten, sie im Hinblick auf das Ziel abzuwägen sowie die erzielten Ergebnisse mit dem angestrebten Optimum zu vergleichen.

Die Ergebnisse der jeweils vorangegangenen Entscheidungen ermöglichen es, darauf neue Entscheidungen aufzubauen. Dabei kann der Erfolg der eigenen Entscheidungen aus den entstehenden Entscheidungs-Ereignis-Ketten abgeleitet werden.

Vgl. Bloech, J.; Rüscher, H., (BAP 1987), S. 6 f.

Vgl. Heidack, C., (Planspiel-Praxis 1980), S. 30.

Vgl. Robinson, J., (Research 1962) zitiert nach Dill, W.R.; Doppelt, N., (Acquisition 1963), S. 30.

Vgl. Fabel, M., (Fortentwicklungsperspektiven 1993), S. 49.

Vgl. Heidack, C., (Planspiel-Praxis 1980), S. 38 f. 
KORREKTURFÄHIGKEIT: Durch den rekursiven Prozeß erlaubt das Planspiel, anders als im Leben, eine Überprüfung der Entscheidungsergebnisse und die Durchführung eines neuen, besseren Entscheidungsversuches.

ZEITRAFFEREFFEKT:

Durch die Zeitraffung im Planspiel werden Ergebnisse schneller einsichtig als in der Realität. Hier dauert es zum Teil Wochen oder Jahre, bis die Ergebnisse vollzogener Entscheidungen ersichtlich werden. Im Planspiel können solch lange Zeiträume auf wenige Tage komprimiert werden, wodurch dem Teilnehmer die Konsequenzen seiner Entscheidungen deutlicher bewußt werden.

ÜBUNGSEFFEKT: Dadurch, daß die Entscheidungen mehrfach durchgespielt werden, ergibt sich zusätzlich zum Lerneffekt ein Übungs- und Gewöhnungseffekt.

\subsection{2}

\section{Zur Relevanz von Komplexität im Unternehmensplanspiel}

\subsubsection{Der Komplexitätsbegriff}

Oft wird die Welt mit den Begriffen Ordnung und Unordnung beschrieben. Die ledigliche Nutzung dieser, zwei gegensätzliche Pole beschreibenden Begriffe ist jedoch wenig interessant. So ist Unordnung nicht klar zu umschreiben und ist das, was sie selbst offenbart. Ordnung ist hingegen schnell zu beschreiben, wird rasch trivial und in der Folge schnell uninteressant. ${ }^{96}$ Daher kann das Folgende angenommen werden: „Es muß etwas Drittes geben, das weder totale Unordnung noch vollkommene Ordnung ist, etwas, das nicht trivial, sondern kompliziert ist, ohne ungeordnet zu sein: Komplexität.“97 Dabei meint Komplexität stets etwas Drittes, das sich zwischen zwei entgegengesetzten Polen befindet. Etwas Drittes, daß Nørretranders als den Kontinent der Komplexität bezeichnet; und worüber es keinen gesicherten Begriff gibt. ${ }^{98}$ 
Die Wurzeln des Begriffes Komplexität finden sich im Griechischen und Lateinischen. Der Griechische Begriff ,,$\lambda \varepsilon \kappa \omega \omega^{\circ}$ bedeutet „flechten, stricken“.99 Das Lateinische „,complico“ kann mit „zusammenfalten“ übersetzt werden. ${ }^{100}$ Demnach wären Objekte, die als komplex zu bezeichnen sind, in irgendeiner Weise verflochten und zusammengefaltet. Wollte man Komplexität als die Abwesenheit von Einfachheit, der gegensätzliche Begriff zu Komplexität, bezeichnen, so erforderte dies, zu bestimmen, was Einfachheit bedeutet. Das ist wiederum eine komplexe Angelegenheit. Der ehemals gebräuchliche englische Begriff „simplicity“ beschreibt den Zustand der Einfachheit und leitet sich von einem Ausdruck her, der einmal gefaltet bedeutet. Die indoeuropäischen Begriffsstämme ${ }_{2}$ plic “ $^{“}$ und „plex““ gehen wiederum auf den gleichen indoeuropäischen Begriffsstamm „plek“ zurück. ${ }^{101}$ Könnte etwas Komplexes demnach auch einfach sein, wenn es nur einmal gefaltet ist?

Es zeigt sich, daß der Begriff Komplexität in sich selbst bereits komplex gebaut ist. ${ }^{102}$ Einer allgemeinen Definition scheint er sich offensichtlich zu entziehen. Nach Gell-Mann ist ein einziger Komplexitätsbegriff möglicherweise aber auch nicht ausreichend, ,[...] um unsere intuitiven Vorstellungen von der Bedeutung des Wortes wiederzugeben. Vielleicht müssen wir mehrere verschiedene Arten von Komplexität definieren, von denen wir uns einige noch nicht einmal vorstellen können"103.

Will man verschiedene Arten von Komplexität definieren, so ist ein Aspekt, der stets auftaucht, deren Kontextabhängigkeit. ${ }^{104}$ So existieren auch in unterschiedlichen Disziplinen verschiedene spezifische Definitionen und Begriffe. ${ }^{105}$ Andere Aspekte weisen wiederum darauf hin, daß die Komplexität auch kontextunabhängige Dimensionen beinhaltet. ${ }^{106}$ Der Bezugsrahmen des Komplexitätsbegriffes ist demgemäß offensichtlich mehrdimensional;107 und es läßt sich zunächst festhal-

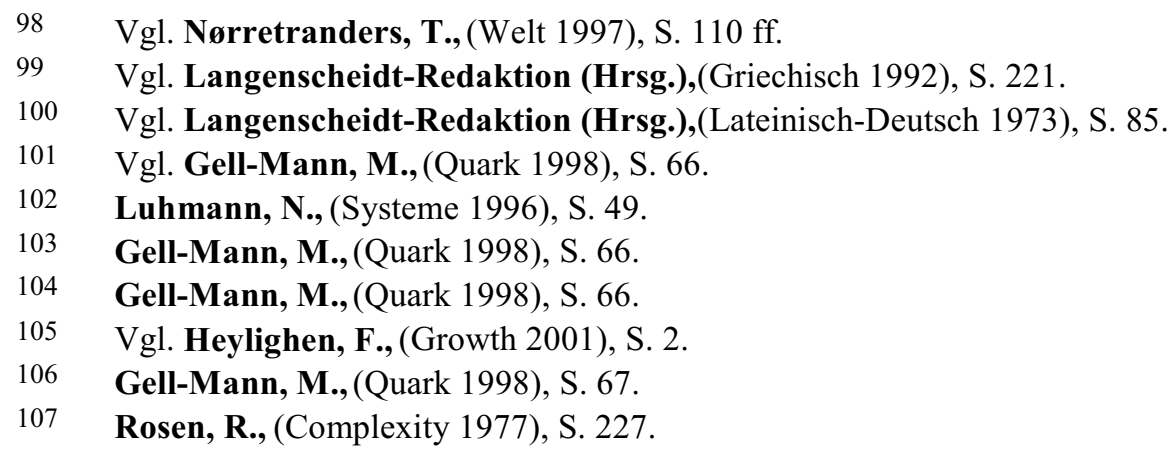


ten, daß er unter kontextabhängigen und kontextunabhängigen Gesichtspunkten zu beleuchten ist.

\subsubsection{Mehrdimensionalität von Komplexität im Unternehmensplan- spiel}

Die traditionellen Ausbildungsmethoden, wie Vorlesungen, Seminare und Übungen sind vorwiegend auf die Vermittlung von Wissen ausgerichtet. Beim Einsatz von Unternehmungsspielen stehen hingegen im allgemeinen ,[...] die Transformation von Wissen in Können und die Gewinnung persönlicher Haltung ${ }^{108}$ im Vordergrund"109. Das im Vorfeld erworbene und verarbeitete Wissen soll hier zur Anwendung gelangen. ${ }^{110}$ In diesem Zusammenhang wird bei Einsatz eines Planspiels maßgeblich das Lernziel verfolgt, komplexes Denken zu schulen. ${ }^{111}$

Die Fähigkeit zum komplexen Denken sowie dessen Training wird heute immer wichtiger, da die Welt zunehmend komplexer wahrgenommen wird. Bereits in der Vergangenheit wurde zunehmende Komplexität mit Evolution assoziiert: „At least, since the days of Darwin, the idea of evolution has been associated with the increase of complexity: if we go back in time we see originally simple systems (elementary particles, atoms, molecules, unicellular organisms) while more and more complex systems (multicellular organisms, vertebrates, mammals, human beings) appear in later stages." 112

Das führt dazu, daß Aufgaben und Probleme heutzutage immer öfter zuerst unter dem Begriff Komplexität subsumiert werden. Die in diesem Zusammenhang erwarteten Lösungsschwierigkeiten führen oft von vornherein $\mathrm{zu}$ Resignation, die

108 Unter persönlicher Haltung versteht Prehm, in Anlehnung an Blohm, die Fähigkeit, Wissen nicht nur zur Ausarbeitung praktischer Maßnahmen zu nutzen, sondern auch die Fähigkeit der Präsentation und Durchsetzung im Entscheidungsgremium. Vgl. Prehm, H.-J., (MARKUS 1995), S. 32; Blohm, H., (Planspiele 1966), S. 2155.

Prehm, H.-J., (MARKUS 1995), S. 32. Nach Ryle ist es ein Unterschied, ob eine Person um bestimmte Tatsachen weiß oder eine Tätigkeit ausführen kann. Vgl. Ryle, G., (Begriff 1969), S. 31. Ein Planspiel eignet sich jedoch prinzipiell sowohl zur Vermittlung betriebswirtschaftlichen Faktenwissens als auch zur Entwicklung des Zusammenhangswissens. Vgl. Bloech, J.; Döhne, S.R.; Hartung, S.; Orth, C.,(Einsatz 1998), S. 59.

110 Vgl. Bleicher, K., (Entscheidungsprozesse 1965), S. 17 ff.

111 Vgl. Graf, J., (Prinzip 1992), S. 12.

112 Heylighen, F., (Growth 2001), S. 1. 
unsachgemäße Vereinfachungen oder möglicherweise auch die Unterlassung von Problemlösungen zur Folge hat. Hier ist es offensichtlich ein Erfordernis unserer Zeit, Beschränkungen seitens des Individuums aufzudecken, um Komplexität in einer angemessenen Weise zu reduzieren, denn das menschliche Antizipationsvermögen stellt sich häufig als limitierender Faktor dar. ${ }^{113}$ Gelingt dies, so könnte einer zunehmenden Komplexität mit geeigneten Maßnahmen entgegengetreten werden, die eine Problemstellung handhabbar erscheinen lassen, da durch eine versäumte Anpassung von Aufgaben und Problemen an eine komplexere Wahrnehmung der Umwelt vielfache Probleme erwachsen können. ${ }^{114}$

Beispielhaft für eine solche Lösung ist das Vorgehen des Unternehmens Gore Associates. So kann ein Individuum scheinbar zu einer maximalen Zahl von 150 Individuen eine sinnvolle Beziehung unterhalten. Schon diese, auf den ersten Blick klein wirkende Zahl, erfordert die Bewältigung von 11175 Wechselbeziehungen durch ein Individuum; eine vergleichsweise beeindruckende Zahl. Das in Delaware ansässige Unternehmen Gore Associates, ein Unternehmen mit Milliarden-Umsätzen und Tausenden von Angestellten, berücksichtigt dieses Wissen in der Weise, daß keine Mitarbeitereinheit mehr als 150 Menschen umfaßt. ${ }^{115}$

Nicht immer ist jedoch das limitierte menschliche Antizipationsvermögen für unzureichende oder unterlassene Problemlösungen verantwortlich. So ist die menschliche Fähigkeit zum komplexen und ganzheitlichen Denken offensichtlich entwicklungsfähig, ${ }^{116}$ aber vielfach unzureichend ausgeprägt. ${ }^{117}$ Dies ist möglicherweise eine Ursache, die zu Ignoranz erforderlicher Problemlösungen führt. „A cynic might say that complexity is a euphemism for ignorance: what we don't understand is complex."118 Die heutige Zeit erfordert jedoch zunehmend ein Denken in komplexen Zusammenhängen, ein »Thinking in complexity«, um adäquate Problemlösungen herbeizuführen. ${ }^{119}$ Dazu ist es notwendig, daß »Prinzip der Komplexität« nachvollziehen zu können. ${ }^{120}$ „Wer [...] Prinzipien versteht, kann

\footnotetext{
113 Vgl. Grossmann, C., (Komplexitätsbewältigung 1992), S. 17.

114 Vgl. Schroder, H.M., (Bedeutsamkeit 1978), S. 35.

115 Vgl. Keese, C., (Firmen 2000), S. 30.

116 Vgl. Abschnitt 6.6.1.3: Metakognition: Die Steuerung des eigenen Denkens.

117 Vgl. Graf, J., (Prinzip 1992), S. 12.

118 Yates, F.E., (Complexity 1978), S. R 201.

119 Vgl. Bliss, C., (Management 2000), S. 259 f.

120 Vgl. Graf, J., (Prinzip 1992), S. 18.
} 
abstrahieren und denkt komplex. Wer wiederum prinzipiell nachvollziehen kann, wie komplexe Prozesse ablaufen und in welchen Strukturen dies geschieht, gewinnt Mut zum Handeln. Und darauf kommt es schließlich bei den Herausforderungen der Zukunft entscheidend an." ${ }^{\text {"121 }}$

\subsubsection{Zum Zusammenhang von Komplexität, Lernzielen und Ler- nerfolg im Unternehmensplanspiel}

Im Hinblick auf den so schwer zu fassenden Begriff der Komplexität ${ }^{122}$ sowie dessen Mehrdimensionalität im Unternehmensplanspiel ${ }^{123}$ ergeben sich nun einige Fragestellungen.

Zunächst existieren in der Literatur verschiedene Meinungen dazu, wodurch die Komplexität eines Unternehmensplanspielmodells determiniert wird. ${ }^{124}$ Ein Index, der allgemeingültige Aussagen darüber zuläßt, wie komplex ein Planspiel ist, existiert bisher nicht. „No generally accepted index of game complexity exists. Still, the number of game characteristics of a simulation reflects the complexity level for a particular game." 125 In diesem Zusammenhang finden sich ungenaue Klassifikationen wie einfache und komplexe Planspiele, ${ }^{126}$ Planspiele unterschiedlicher Komplexität, wobei dabei auch auf deren unterschiedliche Schwierigkeit geschlossen wird, ${ }^{127}$ oder im anglo-amerikanischen auch simple and complex ga$\operatorname{mes}^{128}$.

Um in diesem Zusammenhang zu einer Annäherung zu gelangen, wird in dieser Arbeit die Frage aufgeworfen worin das Prinzip der Komplexität, in Zusammenhang mit Unternehmensplanspielen, überhaupt besteht. Dabei ist auf die spezifische Simulationstechnik beim Planspiel bezug zu nehmen. Sie konnte nach Simulationsmodell ${ }^{129}$ und Entscheidungsspiel ${ }^{130}$ unterschieden sowie weiterhin als

\footnotetext{
121 Vgl. Graf, J., (Prinzip 1992), S. 18.

122 Vgl. Abschnitt 2.2.2.1: Der Komplexitätsbegriff.

123 Vgl. Abschnitt 2.2.2.2: Mehrdimensionalität von Komplexität im Unternehmensplanspiel.

124 Vgl. Orth, C., (Unternehmensplanspiele 1999), S. 70.

125 Golas, B., (Complexity 1981), S. 296.

126 Vgl. Prehm, H.-J., (MARKUS 1995), S. 16 f.

127 Vgl. Sonderegger, W., (Entwurf 1982), S. 37.

128 Vgl. Dill, W.R.; Doppelt, N., (Acquisition 1963), S. 32.

129 Vgl. Abschnitt 2.1.2.1: Das Unternehmensplanspiel: Ein Simulationsmodell.
} 
eine Mensch-Maschine-Simulation ${ }^{131}$ interpretiert werden. Daraus resultieren zwei Funktionen des Unternehmensplanspiels: Zum einen die Funktion des Unternehmensplanspiels als Modell über einen Gegenstandsbereich sowie zum anderen die Funktion als Lehr-Lern-Instrument.

Durch diese Unterscheidung gelingt es, den Komplexitätsbegriff im Unternehmensplanspiel zunächst in zweifacher Weise zu verankern: So ist Komplexität in Zusammenhang mit der Modellbildung als attributive Eigenschaft von Systemen ${ }^{132} \mathrm{zu}$ interpretieren. In Zusammenhang mit der Funktion als Lehr-LernInstrument ist Komplexität als attributive Eigenschaft von Entscheidungen ${ }^{133}$ anzusehen. Eine solche Sichtweise ermöglicht wiederum die Betrachtung weiterer Dimensionen von Komplexität im Unternehmensplanspiel in zwei Untersuchungsbereichen: Im Bereich der Modellbildung wird die Untersuchung objektiver Eigenschaften von Komplexität, unter Berücksichtigung der Systemtheorie, möglich; im Bereich der Lehr-Lern-Forschung wird, unter Berücksichtigung kognitionspsychologischer und entscheidungstheoretischer Forschung, die Untersuchung subjektiver Wahrnehmungen von Komplexität und des daraus resultierenden Umgangs von Personen mit Unternehmensplanspielen möglich.

Für den weiteren Fortgang der Arbeit ist zunächst der Zusammenhang von Komplexität mit den im Unternehmensplanspiel verfolgten Zielen in der Lehr-LernForschung wichtig. So implizieren offensichtlich die mit dem Einsatz eines Unternehmensplanspiels verfolgten Lernziele den Grad an Komplexität des Unternehmensplanspiels; zum anderen nimmt die Komplexität eines Unternehmensplanspiels Einfluß auf den Lernerfolg der Teilnehmer. Daher soll, in Ergänzung der theoretischen Grundlagen, in den nächsten beiden Gliederungspunkten bezug auf den Zusammenhang von Komplexität und Lernzielen sowie weiterhin den Zusammenhang von Komplexität und Lernerfolgen eingegangen werden. 


\subsection{Zum Zusammenhang von Komplexität und Lernzielen}

Die Entwicklung eines Unternehmensplanspiels, das in der Lehre eingesetzt werden soll, setzt zunächst die Formulierung von Lernzielen voraus. ${ }^{134}$ Als Lernziele werden dabei Zielvorstellungen bezüglich der Fähigkeiten bezeichnet, die von Lernenden in Zusammenhang mit einer Unterrichtsmethode erworben werden können. ${ }^{135}$ Aufgrund der komplexen Anforderungssituation im Planspiel können dabei alle Lernzielebenen erreicht werden. So schaffen Planspiele aufgrund ihrer komplexen und weiterhin interdependenten Problemstellungen die Basis für die Lernebenen der »Analyse «, der »Synthese« als auch des »Bewertens « ${ }^{136}$ Offensichtlich besteht daher der folgende Zusammenhang: Je komplexer das Planspiel um so mehr Lernziele können mit einem Planspiel verfolgt werden.

Mit einfachen ${ }^{137}$ Spielen wird nun bspw. das Ziel verfolgt, den Spielern aufzuzeigen, daß Entscheidungen in unterschiedlichen Bereichen und zu unterschiedlichen Zeitpunkten Verknüpfungen aufweisen. Außerdem, daß Entscheidungsorganisation und Entscheidungsprozeduren Konsequenzen bezüglich der Lösungsqualität haben. ${ }^{138}$

Bei Einsatz eines Planspiels mit einem großen Ausmaß an Daten wird das Individuum zusätzlich der Notwendigkeit der Informationsbewältigung ausgesetzt, wodurch die Fähigkeit zur persönlichen Informationsverarbeitung trainiert wird. ${ }^{139}$ Diesem Zweck diente bspw. die Entwicklung des „Carnegie Tech Management Game“, das bewußt komplexer gestaltet wurde als andere, bis zu diesem Zeit-

133 Vgl. Abschnitt 6.4: Komplexität als attributive Eigenschaft von Entscheidungen.

134 Vgl. Achtenhagen, F.; Meyer, H.L.,(Curriculumrevision 1971), S. 159 ff.

135 Vgl. Posch, P.; Schneider, W.; Mann, W.E.,(Unterrichtsplanung 1977), S. 5 f.

136 Vgl. Böhret, C.; Wordelmann, P.,(Planspiel 1975), S. 151.

137 Wie die Ausführungen zum Komplexitätsbegriff zeigen konnten, impliziert der Terminus einfach nicht zwangsläufig auch wenig komplex. Vgl. Abschnitt 2.2.2.1: Der Komplexitätsbegriff. Da jedoch zu diesem Zeitpunkt nicht mehr über den Zusammenhang ausgesagt werden kann, werden die Termini so gebraucht, wie sie in der vorliegenden Literatur vorzufinden sind.

138 Vgl. Dill, W.R.; Doppelt, N., (Acquisition 1963), S. 32.

139 Vgl. Bloech, J., (Modellierung 1989), S. 107. 
punkt vorliegende, Simulationen. Darüber hinaus diente es auch der Entwicklung der folgenden Fähigkeiten: ${ }^{140}$

Die Fähigkeit antizipieren, planen und handeln zu können, um geplante Ergebnisse in einer komplexen Situation zu erzielen.

$>$ Die Rolle des Generalisten sowie des Spezialisten kombinieren zu können.

Mit anderen Menschen effektiv zusammenarbeiten zu können.

Daneben werden unter anderem die folgenden Lernziele in Zusammenhang mit der Planspielmethode in der Literatur genannt: ${ }^{141}$

- $\quad$ Die Förderung von ganzheitlichem und handlungsorientiertem Denken.

- Die Erhöhung der Fähigkeit zum Problemlösen.

- $\quad$ Die Identifikation und Formulierung von Zielen.

- $\quad$ Die Entwicklung und Durchsetzung von Strategien zur Zielerreichung.

- $\quad$ Gegebene, zumeist umfangreiche, Informationen analysieren können.

- Die entscheidungsorientierte Aufbereitung der Informationen.

- Die geeignete Auswahl der zur Verfügung stehenden Instrumente und deren entscheidungsorientierte Anwendung auf das Informationsmaterial.

- $\quad$ Entscheidungsbereiche in Abhängigkeit vom Unternehmensziel lokalisieren.

- Die in den Entscheidungsbereichen enthaltenen Planungsprobleme erkennen und Teilziele formulieren.

- $\quad$ Erkennen, daß Einzelentscheidungen auf Teilbereiche des Unternehmens wirken und interdependent zu anderen Unternehmensbereichen sind.

- Durch Berücksichtigung des Zeitfaktors die dynamische Entwicklung von Systemen aufzeigen.

- Durch häufiges Treffen von Entscheidungen die Entscheidungsfreudigkeit steigern.

- $\quad$ Darstellung der Dynamik von Entscheidungsprozessen.

140 Vgl. Dill, W.R.; Doppelt, N., (Acquisition 1963), S. 32. Auch Cohen, K.J.; u.a., (Management Game 1960).

$141 \mathrm{Zu}$ diesen und weiteren Lernzielen, die durch den Einsatz von Unternehmensplanspielen angestrebt werden vgl. bspw. Bloech, J., (Modellierung 1989), S. 106 f.; Goertzen, H., (Simultanplanung 1990), S. 98 ff.; Prehm, H.-J., (MARKUS 1995), S. 32 ff.; Rohn, W.E., (Methodik 1980), S. 20; Taddicken, U., (Simulationsspiele 1977), S. 13 ff.; Baetge, J.; Biethahn, J.; Bokranz, R., (Information 1997), S. 3; Högsdal, B., (Planspiele 1996), S. 14. 
- Verbesserung der Kenntnisse zum Umgang mit Instrumenten und deren Grenzen erkennen können.

- $\quad$ In der Lage sein, Entscheidungen unter Unsicherheit vorbereiten zu können.

- $\quad$ Durch das Spiel wird intendiert, die Lernmotivation zu steigern.

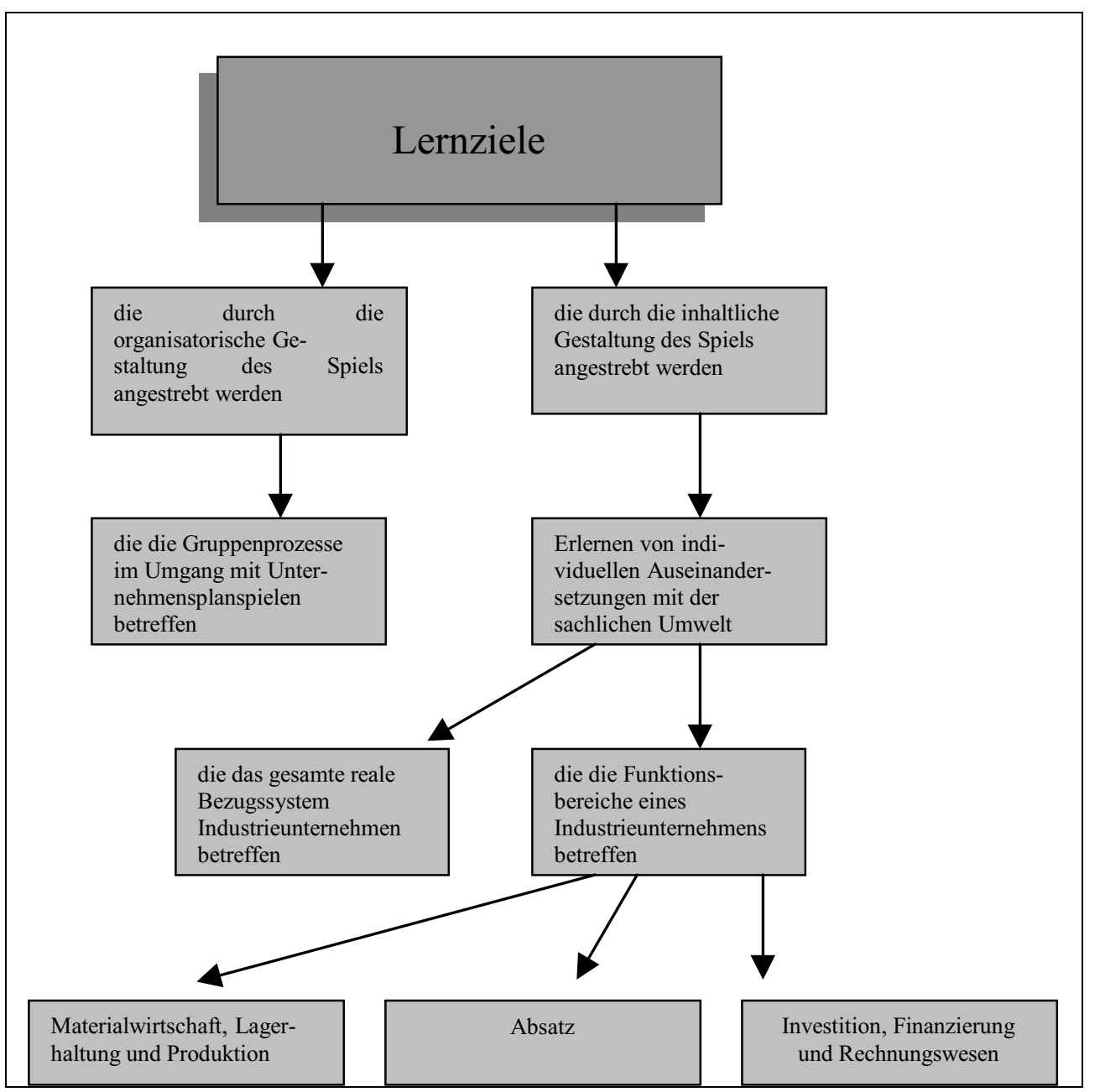

\section{Abbildung 2-5: Systematisierung von Lernzielen im Hinblick auf die Ent- wicklung eines Unternehmensplanspiels ${ }^{142}$}

Aufgrund der Vielzahl von Lernzielen, die mit einem Planspiel verfolgt werden können, ist es sinnvoll die Lernziele zu systematisieren. Orth geht in diesem $\mathrm{Zu}$ sammenhang bspw. von allgemeinen Lernzieltaxonomien aus; und entwickelt eine Taxonomie kognitiver und affektiver Lernziele. ${ }^{143}$ Weiterhin stehen die Lernziele

142 Goertzen, H., (Simultanplanung 1990), S. 99.

143 Vgl. Orth, C., (Unternehmensplanspiele 1999), S. 54 ff. Auch in der Arbeit von Orth liefert das Konzept der Schlüsselqualifikationen, neben den kognitiven und affektiven Lernzielen, einen Ansatz zur Beschreibung von Lernzielen im Unternehmensplanspiel. Vgl. ders., (Unternehmensplanspiele 1999), S. 54 ff. 
im Unternehmensplanspiel auch in Zusammenhang mit dem Konzept der Schlüsselqualifikationen, auf deren Vermittlung abgezielt wird; und die damit einen Rahmen zur Systematisierung bieten. ${ }^{144}$

Am konkreten Beispiel kann eine Systematisierung der Lernziele im Hinblick auf die Entwicklung eines Unternehmensplanspiels bspw. in der von Goertzen vorgenommenen Weise erfolgen. Sie hat eine Gruppierung von Lernzielen vorgenommen, die unter dem Gesichtspunkt der Entwicklung eines Unternehmensplanspiels erfolgte. ${ }^{145}$ [Vgl. Abbildung 2-5].

\subsection{Zum Zusammenhang von Komplexität und Lernerfolg}

Unternehmenspolitische Entscheidungsprobleme werden zum einen von den relevanten ökonomischen Variablen sowie zum anderen vom Verhalten der problemlösenden Menschen dominant beeinflußt. ${ }^{146}$ Dabei hängt der erfolglose versus erfolgreiche Umgang von Individuen mit einem Planspiel oftmals von der Komplexität des Planspiels ab. ${ }^{147}$ Je nach Kenntnis, die ein Betrachter eines Systems über die Menge, die Qualität sowie die Variabilität von Elementen und Relationen besitzt, stellt sich ihm ein System in unterschiedlicher Transparenz dar. Komplexität, als attributive Eigenschaft von Systemen, ${ }^{148}$ ergibt sich demgemäß nicht nur aus objektiven Eigenschaften, sondern auch aus subjektiven Wahrnehmungen, ${ }^{149}$ was dazu führt, daß Menschen unterschiedlicher Qualifikation zu unterschiedlichen Ergebnissen gelangen. ${ }^{150}$

In der Vergangenheit wurden zahlreiche Untersuchungen zum Zusammenhang des Komplexitätsgrades eines Unternehmensplanspieles mit dem Lernerfolg eines Teilnehmers durchgeführt. Obgleich diese Untersuchungen zu unterschiedlichen

144 Vgl. Hartung, S., (Förderung 2000), S. 29 ff. Vgl. auch Bloech, J.; Hartung, S.; Kauer, G.; Orth, C., (Einsatz 1996), S. 16 ff. Hartung führt weiterhin auch Konzepte zur Planung von Lernzielen in der beruflichen Bildung aus. Vgl. Hartung, S., (Förderung 2000), S. 35 ff.

Vgl. Goertzen, H., (Simultanplanung 1990), S. 98 ff. Auf diesem Lernzielkatalog basiert das von ihr entwickelte Simulationsmodell SUBPRO. Vgl. dies., (Simultanplanung 1990), S. 98 u. S. $105 \mathrm{ff}$.

Witte, E., (Unternehmungsspiel 1974), S. 16.

Vgl. Orth, C., (Unternehmensplanspiele 1999), S. 63 ff.

Vgl. Abschnitt 4.2.2: Komplexität als attributive Eigenschaft von Systemen.

Vgl. Bronner, R., (Komplexität 1992), Sp. 1122.

Vgl. Abschnitt 3.3: Umgang von Personen mit komplexen Systemen. 
Ergebnissen gelangten, ${ }^{151}$ läßt sich doch zusammenfassend festhalten, daß der Komplexitätsgrad bei Einsatz eines Planspiels in der Ausbildung dem Vorwissen der Teilnehmer angepaßt werden sollte, um die Grundlage für einen effizienten Lernerfolg $\mathrm{zu}$ schaffen. ${ }^{152}$ Davon geht auch Hauschildt aus; und bezeichnet die Komplexität, die den individuellen Lernerfolg im Planspiel maximiert, als Konfrontation eines Teilnehmers mit der für ihn »optimalen Komplexität«, ${ }^{153}$ was beispielhaft in der von Achtenhagen, Tramm, Preiss, Seemann-Weimar \& Schunck beschriebenen Weise erfolgen kann: Sollen komplexe Systeme lernend erschlossen werden, so ist es zunächst sinnvoll die Funktion und die in der Struktur des Systems angelegte spezifische Problemlösung unter Heranziehung vereinfachter Modelle zu verdeutlichen. Eine solche Vorgehensweise ermöglicht es, frühzeitig die konstitutiven Merkmale des Systems, ganzheitlich-analytisch sowie gleichsam induktiv, am konkreten Beispiel zu erarbeiten. Weiterhin kann ein solches Grundmodell dann in zunehmendem Maße ausdifferenziert und verdichtet werden, wodurch dann auch ein elementenhaft-synthetisches Vorgehen möglich wird. Hier ist es dem Lerner möglich sich auf einzelne Bausteine zu konzentrieren, ohne den Gesamtzusammenhang aus den Augen zu verlieren. ${ }^{154}$

Aufgrund aktueller Zusammenfassungen existierender Untersuchungen, die, wie ausgeführt, im Hinblick auf die Notwendigkeit einer Anpassung der Komplexität an das Vorwissen der Teilnehmer auf ein einheitliches Ergebnis zurückgeführt werden können, ${ }^{155}$ wird an dieser Stelle auf eine erneute Zusammenfassung verzichtet; und beispielhaft eine Untersuchung von Hartung dargestellt. Diese Untersuchung ,[...] entstand aus dem von der Deutschen Forschungsgemeinschaft 1994-1997 geförderten Forschungsprojekt 'Lehren und Lernen mit Hilfe computergestützter Simulationen zur Unternehmensführung, insbesondere zur Investiti-

151 Vgl. Orth, C., (Unternehmensplanspiele 1999), S. 63 ff.; Bloech, J.; Kauer, G.; Orth, C., (Unternehmensplanspiele 1996), S. 39.

152 Vgl. Orth, C., (Unternehmensplanspiele 1999), S. 63 ff. sowie S. 75 und die dort angegebene Literatur. Zum menschlichen Vorwissen vgl. Abschnitt 6.2.2: Vorwissen: Spiegel des Erfahrungshintergrundes eines Individuums.

153 Vgl. Hauschildt, J., (Komplexität 1990), S. 135 f. Dies ist zum Beispiel deshalb sinnvoll, da es beim Versagen im Umgang mit Komplexität bspw. zum Abbau intrinsischer Motivation und zum Rückgang der Kohäsion zwischen gemeinsam arbeitenden Gruppen oder Teams kommen kann. Vgl. Schroder, H.M.,(Bedeutsamkeit 1978), S. 35.

154 Vgl. Achtenhagen, F.; Tramm, T.; Preiss, P.; Seemann-Weimar, H.; John, E.G.; Schunck, A., (Lernhandeln 1992), S. 206.

155 Vgl. Orth, C., (Unternehmensplanspiele 1999), S. 63 ff.; Hartung, S., (Förderung 2000), S. 89 ff. 
ons- und Produktionsplanung ${ }^{` 156}$ heraus“. ${ }^{157}$ Unter Heranziehung der in diesem Forschungsprojekt bereits eingesetzten und weiterentwickelten Instrumente war das übergeordnete Ziel dieser Arbeit die lerneffiziente Gestaltung des Einsatzes eines Unternehmensplanspiels in der beruflichen Fortbildung zu untersuchen. Dazu wurde zum einen durch Anpassungen der Komplexität des Planspieles an das Vorwissen sowie den jeweiligen Lernfortschritt der Teilnehmer der Einfluß der Entscheidungskomplexität auf den Lernerfolg untersucht; zum anderen wurden Maßnahmen entwickelt, welche die Reflexivität der Teilnehmenden förderte, ${ }^{158}$ wodurch ein positiver Effekt auf den Lernerfolg angenommen wurde. ${ }^{159}$ Die Untersuchungen wurden dabei auf Basis des Unternehmensplanspiels EpUS, ${ }^{160}$ das sowohl zu Lern- als auch zu Experimentierzwecken eingesetzt werden kann, ${ }^{161}$ durchgeführt. Der Versuchsaufbau gestaltete sich dabei wie folgt:162 [Vgl. Abbildung 2-6].

\begin{tabular}{|c|c|c|c|c|}
\hline Prätest & \multicolumn{2}{|c|}{ Experimentelle Phase } & Testphase & Posttest \\
\hline \multirow{3}{*}{$\begin{array}{ll}\text { - } & \text { Multiple } \\
& \text { Choice- } \\
& \text { Fragebogen } \\
\text { - } & \text { Netzwerk } \\
\text { - } & \text { Fallstudie }\end{array}$} & modular & herkömmlich & neue Situation & \multirow{3}{*}{$\begin{array}{ll}\text { - } & \text { Multiple } \\
\text { Choice- } \\
\text { Fragebogen } \\
\text { - } & \text { Netzwerk } \\
\text { - } & \text { Fallstudie }\end{array}$} \\
\hline & $\begin{array}{l}\text { sukzessive } \\
\text { Steigerung }\end{array}$ & $\begin{array}{c}\text { volle } \\
\text { Komplexität }\end{array}$ & $\begin{array}{c}\text { volle } \\
\text { Komplexität }\end{array}$ & \\
\hline & \multicolumn{3}{|c|}{ gekreuzter Einsatz der zusätzlichen Fragebögen } & \\
\hline Individuell & \multicolumn{3}{|c|}{ Gruppenarbeit } & Individuell \\
\hline \multicolumn{5}{|c|}{ begleitende Lehre (Vorlesung) } \\
\hline
\end{tabular}

Abbildung 2-6: Ablauf der Untersuchung163

156 Das Projekt wurde im Rahmen des Schwerpunktprogramms 'Lehr-Lern-Prozesse in der kaufmännischen Erstausbildung `(Az. Bl 133/4) gefördert.

157 Hartung, S., (Förderung 2000), S. 5. Zu den in diesem Zusammenhang entstandenen Arbeiten vgl. auch Bloech, J.; Hartung, S.; Kauer, G.; Orth, C.,(Einsatz 1996); Bloech, J.; Kauer, G.; Orth, C., (Unternehmensplanspiele 1996); Bloech, J.; Döhne, S.R.; Hartung, S.; Orth, C., (Einsatz 1998); Bloech, J.; Hartung, S.; Orth, C., (Unternehmensplanspiele 1998); Bloech, J.; Hartung, S.; Orth, C.,(Einsatz 1999); Bloech, J.; Hartung, S.; Orth, C., (Förderung 1999).

158 Vgl. zu diesem Aspekt Abschnitt 6.6.1.3: Metakognition: Die Steuerung des eigenen Denkens.

159 Vgl. Hartung, S., (Förderung 2000), S. 5 ff.

160 Vgl. zum Unternehmensplanspiel EpUS Abschnitt 3.4.5.1: Darstellung des Unternehmensplanspiels EpUS.

161 Vgl. Bloech, J.; Rüscher, H.,(EPUS 1992), S. 268.

162 Vgl. Hartung, S., (Förderung 2000), S.

163 Hartung, S., (Förderung 2000), S. 141. 
Die Untersuchung beinhaltete einen Prätest, eine experimentelle Phase und eine Testphase sowie schließlich einen Posttest. Im Prätest wurde zunächst das Vorwissen der Teilnehmer jeweils individuell erhoben. So erfolgte mittels eines Multiple-Choice-Fragebogens die Erhebung des Faktenwissens, mittels einer Netzwerk-Erhebung die des Zusammenhangswissen. Weiterhin wurde die Transferleistung mittels einer Fallstudie erhoben.

Zur Durchführung der experimentellen Phase wurde das Planspiel in zwei unterschiedlichen Versuchsanordnungen dargeboten. Bei der ersten Versuchsanordnung, die als herkömmliche Durchführung bezeichnet wurde, blieb die Komplexität über den gesamten Versuchsaufbau hinweg konstant und die Teilnehmer entschieden über den gesamten Spielzeitraum von fünf Perioden über alle Variablen. In der zweiten Versuchsanordnung, als modulare Durchführung bezeichnet, wurde die Komplexität hingegen in der folgenden Weise sukzessive gesteigert: ${ }^{164}$

$>$ 1. STUFE: In der ersten Stufe wurden den Teilnehmern die Produktionsmengen vorgegeben. Die Teilnehmer waren durch den Kauf oder den Verkauf von Maschinen sowie der Disposition des Personals aufgefordert eine ausreichende Produktionskapazität bereitzustellen, sowie außerdem die notwendigen Rohstoffmengen bereitzustellen.

2. STUFE: Zu den Entscheidungen der ersten Stufe traten zusätzlich die Produktionsmengen.

> 3. STUFE: Zum Absatz der geplanten Produktionsmengen waren die Marketing-Instrumente durch die Teilnehmer zu gestalten. So mußten auf dieser Stufe die Verkaufspreise der Produkte festgelegt werden.

$>$ 4. STUFE: Hier wurde es erforderlich, daß die Teilnehmer zusätzlich die Entscheidungen bezüglich der Aufwendungen für Werbung und Kundendienst berücksichtigten.

$>$ 5. STUFE: Im Finanzierungs-Bereich war schließlich über die Aufnahme und Tilgung von Krediten sowie über eine kurzfristige Terminanlage für Einzahlungs-Überschüsse zu entscheiden. 
In der Testphase kam das Planspiel lediglich in der herkömmlichen Durchführung zur Anwendung. Dabei wurden in beiden Phasen unterschiedliche Ausgangssituationen ${ }^{165}$ konzipiert. In einem Posttest wurden mit den gleichen Erhebungsinstrumenten wie im Prätest, wiederum individuell, die angeführten Wissensarten erhoben, wobei es diesmal darum ging den Wissenserwerb auf seiten der Teilnehmer festzustellen.

Die Untersuchung der Lernerfolge erfolgte dann in Abhängigkeit von Lernzielen, die in Zusammenhang mit jeder der fünf Stufen formuliert wurden. ${ }^{166}$ Hier konnte insgesamt festgestellt werden, daß die Gruppen, die in der modularen Durchführung spielten, zu besseren Ergebnissen gelangten als diejenigen, die in der herkömmlichen Durchführung spielten. Es konnte daher gefolgert werden, daß die modulare Durchführung zu besseren Ergebnissen bei den Teilnehmern führt.

Weiterhin konnte jedoch auch festgestellt werden, daß die Teilnehmer der modularen Bedingung sich weniger risikofreudig zeigten und in den neuen Bedingungen der Testphase zu eher vorsichtigeren Entscheidungen neigten. Darüber hinaus schien die modulare Bedingung zu einem kongruenteren Entscheidungsverhalten zu führen. ${ }^{167}$

Im gleichen Zusammenhang wird nun bezug auf die Problemlöseforschung, einem Teilgebiet der Denkpsychologie genommen, die den Umgang von Versuchspersonen mit Komplexen Systemen untersucht.

In der experimentellen Phase wurden die ersten drei Perioden vorgespielt. Es standen den Teilnehmern daher bereits Rohstoffe und Maschinen sowie weiterhin ein kleiner Vorrat an Produkten zur Verfügung. Über die Unternehmensergebnisse zum Ende der dritten Periode wurden die Teilnehmer entsprechend informiert und trafen ihre Entscheidungen für die vierte Periode. Vgl. Hartung, S., (Förderung 2000), S. 172 f. In der Testphase haben die Teilnehmer ein Modellunternehmen U1 zu führen. Es wurde die achte Periode vorgespielt; und die Teilnehmer befinden sich bezüglich des einen Produktes in der Marktsättigung, bezüglich des anderen in der Degenerationsphase. In der neunten Periode greift eine Rationalisierungsmaßnahme im Produktionsbereich. Weiterhin sind verschiedene Strategien der Konkurrenzunternehmen deutlich erkennen und gegeneinander abgrenzen. Vgl. dies., (Förderung 2000), S. $181 \mathrm{ff}$. 
Im Forschungsfeld des Komplexen Problemlösens findet sich eine zunächst scheinbar verwirrende Vielzahl von Forschungsthemen. Hier soll daher ein kurzer Überblick gegeben werden.

Grundsätzlicher Gegenstand der Forschung zum komplexen Problemlösen ist der Umgang von Personen mit komplexen Systemen und ihre Fähigkeit der Bewältigung der an sie gestellten Anforderungen. Dörner, Kreuzig, Reither \& Stäudel erachten vier Generalfragen in diesem Zusammenhang als wichtig: ${ }^{168}$

1. Die EFFEKTFRAGE: Sie behandelt den Erfolg von Versuchspersonen im Umgang mit Unbestimmtheit und Komplexität. Dieser wird dazu mit Hilfe verschiedener Produktindikatoren, wie bspw. kritischen Variablen oder deren Verlaufsmerkmalen gemessen.

2. Die PROZEßFRAGE: Sie behandelt die Rückführung der erzielten Effekte auf Merkmale der Denk-, Planungs- und Entscheidungsprozesse.

3. Die BedingungSFRAGE: Sie befaßt sich mit situativen Bedingungen, die sich aus der Versuchsanordnung bedingen sowie persönlichen Bedingungen der Versuchspersonen und deren möglichen Einfluß auf individuell unterschiedliche Arten des Denkens, Planens und Entscheidens.

4. Die theoriefrage: Sie umfaßt das theoretische System, welches die Prognose individuellen Verhaltens ermöglicht.

Bei Vogt findet sich weiterhin eine Übersicht existierender Arbeitsgruppen zum komplexen Problemlösen und ihrer zentralen Themen: ${ }^{169}$

167 Vgl. Hartung, S., (Förderung 2000), S. 238.

168 Vgl. Dörner, D.; Kreuzig, H.W.; Reither, F.; Stäudel, T. (Hrsg.),(Lohhausen 1983), S. $115 \mathrm{ff}$.

169 Vgl. Vogt, H.M., (Persönlichkeitsmerkmale 1998), S. 30 ff. und die dort angegebene Literatur. 
- Bei Versuchspersonen sind verschiedene kognitive, emotionale und motivationale Prozesse zu beobachten. Die BAMBERGER ARBEITSGRUPPE um Dörner verfolgt in diesem Zusammenhang das Ziel, Regelmäßigkeiten und Gesetze zu erforschen. Darüber hinaus sollen Fehlleistungen, die beim Umgang mit komplexen Systemen auftreten, aufgedeckt werden sowie in Dörners Konzept der „Logik des Mißlingens“ deren Hintergründe und Ursachen geklärt werden. ${ }^{170}$

- Ein weiterer Schwerpunkt der Problemlöseforschung ist der Zusammenhang von Testintelligenz und erzielten Problemlöseleistungen. Die BAYREUTHER ARBEITSGRUPPE um Putz-Osterloh beschäftigt dabei in erster Linie die Frage des Zusammenhangs zwischen Testintelligenz und bereichsspezifischem sowie bereichsunspezifischem Wissen mit dem Umgang von Versuchspersonen mit komplexen Problemen. ${ }^{171}$

- Das Ziel der BONNER ARBEITSGRUPPE um Funke ist es, aus experimentellen Untersuchungen Systematisierungen komplexer dynamischer Systeme abzuleiten. ${ }^{172}$ Dabei wird im wesentlichen eine Diagnostik des Systemwissens angestrebt, die prozeßbegleitend durchgeführt wird; und der Untersuchung von Wissenserwerbsprozessen sowie der Wissensanwendung dient.

- Der hamburger ARBEITSKReis um Kluwe sieht es als vorteilhaft an, mit Systemen zu arbeiten, die zum einen experimentell manipulierbar und weiterhin mathematisch genau zu beschreiben sind. Dadurch wird gewährleistet, daß alle kritischen Systemgrößen annähernd kontrolliert werden können und Einflüsse des Vorwissens auszuschließen sind. Zentrale Inhalte der Untersuchungen sind dabei Lern- und Wissenserwerbprozesse, wobei untersucht wird, in welchem Zusammenhang Wissensqualitäten und Problemlösegüte stehen.

\footnotetext{
170 Vgl. Abschnitt 3.4: Das S-H-E-L-L - Modell.

171 Vgl. Abschnitt 6.2.2.1: Methoden zur Erhebung von Vorwissen und Abschnitt 3.3.3: Untersuchungsdesigns zum Umgang von Personen mit komplexen betriebswirtschaftlichen Systemen.

172 Vgl. Abschnitt 2.3.3: Einsatz von Simulationsmodellen im Forschungsfeld des Komplexen Problemlösens.
} 
- Auch ANDERE NATIONALE ARBEITSGRUPPEN beschäftigen sich intensiv mit dem Themengebiet. Dabei legen Hasselmann sowie Strau $\beta$ ihren Schwerpunkt auf Anwendungsmöglichkeiten in der Managementdiagnostik. ${ }^{173}$

- Hussy versucht, ebenso wie Putz-Osterloh, Zusammenhänge zwischen Aspekten des komplexen Problemlösens und Komponenten der Testintelligenz zu klären. ${ }^{174}$ Demgegenüber orientiert sich Leutner eher an pädagogischpsychologischen Konzepten. In diesem Zusammenhang untersucht dieser im Bezugsrahmen des Wissenserwerbs Interdependenzen zwischen Personmerkmalen und Situationsmerkmalen. Weiterhin beleuchtet er auch den Aspekt von Anwendungsmöglichkeiten computersimulierter Szenarien näher.

- Süß, Kersting \& Oberauer erforschen ebenfalls Aspekte der menschlichen Intelligenz. Dabei basiert ihre Forschung auf dem Ansatz der Intelligenzforscher um Jäger. Sie können substantielle Zusammenhänge zwischen Intelligenzkonzepten und Konzepten des komplexen Problemlösens belegen.

- Die Arbeiten der OXFORDER ARBEITSGRUPPE um Broadbent hatten zunächst Dissoziationsphänomene zum Gegenstand. Dabei bezeichnet der Begriff Dissoziationsphänomen eine Diskrepanz zwischen dem komplexen Problemlöseverhalten von Versuchspersonen und dessen verbaler Beschreibung. Später suchte die Arbeitsgruppe nach Erklärungsformen sowie Arten der Wissenspräsentation, die den Problemlöser bei komplexen Suchproblemen unterstützen können.

- Interessengebiet der BRÜSSELER ARBEITSGRUPPE von Cleeremans \& Karnas ist ebenso das Phänomen der Dissoziation.

- Die Untersuchungen der SYSTEMS-DYNAMICS-GRUPPE am MIT in Cambridge haben schließlich den Zusammenhang Wissensumfang sowie weiterhin von System- und Umweltbedingungen und Problemlöseprozeß zum Gegenstand.

173 Vgl. Abschnitt 3.3.3: Untersuchungsdesigns zum Umgang von Personen mit komplexen betriebswirtschaftlichen Systemen.

174 Vgl. Abschnitt 6.2.2.1: Methoden zur Erhebung von Vorwissen. 
Außerdem beschäftigt sie sich mit der Möglichkeit den Umgang mit dynamischen Systemen zu erlernen.

\subsubsection{Einfache und komplexe Probleme}

Das menschliche Problemlösen muß als ein in der Zeit sich erstreckender Prozeß verstanden werden, der am besten als Informationsverarbeitungsprozeß zu verstehen ist. 175 Menschliches Problemlösen nimmt seinen Anfang bei der Wahrnehmung eines Problems. Daher soll hier zunächst auf den Problembegriff sowie Problemlöseprozeß eingegangen werden. Duncker definierte diese, bereits 1935, wie folgt: 176

„Ein „Problem“ entsteht z.B. dann, wenn ein Lebewesen ein Ziel hat und nicht „weiß“, wie es dieses Ziel erreichen soll. Wo immer der gegebene Zustand sich nicht durch bloßes Handeln (Ausführen selbstverständlicher Operationen) in den erstrebten Zustand überführen läßt, wird das Denken auf den Plan gerufen. Ihm obliegt es, ein vermittelndes Handeln allererst zu konzipieren. Die „Lösung“ eines solchen praktischen Problems hat somit zwei Forderungen zu genügen: ihre Verwirklichung (Umsetzung in die Praxis) muß erstens die Realisierung des erstrebten Zustands zur Folge haben und zweitens vom gegebenen Zustand aus dann durch „bloßes Handeln“ erreichbar sein.“

Heutige Definitionen des Problembegriffes spiegeln die Gedanken Dunckers wieder. ${ }^{177}$ Hier sei auf Dörner verwiesen, der ausführt, daß bezüglich der Definition des Problembegriffes in der Psychologie Einigkeit besteht. Nach Dörner werden durch die folgende Definition von Süllwold die unterschiedlichen Versuche, den Problembegriff zu definieren fokussiert sowie durch Klix spezifiziert: 178

175 Vgl. Lass, U.; Lüer, G., (Problemlöseforschung 1990).

176 Duncker, K., (Psychologie 1963), S. 1.

177 Vgl. Hasselmann, D., (Problemstellungen 1993), S. 31. 
„Ein Problem sei dann gegeben, wenn ein Individuum ein bestimmtes Ziel erreichen will, jedoch nicht weiß, wie es zu diesem Ziel gelangen kann, also nicht auf wohlbekannte spezifische Verfahren, spezifische Techniken und Operationen zurückzugreifen vermag." ${ }^{179}$

1. Ein Problem weist einen Anfangszustand mit bestimmten physikalischen oder abstrakten Eigenschaften auf. Diese Eigenschaften werden als Merkmale mit spezifischen Transformationseigenschaften wahrgenommen.

2. Aus dem Anfangszustand ist der Zielzustand des Problems herzustellen.

3. Zwischen Anfangszustand und Zielzustand liegt eine Barriere ${ }^{180}$. Diese muß überwunden werden, um eine Überführung der Anfangssituation in die angestrebte Zielsituation zu erreichen. ${ }^{181}$

Zur Klassifikation von Problemen existieren unterschiedliche Vorschläge. Dörner unterscheidet folgende Kategorien von Problemen: ${ }^{182}$

$\mathrm{P}_{1} \quad$ OfFEnheit DeR ANFAngSSituation: Die Offenheit der Anfangssituation hängt davon ab, wie groß die Anzahl der Alternativen ist, die nach Einschätzung des Individuums als Anfangssituation in Betracht gezogen werden können. Liegt lediglich eine in Frage kommende Anfangssituation vor, so handelt es sich um eine geschlossene Anfangssituation. Die Offenheit wächst dann mit zunehmender Anzahl an Al-

178 Vgl. Dörner, D., (Organisation 1974), S. 20.

179 Süllwold, F., (Bedingungen 1960), S. 96.

180 Die Bezeichnung Barriere stammt von Dörner. Vgl. Dörner, D., (Organisation 1974), S. 20.

181 Vgl. Klix, F., (Information 1976), S. 640. Damit sind die Teile, aus denen sich ein Problemlösungsprozeß zusammensetzt benannt. Sie bilden die Grundpfeiler einer Theorie des Denkens. Dabei kann der Ausgangszustand in seinen Bestandteilen entweder fest definiert sein oder auch nicht. Wird bspw. von einer Schachpartie ausgegangen, so ist der Ausgangzustand zu Beginn einer Partie präzise zu benennen. Genauso können auch im Verlauf einer Partie erreichte Spielstände als Ausgangszustände ausgewählt werden, die auch genau zu definieren sind. Es ist weiterhin denkbar, daß alternative Ausgangspunkte zur Verfügung stehen, die den Ausgangspunkt für den weiteren Problemlöseprozeß darstellen sowie let丸lich Ausgangszustände, die nicht so leicht zu beschreiben sind wie beim Schachbeispiel. Das gleiche gilt für den Zielzustand. Er kann ebenso aus mehreren Alternativen bestehen oder nicht sowie unterschiedliche Stufen der Genauigkeit aufweisen. Vgl. Lüer, G., (Denkabläufe 1973), S. $11 \mathrm{f}$.

182 Vgl. Dörner, D., (Organisation 1974), S. 43 ff. Zu einer Einteilung von Problemtypen vgl. auch Schaub, H., (Modellierung 1993), S. 43. 
ternativen, die für eine Anfangssituation in Frage kommen sowie der Anzahl an Alternativen, die durch das Individuum als wenig erfolgversprechend eingeschätzt werden. Ist die Anfangssituation geschlossen, gibt es also nur eine Startsituation wie bspw. beim Schach. Hier weist Dörner auf die Wahl einer erfolgversprechenden Ausgangsstellung hin.

$\mathrm{P}_{2}$ OFFENHEIT DER ZIELSITUATION: Auch die Offenheit der Zielsituation richtet sich nach der Anzahl der Alternativen, die nach Meinung des Individuums als Zielsituation in Frage kommen. Der Grad der Offenheit kann nach drei charakteristischen Situationen unterschieden werden: Eine geschlossene Zielsituation liegt dann vor, wenn dem Individuum das Zielobjekt genau bekannt ist. Ist das Zielobjekt nicht genau bekannt, jedoch ein positiv damit korrelierendes Merkmal, so ist das Problem weder geschlossen noch gänzlich offen. Im Falle einer offenen Zielsituation ist es dem Problemlöser nicht möglich, das Ziel zu benennen. Die Merkmale der Zielsituation sind ihm gänzlich unbekannt.

$\mathrm{P}_{3} \quad$ VERFÜGBARKEIT ÜBER DIE OPERATOREN: Diese Dimension betrifft das Ausmaß, in dem der Problemlöser weiß oder zu wissen glaubt, daß er über die Mittel zur Transformation der Startsituation in die Zielsituation verfügt.

Demnach richtet sich ein Problemtyp im Sinne von Dörner nach der Klarheit der Anfangs- und Zielkriterien sowie dem Bekanntheitsgrad der Mittel, welche zur Transformation der Anfangs- in die Zielsituation geeignet sind. Dörner spricht in diesem Zusammenhang auch von Interpolations-, Synthese- sowie dialektischen Problemen. Ein Interpolationsproblem liegt vor, wenn dem Problemlöser Anfangs- und Zielzustand sowie die erforderlichen Operatoren bekannt sind, die richtige Kombination der Operatoren hingegen nicht. Sind Anfangszustand und Ziel klar, der Bekanntheitsgrad der Mittel jedoch nicht, so liegt ein Syntheseproblem vor. Im Falle eines dialektischen Problems verfügt der Problemlöser darüber 
hinaus über keine konkrete Vorstellung bezüglich des angestrebten Ziels. ${ }^{183}$ [Vgl. Abbildung 2-7].

\begin{tabular}{|c|c|c|c|}
\hline \multirow{4}{*}{$\begin{array}{l}\text { Bekanntheits- } \\
\text { grad der Mittel }\end{array}$} & & \multicolumn{2}{|c|}{ Klarheit der Zielkriterien } \\
\hline & & hoch & gering \\
\hline & hoch & $\begin{array}{c}\text { Interpolations- } \\
\text { Barriere }\end{array}$ & $\begin{array}{l}\text { dialektische } \\
\text { Barriere }\end{array}$ \\
\hline & gering & $\begin{array}{l}\text { Synthese- } \\
\text { Barriere }\end{array}$ & $\begin{array}{c}\text { dialektische } \\
\text { Barriere und } \\
\text { Synthese-Barriere }\end{array}$ \\
\hline
\end{tabular}

\section{Abbildung 2-7: $\quad$ Klassifikation von Barrieretypen 184}

Gomez \& Probst unterscheiden nach einfachen, komplizierten und komplexen Problemen. Einfache Probleme werden von ihnen als solche klassifiziert, die sich durch eine geringe Anzahl an Einflußgrößen, eine geringe Dichte vorliegender Verknüpfungen zwischen diesen Größen und einen weitgehenden Grad an Stabilität dieser Beziehungen auszeichnen. Komplizierte Probleme hingegen weisen eine hohe Anzahl an Einflußfaktoren auf, die auch relativ stark untereinander verknüpft sind. Dabei verändert sich das Verknüpfungs- und Interaktionsmuster im Zeitablauf nicht wesentlich. Komplexe Probleme unterscheiden sich von den komplizierten Problemen schließlich noch darin, daß sie durch Dynamik charakterisiert sind. ${ }^{185}$ Diesen, und andere Aspekte führen auch Dörner, Kreuzig, Reither \& Stäudel als kennzeichnend für komplexe Probleme aus: ${ }^{186}$

KOMPLEXITÄT: Die Komplexität einer Situation wird durch die Vielzahl zu berücksichtigender Aspekte konstituiert.

INTRANSPARENZ: Die Situation stellt sich als intransparent dar, da notwendige Informationen nicht verfügbar sind und aus zeitlichen Gründen oder wegen

183 Vgl. Dörner, D., (Problemlösen 1976), S. 11 ff.

184 Dörner, D., (Problemlösen 1976), S. 14.

185 Vgl. Gomez, P.; Probst, G.,(Praxis 1995), S. 17 ff.

186 Vgl. Dörner, D.; Kreuzig, H.W.; Reither, F.; Stäudel, T. (Hrsg.),(Lohhausen 1983), S. $19 \mathrm{ff}$. 
unzulänglicher Daten auch nicht beschafft werden können. Außerdem können vorhandene Informationen falsch sein, werden aber als richtig eingeschätzt.

> VERnETZTHEIT: Die unterschiedlichen Aspekte der Situation sind voneinander abhängig und müssen daher unter dem Aspekt ihrer Vernetztheit betrachtet werden.

EIGENDYNAMIK: Die Situation entwickelt sich selbstgesteuert weiter, so daß der Akteur unabhängig von einer eigenen Einflußnahme im Zeitablauf einer veränderten Situation gegenübersteht.

POLYTELIE: In der Situation sind mehrere Ziele gleichzeitig zu berücksichtigen, die sich zumeist auch gegenläufig zueinander verhalten.

UNBESTIMMTHEIT: Das zu erreichende Ziel kristallisiert sich erst im Zeitablauf heraus, so daß der Akteur einem hohen Maß an Unbestimmtheit ausgesetzt ist.

\subsection{3}

\section{Einsatz von Simulationsmodellen im Forschungsfeld des Kom- plexen Problemlösens}

Komplexe Systeme liegen in der Realität in vielfältigen Ausdrucksformen vor. Unter anderem sind sie in den Realitätsbereichen der Technik, Politik, Ökologie, Ökonomie oder Biologie vorzufinden. Dabei wirken sich die Entscheidungen und Eingriffe von Menschen auf die Zustände solcher technischer, ökologischer oder auch anderer Systeme aus. Ein zentrales Charakteristikum dieser Systeme ist, daß die Auswirkungen von Eingriffen auf das System und damit die für Eingriffe erforderlichen Informationen nicht gleich verfügbar sind und eigens der Suche bedürfen. Es handelt sich daher um eine Entscheidungssituation unter Unsicherheit. ${ }^{187}$

In den vergangenen Jahren konnten mit Hilfe computersimulierter Systeme Ausschnitte der Realität nachgebildet werden. ${ }^{188}$ Derartige Simulationsprogramme finden Verwendung im Forschungsfeld des komplexen Problemlösens. So werden hier komplexe Systeme in Form von Computersimulationen operationalisiert, die von Versuchspersonen zu kontrollieren sind. ${ }^{189}$ Inzwischen liegt eine Reihe von Untersuchungen zum Umgang von Versuchspersonen mit komplexen Systemen

\footnotetext{
187 Kluwe, R.H., (Kontrolle 1990), S. 236 ff.

188 Vgl. Funke, J., (Steuerung 1985), S. 1.

189 Vgl. Opwis, K., (Modelle 1985), S. 13.
} 
vor. ${ }^{190}$ Diesen Designs ist das Ziel, komplexes Problemlösen zu untersuchen, gemeinsam. Sie weisen spezifische Problemcharakteristika auf, deren Erforschung auf Dörner zurückgeht. Dörner untersucht solche Systeme als Probleme, zu deren Handhabung Personen komplexe Denk- und Entscheidungsstrategien zu nutzen haben. ${ }^{191}$ Dadurch unterscheiden sie sich auch gleichzeitig von traditionellen Untersuchungsdesigns wie dem „TURM-VON-HANOI“-Problem ${ }^{192}$ oder dem „MISSIONAR-KANNIBALEN"-Problem ${ }^{193}$, die zwar auch der Analyse von Problemlöseaktivitäten galten, aber einfache Denksportaufgaben darstellten. ${ }^{194}$

Solchen Untersuchungsdesigns liegen auch ökonomische Sachverhalte zu Grunde. ${ }^{195}$ Dies ist nach Petzing ein Grund, die hier bestehende Schnittstelle psychologischer und wirtschaftswissenschaftlicher Forschung in den Wirtschaftswissenschaften nicht weiter zu ignorieren, wie es bisher scheinbar weitestgehend der Fall ist. Daneben nennt er noch zwei weitere Gründe: Zum einen berühren Planspielziele sowie Vorteile der Planspielmethode oft Konzepte der psychologischen Forschung. Zum anderen hängt die Erreichbarkeit von Planspielzielen oft von deren kognitiven Anforderungen ab. ${ }^{196}$

190 Eine Übersicht sowie detailliertere Beschreibung der bis 1984 durchgeführten deutschsprachigen Untersuchungen findet sich bei Funke, J., (Problemlösen 1984), S. 5 ff. Hier seien beispielhaft genannt: DORI Hesse, F.W., (Effekte 1982); HAMURABI Gediga, G.; Schöttke, H.; Tücke, M., (Problemlösen 1982) und MONDlandung, Funke, J., (Mondlandung 1981). Des weiteren findet sich eine tabellarische Übersicht mit einer Unterteilung der Smulationsprogramme nach Variablenumfang bei Funke, J., (Problemlösen 1985), S. 119. Vgl. auch den kurzen Überblick bei Eyferth, K.; Schömann, M.; Widowski, D.,(Umgang 1986), S. 11.

191 Vgl. bspw. Dörner, D.; Drewes, U.; Reither, F.,(Problemlösen 1975).

192 Das ,TURM-VON-HANOI“-Problem veranschaulicht, daß auch in einfachen Problemumwelten eine Vielzahl von Lösungsstrategien möglich sind und unterschiedliche Individuen sich voneinander unterscheidende Lösungsstrategien aneignen können. Vgl. z.B. Klix, F., (Information 1976), S. $704 \mathrm{ff}$.

Das „MISSIONAR-KANNIBALEN“-Problem ist eines von einer Vielzahl von „RIVERCROSSING“'-Problemen. Aufgabe ist es, ein Boot mit einer Gruppe von Reisenden zu besetzen, das diese vom einen zum anderen Ufer eines Flusses transportieren soll. Die Problematik dieser Aufgabe besteht in der Kapazitätsgrenze des Bootes und Restriktionen, die dnige Kombinationen von Reisenden unmöglich machen. Vgl. Jeffries, R.; Polson, P.G.; Razran, L.; Atwood, M.E., (Process 1977), S. 413. Dieses wird heute, in Anlehnung an Tolkien's Herr der Ringe, ,HOBBITS- und ORCS“- Problem genannt. Vgl. Anderson, J.R., (Psychologie 1996), S. 247 f. Auf Grundlage des ,HOBBITS- und ORCS“6- Problems untersuchte Greeno sequentielles Problemlösen. Vgl. Greeno, J.G., (Hobbits 1974), S. 270 ff. Opwis, K., (Modelle 1985), S. 13.

195 Vgl. Abschnitt 3.3.3: Untersuchungsdesigns zum Umgang von Versuchspersonen mit betriebswirtschaftlichen Systemen.

196 Vgl. Petzing, F., (Untersuchung 1993), S. 19. 
Weiterhin wirft Achtenhagen die Frage auf, ,[...] warum die Problemlöseforschung denn nicht auf die in großer Zahl vorliegenden ökonomischen oder militärischen Planspiele zurückgegriffen hat"“. ${ }^{197}$ Dies wäre schon deshalb sinnvoll, da in den Kognitionswissenschaften identische Simulationsprogramme, lediglich unter Berücksichtigung einiger Modifikationen, zur Darstellung unterschiedlicher Realitätsausschnitte genutzt werden. Ein Simulationsprogramm, welches einmal der Darstellung eines Gebietes in der Sahelzone dient, wird an anderer Stelle zur Simulation des Verlaufs einer Grippe- und Pockenepidemie genutzt. ${ }^{198}$ So wird eine Zusammenarbeit aber auch in der Psychologie als wünschenswert erachtet; ${ }^{199}$ und zumeist die Möglichkeit einer Einbeziehung wirtschaftswissenschaftlicher Erkenntnisse sowie weiterhin der Austausch mit wirtschaftswissenschaftlichen Experten diskutiert. ${ }^{200}$ 
In Kapitel drei wird, unter Berücksichtigung des Grundgedankens der Allgemeinen Systemtheorie, ${ }^{201}$ deren Systembegriff dem Planspiel als einer allgemeinen Methode zugrunde gelegt wurde,202 versucht, Erkenntnisse der betriebswirtschaftlichen sowie der psychologischen Forschung in einen gemeinsamen Bezugsrahmen zu stellen. Dies kann zum einen unter Berücksichtigung der in Kapitel zwei ausgeführten Diskussion bezüglich einer wünschenswerten Zusammenarbeit zwischen der Betriebswirtschaftslehre und der Psychologie gesehen werden, ${ }^{203}$ geschieht jedoch vordergründig im Hinblick auf den in Kapitel sechs ausgeführten Aspekt, daß die Teilnehmer an einem Unternehmensplanspiel ihr in Seminaren und Vorlesungen erworbenes Sachwissen nicht anwenden.204 Dabei können die Betrachtungen in Kapitel zwei in der folgenden Weise als Ausgangspunkt der Betrachtung angesehen werden:

Zunächst ist davon auszugehen, daß ein Planspiel als Modell im Sinne einer Mensch-Maschine-Simulation interpretiert werden kann. ${ }^{205}$ Unter diesem Aspekt wird das Unternehmensplanspiel als Mensch-Maschine-System verstanden, wodurch der menschliche Verhaltensfaktor, zusätzlich zum Modellaspekt, ein Gegenstand des Bezugsrahmens wird. Die objektiven Charakteristika von Planspielen stehen also in Wechselwirkung mit dem subjektiven Verständnis von Menschen, die sich mit einem Planspiel auseinandersetzen, womit die folgenden Aspekte impliziert werden:

Zum einen wird in Zusammenhang mit der Modellbildung beim Unternehmensplanspiel die Berücksichtigung der Systemtheorie impliziert.

$>$ Weiterhin wird durch die Relevanz des Menschen, hier in der Funktion als Teilnehmer an einem Unternehmensplanspiel, die Notwendigkeit einer Inte-

201 Vgl. Abschnitt 2.1.2.1: Das Unternehmensplanspiel: Ein Simulationsmodell und Abschnitt 3.4.2: Das S-H-E-L-L - Modell als eine Möglichkeit zur Darstellung der Natur menschlichen Fehlverhaltens.

202 Vgl. Abschnitt 2.1.2: Simulationstechnik beim Unternehmensplanspiel.

203 Vgl. Abschnitt 2.3.3: Einsatz von Simulationsmodellen im Forschungsfeld des Komplexen Problemlösens.

204 Vgl. Abschnitt 6.3: Die unternehmerische Handlungskompetenz.

205 Vgl. Abschnitt 2.1.2.3: Interpretation der Simulationstechnik beim Unternehmensplanspiel. 
gration der Psychologie impliziert, wobei an dieser Stelle zunächst die Erkenntnisse der Problemlöseforschung relevant sind.

In diesem Zusammenhang wird an dieser Stelle das in der Luft- und Raumfahrttechnik entwickelte S-H-E-L-L - Modell vorgestellt. Bei diesem Modell handelt es sich um ein Mensch-Maschine-System, wodurch es als geeigneter Rahmen angesehen wird, die beim Planspiel existierende Schnittstelle von Mensch und Maschine darzustellen. Die Darstellung erfolgt dabei unter folgenden Gesichtspunkten:

Als problematisch erweist sich zunächst, daß sich aus der grundlegenden Simulationstechnik beim Unternehmensplanspiel eine Vielzahl unterschiedlicher Modellkonstruktionen entwickelt hat, wodurch sich die Planspielteilnehmer in jeweils verschiedenen experimentellen Situationen befinden. ${ }^{206}$ Eine Systembetrachtung unter explizitem Bezug auf die Allgemeine Systemtheorie bietet nun den Vorteil eine Betrachtung unter allgemeingültigen Kriterien vorzunehmen. Hier bietet das S-H-E-L-L - Modells den Vorteil, daß es die Möglichkeit bietet, eine Modellbetrachtung vorzunehmen, die unter Berücksichtigung grundlegender Systemkomponenten erfolgt.

Weiterhin besteht durch die Berücksichtigung des S-H-E-L-L - Modells die Möglichkeit, die in der Problemlöseforschung gewonnenen Erkenntnisse zu menschlichem Fehlverhalten im Umgang mit komplexen Systemen in einen gemeinsamen Bezugsrahmen zu stellen.

Dadurch werden vier Aspekte gewährleistet:

1. Es erfolgt die gewünschte Gegenüberstellung betriebswirtschaftlicher und psychologischer Forschungserkenntnisse.

2. Das Unternehmensplanspiel wird in seinen grundlegenden systemtheoretischen Dimensionen erfaßt.

3. Das Individuum wird, in seiner Funktion als Teilnehmer an einem Planspiel, durch die Interpretation als handelndes Subjekt berücksichtigt.

4. Es werden mögliche Fehlermomente des Menschen im Umgang mit komplexen Systemen aufgezeigt. 
Insgesamt kann erwartet werden, daß das betriebswirtschaftliche Handlungsfeld und in diesem mögliche Fehlleistungen näher spezifiziert werden kann. Um in diesem Zusammenhang die notwendigen theoretischen Grundlagen für die weiteren Betrachtungen zu schaffen, ist zunächst aufzuzeigen, daß die Unternehmung einer Anwendung der Allgemeinen Systemtheorie zugänglich ist. Weiterhin ist auf Arten und Ablauf von Unternehmensplanspielen sowie Konzepte der Problemlöseforschung einzugehen.

\subsection{Das System der Unternehmung}

Es ist zunächst davon auszugehen, daß die Unternehmung einer Anwendung der Allgemeinen Systemtheorie zugänglich ist. Von dieser Möglichkeit wird in der Psychologie und in der Soziologie schon seit längerem Gebrauch gemacht, während deren begriffliches Instrumentarium im Rahmen der Wirtschaftswissenschaft erst in jüngerer Zeit Berücksichtigung findet. ${ }^{207}$

Die Unternehmung wird dabei dadurch einer Betrachtung als System zugänglich, daß Sachaufgaben (Bedarfsdeckungsaufgaben) definiert werden und die Unternehmung sich dadurch gegenüber ihrer Umwelt profiliert. Somit steht dem System der Unternehmung das Supersystem Umwelt gegenüber, zu welchem es in Austauschbeziehungen steht, ${ }^{208}$ wobei nach Luhmann in der Betriebswirtschaftslehre ,[...] die Vorstellung eines Systems, das Beziehungen zu mehreren Umwelten unterhält und deren Interessengefälle ausgleicht $[\ldots]^{“}$, am weitreichendsten ausgearbeitet ist. ${ }^{209}$

206 Vgl. Abschnitt 3.2.1: Arten von Unternehmensplanspielen.

207 Fuchs, H., (Systemtheorie 1969), Sp. 1623. Nach Albach kann hier von der dritten Generation der Betriebswirtschaftslehre gesprochen werden. Vgl. Albach, H., (Aufgaben 1967), S. 447 ff. Vordergründig wurde der von der Allgemeinen Systemtheorie behandelte Problemkreis dabei von der betriebswirtschaftlichen Organisationslehre aufgegriffen. Vgl. bspw. Johnson, R.A.; Kast, F.E.; Rosenzweig, J.E., (Theory 1967), S. 50 ff.; Grochla, E., (Automation 1966), S. 121 ff.; Grochla, E., (Systemtheorie 1970), S. 10 ff.; Bleicher, K., (Entwicklung -Heft 1- 1970), S. 3 ff. Grochla ist der Ansicht, daß sich die Notwendigkeit, die Ansätze der Organisationstheorie zu überdenken durch die Automation bedingt. Ausgehend von US-amerikanischen Diskussionen stellt er eine vergleichende Betrachtung organisationstheoretischer Ansätze mit systemtheoretischen Ansätzen an und zeigt deren Gemensamkeiten auf. Vgl. Grochla, E., (Automation 1966), S. 123 f.

209 Luhmann, N., (Theorie 1966), S. 36. 
Unter Bezug auf die Allgemeine Systemtheorie kann die Unternehmung in diesem Sinne als vieldimensionale Ganzheit verstanden werden, die als strukturierter Aufbau von Elementen sowohl zweck- als auch zielorientiert gegenüber ihrer Umwelt handelt. Dabei wird das System der Unternehmung in seiner statischen Dimension durch den Aufbau sowie die wechselseitigen Interdependenzen der Unternehmenselemente beschrieben. Gegenüber dieser betrieblichen Beziehungsstruktur verkörpert die Prozeßstruktur die dynamische Dimension des Unternehmenssystems. Sie umfaßt die sowohl zeitlich als auch logisch gerichteten Aktivitäts- und Kommunikationsbeziehungen. Insgesamt wird der systemorientierte Ansatz der Betriebswirtschaftslehre schließlich als Lehre von der Gestaltung und Lenkung betrieblicher Systeme interpretiert, ${ }^{210}$ wobei die Systemtheorie ,[...] Begriffe zur Entwicklung eines einheitlichen und umfassenden Grundmodells der Betriebswirtschaft" ${ }^{* 211}$ liefert.

Zusammenfassend kann festgehalten werden, daß die Unternehmung als System angesehen werden kann, wobei die Allgemeine Systemtheorie ein begriffliches Instrumentarium liefert das System zu beschreiben. Da das Unternehmensplanspiel ein Modell über das System der Unternehmung darstellt, wird an dieser Stelle davon ausgegangen, daß das begriffliche Instrumentarium der Allgemeinen Systemtheorie auch in der Lage ist das Unternehmensplanspielmodell $\mathrm{zu}$ beschreiben.

\section{2}

\section{Arten und Ablauf von Unternehmensplanspielen}

\subsection{1}

\section{Arten von Unternehmensplanspielen}

Die grundlegende Simulationstechnik bei Unternehmensplanspielen wurde bereits ausgeführt. Danach kann ein Unternehmensplanspiel als eine Mensch-MaschineSimulation aufgefaßt werden, die einen Aktions- und einen Reaktionsbereich aufweist. Über einen vorgegebenen, simulierten Zeitraum sind Personen im Aktionsbereich dazu aufgefordert, Entscheidungen zu treffen, welche durch den Reaktionsbereich verarbeitet und in Form von Berichten an die Planspieler zu-

210 Vgl. Ulrich, H., (Unternehmung 1970), S. 153 ff. zitiert nach Bliss, C., (Komplexität 2000), S. 87. 
rückgemeldet werden. ${ }^{212}$ Auf Grundlage dieser wirtschaftlichen Entscheidungssimulation hat sich inzwischen eine Vielzahl von Modellkonstruktionen entwikkelt, ${ }^{213}$ wodurch sich die Planspielteilnehmer in jeweils verschiedenen experimentellen Situationen befinden. ${ }^{214} \mathrm{Zu}$ ihrer Klassifikation existieren keine allgemein anerkannten Ansätze. Es lassen sich jedoch einige zentrale Merkmale wirtschaftlicher Planspiele festhalten. ${ }^{215}$

Häufigster Planspieltyp ist derjenige, in dem ein einzelnes Unternehmen einer Branche simuliert wird. Wird ein einzelnes Unternehmen in allen seinen betrieblichen Teilbereichen dargestellt, so handelt es sich um Unternehmensplanspiele im eigentlichen Sinn, die als generelle Planspiele bezeichnet werden. Unternehmensplanspiele, die lediglich ausgesuchte betriebliche Teilbereiche zum Gegenstand haben, werden als spezielle Planspiele bezeichnet. ${ }^{216}$ Diese Unterscheidung trifft auch Bleicher und bezeichnet gekoppelte Modelle aus generellen und speziellen Modellen schließlich als hierarchische Modelle, wobei der Vorteil dieser Modelle darin besteht, daß durch sie eine höhere Realitätsnähe gelingt. ${ }^{217}$

Wesentlich ist auch der Konkretisierungsgrad des Unternehmensplanspiels. Hier sind solche mit abstrakten und solche mit konkreten Daten zu unterscheiden. Bei einem Unternehmensplanspiel mit abstrakten Daten werden die Erzeugnisse nicht näher bezeichnet, und der Absatz erfolgt auf anonymen Märkten. Bei konkreten Daten werden die Erzeugnisse und Märkte hingegen eindeutig bestimmt. Bei der Verwendung von abstrakten Daten besteht die Möglichkeit der Verwendung für beliebige Unternehmen oder Branchen. Konkrete Daten setzen hingegen eine spe-

211 Meffert, H., (Systemtheorie 1971), S. 176.

212 Vgl. Abschnitt 2.1.2.2: Das Unternehmensplanspiel: Ein Entscheidungsspiel.

213 Vgl. Bleicher, K., (Entscheidungssimulation 1962), S. 21.

214 Vgl. Koller, H., (Planspieltechnik 1969), S. 104.

215 Vgl. Koller, H., (Planspieltechnik 1974), S. 11.

216 Vgl. Rohn, W.E., (Führungsentscheidungen 1964), S. 50. Koller bezeichnet spezielle Planspiele als funktionelle Planspiele. Als spezielle Planspiele faßt er Planspiele auf, welchen ,[...] die besonderen Gegebenheiten und Probleme einzelner Unternehmungsarten oder Wirtschaftszweige zugrunde gelegt wurden“. Koller, H., (Planspieltechnik 1974), S. 11. Vgl. auch Koller, H., (Planspieltechnik 1969), S. 105 f.

217 Vgl. Bleicher, K., (Unternehmungsspiele 1969), Sp. 1688 f. Gemäß Högsdal sind generelle Planspiele als Standardplanspiele anzusehen, die von firmenspezifischen Planspielen, welche die Gegebenheiten bestimmter Unternehmen abbilden, zu unterscheiden sind. Vgl. Högsdal, B., (Planspiele 1996), S. 15. 
zifische Verwendung voraus, gewährleisten aber auch eine höhere Realitätsnähe. ${ }^{218}$

Bezüglich der Determinierung des Simulationsmodells kann nach deterministischen und stochastischen Planspielen unterschieden werden. Während in deterministischen Planspielen die Ergebnisse eindeutig von den Entscheidungen der Spieler abhängen, werden in stochastischen Planspielen Zufallselemente berücksichtigt, die dazu führen, daß die Ergebnisse auch durch die Zufallselemente beeinflußt werden - und damit nicht mehr einfach nur von den Entscheidungen der Spieler abhängen. Der Vorteil stochastischer Planspiele wird darin gesehen, daß sie aufgrund der eingebauten Zufallselemente eine höhere Realitätsnähe aufweisen. ${ }^{219}$ Dabei wird die Verwendung von Zufallselementen dadurch begründet, daß auch in der Realität keine genaue Vorhersage bezüglich der Auswirkungen von Entscheidungen möglich ist. 220

Unternehmensplanspiele können weiterhin nach ihrer Komplexität unterschieden werden. Diese stellt, wie bereits ausgeführt, im Rahmen der Ausbildung ein zentrales Kriterium dar. ${ }^{221}$ Die diesbezüglichen Klassifikationen sind jedoch wenig genau. Einige Autoren unterscheiden zwischen einfachen und komplexen Planspielen. $^{222}$ Andere differenzieren unterschiedliche Schwierigkeitsstufen. ${ }^{223}$

Vgl. Bleicher, K., (Unternehmungsspiele 1962), S. 53. An anderer Stelle spricht Bleicher auch von 'anonymen' und 'konkreten' Daten. Vgl. Bleicher, K., (Unternehmungsspiele 1969), Sp. 1690. Wie ausgeführt unterscheidet Högsdal in diesem Zusammenhang nach Standardplanspielen und firmenspezifischen Planspielen. Vgl. Högsdal, B., (Planspiele 1996), S. 15.

219 Vgl. Rohn, W.E., (Führungsentscheidungen 1964), S. 51; Koller, H., (Planspieltechnik 1974), S. 12.

220 Vgl. Koller, H., (Planspieltechnik 1969), S. 108. Koller wendet jedoch ein, daß bereits das nicht vorhersehbare Verhalten anderer Spielgruppen sowie die Undurchsichtigkeit des komplexen Modells als solchem stochastisch auf die Spielteilnehmer einwirken, so daß durch die Berücksichtigung von Zufallselementen die Analyse noch zusätzlich erschwert wird. Vgl. ders., (Planspieltechnik 1969), S. 108.

221 Vgl. Abschnitt 2.2.2: Zur Relevanz von Komplexität im Unternehmensplanspiel. Nach Koller hängt bspw. der Schwierigkeitsgrad der Entscheidungsaufgaben von der Komplexität des Planspiels ab. Er differenziert nach einfachen und äußerst komplexen Planspielen. Als Beispiel für einfache Planspiele führt er solche an, in denen lediglich eine Entscheidung bspw. zur Bestimmung optimaler Losgrößen gefällt werden muß. Als äußerst schwieriges Planspiel führt er das „Carnegie Tech Management Game“ an, in dem mehr als 300 Entscheidungen zu treffen sind. Andere Planspiele lassen sich seiner Ansicht nach irgendwo zwischen diesen beiden Extremfällen einordnen. Vgl. Koller, H., (Planspieltechnik 1969), S. $110 \mathrm{f}$. und die dort angegebene Literatur. 
Högsdal nimmt für den Grad der Komplexität eines Planspiels die pro Periode zu treffende Anzahl an Entscheidungen an und differenziert weiterhin Komplexität vs. Kompliziertheit, als den Grad der Vernetzung der Entscheidungen. ${ }^{224}$ Bezüglich der Variierbarkeit der Komplexität unterscheidet Högsdal zwischen Standardplanspielen und modularen Planspielen. ${ }^{225}$ Es kann jedoch auch von herkömmlichen und modularen Planspielen gesprochen werden. ${ }^{226}$

Eine Klassifikation bezüglich des Freiheitsgrades des Entscheidungsbereiches der Spieler erfolgt durch die Unterscheidung von freien und starren Planspielen. Beim freien Spiel werden die Entscheidungen der Spieler durch eine Schiedsrichtergruppe bewertet. Auf deren Grundlage werden die resultierenden Wirkungen und folgenden Entscheidungssituationen festgelegt. Beim starren Spiel, welches die neuere Ausprägungsform darstellt, sind Entscheidungsrahmen und Spielregeln hingegen mathematisch formuliert, wodurch die Ergebnisse objektiv rückgekoppelt werden. ${ }^{227}$

Im Hinblick auf die Auswertung der Entscheidungen wird zwischen manuellen und computergestützten Planspielen unterschieden. Bei manuellen Spielen erfolgt die Berechnung der Ergebnisse von Hand sowie unter Heranziehung herkömmlicher Rechenhilfen wie bspw. Tischrechnern und Rechenmaschinen. Bei der Verwendung von Computerspielen wurde das Spielmodell als Maschinenprogramm

223 Bleicher ist der Ansicht, daß je nach Qualifikation der Führungskräfte, Planspielmodelle unterschiedlichen Schwierigkeitsgrades zum Einsatz kommen sollten. Die Planspiele UB-4 bis UB-7, deren Schwierigkeitsgrad graduell zunimmt, sind in diesem Zusammenhang von Bleicher als "Grundmodelle“ für eine erste Ausbildungsstufe konzipiert worden. Das schwierigere Modell UB-10 ist dagegen für eine Anwendung in einer zweiten Ausbildungsstufe gedacht. Vgl. Bleicher, K., (Entscheidungsprozesse 1965), S. 21 f. Vgl. zur Konzeption des Planspiels UB-5 auch Bleicher, K., (Planspiel UB-5 1962), S. 23 ff. Zum Planspiel UB-10 vgl. Bleicher, K., (Planspiel UB-10 1962), S. 13 ff. Auch Sonderegger unterscheidet nach dem Merkmal Schwierigkeit und nimmt drei Schwierigkeitsstufen an. Vgl. Sonderegger, W., (Entwurf 1982), S. 11.

224 Vgl. Högsdal, B., (Planspiele 1996), S. 15. Für das Kriterium der Komplexität kann weiterhin auch die Anzahl der Produkte sowie die der Märkte angenommen werden. Vgl.ders, (Planspiele 1996), S. 15.

225 Vgl. Högsdal, B., (Planspiele 1996), S. 15.

226 Vgl. Hartung, S., (Förderung 2000), S. 130 ff. Vgl. auch Abschnitt 2.2.2.3.2: Zum Zusammenhang von Komplexität und Lernerfolg.

227 Vgl. Koller, H., (Planspieltechnik 1974), S. 11 f. 
formuliert, und die Ergebnisse können daher automatisch mit der elektronischen digitalen Datenverarbeitung berechnet werden. ${ }^{228}$

Nach der Anzahl der Teilnehmergruppen am Planspiel können Solo- und Konkurrenzplanspiele unterschieden werden. Beim Solospiel ist nur eine Planspielgruppe aktiv. Beim Konkurrenzplanspiel agieren mehrere Gruppen im Wettbewerb zueinander. ${ }^{229}$ Darüber hinaus sind auch Einzelspiele denkbar, bei denen nur eine einzelne Person agiert. Für Einzelspiele eignen sich jedoch nur sehr einfache Planspiele. ${ }^{230}$

Im Hinblick auf die gegenseitige Beeinflußbarkeit der Entscheidungen untereinander kann zwischen interaktiven und nicht-interaktiven Planspielen unterschieden werden. Bei interaktiven Planspielen nehmen die Entscheidungen der jeweiligen Planspielgruppen Einfluß aufeinander. Das ist bei nicht-interaktiven Spielen nicht der Fall. Die Entscheidungen beeinflussen sich nicht gegenseitig. ${ }^{231}$ Interaktive Spiele können darüber hinaus offen oder geschlossen sein. In offenen Spielen sind die Spieler in der Lage, untereinander in Kontakt zu treten, während diese in geschlossenen Planspielen voneinander getrennt agieren. ${ }^{232}$ 1962), S. 53. Rohn verwendet in diesem Zusammenhang die Ausdrücke Hand- und Maschinenspiele. Vgl. Rohn, W.E., (Führungsentscheidungen 1964), S. 53.

231 Vgl. Bleicher, K., (Unternehmungsspiele 1962), S. 53; Koller, H., (Planspieltechnik 1969), S. 109; Koller, H., (Planspieltechnik 1974), S. 12.

232 Vgl. Rohn, W.E., (Führungsentscheidungen 1964), S. 52; Koller, H., (Planspieltechnik 1969), S. 110; Koller, H., (Planspieltechnik 1974), S. 12. 
Insgesamt ergibt sich die folgende Merkmalsübersicht:233 [Vgl. Abbildung 3-1].

\begin{tabular}{|c|c|c|c|}
\hline Unterscheidungsmerkmal & \multicolumn{3}{|c|}{ Ausprägung } \\
\hline Abstraktionsniveau & generell & speziell & hierarchisch \\
\hline Konkretisierungsgrad & \multicolumn{2}{|c|}{ abstrakt } & konkret \\
\hline Determinierung & \multicolumn{2}{|c|}{ deterministisch } & stochastisch \\
\hline Komplexität & einfach & komplex & $\begin{array}{l}\text { Anzahl an Ent- } \\
\text { scheidungen }\end{array}$ \\
\hline Variierbarkeit der Komplexität & \multicolumn{2}{|c|}{ herkömmlich } & modular \\
\hline $\begin{array}{l}\text { Vernetzungsgrad der Entschei- } \\
\text { dungen }\end{array}$ & \multicolumn{2}{|c|}{ Komplexität } & Kompliziertheit \\
\hline $\begin{array}{c}\text { Freiheitsgrad des Entscheidungs- } \\
\text { bereiches }\end{array}$ & \multicolumn{2}{|c|}{ starr } & frei \\
\hline Auswertung der Entscheidungen & \multicolumn{2}{|c|}{ manuell } & mputergestützt \\
\hline Anzahl der Teilnehmer & \multicolumn{2}{|c|}{ Einzelspiel } & onkurrenzspiel \\
\hline Stellung der Teilnehmer & \multicolumn{2}{|c|}{ interaktiv } & icht-interaktiv \\
\hline
\end{tabular}

\section{Abbildung 3-1: Arten von Unternehmensplanspielen}

\subsubsection{Ablauf von Unternehmensplanspielen}

Der Einsatz von Unternehmensplanspielen ist sehr aufwendig. So bedürfen sie bereits vor ihrem Einsatz in der Aus- und Weiterbildung einer umfangreichen Vorbereitung. Diese widmet sich vor allem der Auswahl und Beschaffung des geeigneten Modells, dessen Erprobung in Testläufen sowie der Qualifizierung des Spielleiters. Darüber hinaus ist aber auch für die räumliche Ausstattung zur Durchführung des Planspiels sowie für die Erstellung und Bereitstellung der erforderlichen Spielunterlagen zu sorgen. ${ }^{234}$

233 Vgl. zu weiteren Übersichten auch Prehm, H.-J., (Markus 1995), S. 11; Högsdal, B., (Planspiele 1996), S. 15; Hartung, S., (Förderung 2000), S. 56.

234 Vgl. hierzu sowie zu umfassenderen Ausführungen Rohn, W.E., (Führungsentscheidungen 1964), S. 82 ff. Vgl. auch Orth, C., (Unternehmensplanspiele 1999), S. 25 ff. und Prehm, H.-J., (MARKUS 1995), S. 19 ff. sowie die jeweils dort angegebene Literatur. 
Im Rahmen der eigentlichen Ausbildungsmaßnahme vollzieht sich ein Planspieleinsatz dann in drei Phasen. Diese Phasen stellen die Einführungsphase, die Spielphase sowie die Auswertungsphase dar. ${ }^{235}$

Zur zweckmäßigen Gestaltung der Einführungsphase sieht Ceppi drei Elemente als wesentlich an. Erstens wird den Teilnehmern eine vom Modell unabhängige, grundlegende Einführung in Unternehmensspiele gegeben. Zweitens werden sowohl praktische als auch theoretische Unternehmenszielsetzungen erläutert, da Zielsetzungen und quantitative Zielobjekte wesentlich für das Treffen von Entscheidungen sind. Drittens wird schließlich das jeweils vorliegende Modell bezüglich der Ausgangssituation sowie der Spielregeln erläutert. 236 In der Einführungsphase werden auch die Spielgruppen gebildet. ${ }^{237}$ Dabei spielt die Gruppengröße eine wichtige Rolle. Sie richtet sich nach dem vorliegenden Spielmodell,238 aber auch danach, eine effiziente Zusammenarbeit zu gewährleisten. So wird mit zunehmender Gruppengröße das Beziehungsgeflecht immer größer, so daß die Effizienz der Gruppe abnimmt. Heidack verdeutlicht dies durch Beziehungsdiagramme, in welchen die vielfältig möglichen Wechselbeziehungen ersichtlich werden. ${ }^{239}$ Es empfiehlt sich eine Gruppengröße von mindestens 3 bis 5 Teilnehmern, jedoch nicht mehr als 6 bis 10 Teilnehmern. ${ }^{240}$ Auch die Organisation innerhalb der Gruppe kann eine wesentliche Rolle spielen. Dies ist vor allem dann der Fall, wenn sie der Gruppe nicht selbst überlassen bleibt, sondern formal festgelegt wird. Dies geschieht dann in der Absicht, den Teilnehmern personelle Organisationsprinzipien zu vermitteln. ${ }^{241}$ Nach Bildung der Spielgruppen werden die Teilnehmer für gewöhnlich dazu aufgefordert, ihre Unternehmenspolitik schriftlich zu fixieren. ${ }^{242}$

Vgl. Orth, C., (Unternehmensplanspiele 1999), S. 30.

Vgl. Ceppi, C., (Erfahrungen 1970), S. 307.

Vgl. Bleicher, K., (Unternehmungsspiele 1962), S. 75.

Vgl. Ceppi, C., (Erfahrungen 1970), S. 307.

Vgl. Heidack, C., (Planspiel-Praxis 1980), S. 144 ff.

Vgl. Ceppi, C., (Erfahrungen 1970), S. 307.

Vgl. Rohn, W.E., (Führungsentscheidungen 1964), S. 88.

Vgl. Bleicher, K., (Unternehmungsspiele 1962), S. 75.
} 
Mit der Planung für die erste Periode beginnt die eigentliche Spielphase. ${ }^{243}$ Hier analysieren die Teilnehmer die aktuelle Situation und treffen auf dieser Basis ihre Entscheidungen. Diese Entscheidungen werden durch das Simulationsmodell verarbeitet und die erzielten Ergebnisse den Teilnehmern zur Verfügung gestellt. ${ }^{244}$ Neben der Beeinflussung des Spielverlaufs durch die Teilnehmer erfolgt auch eine Beeinflussung durch den Spielleiter. Diese sollte zunächst sehr gering gehalten werden. Im weiteren Spielverlauf bietet sich für den Spielleiter jedoch die Möglichkeit, Parameterveränderungen durchzuführen. ${ }^{245}$ Als problematisch erweist sich zumeist die Beendigung des Spiels. In dem Falle, daß das Ende des Spiels den Teilnehmern von vornherein bekannt ist, wird im Gegensatz zu einer vorherigen langfristigen Politik zum Ende des Spiels zumeist eine kurzfristige Politik betrieben. So werden kapitalintensive Maßnahmen wie bspw. Investitionen in Forschungs- und Entwicklungsprojekte oder Erweiterungen der Kapazität im Hinblick auf das Ende des Spiels eingestellt. ${ }^{246}$

Die Auswertungsphase wird von Rohn als die wichtigste Phase im Planspielverlauf betrachtet. Ihre Vorbereitung muß im wesentlichen vom Spielleiter während des Planspielverlaufes vorgenommen werden. Dabei kann dieser routinemäßig auf seine Erfahrungen zurückgreifen, muß aber auch die Aspekte berücksichtigen, die sich speziell in dem jeweiligen Spieldurchlauf ergeben. ${ }^{247}$ Auf dieser Grundlage werden dann der Spielverlauf und die erreichten Ergebnisse von der Spielleitung und den Teilnehmern gemeinsam analysiert. Solch eine Analyse kann durch den Vergleich der durch die Teilnehmergruppen verfolgten Strategien mit den erziel-

243 Vgl. Bleicher, K., (Unternehmungsspiele 1962), S. 76. Dabei lassen sich als Ausgangssituation zwei grundsätzliche Spieltypen unterscheiden. Zum einen kann von einem laufenden Geschäftsbetrieb ausgegangen werden. Zum anderen besteht die Möglichkeit, daß das Unternehmen erst von den Spielern zu gründen ist. Amerikanische Unternehmungsspiele basieren zumeist auf der ersten Ausgangssituation. Einiges spricht aber auch für die Gründungsvariante. So sind die Spieler zunächst mit einem übersichtlichen Entscheidungsrahmen konfrontiert. Dann erfolgt eine Aufteilung des Spielverlaufes in eine Gründungs-, Konsolidierungs- und Wettbewerbsphase. Schließlich wird eine langfristige Planung seitens der Spieler zwingend erforderlich. Vgl. ders., (Unternehmungsspiele 1962), S. 76.

Vgl. Geuting, M., (Planspiel 1992), S. 88.

245 Vgl. Ceppi, C., (Erfahrungen 1970), S. 308.

246 Vgl. Bleicher, K., (Unternehmungsspiele 1962), S. 77 f. Um die Spieler für eine Fortsetzung der langfristigen Unternehmenspolitik zu motivieren, empfiehlt Bleicher, in den letzten Spielperioden entsprechende Anreize zu schaffen. Vgl. ders., (Unternehmungsspiele 1962), S. 78.

Vgl. Rohn, W.E., (Führungsentscheidungen 1964), S. 99. 
ten Ergebnissen geschehen. ${ }^{248}$ Im Rückblick läuft das Spiel, gewissermaßen vor dem inneren Auge, noch einmal ab. ${ }^{249}$ Ziel ist es dabei, den Teilnehmern zu verdeutlichen, daß es in dynamischen Modellen wie einem Planspiel keine Musterlösung gibt, sondern unterschiedliche Strategien zu einer erfolgreichen Unternehmensleitung führen können. ${ }^{250}$ In dieser Phase kann auch Kritik an der Gestaltung des Planspiels geübt werden, die eventuell in einer Modifikation mündet. ${ }^{251}$

In diesem Zusammenhang führen interindividuelle Unterschiede bezüglich des Vorwissens beim Menschen zu einem unterschiedlich erfolgreichem Umgang mit komplexen Systemen. Es wurde bereits ausgeführt, daß es daher notwendig ist, den Teilnehmer einer Ausbildungsmaßnahme mit einer für ihn optimalen Komplexität zu konfrontieren, um einen optimalen Lernerfolg zu gewährleisten. ${ }^{252}$ Damit wird jedoch noch nichts über unterschiedliche Qualitäten des Wissens ausgesagt. Daher soll im folgenden auf die Expertiseforschung eingegangen werden, die diesen Umstand mit der Methode der Experten-Novizen-Vergleiche untersucht.

Die deutsche Expertiseforschung blickt auf eine 35-jährige Tradition zurück. Sie kann neben der amerikanischen Forschung, die im Bereich der Expertiseforschung

248 Vgl. Kaiser, F.-J.; Kaminski, H., (Methodik 1994). Dieser Strategie entspricht im wesentlichen auch das von Rohn als Muster beispielhaft ausgeführte Auswertungsschema des „Pillsbury Management Game“. Vgl. Rohn, W.E., (Führungsentscheidungen 1964), S. 100 ff.

249 Vgl. Geuting, M., (Planspiel 1992), S. 366.

250 Vgl. Geuting, M., (Planspiel 1992), S. 366. Insbesondere am Ende des Spiels treten die unterschiedlichen, oft auch gegensätzlichen Auffassungen und Argumente der Teilnehmergruppen anschaulich hervor. ,Wenn den Spielteilnehmern hierbei die subjektive Relativität dessen, was jeweils als 'Wahrheit' angesehen wurde, selbstkritisch bewußt wird, sind sie vielleicht eher bereit, angesichts prinzipieller Erfahrungsgrenzen ihren eigenen Urteilen zu mißtrauen.“ ders., (Planspiel 1992), S. 366.

251 Vgl. Kaiser, F.-J.; Kaminski, H., (Methodik 1994). Im Unternehmensplanspiel gibt es keine Gewinner oder Verlierer. Im Vordergrund steht der Lerneffekt. So hat die Spielkritik den Sinn, Fehler aufzudecken, jedoch im eigentlichen Sinne nicht den Charakter einer Bewertung. Vgl. Ceppi, C., (Erfahrungen 1970), S. 308. 
durchaus eine Vorreiterrolle innehat, als führend in der Expertiseforschung angesehen werden. ${ }^{253}$

In den vergangenen zehn Jahren hat die Expertiseforschung nun eine zunehmende Bedeutung als eine Domäne psychologischer Forschung erfahren. ${ }^{254}$ Durch die kognitionspsychologische Orientierung erhielt die Expertiseforschung die entscheidenden Impulse. Dort erfaßte man in spezifischen Studien interindividuelle Unterschiede beim Lösen von Problemen, die auf unterschiedliches Vorwissen zurückzuführen zu sein schienen. ${ }^{255}$

In der Literatur findet sich keine explizite Definition des Expertenbegriffes oder des Begriffs der Expertise. Eher ist sie eine implizite Einschätzung, die sehr stark mit dem jeweiligen Untersuchungsdesign korreliert. ${ }^{256}$ Nach den Befunden der Expertise-Forschung beruhen die kognitiven Leistungen von Experten fast ausschließlich auf dem bereichsspezifischen Wissen der Experten. ${ }^{257}$ Die Meinungen dazu, ob Expertise in unterschiedlichen Bereichen als generell vergleichbar anzusehen ist oder nicht, sind jedoch geteilt.

So führten Larkin, McDermott, Simon \& Simon Untersuchungen in unterschiedlichen Domänen durch und halten die Expertise in den verschiedenen Domänen für prinzipiell vergleichbar. ${ }^{258}$ Anderson sieht Expertise hingegen durch spezifische Domänencharakteristika bestimmt. So führt er die folgende Sicht eines Experten an: „Wir sehen Experten als Menschen an, die ein umfassendes Wissen über Konzepte in ihrer Expertisedomäne haben $[\ldots]^{] “ .259}$

253 Vgl. Gruber, H.; Ziegler, A., (Expertise 1996), S. 7. Die Expertiseforschung weist einen engen Zusammenhang zur Begabtenforschung auf, mit der sie das Interesse an der Leistungseminenz teilt. Während die Begabtenforschung jedoch fragt: „Wo gehen meine Begabten hin?“, fragt die Expertiseforschung „Woher kommen meine Experten?“. dies., (Expertise 1996), S. 7.

254 Vgl. Gruber, H.; Ziegler, A., (Expertise 1996), S. 10.

255 Vgl. Abschnitt 3.3.3: Untersuchungsdesigns zum Umgang von Versuchspersonen mit komplexen betriebswirtschaftlichen Systemen.

256 Vgl. Gruber, H.; Ziegler, A., (Expertise 1996), S. 8.

257 Vgl. Ceci, S.J.; Liker, J.K., (Study 1986), S. 255 ff.

258 Vgl. Larkin, J.; McDermott, J.; Simon, D.P.; Simon, H.A.,(Expert 1980), S. 1336.

259 Vgl. Anderson, J.R., (Character 1990), S. 70. 


\subsection{2}

\section{Kontrastive Methode der Experten-Novizen-Vergleiche}

Durch die Untersuchungen von Personen bezüglich ihres Umgangs mit komplexen Systemen gewann die Methode der Experten-Novizen-Vergleiche ein besonderes Gewicht innerhalb der Expertiseforschung. Die Experten-NovizenForschung verfolgt in diesem Zusammenhang das Ziel, festzustellen, wie sich Personen unterschiedlicher Wissensvoraussetzungen bei der Bearbeitung von Problemen unterscheiden. ${ }^{260}$ Experten-Novizen-Vergleiche werden dabei bereits in unterschiedlichsten Domänen, wie der Mathematik, ${ }^{261}$ der Physik, ${ }^{262}$ der Politik ${ }^{263}$ oder auch der Computerprogrammierung, ${ }^{264}$ durchgeführt.

Experten-Novizen-Vergleiche sind querschnittlich angelegt und arbeiten mit dem kontrastiven Ansatz, der im Prinzip sehr einfach ist. So ist es im allgemeinen bei jeder Aufgabe so, daß diese von einem Teil der Personen gut, von einem anderen schlecht gelöst wird, so daß mit kontrastiven Untersuchungen die folgenden Ziele verfolgt werden können: 265

ERSTES ZIEL Welches Wissen und welche Fähigkeiten haben diejenigen Personen, die Aufgaben gut bewältigen? Welche Kompetenz weisen diejenigen auf, die Aufgaben schlecht bewältigen?

ZWEITES ZIEL Wie können jene Personen, die Aufgaben unbefriedigend erledigen, zu Experten werden?

DRITTES ZIEL Was charakterisiert Experten - Fähigkeiten, und wie ist deren Simulation möglich?

260 Vgl. Fürstenau, B., (Problemlösen 1994), S. 23.

261 Vgl. bspw. Lewis, C., (Skill 1981).

262 Vgl. bspw. Chi, M.T.H.; Feltovich, P.J.; Glaser, R., (Categorization 1981); Hardiman, P.T.; Dufresne, R.; Mestre, J.P.,(Relation 1989).

263 Vgl. Fiske, S.T.; Kinder, D.R.; Larter, W.M.,(Novice 1983).

264 Vgl. bspw. Adelson, B., (Problem 1981); Adelson, B., (Difficulty 1984); Weiser, M.; Shertz, J., (Problem 1983). Weiterhin gibt Allwood einen Überblick über den Stand der Forschung zum Umgang von Novizen mit Personalcomputern, mit gelegentlichen Vergleichen zu Experten. Vgl. Allwood, C.M., (Novices 1986).

265 Vgl. Voss, J.F.; Fincher-Kiefer, R.H.; Greene, T.R.; Post, T.A.,(Performance 1986), S. 298. 
Untersuchungsdesigns zum Umgang von Personen mit komplexen betriebswirtschaftlichen Systemen

In diesem Zusammenhang kontrastiert die Experten-Novizen-Forschung in ihren Untersuchungen Experten, Laien und fachfremde Experten. Dabei wird davon ausgegangen, daß sich mit zunehmender Erfahrung beim Problemlösen die Organisationsstruktur des Wissens wandelt; die Personen daher über unterschiedliche Wissensvoraussetzungen verfügen. Weiter wird davon ausgegangen, daß der Vorgang des Problemlösens als Anwendung und Erwerb von Wissen anzusehen ist, ${ }^{266}$ wobei Anwendung und Erwerb von Wissen in bezug auf verschiedene Wissensarten erfolgen, deren Abgrenzung innerhalb des menschlichen kognitiven Systems wegen unterscheidbarer Funktionen des jeweiligen Wissens in der Kognitionspsychologie anerkannt ist. ${ }^{267}$

Hier existieren auch Untersuchungen, die auf betriebswirtschaftlichen Sachverhalten basieren. Bevor jedoch einige solcher kontrastiven Untersuchungen, die auf betriebswirtschaftlichen Sachverhalten beruhen, vorgestellt werden, soll an dieser Stelle kurz auf einige unterschiedliche Wissensarten eingegangen werden, die als bedeutsam erachtet werden:268

Zunächst nimmt die Unterscheidung zwischen deklarativem und prozeduralem Wissen eine zentrale Stellung ein. ${ }^{269}$ Nach Putz-Osterloh sind diese zwei Wissensarten auch grundlegend für das Problemlösen. So dient das deklarative $\mathrm{Zu}-$ standswissen dazu, Problemzustände zu identifizieren, was bedeutet, daß sie verstanden werden. Veränderungen in Hinblick auf den „Lösungszustand“ können jedoch erst unter Aktivierung des operativen Veränderungswissens durchgeführt werden. Gemeinsam bestimmen beide Wissensarten die Beschaffenheit und den Ablauf des Problemlösens: „Von Zustandsrepräsentationen ist abhängig, welche verfügbaren Operationen anwendbar sind - und verfügbare Operationen determi-

266 Vgl. Abschnitt 5.2: Komplexes Problemlösen und Wissen.

267 Vgl. Oswald, M.; Gadenne, V.,(Wissen 1984), S. 178 ff.

268 Vgl. ausführlich dazu: Kapitel 5: Das Unternehmensplanspiel als Lehr-Lern-Instrument.

269 Deklaratives Wissen kann durch Mitteilung erworben werden, ist abrufbar und verbalisierbar; prozedurales Wissen wird durch Übung angeeignet, seine Einzelkomponenten sind nicht in gleicher Weise in das Bewußtsein abrufbar und verbalisierbar. Vgl. Oswald, M.; Gadenne, V., (Wissen 1984), S. 173. 
nieren, welche Zustände erreichbar sind“. ${ }^{270}$ Das Veränderungswissen kann weiterhin nach der Bereichsspezifität unterschieden werden. Dies ist eine Unterscheidung nach bereichsspezifischen Algorithmen sowie allgemeinen heuristischen Strategien. ${ }^{271}$ Auch sie spielen sowohl bei der Problembearbeitung als auch beim Wissenserwerb eine tragende Rolle. Dabei beinhaltet heuristisches Wissen ein Repertoire an sehr allgemein anwendbaren Operatoren zum Umgang mit unbekannten, komplexen und dynamischen Systemen. Vor allem die konsequente Analyse der Problemsituation, die verstärkte Beachtung von Systemstrukturen, die Explikation von Hypothesen und die Planung des eigenen Vorgehens. ${ }^{272}$

\section{SIMULATIONSDESIGN MANUTEX \& MORO}

In einer von Strohschneider \& Schaub durchgeführten Studie mit den Simulationen MANUTEX ${ }^{273}$ und MORO $^{274}$ wurde das Problemlöseverhalten von 45 Studenten aus vorwiegend geistes- und sozialwissenschaftlichen Fächern mit dem von 45 erfahrenen Wirtschaftsmanagern verglichen. ${ }^{275}$ Der Vergleich geschah hier unter Heranziehung von Gütemaßen zur Problemlösegüte. Im Hinblick auf das Szenario MORO wurde dazu ein von Putz-Osterloh entwickeltes Verfahren adaptiert, das die Versuchspersonen an Hand von sechs Zentralvariablen in Gütegruppen einsortiert. ${ }^{276}$ Für das Szenario MANUTEX wird ,[...] mit eindeutig »kapitalistischer«

\footnotetext{
270 Putz-Osterloh, W., (Experten 1987), S. 63.

271 Vgl. Putz-Osterloh, W., (Experten 1987), S. 63.

272 Vgl. Strohschneider, S., (Wissenserwerb 1990), S. 94 ff.

273 Es handelt sich bei MANUTEX um eine interaktive Simulation einer kleinen Textilfabrik in Kuala Lumpur, in der die Beschäftigten, dreißig Arbeiter und Angestellte, Blusen, Hemden und Hosen produzieren. Die Versuchsperson in der Funktion eines jungen deutschen Managers hat diese von ihrem Onkel geerbt und hat nun die Aufgabe, das Unternehmen, wdches sich durch den Tod des Onkels in einem problematischen Zustand befindet, während einer Periode von 24 Monaten zu managen. Während dieser Zeit soll sie Profite erzielen und - wenn möglich - die Anzahl der Beschäftigten sowie die Arbeitsentgelte erhöhen. Dazu steht ihr zunächst eine Summe von einer Million malaiischen Dollar zur Verfügung. Vgl. Schaub, H., (Fehler 2001), S. 3; Ramnarayan, S.; Strohschneider, S.; Schaub, H., (Trappings 1997), S. 30 f Hier wurde die von Schaub entwickelte Simulation MANUTEX für die von Strohschneider \& Schaub durchgeführte Untersuchung modifiziert. Durch diese Modifikation wurde eine Anforderungssituation geschaffen, die dem speziellen Fachwissen der Manager-Untersuchungsgruppe entsprach. Vgl. Strohschneider, S.; Schaub, H., (Manager 1991), S. 327 f. und die dort angegebene Literatur.

274 Bei MORO handelt es sich um die Simulation des Lebens eines Halbnomadenstammes am Südrand der Sahelzone. Vgl. Strohschneider, S.; Schaub, H.,(Manager 1991), S. 327.

275 Vgl. Strohschneider, S.; Schaub, H.,(Manager 1991), S. 327.

276 Vgl. Strohschneider, S.; Schaub, H., (Manager 1991), S. 329 und die dort angegebene Literatur.
} 
Zielvorgabe das Flüssigkapital und das Gesamtvermögen der Firma“ herangezogen. 277

In einer ersten Untersuchung wurde nur das Szenario MORO eingesetzt. Dieses Simulationsdesign weist einige zentrale Schwierigkeiten auf. Zum einen müssen die Versuchspersonen umfangreiche Informationen verarbeiten und $\mathrm{zu}$ einem möglichst geschlossenen Gesamtbild der betrachteten Situation zusammenfügen. Zum anderen ist es bei bestimmten Maßnahmen möglicherweise schwierig, deren langfristige Auswirkungen abzuschätzen. Außerdem muß die gegenseitige Beeinflussung der Variablen untereinander generell ausbalanciert werden. Dieses Szenario war von allen Probanden aus beiden Gruppen zu absolvieren. Da die Teilnehmer bezüglich dieses Szenarios über keinerlei spezifisches Wissen verfügten, wurde davon ausgegangen, daß die Versuchspersonen auf der Grundlage ihres allgemeinen »Weltwissens« werden operieren müssen und sich Unterschiede im Hinblick auf den Umgang mit der Problemstellung auf unterschiedliche heuristische Strategien zurückführen lassen. ${ }^{278}$

In einer Teilstichprobe mit 23 Managern und einer neuen Stichprobe von 25 Studenten wurde deren Steuerungsverhalten verglichen. Zusätzlich wurde hier neben MORO die Simulation MANUTEX eingesetzt. Die zentrale Schwierigkeit dieses Szenarios liegt vor allem darin, daß der Zeithorizont von zweieinhalb Stunden zu knapp bemessen ist. Daher ist es nicht möglich, alle relevanten Daten zu sammeln und zu integrieren. Es besteht somit die Notwendigkeit, oberflächlicher zu handeln oder sich auf Teilgebiete zu konzentrieren. Während die studentische Gruppe nur MANUTEX steuerte, steuerten die 23 Manager zunächst MORO und später MANUTEX. Bei MANUTEX können die Manager nun auch verstärkt unter Einsatz ihres Fachwissens operieren. Dadurch ergibt sich die Möglichkeit, Interaktionen zwischen bereichsspezifischem und heuristischem Wissen zu untersuchen. ${ }^{279}$

Die Auswertung der Untersuchungen ergab die folgenden Ergebnisse: Die Manager explorieren die Problemstellung bei beiden Versuchsanordnungen intensiver und realisieren dadurch die Unbestimmtheit des Problems. In Folge dessen reagie- 
ren sie - im Gegensatz zu den Studenten, die zu einem wagemutigeren, aktionistischeren Vorgehen neigen - mit entsprechender Zurückhaltung. Dieser durchgehende Gegensatz in der Strategie der Beschäftigung mit dem Szenario wird von Strohschneider \& Schaub als primär auf das unterschiedliche heuristische Wissen zurückzuführend, interpretiert. So sammeln Manager im Verlauf ihrer praktischen beruflichen Tätigkeit im Umgang mit verschiedenartigen Problemen ein Potential an unterschiedlichen Strategien an. Sie analysieren das Problem zunächst mit der Intention, die entsprechende Handlungsstrategie zu generieren und operieren erst dann auf Grundlage derselben. Dieses Wissen ist bei den Studenten weniger ausgeprägt. So läßt die Tatsache, daß die Studenten weniger Zeit auf die Exploration des Problems verwenden, Rückschlüsse darauf $\mathrm{zu}$, daß es ihnen nicht notwendig erscheint, ihre Vorgehensweise an die konkrete Problemsituation anzupassen. Im Design MANUTEX gestalten die Manager ihre globale Vorgehensweise, deren Wahl offenbar nicht vom Fachwissen abhängig ist, zusätzlich durch den gezielten Einsatz bereichsspezifischen Wissens inhaltlich aus. ${ }^{280}$

\section{SIMULATIONSDESIGN TEXTILFABRIK}

In einer Untersuchung mit dem ökonomischen Simulationsprogramm TEXTILFABRIK verglich Hasselmann 17 Führungsnachwuchskräfte einer Bank, 11 Studenten der Betriebswirtschaftslehre und 41 Studenten diverser Fachrichtungen bezüglich ihrer Steuerungsleistung.

Das Simulationsprogramm TEXTILFABRIK simuliert eine kleinere Textilfabrik, die Hemden produziert. Aufgabe der Versuchspersonen ist es, diese Fabrik über einen vorgegebenen simulierten Zeitraum zu leiten. Die Versuchspersonen können über insgesamt 16 unterschiedliche Eingriffsvariablen Einfluß auf das System nehmen. Sie entscheiden bspw. über Rohstoffeinkauf, Mitarbeiterbeschaffung und entlassung, Maschineneinkauf und -verkauf. Dabei ist es ihr Ziel, das Firmenkapital zu maximieren.

Die Bankangestellten steuerten das System signifikant besser als die gemischte Studentengruppe. Im Vergleich zu den BWL-Studenten war die Leistung der 
Banker nur wenig besser, das vorab festgelegte Signifikanzniveau wurde nur knapp verfehlt. Der Unterschied zwischen den Steuerungsleistungen der beiden Studentengruppen war nicht signifikant. Wie Strohschneider \& Schaub gelangt Hasselmann zu dem Ergebnis, daß die Überlegenheit der Banker aus dem umfangreichen heuristischen Wissen resultieren müsse. Aus den Leistungen der beiden Studentengruppen, welche sich nicht unterschieden, schließt er, daß fachspezifisches Wissen keine oder nur eine geringfügige Bedeutung für die Steuerungsleistung hat. ${ }^{281}$

\section{SIMULATIONSDESIGN SCHOKOMAX}

Bei SCHOKOMAX handelt es sich um die Simulation eines mittelständischen Unternehmens. Diese realitätsnahe und sehr komplexe Simulation einer Schokoladenfabrik mit 100 Mitarbeitern und einem Umsatz von 25 Millionen ist von den Versuchspersonen über einen simulierten Zeitraum von 12-24 Monaten zu leiten.

Reichert \& Stäudel verglichen die Leistungen und Vorgehensweisen von Managern, Wirtschaftsstudenten und Laien. ${ }^{282}$ Nach einem komplexen Gütemaß ${ }^{283}$ erzielten die Manager signifikant bessere Steuerungsleistungen als die beiden Studentengruppen. Die Leistung der Manager korreliert dabei mit ihrem strategischen Verhalten. So nahmen diese eine wesentlich intensivere Systemanalyse vor, wodurch sie einen besseren Überblick erzielten als die Vergleichsgruppen, ohne sich dabei im Detail zu verlieren. ${ }^{284}$

\section{SIMULATIONSDESIGN SCHNEIDERWERKSTATT \& MORO}

Putz-Osterloh untersuchte, wie sich heuristisches Wissen auf Wissenserwerb und Problemlöseleistung auswirkt. Die SCHNEIDERWERKSTATT ist ein betriebswirtschaftlich orientiertes Szenario, in dem Hemden produziert und verkauft werden

281 Vgl. Hasselmann, D., (Problemstellungen 1993), S. 166 ff. Das Simulationsdesign TEXTILFABRIK wird auch in Assessmentcentern zur Personalauswahl und Personalentwicklung von Kreditinstituten eingesetzt. Vgl. Hasselmann, D., (Einsatz 1990), S. 64 f.

Sie verwendeten für ihre Diagnose durch Maschinen automatisch bereitgestellte Protokolle, die System- und Verhaltensdaten auswiesen. Außerdem nahmen geschulte Experten auf Grundlage von wissenschaftlich entwickelten Diagnosesystemen eine Verhaltensbeobachtung vor. Vgl. Reichert, U.; Stäudel, T., (Diagnostik 1991), S. 103.

Hier wird zum Vergleich der Verlauf der flüssigen Mittel über zwölf simulierte Monate dargestellt. Das komplexe Gütemaß wurde aus Platzgründen nicht weiter ausgeführt. Vgl. Reichert, U.; Stäudel, T.,(Diagnostik 1991), S. 104. 
müssen. Im Szenario MORO geht es - wie bereits dargestellt - darum, die Lebensbedingungen eines Nomadenstammes zu verbessern. Aufgabe der Versuchspersonen ist es, in das System im Hinblick auf eine Veränderung der Zielvariablen ${ }^{285}$ einzugreifen. Dabei bekleidet die Versuchsperson im Szenario SCHNEIDERWERKSTATT die Funktion der Geschäftsleitung, im Szenario MORO die eines Entwicklungshelfers. ${ }^{286}$

Putz-Osterloh verglich sieben Professoren der Betriebswirtschaftslehre mit dreiBig unausgewählten Studenten, wobei von den Versuchspersonen zunächst das System SCHNEIDERWERKSTATT und dann das MORO-System in jeweils zwei- bis dreistündigen Sitzungen bearbeitet wurde. ${ }^{287}$

Bei der Auswertung der Ergebnisse dienen die im Hinblick auf die Zielvariablen erzielten Werte als Gütekriterien. Bei der Bearbeitung der SCHNEIDERWERKSTATT schneiden die Experten hinsichtlich aller drei Gütekriterien besser ab als die Laien. Dies ist bei MORO nicht der Fall. Hier ergeben sich hinsichtlich der Gütekriterien keine Unterschiede zwischen den beiden Gruppen. ${ }^{288}$ Außerdem wurde anhand der Protokolle lauten Denkens das Systemwissen erhoben. ${ }^{289}$ Da den Versuchspersonen gegenüber keine Angaben bezüglich der Verknüpfungen im System gemacht wurden, waren sie aufgefordert, selbst Hypothesen über Systemvariablen und deren Verknüpfungen vorzunehmen. Diese Hypothesen können dann anhand der Reaktionen des Systems überprüft werden, wodurch Ausbau und Korrektur des Systemwissens gelingt. ${ }^{290}$ Es konnte festgestellt werden, daß die Gruppen in beiden Szenarien hinsichtlich des Systemwissens unterschiedlich abschneiden. Sowohl bei der SCHNEIDERWERKSTATT als auch bei MORO analysieren

Im System SCHNEIDERWERKSTATT bekommen die Versuchspersonen die folgenden Zielkriterien vorgegeben: erstens der am Ende der Bearbeitung ausgewiesene Kontostand; zweitens die Gewinne, die im Verlauf der Bearbeitung erzielt werden; drittens der an die Mitarbeiter gezahlte Lohn. Im System MORO sind durch die Versuchspersonen folgende Zielvariablen zu beachten: erstens der Umfang des Weidelandes, zweitens die Zahl der Rinder sowie die der MOROS, drittens das vorhandene Grundwasser, viertens das vorhandene Kapital. Vgl. Putz-Osterloh, W., (Experten 1987), S. 67.

Vgl. Putz-Osterloh, W., (Experten 1987), S. 63 f.

Vgl. Putz-Osterloh, W., (Experten 1987), S. 70.

Vgl. Putz-Osterloh, W., (Experten 1987), S. 73 ff.

Es wurde für beide Szenarien geprüft, ob die Professoren mehr Systemwissen erwerben als die Studenten, und ob die Professoren über mögliche Variablen mehr Ideen generieren. Die Interkorrelationen sollten Aufschluß darüber geben, ob der Wissenserwerb generalisierbar ist. Vgl. Putz-Osterloh, W., (Experten 1987), S. 72. 
die Experten mehr Zusammenhänge zwischen Variablen und erkennen mehr richtige Zusammenhänge im System. ${ }^{291}$ Dies gilt auch für die eingesetzten Problemlösestrategien. Die Experten bilden mehr Hypothesen als die Laien, sie analysieren die Probleme genauer, wobei ihr Vorgehen geplanter erfolgt. ${ }^{292}$

Insgesamt lassen sich die Ergebnisse wie folgt zusammenfassen: Wirtschaftswissenschaftliche Experten unterscheiden sich sowohl bei einem ökonomischen als auch bei einem ökologischen System von studentischen Nichtexperten. So unterschieden sich der Wissenserwerb als auch die Problemstrategien der Experten wie erwartet von studentischen Nichtexperten. Das führt dazu, daß die Experten insgesamt systematisch bessere Leistungen erzielen. Dabei lassen sich diese Unterschiede nicht durch bereichsspezifisches Wissen erklären. Sie müssen auf generalisierbares Systemwissen sowie auf Unterschiede in heuristischen Strategien zurückgeführt werden. ${ }^{293}$ Es läßt sich daher festhalten:

$>$ Die Experten-Novizen-Forschung kontrastiert in ihren Untersuchungen Experten und Novizen.

$>$ Expertenwissen stellt eine notwendige Voraussetzung für das Problemlösen dar.

$>$ Ein betriebswirtschaftlicher Experte kann definiert werden als eine Person, die sowohl über ein umfangreiches bereichsspezifisches Wissen, als auch über generalisierbares Systemwissen sowie weiterhin heuristisches Wissen verfügt.

$>$ Die Denkpsychologie betrachtet den Menschen im Umgang mit komplexen Systemen als einen Problemlöser. Der Problemlöseprozeß wird dabei als Anwendung und Erwerb von Wissen angesehen.

Nun stellt der Mensch aufgrund seiner Fehlleistungen den eigentlich zu eliminierenden Faktor in Systemen dar. Dabei haben die ausgeführten Untersuchungen gezeigt, daß davon auszugehen ist, daß Novizentum in einem stärkeren Umfang von Fehlleistungen geprägt ist als Expertentum. Wurde ein angestrebtes Ergebnis

290 Vgl. Putz-Osterloh, W., (Experten 1987), S. 64.

291 Vgl. Putz-Osterloh, W., (Experten 1987), S. 77. Dies, obgleich sie sich hinsichtlich ihres Frageverhaltens nicht von den Studenten unterscheiden, d.h. nicht mehr Informationen durch häufigeres Fragen erhalten. Vgl. dies., (Experten 1987), S. 80. 
nicht erzielt, so ergibt sich die Notwendigkeit, sein bisheriges Verhalten zu reflektieren und in Beziehung zu den erzielten Ergebnissen zu setzen. Es ist notwendig, die Ursache für das verfehlte Ergebnis zu ermitteln. Dazu sind die Fehler in einen Zusammenhang zu dem sie erzeugenden Verhalten zu bringen; und fehlerhaftes Verhalten zu modifizieren.

Hier wird daher davon ausgegangen, daß Fehlleistungen sowohl das Ende eines verfehlten Problemlöseprozesses als auch Anfang eines erneuten Problemlöseversuches sein können; und demnach auch »Motor« für den kognitiven Entwicklungsprozeß sein können. Hier ist es wichtig Aufschluß über Systemmerkmale von Unternehmensplanspielen sowie typische Fehler im Umgang mit komplexen Systemen zu gewinnen. Daher wird im folgenden der Versuch unternommen, betriebswirtschaftliche Erkenntnisse zu Unternehmensplanspielen sowie Komplexer Problemlöseforschung zu Fehlleistungen den im S-H-E-L-L - Modell modellierten Systemkomponenten zuzuordnen. Dadurch soll es gelingen, das betriebswirtschaftliche Handlungsfeld und in diesem mögliche Fehlleistungen näher zu spezifizieren. 


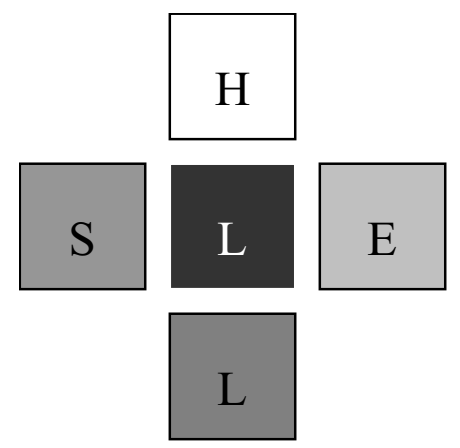

\subsection{1}

\section{Die Natur menschlichen Fehlverhaltens}

Die Voraussetzung für eine menschliche Handlung bildet die menschliche Entscheidung. Sie, die menschliche Entscheidung, wirkt sich täglich in realen Situationen auf unterschiedlichste Systeme aus. In Folge getroffener Entscheidungen in wirtschaftlichen, politischen, technischen oder anderen Systemen kann es zu Katastrophen, Fehlentwicklungen, Stör- und Unglücksfällen kommen. Dies gibt in der Regel Anlaß dazu zu fragen, was Menschen falsch gemacht haben könnten.294 Besonders technische Systeme sind oftmals mit einem hohen Risiko für Menschen verbunden. Hier haben Stör- und Unglücksfälle, die durch Fehler und Irrtümer von Menschen bei der Systemkontrolle und -steuerung verursacht wurden, verstärkt die Untersuchung der kognitiven Strukturen und Prozesse von Menschen in den Mittelpunkt des Interesses gerückt. ${ }^{295}$ So haben statistische Untersuchungen von Katastrophen und Unglücken gezeigt, daß menschliches Versagen zu 60-90 Prozent deren Auftreten verursacht. Demgemäß hat sich eine Sichtweise durchgesetzt, daß die Menschen die eigentlich kritischen Systemkomponenten darstellen, die es zu ersetzen gilt. ${ }^{296}$

Ein Fehler kann zunächst grundlegend als eine beabsichtigte Handlung betrachtet werden, die in einem nicht beabsichtigtem Sinne ausgeführt wurde. Auf einer einfachen, aber oftmals unverständlichen Ebene, sind Fehler solche Handlungen, die

\footnotetext{
294 Vgl. Kluwe, R.H., (Kontrolle 1990), S. 241 ff.

295 Vgl. Kluwe, R.H.; Haider, H., (Modelle 1990), S. 174.

296 Vgl. Kluwe, R.H., (Analysen 1991), S. 275.
} 
durch verschiedene bewußte oder unbewußte Fehler genährt werden. ${ }^{297}$ Auf einer höchst transparenten Ebene können Fehler als jegliche Handlungen betrachtet werden, die zu Zwischen- oder Unglücksfällen führen, die menschliche Verletzungen, Tod oder Sachschaden nach sich ziehen. ${ }^{298}$

Dabei sind Fehler für gewöhnlich sehr schwer zu entdecken. Werden sie entdeckt, dann zumeist erst spät. ${ }^{299}$ Von der Kognitionspsychologie wird in diesem Zusammenhang erwartet, daß sie auf Grund ihrer Forschungen im Bereich menschlicher Informationsverarbeitung Entscheidungsverhalten erklären kann, vielleicht sogar einen Beitrag dazu leisten kann, menschlichen Fehlern entgegenzuwirken. ${ }^{300}$

\subsection{2}

\section{Das S-H-E-L-L - Modell als eine Möglichkeit zur Beschreibung der Natur menschlichen Fehlverhaltens}

Aus der grundlegenden Simulationstechnik beim Unternehmensplanspiel, ${ }^{301}$ haben sich unterschiedliche Planspielarten entwickelt, so daß sich die Planspielteilnehmer in jeweils unterschiedlichen experimentellen Planspielsituationen befinden. ${ }^{302}$ Die objektiven Charakteristika von Unternehmensplanspielen stehen dabei in Wechselwirkung mit dem subjektiven Verständnis der Planspielteilnehmer. In einer extremen Ausprägung führt dieses subjektive Verständnis zu Fehlverhalten im Umgang mit komplexen Systemen. So ist der Mensch der eigentlich zu eliminierende Faktor in Systemen. ${ }^{303}$ Die Forschung zum Komplexen Problemlösen unternimmt in diesem Zusammenhang den Versuch, Fehlleistungen, die beim Umgang mit komplexen Systemen auftreten, aufzudecken sowie deren Hintergründe und Ursachen zu klären. ${ }^{304}$ Dabei werden auch diese Untersuchungen an unterschiedlichen experimentellen Designs durchgeführt.

\footnotetext{
297 Vgl. Isaac, A.R.; Ruitenberg, B., (Air 1999), S. 11.

298 Vgl. Isaac, A.R.; Ruitenberg, B.,(Air 1999), S. 11.

299 Vgl. Kluwe, R.H., (Analysen 1991), S. 277.

300 Vgl. Kluwe, R.H., (Analysen 1991), S. 275.

301 Vgl. Abschnitt 2.1.2: Simulationstechnik beim Unternehmensplanspiel.

302 Vgl. Abschnitt 3.2.1: Arten von Unternehmensplanspielen.

303 Vgl. Abschnitt 3.4.1: Die Natur menschlichen Fehlverhaltens.

304 Vgl. Abschnitt 2.3.1: Komplexes Problemlösen - Ein Aufriß.
} 
Hier soll nun die betriebswirtschaftliche und kognitionspsychologische Forschung in einen gemeinsamen Bezugsrahmen gestellt werden, wobei es sich als problematisch erweist, daß im Zusammenhang mit Unternehmensplanspielen von unterschiedlichen Modellsituationen auszugehen ist sowie weiterhin in der psychologischen Forschung unterschiedliche experimentelle Designs herangezogen werden.

Um in diesem Zusammenhang eine Gegenüberstellung vornehmen zu können, ist zunächst zu berücksichtigen, daß dem Unternehmensplanspiel als einer allgemeinen Methode der Systembegriff der Allgemeinen Systemtheorie zu Grunde gelegt werden konnte. ${ }^{305}$ Der Grundgedanke der Allgemeinen Systemtheorie war, „[...], daß materiell verschiedenartige Objektsysteme häufig durch formal-isomorphe Systemgesetze erklärt werden können. Entsprechend sieht sie ihre Aufgabe darin, formale Isomorphien in den Strukturen von Theorien über materiell unterschiedliche Sachverhalte aufzudecken, in einer einheitlichen Terminologie zu beschreiben und $\mathrm{zu}$ interdisziplinär verwendbaren, generalisierten Theoriesystemen zusammenzufassen, wobei eine mathematische Formulierung dieser Theoriesysteme angestrebt werden soll." ${ }^{306}$ So können, bedingt durch die von Bertalanffy vollzogene Verallgemeinerung des Offenen-System-Konzepts, neben der Erklärung naturwissenschaftlicher Sachverhalte auch nicht-naturwissenschaftliche Problemstellungen anhand des Modells und der Theorie offener Systeme abgehandelt werden. ${ }^{307}$

In diesem Sinne kann die Allgemeine Systemtheorie nach Luhmann auch als eine »Supertheorie« aufgefaßt werden. ${ }^{308}$ Oder nach Boulding als das Skelett der Wissenschaften: „Die Theorie allgemeiner Systeme ist in dem Sinn ein Skelett der Wissenschaften, als sie die Struktur von Systemen darstellt, die Fleisch und Blut unterschiedlicher Fächer zusammenhält, d.h. die Forschungsgegenstände in einem

305 Vgl. Abschnitt 2.1.2.1: Das Unternehmensplanspiel: Ein Simulationsmodell.

306 Grochla, E., (Systemtheorie 1970), S. 6 ff.

307 Vgl. Fuchs, H., (Systemtheorie 1969), Sp. 1623. In diesem Zusammenhang wird oft eingewendet, daß die Gefahr besteht, daß eine Allgemeine Systemtheorie in bedeutungslosen Analogien endet. Bertalanffy selbst diskutiert diesen Einwand und führt aus, daß diese Gefahr in der Tat besteht. So ist es zum Beispiel eine weitverbreitete Idee, einen Staat oder dne Nation als einen übergeordneten Organismus zu betrachten. Eine solche Theorie würde das Fundament eines totalitären Staates konstituieren, in dem das menschliche Individuum eine unbedeutsame Zelle in einem Organismus oder ein unwichtiger Arbeiter in einem Bienenstock wäre. Vgl. Bertalanffy, L. v., (System 1988), S. 35. 
geordneten oder zusammenhängenden Wissenskörper vereint. “309 In diesem Zusammenhang ist auch die Unternehmung einer Anwendung der allgemeinen Systemtheorie zugänglich. ${ }^{310}$

Unter explizitem Bezug auf die Allgemeine Systemtheorie besteht daher die Möglichkeit, das Unternehmensplanspiel sowie die Natur menschlichen Fehlverhaltens in Zusammenhang mit Basiselementen eines Systems zu beschreiben. ${ }^{311}$ Dazu wird ein Modell, welches von der International Civil Aviation Organisation [ICAO] genutzt wurde, herangezogen. Es handelt sich hierbei um ein MenschMaschine-Modell, wodurch die komplexe Interaktion zwischen dem Menschen und der Maschine dargestellt werden kann. ${ }^{312}$

Dieses Modell wurde von Edwards entwickelt und bekannt als das SHEL-Modell. Im SHEL-Modell geht Edwards davon aus, daß Systeme sich aus drei Basiselementen zusammensetzen. Diese drei Basiselemente stellen Liveware, Software und Hardware dar. Sie existieren in einer Umwelt [Environment], welche auf die Elemente in einer Vielzahl von Möglichkeiten einwirkt. Der Ausdruck SHEL ist dabei ein Akronym der Anfangsbuchstaben der insgesamt vier Komponenten Software, Hardware, Environment und Liveware. Die Software stellt die Regeln, Prozeduren, Worte etc. dar, die Teil und Ganzes standardisierter Operatorprozeduren sind. Die vorhandene Ausstattung bildet die Hardware. Dies sind in der von Edwards betrachteten Domäne z.B. Reifen, Tragflächen, Flugschreiber etc. Das Environment ist zum einen das existierende soziale und ökonomische Klima, wie zum anderen die natürliche Umgebung. Die Liveware schließlich bilden die Menschen, wie z.B. Manager oder Bürokraten innerhalb des Systems. ${ }^{313}$

Die traditionelle, von Edwards dargestellte, Vorgehensweise ist die Entwicklung eines linearen Modells. Sie erfolgt in einer Sequenz von Schritten. So erwächst zunächst ein Bedarf in der Umwelt. Die Entwicklung eines Systems wird dann

Boulding, K.E., (Systemtheorie 1975), S. 31. Auch Boulding, K.E., (Systems 1956), S. 208.

310 Vgl. Abschnitt 3.1: Das System der Unternehmung.

311 Der Aspekt der Verwendung unterschiedlicher experimenteller Designs in der psychologschen Forschung kann an dieser Stelle vernachlässigt werden, da das S-H-E-L-L - Modell bereits per definitionem das Ziel verfolgt, menschliches Fehlverhalten zu modellieren. 
vom Stand der Technik dominiert. Aus dem Design der Hardware-Komponente entsteht der Bedarf an Software, um das System zu managen. Diese zwei Komponenten legen schließlich fest, was vom Faktor Mensch zu erbringen ist. Dem gegenüber erhält die Komponente Liveware nur geringe Beachtung. ${ }^{314}$ [Vgl. Abbildung 3-2].

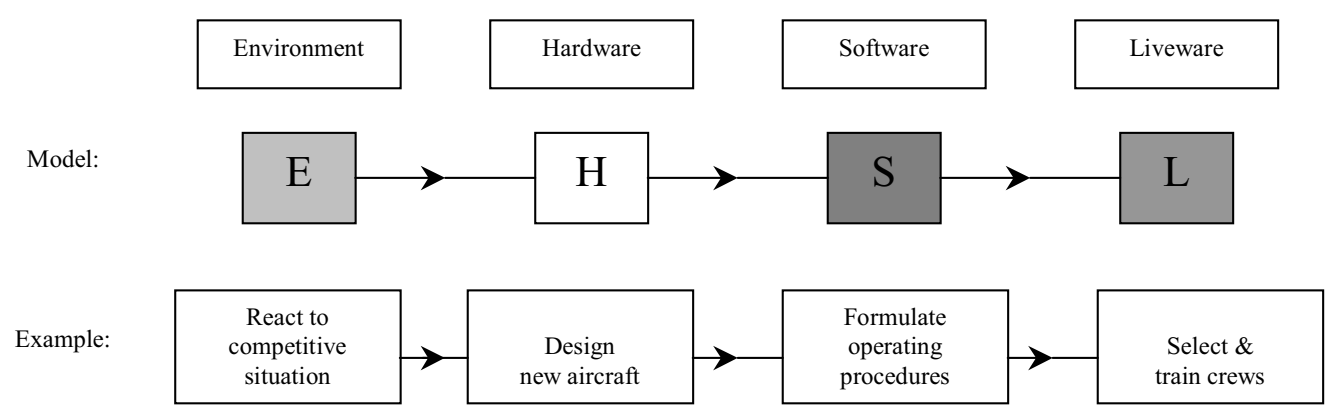

Abbildung 3-2: The traditional sequence in system design in the domain aircraft $^{315}$

Die lineare Vorgehensweise stellt jedoch einen Anachronismus dar. In anwendungsbezogener Weise werden die Komponenten Software, Liveware und Hardware in interdependenter Form dargestellt und existieren in einer dynamischen Umwelt [Environment]. Jede Komponente muß notwendigerweise mit jeder anderen Komponente verbunden werden, so daß sich am Ende ein integriertes und optimiertes Ganzes ergibt. ${ }^{316}$ [Vgl. Abbildung 3-3].

Vgl. Edwards, E., (Machine 1972), S. 22; Isaac, A.R.; Ruitenberg, B.,(Air 1999), S. 13.

314 Vgl. Edwards, E., (Machine 1972), S. 22 ff. und die dort geführte Diskussion zur Dominanz der Hardware-Komponente und der vergleichsweise geringen Beachtung der Komponente Liveware.

315 Vgl. Edwards, E., (Machine 1972), S. 23.

316 Vgl. Isaac, A.R.; Ruitenberg, B., (Air 1999), S. 13. 


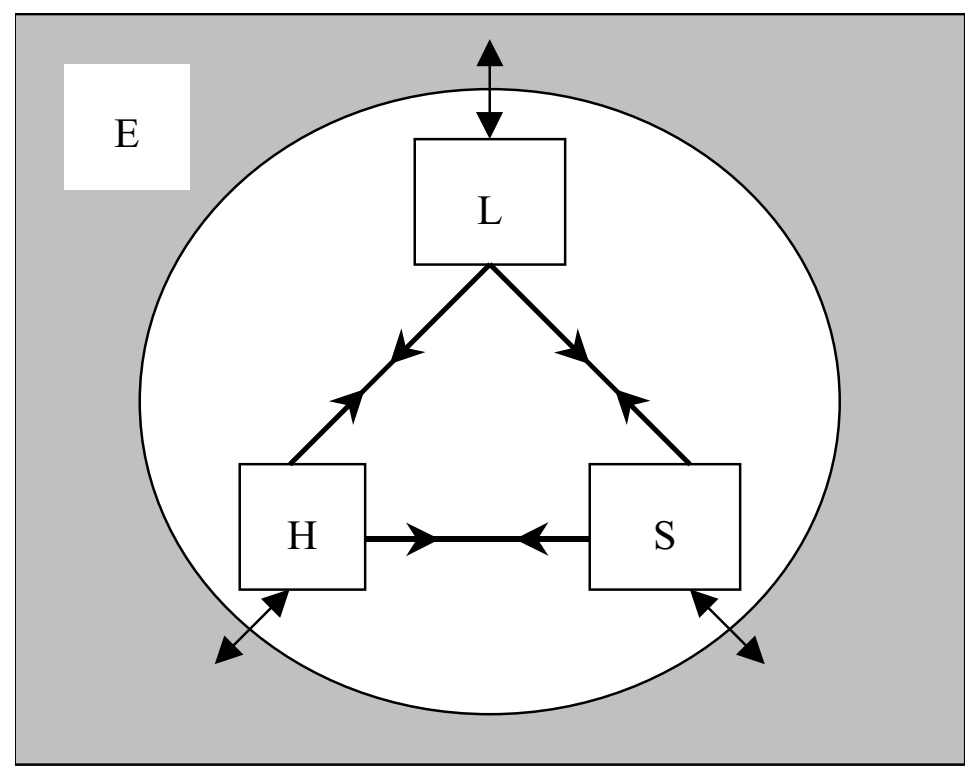

Abbildung 3-3: The dynamic HELS system, showing the interactions between Hardware, Software and Liveware components, and between each of these and the Environment ${ }^{317}$

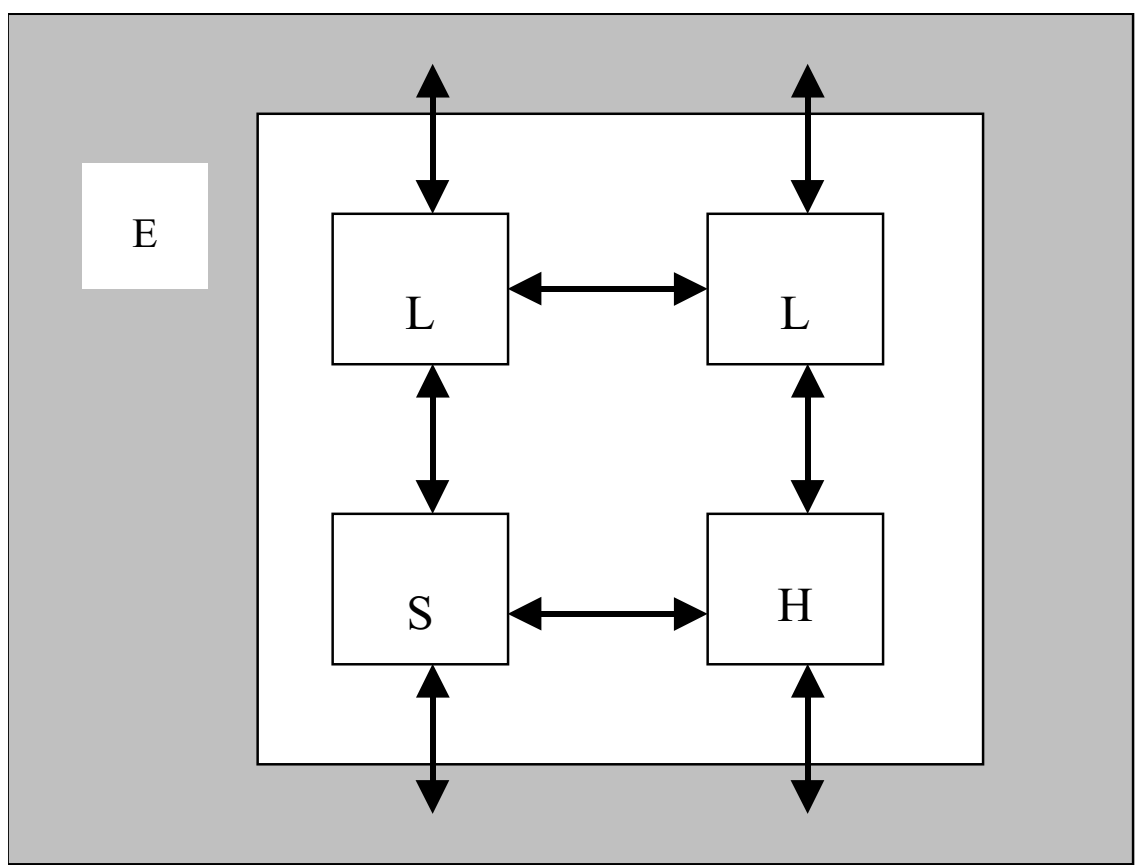

Abbildung 3-4: The dynamic HELLS system, showing the interactions between Hardware, Software, Liveware and Liveware components, and between each of these and the Environment 
Später modifizierte Hawkins dieses Modell. In seinem Modell liegt der kritische Fokus auf dem in einem System agierenden Menschen, oder Liveware. Denn abgesehen von allen anderen Dimensionen des Modells ist der Mensch derjenige, welcher letztlich am meisten empfindsam auf interne und externe Veränderungen reagiert. Der Mensch, oder Liveware, wird hier als Controller des Systems betrachtet und stellt gleichsam die zentrale Komponente des Modells dar. Zusätzlich erweiterte er es um den Aspekt der Schnittstelle zwischen Menschen. Er nannte dieses Modell S-H-E-L-L, wiederum ein Akronym zu den jetzt fünf Komponenten

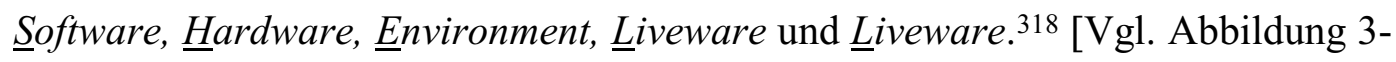
4].

\section{$\mathrm{L}$}

\subsubsection{Liveware}

\subsubsection{Der Einfluß von Personenmerkmalen auf den Umgang mit komplexen Systemen}

Personenmerkmale nehmen Einfluß auf den Umgang mit komplexen Systemen. Dabei werden unter das Kriterium Personenmerkmale alle Faktoren subsumiert, die eine Person in eine vorgegebene Situation mitbringt. Außerdem diejenigen, die sie in der betrachteten Situation erwirbt. ${ }^{319}$ In Zusammenhang mit dem Umgang einer Person mit einem komplexen System werden diese Faktoren in folgender Weise als bedeutsam erachtet: ${ }^{320}$

\section{KOGNITIVE MERKMALE}

Menschen verfügen über Wissen, das in Verbindung mit Sachverhalten und Abläufen der Realität steht. Dieses Wissen sowie seine Beziehung zu Umweltvariablen wird durch direkte Lernvorgänge, durch die Beeinflussung von Medien und

\footnotetext{
318 Vgl. Hawkins, F.H., (Human 1984), S. 334 ff.

319 Vgl. Funke, J., (Systemmerkmale 1990), S. 146.

320 Vgl. Funke, J., (Systemmerkmale 1990), S. 146.
} 
Beobachtungslernen erworben und im Verlauf des Lebens in vielfältiger Weise verändert. ${ }^{321}$ Kognitive Merkmale üben daher direkten Einfluß auf das Verhalten eines Individuums aus. Sie sollten deshalb eine bedeutsame Rolle im Umgang mit einem dynamischen System spielen. Funke nennt hier zum einen die von Dörner eingeführte Unterscheidung von epistemischen und heuristischen Gedächtnisstrukturen und das darauf beruhende Konzept einer heuristischen bzw. epistemischen Kompetenz. ${ }^{322}$ Zum anderen zählt er hierzu die Verwendung bereits vorhandener und die Ausbildung neuer mentaler Modelle, deren Diagnostik jedoch immer noch unklar ist. Daneben den Bereich kognitiver Stile, wie letztlich denjenigen der Intelligenz. ${ }^{323}$ Hierfür schlägt Fiedler in Anlehnung an Innerhofer ${ }^{324}$ den Begriff Kognitive Strukturvariable vor. Er versteht ,[...] darunter jene kognitiven Mediatoren, die als Erziehungsziele und Werthaltungen, als Befürchtungen und Erwartungen, als Anweisungen und Instruktionen einen unmittelbaren oder mittelbaren Einfluß auf das Verhalten ausüben. “325 Daneben zählt er dazu Variablen wie geschlechtsspezifische Eigenschaften, individuumspezifische Eigenheiten der Wahrnehmung der physikalischen Umwelt, soziale Normen, Regeln und Konventionen, persönliche Konstrukte seiner selbst und anderer, wobei sich diese Variablenvielfalt unter lerntheoretischen Gesichtspunkten zu drei Bereichen zusammenfassen läßt: Erwartungshaltungen, Werthaltungen und Verhaltensregeln. ${ }^{326}$

\section{EMOTIONALE UND MOTIVATIONALE MERKMALE}

In der bisherigen Forschung der Problemlösepsychologie wurden motivationale Aspekte wenig berücksichtigt. Da davon ausgegangen wurde, daß Motivation stets ausreichend vorhanden ist, wurden die kognitiven Operationen von Individuen als wesentlich für Problembearbeitungen angesehen. ${ }^{327}$ Es konnte jedoch gezeigt werden, daß neben kognitiven Operationen motivationale ebenso wie auch emotionale Operationen wesentlich für Problembearbeitungen sind. Funke verweist hier auf die von Dörner, Kreuzig, Reither \& Stäudel berichteten Notfallre-

\footnotetext{
321 Vgl. Kluwe, R.H.; Spada, H., (Wissen 1981), S. 284.

322 Vgl. Abschnitt 5.5.1: Dörner's Theorie der Absichtsbehandlung.

323 Vgl. Funke, J., (Systemmerkmale 1990), S. 146.

324 Vgl. Innerhofer, P., (Regelmodell 1974), S. 11.

325 Vgl. Fiedler, P.A., (Funktion 1976), S. 31.

326 Vgl. Fiedler, P.A., (Funktion 1976), S. 31 f. und die dort angegebene Literatur.

327 Vgl. Hesse, F.W.; Spies, K.; Lüer, G., (Einfluß 1983), S. 400 f.
} 
aktionen des kognitiven Systems, die auf den Stellenwert dieser Faktoren hingewiesen haben. ${ }^{328}$ Demnach ist es durchaus nicht als ausreichend anzusehen, eine isolierte Betrachtung rein kognitiver Leistungsmerkmale beim Bearbeiten komplexer Probleme vorzunehmen, um zu einer ausreichenden Beschreibung des beobachtbaren Verhaltens zu gelangen. ${ }^{329}$ Auch Hesse, Spies \& Lüer demonstrierten die Abhängigkeit der Lösungsgüte von der Betroffenheit der Teilnehmer. Die unabhängige Variable "Stärke der persönlichen Betroffenheit" wurde in zwei Stufen variiert, und zwar nach der Gefährlichkeit der ausgebrochenen Epidemie. ${ }^{330}$ So waren die Ergebnisse von Personen, die davon ausgingen, eine Pockenepidemie zu bearbeiten, unterschiedlich zu den Ergebnissen einer Gruppe, welche annahm, es handele sich um eine Grippeepidemie. Offensichtlich nahm hier das Ausmaß der persönlichen Betroffenheit Einfluß auf die Problembearbeitung. So erzielten die Personen, die von einer Pockenepidemie ausgingen, bessere Ergebnisse als diejenigen, die von einer weniger gefährlichen Grippeepidemie ausgingen. ${ }^{331}$

\section{PERSÖNLICHKEITSMERKMALE IM ENGEREN SINN}

Hierzu zählen Dimensionen, die in der persönlichkeitspsychologischen Forschungstradition eruiert wurden. Es wird angenommen, daß Faktoren wie Selbstsicherheit und Ängstlichkeit einen Einfluß auf den Umgang mit komplexen Systemen haben könnten. Funke verweist hier auf Dörner, Kreuzig, Reither \& Stäudel, die einen solchen Zusammenhang in der LOHHAUSEN-Studie aufzeigten ${ }^{332}$. So scheint ein zentrales Merkmal im Denken von Versuchspersonen, die schlechte Ergebnisse erzielten, eine niedrige Einschätzung der eigenen Handlungskompetenz zu sein. Nicht klar ist dabei, ob dies ein Merkmal ist, welches von vornherein in Zusammenhang mit der Persönlichkeit der Versuchsperson steht, oder ob es durch die Mißerfolge hervorgerufen wird, die im Versuchsverlauf eintreten. ${ }^{333}$ Einen Zusammenhang zwischen Selbstsicherheit und Steuerungserfolg konnten auch Ramnarayan, Strohschneider \& Schaub in einer Untersuchung mit dem be-

\footnotetext{
328 Vgl. Dörner, D.; Kreuzig, H.W.; Reither, F.; Stäudel, T. (Hrsg.),(Lohhausen 1983), S. $426 \mathrm{ff}$.

329 Vgl. Funke, J., (Systemmerkmale 1990), S. 146.

330 Vgl. Hesse, F.W.; Spies, K.; Lüer, G.,(Einfluß 1983), S. 408.

331 Vgl. Hesse, F.W.; Spies, K.; Lüer, G.,(Einfluß 1983), S. 410 f.

332 Vgl. Dörner, D.; Kreuzig, H.W.; Reither, F.; Stäudel, T. (Hrsg.),(Lohhausen 1983).

333 Vgl. Dörner, D., (Schwierigkeiten 1981), S. 168.
} 
triebswirtschaftlichen Simulationsdesign MANUTEX feststellen. ${ }^{334}$ Sie beobachteten in einer Laborstudie 60 Teilnehmer in 20 Gruppen mit jeweils drei Teilnehmern, die alle eine Ausbildung in «business management» besaßen, während sie versuchten, das Szenario MANUTEX zu steuern. Alle 60 Teilnehmer waren fortgeschrittene Studenten einer angesehenen indischen Managementschule, die sämtliche Grundlagenscheine absolviert hatten. ${ }^{335}$ Die Untersuchung ergab, daß es erforderlich ist, das Bewußtsein über die eigenen Fähigkeiten zu bewahren, da zielgerichtetes Verhalten ansonsten unmöglich wird. Dies ganz besonders im Angesicht von Schwierigkeiten und Fehlern. 336

\subsubsection{Der Spieler als Handlungssubjekt im Unternehmensplanspiel}

\subsection{Das epistemologische Subjektmodell}

Auch im Unternehmensplanspiel nehmen Personenmerkmale Einfluß auf das Spielgeschehen. So bereits ausgeführt, daß das Simulationsmodell im Spiel zum Gegenstand der vorgesehenen Geschäftsmaßnahmen wird, wobei das Spielverhalten durch das Handeln des Spielers hervorgerufen wird. ${ }^{337}$ Entsprechend soll hier die Liveware-Komponente im S-H-E-L-L - Modell als der Teilnehmer an einem Planspiel interpretiert werden, der sich aktiv mit den Gegebenheiten des Simulationsmodells auseinandersetzt, respektive sich darin auf eine bestimmte Weise «verhält».338

334 Das Untersuchungsdesign der Simulation MANUTEX wurde bereits skizziert. Vgl. Abschnitt 3.3.3: Untersuchungsdesigns zum Umgang von Personen mit komplexen betriebswirtschaftlichen Systemen.

335 Vgl. Ramnarayan, S.; Strohschneider, S.; Schaub, H.,(Trappings 1997), S. 30 f.

336 Vgl. Ramnarayan, S.; Strohschneider, S.; Schaub, H.,(Trappings 1997), S. 41.

337 Vgl. Abschnitt 2.1.2.2: Das Unternehmensplanspiel: Ein Entscheidungsspiel.

338 In diesem Zusammenhang ist es wichtig, daß der Spieler sich gleichsam als Element, wie auch als Kontrolleur des von ihm analysierten Systems begreift. So versteht das S-H-E-L-L - Modell die von ihm dargestellten Komponenten als grundlegende Elemente des Systems, die in einer Umwelt existieren. Vgl. Abschnitt 3.4.2: Das S-H-E-L-L - Modell als eine Möglichkeit zur Beschreibung der Natur menschlichen Fehlverhaltens. Auf der Metaebene ist der Mensch jedoch auch gleichsam derjenige, der ein System aktiv gestaltet. In diesem Zusammenhang wird später der Aspekt der Metakognition wichtig, der an der in diesem Abschnitt beschriebenen Selbstreflexivität des Subjekts anknüpft. Vgl. Abschnitt 6.6.1.3: Metakognition: Die Steuerung des eigenen Denkens. 
Verhalten stellt für die Psychologie nun die erste objektive Gegebenheit des Menschen dar. ${ }^{339}$ Dabei ist Voraussetzung aller Theorien menschlichen Verhaltens eine bestimmte Grundauffassung vom Menschen. Das bedeutet, daß sich die Psychologie ein Bild vom Menschen macht. Diese Menschenbilder übernimmt die Psychologie von der jeweils vorherrschenden Weltanschauung. Sie sind nicht selbst durch Beobachtung und Experiment empirisch überprüfbar, dienen jedoch als allgemeine Grundlage der Theoriebildung. Von dieser Grundlage ausgehend werden dann empirisch überprüfbare Theorien konstruiert und weiterentwickelt. Sie sollen der Erklärung und Prognose menschlicher Verhaltensweisen dienen, womit die Psychologie wiederum auf die Gesellschaft zurückwirkt. ${ }^{340}$

Verhaltenstheorien liegt dabei ein behaviorales Menschenbild zugrunde. Dieses Menschenbild beinhaltet, daß der Mensch, ein grundsätzlich passives Wesen, als ein ausschließlich durch externe Reize oder physiologisch hervorgerufene Triebreize in Bewegung versetzter Organismus betrachtet wird;341 und damit die Rolle eines ausschließlich durch Umwelt-Reize kontrollierten Subjekts einnimmt. ${ }^{342}$ Eine solche Sichtweise entwickelte sich in der Zeit nach dem zweiten Weltkrieg, im Sinne des methodologischen Behaviorismus, zum beherrschenden Forschungsmodell. ${ }^{343}$ Groeben $\&$ Scheele kennzeichnen dies auch schlagwortartig als behaviorales Subjektmodell. ${ }^{344}$ Dieses Menschenbild wird für diese Ausführungen jedoch nicht als sinnvoll erachtet, da das Lernen im Unternehmensplanspiel als Lernhandeln begriffen wird ${ }^{345}$ und daher einer Untersuchung unter explizitem Bezug auf menschliches Handeln bedarf, dem seitens der Psychologie ein anderes Menschenbild zugrunde gelegt wird.

So liegt Handlungstheorien ein epistemologisches Subjektmodell zugrunde. Das epistemologische Subjektmodell modelliert den Menschen als ein auf die Zukunft hin orientiertes Wesen. Dieses setzt sich eigene Ziele und stellt Annahmen über sich und seine Umwelt auf. Es richtet dann sein Handeln an den selbst gesteckten

\footnotetext{
339 Vgl. Aebli, H., (Denken 1980), S. 18.

340 Vgl. Werbik, H., (Handlungstheorien 1978), S. 11.

341 Vgl. Werbik, H., (Handlungstheorien 1978), S. 11.

342 Vgl. Skinner, B.F., (Freiheit 1973), S. 80 ff. und S. 188 f.

343 Vgl. Groeben, N.; Scheele, B., (Argumente 1977), S. 1.

344 Vgl. Groeben, N.; Scheele, B., (Argumente 1977), S. 3 und S. 6 ff.

345 Vgl. Abschnitt 5.1: Das Unternehmensplanspiel: Ein komplexes Lehr-Lern-Arrangement.
} 
Zielen aus; und verfolgt diese unter Nutzung des eigenen Wissens, der eigenen Reflexivität sowie der kognitiven Konstruktivität. Dies ist eine „Sicht von innen“, die sich auf den Sinn bezieht, den eine Handlung für den Handelnden hat. ${ }^{346}$

„Als handelndes Subjekt ist jeder Mensch in individuell unterschiedlichen Ausprägungen durch die Merkmale der Intentionalität, Reflexivität, potentiellen Rationalität und sprachlichen Kommunikationsfähigkeit gekennzeichnet. Dabei wird die Reflexivität in den Mittelpunkt der Kernannahmen des epistemologischen Subjektmodells gestellt. Sie, die Reflexivität, wird als Manifestation der Existenzweise des Menschen angesehen." 347 Dabei bedeutet Reflexivität die Fähigkeit des Individuums, das eigene Denken und Handeln im Lichte der erreichten Ergebnisse selbst zu beobachten, ${ }^{348}$ womit Denken, oder auch Reflexion, eine Metatätigkeit über dem konkreten Handeln darstellt. ${ }^{349}$ Dadurch wird dem Menschen die Fähigkeit zugestanden, in seiner praktischen Tätigkeit innehalten zu können und sein praktisches Tun gegebenenfalls gegen eine Tätigkeitsform auszutauschen, welche die Analyse der Struktur erleichtert. ${ }^{350}$

Der Mensch ersetzt in diesem Sinne jedoch nicht sein bisheriges Verhalten gegen ein gänzlich neues Verhalten, sondern er entwickelt, sozusagen aus dem gleichen Verhalten, ein verfeinertes, dem Zwecke besser angepaßtes Verhalten. ${ }^{351}$ Wobei der Motor dazu auch Fehlleistungen sein können, ${ }^{352}$ auf die im folgenden im $\mathrm{Zu}$ sammenhang mit komplexen Systemen eingegangen werden soll.

346 Vgl. Groeben, N., (Handlungsperspektive 1981), S. 20 ff. und die dort angegebene Literatur.

347 Groeben, N.;Scheele, B., (Argumente 1977), S. 65.

348 Aebli, H., (Denken 1980).

349 Vgl. Aebli, H., (Denken 1980), S. 22.

350 Vgl. Aebli, H., (Denken 1980), S. 21 f.

351 Vgl. Aebli, H., (Denken 1980), S. 22. Diesen Prozeß ist der Mensch offensichtlich so lange bestrebt fortzusetzen, bis er zur Ordnung gelangt: „Wir sehen im Handeln ein Streben nach Ordnung und Struktur, das sich im Denken in reinerer Form fortsetzt. So ist das Handeln auf ein Ziel der Transparenz und der Ordnung hin angelegt. Das Streben nach ihm motiviert den Handelnden und den Denker, denn wo Ordnung ist, da ist Leben; Verwirrung und Claos bedeuten Tod.“ ders, (Denken 1980), S. 16. Offensichtlich erreicht den Menschen mit der Ordnung aber auch die Langeweile, denn den Ausführungen Nørretrander's zu Folge wird Ordnung als langweilig empfunden. Dies mag der Grund dafür sein, warum der Mensch sich stets neuen Herausforderungen zuwendet. Vgl. Abschnitt 2.2.2.1: Der Komplexitätsbegriff.

352 Vgl. Abschnitt 3.3.3: Untersuchungsdesigns zum Umgang von Personen mit komplexen betriebswirtschaftlichen Systemen. 


\subsection{Fehlverhalten in Zusammenhang mit komplexen Systemen}

Die thematischen Schwerpunkte existierender Arbeitsgruppen im Bereich des komplexen Problemlösens wurden bereits im Überblick dargestellt. ${ }^{353}$ Einen Bereich stellte der Umgang von Personen mit komplexen Systemen dar. Hier zeigten verschiedene Untersuchungen, daß sich im Umgang mit komplexen Systemen typische Verhaltensdefizite feststellen lassen. So machen die meisten Menschen im Umgang mit komplexen, vernetzten und dynamischen Systemen typische Fehler, die Reichert \& Stäudel auf drei Gründe zurückführen: ${ }^{354}$

1. Die Problemlöser kennen die Merkmale und Anforderungen komplexer, vernetzter und dynamischer Probleme nicht. Daher handeln sie so, als hätten sie ein einfaches, statisches Problem zu bewältigen.

2. Vielen Problemlösern mangelt es an effizienten Methoden, um mit den an sie gestellten Anforderungen angemessen umzugehen. Herkömmliche Methoden der Entscheidungsfindung und Planung führen hinsichtlich vernetzter und dynamischer Systeme unweigerlich zu unerwünschten Fehlern.

3. Problemlösendes Handeln stellt einen ganzheitlichen Prozeß dar. Dadurch, daß die Komplexität von Systemen Unbestimmtheit und damit emotionale Belastung hervorruft, tendiert der Mensch zu Notfallreaktionen, die meist fatale Folgen nach sich ziehen.

Dörner führt eine Reihe möglicher Fehler von Menschen an, die im Umgang mit komplexen Systemen bedeutsam sind: ${ }^{355}$

Als PRIMÄRFEHLER bezeichnet Dörner einige Fehler, die fast alle Versuchspersonen machen. Als erstes nennt er die unzureichende Betrachtung von Zeitverläufen. So lenken Personen in komplexen Aufgabenstellungen ihr Augenmerk zumeist auf den aktuellen Zustand, weniger auf Trends und Entwicklungstendenzen.356 „Die Ermittlung der Struktur von «Zeitgestalten» durch Beobachtung derselben

353 Vgl. Abschnitt 2.3.1: Komplexes Problemlösen - Ein Aufriß.

354 Vgl. Reichert, U.; Stäudel, T.,(Diagnostik 1991), S. 102 f.

355 Vgl. Dörner, D., (Schwierigkeiten 1981), S. 166 ff. Dieser Artikel wurde auch in Englisch veröffentlicht. Vgl. dann Dörner, D., (Difficulties 1987), S. 99 ff.

356 Vgl. Dörner, D., (Schwierigkeiten 1981), S. 166. 
scheint Menschen schwerer zu fallen als die Ermittlung der Charakteristika von räumlichen Konfigurationen.“357 Reichert \& Dörner vermuten, daß die Ursachen solcher Schwierigkeiten wohl darin zu suchen sind, daß zeitlich gestreute Information dem Betrachter nicht in dem Umfang zur Verfügung steht, wie räumliche Information. Diese hat den Vorteil, daß sie über längere Zeit hinweg unverändert bleibt und demzufolge stets wieder betrachtet werden kann. Damit belasten in der Zeit variierende Informationen die kognitive Kapazität in viel höherem Grade, als räumliche Konstellationen, da diese in viel geringerem Maße Anforderungen an die Kapazität der Informationsverarbeitungsschritte pro Zeiteinheit stellen. Demzufolge haben Menschen erhebliche Probleme bei der richtigen Organisation ihrer Handlungen, wenn die Rückmeldungen auf diese Handlungen verzögert erfolgen, denn der Zusammenhang zwischen dem eigenen Verhalten und der Rückmeldung ist dann nicht mehr sinnfällig. 358

Als zweiten PRIMÄRFEHLER führt Dörner die Schwierigkeit im Umgang mit exponentiellen Verläufen an. ${ }^{359}$ Auch Wagenaar \& Sagaria schildern, daß Versuchspersonen Exponentialverläufe einzelner Variablen falsch einschätzen. Eine Vielzahl weltweiter Probleme stehen jedoch in Zusammenhang mit Wachstum. Wirtschaftliches Wachstum und Bevölkerungswachstum induzieren Knappheit von Energiereserven und Rohstoffvorkommen, sowie den Anstieg von Lebenshaltungskosten und Umweltverschmutzung. Die subjektiven Extrapolationen unterschätzen die tatsächliche Wachstumsrate bei Exponentialfunktionen. ${ }^{360}$ Eine adäquate Einschätzung scheint jedoch wichtig, denn: „Any attempt to control these processes will depend on the cooperation of individual citizens; they should first appreciate how fast a growth process will be, before they can reasonably weigh the growth problem against a number of alternative factors, such as religious beliefs or personal comfort.“"361

Reichert, U.; Dörner, D.,(Heurismen 1988), S. 12.

Vgl. Reichert, U.; Dörner, D.,(Heurismen 1988), S. 12.

Vgl. Dörner, D., (Schwierigkeiten 1981), S. 166 f.

Vgl. Wagenaar, W.A.; Sagaria, S.D.,(Misperception 1975), S. 419. Wagenaar \& Sagaria unternahmen dazu zwei Experimente. In einem ersten Experiment boten sie den Versuchspersonen die Informationen in numerischer, bei einem zweiten Experiment in graphischer Form an. Die Versuchspersonen unterschätzten die Exponentialverläufe jedoch in beiden Versuchsreihen. Vgl. dies., (Misperception 1975), S. 422. Das ist besonders interessant, da es seit jeher eine beliebte Methode ist, zeitliche Verläufe in Diagrammen graphisch darzustellen. Vgl. Reichert, U.; Dörner, D.,(Heurismen 1988), S. 12.

Wagenaar, W.A.; Sagaria, S.D.,(Misperception 1975), S. 416. 
Als dritter PRIMÄRFEHLER wird schließlich das Denken in kausalen Serien statt in kausalen Netzen angeführt. Versuchspersonen realisieren nur den anvisierten Haupteffekt der Maßnahme, nicht aber die Nebeneffekte.

Als Fehler 'schlechter’ Versuchspersonen identifiziert Dörner thematisches Vagabundieren, Verkapselung, eine abnehmende Bereitschaft, Entscheidungen zu treffen, die Tendenz, Entscheidungen zu delegieren sowie eine Exkulpationstendenz. Diese Merkmale werden nun näher ausgeführt:362

> Das thematische Vagabundieren wird als Fluchtverhalten interpretiert. So „flüchten“ solche Versuchspersonen vor der Auseinandersetzung mit einem Thema, sobald die ersten Schwierigkeiten auftreten. Die Behandlung des Themas bleibt oberflächlich und ein anderes Thema wird ausgewählt. ${ }^{363}$

Die Verkapselung bedeutet das Steckenbleiben in einem Themenkomplex, der keinen Widerstand bietet. Dadurch verstellt sich der Blick für Problembereiche, die nun nicht mehr behandelt werden. ${ }^{364}$

$>$ In Zusammenhang mit einer mangelnden Bereitschaft, Entscheidungen zu treffen, nimmt die Anzahl der vorgenommenen Entscheidungen im Zeitverlauf $\mathrm{ab}$.

$>$ Die Tendenz zu delegieren steht in einem engen Zusammenhang mit der mangelnden Entscheidungsbereitschaft. Hier wird die Verantwortlichkeit, Entscheidungen durchzuführen, an andere delegiert.

Die Exkulpationstendenz hängt wiederum eng mit der Tendenz zu delegieren zusammen. Die Versuchspersonen suchen die Verantwortlichkeit in ihrem Umfeld oder bei anderen Personen.

\footnotetext{
362 Vgl. Dörner, D., (Schwierigkeiten 1981), S. 168 ff.

363 Vgl. auch Schaub, H., (Fehler 2001), S. 2.

364 Schaub spricht hier von Einkapselung. Vgl. Schaub, H., (Fehler 2001), S. 2.
} 

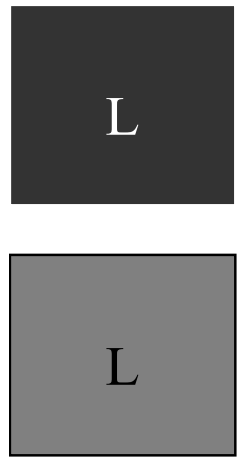

\subsubsection{Liveware-Liveware}

\subsubsection{Umgang mit Unternehmensplanspielen in Gruppen}

Die Liveware-Komponente im S-H-E-L-L - Modell konnte als der Teilnehmer im Unternehmensplanspiel interpretiert werden, der sich aktiv mit den Gegebenheiten des Planspielmodells auseinandersetzt. ${ }^{365}$ Nun erfolgt im zeitlichen Ablauf von Unternehmensplanspielen die Bildung von Spielgruppen, ${ }^{366}$ so daß die LivewareLiveware-Komponente im S-H-E-L-L - Modell hier als der aktive Umgang mit den Gegebenheiten des Planspielmodells in Gruppen interpretiert wird.

Durch die Gruppenbildung wird im Unternehmensplanspiel ein Handeln in Gruppen notwendig, welches gemeinsame Entscheidungen verlangt. ${ }^{367}$ Eine Gruppe ist dabei dadurch gekennzeichnet, daß, ,[... zwei oder mehr Individuen für eine gewisse Zeit in aktiver wechselseitiger Beziehung stehen und in gemeinsamer Anstrengung ein Ziel verfolgen“. ${ }^{368}$ Somit ist das Planspiel nicht nur subjektiver Ausdruck individueller Selbstverwirklichung, sondern vielmehr Expression einer im Rahmen der Spielregeln gefällten Gruppenentscheidung. ${ }^{369}$

Haben mehrere Personen Anteil an einer Entscheidung, so kann eine Gruppe von Personen auch als Entscheidungsgremium aufgefaßt werden. Dieses Entscheidungsgremium gelangt dann durch Informationsaustausch zur Formulierung des

365 Vgl. Abschnitt 3.4.3.2: Der Spieler als Handlungssubjekt im Unternehmensplanspiel.

366 Vgl. Abschnitt 3.2.2: Ablauf von Unternehmensplanspielen. Das Einzelspiel gilt als Ausnahmefall. Vgl. ebenfalls Abschnitt 3.2.2: Ablauf von Unternehmensplanspielen.

367 Vgl. Freudenreich, D., unter Mitarbeit von Großhans, V., (Planspiel 1979), S. 42 f.

368 Huck, K., (Analyse 1975), S. 7.

369 Vgl. Geuting, M., (Planspiel 1992), S. 88. 
Entscheidungsvorhabens. ${ }^{370}$ Auch eine Planspielgruppe kann demnach als Entscheidungsgremium verstanden werden, wodurch die Vermutung naheliegt, daß in diesem Zusammenhang, gleichermaßen wie im Entscheidungsgremium, sowohl gewisse gruppendynamische Effekte als auch Veränderungen im individuellen Risikoverhalten auftreten, welche die Berücksichtigung von Gruppenentscheidungsregeln erforderlich machen. ${ }^{371}$

In diesem Zusammenhang führt Högsdal aus, daß das Planspiel innerhalb der einzelnen Teilnehmergruppen einen hohen Aktivierungsgrad bewirkt, wodurch der Verhaltensaspekt sowohl in Zusammenhang mit der Entscheidungsfindung als auch der Ergebnisanalyse eine herausragende Rolle spielt. ${ }^{372}$ Er führt weiterhin die Förderung ,[...] des Verständnisses für die Auswirkung gruppendynamischer Prozesse auf die Entscheidungsergebnisse“ als eines der primär mit Planspielen verfolgten Lernziele an. ${ }^{373}$

Nach Ansicht von Geuting muß jedoch darauf geachtet werden, daß die Gruppenbildung im Planspiel nicht überbewertet wird. Sozialpsychologische Analysen des Planspiels bergen leicht die Gefahr, theoretische, gruppendynamische Gesichtspunkte auf die Planspielsituation zu transferieren. Die einfachen Rahmenbedingungen des Planspiels müssen dabei jedoch Beachtung finden. So ist die Gruppenbildung beim Planspiel nur kurzfristig, so daß psychosoziale Effekte sich nur eingeschränkt entfalten können. Außerdem ist der Verhaltensraum der Gruppenmitglieder durch die Spielregeln eingeschränkt. Schließlich sind die Verhaltensweisen in der Planspielsituation nicht in Gänze mit realem Verhalten vergleichbar. ${ }^{374}$ Geuting erachtet aber vier Gesichtspunkte als wichtig: ${ }^{375}$

1. Die interne Gruppenstruktur. Das bedeutet die gruppeninternen Interaktionen. Die externe Gruppenstruktur.

2. Die Interaktionen der jeweiligen Spielgruppen untereinander.

\footnotetext{
370 Vgl. Meyer, R., (Entscheidungstheorie 2000), S. 135 ff.

371 Vgl. Meyer, R., (Entscheidungstheorie 2000), S. 138. Vgl. zur Entscheidungsfindung in Gruppen bspw. Boos, M., (Entscheidungsfindung 1996).

372 Vgl. Högsdal, B., (Planspiele 1996), S. 13.

373 Högsdal, B., (Planspiele 1996), S. 14.

374 Vgl. Geuting, M., (Planspiel 1992), S. 81.

375 Vgl. Geuting, M., (Planspiel 1992), S. 81 f.
} 
3. Die Form oder der Ausdruck der Aktivitäten.

4. Das Erleben der Gemeinschaft durch die Gruppenmitglieder.

Demnach liegt, trotz des eingeschränkten Verhaltensraumes, ein besonderes Gewicht auf der Interaktion in und zwischen den Gruppen. Das Individuum steht hier einer expliziten Umwelt gegenüber, das heißt einer sozialen Präsenz anderer Personen mit unmittelbaren Interaktionen, ${ }^{376}$ so daß die Entscheidungsfähigkeit offensichtlich doch $\mathrm{zu}$ einem nicht unwesentlichen Anteil von den Interaktionen innerhalb der Gruppe abhängt. Der einzelne muß bspw. in der Lage sein, Kontakt $\mathrm{zu}$ anderen aufzunehmen sowie seine Interessen darzustellen und $\mathrm{zu}$ vertreten. Darüber hinaus muß der einzelne bereit sein, dem andern zuzuhören und dessen Gefühle und Interessen zu achten. Außerdem wirft die Zusammenarbeit auch Fragen nach Führungsstil sowie nach der Verteilung von Macht und Einfluß in den Gruppen auf. 377

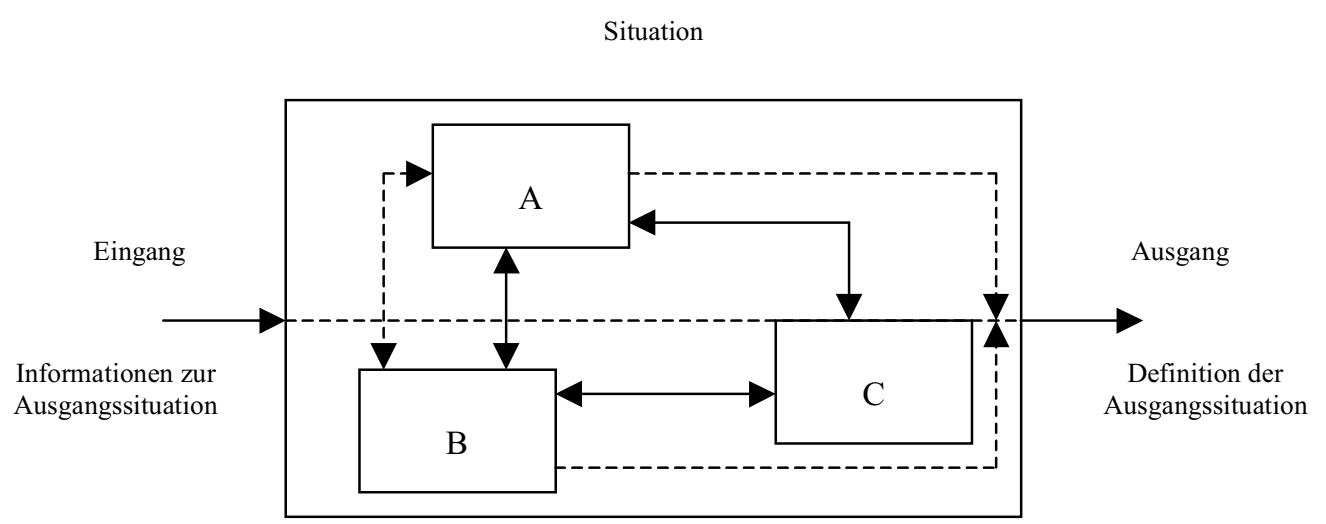

\section{Abbildung 3-5: Aufnahme und Austausch der Informationen in einer Gruppe während der Situationsanalyse ${ }^{378}$ \\ Kommunikationen \\ Aufnahme der Informationen und Beiträge zum hypothetisch angenommenen gemeinsamen Denkprozeß}

376 Vgl. Huck, K., (Analyse 1975), S. 7.

377 Vgl. Freudenreich, D., unter Mitarbeit von Großhans, V., (Planspiel 1979), S. 42 f. Vgl. zu Interaktionsprozessen in Gruppen sowie Verfahren zu deren Analyse und Organisationsentwicklung bspw. Fassheber, P.; Terjung, B., (Symlog-Teamdiagnostik 1986). Zu interpersonellen Untersuchungen mit den Dimensionen Selbstbild, vermutetes Fremdbild und Fremdbild vgl. bspw. Fassheber, P.; Terjung, B., (Symlog-Rating-Daten 1989). 
Als wesentlicher Bestandteil der Interaktion innerhalb der einzelnen Spielgruppen sowie der Interaktion mit anderen Gruppen ist die Kommunikation anzusehen. So sind die Spieler, durch den gegenseitigen kommunikativen Austausch, dazu aufgefordert, gemeinsam zu lernen. ${ }^{379}$ [Vgl. Abbildung 3-5].

\subsubsection{Mögliches Fehlverhalten in der Mensch-Mensch-Interaktion}

Definitionen zur menschlichen Kommunikation existieren in der Literatur in groBer Vielfalt. ${ }^{380}$ Auf einer sehr allgemeinen, abstrakten Ebene meint Kommunikation im einfachen Sinne, Mitteilungen zu übermitteln und auszutauschen. Dieser Austausch findet jeweils zwischen einem Sender, welcher die Nachricht sendet, und einem Empfänger, der diese Nachricht erhält, statt. ${ }^{381}$ [Vgl. Abbildung 3-6].

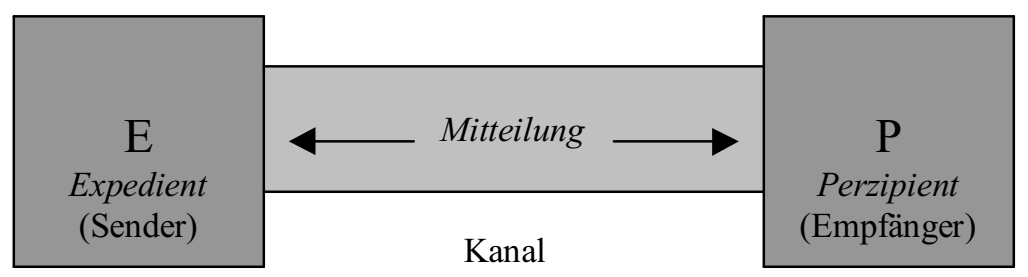

\section{Abbildung 3-6: $\quad$ Schema des Kommunikationsvorganges ${ }^{382}$}

Kommunikation heißt also Mitteilung, heißt Verständigung durch Informationsaustausch. Die Informationen werden abgegeben durch Kodierung in Zeichen und Nachrichten. Informationen werden transportiert und aufgenommen, und letztlich wird kontrolliert, inwieweit die vom Empfänger aufgenommene Information mit der vom Sender abgegebenen übereinstimmt. Dadurch wird Information zu etwas Gemeinsamem, und der Austausch geschieht mit dem Ziel, bestimmte Handlungen und Verhaltensweisen $\mathrm{zu}$ erzielen. ${ }^{383}$ Dabei kann Kommunikation einer Handlung sowohl vorangehen als auch nachfolgen. Wenn sie der Handlung vor-

379 Vgl. Sonderegger, W., (Entwurf 1982), S. 28 f. und die dort angegebene Literatur.

380 Vgl. bspw. Manstetten, R., (Kommunikation 1982), S. 33 ff; Pürer, H., (Einführung 1993), S. 17 ff; Noelle-Neumann, E.; Schulz, W.; Wilke, J. (Hrsg.),(Publizistik 1994), S. $140 \mathrm{ff}$.

381 Vgl. Maser, S., (Grundlagen 1972), S. 9.

382 Maser, S., (Grundlagen 1972), S. 10.

383 Vgl. Maser, S., (Grundlagen 1972), S. 168. 
angeht, stellt sie die Argumente, die Gründe bereit, die menschlichem Verhalten zugrunde liegen. In dem Falle, daß Kommunikation einer Handlung nachfolgt, liefert sie eine kritische Rechtfertigung oder Mißbilligung. 384

In Zusammenhang mit menschlicher Interaktion nimmt Watzlawick verschiedene Formen fehlgeleiteter Kommunikation an. Diese führen letztlich dazu, daß ein Empfänger die von einem Sender dargebrachte Mitteilung nicht in der von diesem gewünschten Form versteht. Die Überlegungen, die Watzlawick anstellt, basieren zunächst auf zwei Formen von Wirklichkeit; die Wirklichkeit erster und zweiter Ordnung. Die Wirklichkeit erster Ordnung ist eine objektive Wirklichkeit, deren Wahrnehmung auf einem allgemeinen Konsensus beruht, vor allem jedoch auf experimentell nachweisbaren Ergebnissen. Die Wirklichkeit zweiter Ordnung ist eine subjektive Wirklichkeit, die keiner Bewertung in Form von wahr oder falsch zugänglich ist, da Kulturen und Personen eigene Wertvorstellungen prägen und darüber kein objektiver Nachweis möglich ist was 'besser oder schlechter' ist. ${ }^{385}$ In diesem Zusammenhang sind die Grundvoraussetzungen menschlicher Kommunikation vorhanden, problematisch ist jedoch das ,[...] Auftreten bestimmter Hindernisse, die den Austausch von Kommunikation erschweren oder verunmöglichen und dadurch die Kommunikation zur Zuschreibung widersprüchlicher Bedeutungen oder Werte an die gemeinsam erlebte Situation führen“. Ist das Hindernis, Konfusion und Paradoxie, jedoch aus dem Weg geräumt, ist der Weg geebnet für ungehinderte Kommunikation: ${ }^{386}$

Konfusion in Folge einer Störung des Übertragungsvorganges.

Der Zustand einer Konfusion kann als Spiegel der Kommunikation aufgefaßt werden. „Mit dieser sehr allgemeinen Definition sei einfach dies gemeint: Wenn ein sogenannter erfolgreicher Kommunikationsvorgang in der korrekten Übermittlung von Informationen besteht und damit die beabsichtigte Wirkung auf den Empfänger hat, so ist Konfusion die Folge

\footnotetext{
384 Vgl. Maser, S., (Grundlagen 1972), S. 168.

385 Vgl. Watzlawick, P., (Wirklichkeit 1995), S. $142 \mathrm{ff}$.

386 Watzlawick, P., (Wirklichkeit 1995), S. 147. Davon grundsätzlich zu unterscheiden sind Situationen, in denen die Basis für Kommunikation erst noch zu schaffen ist. Vgl. ders., (Wirklichkeit 1995), S. 147.
} 
gescheiterter Kommunikation und hinterläßt den Empfänger in einem Zustand der Ungewißheit oder eines Mißverständnisses.“387

Paradoxie in Folge einer Störung in der Struktur der Mitteilung.

Eine Mitteilung ist so geartet, daß sie sich selbst, also ihrer eigenen Bedeutung widerspricht, sich damit selbst entwertet und eine Paradoxie nach sich zieht. 388
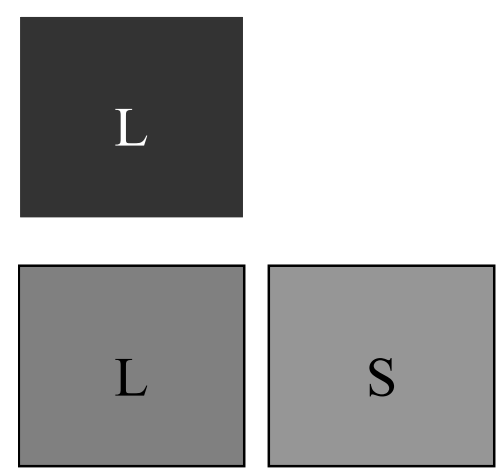

\subsubsection{Liveware-Software}

\subsubsection{Darstellung des Unternehmensplanspiels EpUS}

Die Software-Komponente des S-H-E-L-L - Modells wird als die Entscheidungssituation im Planspiel begriffen, mit der sich der Teilnehmer auseinanderzusetzen hat, wobei für diese Betrachtung das Unternehmensplanspiel EpUS herangezogen wird.

Das Planspiel EpUS, ein Akronym für Ein-Platz-Unternehmens-Simulation, wurde am Institut für betriebswirtschaftliche Produktions- und Investitionsforschung Abteilung für Unternehmensplanung- der Universität Göttingen durch Bloech, in Zusammenarbeit mit Rüscher \& Rill entwickelt. 389

387 Watzlawick, P., (Wirklichkeit 1995), S. 13. Watzlawick führt dazu verschiedene Beispiele an. Vgl. ders., (Wirklichkeit 1995), S. 14 ff.

388 Vgl. Watzlawick, P., (Wirklichkeit 1995), S. 57 u. S. 25 ff.

389 Vgl. Bloech, J., in Zusammenarbeit mit Rüscher, H.; Rill, W., (EpUS 1991), S. 3 ff.; Bloech, J.; Rüscher, H., (EpUS 1992), S. 269 ff. Auch Hartung, S., (Förderung 2000), S. $164 \mathrm{ff}$. 
Das Planspiel EpUS stellt ein vollständiges Unternehmensmodell einer Aktiengesellschaft der Industrie dar. Das Experimentieren ist für ein Unternehmen, welches in seinen wesentlichen Funktionen und Abläufe dargestellt ist, vorgesehen. Dieses Unternehmen produziert zwei Endprodukte und hat sich gegen vier gleichstrukturierte, fiktive Unternehmen zu behaupten und in einem Zeitraum von 5-12 Entscheidungsrunden seinen Unternehmenserfolg zu maximieren.

Wesentlich im Unternehmensplanspiel EpUS sind zunächst zwei Ebenen: Die Unternehmenssituation sowie die Beziehungen des Unternehmens zur Umwelt.

DIE UNTERNEHMENSSITUATION:

Die Unternehmen produzieren zwei Endprodukte, die im Unternehmen in einem einstufigen Produktionsprozeß aus zwei verschiedenen Rohstoffen hergestellt werden. Dabei wird der erforderliche Einsatz an Rohstoffen und an Fertigungskapazität durch eine Stückliste vorgegeben. Die für die Produktion benötigten Rohstoffe einer Periode müssen eine Periode vorher bestellt werden. Die Lieferung erfolgt zum Ende der Bestellperiode und die Zugänge werden im Lagerbestand erfaßt. In der folgenden Periode kann dann der Einsatz der Rohstoffe in der Produktion erfolgen. Die Bezahlung der Rohstoffe erfolgt jeweils nach Lieferung, eine Periode nach der Bestellung. Transport- und Bestellkosten sind in den Beschaffungskosten enthalten. Im Modell werden dabei die vorgegebenen Produktionsmengen immer bereitgestellt. So stehen die Produktionsmengen und der Lagerendbestand der laufenden Periode für den Absatz in der nächsten Periode auf jeden Fall zur Verfügung. Für den Fall, daß die vorgegebene Produktionsmenge mit den vorhandenen Anlagen wegen Personal- oder Faktormangel nicht durchgeführt werden kann, erfolgt eine automatische Einstellung von Leiharbeitern oder/und eine Notbeschaffung von Material. Die sofortige Bereitstellung von fehlendem Personal und fehlenden Rohstoffen sichert dabei zwar die geplante Auslastung der vorhandenen Anlagen, ist jedoch erheblich teurer als eine planmäßige, rechtzeitige Beschaffung. Liegt die Produktionsmenge über der vorhandenen Produktionskapazität, werden Fertigerzeugnisse auf dem Weltmarkt beschafft. Auch das ist entsprechend teurer. Die bei- 
den Produktarten werden auf einem Maschinentyp produziert. Der Kapazitätsbedarf einer Periode errechnet sich aus den Leistungseinheiten der Stückliste und der vorgegebenen Produktionsmenge.

DIE BEZIEHUNGEN ZUR UMWELT:

\section{Die Konkurrenzsituation auf dem Absatzmarkt:}

Die beiden Endprodukte werden sowohl von den durch die Teilnehmer geleiteten Unternehmen, als auch von den Konkurrenzunternehmen auf zwei getrennten Märkten angeboten, wodurch sich eine oligopolistische Marktform ergibt. Die Kunden orientieren sich bei ihren Kaufentscheidungen an den Preisen der einzelnen Unternehmen und dem Marktdurchschnittspreis, außerdem an den Werbeausgaben sowie an den durchschnittlichen Ausgaben für den Kundendienst. Die Absatzmenge eines Unternehmens kann daher durch eine aktive Preispolitik und durch den Einsatz von Werbung und Kundendienst beeinflußt werden.

2. Weitere Umweltbeziehungen:

Arbeitskräfte können über den Arbeitsmarkt beschafft und auch auf diesen entlassen werden. Dabei wird der erforderliche Personalbedarf durch die technischen Daten des Maschinentyps festgelegt. So sind zur Bedienung jeder eingesetzten Maschine jeweils zwei Arbeitskräfte notwendig. Auf dem Beschaffungsmarkt können die für die Produktion benötigten Komponenten bzw. Rohstoffe zu vorgegebenen Preisen eingekauft werden. An den Staat sind Steuern zu entrichten. Mit den Banken ist die Finanzierung zu regeln. An die Eigentümer sind schließlich die Dividenden zu zahlen.

\subsubsection{Darstellung der Entscheidungssituation im Planspiel EpUS}

Im Planspiel EpUS können ohne Unterbrechungen und ohne Eingriffe von außen über einen vorgegebenen Zeitraum Entscheidungen getroffen werden. Die Konsequenzen können dabei unmittelbar erkannt und verarbeitet werden. Dazu ist der Ablauf des Planspiels in kleinere Zeitintervalle unterteilt, die als Perioden bezeichnet werden. Die tatsächliche Länge dieser Perioden wurde dabei nicht genau 
definiert; sie kann jedoch mit einem Monat vorgestellt werden. In jeder Periode werden von den Teilnehmern nun Entscheidungen erwartet, die den Betriebsablauf steuern. Dabei kann das Betriebsgeschehen von bspw. einem Jahr durch die Annahme von 12 Spielperioden in sehr kurzer Zeit, quasi im Zeitraffereffekt, simuliert werden.

Zur Steuerung sämtlicher Betriebsabläufe sind die Entscheidungen zu Beginn eines Monats festzulegen, wobei es sich bei dieser Periode um die aktuelle Periode handelt. Dabei sind gleichzeitig Entscheidungen in allen wesentlichen betrieblichen Bereichen zu fällen. Dies sind die Bereiche Absatz, Produktion, Beschaffung, Investition, Personal und Finanzierung. Die durch den Planspieler beeinflußbaren Variablen stellen sich im Planspiel EpUS wie folgt dar: [Vgl. Abbildung 3-7].

\begin{tabular}{|l|l|l|l|l|}
\hline Produkt 1 (DM) & Markt 1 & Markt 2 & Produktionsmenge (in Stück) \\
\hline Verkaufspreis & & & Produkt 1 & \\
\hline Werbung & & Produkt 2 & \multicolumn{3}{|c|}{$\begin{array}{r}\text { Bei fehlender Produktionskapazität werden die } \\
\text { Produkte automatisch zugekauft }\end{array}$} \\
\hline Kundendienst & & & \multicolumn{3}{|c|}{} \\
\hline
\end{tabular}

\begin{tabular}{|c|c|c|c|c|}
\hline Produkt 2 (DM) & Markt 1 & Markt 2 & \multicolumn{2}{|c|}{ Rohstoffbeschaffung (in Stück) } \\
\hline Verkaufspreis & & & Rohstoff 1 & \\
\hline Werbung & & & Rohstoff 2 & \\
\hline Kundendienst & & & \multicolumn{2}{|c|}{$\begin{array}{l}\text { Bei fehlenden Rohstoffen erfolgt ein Zukauf } \\
\text { (Notbeschaffung) automatisch }\end{array}$} \\
\hline \multicolumn{3}{|c|}{ Marktforschung (ja/nein): } & \multicolumn{2}{|c|}{ Investitionen (Anzahl) } \\
\hline & & & \multicolumn{2}{|l|}{ Maschinenkauf } \\
\hline & & & \multicolumn{2}{|l|}{ Maschinenverkauf } \\
\hline \multicolumn{3}{|c|}{ Finanzierung (in DM) } & \multicolumn{2}{|c|}{ Personal (Anzahl Mitarbeiter) } \\
\hline \multicolumn{2}{|l|}{ Kreditaufnahme } & & \multicolumn{2}{|l|}{ Einstellungen: } \\
\hline \multicolumn{2}{|l|}{ Kredittilgung } & & \multicolumn{2}{|l|}{ Entlassungen: } \\
\hline \multicolumn{3}{|l|}{ Terminanlage } & \multicolumn{2}{|c|}{$\begin{array}{l}\text { Bei fehlenden Arbeitskräften erfolgt die kurzfristi- } \\
\text { ge Einstellung von Aushilfskräften }\end{array}$} \\
\hline
\end{tabular}

Abbildung 3-7: $\quad$ Modellentscheidungen im Planspiel EpUS390

390 Entscheidungsblatt aus den Planspielunterlagen zum Planspiel EpUS. Vgl. zu weiteren Planspielunterlagen Hartung, S., (Förderung 2000), S. 168 f. 
Zunächst sind für den Absatzbereich die Angebotspreise festzulegen und die Ausgaben für die absatzpolitischen Instrumente Werbung und Kundendienst zu bestimmen. Weiterhin ist darüber $\mathrm{zu}$ entscheiden, ob ein Marktforschungsbericht angefordert werden soll. Dieser liefert, bei Anforderung, Informationen über Preise, Umsatz und Absatzmengen der Konkurrenten.

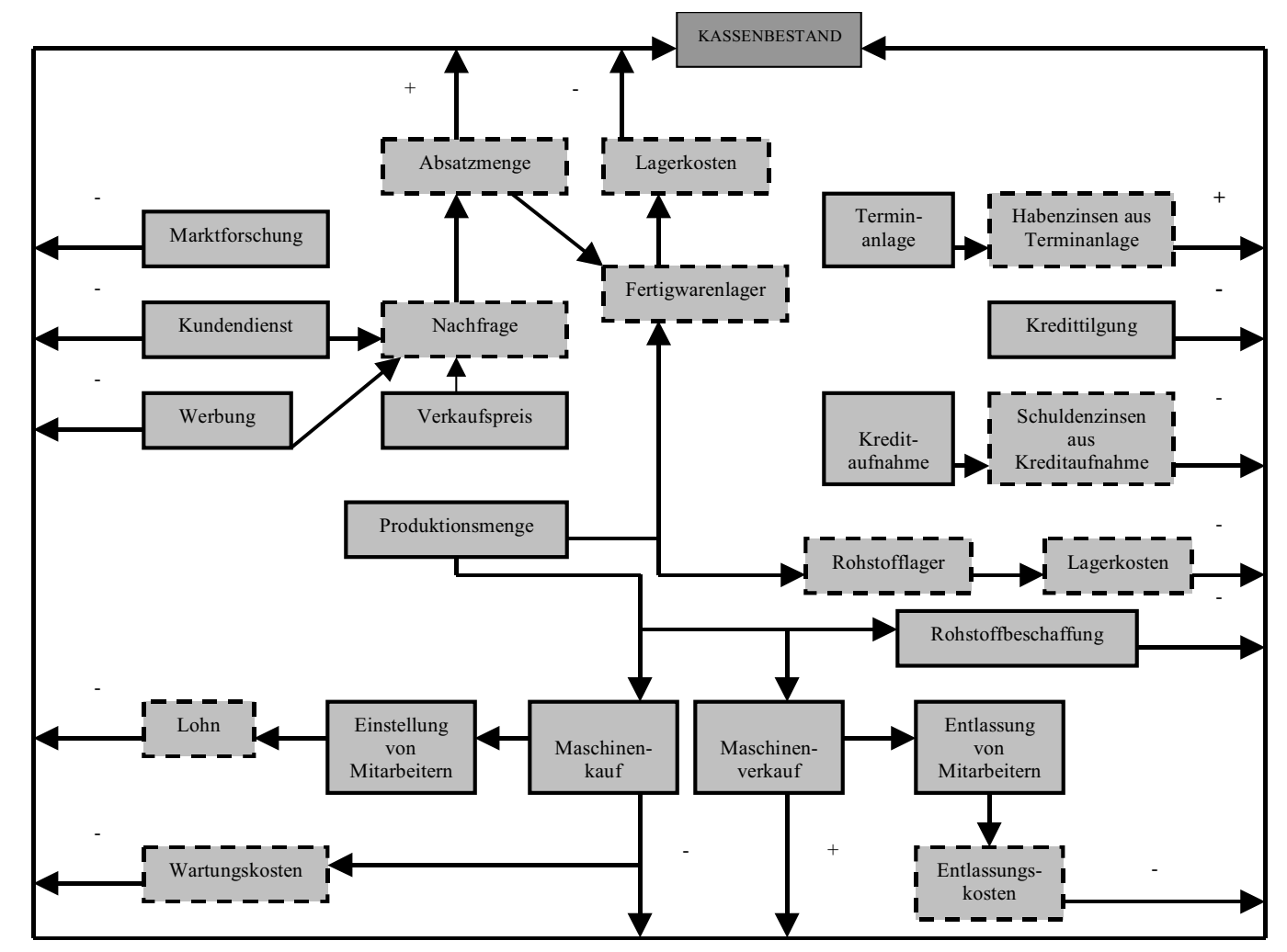

Abbildung 3-8: $\quad$ Entscheidungssituation im Planspiel EpUS mit

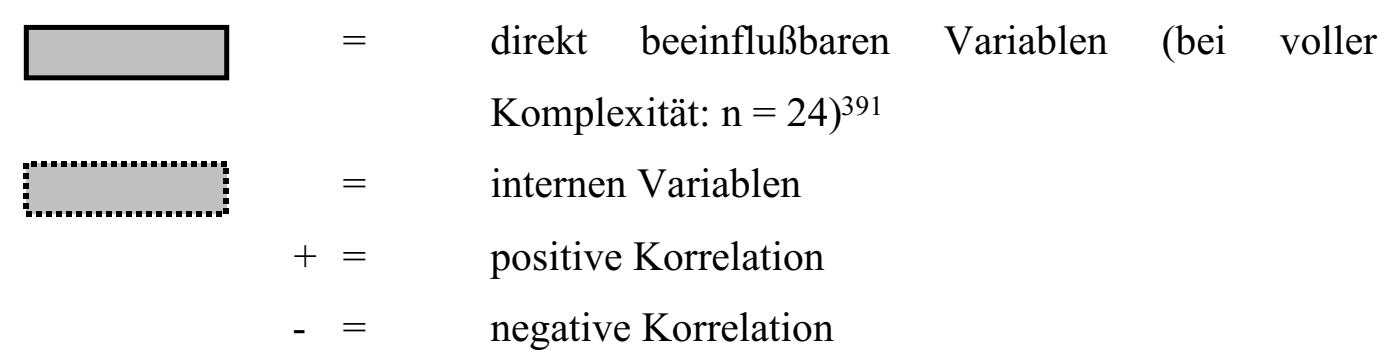

Für den Produktionsbereich ist zu entscheiden, welche Produktionsmenge produziert werden soll, wobei es sich hier gewissermaßen um eine Schlüsselentscheidung handelt, da sie sowohl für den Absatz- als auch für den Beschaffungsbereich bedeutsam ist. So wird dadurch zum einen die Angebotsmenge wesentlich be-

391 Da zwei Produkte abgesetzt werden und der Absatz auf zwei Märkten erfolgt, ergeben sich aus den zu beeinflussenden Variablen Verkaufspreis, Werbung und Kundendienst für den 
stimmt als auch die notwendigen Beschaffungsmengen. Dabei sind im Beschaffungsbereich Entscheidungen bezüglich verschiedener Faktoren zu treffen. Es ist über die erforderliche Menge an Rohstoffen, über die Produktionskapazität durch $\mathrm{Zu}$ - oder Verkauf von Maschinen als auch über die damit in direktem Zusammenhang Zahl an Mitarbeitern zu entscheiden.

Die Entscheidungen im Finanzierungsbereich erfolgen ausgehend von einem zu Beginn des Spiels zur Verfügung gestellten Kassenbestandes. Zur weiteren Finanzierung können die Teilnehmer sowohl über die Aufnahme von Krediten als auch über eine zinsbringende Anlage verfügbarer Kassenbestände am Kapitalmarkt befinden.

In einem interdependenten Zusammenhang stellt sich die Entscheidungssituation, unter Berücksichtigung sowohl direkt beeinflußbarer als auch interner Variablen in Bezug auf den Kassenbestand schließlich wie oben aufgezeigt dar. [Vgl. Abbildung 3-8].

\subsubsection{Fehlverhalten in Entscheidungssituationen}

Albach unterscheidet drei Möglichkeiten, die dazu führen, daß es nicht zu einer rationalen Entscheidung kommt: 392

\section{VERSTOß GEGEN DIE ENTSCHEIDUNGSFUNKTION}

Es besteht die Möglichkeit, daß mit der Entscheidung betraute Personen gegen die Entscheidungsfunktion verstoßen. In dem Fall, daß objektiv, operational definiert ist, ist davon auszugehen, daß dieser Fall nur dann auftreten kann, wenn die Entscheidungsfunktion bewußt fehlinterpretiert wird. Dabei wird diese Möglichkeit von der Kontrollfunktion im Rahmen der Unternehmensorganisation sowieso ausgeschlossen. 


\section{VERSTOß GEGEN DIE DEFINITIONSFUNKTION}

$>$ Es ist den Personen, die mit der Entscheidung betraut werden, nicht möglich, die Definitionsfunktion nachzuvollziehen, wobei zwei Gründe dafür ausschlaggebend sein können: Zum einen ist es möglich, daß die Personen Flüchtigkeitsfehler machen. Zum anderen können Denkfehler vorliegen.

\section{KEINE ERKLÄRUNGSFUNKTION}

$>$ Die Personen, welche die zu treffende Entscheidung fällen sollen, können keine Erklärungsfunktion erstellen. Daher entwickeln sie auch keine Erklärungsfunktion, wobei die Grenzen der Rationalität betrieblicher Entscheidungen an dieser Stelle in den Möglichkeiten der Informationsgewinnung zu finden sind.

Schaub führt weiterhin das sogenannte »ballistische Entscheidungsverhalten « 393 an. In diesem Fall wird eine Entscheidung zwar vorgenommen, ihr Verlauf unterliegt jedoch nicht mehr einer Beobachtung. Das führt dazu, daß Entscheidungen unter Umständen versanden und die mit ihnen angestrebte Entwicklung nicht vollzogen wird. Problematisch ist in diesem Zusammenhang weiterhin, daß der Entscheidungsträger eine mögliche Fehlentwicklung zumeist sehr spät, möglicherweise auch überhaupt nicht zurückgemeldet bekommt. Dies begründet sich auch dadurch, daß er seine Entscheidung bereits wieder vergessen hat. ${ }^{394}$

393 Dieses Entscheidungsverhalten wird als »ballistisches Entscheidungsverhalten« bezeichnet, da Entscheidungen wie eine Kanonenkugel abgefeuert werden, ihr Verlauf jedoch, wie bei einer Kanonenkugel weder gesteuert noch kontrolliert wird. Vgl. Schaub, H., (Fehler 2001), S. 2. 

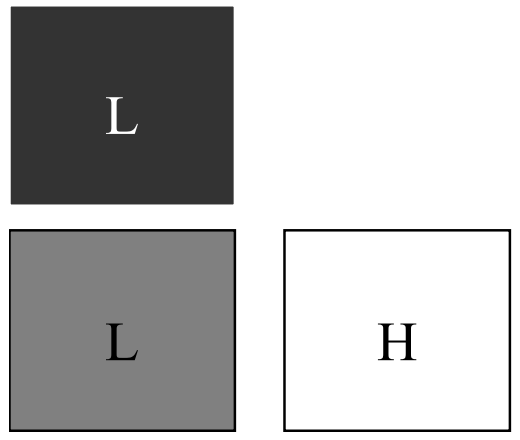

3.4.6

Liveware-Hardware

3.4.6.1

Instrumente zur Entscheidungsvorbereitung

\subsection{Allgemeiner Überblick über das Unternehmensplanspiel MARKUS}

Der Teilnehmer an einem Planspiel hat die Aufgabe, betriebswirtschaftliche Planungsaufgaben ${ }^{395}$ unter Einsatz von Instrumenten zur Entscheidungsvorbereitung zu lösen. Daher wird die Liveware-Hardware-Komponente des S-H-E-L-L - Modells als die Auseinandersetzung eines Teilnehmers respektive einer Gruppe mit Methoden zur Entscheidungsfindung interpretiert, welche die Teilnehmer anzuwenden haben, um ihre Entscheidungen vorzunehmen. Dabei wird auf die Komplexität des Planungsprozesses abgestellt, den der Teilnehmer zu durchlaufen hat, bis er eine mögliche Entscheidung fällen kann.

In der Arbeit von Prehm wird die Beschreibung von Planungsinstrumenten und deren Einsatzmöglichkeiten, zum Teil mit Ergänzung durch Fallbeispiele, fokussiert. Dies dient der Vertiefung der Methodenkenntnis von Planspielteilnehmern und der Umwandlung von Wissen in Können. ${ }^{396}$ Beim Unternehmensplanspiel MARKUS, ein Akronym für Marketing-ㅂnternehmensspiel, ${ }^{397}$ handelt es sich um ein , ,...] generelles, allgemeines, mit abstrakten Daten und Problemen arbeiten-

395 Vgl. zum Planungsbegriff sowie zu Möglichkeiten der Dekomposition einer Planungsaufgabe Abschnitt 6.8.2: Dekomposition einer Planungsaufgabe.

396 Vgl. Prehm, H.-J., (MARKUS 1995), S. 40.

397 Vgl. Prehm, H.-J., (MARKUS 1995), S. 32. 
des, deterministisches, komplexes, computerunterstütztes Interaktionsspiel, dessen Schwerpunkt auf Marketingentscheidungen liegt"“.398

Im Unternehmensplanspiel MARKUS übernehmen die Teilnehmer die Geschäftsführung von neun gleichstrukturierten Unternehmen, wobei dies in Gruppen von drei bis sechs Personen geschieht. In dieser Funktion sind sie dazu aufgefordert, Entscheidungen in allen wesentlichen betrieblichen Funktionsbereichen zu treffen und bezüglich aller Planungsaufgaben die Unternehmenszielsetzung "Maximierung der bis zum Ende der letzten Spielperiode kumulierten Periodenüberschüsse vor Steuern" zu verfolgen. ${ }^{399}$

Es ist in diesem Zusammenhang möglich, jedes Unternehmen im Planspiel MARKUS in vier Funktionsbereiche zu gliedern. Dies sind die Bereiche Materialwirtschaft, Produktion, Marketing sowie Finanzwirtschaft und Rechnungswesen. Der Bereich Materialwirtschaft umfaßt dabei wiederum die Teilbereiche Lagerhaltung und Beschaffung.

Im Bereich Materialwirtschaft können in diesem Zusammenhang die benötigten Rohstoffe stets ausreichend und in gleichbleibender Qualität beschafft werden. Bei dem im Planspiel abgebildeten Lager handelt es sich um ein Eigenlager, welches jedoch kapazitätsmäßig begrenzt ist. Zur Kapazitätserweiterung besteht aber die Möglichkeit, Lagerkapazitäten in Fremdlägern anzumieten. Die Beschaffung von Lagerarbeitern erfolgt dabei durch eine Bereichsleitung.

Im Produktionsbereich werden die Produkte in einer drei- bis fünfstufigen Sortenfertigung produziert sowie weiterhin verpackt, markiert und letztendlich versandfertig gemacht. In diesem Zusammenhang müssen die Erzeugnismengen geplant sowie weiterhin eine Reihenfolgeplanung vorgenommen werden. Diese Planung spielt eine zentrale Rolle im Produktionsbereich, da an den Maschinen stets Umrüstarbeiten anfallen, wenn eine neue Sorte gefertigt werden soll. Dabei stehen zur Fertigung zeitlich begrenzt nutzbare Maschinen in drei Leistungsvarianten zur Verfügung, wobei die Anlagenkonfiguration kurzfristig austauschbar ist. 
Der Schwerpunkt liegt im Unternehmensplanspiel MARKUS auf dem Marketingbereich. Hier finden sich zwei Abnehmergruppen für die gefertigten und zu veräußernden Produkte. So werden die Produkte zum einen durch gewerbliche Großabnehmer nachgefragt, zum anderen durch Konsumenten. In diesem Zusammenhang richten die Großabnehmer ihre Nachfrage entweder an ein Unternehmen oder gegebenenfalls auch an mehrere Unternehmen. Die Konsumenten können hingegen ihre Produkte nur unternehmensindividuell beziehen, entweder über direkte Vertriebswege wie Versand und Filialen oder über indirekte Vertriebswege wie Einzel- und Großhandelsbetriebe, wobei in diesem Zusammenhang von den Unternehmen ein physisches Distributionssystem einzurichten ist.

Im Bereich Finanzwirtschaft und Rechnungswesen steht schließlich die Planung der Finanzen sowie die Wahrung der Liquidität im Vordergrund. In diesem Zusammenhang können liquide Mittel beschafft und angelegt werden. Eine Periodenabschlußrechnung wird in Form einer Bilanz sowie einer Gewinn- und Verlustrechnung vorgenommen und den Teilnehmer als Anteil eines Ergebnisberichtes zur Verfügung gestellt.

\subsection{Entscheidungsvorbereitung im Planspiel MARKUS}

In der betriebswirtschaftlichen Literatur werden vielfältige Instrumente zur Entscheidungsvorbereitung vorgeschlagen. ${ }^{400}$ Prehm versteht darunter in Anlehnung an Töpfer einen Sammelbegriff für Verfahren, weiterhin Methoden als auch Techniken und Modelle. ${ }^{401}$

Im Unternehmensplanspiel MARKUS haben die Teilnehmer nun Entscheidungen in den ausgeführten Funktionsbereichen zu fällen sowie weiterhin, unter Nutzung der Instrumente zur Entscheidungsvorbereitung, zu planen. Dabei wird darauf

400 Vgl. bspw. Kreikebaum, H., (Unternehmensplanung 1993), S. 63 ff.; Götze, U.; Rudolph, F., (Instrumente 1994), S. 16 ff; Welge, M.K., unter Beteiligung von Rüth, D., (Unternehmensführung 1985), S. 317 ff.; Horvarth, P., (Controlling, 1994), S. 206 ff.; Weber, J., (Einführung 1990), S. 41 ff.; Bramsemann, R., (Controlling 1990), S. 227 ff.; Hopfenbeck, W., (Unternehmen 1989), S. 608 ff.; Wild, J., (Grundlagen 1982), S. 146 ff. 
abgestellt, daß die Teilnehmer die Planungsaufgaben erkennen und lösen. ${ }^{402}$ Diese Planungsaufgaben stellen sich in den angeführten Bereichen wie folgt dar:403

Der Bereich Materialwirtschaft

- Die Beschaffung der Rohstoffe und Materialien wird unter dem Aspekt der wirtschaftlich günstigsten Beschaffungsart geplant

- Es ist erforderlich, die Bestellmengen zu ermitteln

- Weiterhin hat eine Planung der Lagerkapazitäten und des Lagerpersonals zu erfolgen

Der Bereich Produktion

- Es ist eine Produktionsprogrammplanung vorzunehmen

- Weiterhin müssen zum einen die Nutzungsdauern, zum anderen die Ersatzzeitpunkte der Produktionsanlagen geplant werden

- Schließlich ist die Reihenfolgeplanung vorzunehmen, die aufgrund der Sortenfertigung als zentral anzusehen ist, da zur Produktion der Sorten jeweils eine Umrüstung der Maschinen erforderlich wird

Der Bereich Marketing

- Hier muß eine Planung des Absatzprogramms vorgenommen werden

- Weiterhin muß auch der Einsatz der übrigen Instrumente des Marketing-Mix geplant werden

Der Bereich Finanzwirtschaft und Rechnungswesen

- Vornahme einer Finanz- und Erfolgsplanung

Auf diesem Niveau bleibt die Betrachtung noch weitgehend abstrakt, so daß davon ausgegangen werden kann, daß sich ein Teilnehmer einen schnellen Überblick verschaffen kann. Hat der Teilnehmer sich die anstehenden Planungsaufgaben bewußt gemacht, so ist er weiterhin dazu aufgefordert, die damit in Zusammenhang stehenden Variablen entsprechend zu organisieren, was bedeutet, daß er sich in einem Stadium der differenzierteren Informationsaufnahme befindet, wo- 
bei im folgenden deutlich werden soll, daß bereits bezüglich einer Planungsaufgabe eine Reihe von Informationen zu berücksichtigen sind:

Für diese Betrachtung wird an dieser Stelle beispielhaft der Bereich Materialwirtschaft herangezogen und die Merkmale der Planungsaufgabe »Bestimmung der wirtschaftlich günstigsten Güterbeschaffungsart« dargestellt. ${ }^{404}$

Zur Beschaffung der Objekte der Materialwirtschaft, die Basis- und Zusatzfaktoren sowie weiterhin Erzeugnisse umfassen, stehen drei Beschaffungsalternativen zur Auswahl. Dies sind die Vorratsbeschaffung, die bedarfssynchrone Beschaffung sowie schließlich die Beschaffung von Konkurrenzunternehmen, wobei die Beurteilung der Alternativen unter dem Kriterium des ,[...] Güterbereitstellungswertes bei Fremdbezug auf der Grundlage der entscheidungsrelevanten Kosten“ 405 erfolgt. Dabei umfaßt der Güterbereitstellungswert bei Fremdbezug den Gütereinstandswert sowie weiterhin die relevanten Lagerkosten und die Kapitalbindungskosten.

In den Vorteilhaftigkeitsvergleich sind daher die folgenden Größen einzubeziehen:

FÜR DIE GÜTEREINSTANDSWERTE

- Die mit Preisen bewerteten Beschaffungsmengen

- Die Transportkosten, die durch die Nutzung von Speditionsleistungen entstehen und durch die Teilnehmer exakt berechnet werden können.

Die Gütereinstandswerte setzen sich dabei aus den mit Preisen bewerteten Beschaffungsmengen sowie den Transportkosten zusammen. Nehmen die Teilnehmer eine Prognose der Güterpreise vor, so haben sie zu berücksichtigen, daß eine Vorratsbeschaffung gegenüber einer bedarfssynchronen Beschaffung mindestens eine Periode vorher vollzogen werden muß, wobei von einer Abweichung der Marktpreise in den Betrachtungsperioden auszugehen ist. Weiterhin ist ein Auf-

\footnotetext{
403 Vgl. Prehm, H.-J., (MARKUS 1995), S. 39 f.

404 Vgl. Prehm, H.-J., (MARKUS 1995), S. 85 ff.

405 Vgl. Prehm, H.-J., (MARKUS 1995), S. 86.
} 
schlag, der in Zusammenhang mit einer bedarfssynchronen Beschaffung auferlegt wird und als Aufschlag auf den Marktpreis erfolgt, in die Prognose mit einzubeziehen. Sicherheit bezüglich der Preise besteht lediglich im Hinblick auf eine Beschaffung bei einem konkurrierenden Unternehmen.

\section{SOWIE WEITERHIN}

- Die von der Beschaffungsart abhängigen relevanten Lagerkosten, die unter Berücksichtigung des Lagerraumnutzungsgrades oder durch eine Grenzkostenbetrachtung berechnet werden können.

- Die relevanten Kapitalbindungskosten sind in den Vorteilhaftigkeitsvergleich einzubeziehen, falls zu unterschiedlichen Zeitpunkten und in unterschiedlicher Höhe liquide Mittel gebunden sind.

Es konnte deutlich gemacht werden, daß bereits bezüglich einer einzelnen Planungsaufgabe eine Reihe von Informationen zu berücksichtigen sind. In diesem Zusammenhang wird weiterhin deutlich, daß der Teilnehmer im Hinblick auf alle genannten Planungsaufgaben eine Vielzahl von Aspekten in einen Sinnzusammenhang zu bringen hat.
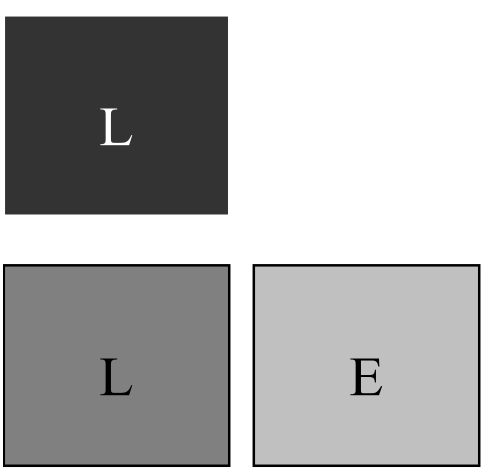

\subsection{7}

\section{Liveware-Environment}

\subsubsection{1}

\section{Darstellung des Unternehmensplanspiels PENTA}

Das S-H-E-L-L - Modell geht davon aus, daß die in ihm angenommenen Basiselemente eines Systems in einer Umwelt existieren. Auch das System Unternehmung existiert in einer Umwelt. So führt Bramsemann in bezug auf das System Unternehmung aus: „Jede Unternehmung ist in ein Beziehungsnetz wirt- 
schaftlicher, sozialer, juristischer, technologischer, logistischer und politischer Entwicklungen eingebunden." 406 So richten sich alle strategischen Entscheidungen sowohl am Unternehmen als auch an dessen Umweltbedingungen aus. ${ }^{407}$ Die Liveware-Environment-Komponente des S-H-E-L-L - Modells wird daher hier als Umfang verstanden, in dem ein Teilnehmer im Unternehmensplanspiel mit Randelementen des Unternehmungssystems konfrontiert ist, die mit ihren Relationen zur Umwelt im Modell abgebildet werden.

In diesem Zusammenhang soll auf ein weiteres Unternehmensplanspiel bezug genommen werden, dessen Schwerpunkt im Bereich der Umweltentscheidungen liegt. Dabei handelt es sich um das Unternehmensplanspiel PENTA. Das Unternehmensplanspiel PENTA, ein Akronym für Planspiel Entsorgung und A Abfallwirtschaft, wurde am Institut für betriebswirtschaftliche Produktions- und Investitionsforschung, Abteilung Unternehmensplanung, durch Bloech in Zusammenarbeit mit Rüscher \& Schubert entwickelt. 408

Das Planspiel PENTA stellt ein allgemeines Konkurrenzplanspiel dar, welches insbesondere der Aus- und Weiterbildung dient. Es werden hier alle wesentlichen betrieblichen Teilbereiche simuliert, wobei der Schwerpunkt in der Berücksichtigung der Abfall- und Entsorgungswirtschaft liegt, die bereits seit Jahren eine hohe Brisanz aufweist, da Unternehmen immer häufiger Umweltauflagen zu berücksichtigen haben. Im Planspiel PENTA finden in besonderem Maße produktionsbedingt verursachte Emissionen und Abfallstoffe, inklusive zur Verfügung stehender Maßnahmen zu ihrer Beseitigung, Aufbereitung und Entsorgung, aber auch zu ihrer Vermeidung Berücksichtigung, ${ }^{409}$ wobei sich die Gesamtsituation wie folgt darstellt:410 [Vgl. Abbildung 3-9].

Mit der Produktion der Fertigprodukte X und Y sind Verunreinigungen von Luft und Wasser verbunden, zudem entstehen Lärmemissionen, und es fallen feste Abfallstoffe an. Die Aufgabe der Unternehmen besteht in diesem Zusammenhang

\footnotetext{
406 Bramsemann, R., (Handbuch 1990), S. 29.

407 Vgl. Kreikebaum, H., (Unternehmensplanung 1993), S. 34.

408 Vgl. Bloech, J., in Zusammenarbeit mit Rüscher, H.; Schubert, U., (PENTA 1991);

Bloech, J.; Rüscher, H., (PENTA 1992), S. 75.

409 Vgl. Bloech, J.; Rüscher, H.,(PENTA 1992), S. 67 f.
} 
darin, die genannten Faktoren aufzubereiten, $\mathrm{zu}$ vermindern und $\mathrm{zu}$ entsorgen. Bezüglich aller Umweltbelastungen bestehen dabei gesetzlich vorgeschriebene Grenzwerte, die bei Überschreitung mit Geldstrafen geahndet werden oder zu Produktionskürzungen führen. Die Entsorgung kann durch eigene Maßnahmen oder auch durch Abgabe an eine Abfallbörse, sprich durch Aufbereitung seitens anderer Unternehmen, realisiert werden. Jedes Unternehmen ist aber auch selbst in der Lage über die Abfallbörse Entsorgungen durchzuführen. Demgemäß entstehen einem Unternehmen Kosten bei Fremdentsorgung, es kann aber auch Erträge durch die Entsorgung für andere Unternehmen erwirtschaften.

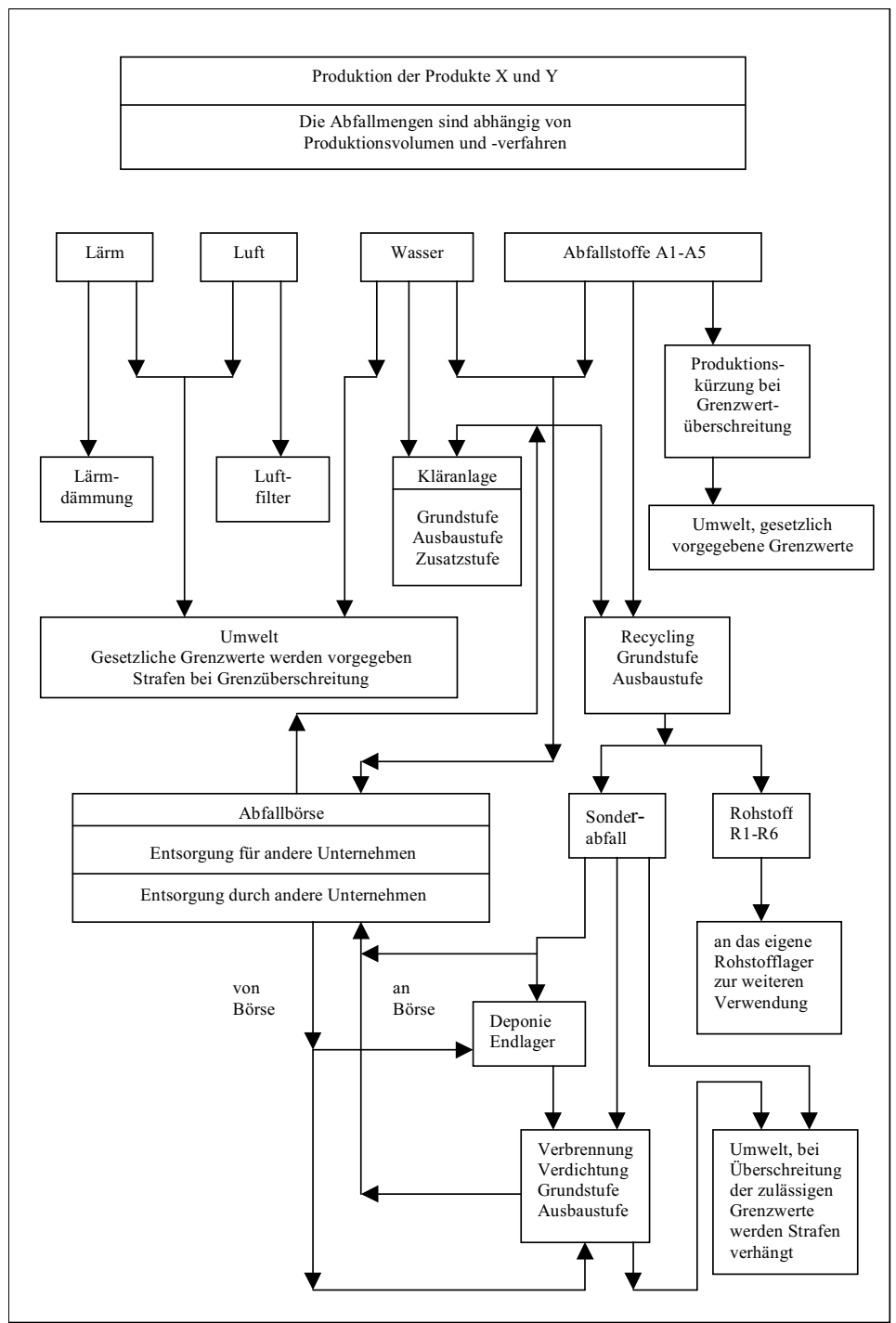

Abbildung 3-9: Entsorgungs- und Abfallwirtschaft im Planspiel PENTA $^{411}$ 
Aus der Produktionstätigkeit erwachsen nun im wesentlichen die nachfolgend ausgeführten Umweltbelastungen:412

\section{LÄRMBELASTUNG}

Die durch das Unternehmen eingesetzten Fertigungsanlagen erzeugen eine in Dezibel gemessene Grundlärmbelastung, die sich logarithmisch mit der Anzahl der eingesetzten Maschinen erhöht. Eine Reduzierung des Lärms ist durch den Einbau von Anlagen zur Lärmdämmung möglich.

\section{LUFTVERSCHMUTZUNG}

Das Ausmaß an Luftverschmutzung und die Menge an verbrauchter Luft wird zum einen durch die Abfallstückliste, zum anderen durch das eingesetzte Fertigungsverfahren bestimmt. Die Verschmutzung der Luft kann durch den Einbau von Luftfiltern schrittweise reduziert werden.

\section{WASSERVERSCHMUTZUNG}

Durch Produktionsmenge und Produktionsverfahren wird der Umfang an anfallendem Schutzwasser bestimmt, wobei verschiedene Maßnahmen gleichzeitig nutzbar sind, um das Schmutzwasser zu entsorgen bzw. das Maß an Wasserverschmutzung zu vermindern:

- Entsorgung durch eine ungereinigte Abgabe an die Umwelt, als wilde Entsorgung bezeichnet.

- Entsorgung durch Abgabe an die Abfallbörse oder durch direkte Abgabe an Konkurrenten.

- Durch Errichtung einer eigenen Kläranlage, in der das Schmutzwasser mit unterschiedlichen Aufbereitungsgraden geklärt werden kann.

\section{FESTE ABFALLSTOFFE}

Auch die Menge an festen Abfallstoffen wird durch die Produktionsmengen sowie durch die Produktionsverfahren determiniert, wobei hier weiterhin ebenfalls die Möglichkeit einer wilden Entsorgung und die Abgabe an die Abfallbörse oder

412 Vgl. Bloech, J.; Rüscher, H., (PENTA 1992), S. 89 ff; Bloech, J., in Zusammenarbeit mit Rüscher, H.; Schubert, U.,(PENTA 1991), S. 32 ff. 
Konkurrenten möglich ist. Als dritte Entsorgungsmöglichkeit kann schließlich eine Aufarbeitung der Abfallstoffe durch Recycling vorgenommen werden.

SONDERABFALL

Durch das Recycling der Abfallstoffe kommt es weiterhin zu Sonderabfällen, die ebenfalls auf drei Wegen entsorgt werden können. So kann zum einen eine wilde Entsorgung vorgenommen werden, zweitens kann eine Abgabe an die Abfallbörse oder direkt an die Konkurrenten erfolgen. Drittens schließlich ist eine Deponierung des Sonderabfalls in einer eigenen Deponie vorgesehen, die jedoch Personalund Wartungskosten verursacht und auch nach ihrer Schließung noch Unterhaltskosten hervorruft. 

bereich

Unter Berücksichtigung des Grundgedankens der Allgemeinen Systemtheorie, ${ }^{413}$ deren Systembegriff dem Planspiel als einer allgemeinen Methode zugrunde gelegt wurde, ${ }^{414}$ erfolgte in Kapitel drei die Betrachtung des Unternehmensplanspiels, in Zusammenhang mit grundlegenden Systembausteinen. Dies geschah unter Berücksichtigung des S-H-E-L-L - Modells, das weiterhin die Möglichkeit bot, die in der Problemlöseforschung gewonnenen Erkenntnisse zu menschlichem Fehlverhalten im Umgang mit komplexen Systemen in einen gemeinsamen Bezugsrahmen zu stellen. ${ }^{415}$

In diesem Zusammenhang konnten die im S-H-E-L-L - Modell repräsentierten Komponenten Liveware, Liveware, Software, Hardware und Environment wie folgt interpretiert werden:

- Die Liveware-Komponente des S-H-E-L-L - Modells wurde als der Teilnehmer an einem Planspiel interpretiert, der sich aktiv mit den Gegebenheiten des Simulationsmodells auseinandersetzt, respektive sich «handelnd» mit dem Planspiel auseinandersetzt. Dabei wurde der Spieler als Handlungssubjekt im Planspiel angenommen.

Demgemäß konnte davon ausgegangen werden, daß der Spieler als Handlungssubjekt in Korrespondenz mit den übrigen Komponenten des S-H-E-L-L - Modells steht, respektive als Kontrolleur des Systems, wobei er sich gleichsam jedoch auch selbst als Systemelement zu begreifen hat. In diesem Zusammenhang wurde aufgezeigt, daß die Liveware-Komponente im folgenden als interagierend mit den übrigen Komponenten zu betrachten ist, wobei der Liveware-Liveware Beziehung eine Doppelfunktion zugewiesen werden kann.

\footnotetext{
413 Vgl. Abschnitt 2.1.2.1: Das Unternehmensplanspiel: Ein Simulationsmodell und Abschnitt 3.4.2: Das S-H-E-L-L - Modell als eine Möglichkeit zur Darstellung der Natur menschlichen Fehlverhaltens.

414 Vgl. Abschnitt 2.1.2: Simulationstechnik beim Unternehmensplanspiel.

415 Vgl. Abschnitt 3.4: Das S-H-E-L-L - Modell.
} 
- So ist die Liveware-Liveware-Komponente des S-H-E-L-L - Modells, in Zusammenhang mit der im Unternehmensplanspiel erfolgenden Gruppenbildung, als die Auseinandersetzung mit einem Planspiel in Gruppen anzusehen. Weiterhin stehen die Teilnehmer jedoch auch innerhalb der Gruppe in Wechselbeziehung mit den anderen Gruppenmitgliedern, darüber hinaus auch in Wechselbeziehung mit den konkurrierenden Planspielgruppen, wodurch gewisse gruppendynamische Effekte hervorgerufen werden.

Im weiteren konnte davon ausgegangen werden, daß der Teilnehmer an einem Planspiel sich im System zum einen als Einzelperson, zum anderen jedoch auch als Element der Gruppe zu begreifen hat. Daher kann sowohl die Liveware-Komponente, in ihrer Funktion als individuelles Handlungssubjekt, als auch die Liveware-Liveware-Komponente, in ihrer Funktion als Gruppe, als interagierend mit den übrigen Komponenten angesehen werden. So agiert auf der einen Seite jeder Spieler individuell, indem er sich aktiv in den Gruppenprozeß einbringt, während die getroffene Entscheidung auf der anderen Seite eine Expression des Gruppenprozesses darstellt. Wobei auch hier festgehalten werden muß, daß sich sowohl der einzelne Teilnehmer als auch die teilnehmende Gruppe gleichsam als »Kontrolleur« sowie als Bestandteil des Systems zu begreifen hat.

- Die Liveware-Software-Komponente wurde als die Entscheidungssituation im Unternehmensplanspiel begriffen, mit der sich die Teilnehmer auseinanderzusetzen haben.

- Die Liveware-Hardware-Komponente des S-H-E-L-L - Modells wurde als die Auseinandersetzung eines Teilnehmers respektive einer Gruppe mit Methoden zur Entscheidungsfindung interpretiert, welche die Teilnehmer anzuwenden haben, um ihre Entscheidungen vorzubereiten. Dabei wird auf die Komplexität des Planungsprozesses abgestellt, den der Teilnehmer zu durchlaufen hat, bis er eine mögliche Entscheidung fällen kann. Diese Komponente kennzeichnet daher die Planungssituation.

- Die Liveware-Environment-Komponente des S-H-E-L-L - Modells wurde schließlich als Umfang verstanden, in dem ein Teilnehmer im Unternehmens- 
planspiel mit Elementen des Unternehmungssystem konfrontiert ist, die mit ihren Relationen zur Umwelt im Modell abgebildet werden. Diese Komponente kennzeichnet daher die Unternehmenssituation in ihrem Bezug zur Umwelt.

Die konstitutiven Elemente eines Unternehmensplanspielsystems können daher, unter Berücksichtigung der Erkenntnisse aus Kapitel drei, in der folgenden Weise voneinander abgegrenzt werden:

- Der individuelle Teilnehmer an einem Planspiel

- Der individuelle Teilnehmer an einem Planspiel als Teil einer Planspielgruppe sowie deren konkurrierendes Verhalten

- Das Planspielmodell mit

a) Entscheidungssituation

b) Planungssituation

c) Unternehmenssituation in bezug auf die Umwelt

Nach Orth könnte es auch um die folgenden Aspekte ergänzt werden:416

- Der Spielstand

- Der Spielleiter

Weiterhin gelang es, unter Berücksichtigung der dargestellten Unternehmensplanspiele, das durch die Modellbildung erfaßte Ausmaß an betrieblicher Komplexität sowie Fehlleistungen im Umgang mit dieser Komplexität aufzuzeigen. An dieser Stelle erscheint es nun wichtig, daß Dörner die unterschiedlichen Formen von Fehlverhalten insgesamt auf den Umgang mit Komplexität zurückführt. Sie, die Komplexität, stellt möglicherweise den Ausgangspunkt für Mißerfolge dar, die sich wiederum aus der Unfähigkeit von Personen begründen, mit Komplexität umzugehen. „Manche können daraus lernen und ihr Denken den Anforderungen adaptieren; andere schaffen das nicht"“.417

Da davon auszugehen ist, daß differenziertere Aussagen über die Komplexität als solche dazu führen Komplexität besser zu verstehen und demzufolge das Denken

\footnotetext{
416 Vgl. Orth, C., (Unternehmensplanspiele 1999), S. 71 ff. Orth berücksichtigt daneben auch die Aspekte des Planspielmodells sowie der konkurrierenden Planspielgruppen. 
den Anforderungen besser zu adaptieren ist, wird im folgenden der Systemgedanke erneut aufgegriffen. Dabei wird das durch die Modellbildung erfaßte Ausmaß an Komplexität als attributive Eigenschaft von Systemen angenommen; und der Versuch unternommen Komplexität unter kontextunabhängigen Gesichtspunkten näher zu spezifizieren und kontextabhängig anzuwenden.

\subsection{Die Allgemeine Systemtheorie}

Um zu differenzierteren Aussagen über die Komplexität im Unternehmensplanspiel zu gelangen, sind zunächst weitere Aussagen darüber zu machen, in welcher Weise die Allgemeine Systemtheorie in der Lage ist das Unternehmensplanspielmodell zu beschreiben. Dazu ist zunächst auf konstituierende Merkmale derselben einzugehen. Sie verdeutlichen, unter welchen Gesichtspunkten die Allgemeine Systemtheorie zu ihrem universellen Anspruch ${ }^{418}$ gelangt und bilden weiterhin die Grundlage für die sich anschließenden Betrachtungen.

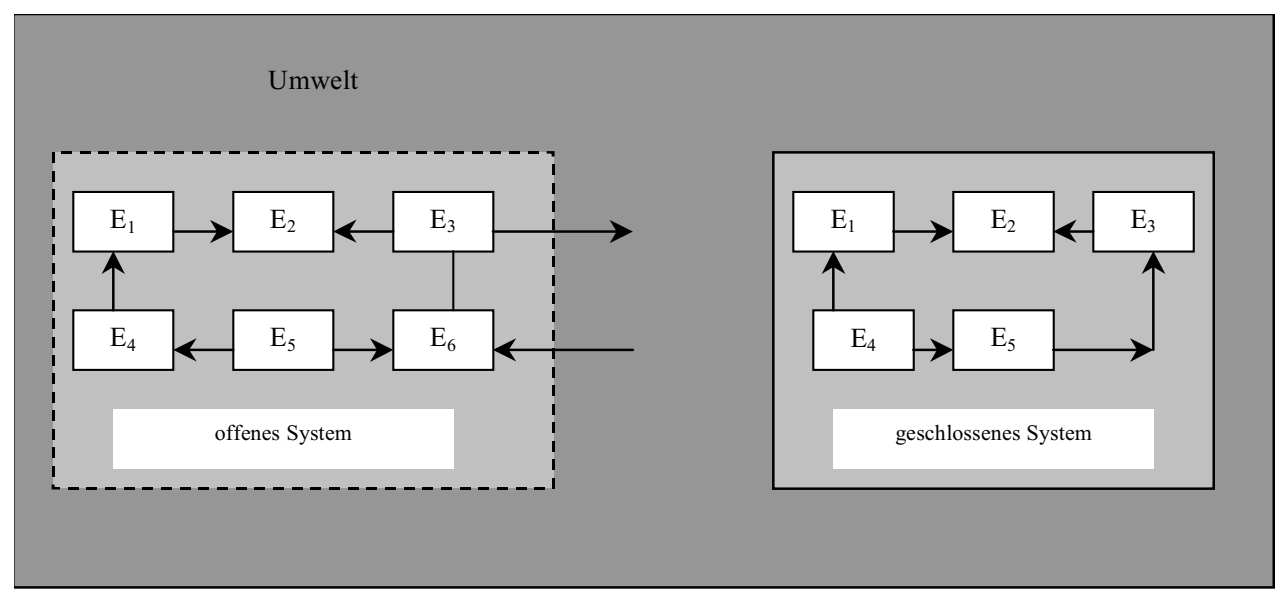

Abbildung 4-1: $\quad$ Offenes und geschlossenes System ${ }^{419}$

Wichtig in Zusammenhang mit dem Systembegriff der Allgemeinen Systemtheorie ist zunächst der Gegensatz zwischen geschlossenem und offenem System, aus dem Bertalanffy seine Auffassung von Wissenschaft herleitet. ${ }^{420}$ [Vgl. Abbildung 4-1].

\footnotetext{
418 Zum universellen Anspruch der Allgemeinen Systemtheorie vgl. Abschnitt 2.1.2: Simulationstechnik beim Unternehmensplanspiel und Abschnitt 3.4.2: Das S-H-E-L-L - Modell als eine Möglichkeit zur Beschreibung der Natur menschlichen Fehlverhaltens.

419 In Anlehnung an Dworatschek, S., (Management-Informations-Systeme 1971), S. 31.

420 Vgl. Müller, K., (Systemtheorie 1996), S. 68.
} 
So führte Bertalanffy Zweifel gegenüber dem physikalischen Konzept eines geschlossenen Systems und dem damit verbundenen phänomenologischen Entropiebegriff der Thermodynamik an. ${ }^{421}$ Im Bereich geschlossener Systeme gilt der Zweite Thermodynamische Hauptsatz, demzufolge Arbeit stets in Wärme, Wärme jedoch stets nur zu einem Bruchteil in Arbeit überführt werden kann, uneingeschränkt. Entropie stellt in diesem Zusammenhang ein Maß für die in einem System enthaltene Energie dar, die jedoch nicht in Arbeit umgewandelt werden kann, sondern lediglich in Wärme. Bertalanffy bezeichnet diesen Anteil an Energie in einem System als gebundene Energie, die dem Anteil an Energie gegenübersteht, die in Arbeit umgewandelt und als freie Energie bezeichnet werden kann. ${ }^{422}$

Der Systemzustand des Organismus macht es nun erforderlich, von geschlossenen $\mathrm{zu}$ offenen Systemen überzugehen. „Denn es ist das wichtigste Kennzeichen der organischen Systeme, daß sie nicht geschlossene Systeme in einem echten Gleichgewicht, sondern offene in einem (quasi-) stationären Zustand darstellen. Dieser Charakter als eines Systems, das sich im Wechsel seiner Bestandteile erhält, kommt allen biologischen Systemen zu, seien diese nun Stoffwechselgeschehnisse, Zellen, vielzellige Lebewesen, Vergesellschaftungen von Einzelwesen." $" 423$

So berücksichtigt die Thermodynamik offener Systeme, im Gegensatz zu derjenigen geschlossener Systeme, sowohl Entropieänderungen aufgrund interner Arbeit als auch den Transport von energiehaltigem Material in ein System. Offene Systeme zeichnen sich durch eine, von der klassischen Physik her nicht zu erwartende, abnehmende Entropie aus: durch den paradox erscheinenden Vorgang zunehmender Ordnung. Sie geben zerfallene Bausteine ab und bauen neue Vorgänge auf, indem sie die freie Energie komplexer organischer Moleküle in ihrer Umwelt nutzen. Ihr innerer Zustand ist nicht der eines statischen (chemischen) Gleichgewichts, in dem sie arbeitsunfähig wären, sondern ein stationärer Prozeß.

\footnotetext{
421 Vgl. Bertalanffy, L. v., (Biologie 1942), S. 12 ff.

422 Vgl. Bertalanffy, L. v., (Biologie 1942), S. 14 f. Zu verschiedenen Entropie-Begriffen vgl. bspw. Greulich, W. (Hrsg.), (Lexikon 1999), S. 251 f. 
Um diesen zu kennzeichnen, führt Bertalanffy eine Kategorie ein, die geeignet scheint, die Differenz von offenen zu geschlossenen Systemen klar herauszustellen. Stationäre Prozesse, die sich weitab von maximaler Entropie bewegen, befinden sich danach in einem fließenden Gleichgewicht, das definiert ist durch eine gegen null gehende Entropieproduktion. ${ }^{424}$

Weiterhin ist für die Allgemeine Systemtheorie konstituierend, daß der Systembegriff durch die Existenz von Beziehungen zwischen den Systemelementen festgelegt wird. Dadurch unterscheidet er sich gegenüber einer Menge.425 Dieser Zusammenhang impliziert, daß sich die allgemeinen Systemeigenschaften gerade durch die Unterscheidung gegenüber einer Menge konstituieren. ${ }^{426}$ [Vgl. Abbildung 4-2].

So sind die Elemente in einem System verbunden und agieren als Einheit, während die Elemente in einer Menge eine beziehungslose Ansammlung darstellen. Die Beziehungen in einem System werden dabei als Struktur des Systems bezeichnet, wodurch ein System stets aus einer Menge von Elementen und einer damit aufgebauten Struktur besteht. ${ }^{427}$ Die Elemente des Systems offenbaren daher ihre Eigenschaften nur als Teil des Ganzen und nicht bei Abstraktion über die Relationen.

Flechtner führt hingegen aus, daß sich die Unterscheidung zwischen System und Menge wesentlich im Methodischen begründet, sie genauer gesagt eine Frage der Betrachtungsweise ist. Im Realen existiert kein einfaches Nebeneinander im strengen Sinne, im Gegenteil beinhaltet jedes Nebeneinander realer Objekte deren Beziehungen untereinander. In bestimmten Fragestellungen ist es aber möglich, Systeme als Mengen zu betrachten. In dem Falle wird von allen Beziehungen innerhalb des Nebeneinanders abstrahiert. Desgleichen ist es möglich, höher organisierte Ganzheiten als bloße Systeme zu betrachten. Hier werden dann lediglich die existierenden Beziehungen zwischen den Objekten betrachtet. ${ }^{428}$

\footnotetext{
424 Vgl. Bertalanffy, L. v., (Biologie 1942), S. 25 ff.

425 Vgl. Abschnitt 2.1.2.1: Das Unternehmensplanspiel: Ein Simulationsmodell.

426 Vgl. Bliss, C., (Management 2000), S. 82.

427 Vgl. Dworatscheck, S., (Management-Informations-Systeme 1971), S. 29.

428 Vgl. Flechtner, H.-J., (Grundbegriffe 1969), S. 12.
} 


\begin{tabular}{|c|c|c|c|}
\hline & & System & Menge von Elementen \\
\hline 常 & $\frac{\infty}{\frac{D}{D}}$ & $\begin{array}{l}\text { Miteinander verbundene Elemente agieren } \\
\text { als Einheit. }\end{array}$ & $\begin{array}{l}\text { Beziehungslose Ansammlung } \\
\text { von Elementen. }\end{array}$ \\
\hline 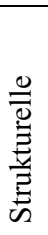 & 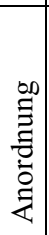 & $\begin{array}{l}\text { Die Anordnung der Elemente ist entschei- } \\
\text { dend. Eine Veränderung dieser Struktur } \\
\text { verändert auch die Eigenschaften bzw. das } \\
\text { Verhalten des Systems }\end{array}$ & $\begin{array}{l}\text { Die Anordnung der Elemente ist irrelevant. } \\
\text { Mengen besitzen keine Struktur. }\end{array}$ \\
\hline 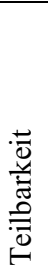 & & $\begin{array}{l}\text { Das Hinzufügen oder Eliminieren verändert } \\
\text { potentielle Eigenschaften. Die Spaltung } \\
\text { eines Systems generiert keinesfalls zwei } \\
\text { Systeme, sondern ein potentiell beschädigtes } \\
\text { System mit Funktionsdefekten. }\end{array}$ & $\begin{array}{l}\text { Das Hinzufügen oder Eliminieren von Ele- } \\
\text { menten tangiert keine essentiellen Eigen- } \\
\text { schaften. Die Teilung einer Menge erzeugt } \\
\text { zwei Mengen. }\end{array}$ \\
\hline 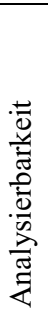 & & $\begin{array}{l}\text { Die Elemente sind über Relationen verbun- } \\
\text { den und offenbaren ihre Funktion nur als } \\
\text { Teil des Ganzen. Die Eigenschaften des } \\
\text { Systems lassen sich nur unzureichend über } \\
\text { eine isolierte Charakterisierung ihrer Sy- } \\
\text { stemelemente beschreiben. }\end{array}$ & $\begin{array}{l}\text { Die Elemente sind nicht verbunden und sind } \\
\text { daher isoliert analysierbar und bewertbar. } \\
\text { Die isolierte Charakterisierung der Elemente } \\
\text { beschreibt in der Summe die Menge. }\end{array}$ \\
\hline 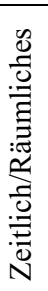 & 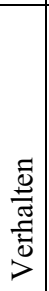 & $\begin{array}{l}\text { Die relative Anordnung der Elemente er- } \\
\text { möglicht unterschiedliche Systemzustände. } \\
\text { Systeme besitzen damit die Fähigkeit einer } \\
\text { dynamischen Veränderlichkeit und zeigen } \\
\text { hierbei ein Systemverhalten. }\end{array}$ & $\begin{array}{l}\text { Mengen verharren ausschließlich statisch. } \\
\text { Sie verfügen über keine Zustände und zeigen } \\
\text { keinerlei Verhalten. }\end{array}$ \\
\hline
\end{tabular}

\section{Abbildung 4-2: Allgemeine Systemeigenschaften in Abgrenzung zu einer bloßen Menge von Elementen ${ }^{429}$}

Diese Sichtweisen sind in Zusammenhang mit der Unterscheidung von geschlossenem und offenem System zu beleuchten. So fällt in die Gattung geschlossener Systeme jede mögliche Anordnung von Objekten, die dem Einfluß bestimmter Gesetze unterliegen. Gemäß dieser Gesetze gelten gewisse Identitätsbedingungen bereits auf der Ebene von Elementen: „Es ist möglich die Elemente solcher Systeme je für sich zu betrachten. Elementare Eigenschaften sind unabhängig vom Ort der Teile innerhalb des Systemganzen und unabhängig von ihrer zeitlichen Position in der Systemgeschichte gegeben." 430 In offenen Systemen sind die konstitutiven Eigenschaften hingegen nicht an den Elementen selbst ablesbar, sondern 1997). 
stellen ein Resultat ihrer Konfiguration dar. Hier bilden die kausal wechselwirkenden Elemente einen Funktionszusammenhang höherer Ordnung, der auf jedes einzelne Element zurückwirkt. ${ }^{431}$

Es resultiert daher, daß die Unterscheidung zwischen System und Menge nicht lediglich eine unterschiedliche Form der methodischen Betrachtung darstellt, sondern die Möglichkeit zur isolierten Betrachtung von Elementen von der Gestalt des betrachteten Systems abhängig ist. So ist in geschlossenen Systemen eine isolierte Betrachtung von Elementen möglich, wohingegen dies in offenen Systemen nicht der Fall ist. Daher erlangen die, von Bliss in weiter Anlehnung an O'Connor ausgeführten, Allgemeinen Systemeigenschaften in Abgrenzung zu einer bloßen Menge von Elementen Gültigkeit in offenen Systemen. In geschlossenen Systemen ist hingegen davon auszugehen, daß hier auch eine isolierte Betrachtung von Elementen möglich ist.

Es ist jedoch anzumerken, daß auch in der Allgemeinen Systemtheorie geschlossene Systeme lediglich zur untergeordneten Klasse einer allgemeineren Klasse von offenen Systemen werden. Hier konvergiert zum einen der zwischen System und Umwelt existierende energetische Ausdruck gegen Null. Weiterhin entfalten auch die Wechselwirkungen zwischen den Elementen des Systems eine nur schwache Kausalität. Die an offenen Systemen zu beobachtenden thermodynamischen Erscheinungen sind hingegen ein Zeichen einer besonderen Form der Organisation: von organisierter Komplexität. ${ }^{432}$

Im folgenden werden nun zwei Möglichkeiten zur Dekomposition eines offenen Systems, mit dem Ziel sie auf das Unternehmensplanspielmodell anzuwenden, ausgeführt.

\subsection{Systemdifferenzierung und Systemkomplexität}

- Luhmann leitet aus der Differenz von System und Umwelt sowie Element und Relation zwei Möglichkeiten zur Dekomposition eines Systems ab. Dabei

\footnotetext{
431 Vgl. Müller, K., (Systemtheorie 1996), S. 86 f.

432 Vgl. Müller, K., (Systemtheorie 1996), S. 68 ff und die dort angegebene Literatur.
} 
richtet sich die eine Möglichkeit auf die Bildung von Teilsystemen im System. Die andere Möglichkeit besteht in der Dekomposition in Elemente und Relationen. ${ }^{433}$

- „Im einen Falle geht es um die Zimmer des Hauses, im anderen Falle um die Steine, Balken, Nägel usw. Die erste Art der Dekomposition wird in einer Theorie der Systemdifferenzierung fortgeführt. Die andere mündet in eine Theorie der Systemkomplexität.“434

- Diese Theorien, die der Systemdifferenzierung sowie die der Systemkomplexität, bilden die Grundlage der weiterführenden Betrachtung.

„Die Theorie ist das Netz, das wir auswerfen, um „die Welt“ einzufangen, - sie zu rationalisieren, zu erklären und zu beherrschen. Wir arbeiten daran, die Maschen des Netzes immer enger zu machen. “435 Es kann jedoch nicht gelingen, die Welt in Gänze zu erfassen. Denn: „Nur die Welt selbst ist groß genug, um die ganze Welt zu begreifen. Es ist keine Karte von der ganzen Welt möglich, auf der alles verzeichnet wäre - es sei denn, die Karte wäre das Gelände selbst. Aber dann wäre sie keine Karte.“436 Diesen Zusammenhang verdeutlicht der „Satz von der Unvollständigkeit“" von Gödel.437 So weist er darin nach, daß in einem existenten formalen System die axiomatischen Aussagen nur dann belegbar sind, wenn sie unwahr sind. ${ }^{438}$ Nørretranders bezieht sich auf Gödel und führt aus: „Gödels Satz macht die Grenzen aller Erkenntnis sichtbar und damit, in gewissem Sinne, die einzige Gewißheit, die wir je haben werden: daß sich eine Unendlichkeit der Wahrheit nicht mit einer endlichen Theorie erfassen läßt“"439

\footnotetext{
433 Vgl. Luhmann, N., (Systeme 1996), S. 41.

434 Luhmann, N., (Systeme 1996), S. 41.

435 Popper, K.R., (Logik 1994), S. 31.

436 Nørretranders, T., (Welt 1997), S. 79.

437 Vgl. Gödel, K., (Sätze 1931).

438 Vgl. Chaitin, G.J., (Information 1982), S. $941 \mathrm{ff}$.

439 Nørretranders, T., (Welt 1997), S. 78 f.
} 
Luhmann geht jedoch davon aus, daß Systeme existieren und, in einem engeren Sinne, davon, daß selbstreferentielle Systeme existieren. Dies bedeutet in einem ganz allgemeinen Sinne, daß Systeme existieren, die in der Lage sind, eine Beziehung zu sich selbst herzustellen und darüber hinaus in der Lage sind, diese Beziehungen gegenüber Beziehungen zu ihrer Umwelt zu differenzieren, 440 wobei fachlicher Konsens darüber besteht, daß diese Existenz von Systemen in einer Umwelt und ihre Differenz zueinander als Ausgangspunkt jeder systemtheoretischen Analyse anzusehen ist. ${ }^{441}$ Luhmann verweist hier auf Foerster, der ausführt, daß Systeme nicht lediglich als gelegentlich und nicht nur als adaptiv anzusehen sind, ${ }^{442}$ sondern als strukturell an ihrer Umwelt orientiert. Ohne Umwelt könnten Systeme demgemäß nicht bestehen, sondern sie konstituieren und erhalten sich durch Herstellung und Wahrung einer Differenz zur Umwelt, wobei sie ihre Grenzen weiterhin dazu benutzen, die Differenz zu regulieren. ${ }^{443}$ Luhmann folgert daraus, daß Grenzerhaltung in diesem Sinne Systemerhaltung bedeutet. ${ }^{444}$

In diesem Zusammenhang ist davon auszugehen, daß die Umwelt all das verkörpert, was außerhalb des Systems liegt. Sie ist der »Rest der Welt« und verkörpert jenseits der definierten Systemgrenze »alles andere «. ${ }^{445}$ Dieses andere darf jedoch nicht außerhalb der Betrachtung bleiben, sondern dient dem Verständnis des Systems. So formuliert Luhmann: „Die Betrachtung eines Innen hat nur Sinn, wenn es ein Außen gibt. Dieses Außen muß im Systembegriff mitthematisiert werden, weil anders das Innen nicht verständlich gemacht werden kann." ${ }^{446}$

Weiterhin ist es notwendig, die Differenz von System und Umwelt von einer zweiten, ebenfalls konstitutiven Differenz, zu unterscheiden. Dies ist die Diffe-

440 Vgl. Luhmann, N., (Systeme 1996), S. 30 f. Es ist daher notwendig, die Systemtheorie als Theorie selbstreferentieller Systeme anzusehen. Bereits dadurch wird impliziert, daß die Systemtheorie stets sich selbst als einen ihrer Gegenstände zu begreifen hat. Vgl. ders., (Systeme 1996), S. 30 f.

441 Vgl. Luhmann, N., (Systeme 1996), S. 35.

442 Ein adaptives System ist ein System, welches gleichzeitig Informationen über seine Umwelt sowie die eigene Wechselwirkung mit der Umwelt aufnimmt. In den Informationen werden dann Regelmäßigkeiten erkannt, die bspw. in einem Modell verdichtet werden, welches dann die Grundlage für erkennbares Handeln in der realen Welt darstellt. Vgl. Gell-Mann, M., (Quark 1998), S. 53.

443 Vgl. Foerster, H. v., (Constructing 1973), S. 35 ff. zitiert nach Luhmann, N., (Systeme 1996), S. 35.

444 Vgl. Luhmann, N., (Systeme 1996), S. 35.

445 Porr, B., (Definitionen 2001), S. 5 f. 
renz von Element und Relation. Genauso wie Systeme nie ohne Umwelten oder auch Umwelten nie ohne Systeme existieren, existieren auch keine Elemente ohne Verknüpfungen oder Verknüpfungen ohne Elemente. Dabei stellt die Differenz in beiden Fällen eine Einheit dar, sie wirkt jedoch nur als Differenz. Denn nur als Differenz ermöglicht sie es, Informationsverarbeitungsprozesse anzuschließen. So muß sowohl die Differenz als auch die Einheit der Differenz in beiden Fällen als konstitutiv gedacht werden. ${ }^{447}$

Dadurch, daß Elemente relational in Anspruch genommen, also aufeinander bezogen werden, gewinnen sie zudem auch an Qualität. Dies kann ab einer verhältnismäßig geringen Größe in realen Systemen nur selektiv geschehen. Das bedeutet, daß andere, auch denkbare Relationen weggelassen werden müssen. Damit ist Qualität nur durch Selektion möglich. Die Notwendigkeit der Selektion ergibt sich hingegen durch Komplexität. 448

Dabei erscheint die Selektivität in Systemen, die eine sinnvolle Beziehung zu sich selbst herstellen können, als Kontingenz. Hier bedeutet Kontingenz zum einen, daß die Realität als auch anders möglich bewußt wird. Zudem kann sie auch als eingeschränkt verstanden werden, was bedeutet, daß die realisierten Strukturen und Prozesse von Bedingungen abhängen. Es läßt sich schließlich folgern, daß beide Annahmen beschränkend wirken. Sie agieren reduktiv und wirken damit beschränkend auf die potentielle Verknüpfung von allem mit allem. ${ }^{449}$

Zusammenfassend kann festgehalten werden, daß nach Luhmann davon auszugehen ist, daß sich ein realer Objektbereich aus Systemen aufgebaut denken läßt. Wird von offenen, dynamischen Systemen ausgegangen, so besitzt jedes einzelne der Systeme Beziehungen zu anderen Systemen. Diese bilden die Umgebung des Systems und wirken auf das betrachtete System über bestimmte Eingänge ein. Das betrachtete System wiederum wirkt auf die Umgebung über bestimmte Ausgänge ein. Das System selbst ist wieder zusammengesetzt aus Untersystemen und besitzt bestimmte Zustände. Diese Zustände, wie auch die Ein- und Ausgänge, können

\footnotetext{
446 Luhmann, N., (Zweckbegriff 1968), S. 120.

447 Vgl. Luhmann, N., (Systeme 1996), S. 41.

448 Vgl. Luhmann, N., (Systeme 1996), S. 42.

449 Vgl. Luhmann, N., (Komplexität 1980), Sp. 1065 f.
} 
sich im Zeitablauf ändern. Weiterhin ist festzuhalten, daß Luhmann davon ausgeht, daß in offenen, dynamischen Systemen relationale Verknüpfungen zwischen den Systemelementen existieren, die eine konstitutive Eigenschaft des Systems darstellen und sich ebenfalls im Zeitablauf verändern können. Diese Verknüpfungen sind dabei relational, da mit zunehmender Größe von Systemen eine wachsende Anzahl von Verknüpfungen zwischen den Elementen möglich wird. Es ergibt sich daher ein Selektionszwang, der es ermöglicht, daß Elemente Qualität gewinnen können. Dieser manifestiert sich als Kontingenz.

\section{Komplexität als attributive Eigenschaft von Systemen}

Es wurde bereits versucht, sich dem Komplexitätsbegriff grundlegend anzunähern, wobei festgehalten werden konnte, daß der Begriff der Komplexität zum einen unter kontextabhängigen, zum anderen unter kontextunabhängigen Aspekten $\mathrm{zu}$ beleuchten ist. ${ }^{450}$ Unter Rückgriff auf die Allgemeine Systemtheorie wird hier nun eine kontextunabhängige Betrachtung des Komplexitätsbegriffes verfolgt, und zwar mit dem Ziel, Aufschluß über allgemeine Merkmale von Komplexität zu erhalten. Dies kann dadurch gelingen, daß Komplexität als attributive Eigenschaft von Systemen angesehen wird. ${ }^{451}$

Zentrale Bedeutung hat dabei zunächst die Unterscheidung zwischen offenem und geschlossenem System, ${ }^{452}$ wobei im Zusammenhang mit den an dieser Stelle interessierenden Aussagen über Komplexität von dem Luhmann'schen SystemUmwelt-Paradigma auszugehen ist, in dem zwischen dem System als Einheit und dem System in der Systemumwelt unterschieden wird. ${ }^{453}$ So besteht zwischen dem System und der Systemumwelt ein Komplexitätsgefälle, welches als unabdingbar gegeben anzunehmen ist. ${ }^{454}$ Es ergibt sich durch die Unterscheidung von Außen und Innen. So entstehen durch Grenzziehung und Konstituierung der Differenz Bereiche von unterschiedlicher Komplexität. Dabei ist die Welt jedoch

$450 \quad$ Vgl. Abschnitt 2.2.2.1: Der Komplexitätsbegriff.

451 Vgl. zu der Verwendung von Komplexität als attributive Eigenschaft eines Systems Bliss, C., (Management 2000), S. 81.

452 Vgl. Abschnitt 4.1: Die Allgemeine Systemtheorie.

453 Vgl. Abschnitt 4.2.1: Differenzierung als konstitutive Eigenschaft von Systemen.

454 Vgl. Luhmann, N., (Zweckbegriff 1968), S. 121 f.; Luhmann, N., (Systeme 1996), S. 47 ff. 
immer komplexer als jedes System in der Welt. Das führt dazu, daß in der Welt stets mehr Ereignisse möglich sind als im System, da sie im Vergleich zum System in der Lage ist, mehr Zustände anzunehmen. ${ }^{455}$

Mithin schließt ein System für sich selbst mehr Möglichkeiten aus. Das System reduziert Komplexität, bildet dadurch eine höhere Ordnung mit weniger Möglichkeiten an denen sich das Erleben und Handeln des Menschen besser orientieren kann. Es läßt sich daher festhalten, daß die Trennung von Außen und Innen ein Gefälle der Komplexität stabilisiert. Dies führt dazu, daß dem Erleben und Handeln jetzt lediglich eine begrenzte Auswahl an Möglichkeiten näher gebracht wird. ${ }^{456}$ Wobei trotz einer begrenzten Auswahl von Möglichkeiten ein komplexes System weiterhin eine komplexe Angelegenheit bleibt: „In short, a complex system is something quite complex.“"457

Zentrale Bedeutung in Zusammenhang mit dem Verständnis komplexer Systeme hat nach Simon auch die Unterscheidung zwischen statischer und dynamischer Komplexität: „It is a familiar proposition that the task of science is to make use of the world's redundancy to describe that world simply. I shall not pursue the general methodological point here, but shall instead take a closer look at two main types of description that seem to be available to us in seeking an understanding of complex systems. I shall call these state description and process description, respectively. “458

Dabei handelt es sich bei einem statischen System um ein System, welches seinen Zustand nicht verändert. ${ }^{459}$ Die statische Komplexität beschreibt die strukturellen Eigenschaften des Systems sowie der Subsysteme ${ }^{460}$ zu einem Betrachtungszeitpunkt. Sie umfaßt Eigenschaften ,„...] wie die hierarchische Struktur des Systems, die Verbindungsmuster der Variablen, die Verschiedenheit der Komponenten sowie die Stärke der Interaktionen." ${ }^{6} 61$

\footnotetext{
455 Vgl. Luhmann, N., (Zweckbegriff 1968), S. 121.

456 Vgl. Luhmann, N., (Zweckbegriff 1968), S. 121.

457 Casti, J., (Connectivity 1979), S. 40.

458 Simon, H.A., (Architecture 1962), S. 479.

459 Vgl. Strohner, H., (Systeme 1995), S. 30.

460 Vgl. Funke, J., (Problemlösen 1984), S. 26.

461 Vgl. Funke, J., (Problemlösen 1984), S. 26.
} 
In diesem Zusammenhang wird die Anzahl von Variablen eines Systems oft als ein zentraler Indikator für dessen Komplexität angesehen. Ein solcher Indikator findet sich bspw. bei Vogt, der die Größe von Systemen anhand der Variablenanzahl differenziert und nach kleinen, mittelgroßen und großen Systemen unterscheidet. ${ }^{462}$ Wenn die Anzahl der Elemente gezählt wird, ergibt sich eine quantitative Dimension von Komplexität, und man könnte sich fragen, ob denn ein 12 Variablen umfassendes System halb so schwer sei wie ein System mit 24 Variablen? Eines mit mehr als 2000 Variablen möglicherweise um das Hundertfache schwerer als das mit 24 Variablen? ${ }^{463}$

So einfach verhält sich die Sache offensichtlich nicht. Das begründet sich dadurch, daß das reine Zählen von Elementen den qualitativen Einblick verwehrt, da die zwischen den Elementen existierenden Relationen nicht berücksichtigt werden. ${ }^{464}$ Auch diese Relationen werden im Hinblick auf die Komplexität jedoch als wichtig angesehen. So führt Malik aus: „Komplexität hat ihre Ursache im wesentlichen in den Interaktionen von Elementen.“465 Demnach wird die Komplexität der Systemstruktur durch die Anzahl der Elemente und ihrer Beziehungen bestimmt. Es ist also nicht allein eine große Zahl von Elementen ausreichend, sondern diese müssen miteinander verknüpft sein, damit ein System komplex wird. Nach Luhmann müssen mindestens drei Dimensionen bestimmt werden, um das Ausmaß der Komplexität eines Systems zu bestimmen. Dies sind die beiden vorgenannten Dimensionen der Zahl der Elemente sowie der Zahl der im System möglichen Beziehungen zwischen den Elementen - und zusätzlich, als dritte Dimension, die Verschiedenartigkeit der Beziehungen.

\begin{tabular}{|c|c|c|}
\hline & Komplexität & Kompliziertheit \\
\hline Elemente & Zahl der Elemente & Verschiedenartigkeit der Elemente \\
\hline Relationen & Zahl der Relationen & Verschiedenartigkeit der Relationen \\
\hline
\end{tabular}

$\begin{array}{ll}\text { Abbildung 4-3: } & \text { Statische Dimensionen systemtheoretischer Komplexität } \\ & \text { und Kompliziertheit }\end{array}$


Bronner subsumiert den letztgenannten Aspekt der Verschiedenartigkeit der Beziehungen zusammen mit der Verschiedenartigkeit der Systemelemente unter dem Begriff Kompliziertheit, der damit auch gegenüber dem Begriff der Komplexität, versteht man darunter die Dimensionen der Zahl der Elemente und der Zahl der Beziehungen, abgegrenzt werden kann. ${ }^{466}$ [Vgl. Abbildung 4-3].

\begin{tabular}{|c|c|c|}
\hline & Komplexität & Kompliziertheit \\
\hline Elemente & Variation der Anzahl & Variation der \\
& der Elemente im Zeitablauf & Verschiedenartigkeit der \\
& & Elemente im Zeitablauf \\
\hline Relationen & Variation der Anzahl & Variation der \\
& der Relationen im Zeitablauf & Verschiedenartigkeit der \\
& & Relationen im Zeitablauf \\
\hline
\end{tabular}

\section{Abbildung 4-4: Dynamische Dimensionen systemtheoretischer Komplexi- tät und Kompliziertheit}

Unter dem Hinweis auf die Dynamik eines komplexen Systems begegnet man einer anderen Form von Komplexität. ${ }^{467}$ Ein dynamisches System ist in der Lage, im Zeitablauf seinen Zustand zu verändern. Nimmt man für ein System zu einem beliebigen Zeitpunkt $\mathrm{t}$ den Zustand $\mathrm{z}_{\mathrm{t}}$ an, so kann der darauf folgende Zustand mit $\mathrm{Z}_{\mathrm{t}+1}$ beschrieben werden. Die Veränderung des Zustandes ist in der Regel vom Ausgangszustand sowie dem Input $x_{t}$ abhängig. Beschrieben wird die Veränderung mit der Übergangsfunktion $\mathrm{f}: \mathrm{z}_{\mathrm{t}+1}=\mathrm{f}\left(\mathrm{z}_{\mathrm{t}}, \mathrm{x}_{\mathrm{t}}\right) .{ }^{468}$ Nicht immer ist der Systemoutput jedoch eine einfache Funktion von dessen Input. So kann ein System strukturell durchaus einfach sein, dynamisch jedoch hoch komplex. ${ }^{469}$

Nach Luhmann sind Begriffsfassungen, die auch den Zeitaspekt berücksichtigen, als anspruchsvollere Begriffsfassungen anzusehen. Dabei nimmt die temporale Komplexität eines Systems in dem Maße zu, wie die Komplexitätsbestimmungen pro Zeiteinheit variieren. [So sind bspw. die Größenverhältnisse wie Anzahl der Beschäftigten oder Anzahl zu treffender Entscheidungen Schwankungen im

\footnotetext{
465 Malik, F., (Strategie 1989), S. 186.

466 Vgl. Bronner, R., (Komplexität 1992), Sp. 1122.

467 Vgl. Funke, J., (Problemlösen 1984), S. 28.

468 Vgl. Strohner, H., (Systeme 1995), S. 30 f.

469 Vgl. Casti, J., (Connectivity 1979), S. 102.
} 
Zeitablauf ausgesetzt.] Das System erinnert und antizipiert sich jedoch zu jedem Zeitpunkt selbst. Dies muß mit in Betracht gezogen werden, da hierdurch die Zeitkomplexität, wenn auch nur selektiv, zur Situationskomplexität wird. ${ }^{470}$ In einer grundlegenden Zusammenfassung können daher die Dimensionen statischer Komplexität und Kompliziertheit herangezogen und unter Einbeziehung des Zeitaspektes dargestellt werden. [Vgl. Abbildung 4-4].

\subsection{Das Unternehmensplanspiel als Modell über das komplexe Sy- stem der Unternehmung}

Die kontextunabhängigen Betrachtungen haben gezeigt, daß sich ein komplexer Gegenstandsbereich aus Systemen aufgebaut denken läßt; und verschiedene Arten von Komplexität aufweist. Im folgenden soll nun die Möglichkeit untersucht werden, mit dem Instrument der Systemdifferenzierung und Systemkomplexität ein Unternehmensplanspielmodell $\mathrm{zu}$ differenzieren, dessen Komplexitätsdimensionen zu benennen und ein Komplexitätsmaß abzuleiten.

Es erfolgt daher an dieser Stelle eine Betrachtung unter kontextabhängigen Gesichtspunkten, wobei davon auszugehen ist, daß eine kontextabhängige Betrachtung unter zwei Gesichtspunkten möglich ist: Zum einen ist auch hier davon auszugehen, daß das System der Unternehmung einer Anwendung der Allgemeinen Systemtheorie zugänglich ist. ${ }^{471}$ Weiterhin ist davon auszugehen, daß die betriebliche Komplexität ein zentrales Moment der Unternehmung darstellt. ${ }^{472}$ Es ist daher zu folgern: Unter kontextabhängigen Gesichtspunkten kann Komplexität als attributive Eigenschaft des Systems der Unternehmung angesehen werden, welches einer Anwendung der Allgemeinen Systemtheorie zugänglich ist.

\footnotetext{
$470 \quad$ Vgl. Luhmann, N., (Komplexität 1980), Sp. 1065.
}

471 Vgl. Abschnitt 3.1: Das System der Unternehmung. 


\subsection{1}

Differenzierung und Komplexität im Unternehmensplanspiel

\subsubsection{Differenzierung im Unternehmensplanspiel}

Es konnte festgehalten werden, daß Luhmann davon ausgeht, daß Systeme differenzierbar sind. In diesem Zusammenhang wurde bereits ausgeführt, daß die Differenz von System und Umwelt in einer Theorie der Systemdifferenzierung mündet. ${ }^{473}$ An dieser Stelle soll nun aufgezeigt werden, daß die Theorie der Systemdifferenzierung die Möglichkeit bietet, ein Unternehmensplanspielmodell zu differenzieren.

Es ist hier die Tatsache entscheidend, daß ein Systemmodell kein isomorphes Abbild des Realsystems sein muß, wofür zwei Gründe anzuführen sind: Zum einen kann der Umgang mit einem Modell nur dann sinnvoll sein, wenn dies eine Abstraktion des Realsystems darstellt, zum anderen kann es nur selten gelingen, Realsysteme in allen ihren Ausprägungen vollständig zu erfassen, so daß Modelle notwendigerweise unvollständig sein müssen. Ausreichend ist es, Homomorphie zu fordern, womit auch der Systemansatz Modelle nicht von der ceteris-paribusKlausel befreit. ${ }^{474}$

Es kann daher im weiteren davon ausgegangen werden, daß das Unternehmensplanspielmodell verschiedene Abstraktionsniveaus aufweist und damit einer Differenzierung zugänglich ist. Dieser Differenzierung liegen dabei zwei unterschiedliche Systemansätze zugrunde. Dies ist im Hinblick auf die bottom-upBetrachtung der extraspektive Systemansatz, bei dem alle Teile des Systems als Subsysteme verstanden werden, die durch Vereinigung das Gesamtsystem ergeben. Im Hinblick auf die top-down-Betrachtung ist dies der introspektive Systemansatz, der von einer systematischen Zerlegung des Gesamtsystems in seine Subsysteme ausgeht. ${ }^{475}$

\footnotetext{
472 Vgl. Malik, F., (Strategie 1989).

473 Vgl. Abschnitt 4.2.1: Differenzierung als konstitutive Eigenschaft von Systemen.

474 Vgl. Abschnitt 2.1.2.1: Das Unternehmensplanspiel: Ein Simualtionsmodell.

475 Vgl. Müller-Merbach, H., (Arten 1992), S. 857 ff. Für die Betrachtungen in Kapitel sechs kann später der konstruktive Systemansatz angenommen werden, der die beiden hier dargestellten Ansätze zu einem ganzheitlichen Systemverständnis vereint. Vgl. Abschnitt 6.3: Die unternehmerische Handlungskompetenz.
} 
Dabei kann das System der Unternehmung, im Sinne einer bottom-upBetrachtung ${ }^{476}$, als Ergebnis einer Synthese aus verschiedenen Subsystemen angenommen werden. Die kleinsten Beziehungskomplexe, die in diesen Systemen betrachtet werden, lassen sich hier als Elemente kennzeichnen, wobei Elemente sehr wohl eigene komplexe Systeme sein können. Sie werden jedoch dann als Elemente bezeichnet, wenn ihre interne Struktur nicht mehr Betrachtungsgegenstand ist, sondern nur die Wirkungen ihrer Verhaltensweisen auf das zu untersuchende System interessieren. ${ }^{477}$ Dies kann beispielhaft anhand einer horizontalen Differenzierung dargestellt werden. [Vgl. Abbildung 4-5].

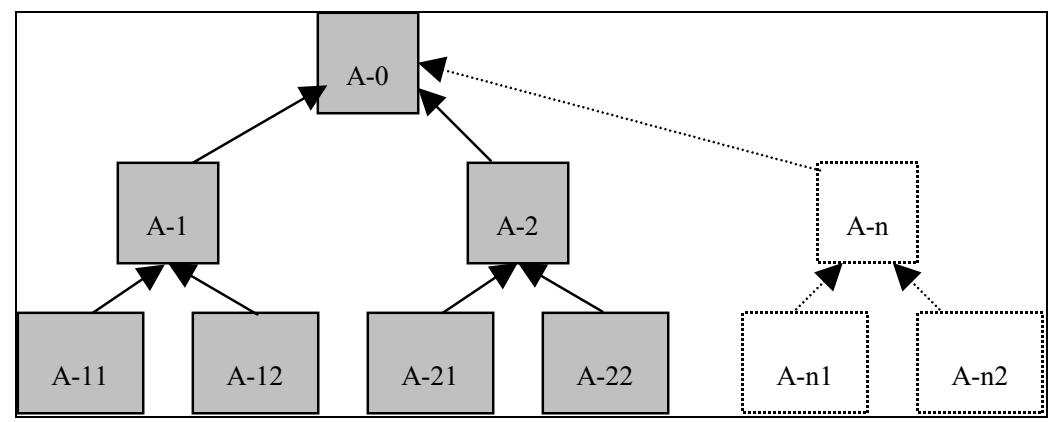

\section{Abbildung 4-5: Horizontale Differenzierung im Sinne einer bottom-up- Betrachtung $^{478}$}

Im Sinne einer top-down-Betrachtung ${ }^{479}$ kann das System Unternehmung demgegenüber als Zerlegung in verschiedene Subsysteme angenommen werden. Dies kann beispielhaft anhand einer vertikalen Differenzierung dargestellt werden. [Vgl. Abbildung 4-6].

476 Bei der Bottom-Up-Methode soll durch den Aufbau von Teillösungen eine neue Gesamtbsung konstruiert werden. Vgl. Biethahn, J.; Mucksch, H.; Ruf, W., (Informationsmanagement 1991), S. 303

477 Bleicher, K., (Entwicklung -Heft 1- 1970), S. 3 f.

478 Die Art der Darstellung erfolgt hier in Anlehnung an Klotzbücher. Klotzbücher, R., (Planspielentwicklung 1996), S. 101. Dieser spricht jedoch in diesem Zusammenhang von horizontaler Systemkomplexität. Vgl. ders., (Planspielentwicklung 1996), S. 101.

479 Bei der Top-Down-Methode werden Teilprobleme konstruiert, die ihren Ursprung in einem Ausgangsproblem haben. Vgl. Biethahn, J.; Mucksch, H.; Ruf, W., (Informationsmanagement 1991), S. 300. 


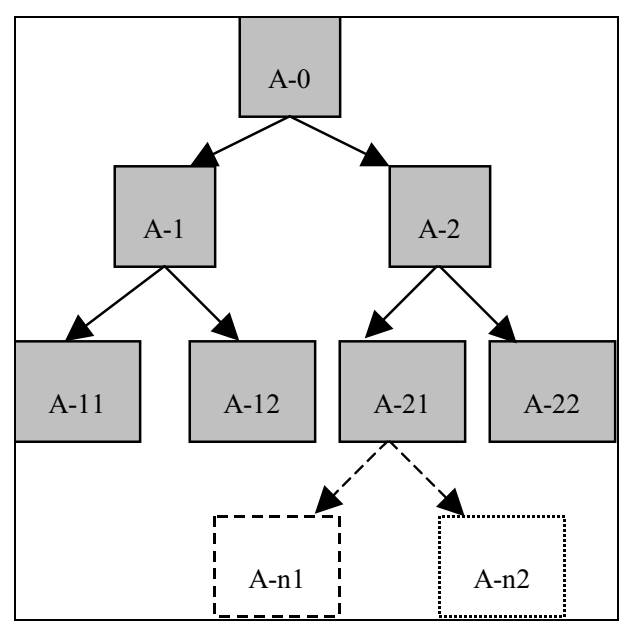

\section{Abbildung 4-6: Vertikale Differenzierung im Sinne einer top-down- Betrachtung ${ }^{480}$}

\subsubsection{Komplexität im Unternehmensplanspiel}

Es konnte weiterhin festgehalten werden, daß Luhmann davon ausgeht, daß zwischen Element und Relation eine Differenz existiert. In diesem Zusammenhang wurde bereits ausgeführt, daß die Differenz von Element und Relation in einer Theorie der Systemkomplexität mündet. ${ }^{481}$ An dieser Stelle soll nun aufgezeigt werden, daß die Theorie der Systemkomplexität die Möglichkeit bietet, Komplexitätsdimensionen für ein Unternehmensplanspielmodell zu bestimmen.

Auch an dieser Stelle ist, in bezug auf das Unternehmensplanspiel zunächst davon auszugehen, daß durch die Anwendung der Simulation auf das Systemmodell, welches die Realität in den - im Hinblick auf die Fragestellung - als wichtig erachteten Aspekten abbildet, ein Simulationsmodell entsteht. Da es die Realität jedoch nur in ausgewählten Aspekten erfaßt, wird lediglich vorgetäuscht, daß die Abbildung der Wirklichkeit entspricht. Durch die Modellbildung erfolgt daher eine Abstraktion über die realen Gegebenheiten, wobei diese Art der Komplexität als Abbildungskomplexität bezeichnet werden kann.

Die Art der Darstellung erfolgt hier in Anlehnung an Klotzbücher. Klotzbücher, R., (Planspielentwicklung 1996), S. 101. Dieser spricht jedoch in diesem Zusammenhang von verikaler Systemkomplexität. Vgl. ders., (Planspielentwicklung 1996), S. 101. 
Weiterhin ist in bezug auf die Unternehmung festzuhalten, daß es sich hier um ein offenes System handelt. ${ }^{482}$ In diesem Zusammenhang wird der Aspekt wichtig, daß Systeme Grenzen aufweisen, die jedoch nicht ohne ein dahinter zu denken sind; und demgemäß die Realität des Jenseits und damit die Möglichkeit des Überschreitens voraussetzen. Sie weisen also nach allgemeinem Verständnis eine Doppelfunktion, im Sinne einer gleichermaßen Trennung und Verbindung von System und Umwelt auf. ${ }^{483}$ Durch Grenzdefinitionen sind Systeme daher in der Lage sich gleichsam zu öffnen und zu schließen, da infolgedessen interne Relationen von externen Relationen unterschieden, gleichzeitig aber auch aufeinander bezogen werden können. ${ }^{484}$ Die Konstitution einer Grenze vom System zur Umwelt führt dabei dazu, daß Elemente konsequent dem System oder der Umwelt zuzurechnen sind. Die Relationen können demgegenüber auch zwischen System und Umwelt bestehen. ${ }^{485}$

Das bedeutet für das System der Unternehmung, daß die in ihm existierenden Elemente gegenüber der Umwelt abgegrenzt werden können und damit eine konsequente Zurechnung zum System der Unternehmung ermöglichen. Weiterhin können auch die zwischen den Elementen existierenden Relationen konsequent dem System der Unternehmung zugerechnet werden. Innerhalb der Gesamtzahl der im System der Unternehmung existierenden Elemente weisen einige der Elemente jedoch Relationen zur Umwelt auf. Dies können bspw. Elemente wie Marktforschung oder Beschaffung sein. Es ist daher davon auszugehen, daß das System Unternehmung einen Grad an Komplexität aufweist, der konsequent dem System zuzurechnen ist, wenn, unter Berücksichtigung einer Grenzdefinition, die Unternehmung als geschlossenes System betrachtet wird.

Unter Vernachlässigung der Grenzdefinition erhöht sich dieser Grad an Komplexität jedoch um einen Anteil, der sich aus dem Charakter der Unternehmung als offenes System ergibt. So werden hier zusätzlich die Relationen der Elemente, die Relationen zu Umweltelementen aufweisen, berücksichtigt, was impliziert, daß auch die Umweltelemente Eingang in die Betrachtung finden. Bei einer isolierten

\footnotetext{
482 Vgl. Abschnitt 3.4.7: Liveware-Environment.

483 Vgl. Luhmann, N., (Systeme 1996), S. 52.

484 Vgl. Luhmann, N., (Systeme 1996), S. 52.

485 Vgl. Luhmann, N., (Systeme 1996), S. 52.
} 
Betrachtung der Unternehmung als geschlossenes System, die durch Grenzdefinition möglich ist, ist daher von der Komplexitätsdimension Systemkomplexität auszugehen. Bei einer offenen Systembetrachtung ist demgegenüber zusätzlich die Komplexitätsdimension Umweltkomplexität, in bezug auf die für das System relevante Komplexität, anzuführen.

Die Unternehmensstruktur ist nun zum einen in statischer Sicht komplexer Natur. ${ }^{486}$ Die statische Komplexität der Unternehmensstruktur wird im Unternehmensplanspielmodell durch die Struktur des Modells repräsentiert und hängt von der Anzahl der berücksichtigten Elemente sowie dem Grad ihrer Vernetztheit ab. 487 Dieser Aspekt kann unter Berücksichtigung der konstituierenden Merkmale von Systemen näher beleuchtet werden.

So folgert Luhmann aus der Annahme, daß Systeme in der Lage sind, sich gleichsam zu öffnen und zu schließen, daß die Unterscheidung von offenem und geschlossenem System nun keinen Typengegensatz mehr darstellt, sondern ein Steigerungsverhältnis. ${ }^{488}$ In diesem Zusammenhang können, unter Vernachlässigung der zwischen den Randelementen existierenden Beziehungen zur Umwelt, alle Elemente konsequent dem System zugerechnet werden, wodurch es den Charakter eines geschlossenen Systems erhält. Hier ist dann eine Betrachtung der Elemente unter Vernachlässigung der zwischen den Elementen existierenden Relationen möglich. In diesem Zusammenhang kann daher die Anzahl der im Systemmodell abgebildeten Elemente als Elementekomplexität bezeichnet werden. Die Anzahl der Relationen kann als Relationenkomplexität bezeichnet werden.

Werden jedoch auch die Relationen zwischen den Randelementen und der Umwelt berücksichtigt, so ist die Unterscheidung zwischen Elemente- und Relationenkomplexität aufzuheben. Dies begründet sich in Zusammenhang mit dem oben ausgeführten Aspekt, daß in offenen Systemen keine isolierte Betrachtung der Elemente möglich ist, sondern diese als verknüpft anzusehen sind. ${ }^{489}$ Wird die

\footnotetext{
486 Vgl. Ulrich, H., (Unternehmung 1970), S. 157.

487 Vgl. Orth, C., (Unternehmensplanspiele 1999), S. 70.

488 Vgl. Morin, E., (Méthode 1977), insbes. S. 197 ff. zitiert nach Luhmann, N., (Systeme 1996), S. 52.

489 Vgl. Abschnitt 4.1: Die Allgemeine Systemtheorie.
} 
Unternehmung im Unternehmensplanspielmodell in ihrem Charakter als offenes System abgebildet, so ist in Zusammenhang mit einer Betrachtung des Gesamtmodells davon auszugehen, daß Elemente- und Relationenkomplexität nicht unabhängig voneinander betrachtet werden können.

Bliss leitet in diesem Zusammenhang aus dem Verständnis eines Komplexitätsgefälles nach Luhmann ${ }^{490}$ ein Substitutionsverhältnis zwischen Elemente- und Relationenkomplexität ab und bezeichnet dieses als Komplexitätsgrad eines Systems. ${ }^{491}$ In offenen Systemen ist daher von Komplexitätsgraden auszugehen. [Vgl. Abbildung 4-7].

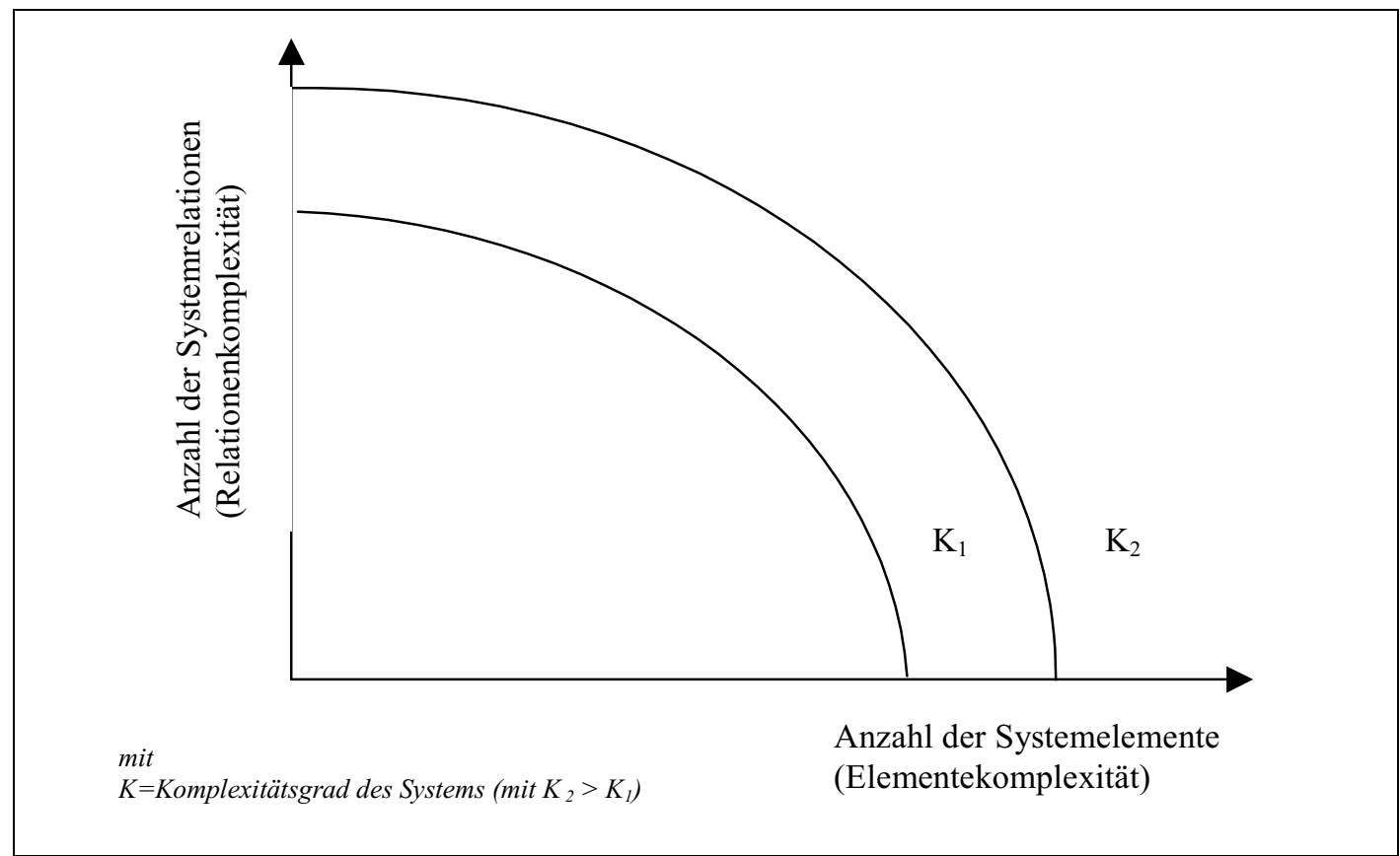

Abbildung 4-7: $\quad$ Das Konzept der Iso-Komplexitätskurven ${ }^{492}$

Die Unternehmensstruktur ist weiterhin auch in dynamischer Sicht komplexer Natur. ${ }^{493}$ So unterliegt die Unternehmung Veränderungen im Zeitablauf. Diesen Veränderungen liegen Steuerungs- und Regelungsvorgänge zugrunde, die zu besonderen Anforderungen im Hinblick auf deren Analyse führen. Es ist daher nicht hinlänglich, lediglich punktuelle Betrachtungen von Unternehmungen durchzuführen. Vielmehr ist es notwendig auch Struktur- und Verhaltensänderungen im

\footnotetext{
490 Vgl. Abschnitt 4.2.2: Komplexität als attributive Eigenschaft von Systemen.

491 Vgl. Bliss, C., (Management 2000), S. 92 ff.

492 Bliss, C., (Management 2000), S. 94.

493 Vgl. Ulrich, H., (Unternehmung 1970), S. 158 ff.
} 
Zeitablauf zu benennen. ${ }^{494}$ In Zusammenhang mit einer dynamischen Betrachtung des im Unternehmensplanspiel betrachteten Systemausschnittes können die identifizierten Planspielkomponenten herangezogen werden. 495 So ist es bspw. möglich, Aussagen zu Veränderungen im Zeitverlauf bezüglich des Spielstandes, zur Situation in den Planspielgruppen und ihres Einflusses auf den Spielverlauf, zur Situation des Spielleiters sowie auch zur individuellen Situation eines Spielers vorzunehmen. ${ }^{496}$

Für ein Unternehmensplanspielmodell, das die Unternehmung als offenes System abbildet, ergeben sich insgesamt folgende Dimensionen von Komplexität:

KOMPLEXITÄTSDIMENSION I

KOMPLEXITÄTSDIMENSION II

KOMPLEXITÄTSDIMENSION III

KOMPLEXITÄTSDIMENSION IV

KOMPLEXITÄTSDIMENSION V

\section{Abbildungskomplexität}

Komplexitätsgrad K II: das substitutive Verhältnis von Elemente- und Relationenkomplexität, in bezug auf die Umwelt: Anzahl der im Modell berücksichtigten Umweltelemente sowie ihre Relationen zu Randelementen des Systems.

Komplexitätsgrad K III: das substitutive Verhältnis von Elemente- und Relationenkomplexität, in bezug auf das System: Anzahl der im Modell berücksichtigten Systemelemente und Relationen.

Komplexitätsgrad K IV: Die Veränderlichkeit des Komplexitätsgrades $K I I$

Komplexitätsgrad K V: Die Veränderlichkeit des Komplexitätsgrades K III

\footnotetext{
494 Vgl. Fuchs-Wegner, G., („Systemanalyse“ 1974), S. 70. Das setzt spezifische Verfahren voraus, wobei das Instrumentarium der Regelungstheorie von besonderer Wichtigkeit ist. Vgl. dies., (,Systemanalyse“ 1974), S. 70 und die dort angegebene Literatur.

496 Vgl. auch Orth, C., (Unternehmensplanspiele 1999), S. 73.
} 


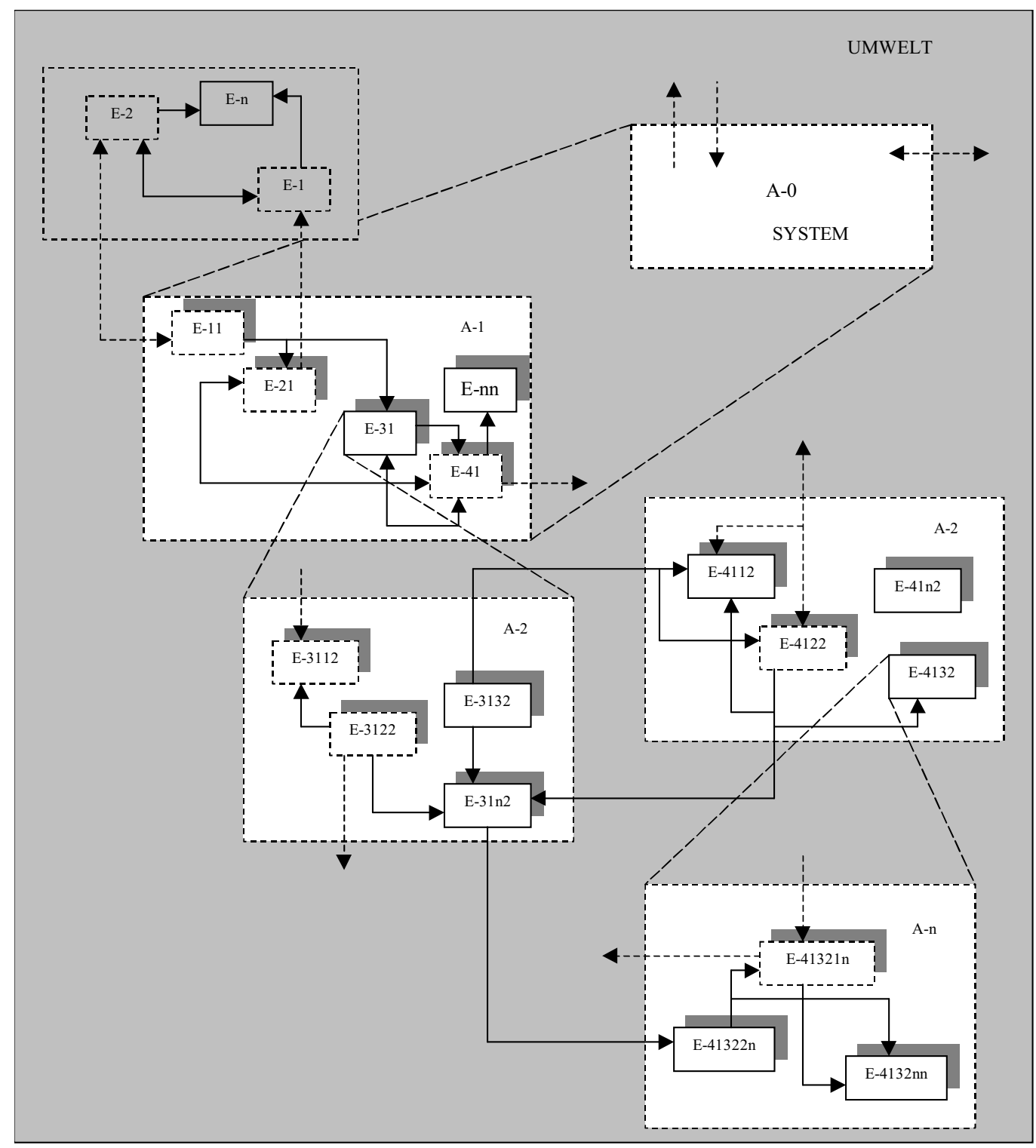

Abbildung 4-8: $\quad$ Top-Down-Zerlegung eines offenen Systems mit ${ }^{497}$

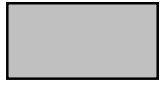

E-nn

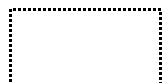

$=$ Systemumwelt

$=$ Umweltelement $\mathrm{n}$, das auf dem Abstraktionsniveau $\mathrm{n}$ eine Verknüpfung zum offenen System aufweist

$=$ Offenes Systemmodell

Die Überlegungen zur Entwicklung des vorliegenden Modells wurden durch die SADTMethode [»Structural Analysis and Design Technique«] angeregt, die von Ross in den ausgehenden 60er Jahren entwickelt wurde und eine graphische Sprachform zur Unterstützung der EDV-System-Entwicklung darstellt. Vgl. Ross, D.T., (Applications 1985). Mit ihr kann die Struktur und der Aufbau eines Systems beschrieben werden, und sie kann prinzipiell auf beliebige Systeme angewendet werden. Die fortschreitenden Überlegungen zeigten jedoch, $\mathrm{da} \beta$ eine Übertragbarkeit in diesem Zusammenhang nicht möglich ist, da die SADTMethode unter einem System eine nach außen [Umwelt] und innen [Detaillierungsgrad] bgegrenzte, geordnete Menge von Elementen, zwischen denen Beziehungen und Abhänggkeiten bestehen, verstanden wird. Vgl. Marca, D.A.; McGowan, C.L., (SADT 1988); Mayrhofer, M., (Analyse 1985), S. 111. 


$\begin{aligned}= & \text { Abstraktionsniveau n } \\ = & \text { Im Modell berücksichtigtes Systemelement n -ohne Umwelt- } \\ & \text { bezug- auf dem Abstraktionsniveau n } \\ = & \text { Im Modell berücksichtigtes Systemelement n -mit } \\ & \text { Umweltbezug- auf dem Abstraktionsniveau n } \\ = & \text { Zwischen zwei Systemelementen existierende, einseitige Be- } \\ & \text { ziehung } \\ = & \text { Zwischen zwei Systemelementen existierende, wechselseitige } \\ & \text { Beziehung } \\ = & \text { Umweltelement n, das auf dem Abstraktionsniveau n keine } \\ & \text { Verknüpfung aufweist, jedoch mit einem Umweltelement } \\ & \text { verknüpft ist, das auf dem Abstraktionsniveau n eine Ver- } \\ & \text { knüpfung zum System aufweist } \\ = & \text { Umweltelement n, das auf dem Abstraktionsniveau n eine } \\ & \text { Verknüpfung zum System aufweist } \\ = & \text { Zwischen einem System- und einem Umweltelement existie- } \\ & \text { rende, einseitige Beziehung } \\ = & \text { Zwischen einem System- und einem Umweltelement existie- } \\ & \text { rende, wechselseitige Beziehung }\end{aligned}$

Wird weiterhin von der Möglichkeit einer vertikalen und horizontalen top-downDifferenzierung Gebrauch gemacht, so können die statischen Komplexitätsdimensionen, in einer offenen Systembetrachtung, graphisch wie oben dargestellt werden. [Vgl. Abbildung 4-8].

Im folgenden wird nun auf die Möglichkeit eingegangen, aus den entwickelten Komplexitätsgraden und Komplexitätsdimensionen ein Komplexitätsmaß für ein offenes System zu entwickeln.

\subsubsection{Entwicklung eines Komplexitätsmaßes für das offene System der Unternehmung}

Unter Berücksichtigung der Komplexitätsgrade für das offene System der Unternehmung kann nun an dieser Stelle ein Komplexitätsmaß abgeleitet werden. 
Dabei wird, im Hinblick auf die Meßbarkeit von Komplexitätsunterschieden, auf Ehlken bezug genommen, der die Komplexität von betriebswirtschaftlichen Modellen untersucht. Er beleuchtet verschiedene Ansätze von Komplexität ${ }^{498}$ und identifiziert, unter Berücksichtigung eher problemorientierter Ansätze, die folgenden Komplexitätsdimensionen eines betriebswirtschaftlichen Modells:499

- die Gesamtzahl der Variablen bzw. Parameter

- die Anzahl unterschiedlicher Variablen bzw. Parameter

- der Grad der Unterschiedlichkeit zwischen den Variablen bzw. Parametern

- die Art der Beziehungen zwischen den Variablen bzw. Parametern und

- die Veränderlichkeit der Variablen und der Verknüpfungen zwischen ihnen bzw. den Parametern im Zeitablauf.

Für die weitere Betrachtung wird nun die oben beschriebene Komplexitätsdimension I der Abbildungskomplexität herangezogen und davon ausgegangen, daß sie das in Summe generierte Potential aus den formulierten Komplexitätsgraden eines offenen Systems umfaßt. Die formulierten Komplexitätsgrade lauteten dabei wie folgt: 500

- der Komplexitätsgrad K II

- der Komplexitätsgrad K III

- der Komplexitätsgrad K IV

- der Komplexitätsgrad K V

Unter Heranziehung der von Ehlken ermittelten Dimensionen können weiterhin auch die folgenden Komplexitätsdimensionen angenommen werden:

- Komplexitätsdimension K VI die Anzahl unterschiedlicher Variablen bzw. Parameter

- Komplexitätsdimension K VII der Grad der Unterschiedlichkeit

498 Vgl. Ehlken, J., (Modelleinsatz 1999), S. 153 ff.

499 Vgl. Ehlken, J., (Modelleinsatz 1999), S. 155. Kirsch berücksichtigt den Zeitaspekt in Anlehnung an McFarland. Vgl. Kirsch, W., (Handhabung 1988), S. 205 f.; McFarland, A., (Power 1969), S. 16. 
zwischen den Variablen bzw. Parametern

- Komplexitätsdimension K VIII die Art der Beziehungen zwischen den Variablen und Parametern

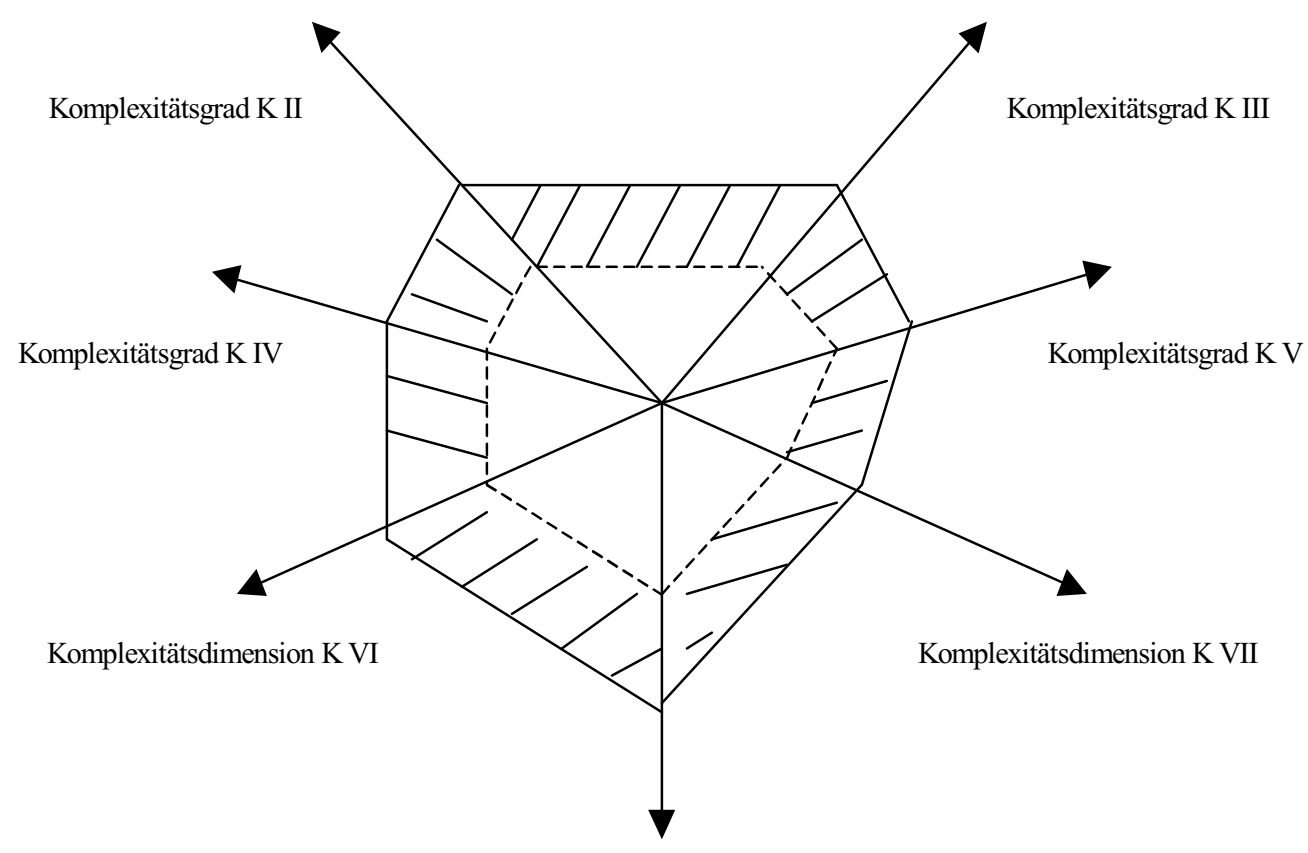

Komplexitätsdimension K VIII

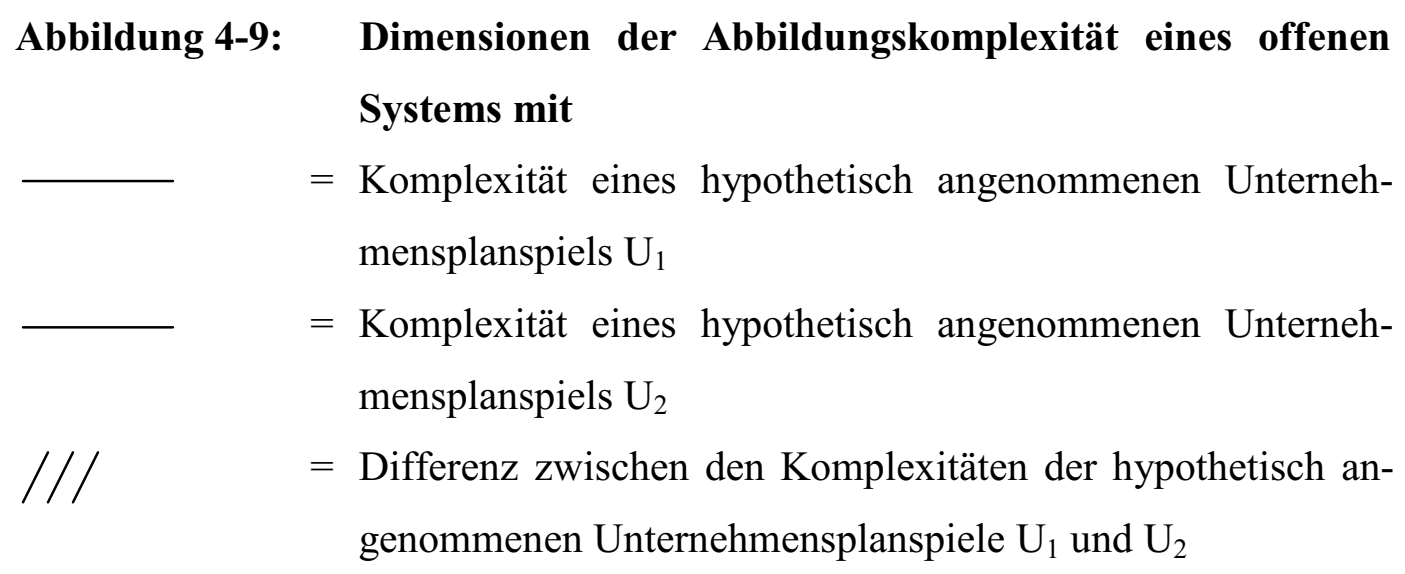

Die formulierten Komplexitätsgrade und Komplexitätsdimensionen können dann weiterhin in einem Polarkoordinatensystem dargestellt werden, wobei die Abbildungskomplexität eines Unternehmensplanspielmodells, welches ein offenes System abbildet, als weniger komplex einzuschätzen ist als die Abbildungskomplexität eines anderen Modells, wenn es bezüglich des in Summe generierten 
Potentials aller genannten Komplexitätsgrade und Komplexitätsdimensionen eine geringere Komplexität aufweist. [Vgl. Abbildung 4-9].

An dieser Stelle soll nun aufgezeigt werden, daß sich die vorgenannten Ergebnisse nun zur ganzheitlichen Strukturierung des Gegenstandes der Unternehmensgründung eignen. In diesem Zusammenhang ist der Gedanke wieder aufzugreifen, daß ein Modell die Realität »nachahmt«; und ein Gegenstand existieren muß, der „nachgeahmt“ werden kann. Dabei ist es entscheidend, daß die Simulation des Systems nicht direkt erfolgt, sondern über ein gedankliches Modell des Systems, welches somit auch als Simulationsmodell bezeichnet werden kann. Um dies zu gewährleisten, müssen das System sowie dessen Prozesse beschrieben werden. Dabei sollen - der zugrundeliegenden Fragestellung entsprechend - meist nur bestimmte Aspekte betrachtet werden. Wichtig ist in diesem Zusammenhang, daß bei der Abstraktion kein wesentlicher Aspekt verlorengeht. 501

Dies ist im Hinblick auf den Gegenstand der Unternehmensgründung besonders wichtig, da sich sowohl die marktlichen als auch die generellen gesellschaftlichen Bedingungen stets verändern und dabei stetig an Komplexität zunehmen. Dies führt für Unternehmen generell, insbesondere jedoch für potentielle Unternehmer, die neu in den Markt eintreten, dazu, daß sie über gegenwärtige und in der $\mathrm{Zu}$ kunft existierende Marktbedingungen nur unvollständig informiert sind;502 was sich besonders auf neu zu gründende Unternehmen auswirkt: „Während mangelnde Transparenz für die bestehende Unternehmung eine Minderung der Überlebenschancen in der Zukunft bedeutet, kann sie bei neuzugründenden Unternehmungen den Geburtsvorgang an sich unmöglich machen." ${ }^{\text {"503 }}$

Zunächst wird, um einen Überblick über den gegenwärtigen Stand der Forschung zu erhalten, dieser in seiner chronologischen Entwicklung dargelegt.

\footnotetext{
501 Vgl. Abschnitt 2.1.2.1: Das Unternehmensplanspiel: ein Simulationsmodell.

502 Vgl. Szyperski, N.; Nathusius, K., (Information 1975), S. 164.
} 


\section{Chronologische Entwicklung der Unternehmensgründungs- forschung}

Nach Klandt beinhaltet Gründungsforschung einen Forschungsbereich, der die Genese und Genetik neuer Unternehmungen untersucht. Danach ist die Gründungsforschung ein Erkenntnisobjekt der betriebswirtschaftlichen, zumindest der wirtschaftswissenschaftlichen Forschung. Bei einer ganzheitlicheren Betrachtung wird darüber hinaus die Soziologie, Sozialpsychologie, Psychologie und die Geschichtswissenschaft mit eingeschlossen. ${ }^{504}$ Nachfolgend wird ein Überblick zum derzeitigen Forschungsstand gegeben. Hierzu erfolgt eine chronologische $\mathrm{Zu}-$ sammenfassung der verschiedenen Standpunkte, die in der Diskussion zur Unternehmensgründung in der veröffentlichten Literatur eingenommen werden:

Bis in die Anfänge der siebziger Jahre hinein kann im deutschsprachigen Raum nur sehr eingeschränkt von einer eigenständigen Gründungsforschung gesprochen werden. ${ }^{505}$ Die Diskussion von Unternehmensgründungen ist zu diesem Zeitpunkt eng verknüpft mit Fragen der Planungs- und Organisationslehre. Im Jahre 1974 dann, unternahm erstmalig Szyperski die Anstrengung, einen Projektbereich Gründungsforschung an der Universität zu Köln einzurichten. ${ }^{506}$ In den folgenden Jahren lassen sowohl die hohe Insolvenzgefährdung junger Unternehmen als auch die seit Jahrzehnten abnehmende Selbständigenzahl507, weiterhin die Entstehung

503 Szyperski, N.; Nathusius, K.,(Information 1975), S. 164.

504 Vgl. Klandt, H., (Aktivität 1984), S. 14. Das Begriffsäquivalent zur Gründungsforschung stellt in englischsprachigen Untersuchungen der Begriff ,ENTREPRENEURSHIP RESEARCH“ dar. Hier wird der Aspekt des unternehmerischen Handelns gegenüber dem der Entstehung und Entwicklung neuer Unternehmen stärker in den Vordergrund gestellt. Vgl. Klandt, H., (Aktivität 1984), S. 14; Barth, S., (Existenzgründer 1995); Manstedten, B.C., (Entwicklung 1997).

505 Vgl. Klandt, H., (Aktivität 1984), S. 14.

506 Vgl. Nathusius, K.; Klandt, H.; Kirschbaum, G. (Hrsg.), (Unternehmensgründung 1984), S. 8.

507 Die zwei sich gegenseitig bedingenden Prozesse marktwirtschaftlicher Steuerung, Gründung und Liquidation, wurden für die Jahre 1973 bis 1979 empirisch untersucht. Diese ampirische Untersuchung beschränkt sich lediglich auf Unternehmensfluktuationen im Bereich Nordrhein-Westfalen; die Autoren unternehmen jedoch den Versuch einer „Hochrechnung“ auf die Unternehmensfluktuation im gesamten Bundesgebiet. Vgl. Szyperski, N.; Kirschbaum, G., (Unternehmensfluktuation 1981). Zu der Forderung nach Daten zur Erfassung des Gründungsgeschehens, auch in den 80er Jahren seitens der amtlichen Statistik vgl. 
neuer Wachstumsbranchen in Japan und den USA durch eine Vielzahl von Unternehmensgründungen sowie schließlich die Probleme bei der Revitalisierung klassischer industrieller Branchen im Inland das Bewußtsein für die Bedeutung von Gründungen neuer Unternehmungen und damit eine zunehmende wissenschaftliche Beschäftigung mit der Entstehung und Entwicklung von Unternehmen, Branchen und Volkswirtschaften wachsen. 508 Es wird im Verlauf der 70er Jahre der Frage nachgegangen, warum ein ungenügender Unternehmungs-Nachwuchs $\mathrm{zu}$ verzeichnen ist und worauf die relativ hohen Insolvenzzahlen in der unternehmerischen Frühentwicklung zurückzuführen sind. ${ }^{509}$ Dazu werden einzelne Problemklassen zur Situation der Unternehmensgründung gebildet und detailliert diskutiert.

Erste Aufgabe der wissenschaftlichen Seite ist es nach Szyperski, die Probleme der Gründungs- und Frühentwicklungsplanung von Unternehmungen zu untersuchen. Dadurch ist gewährleistet, daß nicht Instrumente für Probleme entwickelt werden, die in der Realität keine Probleme sind. Der instrumentelle Aspekt hat hingegen zunächst hinter dieser Aufgabe zurückzustehen. Später entstanden jedoch auch Planungsinstrumente. Diese umfaßten einfache Checklisten, ${ }^{510}$ aber auch modellhafte Abbildungen einer Gründungsunternehmung durch ComputerSimulation. ${ }^{511}$ Diese sollten den Planungsprozeß instrumentell unterstützen und die Erfolgsaussichten eines Gründungsvorhabens beurteilen helfen. Weiterhin wurde auch immer wieder die Forderung nach einer strategischen Sichtweise der Problemstellung erhoben, um eine gewünschte Unternehmensentwicklung zu sichern. ${ }^{512}$ Obwohl auch einige modellhafte Abbildungen vorgenommen wurden, blieben die Ausführungen zumeist sehr deskriptiv und überwiegend univariat. $\mathrm{Zu}$ diesem Ergebnis gelangen Szyperski \& Klandt in einer im Jahre 1981 vorgenommenen Untersuchung der in den 70er Jahren veröffentlichten deutschsprachigen erkenntnisorientierten empirischen Literatur. ${ }^{513}$

Kistner, K.-P.; Südfeld, E.; u. a., (Statistische 1987) und Dahremöller, A., (Existenzgründungsstatistik 1987).

Vgl. Nathusius, K.; Klandt, H.; Kirschbaum, G. (Hrsg.), (Unternehmensgründung 1984), S.8.

509 Vgl. Szyperski, N.; Nathusius, K.,(Probleme 1999), S. 35 ff.

510 Vgl. Kirschbaum, G.; Naujoks, W.,(Erfolgreich 1986), bspw. S. 41 ff.

511 Vgl. Graf, H., (Möglichkeiten 1984), S. 241 ff.

512 Vgl. Szyperski, N.; Nathusius, K.,(Probleme 1999), S. 23 f.

513 Vgl. Szyperski, N.; Klandt, H.,(Research 1981), S. 158 ff. sowie S. 170 f. 
Im Verlauf der 80er Jahre wird versucht, die Gründungsproblematik vorwiegend mit empirischen Untersuchungen zu analysieren, mit dem Ziel, Erfolgsfaktoren vollzogener Gründungen abzuleiten. Ausgesucht soll hier darauf eingegangen werden. Diese Untersuchungen unterscheiden sich zum einen im Hinblick auf ihre Untersuchungsziele zum anderen hinsichtlich ihres Erhebungszeitraums, weiterhin bezüglich der Gründungsarten und Branchen sowie in der Grundgesamtheit zum Teil bedeutend voneinander. Aufgrund dieser Tatsache ist eine Vergleichbarkeit nur eingeschränkt möglich. ${ }^{514}$ Beispielhaft wird eingegangen auf den $\mathrm{Zu}-$ sammenhang bezüglich erfolgreicher Unternehmensgründungen und Gründungsförderung, 515 einzelne Einflußfaktoren, die zum Bereich der Gründerperson und zum mikrosozialen Umfeld zählen, 516 die Förderung technologieorientierter Unternehmensgründungen, ${ }^{517}$ die Identifizierung von Insolvenzgründen, ${ }^{518}$ Strukturbildungsdaten und Gründercharakteristika ${ }^{519}$. Hier ist festzustellen, daß die Benennung von Problemklassen, die es durch Empirien zu untersuchen gilt, dem Vorbild aus den 70er Jahren folgt. Hier lagen die Ausführungen insbesondere den Aussagen aus ersten explorativen Gesprächen mit Experten aus dem Bereich der Unternehmensgründung zugrunde. Dies ist darauf zurückzuführen, daß es neben einigen wenigen wissenschaftlichen Untersuchungsergebnissen nur ein geringes Angebot an betriebswirtschaftlicher Literatur gab. ${ }^{520} \mathrm{Zu}$ folgern ist also, daß man, aufgrund des Forschungsstandes, in der Lage war, Untersuchungsziele zu konkretisieren. Diese konnten jetzt durch umfangreichere empirische Forschung manifestiert oder widerlegt, oder auch ergänzt werden. Kritisch ist jedoch auch hier anzumerken, daß diesen empirischen Untersuchungen ebenfalls eine hohe Deskriptivität gemeinsam ist.

1985 stellt Rentrop als eine Ursache für eine mangelhafte Gründungsqualität das fehlende strategische Denken heraus. ${ }^{521}$ Er stellt fest, daß eine umfassende strategische Sichtweise, die das Zusammenwirken aller für eine Unternehmensgrün-

\footnotetext{
514 Vgl. dazu die kritische Diskussion bei Dietz, J.-W., (Gründung 1989), S. 309 ff.; Klandt, H., (Aktivität 1984).

515 Vgl. May, E., (Existenzgründungen 1981).

516 Vgl. Klandt, H., (Aktivität 1984).

517 Vgl. Berndts, P.; Harmsen, D.-M.,(Technologieorientierte 1985).

518 Vgl. Winkel, A.; Schuster, H.; Trommsdorf, V.,(Insolvenzgründe 1986).

519 Vgl. Kulicke, M., (Technologieorientierte 1987).

520 Vgl. Szyperski, N.; Nathusius, K., (Probleme 1999), S. 36.

521 Vgl. Rentrop, N., (Strategien 1985), S. 1.
} 
dung relevanten Faktoren berücksichtigt, in der Literatur bislang nur unzureichende Aufmerksamkeit fand. Damit nimmt er bezug auf Szyperski \& Nathusius, welche die Bedeutung der Strategie für den Erfolg von Unternehmensgründungen herausstellten, jedoch keine strategischen Ansätze diskutierten. ${ }^{522}$

In seiner Arbeit wird „,[... zum einen mit der Strategie der innovativen Imitation eine eigenständige Entwicklung dargestellt, zum anderen mit dem Konzept der kritischen Erfolgsfaktoren die Übertragbarkeit einer in anderen Managementprozessen bewährten Informationsbedarfsermittlungs-Strategie auf den Gründungsprozeß überprüft.“"523 Die Diskussion dient auch hier der Verbesserung der Qualität von Unternehmensgründungen. Die Strategie der innovativen Imitation gründet sich dabei auf eine bereits zeitlich vorgelagerte Innovation. Dabei ist eine Innovation, streng definiert, als eine Neuheit in dem Sinne anzusehen, daß etwas noch niemals zuvor durchgeführt worden ist. ${ }^{524}$

Die Methode der kritischen Erfolgsfaktoren knüpft gewissermaßen an die Ermittlung von Erfolgsfaktoren auf der Basis von empirischen Untersuchungen an. Ziel der Methode ist es, problemorientierte Steuergrößen zu ermitteln, die sich aus der unternehmenszielorientierten Analyse von kritischen Erfolgsfaktoren ableiten lassen. Diese Steuergrößen lassen sich jedoch nur bezüglich einer Auswahl von Erfolgsfaktoren ermitteln, da bezüglich der Erfolgsfaktoren und einiger sie determinierenden Faktoren von vereinfachten Bedingungen ausgegangen, und damit der Untersuchungsraum eingeschränkt wird. ${ }^{525}$ Es müßte daher nicht nur eine strategische Sichtweise der Problemstellung Berücksichtigung finden, sondern

522 Vgl. Szyperski, N.; Nathusius, K.,(Probleme 1999), S. 23 f.

523 Rentrop, N., (Strategien 1985), S. 1 f. Zur Strategie der innovativen Imitation vgl. auch ders., (Imitation 1990).

524 Vgl. Levitt, T., (Imitation 1966), S. 63.

525 Rentrop reduziert die Komplexität, und nimmt aus Vereinfachungsgründen nur Kapital, Management, Mitarbeiter, Markt und Räumlichkeiten (Räume) als zu berücksichtigende Faktoren für das zu gründende Unternehmen an. Kapital stehe dabei in einem gewissen Umfang zur Verfügung und der Markt sei groß genug. Der Gründer sowie die Ehefrau des Gründers weisen eine Arbeitskapazität auf. So stelle der Gründer Managementkapazität, die Ehefrau des Gründers Mitarbeiterkapazität dar. Von der Wohnung des Gründers aus werden die Aktivitäten gestartet; und der jeweils kleinste Faktor bestimmt den Erfolg (Umsatz) der Unternehmung. Grundlegend ist dabei, daß bei einem der relevanten Faktoren von anem Mangelfaktor ausgegangen wird. Das Wachstum kann im Hinblick auf diesen Mangdfaktor nur in dem Maße beeinflußt werden, in dem man den jeweiligen Mangelfaktor erhöht. Dieser Mangelfaktor ist somit ein kritischer Erfolgsfaktor, der den Erfolg der Unternehmung bestimmt. Vgl. Rentrop, N., (Strategien 1985), S. 54. 
darüber hinaus die Komplexität, unter Berücksichtigung ihrer Interdependenzen, in einem modelltheoretisch gestütztem, systemischem Ansatz bewältigt werden.

Mit Ausgang der 80er Jahre findet sich ein Modell für eine solche Entwicklung bei Dietz, das im folgenden kurz erläutert werden soll. Zunächst wird das Konzept der Erfolgsfaktoren von Dietz erneut aufgegriffen. ${ }^{526}$ Im Gegensatz zu anderen Arbeiten vollzieht er jedoch den Entwurf eines Beurteilungsverfahrens hinsichtlich Unternehmensgründungen (hier stets technologieorientiert) und zeigt Möglichkeiten der Modellierung und Visualisierung auf. Zielsetzung seiner Arbeit ist, die für eine erfolgreiche Unternehmensgründung relevanten Faktoren zu ermitteln und modellgestützt darzustellen. Dietz stellt einzelne Untersuchungen, die im Rahmen der empirischen Gründungsforschung zur Ermittlung von Erfolgsfaktoren durchgeführt wurden, im Überblick dar. Oben wurde darauf bereits kurz eingegangen. Er gelangt zu dem Ergebnis, daß nicht von dem oder den Erfolgsfaktoren ausgegangen werden kann, der oder die übereinstimmend in allen, oder wenigstens der Mehrheit der Veröffentlichungen verwendet wird oder werden. Weiterhin stellen die Erfolgsursachen bei einer ausführlichen Analyse der Realität ein so komplexes System dar, daß es fragwürdig ist, mit einer lediglich ausgesuchten Anzahl von Erfolgsfaktoren „den Erfolgsgrad“ zu bestimmen.527 Dieses Zwischenergebnis veranlaßt Dietz, die Komplexität zu reduzieren. „Um das Dilemma der Abbildung der Komplexität der Realität und der Möglichkeit ihrer wissenschaftlichen Beschreibung sowie der Folgerungen zu vereinfachen, besteht in der Theorie die Möglichkeit zur Modellbildung, als ein vereinfachtes Abbild der Realität, für das eher Aussagen getroffen werden können. "528 Die Grundlage für die Modellbildung bildet eine Auswahl von nur einigen Erfolgsfaktoren. In Anlehnung an die Mathematik ${ }^{529}$ werden zusammenfassend die Möglichkeiten der Modellierung und Visualisierung mittels der Katastrophentheorie ausgeführt. Nach Thom werden bei einer beliebigen Anzahl von Verhaltensvariablen und bei bis zu

\footnotetext{
526 Vgl. Dietz, J.-W., (Gründung 1989), S. 271 ff.

527 Vgl. Dietz, J.-W., (Gründung 1989), S. 347.

528 Dietz, J.-W., (Gründung 1989), S. 347.

529 Allgemeine gedankliche Strukturen sowie insbesondere methodische und instrumentelle Hilfen werden der Gründungsforschung vorwiegend von Idealwissenschaften, wie der Mathematik, Statistik, Informatik, Kybernetik, empirischen Sozialforschung, Philosophie und Wissenschaftstheorie (spez. Erkenntnistheorie), zur Verfügung gestellt. Vgl. Woll, A., (Volkswirtschaftslehre 1971), S. 4.
} 
vier Kontrollvariablen sieben elementare Katastrophen unterschieden. ${ }^{530}$ Dietz stellt die beiden kanonischen Modelle der Spitzen- und der Schmetterlingskatastrophe in den Mittelpunkt seiner Untersuchung. ${ }^{531}$ Während beim ersten Modell der Verhaltensvariable zwei Kontrollvariablen gegenüberstehen, sind es beim zweiten Modell vier. Im Folgenden werden nun der Verhaltensvariable anhand unterschiedlicher Beispiele jeweils spezifische Entscheidungssituationen zugeordnet, die von den Kontrollvariablen abhängen. Im Zeitverlauf wird jetzt die Veränderung der Kontrollvariablen, in Abhängigkeit von den sie beeinflussenden externen Faktoren beobachtet, und anhand ihrer Entwicklung eine Entscheidung gefällt.

Für die 90er Jahre läßt sich festhalten, daß die Gründungsproblematik weiterhin nur in geringem Umfang, und dabei in ähnlicher Form wie in vorangegangenen Untersuchungen wissenschaftstheoretisch diskutiert worden ist. ${ }^{532}$ Mit Beginn der 90er Jahre unterteilen Klandt \& Münch das Forschungsfeld Unternehmensgründung grob in den Bereich der GRÜNDUNGSAKTIVITÄT und den des GRÜNDUNGSERFOLGS und entwerfen einen Katalog von Fragen, die in den angeführten Bereichen der Untersuchung bedürfen. 533

Im Bereich der GRÜNDUNGSAKTIVITÄT, der sich dem Entstehen von Unternehmen widmet, wird gefragt:

$>$ welche Anzahl von Unternehmensgründungen werden in bestimmten Regionen und Zeiträumen vorgenommen,

welche Eigenschaften und Strukturen weisen diese Unternehmensgründungen auf,

welche Merkmale weisen die beteiligten Unternehmungsgründer auf,

\footnotetext{
530 Vgl. Thom, P., (Structural 1975), S. 24 f.

531 Vgl. Dietz, J.-W., (Gründung 1989), S. 535 ff. Vgl. zu der Thematik auch Dietz, J.-W.; Roski, R., (Management 1989). Vgl. zur Spitzenkatastrophe auch Lücke, W. (Hrsg.), unter Mitarbeit von Bloech, J., u.a., (Investitionslexikon 1991), S. 357 f.

532 Vgl. die Ausführungen bei Hebig, M., (Existenzgründungsberatung 1994); Brüderl, J.; Preisendörfer, P.; Ziegler, R.,(Erfolg 1996).

533 Vgl. Klandt, H.; Münch, G., (Gründungsforschung 1990), S. 174 ff.
} 
durch welche Faktoren wird die Entstehung von Unternehmensgründungen unterstützt oder behindert,

wie hoch sollte die Anzahl der zu gründenden Unternehmungen sein,

durch welche Maßnahmen können Unternehmensgründungen gefördert werden.

Im Bereich des GRÜNDUNGSERFOLGS, der sich mit den Einflußgrößen des Erfolgs beschäftigt, wird gefragt:

die Verantwortlichkeit der Gründerpersönlichkeit, sowie der Aufbau des Gründerteams für den Umfang des erzielten Erfolges,

die Relevanz der Gründungsidee sowie die Qualität des Gründungskonzeptes für den Erfolg,

die Bedeutsamkeit externer Hilfen für den Erfolg,

ist der Erfolg einer Unternehmensgründung mit einem akzeptablen Grad an Sicherheit vorhersagbar. Welche Faktoren sind im Falle einer Vorhersagbarkeit dafür verantwortlich. Ist von einem begrenzten Umfang an „kritischen“ Erfolgsfaktoren auszugehen,

ist es möglich den Gründungserfolg zu gestalten, oder ist er zu einem nicht unerheblichen Teil durch Glück und Zufall bestimmt.

Zentral ist in den 90er Jahren jedoch, daß die Forschung sich mit der Notwendigkeit beschäftigt, den Forschungsgegenstand der Unternehmensgründung vermehrt an Hochschulen zu etablieren. In diesem Zusammenhang verweist Szyperski, bereits zum Ende der 70er Jahre, auf die Eignung der Planspielmethode für den Gegenstand Unternehmensgründung. So führt dieser aus, daß ein in Zusammenhang mit der Unternehmensgründung zu entwickelndes Modell zum einen in der Lage sein muß, Ergebnisse des Systemverhaltens zu reproduzieren, sowie zum anderen 
die Wechselbeziehungen aufzuzeigen, die ein bestimmtes Ergebnis hervorbringen. Demnach muß ein entsprechendes Modell dynamischer Natur sein, um Zustandsund Verhaltensänderungen faßbar zu machen und aufzuzeigen. Er gelangt zu dem Schluß, daß die Simulation diesem Anspruch in hohem Maße Rechnung trägt. ${ }^{534}$

\subsubsection{Terminologische Abgrenzungen}

\subsubsection{Der Begriff der Unternehmensgründung}

Der Begriff Gründung bzw. das entsprechende Verb „gründen“ stammen von dem althochdeutschen Wort „grunden“ ab und bedeuten so viel wie „den Grund zu etwas legen, errichten, ins Leben rufen“. 535 Eine Gründung kann weiterhin als die Schaffung eines gegenüber seiner Umwelt qualitativ abgegrenzten und vorher in gleicher Struktur nicht existenten Systems verstanden werden. ${ }^{536}$ Um eine Unternehmungsgründung handelt es sich in dem Moment, wo das System eine Unternehmung darstellt, 537 wobei unter Unternehmungen ,[...] äußerlich selbständige, scheinbar autonome, grundsätzlich auf sich selbst gestellte, unmittelbar nur am eigenen Lebensinteresse orientierte Einheiten“"verstanden werden können. ${ }^{538}$

Die Gründung eines Unternehmens als ein zeitbeanspruchender Proze ${ }^{539}$ umfaßt die Gesamtheit aller Entscheidungen und Handlungen, welche die konstitutionellen Voraussetzungen für das Entstehen des Unternehmens schaffen. So entsteht das Unternehmen im Rahmen der Gründungsvorgänge als ein rechtlich, sozial und wirtschaftlich selbständiges Gebilde, das als Institution eine Funktion im Markt

534 Vgl. Szyperski, N.; Kirschbaum, G., (GRÜMOD 1979), S. 14 f. Diese Sichtweise wird auch in der anglo-amerikanischen Forschung vertreten. So verweisen Ulrich \& Cole darauf, daß eine der Methoden, ,which are best suited to an entrepreneurial learning style“, die der „management simulation“ darstellt. Vgl. Ulrich, T.A.; Cole, G.S., (Entrepreneurs 1987), S. 36 ff. Vgl. auch Abschnitt 6.7.1: Unternehmensgründung als Gegenstand der Lehre.

535 Vgl. Duden, (Etymologie 1963), S. 239.

536 Vgl. Szyperski, N.; Nathusius, K.,(Probleme 1999), S. 25.

537 Der Begriff Unternehmung kann dabei synonym zu Unternehmen verwendet werden. Vgl. Klandt, H., (Aktivität 1984), S. 25 f. und die dort angegebene Literatur. Hier wird im fdgenden der Begriff Unternehmen verwendet.

538 Schumpeter, J., (Unternehmer 1928), S. 476.

539 Vgl. Abschnitt 4.4.2.2: Der Prozeß der Unternehmensgründung. 
übernimmt, womit sie, die Gründung, als ein juristisch-finanzieller Akt angesehen werden kann. 540

In diesem Sinne kann sie auch gegenüber der Errichtung abgegrenzt werden, die, durch Bereitstellung von Produktionsfaktoren, einen technisch-organisatorischen Aufbau darstellt. ${ }^{541}$

\subsubsection{Der Prozeß der Unternehmensgründung}

Die Ausführungen deuteten bereits an, daß sich die Frage nach dem genauen Zeitpunkt der Entstehung eines Unternehmens nicht unmißverständlich klären läßt. ${ }^{542}$ Zunächst sieht Kulicke den Gründungsakt als zeitlich punktuell an und bezeichnet ihn als Schnittpunkt zwischen einer Vorbereitungs- und Planungsphase auf der einen Seite und der Phase des technisch-organisatorischen Aufbaus auf der anderen Seite. ${ }^{543}$

Pörner macht hingegen den Neuheitscharakter der Geschäftsaktivitäten zur Grundlage seiner Betrachtung. In diesem Zusammenhang kann der Gründungsprozeß erst dann als beendet betrachtet werden, wenn die ersten Entscheidungen bezüglich der laufenden Geschäftsaktivitäten ihren generellen Neuheitscharakter verlieren und eine bestimmte Routine aufweisen. ${ }^{544}$

Damit ist eine zeitraumbezogene Betrachtung impliziert, die Jäger vornimmt. Hier verstreicht von der Gründungsidee bis zum Beginn der eigentlichen wirtschaftlichen Tätigkeit im Sinne der Leistungserstellung eine Zeitphase, wobei die zeitliche Erstreckung dieser Phase von einer Vielzahl von Faktoren abhängt. Den

540 Vgl. Jäger, W., (Gründung 1976), Sp. 788.

541 Vgl. Eisele, W., (Gründung 1993), Sp. 1550. Damit bedingen sie sich jedoch auch gegenseitig. Hertlein bezeichnet die Betriebserrichtung als einen innerwirtschaftlichen Vorgang, bei dem die Produktionsfaktoren Kapital und Arbeit diesem Vorgang gewidmet werden, um einen gesetzten Zweck zu erreichen. Demgegenüber stellt die Gründung einen rechtlichen Beginn dar, der die Entstehung einer Unternehmung sowie ihren Einsatz im Außenverkehr beinhaltet. Die Errichtung umfaßt dabei zumeist eine längere Zeit der planvollen Überkgungen und Vorbereitungen, während die Gründung das Ergebnis eines wohlüberlegten Entschlusses darstellt. Vgl. Hertlein, A., (Gründung 1965), Sp. 673.

Vgl. Jäger, W., (Gründung 1976), Sp. 787 f.

543 Vgl. Kulicke, M., (Technologieorientierte 1987), S. 13.

544 Pörner, R., (Management 1989), S. 91. 
Entstehungsprozeß als solches teilt Jäger dabei grob in drei Phasen ein: In die Vorbereitungs-, die Gründungs- und die Errichtungsphase. Dabei fallen in die Vorbereitungsphase alle Planungsüberlegungen und Vorbereitungshandlungen, womit die Voraussetzungen für die Gründungsphase und die Errichtungsphase geschaffen werden. Demnach vollziehen sich in der Gründungsphase die eigentlichen Gründungsvorgänge, wodurch das Unternehmen im Hinblick auf soziale, rechtliche und wirtschaftliche Aspekte als selbständige Einheit ins Leben gerufen wird. ${ }^{545}$

So argumentiert auch Bellinger, der davon ausgeht, daß die Gründung als ein sich in der Zeit erstreckender Prozeß die konstitutiv-konzeptionellen Voraussetzungen schafft, um eine neue Unternehmungseinheit zu entwickeln, aufzubauen und zu starten. 546

Ripsas führt weiterhin aus, daß der unternehmerische Prozeß nicht mit der Gründung endet. 547 Er entwickelt seine Ausführungen bezüglich der betriebswirtschaftlichen und verhaltenswissenschaftlichen Elemente des Entrepreneurships daher ausgehend von einem Phasenmodell des unternehmerischen Prozesses. ${ }^{548}$

So läßt sich der unternehmerische Prozeß bspw. in verschiedene Phasen einteilen, welche sich jeweils wieder weiter unterteilen lassen. Hill unterscheidet dabei vier Stufen: Zum ersten geht er von einer Zeitphase aus, in der Ideen generiert und verfeinert werden, zum zweiten von einer Phase, in der ein Business-Plan ${ }^{549}$ erstellt wird, zum dritten von einem erforderlichen Gründungs- und Wachstumsmanagement sowie viertens schließlich von der Anwendung von Ausstiegs- und Erntestrategien. ${ }^{550}$

Weiterhin kann der unternehmerische Prozeß unter bestimmten Bedingungen auch mit dem Instrumentarium der Produktlebenszyklus-Analyse beschrieben werden. Dadurch wird eine Abgrenzung der Gründungsphase gegenüber der Frühent-

\footnotetext{
545 Vgl. Jäger, W., (Gründung 1976), Sp. 787 f.

546 Vgl. Bellinger, B., (Gründung 1975), Sp. 1722.

547 Vgl. Ripsas, S., (Entrepreneurship 1997), S. 87.

548 Vgl. Ripsas, S., (Entrepreneurship 1997), S. 84 f.

549 Vgl. Abschnitt 6.8.1: Der Geschäftsplan als Instrument der Gründungsplanung.
} 
wicklungsphase möglich, wobei die Gründungsphase, unter dem Gesichtspunkt der Umsatzentwicklung, den Bereich darstellt, in dem noch keine Umsätze gemacht werden. ${ }^{551}$ Werden durch anlaufende Geschäftstätigkeit auch Umsätze realisiert, tritt die Unternehmung dann in die Frühentwicklungsphase ein. Dabei werden zunächst keine Gewinne zu realisieren sein, da Entwicklungs-, Planungs-, Errichtungs- und Markterschließungskosten sowie weiterhin Marktsicherungskosten zu negativen Ergebnissen führen. ${ }^{552}$

\subsubsection{Formen der Unternehmensgründung}

In der Realität finden sich vielfältige Formen von Unternehmensgründungen. In einer ersten terminologischen Abgrenzung können selbständige und unselbständige Gründungen unterschieden werden. Darüber hinaus kann nach einer weiteren terminologischen Abgrenzung von originären und derivativen Gründungen gesprochen werden. Diese Unterscheidung erfolgt nach dem Merkmal Strukturexistenz. Dabei wird unter originärer Gründung der vollständige Neuaufbau eines Unternehmens verstanden ${ }^{553}$, während bei einer derivativen Gründung bereits existierende Wirtschaftseinheiten durch Übernahme, Umgründung oder sonstige Maßnahmen ihre bisherige Identität verlieren.

\begin{tabular}{|c|c|c|}
\cline { 2 - 3 } \multicolumn{1}{c|}{} & derivative Gründungen & originäre Gründungen \\
\hline $\begin{array}{c}\text { unselbständige } \\
\text { Gründung }\end{array}$ & $\begin{array}{c}\text { Fusion/ } \\
\text { Umgründung (1) }\end{array}$ & Betriebsgründung (2) \\
\hline $\begin{array}{c}\text { selbständige } \\
\text { Gründung }\end{array}$ & $\begin{array}{c}\text { Existenzgründung durch } \\
\text { Betriebsübernahme (3) }\end{array}$ & $\begin{array}{c}\text { Unternehmens- } \\
\text { gründung (4) }\end{array}$ \\
\hline
\end{tabular}

\section{Abbildung 4-10: Gründungsformen 554}

Schließlich besteht im Hinblick auf die Merkmale Strukturexistenz und Selbständigkeit die Möglichkeit einer Kombination, so daß eine Matrixdarstellung die

Hill, G.E., (Opportunities 1994), S. 91.

551 Es ist dabei von Umsätzen in Testmärkten abzusehen.

552 Vgl. Szyperski, N.; Nathusius, K, (Probleme 1999), S. 30 ff.

553 Der vollständige Neuaufbau eines Unternehmens wird heute zumeist als Start-up-Gründung bezeichnet. Vgl. bspw. Schoeffling, H.,(Businessplan 2001), S. 36.

Szyperski, N.; Nathusius, K., (Probleme 1999), S. 27. 
Breite möglicher Gründungsformen demonstriert. ${ }^{555}$ [Vgl. Abbildung 4-10]. Neben einer Vielzahl weiterer Gründungsformen können auch Gründungen nach der Rechtsform, nach dem Zeitbezug, nach der Größe, nach der Beteiligungshöhe oder hinsichtlich ihres Angebotes am Markt unterschieden werden. Im Zusammenhang mit ihrem Angebot am Markt werden außerdem innovative sowie imitative Gründungen unterschieden. ${ }^{556}$

Die Alternativen zur Neugründung, nämlich der Erwerb eines Unternehmens, die Teilhaberschaft sowie das Franchising, werden von Schoeffling als bewährte Formen der Selbständigkeit angeführt. Abschließend soll daher noch bezug auf die Unternehmensübernahme sowie das Franchising genommen werden:557

Durch den Erwerb eines Unternehmens ist es dem Gründer möglich, sich den Vorteil eines bereits existierenden Unternehmens $\mathrm{zu}$ sichern. ${ }^{558}$ Unternehmungsübernahmen sind nicht immer genau gegen Unternehmensgründungen abzugrenzen. Es ist möglich, daß in Zusammenhang mit der Übernahme einer Unternehmung die alten Strukturen in so grundlegender Form verändert werden, daß daraus ein gänzlich unterschiedliches Unternehmensgebilde erwächst. ${ }^{559}$ Zumeist agiert der Nachfolger als Existenzgründer, dabei kann es sich sowohl um einen Fremdnachfolger als auch um einen Nachfolger aus der Familie handeln, wobei jedoch immer die Qualifikation des Nachfolgers im Vordergrund steht, nicht dessen Familienzugehörigkeit. 560

Wie oben ausgeführt, kann als weitere Gründungsform auch das Franchising angesehen werden. „Unter Franchise versteht man eine enge geschäftliche Kooperation beim Vertrieb von Waren und/oder Dienstleistungen“"561, wobei Kooperation in diesem Zusammenhang bedeutet, daß rechtlich und wirtschaftlich unabhängige Betriebe mit dem Ziel der Verbesserung ihrer wirtschaftlichen Leistungsfähigkeit zusammenarbeiten. Gleichsam beruht der Zweck einer Franchise-Partnerschaft

\footnotetext{
555 Vgl. Szyperski, N.; Nathusius, K., (Probleme 1999), S. 26 ff.

556 Vgl. dazu die ausführliche Diskussion bei Dietz, J.-W., (Gründung 1989), S. 26 ff.

557 Vgl. Schoeffling, H., (Businessplan 2001), S. 36.

558 Vgl. Schoeffling, H., (Businessplan 2001), S. 36.

559 Vgl. Baldegger, U., (Motivation 1988), S. 19.

560 Vgl. BMWi (Hrsg.), (Unternehmensnachfolge 1999), S. 8.

561 Gregor, C., (Franchising 1990), S. 549.
} 
jedoch auch auf einer strikten Arbeitsteilung, so daß im Idealfall ein Zusammenspiel aus Verbundvorteilen und Vorteilen, die aus einer unabhängigen Funktionserfüllung jedes Systempartners erwachsen, gelingen kann. Die für den Franchise-Nehmer wichtigste Funktion besteht dabei im Verkaufen der Waren, während ihm administrative Aufgaben weitestgehend vom Franchise-Geber abgenommen werden. So ist es möglich, daß sich jeder Systempartner konsequent auf die ihm obliegenden Funktionen konzentrieren kann. Dabei sind drei Arten von Franchise-Systemen zu unterscheiden. Zunächst ist das Investitions-System anzuführen, in dem der Franchise-Nehmer Kapitalgeber, aber keineswegs auch Geschäftsführer im Franchise-Betrieb ist. Beim Management-System ist der Franchise-Nehmer hingegen zusätzlich selbst Geschäftsführer des Franchise-Betriebs, während die dritte Variante, das Master Franchising, schließlich eine weitergehende Form des Franchising darstellt, die zunehmend an Bedeutung gewinnt. Hier übernimmt der Franchise-Nehmer das Recht und auch die Pflicht, eine FranchiseKette für eine Region oder auch ein ganzes Land aufzubauen. ${ }^{562}$

Unabhängig von der Form der Unternehmensgründung, wird Insolvenz als der zentrale Faktor für das Scheitern junger Unternehmen angeführt. Daher soll im folgenden das Element Finanzierung herangezogen und die Anwendbarkeit des oben entwickelten Modells zur Strukturierung des Sachverhaltes und zur Finanzmittelplanung aufgezeigt werden.

\subsection{Insolvenz als zentraler Faktor des Scheiterns junger Unter- nehmen}

Für Unternehmensgründungen wird Insolvenz als der zentrale Grund für das Scheitern angesehen. ${ }^{563}$ Die Heranziehung statistischer Untersuchungen zeigt, daß die seit den 80er Jahren ansteigende Insolvenzzahl vorwiegend eine Problematik neugegründeter Unternehmen darstellt. So wird der Markt von rund einem Drittel der Neugründungen nach drei Jahren, von 50 bis $60 \%$ nach fünf Jahren und von

562 Vgl. Gregor, C., (Franchising 1990), S. 549 ff.

563 Vgl. bspw. Schoeffling, H., (Businessplan 2001), S. 11 u. S. 13. Eine mangelhafte Finanzierung, die Probleme bezüglich der Liquidität nach sich zieht, führt mit 68,6\% die Liste der Gründe an, die zum Scheitern neugegründeter Unternehmen führen. Vgl. dazu ders., (Businessplan 2001), S. 73. 
rund $75 \%$ nach acht Jahren wieder verlassen. ${ }^{564}$ Wobei Liquidität meint: „Liquidität ist die Fähigkeit, jederzeit seinen Zahlungsverpflichtungen nachkommen zu können und dies auch unter Rückgriff auf bestehende Reserven."565 Demgegenüber bedeutet Insolvenz die Unmöglichkeit, seinen Zahlungsverpflichtungen nachzukommen und impliziert damit das Scheitern einer Unternehmung.

Dabei werden innerhalb der verschiedenen Lebensabschnitte eines Unternehmens, unterschiedliche finanzielle Mittel benötigt. Zu Beginn der Überlegungen zu einer Unternehmensgründung benötigt ein Jungunternehmer eine FrühphasenFinanzierung ${ }^{566}$. Die Frühphasen-Finanzierung ist dabei durch einen geringen Kapitalbedarf und ein hohes Finanzierungsrisiko gekennzeichnet. Für das besonders hohe Finanzierungsrisiko der Frühphase nennen Betsch, Groh \& Schmidt folgende Faktoren:567

- Das Unternehmen erwirtschaftet zumeist keine Erträge, wodurch möglicherweise Liquiditätsengpässe auftreten können.

- Vergangenheitswerte über Erfolgsgrößen wie Umsatz, Cash Flow und Gewinne liegen nicht vor. Sie müssen daher ohne Vergleichswerte prognostiziert werden.

- Oftmals verfügen die Unternehmensgründer nicht über die notwendigen betriebswirtschaftlichen Kenntnisse und Führungserfahrungen.

Deshalb ist ein Unternehmen für gewöhnlich nicht ohne Eigenkapital zu gründen, welches mindestens so hoch sein muß, daß

- absehbare oder potentielle wirtschaftliche Anlaufverluste dadurch bewältigt werden können und

\footnotetext{
564 Vgl. Barth, S., (Existenzgründer 1995), S. 1 und 7 f.

565 Schoeffling, H., (Businessplan 2001), S. 73.

566 Vgl. Betsch, O.; Groh, A.P.; Schmidt, K.,(Unternehmen 2000), S. 20.

567 Vgl. Betsch, O.; Groh, A.P.; Schmidt, K., (Unternehmen 2000), S. 20 ff.
} 
- weiterhin die Kredite durch Anlagendeckung oder dingliche Sicherheiten, möglicherweise auch werthaltige Bürgschaften abgesichert werden. ${ }^{568}$

Weiterhin führt Petersen unter anderem die folgenden Möglichkeiten zur Steigerung haftender Mittel des Existenzgründers an:569

\section{EXISTENZGRÜNDUNGSPRÄMIEN}

Die Bundesländer gewähren Existenzgründungsprämien in Höhe von $20 \%$ der eingesetzten Mittel, wobei der maximale Betrag 10000 DM umfaßt. Für innovative Existenzgründungen kann auch von höheren Förderbeträgen ausgegangen werden.

\section{EIGENKAPITALHILFE}

Wenn die Eigenmittel in bezug auf erforderliche Investitionen sowie ein erstes Warenlager mindestens $15 \%$ betragen, so besteht die Möglichkeit, diese auf $40 \%$ aufzustocken, indem Mittel zur Eigenkapitalhilfe der Deutschen Ausgleichsbank in Anspruch genommen werden.

Zur Gewinnung von Fremdkapital führt Petersen unter anderem die folgenden Möglichkeiten an:570

\section{EXISTENZGRÜNDUNGSDARLEHEN}

Bei Existenzgründungsdarlehen handelt es sich um ehemalige Marshall-PlanGelder, wobei ERP ein Akronym für European Recovery Program darstellt. Voraussetzung für die Gewährung eines Existenzgründungsdarlehens stellen dabei Eigenmittel in Höhe von 10 bis $20 \%$ des geplanten Finanzbedarfs dar. Sie werden dabei zur Finanzierung von Anlageinvestitionen sowie weiterhin für ein erstes Warenlager gewährt.

\footnotetext{
568 Vgl. Petersen, U., (Finanzmittelplanung 1990), S. 276.

569 Vgl. Petersen, U., (Finanzmittelplanung 1990), S. 277 ff.
} 


\section{GESCHÄFTSBANKDARLEHEN}

Wichtig wäre hier ein Dispositionskredit, der zum Ausgleich laufender Geschäftsvorfälle dient. Weiterhin besteht die Möglichkeit, Investitionskredite zu beantragen.

\section{WECHSELKREDITE}

Wechselkredite stellen eine interessante Alternative zur kurzfristigen Finanzierung von bis zu drei Monaten dar.

Unter statischen Gesichtspunkten läßt sich nun die in diesem Kapitel entwickelte Top-Down-Zerlegung eines offenen Systems ${ }^{571}$ dazu nutzen, die hier beispielhaft erläuterten Finanzierungsaspekte formal darzustellen.572 Dabei wird von einer potentiellen Zusammensetzung des Eigen- und Fremdkapitals ausgegangen. Der Vorteil besteht darin, daß die übrigen Merkmale, welche die Gründung determinieren nicht benannt werden müssen, aber trotzdem ersichtlich wird, daß die Finanzierungselemente in Interdependenz zu anderen Elementen zu betrachten sind; und gleichsam selbst interdependent zueinander sind. Soll das Modell außerdem um weitere Elemente erweitert werden, so ist dies beliebig möglich. [Vgl. Abbildung 4-11].

570 Vgl. Petersen, U., (Finanzmittelplanung 1990), S. $282 \mathrm{ff}$.

571 Vgl. Abschnitt 4.3.1.2: Komplexität im Unternehmensplanspiel.

572 Zur Möglichkeit einer dynamischen Betrachtung vgl. Abschnitt 6.8.3: Dekomposition einer Finanzmittelplanung im Hinblick auf den Aspekt der Beteiligungsfinanzierung. 


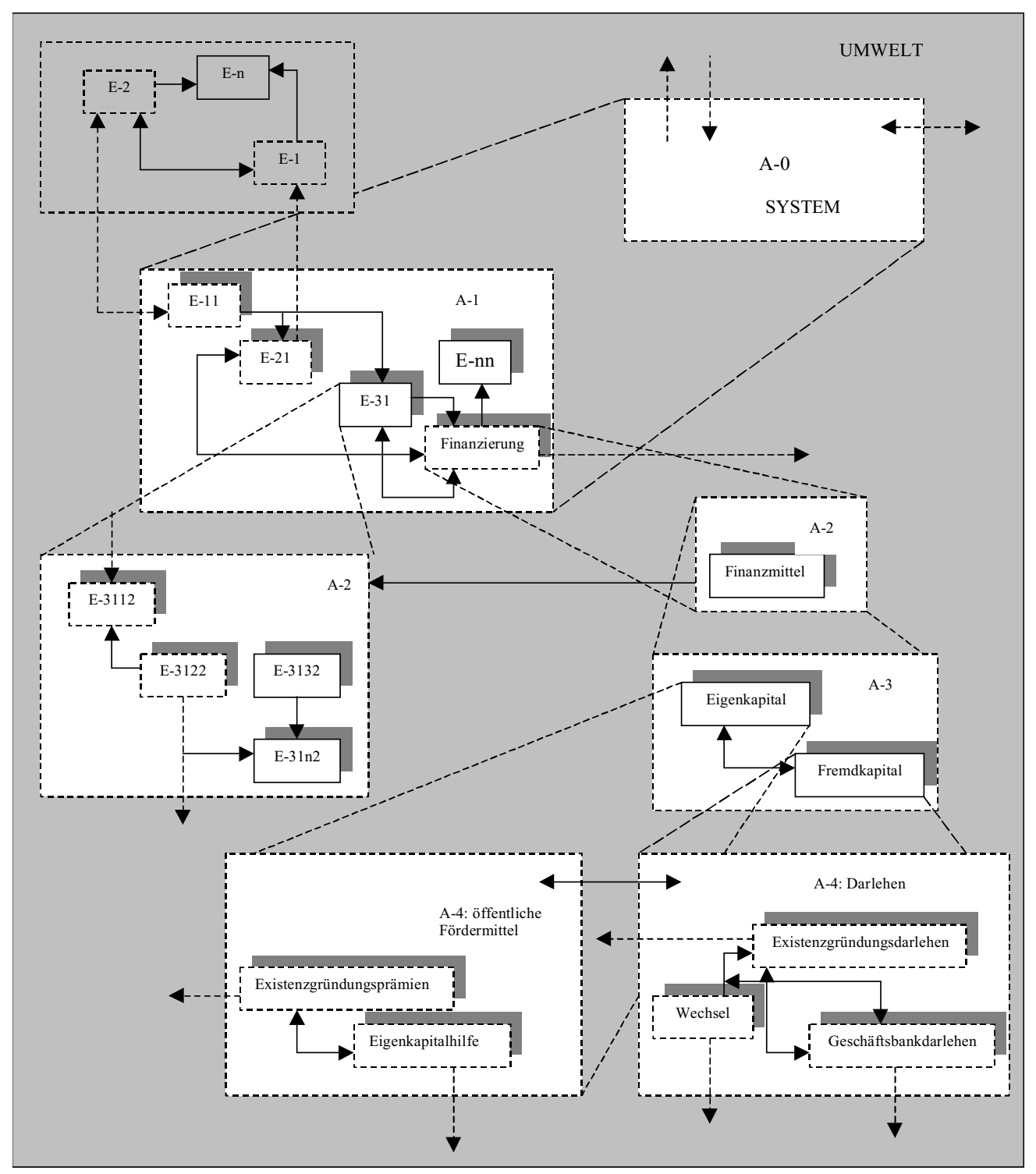

Abbildung 4-11: Zerlegung des Elementes Finanzierung im offenen System der Unternehmung 573

573 Die Elemente wurden bereits erläutert und nach Petersen ausgewählt. Vgl. Petersen, U., (Finanzmittelplanung 1990), S. 276 ff. Vgl. zur Legende des Modells Abschnitt 4.3.1.2: Komplexität im Unternehmensplanspiel. 
Das Unternehmensplanspiel gelangt in der universitären Lehre in Form eines komplexen Lehr-Lern-Arrangements zum Einsatz; wobei die Handlung der Planspielteilnehmer eine zentrale Stellung einnimmt. ${ }^{574}$

In diesem Zusammenhang führen interindividuelle Unterschiede bezüglich des Vorwissens beim Menschen zu einem unterschiedlich erfolgreichem Umgang mit komplexen Systemen. Es wurde bereits ausgeführt, daß es in einer Ausbildungsmaßnahme daher wichtig ist die Teilnehmer mit einer für sie optimalen Komplexität zu konfrontieren, um einen optimalen Lernerfolg zu gewährleisten. ${ }^{575}$ Weiterhin konnte aber auch ausgeführt werden, daß damit noch nichts über die unterschiedlichen Qualitäten des Wissens ausgesagt wird, die in unterschiedlicher Weise Einfluß auf den Umgang mit einem komplexen System nehmen. 576

Wie die Expertiseforschung gezeigt hat, ${ }^{577}$ ist dieser Aspekt jedoch wichtig, so daß an dieser Stelle Ansätze der Wissenspsychologie ausgeführt werden sollen, die einen differenzierteren Einblick in das kognitive System des Menschen gewähren.

5.1

Das Unternehmensplanspiel: Ein komplexes Lehr-Lern-Arrangement

In der jüngeren Vergangenheit haben neuere wirtschaftspädagogische Ansätze, die konstruktivistischen Ursprungs sind, durch das Forschungsprojekt "Lernen, Denken, Handeln in komplexen ökonomischen Situationen" Eingang in die Didaktik der Wirtschaftsfächer gefunden. Dabei werden die damit in Zusammenhang stehenden Unterrichtsmethoden als komplexe oder mehrdimensionale Lehr-LernArrangements bezeichnet, was sich dadurch begründet, daß die Berücksichtigung von neuen Lehr-Lern-Verfahren auch die Berücksichtigung neuer Lehr-Lern-

\footnotetext{
574 Vgl. Abschnitt 5.1: Das Unternehmensplanspiel: Ein komplexes Lehr-Lern-Arrangement.

575 Vgl. Abschnitt 2.2.2.3.2: Zum Zusammenhang von Komplexität und Lernerfolg.

576 Vgl. Abschnitt 3.2.2: Ablauf von Unternehmensplanspielen.

577 Vgl. Abschnitt 3.3: Umgang von Personen mit komplexen Systemen.
} 
Techniken nach sich zieht. ${ }^{578}$ Auch der Einsatz von Unternehmensplanspielen in der universitären Lehre579 wird als Einsatz eines komplexen Lehr-LernArrangements verstanden. ${ }^{580}$ In diesem Zusammenhang wurde bereits darauf eingegangen, daß die Wirtschaftspädagogik dazu aufgefordert ist, die Komplexität des Lernstoffes an das Vorwissen der Teilnehmer anzupassen, ${ }^{581}$ wodurch die Differenz zwischen den Qualifikationsanforderungen, welche das Lernobjekt stellt, und den subjektiven Bedingungen der Lernenden, von Wolff als »pädagogische Lücke« bezeichnet, zum Ausdruck kommt ${ }^{582}$.

Im Planspiel nimmt die Handlung der Planspielteilnehmer eine zentrale Stellung ein. Dies resultiert daraus, daß sie einen entscheidenden Einfluß auf den Lernerfolg hat, gleichsam aber auch selbst ein Lernziel darstellt. ${ }^{583}$ Dabei werden die Handlungen durch die Entscheidungen ausgelöst, welche die Teilnehmer im Planspiel fällen. Die Entscheidungsorientierung des Unternehmensplanspiels repräsentiert dabei das folgende Organisationsverständnis: „Im Vergleich zu andersartigen Systemen können Organisationen begriffen werden als Systeme, die Handlungen so behandeln, als ob es Entscheidungen wären. In Organisationen wird das Zumuten von Entscheidungen institutionalisiert. Man darf voraussetzen, daß Kommunikationen auf Entscheidungen beruhen und daß eigene Entscheidungen andere Entscheidungen auslösen werden. " ${ }^{684}$ Die Entscheidungsorientierung allein ermöglicht es jedoch nicht, das einzelwirtschaftliche System in seiner humanen und wirtschaftlichen Komplexität zu erfassen und zu gestalten. Innerhalb des vielschichtigen Handlungsprozesses wird vielmehr mit dem Wahlakt bei der Entscheidung nur eine Komponente dargestellt. Das Ergebnis des Wahlaktes muß zusätzlich in der operativen Ebene begleitet werden. Es ist auszuführen und umzusetzen. Damit wird dem auf den Ausführungsprozeß gerichteten Willensakt

578 Vgl. Hartung, S., (Förderung 2000), S. 39 f. und die dort angegebene Literatur. Das Lösen komplexer Probleme und Aufgaben kann neben dem Einsatz von Planspielen auch durch Fallstudien, Lernbüros und arbeitsanaloge Lernaufgaben gefördert werden. Vgl. Achtenhagen, F., (Lösen 1990), S. 291. Vgl. Abschnitt 2.2: Einsatz von Unternehmensplanspielen in der Betriebswirtschaftslehre.

580 Vgl. Hartung, S., (Förderung 2000), S. 73.

581 Vgl. Abschnitt 2.2.2.3: Zum Zusammenhang von Komplexität, Lernzielen und Lernerfolg im Unternehmensplanspiel.

582 Vgl. Wolff, K., (Umgang 1994), S. 3.

583 Vgl. Hartung, S., (Förderung 2000), S. 73.

584 Luhmann, N., (Komplexität 1980), Sp. 1066. 
gedient. Mit der handlungsorientierten Komponente werden den Aktionsträgern konkrete sachbezogene Aufgaben in der operativen Ebene als Ergebnisse des Wahlaktes vorgegeben. ${ }^{585}$ Diese sind nach Stachowiak eng miteinander verknüpft. Stachowiak führt aus, daß eine Unterklasse derjenigen Prozesse, die durch die operative Zielgerichtetheit der Informationsumwandlungen gekennzeichnet sind, die Vorgänge operationalen Denkens bilden. Der sie einleitende Abruf von gespeicherten Informationen kann als Vergegenwärtigung bezeichnet werden. Für die durch die Informationsverarbeitung im Vergegenwärtigungsbereich gewonnenen Handlungsantizipationen kann der Versuch unternommen werden, sie auf die Form optimaler Problemlösungen zu bringen. Damit zielt der dem Handeln vorangehende Denk- und Entscheidungsprozeß überwiegend auf die Lösung von Extremalproblemen. ${ }^{586}$ Das Planspiel trägt hier beiden Aspekten Rechnung, da Entscheidungen hier aufgrund der »Quasirealität « ${ }^{587}$ des Planspiels als »Quasihandlungen« begriffen werden können.

Handeln stellt nun eine besondere Art von Verhalten dar. ${ }^{588}$ Wenn von einer Person gesagt wird, daß sie handelt, dann kann auch gesagt werden, daß sie sich verhält. Nicht jedes Verhalten ist jedoch eine Handlung. So ist Handeln als Spezialfall von Verhalten anzusehen.589 Mit den Worten von Kamlah: „Man wird also jedenfalls gut daran tun, die Termini „handeln“ und „sich verhalten“ nicht synonym zu setzen, sondern zu unterscheiden, in der Weise nämlich, daß „handeln“ als ein Spezialfall von „sich verhalten“ zu verstehen ist." 590 Dabei wird unter dem Begriff des Handelns jene Untermenge menschlichen Verhaltens zusammengefaßt, welche die Merkmale der Zielgerichtetheit, der bewußten Zielorientierung, der Erwartungssteuerung und der kognitiven Reguliertheit erfüllt. ${ }^{591}$ Somit umfassen Handlungen außer ihrem operativen Moment, das bedeutet einem manifestem Verhalten, stets auch eine psychische Struktur. ${ }^{992}$ Grundlegend für den Handlungsbegriff ist das Erkennen mehrerer Handlungsmöglichkeiten, das Bedenken

Vgl. Huch, B., (Rechnungswesen 1975), S. 65.

Vgl. Stachowiak, H., (Denken 1965), S. 3.

587 Vgl. Abschnitt 2.2.1.1: Einsatzgebiete von Unternehmensplanspielen.

588 Vgl. auch Abschnitt 3.4.3.2.1: Das epistemologische Subjektmodell.

589 Vgl. Kamlah, W., (Anthropologie 1972), S. 49.

590 Kamlah, W., (Anthropologie 1972), S. 49.

591 Vgl. Achtenhagen, F.; Tramm, T.; Preiss, P.; Seemann-Weimar, H.; John, E.G.; Schunck, A., (Lernhandeln 1992), S. 32.

592 Vgl. Kaminski, G., (Überlegungen 1981). 
zwischen alternativen Handlungsmöglichkeiten, unter Voraussicht der sicheren, wahrscheinlichen und möglichen Folgen der einzelnen Handlungsmöglichkeiten, ${ }^{593}$ und schließlich die Entscheidung, eine bestimmte Handlungsalternative auszuführen, wodurch es letztlich zu »beabsichtigtem Handeln« kommt. ${ }^{594}$

Durch die Handlungsorientierung des Planspiels wird im Planspiel der Lernbegriff zum Begriff des Lernhandelns. Dabei unterscheidet sich „das Handeln, das Lerntätigkeit ist und das zum Ziel hat, sich die Ausführungsverfahren einer Handlung anzueignen, [...], psychologisch gesehen, von dem äußerlich gleichen Handeln, das nicht ein spezielles Lernen ist, sondern ein Tätigsein mit dem Ziel, ein bestimmtes Ergebnis zu erreichen“. 595 Die Beziehung zwischen einer Lernhandlung und der allgemeineren Handlung läßt sich dabei wie folgt beschreiben:596

Das Lernhandeln bezieht sich auf das Handeln. Das Handeln ist eine Strategie, die der Absicht zugrunde liegt zu lernen um dadurch effizienter handeln $\mathrm{zu}$ können.

Das Lernhandeln stellt eine Größe menschlichen Handelns dar, die bei allen Handlungstätigkeiten unter anderem auch Rückmeldungen für den Handelnden beinhaltet, die Lernrelevanz besitzen.

Beim Lernhandeln dreht es sich in dem Moment um Handeln, wenn eine Lerntätigkeit alle wesentlichen Merkmale einer Handlungstätigkeit umfaßt.

Diese Aussagen faßt Flothow wie folgt zusammen: „Lernen weist nach diesen Aussagen einen unterschiedlich stark ausgeprägten Verwandtschaftsgrad zum allgemeinen Handeln auf, bis es schließlich in seiner höchsten Form eine vollkommene Strukturidentität besitzt." 597 Dabei kann im Planspiel das Lernen im Modell sowie das Lernen am Modell unterschieden werden:598

\footnotetext{
593 Vgl. Werbik, H., (Handlungstheorien 1978), S. 19.

594 Kamlah, W., (Anthropologie 1972), S. 52.

595 Rubinstein, S.L., (Grundlagen 1973), S. 742.

596 Vgl. bspw. Bachmann, W., (Grundlagen 1988), S. 58 zitiert nach Flothow, K., (Förderung 1992), S. 93 f.

597 Flothow, K., (Förderung 1992), S. 94.

598 Vgl. Tramm, T., (Übungsfirma 1984), S. 61.
} 


\section{DAS LERNEN IM MODELL}

$>$ Lernprozesse, die direkt an die Qualität des Arbeitshandelns im Modell anknüpfen. Durch die Übernahme von Arbeitsrollen sowie die konkrete Ausführung von Tätigkeiten werden operative Kompetenzen und ein internes Erfahrungsmodell funktionaler Zusammenhangsstrukturen ausgebildet.

\section{LERNEN AM MODELL}

$>$ Aktivitäten, die auf eine systematische Reflexion der Modellstrukturen und Arbeitsprozesse zielen. Auf dieser Ebene wird die Aufmerksamkeit auf die begriffliche Systematisierung, Generalisierung und Überprüfung der im Modell erfolgten Erfahrungsbildung gerichtet.

Damit zielt das Lernhandeln im Unternehmensplanspiel weniger auf den Aufbau von Wissensstrukturen als auf die Umsetzung von Wissen in »Können«. Dabei weist das Lernhandeln im Unternehmensplanspiel zumeist eher den Charakter eines Problems als einer Aufgabe auf. Im folgenden wird daher auf den Zusammenhang von Aktivitäten des Problemlösens und Wissen eingegangen, wobei Problemlösen als Anwendung und Erwerb von Wissen aufgefaßt wird. In diesem Zusammenhang ist hier auszuführen, daß zwischen den Termini Problemlösen und Lernhandeln insofern ein Unterschied besteht, als sie bezüglich der intendierten Dauer der veränderten Gedächtniszustände unterschiedliche Annahmen aufweisen. Während Problemlösen eher der aktuellen Bewältigung einer singulären Anforderungssituation dient, werden durch bewußte Lerntätigkeiten verhältnismäßig langfristige Veränderungen der Gedächtnisstrukturen angestrebt. Dabei können jedoch auch singuläre Problemlöseaktivitäten langfristig zu einer dauerhaften Veränderung bestehender Gedächtnisstrukturen führen. 599

Weiterhin wird durch Lernhandeln die Absicht verfolgt, reale Handlungssituationen in einer Weise vorzubereiten, die dazu führt, daß die als problematisch empfundene Situation langfristig kein Problem mehr darstellt. Damit beinhaltet Lernhandeln auch den Aufbau von Fähigkeiten zum Problemlösen sowie abrufbarer Problemlösungen, ${ }^{600}$ womit $\mathrm{zu}$ folgern ist das Lernhandeln auf Aktivitäten des

\footnotetext{
599 Vgl. Dulisch, F., (Lernen 1986), S. 165 f.

600 Vgl. Dulisch, F., (Lernen 1986), S. 166 ff.
} 
Problemlösens unterstützend wirken kann und umgekehrt. Dabei ist es weiterhin wichtig, daß Problemlöseprozesse als die Anwendung und den Erwerb von Wissen angenommen werden, worauf im folgenden eingegangen werden soll.

Der Gegenstand der Psychologie ist die Analyse der Gestalt und der Charakteristika des Ablaufs der Informationsverarbeitung beim Menschen. ${ }^{601}$ In einem allgemeinen Informationsverarbeitungsparadigma wird der Mensch dabei als ein allgemeines, symbolverarbeitendes System angesehen, in welchem unterschiedliche Teilkomponenten miteinander interagieren ${ }^{602}$ und Informationsverarbeitung dazu führt, Information in andere Information umzuwandeln ${ }^{603}$. Weiterhin spielt die Analogie zum allgemeinen symbolverarbeitenden System Computer eine zentrale Rolle, ${ }^{604}$ denn der Computer dient als eine Metapher dafür, wie Informationen verarbeitet werden. ${ }^{605}$ Lachman, Lachman \& Butterfield formulieren diesen Zusammenhang wie folgt: „Computers take symbolic input, recode it, make decisions about the recoded input, make new expressions from it, store some or all of the input, and give back symbolic output. By analogy, that is most of what cognitive psychology is about. It is about how people take in information, how they recode and remember it, how they make decisions, how they transform their internal knowledge states, and how they translate these states into behavioural output.“606

601 Vgl. Dörner, D., (Informationsverarbeitung 1985), S. 371. Dörner spricht von einer Faszination seitens der Psychologie bezüglich der Informationsverarbeitung, die dazu führt, daß einige Psychologen den Begriff der Informationsverarbeitung , ,[...] geradezu als den zentralen Begriff der Psychologie ansehen“. Ders., (Informationsverarbeitung 1985), S. 371. Vgl. Lachman, R.; Lachman, J.L.; Butterfield, E.C.,(Psychology 1979), S. 99 ff.

603 Vgl. Dörner, D., (Informationsverarbeitung 1985), S. 372. In diesem Zusammenhang werden geistige Prozesse nach einem sehr allgemeinen Verständnis als Transfer von Informationen zwischen unterscheidbaren Systemen verstanden. Dieser Informationsaustausch findet auf verschiedenen Ebenen statt. Vgl. Lüer, G.; Lass, U., (Einleitung 1997), S. 1 f. Vgl. Lachman, R.; Lachman, J.L.; Butterfield, E.C.,(Psychology 1979), S. 99 ff.

605 Vgl. Newell, A.; Shaw, J.C.; Simon, H.A.,(Elements 1958), S. 153. Dabei wird der Computer nicht als analog zum menschlichen Verhalten verstanden, sondern er rückt in den Mittelpunkt des Interesses, weil er bei entsprechender Programmierung, die gleichen $\mathbf{h}$ formationsverarbeitungsschritte ausführt wie der Mensch beim Problemlösen. Vgl. dies., (Elements 1958), S. 153.

Lachman, R.; Lachman, J.L.; Butterfield, E.C.,(Psychology 1979), S. 99. 
Gemäß den Informationsverarbeitungsmodellen kann ein Individuum nun in eine beliebige Umwelt gestellt werden, wo es, mit den immer gleichen kognitiven Prozessen, beliebige Aufgaben bewältigt. ${ }^{607}$ Das Grundkonzept des Informationsverarbeitungsmodells geht dabei davon aus, daß außerhalb des Individuums ein Aufgabenfeld existiert, welches aus einem Problem und dessen Kontext besteht. Demgegenüber ist im Individuum ein Problemraum gegeben, welcher sowohl die potentiellen Zustände des Problems als auch die Problemoperatoren, die dem Individuum dazu dienen, sich von einem zum anderen Zustand zu bewegen, umfaßt. Die Ausgangssituation des Problems ist dabei durch das Aufgabenumfeld sowie die zu lösenden Aufgaben gekennzeichnet und beinhaltet damit das Gegebene. Der gewünschte Endzustand ist dann der Zielzustand, der in dem Moment angenommen wird, in dem der Problemlöser sich durch die Anwendung von Operatoren vom einen zum anderen Zustand bewegt hat. Dabei weist der Problemraum auch Barrieren, wie bspw. die Zielsetzung, auf, welche die Auswahl möglicher Zustände begrenzen. ${ }^{608}$

Dieses Paradigma der Informationsverarbeitung verwendet die Problemlöseforschung als forschungsleitende Grundvorstellung; ${ }^{609}$ und Ansätze zur Erklärung komplexen Problemlösens unternehmen den Versuch, den Problemlöseprozeß als die Anwendung und den Erwerb von Wissen zu beschreiben. ${ }^{610}$ So erfolgt menschliches Problemlösen auf Grundlage und in Wechselwirkung mit äußeren und inneren Wissensbeständen. Dabei haben die verschiedenen Qualitäten des Wissens, auf das zurückgegriffen wird, eine entscheidende Wirkung auf den Vorgang des Problemlösens, welcher sich an einem Modell orientiert, das sich der Problemlöser von der gestellten Anforderung oder der gegebenen Situation macht. Dieses Modell wird als die innere Repräsentation oder die subjektive Abbildung bezeichnet, wobei unterschiedliche Repräsentationen einer gegebenen Problemanforderung zur Anstrengung unterschiedlicher kognitiver Ressourcen sowie $\mathrm{zu}$ unterschiedlichem Problemlösungsverhalten führen. ${ }^{611}$ Von der internen Repräsentation des Systems sowie derjenigen der Kontroll- und Steuerauf-

607 Vgl. Oerter, R., (Informationsverarbeitung 1978).

608 Vgl. Voss, J.F., (Lösen 1990), S. 314.

609 Vgl. Lass, U.; Lüer, G., (Problemlöseforschung 1990), S. 296.

610 Vgl. Süss, H.M., (Intelligenz 1996), S. 67; Kluwe, R.H.; Misiak, C.; Schmidle, R.,(Wissenserwerb 1985), S. 255. 
gabe ist somit weitgehend die Güte und Art der Systemsteuerung abhängig.612 Dabei unterscheiden sich die vorliegenden theoretischen Ansätze nach den angenommenen Arten von Wissen, die Probanden bei der Systemsteuerung erwerben, welches Wissen sie zur Steuerung nutzen und wie vollständig dieses Wissen sein $\mathrm{mu} .{ }^{613}$

So haben Kluwe, Schilde, Fischer \& Oellerer in ihren Arbeiten Hinweise dafür gefunden, daß die Versuchspersonen ein spezifisches System in einfacher, sparsamer Form abbilden. Die Versuchspersonen verwendeten einfache Kontrollund Steuerungsregeln und steuerten das System wie einen Regler mit Soll-IstAbweichungen - sie suchten, wie Verbal- und Verhaltensdaten zeigten, jedoch nicht nach Variablenverknüpfungen. Als Beleg dafür führen Kluwe, Schilde, Fischer \& Oellerer darüber hinaus ingenieurpsychologische Studien an, in denen gezeigt wurde, daß ein Individuum ein technisches System auch auf der Basis eines einfachen und unvollständigen mentalen Modells steuern kann. ${ }^{614}$ Diese Berichte aus ingenieurpsychologischen Feldstudien legen nahe, daß Individuen mit komplexen technischen Systemen auch auf der Basis unvollständigen, vereinfachenden Wissens umgehen können. ${ }^{615}$ Außerdem entwickelten sie ein Simulationsprogramm, ${ }^{616}$ mit dem sie das Steuerungsverhalten ihrer Probanden auf der Basis von Annahmen, die aus Verbal- und Verhaltensdaten gewonnen wurden, simulierten. Dieses Programm stützte weiterhin ihre Befunde. So lieferten die Programmläufe, die ohne implementiertes Strukturwissen abliefen, Kontrollund Steuerungsdaten, die denen der empirischen Erhebungen sehr nahe kamen. ${ }^{617}$

611 Lass, U.; Lüer, G., (Problemlöseforschung 1990), S. 296.

612 Vgl. Kluwe, R.H., (Kontrolle 1990), S. 245.

613 Vgl. Süss, H.M., (Intelligenz 1996), S. 67.

614 Vgl. Kluwe, R.H., Schilde, A.; Fischer, C.; Oellerer, N.,(Problemlöseleistungen 1991), S. $305 \mathrm{f}$.

615 Vgl. bspw. Bainbridge, L., (Equations 1987); Norman, D.A., (Observations 1983), S. 8 f. So führt Norman bspw. aus: „I conclude that most people's understanding of the devices they interact with is surprisingly meager, imprecisely specified, and full of inconsistencies, gaps, and idiosyncratic quirks.“ders., (Observations 1983), S. 8.

616 Hier handelt es sich um das Programm SIMSHELL.001. Vgl. Kluwe, R.H.; Misiak, C.; Haider, H., (Ergebnisse 1989), S. 101.

617 Vgl. Kluwe, R.H., (Kontrolle 1990), S. 245 f. Vgl. auch die differenziertere Erläuterung sowie die graphischen Darstellungen bei Kluwe, R.H.; Misiak, C.; Haider, H., (Ergebnisse 1989), S. 114 ff. 
Eine solche Position hat bereits Ashby vertreten. Danach kann eine Person ein komplexes System erfolgreich steuern, ohne es unbedingt verstehen zu müssen. Vor allem benötigt die Person Wissen über den Umgang mit dem System, weniger einen großen Umfang an Regel-Wissen. Sie muß wissen, wie mit dem System umzugehen ist und erkennen, was passiert. Warum etwas so und nicht anders passiert, ist weniger wichtig. 618 „If a system is too complex to be understood, it may nevertheless still be controllable. For to achieve this, all that the controller wants to find is some action that gives an acceptable result; he is concerned only with what happens, not with why it happens. Often, now matter how complex the system, what the controller wants is comparatively simple: has the patient recovered? - have the profits gone up or down? - has the number of strikes gone up or down?" 619

Demgegenüber wird von Sternberg die Notwendigkeit der Analyse von Systemstrukturen und -prozessen ausgeführt. ${ }^{620}$ Auch Berry \& Broadbent gehen davon aus, daß Probanden im Umgang mit einem dynamischen System Wissen über dessen Struktur erwerben, dieses jedoch implizit ist, da sie in einer Serie von Experimenten ${ }^{621}$ keinen Zusammenhang zwischen verbalisiertem Sachwissen und der ansteigenden Leistung bei der Steuerung des Systems fanden. ${ }^{622}$ Diese Sicht vertreten auch Dörner, Reither, Kreuzig \& Stäudel. Ihrer Ansicht nach legen einige Ansätze den Eindruck nahe, als ob Individuen komplette und richtige Abbildungen der Systeme, insbesondere von deren Struktur entwickeln müßten, um Systeme steuern zu können. Sie sprechen in diesem Zusammenhang von Strukturwissen, das über ein System zu erwerben sei. ${ }^{623}$

618 Vgl. Ashby, W.R., (Variety 1958), S. 97.

619 Ashby, W.R., (Variety 1958), S. 97.

620 Vgl. Sternberg, R., (Reasoning 1982), S. 288 f.

621 In den Experimenten von Berry \& Broadbent hatten die Versuchspersonen eine Zuckerproduktion zu kontrollieren. Vgl. Berry, D.C.; Broadbent, D.E., (Relationship 1984), S. 215 ff.; S. 221 ff. und S. 225 ff.; dies., (Combination 1987), S. 10 ff. und S. 12 ff.

622 Vgl. Berry, D.C.; Broadbent, D.E., (Relationship 1984), S. 229 f.; dies., (Combination 1987), S. 14.

623 Vgl. Dörner, D.; Kreuzig, H.W.; Reither, F.; Stäudel, T. (Hrsg.),(Lohhausen 1983). Ebenso auch deKleer, J.; Brown, J.S., (Assumptions 1983), S. 180 f.; Funke, J., (Systemmerkmale 1990), S. 152. 
Die Befunde und Meinungen sind demnach geteilt. Süß räumt jedoch ein, daß die betont konträre Position von Kluwe, Schilde, Fischer \& Oellerer ${ }^{624}$ bei genauerer Betrachtung keinesfalls so trennscharf ist, wie sie dargestellt wird. So schränkt Kluwe ${ }^{625}$ selbst ein, daß Steuerungsanforderungen, für die umfangreiches Systemwissen notwendig ist, keinesfalls ausgeschlossen sind.626 Ist die Annahme, ein System nur dann erfolgreich steuern zu können, wenn ein isomorphes Modell des Systems verfügbar ist, wohl auch wenig realistisch, ${ }^{627}$ so kann jedoch angenommen werden, daß die Steuerungsleistung um so besser ist, je umfangreicher und genauer das Wissen über die Struktur eines Systems ist. Die Chance, daß Probanden die Handlungsregeln finden, mit denen ein System erfolgreich gesteuert werden kann, ist um so größer, je besser und differenzierter das Wissen über die Struktur eines Systems ist.628 Dafür sprechen auch einige Untersuchungsdesigns, die jedoch für Kluwe, Schilde, Fischer \& Oellerer keine Evidenz haben ${ }^{629}$.

Zusammenfassend läßt sich festhalten, daß zwischen dem erfolgreichen Umgang mit einer kognitiven Anforderungssituation und einer angemessenen Wissensrepräsentation eine positive Korrelation besteht. Dabei unterscheiden sich Individuen bezüglich der Form ihrer Wissensrepräsentation, der Art ihres individuell repräsentierten Wissens, den dazwischen aufzufindenden Relationen und den darüber agierenden individuellen Prozessen. Die individuelle Wissensrepräsentation hängt neben Aspekten wie dem individuellen kognitiven Entwicklungsstand, der Wahrnehmung der Anforderungssituation, den bei der Informationsverarbeitung verfolgten Zielen sowie den vorangegangenen Instruktionen offenbar vom Grad

\footnotetext{
624 Vgl. Kluwe, R.H.; Schilde, A.; Fischer, C.; Oellerer, N.,(Problemlöseleistungen 1991), S. $305 \mathrm{f}$.

625 Vgl. Kluwe, R.H., (Problem 1991), S. 316.

626 Vgl. Süss, H-M., (Intelligenz 1996), S. 68 f.

627 So wäre eine solche Behauptung bei bis zu 2000 Variablen auch wenig überzeugend. Vgl. Süss, H.-M., (Intelligenz 1996), S. 69.

628 Vgl. Süss, H.-M., (Intelligenz 1996), S. 69.

629 Vgl. Süss, H.-M., (Intelligenz 1996), S. 68. So führen die Autoren aus: „Die oft behauptete Suche nach den Variablenverknüpfungen konnten wir weder durch Verbal- noch durch Verhaltensdaten belegen." Kluwe, R.H.; Schilde, A.; Fischer, C.; Oellerer, N., (Problemlöseleistungen 1991), S. 305 f.
} 
der Expertise ab. ${ }^{630} \mathrm{Im}$ folgenden erscheint es daher wichtig auf Modelle der Wissensrepräsentation beim Menschen einzugehen, die Gegenstand der Wissenspsychologie, einem Teilbereich der Kognitionspsychologie, sind.

\section{3}

\section{Mentale Repräsentationen von Wissen}

\subsection{1}

\section{Gegenstand der Wissenspsychologie}

Die Kognitionspsychologie beschäftigt sich sowohl mit dem kognitiven System des Menschen sowie anderer Lebewesen als auch mit simulativen maschinellen Systemen. ${ }^{631}$ Dabei basieren die Theorien der Kognitionswissenschaft auf der Annahme einer mentalen Repräsentation von Informationen. ${ }^{632}$ Sie können daher auch als natürliche, adaptive Systeme verstanden werden, ,[...] die Informationen mit Hilfe eines Zentralnervensystems verarbeiten, oder als künstliche Systeme, die eine derartige Verarbeitung simulieren"633. Um in diesem Zusammenhang zu einer ganzheitlichen Analyse des kognitiven Systems zu gelangen, sind mehrere Fragen an ein kognitives System zu stellen. Dies sind Fragen nach dem Aufbau des Systems, nach den im System ablaufenden Prozessen sowie nach der Entstehung des Systems oder auch nach dessen Tektonik, Dynamik und Genetik. Durch deren Beantwortung wird die vollständige Beschreibbarkeit eines kognitiven Systems möglich. Wird durch die Genetik des Systems gewissermaßen seine Historie beschrieben, so wird durch die Tektonik dessen Aufbau beschrieben, wobei über Veränderungen im Zeitverlauf abstrahiert wird. Diese werden durch die Dynamik aufgegriffen. Hier wird nicht mehr nur, wie in der Tektonik, der Zustand eines Systems zu einem bestimmten Zeitpunkt beschrieben, sondern dessen Veränderung und die sich daraus ergebenden Auswirkungen auf das Verhalten eines Systems. ${ }^{634}$

\footnotetext{
630 Vgl. Tergan, S.-O., (Grundlagen -Teil 1- 1989), S. 156. Vgl. auch Abschnitt 3.3: Umgang von Personen mit komplexen Systemen.

631 Vgl. Strohner, H., (Systeme 1995), S. 23.

632 Vgl. Gerjets, P.; Westermann, R.,(Theorien 1997), S. 263.

633 Strohner, H., (Systeme 1995), S. 34. In diesem Zusammenhang wurde bereits das Paradigma der Informationsverarbeitung erläutert. Vgl. Abschnitt 5.2: Komplexes Problemlösen und Wissen. 
Die Wissenspsychologie stellt nun eines der Kerngebiete der Kognitionspsychologie dar, wobei die Psychologie mit dem menschlichen Wissen vielfältige Fragestellungen verbindet. So wird gefragt, wie menschliches Wissen aus psychologischer Sicht dargestellt werden kann, wie Menschen ihr Wissen ausbauen und umorganisieren und welche menschlichen Leistungen als Indikatoren für bestimmtes Wissen angesehen werden können. ${ }^{635}$ Diese Fragen, nämlich Fragen des Erwerbs und der Anwendung von Wissen, sind seit der kognitiven Wende zentrale Themen der allgemeinpsychologischen Forschung, die unter dem Begriff der Wissenspsychologie subsumiert werden. ${ }^{636}$ Für die in diesem Bereich der Psychologie durchzuführenden, empirischen Untersuchungen werden ebenfalls Problemstellungen genutzt, die als system-vergleichbare Mikrowelten auf Computern implementiert werden. Aufgabe der Versuchspersonen ist es hier, bestimmten Instruktionen entsprechend, diese Mikrowelten zu kontrollieren und zu steuern. ${ }^{637}$

Im Umgang mit solchen Mikrowelten bilden Personen mentale Repräsentationen im Zusammenhang mit dem implementierten System aus, welche den Untersuchungsgegenstand darstellen. Diesbezüglich besteht keine Einigkeit über die Form mentaler Repräsentationen. Der Rahmen populärer Theorien kann jedoch als übereinstimmend angesehen werden. ${ }^{638}$ Zunächst hebt die Kognitionswissenschaft von der individuellen Modellierungsebene die Ebene der psychologischen Modellbildung ab, die als Wissensrepräsentation bezeichnet werden kann. Die Art, in der Gedächtnisinhalte gespeichert werden, bezeichnet man als ihre mentale Repräsentation. Sie können sowohl durch ihre strukturellen als auch durch ihre inhaltlichen Merkmale charakterisiert werden. ${ }^{639}$ So wird in psychologischen Theorien zur Wissensrepräsentation zwischen einer Wissensbasis und den damit arbeitenden Prozessen unterschieden. Dies ist gleichbedeutend mit der Unterscheidung von Struktur und Prozeß oder auch zwischen deklarativem und prozeduralem Wissen. Da es nicht möglich ist, das eine ohne das andere zu erfassen, wird bei experimentellen Überprüfungen der Theorien immer beides untersucht: so-

\footnotetext{
635 Vgl. Kluwe, R.H.; Spada, H., (Wissen 1981), S. 284.

636 Vgl. Mandl, H.; Spada, H.,(Wissenspsychologie 1988), S. 1.

637 Vgl. Kluwe, R.H., (Kontrolle 1990), S. 239 f. sowie auch Abschnitt 3.3: Umgang von Personen mit komplexen Systemen.

638 Vgl. Wender, K.-F., (Netzwerke 1988), S. 55.
} 
wohl die Struktur als auch ein Prozeß - bzw. deklaratives und prozedurales Wissen. ${ }^{640}$

Mit kognitionspsychologischer Forschung wird demnach die Erwartung verbunden, daß sie Analysen der Anforderungen der Kontrolle und Steuerung komplexer Systeme an die menschliche Informationsverarbeitung erarbeitet und daß sie Merkmale und Verlauf der geistigen Aktivität sowie des Kontroll- und Steuerungsverhaltens beschreibt. ${ }^{641}$ Dabei finden sich in der Literatur unterschiedliche Ansätze der Wissensrepräsentation, die sich zunächst nach propositionalen, analogen und regelbasierten Ansätzen unterscheiden lassen.

\subsubsection{Propositionale, analoge und regelbasierte Ansätze der Wissens- repräsentation}

\subsubsection{Propositionale Repräsentationssysteme}

Zur Gruppe der propositionalen Repräsentationssysteme kann die Mehrheit der bisher entwickelten formalen Modelle der Wissensrepräsentation gerechnet werden. In propositionalen Repräsentationssystemen findet Wissen Verwendung, das zunächst meist als sprachlich-begrifflich umschrieben wurde. Beispiele hierfür sind semantische Netzwerkmodelle, Frames und Skripts sowie prädikatenlogische Kalküle. Gemeinsam sind den propositionalen Ansätzen die elementaren Einheiten zur Wissensrepräsentation. Dies sind die diskreten Symbolstrukturen, die sogenannten Propositionen. ${ }^{642}$

In chronologischer Betrachtung sind zunächst die Netzwerkmodelle zu nennen, in denen im allgemeinen jeder Begriff nur an einer Stelle abgespeichert wird. Diese Darstellungsart wurde jedoch als unübersichtlich eingeschätzt, was dazu führte, daß im Laufe der siebziger Jahre eine Reihe von Ansätzen entwickelt wurden, die der eher losen Information im Netzwerkmodell Wissenseinheiten gegenüberstellen, die eine größere interne Kohärenz aufweisen. Diese Ansätze können unter

\footnotetext{
639 Vgl. Gerjets, P.; Westermann, R.,(Theorien 1997), S. 265.

640 Vgl. Wender, K.-F., (Methoden 1992), S. 582.

641 Vgl. Kluwe, R.H., (Kontrolle 1990), S. 239 f.

642 Vgl. Opwis, K., (Modellierung 1992), S. 52.
} 
dem Begriff Schema-Theorie zusammengefaßt werden. ${ }^{643}$ Nach Rumelhardt sind Schemata als Grundelemente des Wissens anzusehen. Es handelt sich um eine Theorie darüber, wie Wissen repräsentiert ist und wie diese Repräsentation den Gebrauch des Wissens vereinfacht. Schema-Theorien zufolge ist jegliches Wissen in Einheiten repräsentiert. Diese Einheiten werden als Schemata bezeichnet. In diese Wissenseinheiten sind, zusätzlich zu dem Wissen selbst, Informationen darüber eingebettet, wie dieses Wissen zu nutzen ist, ${ }^{644}$ womit sie sich als dynamische Wissenselemente klassifizieren lassen. ${ }^{645}$

In der Folge wurden allgemeinere Formalismen entwickelt. So wurden semantische Netze als zu wenig ausdrucksstark angesehen, was zur Entwicklung von Frames führte. ${ }^{646}$ In Frames, oder auch Rahmen, ist nach Minsky Wissen über Begriffe, Objekte, Sachverhalte und Situationen repräsentiert. Rahmen werden dabei als dynamische, hierarchisch organisierte Wissensstrukturen über einen relativ stereotypen Wissensbereich verstanden. Die in Rahmen organisierten Wissenseinheiten sind dabei über vielfältige Relationen verbunden. Darüber hinaus können sie mit denen anderer übergeordneter und untergeordneter Rahmen verbunden sein, wodurch sie in einem sogenannten »frame system « organisiert sind. ${ }^{647}$ Dabei ist als wesentlicher Unterschied zwischen der Netzwerkrepräsentation und der Rahmen-Repräsentation anzusehen, daß ein Konzept innerhalb eines Rahmens zusätzlich nach der Bedeutsamkeit von Informationen klassifiziert wird. So führt Winograd aus: „The frame idea adds an important additional element to this - the idea of importance or centrality. For each frame there is a set of other frames marked as important elements having a specific relation to it.“648

Skripts, eingeführt von Schank \& Abelson ${ }^{649}$, sollten schließlich ,[...] das Textverstehen als eine Integration neuer in bereits bekannte Informationen erklären [...]

\footnotetext{
643 Vgl. Kluwe, R.H.; Spada, H., (Wissen 1981), S. 308 f.

644 Vgl. Rumelhardt, D.E., (Schemata 1980), S. 33 f.

645 Vgl. Tergan, S.-O., (Modelle 1986), S. 104.

646 Vgl. Opwis, K., (Modellierung 1992), S. 64 f.

647 Vgl. Minsky, M., (Framework 1975), S. 212.

648 Winograd, T., (Representations 1975), S. 199.

649 Vgl. Schank, R.C.; Abelson, R.P.,(Scripts 1977), S. 36 ff.
} 
können."650 Ein Skript stellt ein Schema zu wiederholt erfahrenen Ereignissen dar. ${ }^{651}$ In ihrem zeitlichen Ablauf handeln eine oder mehrere Personen und verfolgen dabei ein bestimmtes oder auch mehrere Ziele. ${ }^{652}$ Dabei werden verschiedene Arten von Skripts unterschieden, je nachdem auf welchen Hintergrund sie sich beziehen. ${ }^{653}$

\subsubsection{Analoge Repräsentationssysteme}

Zentrales Charakteristikum analoger Ansätze zur Wissensrepräsentation ist die Annahme, daß das Wissen über Gegenstandsbereiche analog den strukturellen Merkmalen dieser Gegenstandsbereiche repräsentiert sei. Ansätze zur analogen Repräsentation von Wissen wurden im Rahmen der Vorstellungsforschung sowie der Forschung zu mentalen Modellen als die zwei Grundansätze kognitiv orientierter Forschung zur analogen Wissensrepräsentation ${ }^{654}$ entwickelt. ${ }^{655}$ Theoretische und empirische Forschungsarbeiten zeigten jedoch, daß das Konstrukt der Vorstellung, welches sich vorwiegend auf statische Phänomene bezieht ${ }^{656}$, das Verhalten realer, dynamischer Systeme und den Umgang mit diesen nur unzureichend erfaßt. Es erschien daher sinnvoller, in Zusammenhang mit dynamischen Systemen statt von mentaler Vorstellung von einem mentalen Modell zu sprechen - statt von mentaler Transformation von mentaler Simulation ${ }^{657}$. So erweitern mentale Modelle den Rahmen anderer analoger Repräsentationen um den wesentlichen Aspekt des Veranschaulichungsprozesses. ${ }^{658}$ Oder wie Mandl, Friedrich \& Hron es ausdrücken: „Mittels mentaler Modelle ist es möglich, das Verhalten dy-

$650 \quad$ Vgl. Opwis, K., (Modellierung 1992), S. 71.

651 „Immer wieder genannte Beispiele für Skripte sind etwa der Besuch eines Restaurants, das Einkaufen in einem Supermarkt, der Ablauf eines Arzt- oder Kinobesuchs usw."Opwis, K., (Modellierung 1992), S. 71. Bei Schank \& Abelson findet sich bspw. ein RestaurantSkript. Vgl. Schank, R.C.; Abelson, R.P., (Scripts 1977), S. 42 ff.

652 Personale Skripte können auch nicht zielorientiert sein. In diesem Falle handelt es sich bspw. um ein rituelles Verhalten oder eine emotionale Reaktion. Vgl. Schank, R.C.; Abelson, R.P., (Scripts 1977), S. 63.

653 Schank \& Abelson unterscheiden zwischen personal, situational und instrumental scripts. Ein Skript ist demgemäß entweder auf Personen, auf Situationen oder auf Instrumente bezogen. Vgl. Schank, R.C.; Abelson, R.P., (Scripts 1977), S. 61 ff.

Vgl. Tergan, S.-O., (Modelle 1986), S. 185.

655 Vgl. Tergan, S.-O., (Modelle 1986), S. 156.

656 Vgl. Tergan, S.-O., (Modelle 1986), S. 185.

657 Vgl. Tergan, S.-O., (Modelle 1986), S. 163.

658 Vgl. Steiner, G., (Repräsentationen 1988), S. 112. 
namischer Systeme sowie Handlungsvollzüge im Umgang mit diesen Systemen „,vor dem geistigen Auge“ zu simulieren.“659

In Zusammenhang mit der theoretischen Modellierung hat sich die psychologische Forschung Gedanken zur Konzeption eines Rahmenmodells gemacht, welches den Untersuchungen zugrunde gelegt werden kann. Nach Norman ist es wichtig, zwischen dem realen System, einer umfassenden Repräsentation des realen Systems, dem mentalen Modell des Benutzers sowie dem aus diesem abgeleiteten psychologischen Modell zu unterscheiden. So führt dieser aus: „In the consideration of mental models we need really consider four different things: the target system, the conceptual model of that target system, the user's mental model of the target system, and the scientist's conceptualization of that mental model. The system that the person is learning or using is, by definition, the target system. A conceptual model is invented to provide an appropriate representation of the target system, appropriate in the sense of being accurate, consistent, and complete. Conceptual models are invented by teachers, designers, scientists, and engineers." 660

Opwis bezieht sich auf die Ausführungen von Norman und unterscheidet zwischen einem Simulationsmodell eins und zwei. Dabei ist das Simulationsmodell eins das durch den Forscher konzipierte Modell über den zu beschreibenden Realitätsausschnitt, das Simulationsmodell zwei das Modell eines mentalen Modells einer Versuchsperson. ${ }^{661}$

Auch Kluwe unterscheidet zwischen unterschiedlichen Modellarten. Er unterscheidet zwischen einem objektiven Modell, das in formalisierter, wissenschaftlicher Art beschrieben wird. Das individuelle Modell, welches eine Person über das System ausbildet bezeichnet er als mentales Modell. Über diese mentalen Modelle von Individuen entwickelt die psychologische Forschung ein psychologisches Modell. Ein viertes Modell bezeichnet er als Design-und-Instruktions-Modell.

659 Mandl, H.; Friedrich, H.F.; Hron, A.,(Ansätze 1988), S. 146.

660 Norman, D.A., (Observations 1983), S. 7.

661 Vgl. Opwis, K., (Modelle 1985), S. 12 ff.; Opwis, K., (Modelle 1987), S. 265 f.; Opwis, K.; Spada, H.; Schwiersch, M.,(Erwerb 1985), S. 4. 
Funke erweitert den oben vorgestellten Rahmen von Opwis, da seiner Ansicht nach eine weitere Anzahl von Modellen eine Rolle spielen. Er gibt den jeweiligen Bezugsgegenstand der einzelnen Modelle an und zeigt dadurch die Möglichkeit unendlicher Schleifen auf. Durch dieses Vorgehen ergibt sich eine Anzahl von mindestens sechs verschiedenen Modellen. ${ }^{662}$

\subsubsection{Regelbasierte Repräsentationssysteme}

Bei den regelbasierten Systemen handelt es sich schließlich um sogenannte »PHYSICAL SYMBOL SYSTEMS«. Das bedeutet, daß das Wissen des Systems in Form von Symbolen repräsentiert wird. Diese Symbole stellen die grundlegenden Einheiten der Informationsverarbeitung dar. Sie entsprechen in der Regel semantisch interpretierbaren Konzepten, das bedeutet, sie repräsentieren Sachverhalte oder Klassen von Sachverhalten der Innen- und Außenwelt; und sie sind in einer bestimmten Struktur angeordnet. Der Prozeß der Informationsverarbeitung besteht ganz global betrachtet darin, daß Symbole interpretiert und manipuliert werden. Dazu gibt es einen Satz von Regeln, die vorschreiben, wie die Symbole manipuliert werden müssen, damit ein bestimmter neuer Zustand der Symbolstruktur erzeugt wird. Ein regelbasiertes System entspricht damit der allgemeinen Grundstruktur eines Informationsverarbeitungssystems. Die bekannteste Form regelbasierter Repräsentationssysteme sind die Produktionssysteme. ${ }^{663}$ In ihnen sind Annahmen zur Struktur des Gedächtnisses verknüpft mit solchen über Prozesse der Informationsverarbeitung. Die grundlegende Annahme ist, daß menschliche Kognition sich aus einem Set von Bedingungs-Handlungs-Kombinationen zusammensetzt, die als Produktionen bezeichnet werden. ${ }^{664}$

662 Vgl. Funke, J., (Problemlösen 1985), S. 124 f.

663 Die Auseinandersetzung mit regelbasierten Systemen nahm ihren Anfang im Bereich der Berechenbarkeitstheorie. Mit Beginn der 70er Jahre fanden sie durch Newell, der sie als „theory of the control structure of human information processing“ einführte, Eingang in die Psychologie. Vgl. Opwis, K., (Produktionssysteme 1988), S. 79 und die dort angegebene Literatur. $\mathrm{Zu}$ weiteren Ausführungen zur Geschichte der Produktionssysteme vgl. bspw. ders., (Produktionssysteme 1988), S. 79 ff. und Anderson, J.R., (Architecture 1983), S. 5 ff.

Vgl. Anderson, J.R., (Architecture 1983), S. 5. 
Dabei folgt die generelle Struktur eines regelbasierten Systems der dieser Modellklasse inhärenten Unterscheidung von symbolisch repräsentiertem Wissen sowie Regelvorrat und besteht zumeist aus drei Komponenten:665

1. Einem Datenspeicher: Ein Datenspeicher enthält das symbolisch repräsentierte, das „deklarative“ Wissen des Systems. Diese Wissensstruktur ist von sich aus statisch, sie enthält also keine Angaben über Wissensveränderungsoder Wissenserwerbsprozesse.

2. Einem Produktionenspeicher: Dieser Speicher enthält das prozedurale Wissen des Systems in Form einer (ungeordneten) Menge von Produktionen. Produktionen können nicht nur auf die Systemumwelt wirken, sondern auch die Inhalte des Datenspeichers verändern.

3. Einem Interpreter: Ein Interpreter repräsentiert das Kontrollwissen des Systems. Aufgabe des Interpreters ist es, das vorliegende deklarative Wissen zu interpretieren, zu entscheiden, welche Produktionen aktuell anwendbar sind, eine dieser Produktionen nach bestimmten Kriterien auszuwählen und schließlich auch auszuführen.

Im folgenden soll nun auf das mentale Modell als analoges Repräsentationssystem komplexer Systeme sowie das Produktionssystem als eine Form regelbasierter Repräsentationssysteme näher eingegangen werden. Dies ist notwendig um in Kapitel sechs zu Aussagen über betriebswirtschaftliche Lernprozesse im Unternehmensplanspiel zu gelangen. ${ }^{666}$

Mentale Modelle als analoges Repräsentationssystem komplexer Systeme

In Zusammenhang mit komplexen Systemen erreicht der Begriff des mentalen Modells einen hohen Stellenwert. So wird in Zusammenhang mit komplexen Sy-

665 Vgl. Ueckert, H., (Computer-Simulation 1983), S. 564 f.

666 Vgl. Abschnitt 6.6: Betriebswirtschaftliche Lernprozesse im Unternehmensplanspiel. 
stemen davon ausgegangen, daß der Mensch im Umgang mit komplexen Systemen das Wissen darüber in Form eines mentalen Modells im Gedächtnis repräsentiert.

Nach Johnson-Laird, durch den der Begriff des mentalen Modells in die Psychologie eingeführt wurde ${ }^{667}$, entwirft der Mensch ein mentales Modell von der Welt, das den Prämissen des Syllogismus ${ }^{668}$ gerecht wird. Der Mensch analysiert dieses Modell, um festzustellen, ob die Schlußfolgerung gerechtfertigt ist. ${ }^{669}$ Neben dem Begriff des mentalen Modells finden sich in der Literatur auch die Bezeichnungen mental image ${ }^{670}$, user`s model ${ }^{671}$ oder conceptual model ${ }^{672}$. Als vergleichbar in der Bedeutung, führt Stahl daneben noch die Begriffe cognitive frames, cognitive maps oder mental representation an. Weiterhin führt sie aus, daß ,[...] die meistzitierte ökonomische Arbeit zur Bedeutung kognitiver Fragen für individuelles und gemeinschaftliches Handeln [von Denzau \& North] mit dem Begriff des mentalen Modells argumentiert und dieser Begriff daher in der wirtschaftswissenschaftlichen Diskussion die weiteste Verbreitung gefunden hat" “.673 Aus diesem Grund wird auch in dieser Arbeit im weiteren der Begriff mentales Modell verwendet.

Vgl. Strohschneider, S.,(Wissenserwerb 1990), S. 56.

Vgl. Anderson, J.R., (Psychologie 1996), S. 315 ff. Ein Syllogismus kann nach kategorilem und konditionalem Syllogismus unterschieden werden. Beim KATEGORIALEN SYLLOGISMUS handelt es sich um einen Syllogismus, an dem Aussagen mit logischen Quantoren beteiligt sind. So folgt aus zwei Prämissen, welche A und B sowie B und C in Beziehung setzen, daß ein Zusammenhang zwischen $\mathrm{A}$ und $\mathrm{C}$ besteht. Ein Beispiel: Wenn alle Menschen sterblich sind, und Sokrates ein Mensch ist, kann die Schlußfolgerung gezogen werden, daß Sokrates sterblich ist. Beim KONDITIONALEN SYLLOGISMUS handelt es sich um ein Aussagengefüge, dessen Prämissen sich aus einer bedingten Aussage sowie einer dazlgehörigen Behauptung herleiten. Aus ihnen läßt sich dann ein entsprechender Schluß, eine Conclusio herleiten. Vgl. ders., (Psychologie 1996), S. 452. Vgl. auch die „TransitiveChain Theory of syllogistic reasoning" bei Guyote, M.J.; Sternberg, R.S., (Theory 1981), S. 466 ff. Vgl. weiterhin auch Johnson-Laird, P.N.; Steedman, M.,(Psychology 1978), S. $64 \mathrm{ff}$.

Vgl. Johnson-Laird, P.N., (Models 1983), S. 396.

Vgl. Hopkin, V.D., (Human 1995), S. 49. Im Bereich der Fluglotsenforschung ist auch die Bezeichnung 'picture' gebräuchlich und kann ebenfalls als vergleichbar in der Bedeutung angesehen werden. Vgl. bspw. Whitfield, D.; Jackson, A., (Air 1982), S. 37 ff. Hier werden unterschiedliche Wissensarten unter dem Begriff des 'pictures` subsumiert. So erachtet Whitfield bspw. drei Gedächtnisebenen als relevant für das 'picture'des Fluglotsen. Vgl. Whitfield, D., (Study 1979), S. 25.

Vgl. Norman, D.A., (Engineering 1986), S. 46.

Vgl. Wilson, J.R.; Rutherford, A., (Models 1989).

Stahl, S.R., (Beschränkungen 1999), S. 114 und die dort angegebene Literatur. 
Werden die existierenden Definitionen zu mentalen Modellen betrachtet, so kann festgestellt werden, daß diese vielfältig sind - und keine allgemein gültige Definition existiert. So subsumiert bspw. die Mehrzahl der Autoren unterschiedliche Repräsentationsformate - sowohl propositionale, deklarative als auch analoge unter dem Begriff mentale Modelle, da sie sich zumeist als hybride Systeme klassifizieren lassen. ${ }^{674}$

Auch Kluwe \& Haider führen aus, daß die Ansätze zur Beschreibung mentaler Modelle komplexer Systeme verschiedene Wissensformen umfassen, die für ein System erworben werden können, wozu sie zunächst, in Anlehnung an die Merkmale informationsverarbeitender Systeme, ${ }^{675}$ zwischen deklarativem und prozeduralem Wissen unterscheiden. Unter deklarativem Wissen subsumieren sie dann verschiedene Wissensformen, die sie als Systemwissen, Funktionswissen und Weltwissen sowie weiterhin Kontroll- und Steuerungswissen bezeichnen. Demgegenüber verstehen sie unter prozeduralem Wissen sog. geistige Operationen, ,[...] die eine Generierung von System- als auch von System- und Steuerungswissen leisten und die weiter das bereits verfügbare Wissen transformieren“. 676

Wichtig ist zudem, daß es sich bei den in mentalen Modellen repräsentierten Wissensgefügen über komplexe Sachverhalte um subjektive Wissensgefüge handelt. Derlei subjektive Wissensgefüge werden von Personen im Umgang mit diesen Sachverhalten erworben und können nur als begrenzt vollständig und richtig eingeschätzt werden. Mentale Modelle sind dabei funktional, was bedeutet, daß sie das Verstehen von Sachverhalten und den Umgang mit Sachverhalten leiten, wobei sie spezifischen Phänomenen unterliegen, die vordergründig durch Komplexität und geringe Transparenz gekennzeichnet sind. ${ }^{677}$ Darüber hinaus sind mentale Modelle einer Veränderlichkeit im Zeitablauf unterworfen. Sie können daher nicht als fertiges Bild oder Konzept angesehen werden. ${ }^{678}$

\footnotetext{
674 Vgl. Fürstenau, B., (Problemlösen 1994), S. 39 und die dort angegebene Literatur.

675 Vgl. Abschnitt 5.2: Komplexes Problemlösen und Wissen.

676 Kluwe, R.H.; Haider, H., (Modelle 1990), S. 175 f.

677 Kluwe, R.H., (Gedächtnis 1992), S. 157.

678 Vgl. Bierwagen, T., (Fluglotsen 1999), S. 11.
} 
5.5

Produktionssysteme als eine Form regelbasierter Repräsentationssysteme

5.5.1

Dörner's Theorie der Absichtsbehandlung

\subsubsection{1}

Elemente der Theorie der Absichtsbehandlung

Die Theorie der Absichtsbehandlung von Dörner stellt ein Beispiel für ein regelbasiertes Repräsentationsmodell dar. ${ }^{679}$ Dörner's Überlegungen gehen in diesem Modell von der Existenz eines Absichtsgedächtnisses aus. In diesem sind die unerledigten Absichten des Individuums ähnlich einer Auftragsliste gespeichert ${ }^{680}$, und Dörner führt neun Kennzeichen einer Absicht aus: ${ }^{681}$

1. den gewünschten Zielzustand

2. den derzeitigen Anfangszustand

3. die "Geschichte": das Wissen darüber, was bis zum Betrachtungszeitpunkt getan wurde, um das Ziel zu erreichen

4. den Plan: Wissen über die Handlungsmöglichkeiten, die zur Zielerreichung führen

5. die Instrumentalität: Wissen über Beweg- und Hintergrund

6. den Termin: Bestimmung des Zeitpunktes zu dem das Vorhaben beendet sein soll

7. die Wichtigkeit der Absicht, als auch Stärke und Bedeutsamkeit der zugrundeliegenden Motivation

8. die geschätzte Zeit bis zur Erreichung des End- oder Zielzustandes

9. die Kompetenz: Wissen über die eigenen Fähigkeiten als Maßstab für die Erfolgserwartung

Ein Element des Absichtsgedächtnisses setzt sich wiederum aus sieben verschiedenen Gedächtniselementen zusammen: ${ }^{682}$

\footnotetext{
679 Vgl. Dörner, D., (Probleme 1982), S. 57 ff.; Dörner, D., (Wissen 1988), S. 264 ff.

680 Vgl. Dörner, D., (Probleme 1982), S. 57.

681 Vgl. Dörner, D., (Wissen 1988), S. 268.

682 Vgl. Dörner, D., (Probleme 1982), S. 57 f.
} 
1. der Gedächtnisrepräsentation über die derzeitige Situation des Problems,

2. der Gedächtnisrepräsentation über den gewünschten Zielzustand des Problems,

3. der Einschätzung über die Wahrscheinlichkeit einer erfolgreichen Problembewältigung,

4. der Kalkulation der zur Problembewältigung erforderlichen Zeit,

5. der Bedeutsamkeit der Absicht,

6. des Zeitpunktes, zu dem die Absicht zu erledigen ist,

7. der zeitlichen Aktualität der Absicht.

Die Wahrscheinlichkeit einer erfolgreichen Problembewältigung hängt dann zum einen von der epistemischen, zum anderen von der heuristischen Kompetenz des Individuums ab. Die epistemische Kompetenz bezeichnet die individuelle Selbsteinschätzung bezüglich der Möglichkeit, die Anforderung mit dem verfügbaren Fachwissen über den jeweils relevanten Realitätsbereich bewältigen zu können. Die heuristische Kompetenz beschreibt die individuelle Selbsteinschätzung bezüglich der Möglichkeit, unbestimmte Situationen, in denen das Individuum nur unvollständige Kenntnisse zur Transformation des Anfangs- in den Endzustand besitzt, von Dörner als echte Probleme bezeichnet, zu bewältigen. Diese heuristische Kompetenz wird, im Gegensatz zur epistemischen Kompetenz, als allgemeingültig angesehen. Sie steht also nicht in Verbindung mit einem bestimmten Realitätsbereich oder Problemtyp. ${ }^{683}$ In Zusammenhang mit epistemischer und heuristischer Kompetenz auf Seiten des Individuums stehen die Annahmen bezüglich der Wissensrepräsentation. Hier wird zwischen epistemischem und heuristischem Wissen unterschieden. Das epistemische Wissen stellt das Wissen eines Individuums über einen Realitätsbereich dar und wird auch als Wissensbasis bezeichnet. Dieses Wissen wird weiter nach einem Afferenz- und einem Efferenzteil unterschieden, wobei der Afferenzteil das Gedächtnis für Sachverhalte, der Efferenzteil das Gedächtnis für Handlungen darstellt. Das heuristische Wissen, welches auch als Verfahrensbibliothek bezeichnet wird, enthält eine Rei- 
he elementarer kognitiver Prozesse und dient dem Umgang mit dem epistemischen Wissen. ${ }^{684}$

Dieses Wissen ist in Strukturen organisiert. So nimmt Dörner analog zu der Unterscheidung nach epistemischem und heuristischem Wissen, eine Unterscheidung nach heuristischer und epistemischer Struktur an. Dörner geht es jedoch weniger um die Struktur, als um Art und Zweck der Verwendung von Wissen zur „Verhaltensregulation“. Vier parallel arbeitende Informationsverarbeitungssysteme bestimmen dabei die Art und Weise des Verhaltens: Dies ist erstens der Hauptstrang der Absichtsregulation, zum zweiten die allgemeine Lageempfindung zur Modifikation der Absichtsbehandlung, zum dritten der Umgebungskontext als Erwartungsbildung über die nähere und fernere Zukunft sowie viertens schließlich die Protokolleinheit zur Kontrolle der eigenen Tätigkeit und Überprüfung bezüglich defizienter Zustände, ${ }^{685}$ was direkt zu angenommenen Wissenserwerbsprozessen in der Theorie der Absichtsbehandlung hinführt, auf die im folgenden Gliederungspunkt eingegangen werden soll.

\subsubsection{Prozesse des Wissenserwerbs}

Die Ausführungen zum Wissenserwerb im Rahmen der Theorie der Absichtsregulation beziehen sich auf Strohschneider, der ,aus Gründen der Prägnanz und um Verwechslungen mit anderen Ansätzen zu vermeiden, [...]“ von einer „kognitiven Handlungstheorie“" spricht. ${ }^{686}$ In diesem Modell der kognitiven Handlungstheorie geht Strohschneider davon aus, daß zur Gewährleistung von Handlungen drei Arten von Wissen vorauszusetzen sind. Dies ist zum einen das Wissen um herrschende Mangelzustände, zum anderen Realitätswissen, was gleichzeitig die Existenz von heuristischem Wissen impliziert sowie schließlich Wissen, welches in Zusammenhang mit einer konkreten motorischen Ausführbarkeit von Handlungen steht. Diese Annahme wird von Dörner als „Tripelhypothese“ bezeichnet. ${ }^{687}$

684 Dörner, D., (Struktur 1977), S. 39 ff.

685 Vgl. Dörner, D., (Wissen 1988), S. 265 ff.

686 Strohschneider, S., (Wissenserwerb 1990), S. $60 \mathrm{ff}$.

687 Vgl. Strohschneider, S., (Wissenserwerb 1990), S. 65 ff. und die dort angegebene Literatur. 
Der Wissenserwerb kann dann im Rahmen der kognitiven Handlungstheorie auf unterschiedliche Art und Weise erfolgen, was in Zusammenhang mit den unterschiedlichen erforderlichen Wissensarten steht. Als im Vordergrund stehend sieht Strohschneider jedoch Wissenserwerb im Sinne von erfahrungsabhängigen Veränderungen von Aktionsschemata an, der auf die folgenden Arten ablaufen kann: 688

DURCH OPERATORBILDUNG

Eine Sequenz von Aktionsschemata kann zu einem Operator zusammengefaßt werden, wenn die elementaren Aktionsschemata in einer stets wiederkehrenden Abfolge benötigt werden. Dadurch wird es möglich den Operator zukünftig bei Vorliegen des Input-Schemas des ersten Aktionsschemas des Operators automatisch abzurufen und die Handlung bis zum Vorliegen des Erwartungsschemas des letzten Aktionsschemas bis zu Ende zu führen.

\section{DURCH DIFFERENZIERUNG}

Weicht das Erwartungsschema eines vorhandenen Operators immer wieder von der Realität ab, so muß dieser durch das Individuum aufgebrochen werden. Andere Aktionsschemata sind dann ganz bewußt neu zu kombinieren.

\section{DURCH GENERALISIERUNG}

Zur Aktivierung von Aktionsschemata kommt es, wenn entsprechende situative Auslösebedingungen vorliegen. Ein Operator kann vom Individuum generalisiert werden, wodurch unterschiedliche Input-Schemata und ebenso auch unterschiedliche Erwartungskopplungen durch die Wahl desselben Aktionsschemas erledigt werden können und dadurch zu einer allgemeineren Anwendbarkeit gelangen. 


\section{DURCH SPEZIALISIERUNG}

Das Gegenteil ist der Fall, wenn ein Aktionsschema sich nur zeitweise als erfolgreich anwendbar erweist. So sind dann das Input-Schema und das Erwartungsschema zu konkretisieren, so daß spezifischere Bedingungen analysiert werden, die zum Abruf des Aktionsschemas führen.

\section{DURCH NEUKONSTRUKTION VON AKTIONSSCHEMATA}

Existierende Aktionsprogramme können zur Neukonstruktion von Aktionsschemata mit neuen Ausgangs- und Erwartungszuständen verknüpft werden.

\subsubsection{Newell's SOAR-Theorie}

\subsubsection{Die Architektur von SOAR}

Von Newell stammt die, ebenfalls den regelbasierten Systemen zuzurechnende, SOAR-Architektur. ${ }^{689}$ Sie stellt, neben der ACT*-Theorie ${ }^{690}$, die bekannteste kognitive Theorie dar. ${ }^{691}$ Rosenbloom, Laird, Newell \& McCarl unterteilen die SOARArchitektur in drei aufeinander basierende Ebenen, welche die Elemente repräsentieren, über die eine generelle Intelligenz verfügen muß: [Vgl. korrespondierend zu den Ausführungen Abbildung 5-1].

689 Vgl. bspw. Newell, A., (Theories 1994); Newell, A.; Rosenbloom, P.S.; Laird, J.E.,(Architectures 1991); Rosenbloom, P.S.; Laird, J.E.; Newell, A.; McCarl, R., (Analysis 1991). Unter dem Begriff Architektur wird eine fixe Struktur verstanden, die den Rahmen für kognitive Prozesse im Gedächtnis darstellt. Vgl. Newell, A.; Rosenbloom, P.S.; Laird, J.E., (Architectures 1991), S. 93. Die Autoren geben auch einen Überblick bezüglich der generellen Rolle von Architekturen in der Kognitionswissenschaft. Vgl. dies., (Architectures 1991), S. 93 ff.

690 Vgl. zu Anderson's ACT*-Theorie Abschnitt 5.5.3: Anderson's ACT-Theorie.

691 Vgl. Opwis, K., (Modellierung 1992), S. 46. 


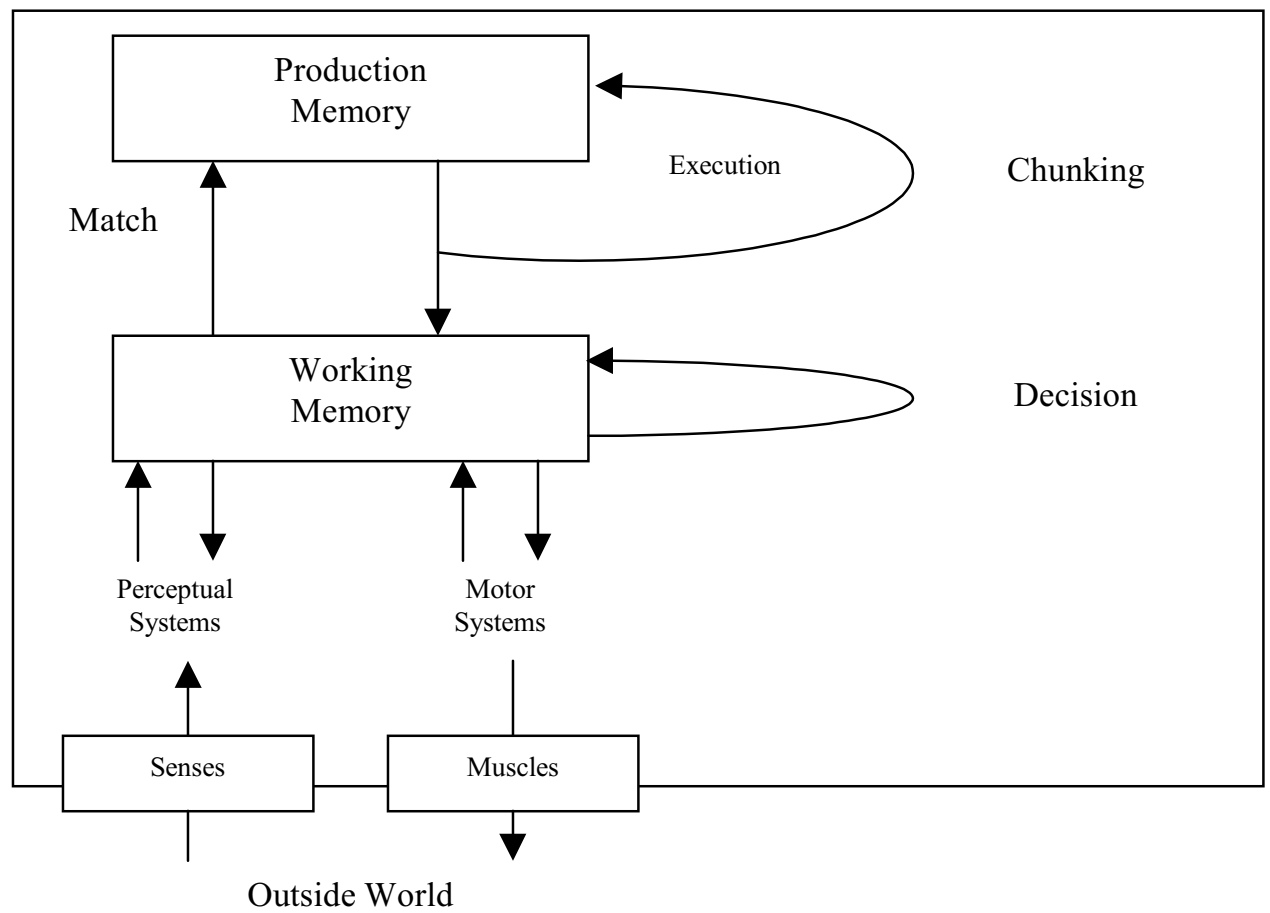

\section{Abbildung 5-1: $\quad$ Überblick über die SOAR-Architektur ${ }^{692}$}

EBENE 1

GEDÄCHTNIS

SOAR besteht aus einem Langzeitgedächtnis [PRODUCTION MEMORY] und einem Arbeitsgedächtnis [WORKING MEMORY] sowie außerdem aus Systemen [PERCEPTUAL SYSTEMS; MOTOR SYSTEMS], die es ermöglichen, die Umgebung [OUTSIDE WORLD] wahrzunehmen und zu beeinflussen. Im Langzeitgedächtnis sind eine Reihe kognitiver Produktionen gespeichert, die sowohl deklaratives, prozedurales als auch episodisches Wissen beinhalten können. Das deklarative Wissen meint dabei Faktenwissen über die Realität sowie außerdem Faktenwissen über Handlungen, die ausgeführt werden können. Das prozedurale Wissen stellt Wissen darüber dar, wie Handlungen vollzogen werden können und umfaßt ebenso Kontrollwissen darüber, welche Handlungen zu welchem Zeitpunkt durchgeführt werden können. Das episodische Wissen stellt schließlich Wissen darüber dar, welche Aktionen zu welchem Zeitpunkt vollzogen wurden. Neben den gespeicherten Wissenselementen weist jede Produktion zudem eine BedingungsHandlungs-Struktur auf, die dazu führt, daß eine Produktion zur Anwendung gelangt, wenn seine Bedingung erfüllt ist. Das Arbeitsgedächtnis dient dem Abruf 
von Informationen aus dem Langzeitgedächtnis. Erfolgt ein solcher Abruf, besteht das Arbeitsgedächtnis aus einem Set von Objekten, die, so lange sie im Arbeitsgedächtnis vorhanden sind, über ein Symbol identifiziert werden können. ${ }^{693}$ Wurden die abgerufenen Produktionen bereits erfolgreich ausgeführt [MATCH], so können sie direkt angewendet werden; wird hingegen keine erfolgreiche Produktion identifiziert, so schließt sich die Ebene der Entscheidungsfindung an. ${ }^{694}$

EBENE 2

ENTSCHEIDUNGEN

Die Entscheidungsebene basiert auf dem Gedächtnis und wird um eine Entscheidungsprozedur ergänzt, welche die Bearbeitung der Problemlöseschritte in einem zweiphasigen Entscheidungszyklus ermöglicht [DECISION]:695

In der Elaborationsphase „feuern“ alle anwendbaren Produktionen des Problemraumes parallel, bis das System zum Stillstand kommt [QUIESCENCE]. Stillstand ist dann erreicht, wenn jedwedes Wissen, das mit der aktuellen Entscheidung in Zusammenhang steht, aus dem Langzeitgedächtnis ins Arbeitsgedächtnis abgerufen wurde.

Nachdem der Stillstand des Systems erreicht wurde, wird in der Entscheidungsphase unter den in das Arbeitsgedächtnis abgerufenen Handlungen diejenige ausgewählt, welcher die höchste Präferenz zugeordnet ist. Dazu müssen die Präferenzen, die den kognitiven Handlungen bei ihrem Erwerb zugeordnet wurden, ${ }^{696}$ ausgewertet werden.

692 Newell, A.; Rosenbloom, P.S.; Laird, J.E.,(Architectures 1991), S. 111.

693 Vgl. Rosenbloom, P.S.; Laird, J.E.; Newell, A.; McCarl, R.,(Analysis 1991), S. 293 ff. Die Autoren führen das folgende Beispiel für ein Objekt an: "For example, an object representing a green cat named Fred might look like (object o025 $\wedge^{\wedge}$ name fred ${ }^{\wedge}$ type cat ${ }^{\wedge}$ color green).” Dies., (Analysis 1991), S. 294. Dabei stellt das Symbol o025 für das Objekt das Identifikationssymbol dar, so lange es im Arbeitsgedächtnis vorhanden ist. Vgl. dies., (Analysis 1991), S. 294.

694 Vgl. Newell, A.; Rosenbloom, P.S.; Laird, J.E.,(Architectures 1991), S. 111.

695 Vgl. Rosenbloom, P.S.; Laird, J.E.; Newell, A.; McCarl, R.,(Analysis 1991), S. 295 f.

696 Die Präferenzstruktur stellt eine spezielle Ausprägung des Arbeitsgedächtnisses dar, die dazu dient, die Akzeptanz und Erwünschtheit von Handlungen durch die Anwendung von 
EBENE 3

ZIELE

Die Zielebene basiert wiederum auf der Entscheidungsebene. So ist zur Generierung von Entscheidungen ein zielgerichtetes Verhalten zwingend erforderlich. Ziele werden dabei immer dann gesetzt, wenn eine Entscheidung, aufgrund eines Entscheidungskonfliktes, nicht getroffen werden kann und demgemäß aktuell keine Handlung zur Erreichung des Zielzustandes identifiziert werden kann. Abhilfe schafft hier die von SOAR selbständig durchgeführte Zerlegung des Problems in Subziele. ${ }^{697}$ Newell unterscheidet hier vier Konfliktsituationen, die Anlaß zur Subzielbildung geben: 698

1. tie impasse: eine Menge von Operatoren wird mit der gleichen Präferenz belegt.

2. no-change impasse: eine Auswertung aller Präferenzen führt zu dem Ergebnis, daß kein Operator akzeptabel ist.

3. reject impasse: die einzige verbleibende Präferenz weist eine vorher getroffene Entscheidung zurück.

4. conflict impasse: ein Operator wird durch eine Präferenzbildung besser als ein zweiter eingestuft, durch eine andere Präferenzbildung erfolgt eine umgekehrte Einschätzung. Dadurch stehen die beiden Operatoren in einem Konflikt zueinander.

\subsubsection{Wissenserwerb durch den CHUNKING-Mechanismus}

In SOAR besteht nun die Möglichkeit, auf der Basis zielorientierten Problemlösens kontinuierlich neue Produktionen zu entwickeln [CHUNKING]. ${ }^{69}$ Dazu verfügt SOAR über einen Mechanismus, den sogenannten Chunking-Mechanismus, der in der Psychologie bereits vielfältig diskutiert wurde 700 und ein Lernschema zur au-

Kontrollwissen zu überprüfen. Vgl. Rosenbloom, P.S.; Laird, J.E.; Newell, A.; McCarl, R., (Analysis 1991), S. 294. 
tomatischen Organisation und zum automatischen Abruf von Erfahrungen darstellt. ${ }^{701}$

Die zentrale Stellung des Chunking-Mechanismus ${ }^{702}$ rührt in SOAR von der Fähigkeit her, für jede Form von Problemlöseverhalten automatisch Ziele zu generieren. ${ }^{703}$ So nehmen Entscheidungskonflikte in SOAR eine zentrale Stellung ein und können durch Subzielbildung sowie Hierarchisierung der Ziele einer Lösung zugeführt werden. ${ }^{704}$ Liegt eine impasse-Situation vor, ist also das verfügbare Wissen unvollständig und kann somit der nächste Schritt der Problemlösung nicht ausgeführt werden, so wird ein Subziel gebildet. Dieses Subziel wird entwickelt, um das erforderliche Wissen zu finden. Durch den Chunking-Mechanismus wird dieses Wissen erworben und steht dann in Zukunft in der gleichen Situation sofort zur Verfügung. SOAR lernt damit die impasse-Situation in Zukunft zu vermeiden, wenn die gleiche Problemstellung vorliegt. Dies geschieht, indem eine neue Produktion, ein Chunk, gebildet wird, der die Problemlösung zusammenfaßt. Dieser Chunk enthält einen mit der Problemlösung verbundenen Aktions- und Bedingungsteil. Die Ergebnisse der Problemlösung sind im Aktionsteil festgehalten, im Bedingungsteil befinden sich diejenigen Elemente des Arbeitsgedächtnisses, die vor Subzielsetzung vorhanden waren und in die Problemlösung eingingen. ${ }^{705}$

Durch direkt verfügbares Wissen ersetzt Chunking somit Suchprozesse und erhöht so die Geschwindigkeit kognitiver Leistungen. Weiterhin findet mit dem Chunking implizit eine Generalisierung statt, da nur die vor der Subzielsetzung im Arbeitsgedächtnis vorhandenen Bedingungen in der neuen Produktion enthalten sind, die auch in die Ergebnisfindung eingingen - alle anderen Arbeitsgedächtnisinhalte sind irrelevant. ${ }^{706}$

\footnotetext{
701 Vgl. Laird, J.E.; Newell, A.; Rosenbloom, P.S.,(SOAR 1987), S. 35.

702 Vgl. bspw. Newell, A., (Theories 1994), S. 185 ff.; Rosenbloom, P.S.; Laird, J.E.; Newell, A.; McCarl, R., (Analysis 1991), S. 298 f.; Laird, J.E.; Newell, A.; Rosenbloom, P.S., (SOAR 1987), S. 35 ff.

703 Vgl. Laird, J.E.; Newell, A.; Rosenbloom, P.S.,(SOAR 1987), S. 35 f.

704 Vgl. Abschnitt 5.5.2.1: Die Architektur von SOAR.

705 Vgl. Laird, J.E.; Newell, A.; Rosenbloom, P.S.,(SOAR 1987), S. 36 ff. Laird, Newell \& Rosenbloom führen ein Beispiel zur Chunk-Entwicklung aus. Vgl. dies., (SOAR 1987), S. $39 \mathrm{ff}$.

706 Vgl. Laird, J.E.; Newell, A.; Rosenbloom, P.S.,(SOAR 1987), S. 35 ff.
} 
Die ACT $^{*}$-Theorie von Anderson, ${ }^{707}$ als weiteres Beispiel für ein regelbasiertes System, gilt schließlich als die derzeit umfassendste Theorie der Kognition ${ }^{708}$. Bereits mehrfach wurde sie, in der englisch- aber auch deutschsprachigen Litera-

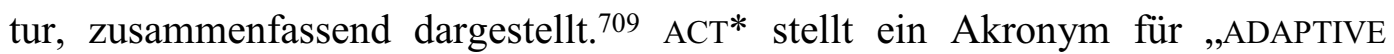
CONTROL OF THOUGHT“ dar. Der Stern weist dabei darauf hin, daß es sich um die bis zu diesem Zeitpunkt elaborierteste Ausarbeitung handelt, ${ }^{710}$ da Anderson die ACT*-Theorie in einem zeitlich ausgedehnten Prozeß entwickelte. Alle Ansätze galten jedoch dem Versuch, eine Theorie über die Ursprünge menschlichen Wissens $\mathrm{zu}$ entwickeln ${ }^{711}$ und wurden im Hinblick auf zwei Gesichtspunkte entwickelt. Zum einen hinsichtlich der Entwicklung einer kognitionspsychologischen Theorie, zum anderen als Computersimulationsprogramm.

707 Vgl. Anderson, J.R., (Architecture 1983).

708 Vgl. Newell, A., (Theories 1994), S. 26.

709 Vgl. bspw. Hesse, F.W., (Review 1985), S. 231-237; Mandl, H.; Friedrich, H.F.; Hron, A., (Psychologie 1986), S. 178-186; Spies, K.; Hesse, F.W., (Interaktion 1986), S. 82-83; Tack, W.H., (Ziele 1987), S. 153-157; Mandl, H.; Friedrich, H.F.; Hron, A., (Ansätze 1988), S. 137-145; Newell, A.; Rosenbloom, P.S.; Laird, J.E., (Architectures 1991), S. 93-131; Newell, A., (Theories 1994), S. 26-29 u.a.; Strohschneider, S., (Wissenserwerb 1990), S. 34-40; Wender, K. F., (Methoden 1992), S. 587-588. Zu Zusammenfassungen der $\mathrm{ACT}^{*}$-Theorie im Hinblick auf die Untersuchung betriebswirtschaftlicher Fragestellungen vgl. bspw. Hartung, S., (Förderung 2000), S. 18 f.; Orth, C., (Unternehmensplanspiele 1999), S. $41 \mathrm{ff}$.

710 Vgl. Anderson, J.R., (Architecture 1983), S. VIII f. sowie S. 17 ff.

711 Vgl. Anderson, J.R., (Theory 1989), S. 313. In chronologischer Reihenfolge legte er zlnächst, in Zusammenarbeit mit Bower, eine Theorie über deklaratives Wissen vor, die sich HAM [HUMAN ASSOCIATIVE MEMORY] nannte. Vgl. Anderson, J.R.; Bower, G.H., (Human 1973). HAM wurde 1976 durch die elaboriertere ACT-Theorie abgelöst. Sie beinhaltet zusätzlich zu der deklarativen Komponente eine prozedurale Komponente in der Form eines Produktionssystems. Vgl. Anderson, J.R., (Language 1976). 1983 resultierte die ACTTheorie in der $\mathrm{ACT}^{*}$-Theorie, welche außerdem eine elaborierte Theorie über den Erwerb von Produktionsregeln beinhaltet. Vgl. Anderson, J.R., (Architecture 1983). Die neuesten Überlegungen liegen schließlich in Form von PUPS, ein Akronym für PenUltimate Production System, vor. Diese Theorie versucht, Defizite der ACT*-Theory bezüglich der Operatordiskriminierung, die Lewis \& Anderson an Hand von drei Experimenten feststellen konnten, zu beseitigen. Gewissermaßen existiert sie jedoch nicht richtig, da an der Entwicklung dieses Produktionssystems noch gearbeitet wird. Vgl. Anderson, J.R.; Thompson, R., (Analogy 1989), S. 268 ff. Zu den Experimenten vonLewis \& Anderson bezüglich der Operatordiskriminierung vgl. Lewis, M.W.; Anderson, J.R., (Discrimination 1985), S. $34 \mathrm{ff}$. 


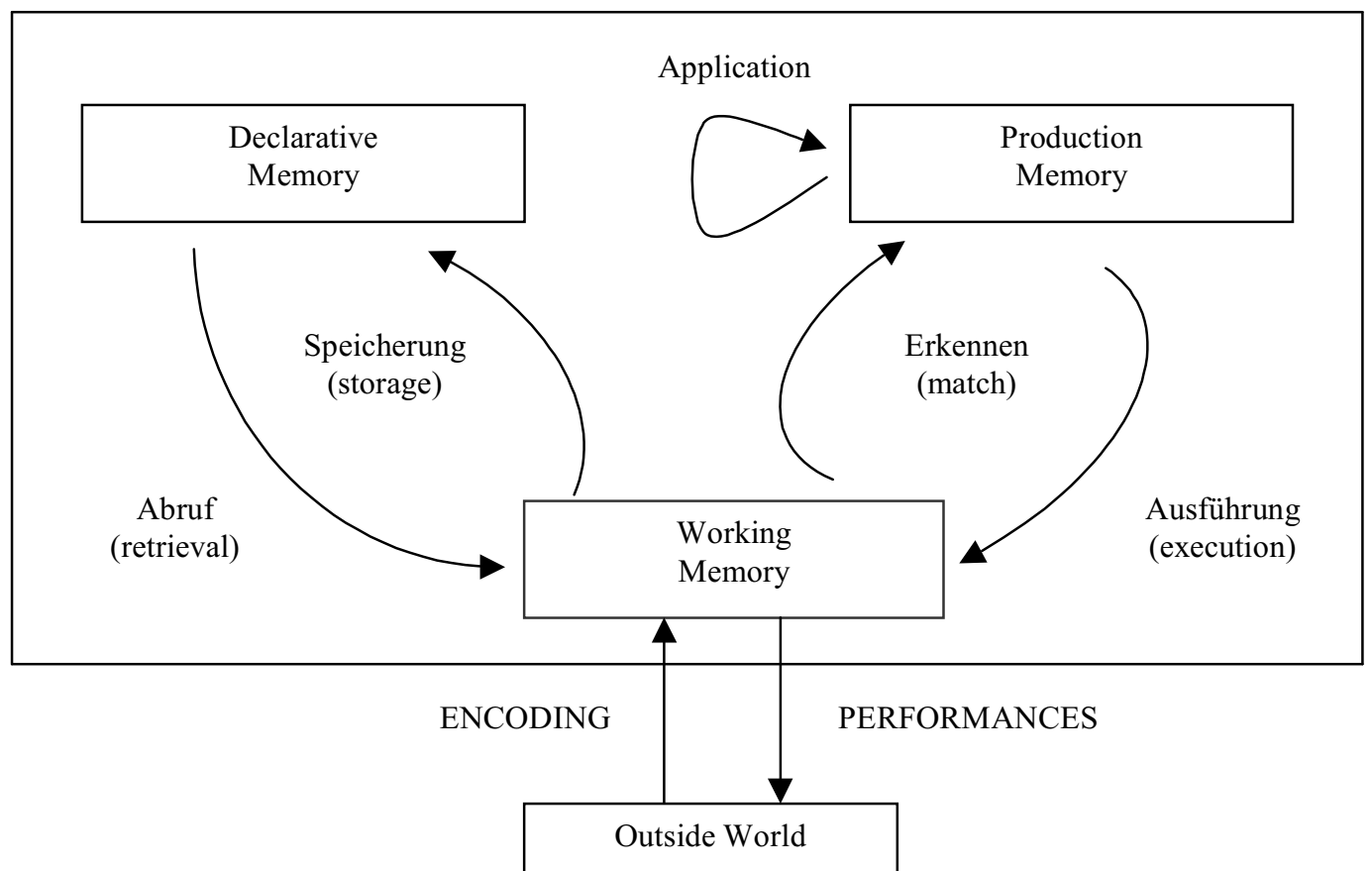

\section{Abbildung 5-2: Ein allgemeiner Rahmen für das ACT- Produktionssystem ${ }^{712}$}

Zunächst basiert $\mathrm{ACT}^{*}$ auf einem generellen Rahmen. In diesem Rahmen konstituiert Anderson eine Dreiteilung des Gedächtnisses in ein deklaratives und prozedurales Gedächtnis sowie ein Arbeitsgedächtnis. Deklaratives und prozedurales Gedächtnis [DECLARATIVE MEMORY AND PRODUCTION MEMORY] werden insgesamt als Langzeitgedächtnis bezeichnet, welches der Speicherung von Langzeitwissen dient, während im Arbeitsgedächtnis [WORKING MEMORY] temporär gerade verfügbares, aktives Wissen gespeichert ist. ${ }^{713}$ [Vgl. Abbildung 5-2].

Für deklaratives und prozedurales Wissen postuliert Anderson zwei unterschiedliche Repräsentationsformate. So ist das deklarative Wissen zunächst in Form eines semantischen Netzes gespeichert, welches wiederum drei Repräsentationsformate aufweist, die dort verknüpft werden. Dies sind zum einen Propositionen [ABSTRACT PROPOSITIONS], zum anderen zeitliche Folgen [TEMPORAL STRINGS] und schließlich räumliche Anordnungen oder Vorstellungsbilder [SPATIAL IMAGES]. Das prozedurale Wissen, oder auch Handlungswissen, wird hingegen in Form von Produktionssystemen repräsentiert. Bei den dort enthaltenen Produktionen handelt 
es sich um Wenn-Dann-Regeln, die aus einer Bedingungs- und einer HandlungsKomponente aufgebaut sind..$^{714}$

Nach Tack wird $\mathrm{ACT}^{*}$ jedoch vor allem durch drei weitere Komponenten zu einem konzeptuellen Modell kognitiver Leistungen. So wird durch diese drei Komponenten aus einem allgemeinen Modell der Wissensrepräsentation insofern ein psychologisches Modell, als dadurch vielfältige reale Phänomene erklärbar werden. Die erste Komponente, die dies gestattet, ist die Theorie der Aktivationsausbreitung sowie der Stärke deklarativer Einheiten; zum zweiten eine Menge von Annahmen über Prozeduralisierung und Stärke von Produktionen; drittens letztlich die Einführung von Zielen mit einer impliziten Zielhierarchie. ${ }^{715}$ Diese drei Komponenten sollen im folgenden erläutert werden:

DIE THEORIE DER AKTIVATIONSAUSBREITUNG UND DER STÄRKE DEKLARATIVER EINHEITEN

Jeder Knoten im deklarativen Gedächtnis weist eine bestimmte Stärke auf. Sie ist Ausdruck der Häufigkeit, mit der diese kognitive Einheit bereits aktiviert wurde. Abhängig von dieser Knotenstärke ist das Ausmaß an Aktivierung im deklarativen Gedächtnis und damit die Wahrscheinlichkeit, daß ein Element im Arbeitsgedächtnis zur Verfügung steht. Durch das Prinzip der sich ausbreitenden Aktivierung regen Arbeitsgedächtnis-Inhalte weitere Elemente des deklarativen Gedächtnisses an. Umgekehrt sind die temporären Arbeitsgedächtnis-Strukturen langfristig als Einheiten speicherbar. ${ }^{716}$

\section{DIE ANNAHMEN ÜBER PROZEDURALISIERUNG UND STÄRKE VON PRODUKTIONEN}

Durch Lernprozesse kann die Menge der Produktionen im Produktionengedächtnis erweitert werden. Dies nennt Anderson Produktionen-Anwendung, da die Anwendung der Produktionen die Voraussetzung für den Erwerb neuer Produktionen darstellt. Die Stärke einer Produktion und der Aktivierungsgrad der Arbeitsgedächtnis-Elemente bestimmen, wie schnell Produktionen-Bedingungen geprüft

\footnotetext{
714 Vgl. Anderson, J.R., (Architecture 1983), S. 10.

715 Vgl. Tack, W.H., (Ziele 1987), S. 154.

716 Vgl. Anderson, J.R., (Architecture 1983), S. 26 ff.
} 
werden und ihr Aktionsteil zur Ausführung gelangt. ${ }^{717}$ Dabei ist es möglich, daß für mehrere Produktionen gleichzeitig die Bedingungen erfüllt sind. Dadurch wird ein Konflikt hervorgerufen, dessen Lösung die Entscheidung darüber beinhaltet, welche Produktion zur Anwendung gelangt. Tritt ein solcher Konflikt auf, so existieren fünf Prinzipien zur Konfliktlösung:718

1.) Grad der Übereinstimmung [DEgREE OF MATCH]: Damit der Aktionsteil der Produktionen ausgeführt werden kann, muß ihr Bedingungsteil nicht unbedingt vollständig erfüllt sein. Bevorzugt zur Anwendung gelangt jedoch die Produktion, die einen höheren Übereinstimmungsgrad aufweist.

2.) Produktionsstärke [PRODUCTION STRENGTH]: Die Stärke der Produktion reflektiert die Häufigkeit erfolgreicher Anwendung in der Vergangenheit. Stärkere Produktionen werden schneller und zuverlässiger angewendet.

3.) Refraktärprinzip [DATA REFRACTORINESS]: Unter Nutzung derselben Daten kann eine Produktion nur einmalig ausgeführt werden.

4.) Spezifität [SPECIFICITY]: Wenn die Bedingungen von zwei Produktionen erfüllt sind, wird die spezifischere bevorzugt. Dabei existieren zwei Möglichkeiten, wann eine Produktion spezifischer ist als eine andere: Zum einen kann die eine Produktion Teilmenge der anderen sein und die allgemeingültigere Produktion durch ein konkretes Beispiel spezifizieren. Zum anderen enthält eine spezifischere Produktion zusätzliche Annahmen in ihrem Bedingungsteil.

5.) Zieldominanz [GOAL-DOMINANCE]: Die Zieldominanz bildet das Primärprinzip in der Konfliktlösung. Im System kann nur ein aktuelles Ziel verfolgt werden. Wenn ein aktuelles Ziel erreicht wurde, konzentriert sich die Aufmerksamkeit auf das nächste Ziel im Zielkatalog. Es gelangt die Produktion zur Anwendung, deren Ziel-Spezifikation zu dem aktuellen Ziel paßt. Paßt die ZielSpezifikation mehrerer Produktionen zu dem aktuellen Ziel, so gelangen die

\footnotetext{
717 Vgl. Anderson, J.R., (Architecture 1983), S. 34.
}

718 Vgl. Anderson, J.R., (Architecture 1983), S. 132 ff. 
Prinzipien STRENGHT, DEGREE OF MATCH \& SPECIFICITY zur Anwendung, um diejenige Produktion auszuwählen, die zur Anwendung gelangt.

\section{DIE EINFÜHRUNG VON ZIELEN MIT EINER IMPLIZITEN ZIELHIERARCHIE.}

Die Produktionen in $\mathrm{ACT}^{*}$ können eine hierarchische Zielstruktur entwerfen und transformieren, welche den aktuellen Aktionsplan widerspiegelt. Dies vereinfacht sowohl die Problemlöseprozesse, als auch den Erwerb von Produktionen wesentlich. Es ist zum einen möglich, ein Ziel durch Aufmerksamkeitsfokussierung zu aktivieren, zum anderen ist es möglich, den Aufmerksamkeitsfokus innerhalb einer Zielstruktur zu variieren. So kann zum Beispiel die Aufmerksamkeit im Hinblick auf ein Ziel, nachdem es erreicht wurde, zugunsten des nächsten aufgegeben werden. Dadurch gelingt es, ein Problem sukzessive einer Lösung zuzuführen. ${ }^{719}$

\subsubsection{Prozeß des Wissenserwerbs in ACT*}

Komplexe Lernmechanismen in $\mathrm{ACT}^{*}$ ermöglichen es, aus bereits vorliegenden Produktionen neue und effektivere Regeln für verschiedene Anwendungsbereiche zu generieren. In einem Produktionssystem wird der Prozeß des Wissenserwerbs im allgemeinen nicht als verbales Lernen, sondern als der Erwerb von Fähigkeiten angenommen. Dies tut auch Anderson und unterscheidet in diesem Wissens- oder damit auch Fähigkeitserwerb drei Phasen:720

Vgl. Anderson, J.R., (Architecture 1983), S. 33 f.

720 Vgl. z.B. Anderson, J.R., (Architecture 1983), S. 216 ff.; Mandl, H.; Friedrich, H.F.; Hron, A., (Ansätze 1988), S. 137 ff. Anderson's Theorie des Wissenserwerbs geht auf die von Fitts unterschiedenen Stadien des Fertigkeitserwerbs zurück. So bezieht Anderson seine Theorie bereits 1982 auf die Ausführungen von Fitts und beschreibt ACT als korrespondierend mit den von Fitts beschriebenen drei Stadien des Fähigkeitserwerbs. Das erste Stadium wird als das kognitive, das zweite als das assoziative und das dritte schließlich als das autonome Stadium bezeichnet: Das kognitive Stadium beinhaltet ein anfängliches enkodieren der Fertigkeit in eine ausreichende Form, die es dem Lerner gestattet, das gewünschte Verhalten zu einer letztlich unfertigen Annäherung auszubauen. In diesem Stadium ist es allgemein notwendig, auf die verbale Vermittlung zu achten, in welcher der Lerner benötigte Informationen für die Ausführung der Fertigkeit wiederholt. Das assoziative Stadium beinhaltet das Training der Fertigkeitsausführung. Fehler im anfänglichen Verständnis der Fertigkeit werden allmählich aufgedeckt und eliminiert. Die verbale Vermittlung wird zunehmend vernachlässigt. Das autonome Stadium ist jene stufenweise fortschreitende Vervollkommnung des Fähigkeitserwerbs. Die Vervollkommnung auf diesem Stadium setzt sich oft unbeschränkt fort. Obgleich diese allgemeinen Beobachtungen bezüglich des Weges der Fertigkeitsentwicklung auf eine breite Menge von Fertigkeiten zuzutreffen scheinen, bedürfen sie einer systematischen theoretischen Analyse. Vgl. Anderson, J.R., (Acquisition 1982), S. 369 und die dort angegebene Literatur. 


\section{Deklarative Phase}

In der ersten Phase des Wissenserwerbs ist der deklarative Teil des Langzeitgedächtnisses von zentraler Bedeutung. Informationen werden deklarativ enkodiert, in den deklarativen Teil des Langzeitgedächtnisses überführt und dort in Form eines semantischen Netzwerkes oder Schemas gespeichert. Aus dem Produktionengedächtnis erfolgt nun ein Abruf allgemeiner Prozeduren, die sich nicht auf den neuen Wissensbereich beziehen, mit deren Hilfe jedoch eine Interpretation des neuen Wissens erfolgt. 721

\section{Phase der Wissenskompilierung}

Werden allgemeine Problemlöseprozeduren auf neue Probleme angewandt, so erlaubt dies einen flexiblen Umgang, ist jedoch kognitiv sehr aufwendig und langsam. Daher werden in der zweiten Phase des Wissenserwerbs spezielle Prozeduren durch das lernende System konstruiert, die in direktem Zusammenhang mit dem neuen Problem stehen. Dieser Prozeß wird von Anderson als Wissenskompilierung bezeichnet, und er erläutert zwei Mechanismen, auf welchen dieser Prozeß basiert. Das ist zum einen der Mechanismus der Prozeduralisierung, welcher der Ausbildung spezifischer Produktionen dient. Auf Grundlage allgemeiner Prozeduren werden hier spezielle Prozeduren erzeugt. Der Aktionsteil allgemeiner Produktionen wird hier so mit dem neuen deklarativen Wissen verknüpft, daß eine neue Produktion entwickelt wird, die das neu erworbene deklarative Wissen als Bedingungsteil enthält. So wird deklaratives Wissen in prozedurales überführt. Zum anderen nennt Anderson den Mechanismus der Kombination. Hier werden sogenannte Makroproduktionen erzeugt, die eine Sequenz von Produktionsanwendungen in einem Schritt erledigen. ${ }^{722}$

\section{Phase der Wissensoptimierung, des ,Tuning“6}

Durch die Phase der Wissenskompilierung liegt nun prozeduralisiertes Wissen vor. Der Prozeß des Fertigkeitserwerbs wird jedoch in dieser Phase noch weiter fortgeführt. Aufgabe der Wissensoptimierung ist es insbesondere, die Suche nach den Produktionen, die für das aktuelle Ziel relevant sind, zu erleichtern. Dazu ist 
eine Feinabstimmung der Produktionen notwendig. Sie erfolgt unter Anwendung dreier Prozesse: GENERALISATION, DISKRIMINATION UND VERSTÄRKUNG.

Durch GENERALISATION werden die identischen Merkmale spezifischer Produktionen zu einer neuen Produktion kombiniert. Diese sind dann wiederum auf neue deklarative Strukturen anwendbar, weil sie durch den Prozeß der GENERALISATION allgemeingültiger geworden sind.

Durch DISKRIMINATION erfolgt ein gegenläufiger Prozeß. Sehr allgemeine Prozeduren werden dahingehend verändert, daß sie eine spezifische Gültigkeit erlangen.

Durch GENERALISATION und DISKRIMINATION wird der Inhalt des Produktionengedächtnisses verändert. Der Mechanismus der VERSTÄRKUNG bewirkt dagegen lediglich Veränderung des Stärkewertes, der jeder Produktion zugeordnet ist. Dies geschieht aufgrund erfolgreicher oder erfolgloser, Anwendung. Dies führt zu einer Erhöhung oder Senkung der Auswahlwahrscheinlichkeit der vorliegenden Produktion. ${ }^{723}$

\subsection{Diskussion und Gegenüberstellung der Produktionssysteme}

Nach Strohschneider unterscheiden sich Theorien zur Erklärung von Wissenserwerbsprozessen bezüglich ihres Geltungsanspruchs, bezüglich der Formen des Wissenserwerbs, auf die sie sich beziehen, und in Hinsicht auf die Erklärungsoder Beschreibungsebene. ${ }^{724}$ Als verschiedene Formen des Wissenserwerbs nennt er den Erwerb sensumotorischer Koordination, das Lernen von Regeln und von Begriffen sowie den Erwerb strategischen Wissens. ${ }^{725}$

\footnotetext{
723 Vgl. Anderson, J.R., (Architecture 1983), S. 241 ff.

724 Bezüglich des Problems der Erklärungsebene liegen mehrere Systematisierungsvorschläge vor. Diese wurden auch bereits gegenübergestellt. Vgl. dazu Anderson, J.R., (Character 1990).

Vgl. Strohschneider, S., (Wissenserwerb 1990), S. 15 ff.
} 
Newell, Rosenbloom \& Laird stellen einen Vergleich der Architekturen von SOAR und ACT im Hinblick auf die Aspekte Gedächtnis, Symbole, Operationen, Interpretation sowie Interaktion mit der Umwelt an. ${ }^{726}$ Die folgenden Ausführungen lehnen sich, unter Berücksichtigung der Aspekte Gedächtnis und Operationen, an diese Vorgehensweise an - und integrieren weiterhin in den betrachteten Punkten die relevanten Aspekte in bezug auf Dörner's Theorie der Absichtsbehandlung. Das Gedächtnis analysieren die Autoren unter dem folgenden Gesichtspunkt: „Memory is to be identified by asking what persits over time that can be created and modified by the system. “727

Zunächst verfügen sowohl SOAR als auch $\mathrm{ACT}^{*}$ über Gedächtnishierarchien, die ein Arbeitsgedächtnis, zur kurzzeitigen Speicherung von Informationen, und ein Langzeitgedächtnis aufweisen. Der Kurzzeitcharakter des Arbeitsgedächtnisses rührt in SOAR von der Verknüpfung mit Zielen und deren Problemräumen her. Sobald ein Problem gelöst wurde, hat das Arbeitsgedächtnis seine Funktion zunächst erfüllt. In $\mathrm{ACT}^{*}$ hängt dieser Aspekt damit zusammen, daß das Arbeitsgedächtnis ein aktiviertes Set deklarativen Wissens darstellt, welches in Zusammenhang mit der Aufmerksamkeitsfokussierung steht. Damit erfolgt ein Abruf von deklarativem Wissen in das Arbeitsgedächtnis nur bei Aufmerksamkeitsfokussierung. Beide Architekturen verfügen weiterhin über ein Langzeitgedächtnis. Während jedoch $\mathrm{ACT}^{*}$ über zwei Langzeitgedächtnisse verfügt, das deklarative und das prozedurale Gedächtnis, verfügt SOAR nur über ein Produktionengedächtnis, welches jedoch für beide Wissensarten, deklaratives und prozedurales Wissen, genutzt werden kann. ${ }^{728}$

726 Vgl. Newell, A.; Rosenbloom, P.S.; Laird, J.E.,(Architectures 1991), S. 109 ff. Auch bei Rosenbloom, Laird, Newell \& Mc Carl findet sich eine umfangreiche Gegenüberstellung von SOAR und ACT*. Vgl. Rosenbloom, P.S.; Laird, J.E.; Newell, A.; McCarl, R., (Analysis 1991), S. 289-32

727 Vgl. Newell, A.; Rosenbloom, P.S.; Laird, J.E.,(Architectures 1991), S. 109 ff.

728 Vgl. Newell, A.; Rosenbloom, P.S.; Laird, J.E.,(Architectures 1991), S. 111 ff. 


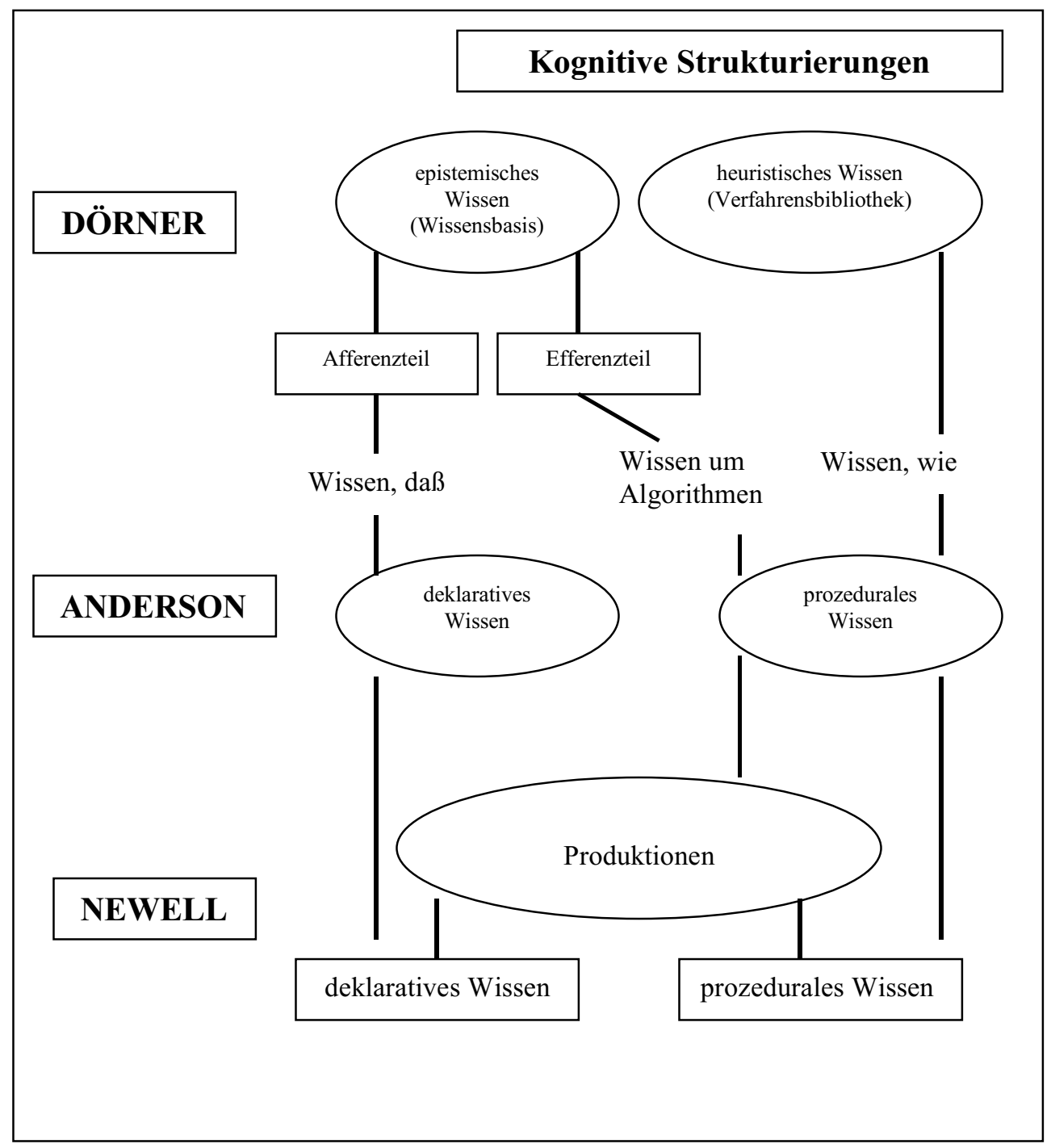

Abbildung 5-3: $\quad$ Kognitive Strukturierungen nach DÖRNER, NEWELL \& ANDERSON729

In bezug auf die betrachteten Ansätze führt weiterhin Paar aus, daß es von besonderer Bedeutung ist, daß sich die Ansätze in ihrer Annahme über die Konzeption deklarativen und prozeduralen Wissens ${ }^{730}$ wie folgt unterscheiden: ${ }^{731}$ [Vgl. Abbildung 5-3].

In Anlehnung an Orth, C., (Unternehmensplanspiele 1999), S. 41 in Anlehnung an Sembill, D., (Problemlösefähigkeit 1992), S. 86.

730 Die Unterscheidung von deklarativem und prozeduralem Wissen ist in der Kognitionspsychologie allgemein anerkannt. Vgl. Oswald, M.; Gadenne, V., (Wissen 1984), S. 178 ff. 
So unterscheidet zunächst Dörner in seiner Theorie der Absichtsregulation zwischen heuristischer und epistemischer Struktur. In der epistemischen Struktur ist das epistemische Wissen repräsentiert, welches das Wissen eines Individuums über einen Realitätsbereich darstellt und auch als Wissensbasis bezeichnet wird. Dieses Wissen wird weiter nach einem Afferenz- und einem Efferenzteil unterschieden, wobei der Afferenzteil das Gedächtnis für Sachverhalte, der Efferenzteil das Gedächtnis für Handlungen darstellt. In der heuristischen Struktur ist das heuristische Wissen, welches auch als Verfahrensbibliothek bezeichnet wird, enthalten. Es wird in einer Reihe elementarer kognitiver Prozesse repräsentiert und dient dem Umgang mit dem epistemischen Wissen. In seiner Theorie ist jegliches Wissen in Form von Aktionsschemata repräsentiert, so daß eine einheitliche Wissensrepräsentation vorgeschlagen wird. ${ }^{732}$

Im Langzeitgedächtnis von SOAR sind hingegen eine Reihe kognitiver Produktionen gespeichert, die jedoch sowohl deklaratives, prozedurales als auch episodisches Wissen beinhalten können. Das deklarative Wissen meint dabei Faktenwissen über die Realität sowie außerdem Faktenwissen über Handlungen, die ausgeführt werden können. Das prozedurale Wissen stellt Wissen darüber dar, wie Handlungen vollzogen werden können und umfaßt ebenso Kontrollwissen darüber, welche Handlungen zu welchem Zeitpunkt durchgeführt werden können. Das episodische Wissen stellt schließlich Wissen darüber dar, welche Aktionen zu welchem Zeitpunkt vollzogen wurden. ${ }^{733}$

Auch in $\mathrm{ACT}^{*}$ wird zwischen deklarativem und prozeduralem Wissen unterschieden. Für deklaratives und prozedurales Wissen postuliert Anderson zwei unterschiedliche Repräsentationsformate. So ist das deklarative Wissen zunächst in Form eines semantischen Netzes gespeichert, welches wiederum drei Repräsentationsformate aufweist, die dort verknüpft werden. Dies sind zum einen Propositionen [ABSTRACT PROPOSITIONS], zum anderen zeitliche Folgen [TEMPORAL STRINGS] und schließlich räumliche Anordnungen oder Vorstellungsbilder [SPATIAL IMAGES]. Das prozedurale Wissen, oder auch Handlungswissen, wird hingegen in Form von Produktionssystemen repräsentiert. Bei den dort enthalte-

732 Vgl. Abschnitt 5.5.1.1: Elemente der Theorie der Absichtsbehandlung.

733 Vgl. Abschnitt 5.5.2.1: Die Architektur von SOAR. 
nen Produktionen handelt es sich um Wenn-Dann-Regeln, die aus einer Bedingungs- und einer Handlungs- Komponente aufgebaut sind. ${ }^{734}$

Paar gelangt zu dem Ergebnis, daß im Hinblick auf die Modellierung deklarativen und prozeduralen Wissens alle drei Lösungen akzeptabel erscheinen, da sie die funktionalen Eigenschaften dieser Wissensarten berücksichtigen. ${ }^{735}$

Operationen untersuchen Newell, Rosenbloom \& Laird wie folgt: „The operations are to be identified by asking how new structures get built and established in the

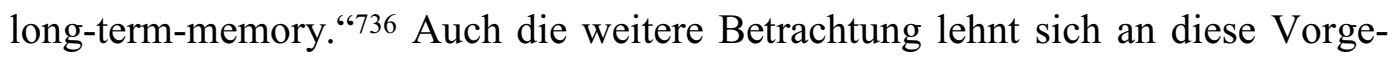
hensweise an.

In $\mathrm{ACT}^{*}$ und SOAR ist das Speichern von Informationen im Langzeitgedächtnis getrennt von der Informationsverarbeitung im Arbeitsgedächtnis. Sie wird als der Aufbau neuer Produktionen verstanden und in SOAR als Chunking, in ACT* als Prozeduralisierung bezeichnet. Dieser Aspekt ist auch Bestandteil Dörner's Theorie und wird als Operatorbildung bezeichnet. ${ }^{737}$

Für eine handelnde Person ist es weiterhin ein Unterschied, ob ein Ziel lediglich vage oder gut definiert vorgegeben ist. ${ }^{738}$ In diesem Zusammenhang ist auch die Berücksichtigung von Zielen bzw. Absichten in den Theorien von Bedeutung. Bezüglich ihrer Verwendung nimmt wiederum Paar einen Vergleich der drei vorgestellten Theorien vor. Sie gelangt zu dem Ergebnis, daß sich die Theorien unter diesem Aspekt auf unterschiedlichen Ebenen einordnen lassen. So ordnet sie ACT* unterhalb der Ebene Dörners ein. Wiederum darunter siedelt sie Newells ZielSubziel-Strukturen an. ${ }^{739} \mathrm{Zu}$ diesem Ergebnis gelangt sie wie folgt:

\footnotetext{
734 Vgl. Abschnitt 5.5.3.1: Architektur von ACT*.

735 Vgl. Paar, I., (Handlungsziel 1993), S. 45.

736 Vgl. Newell, A.; Rosenbloom, P.S.; Laird, J.E.,(Architectures 1991), S. 114.

737 Vgl. Paar, I., (Handlungsziel 1993), S. 46.

738 Vgl. Broadbent, D.E.; Fitzgerald, P.; Broadbent, M.H.P.,(Knowledge 1986); Reichert, U.; Dörner, D., (Heurismen 1988).

739 Vgl. Paar, I., (Handlungsziel 1993), S. 46.
} 
In der Hauptsache geht es Dörner um die Entstehung von Zielen [Absichten]. Weiterhin thematisiert er ihre Veränderung durch die Ergänzung neuer Informationen sowie schließlich auch Unterschiede im Hinblick auf das Verhalten bei der Durchführung einer Absicht, die in Abhängigkeit von Faktoren wie bspw. der Wichtigkeit oder Kompetenz auftreten. Dörner's Kognitive Handlungstheorie wird daher von Paar auf einer sehr hohen Erklärungsebene eingeordnet.

In $\mathrm{ACT}^{*}$ werden hingegen zum Zustandekommen von Zielen oder Zielhierarchien und ihrer zeitlichen Dauer keine Aussagen getroffen. ${ }^{740}$ Die Bedeutung von Zielen wird von Anderson jedoch betont. Dies geschieht zum einen im Hinblick auf die Aufmerksamkeitsfokussierung zur Steuerung von Handlungen sowie weiterhin zur Komposition von Produktionen, die in der prozeduralen Phase des Wissenserwerbs erfolgt. Sie sind daher Bestandteil der Mechanismen seines Systems und erlangen bei der Konfliktlösung eine besondere Bedeutung. So kann mit der Konzeption der hierarchischen und veränderbaren Zielstruktur die Produktions-

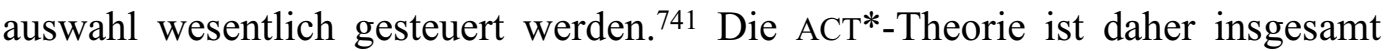
unterhalb der Kognitiven Handlungstheorie von Dörner einzuordnen.

Unterhalb der ACT*-Theorie ordnet Paar schließlich die Ziel-Subziel-Strukturen in der SOAR-Architektur an. Diese Einordnung erfolgt unter dem Aspekt, daß hier die Sub-Ziele in keinerlei Weise mit menschlichen Zielen oder Absichten gleichzusetzen sind.

740 Vgl. Hesse, F.W., (Review 1985), S. 235.

741 Vgl. Paar, I., (Handlungsziel 1993), S. 46. Vgl. auch Anderson, J.R., (Architecture 1983), S. 33 . 
Wird das Unternehmensplanspielmodell nun im Rahmen eines komplexen LehrLern-Arrangements eingesetzt, ${ }^{742}$ so wird es im Spiel zum Gegenstand der vorgesehenen Geschäftsmaßnahmen, da diese vorwiegend zur Simulation von Entscheidungen dienen. So sind die meisten Modelle so konstruiert, daß sie eine systematische Durchführung des Entscheidungsprozesses, seiner Teile, Schritte und Phasen ermöglichen. Die Entscheidungen wirken nun ihrerseits auf das Modell, auf die in ihm gespeicherten Daten und Zusammenhänge ein und verändern das Modell. Durch die mathematische Verknüpfung mit den Modell-Daten werden die Ergebnisse der getroffenen Entscheidungen ausgelöst. ${ }^{743}$

Die Veränderung des Modells wird demgemäß durch das Entscheidungshandeln der Planspielteilnehmer ausgelöst. Dabei muß die Komplexität des Unternehmensplanspiels dem Vorwissen der Teilnehmer angepaßt werden, da der Umgang mit einem Unternehmensplanspiel für diese den Charakter eines Problems aufweist. ${ }^{744}$ Das Entscheidungshandeln im Unternehmensplanspiel stellt demgemäß einen Problemlöseprozeß dar, der durch Anwendung und Erwerb von Wissen vollzogen wird. Dabei wird dieser, ebenso wie die Veränderung des Modells, durch das Entscheidungshandeln vorangetrieben.

In diesem Zusammenhang ist die Qualität des angewandten und erworbenen Wissens wichtig. So weist das Wissen der Planspielteilnehmer unterschiedliche Qualitäten auf und ist auf unterschiedliche Arten repräsentiert. Dabei haben die Ausführungen in Kapitel fünf sowohl einen differenzierten Einblick darüber gewährt, welche Qualitäten Wissen haben kann als auch darüber, wie es repräsentiert sein kann. ${ }^{745}$ In bezug auf die Planspielteilnehmer ist für die weiteren Betrachtungen davon auszugehen, daß die unterschiedlichen Wissensarten in Form eines hier hypothetisch angenommenen mentalen Modells über das Unternehmensplan-

\footnotetext{
742 Vgl. Abschnitt 5.1: Das Unternehmensplanspiel: Ein komplexes Lehr-Lern-Arrangement.

743 Vgl. Abschnitt 2.1.2.2: Das Unternehmensplanspiel: Ein Entscheidungsspiel.

744 Vgl. Abschnitt 2.2.2.3.2: Zum Zusammenhang von Komplexität und Lernerfolg.

745 Vgl. Kapitel 5: Das Unternehmensplanspiel als Lehr-Lern-Instrument.
} 
spielmodell repräsentiert sind, ${ }^{746}$ welches sich, ebenso wie das Planspielmodell, im Zeitablauf verändert.

Demgemäß stellt der Problemlöseprozeß einen Lernprozeß dar, der durch Entscheidungen vorangetrieben wird. Dies impliziert, daß der Umgang mit der Komplexität eines Unternehmensplanspielmodells für den Planspielteilnehmer ein »Management von Komplexität« darstellt, welches über Entscheidungen erfolgt. Damit stellt die Komplexität des Unternehmensplanspielmodells für den Planspielteilnehmer, der an einer Ausbildungsmaßnahme partizipiert, eine Komplexität der Entscheidung dar; weshalb an dieser Stelle eine Analyse der Entscheidungshandlung im Unternehmensplanspiel vorgenommen werden soll.

Hier ist in Zusammenhang mit der Entscheidungsorientierung der Betriebswirtschaftslehre zunächst auf die Entscheidungstheorie einzugehen, welche sich nach normativer und deskriptiver Entscheidungstheorie unterscheiden läßt.

6.1

Der Entscheidungsansatz in der Betriebswirtschaftslehre

\subsection{1}

\section{Der Gegenstand der normativen und deskriptiven Entschei- dungstheorie}

Grundsätzlich kann die Entscheidungstheorie zwei unterschiedlichen Fragestellungen nachgehen, die von der wissenschaftlichen Zielsetzung abhängen. ${ }^{747}$ Nach Luhmann ist eine solche Unterteilung für Wissenschaften, deren Gegenstand das menschliche Handeln betrifft, üblich. Sie werden in deskriptive oder empirischerklärende und präskriptive oder rationale Disziplinen eingeteilt. Dies war bis ins 19. Jahrhundert nicht der Fall. Die Wissenschaften, die das menschliche Handeln betreffen, waren bis dahin in ihrer Grundkonzeption einheitlich und gingen davon

746 Der Vorteil eines mentalen Modells war darin zu sehen, daß es ein hybrides System dastellt, welches unterschiedliche Repräsentationsformate beinhalten kann. Vgl. Abschnitt 5.4: Mentale Modelle als analoges Repräsentationssystem komplexer Systeme.

747 Vgl. bspw. Meyer, R., (Entscheidungstheorie 2000), S. 2 f. und Sieben, G.; Schildbach, T., (Entscheidungstheorie 1990), S. 1 ff. 
aus, daß das menschliche Handeln der Rationalität ${ }^{748}$ unterliegt. Es wurden zwar Unvollkommenheiten zugestanden, sie wurden jedoch als nicht entscheidend für das Wesen des Handelns angenommen, da Fehlhandlungen nicht nur als Verfehlung einer Norm angesehen wurden, sondern gleichsam den 'Abfall vom Sein' darstellten. Daher wurde das Wesen des Handelns durch Zwecke und Mittel als rationale Ordnung des Handlungssinnes entwickelt und erklärt, was bedeutete, daß es insofern zugleich Erkenntnisgegenstand war. Da der Zerfall dieser Sichtweise jedoch eine Tatsache darstellt, sind diejenigen Wissenschaften, die sich um die richtige Beschaffenheit des Handelns bemühen, heute von denen zu trennen, welche das Sein, den faktischen Verlauf des Handeln erforschen, ${ }^{749}$ was für die Entscheidungstheorie bedeutet, daß sie sich in der folgenden Weise unterteilt:

\section{NORMATIVE ENTSCHEIDUNGSTHEORIE}

„Ausgehend einerseits von einem vorgegebenen Ziel oder Zielbündel und andererseits von einer gegebenen Entscheidungssituation, die dem Entscheidungsträger mehr als nur eine Handlungsalternative anbietet, steht bei diesem Zweig der Entscheidungstheorie die Frage im Mittelpunkt, wie aus der Menge möglicher Handlungen diejenige herausgefunden werden kann, welche bezogen auf das vorgegebene Ziel oder Zielbündel am vorteilhaftesten ist. Die Suche nach der unter gegebenen Bedingungen zur größten Zielerfüllung führenden Handlung kann als rationale Wahl bezeichnet werden." 750

\section{DESKRIPTIVE ENTSCHEIDUNGSTHEORIE}

„Fußend auf der Überzeugung, eine Realwissenschaft müsse Aussagen (Hypothesen) erarbeiten, die - weil sie etwas über die Realität aussagen - an der Erfahrung

Eine kurze Diskussion verschiedener Definitionen von Rationalität führt Meyer; und kommt zu dem Ergebnis, daß bezüglich einer Definition des Rationalitätsbegriffs keine Einigkeit herrscht. Vgl. Meyer, R., (Entscheidungstheorie 2000), S. 7 ff.

750 Sieben, G.; Schildbach, T., (Entscheidungstheorie 1990), S. 1. 
scheitern können, sieht sie ihre Aufgabe darin, empirisch gehaltvolle Hypothesen über das menschliche Entscheidungs- und Problemlösungsverhalten zu finden. Nicht die Rationalität als Norm, sondern das tatsächliche Problemlösungsverhalten eines einzelnen Menschen oder einer Gruppe von Menschen in einer Organisation steht im Mittelpunkt.“"751

Diese beiden Richtungen der Entscheidungstheorie besitzen auch Relevanz für die Betriebswirtschaftslehre, worauf im folgenden eingegangen werden soll.

\subsubsection{Zur Relevanz von normativer und deskriptiver Entscheidung- stheorie in der Betriebswirtschaftslehre}

In den 60er Jahren ist die Entwicklung der Betriebswirtschaftslehre von einer Vielzahl verschiedener Ansätze geprägt. ${ }^{752}$ Schanz spricht in diesem Zusammenhang vom Pluralismus der Betriebswirtschaftslehre. ${ }^{753} \mathrm{Zu}$ diesen Ansätzen zählt, neben der Systemtheorie, ${ }^{754}$ auch die Entscheidungstheorie. Dabei nahm das Interesse an der Entscheidungstheorie zu, was sich dadurch bedingte, daß die unternehmerischen Entscheidungen immer stärker in den Mittelpunkt rückten, da sie den Ablauf der Unternehmungstätigkeit wesentlich bestimmen. ${ }^{755}$ Die Entscheidungsorientierung der Betriebswirtschaftslehre manifestiert sich in diesem $\mathrm{Zu}$ sammenhang in dem Versuch, eine Darstellung betriebswirtschaftlicher Probleme als Entscheidungsprobleme zu ermöglichen und diese einer Lösung zuzuführen, ${ }^{756}$ wobei als Wissenschaftsziel der entscheidungsorientierten Betriebswirtschaftslehre die Erklärung des betriebswirtschaftlichen Entscheidungsverhaltens sowie die

751 Sieben, G.; Schildbach, T.,(Entscheidungstheorie 1990), S. 3.

752 Vgl. bspw. Albach, H., (Aufgaben 1967), S. 447 ff.; Szyperski, N., (Orientierung 1971), S. $263 \mathrm{ff}$.

753 Vgl. Schanz, G., (Pluralismus 1973), S. 139 ff. Ein solcher wissenschaftlicher Pluralismus ist dabei als wünschenswert anzusehen, da dieser einen methodologischen Zweifel impliziert und dadurch erkenntnisfördernd wirkt. Vgl. ders., (Pluralismus 1973), S. 135 ff. und die dort angegebene Literatur.

754 Vgl. Kapitel 4: Unternehmensplanspiele als Modelle über einen Gegenstandsbereich.

755 Vgl. Hax, K., (Entwicklung 1959), S. 23. Auch Heinen, E., (Wissenschaftsprogramm 1969), S. 208. Dabei ist im wesentlichen die Art und Weise, wie die Betriebswirtschaftslehre von nun an Entscheidungen untersucht, neu, nicht jedoch die Tatsache, daß sie sich überhaupt mit Entscheidungen befaßt. Vgl. ders., (Wissenschaftsprogramm 1969), S. 208. 
Entwicklung von Verhaltensempfehlungen bei der Entscheidungsfindung anzusehen ist. ${ }^{757}$

Die Betriebswirtschaftslehre, als jene wirtschaftswissenschaftliche Disziplin, die aus einzelwirtschaftlicher Sicht den rationalen Umgang mit knappen Gütern untersucht, bezeichnet zunächst eine speziell normative Entscheidungstheorie. ${ }^{758}$ In diesem Zusammenhang ist für die Betriebswirtschaftslehre von besonderem Interesse, ,[...] wie es zu Entscheidungen kommt und welche Bedeutung ihnen im einzelnen beizumessen ist" ${ }^{759}$ wobei die Betriebswirtschaft als ein zielorientiertes, produktives, sozio-technisches System betrachtet wird, in dem Entscheidungsprozesse zur Gestaltung und Lenkung des Systems ablaufen. ${ }^{760}$

Weiterhin kann die Aufgabe der Betriebswirtschaftslehre aber auch darin gesehen werden, das tatsächliche Verhalten von Menschen in Betrieben zu erklären. Eine derartige, deskriptiv ausgerichtete Entscheidungstheorie, wird dann als eine Theorie kognitiver Entscheidungs- oder Problemlösungsprozesse angesehen ${ }^{761}$ und kann als Gesamtheit aller Vorgänge betrachtet werden, in denen Menschen unter Einsatz ihrer geistigen Fähigkeiten Probleme lösen. ${ }^{762}$ Analog zu den normativen Ausprägungen ist sie also als spezielle empirisch-realistische Entscheidungstheorie anzusehen, die sich mit dem kognitiven Problemlösungsverhalten von Menschen in Betrieben befaßt. ${ }^{763}$

So kann die Betriebswirtschaftslehre, ob als empirisch-realistische oder als normative Wissenschaft, zusammenfassend als jeweils spezielle Disziplin der korrespondierenden Entscheidungstheorie angesehen werden. ${ }^{764}$

\footnotetext{
756 Vgl. Pfohl, H.-C., (Entscheidungsfindung 1977), S. 30.

757 Vgl. Heinen, E., (Wissenschaftsprogramm 1969), S. 209 ff.

758 Vgl. Sieben, G.; Schildbach, T.,(Entscheidungstheorie 1990), S. 5.

759 Kloidt, H.; Dubberke, H.-A.; Göldner, J.,(Problematik 1959), S. 11 f.

760 Vgl. Heinen, E., (Ansatz 1971), S. 432 f.

761 Vgl. Kirsch, W., (Entscheidungsprozesse -Band 1- 1970), S. 68 f.; Hesselbach, J., (Verhaltensforschung 1970), S. $647 \mathrm{ff}$.

762 Vgl. Sieben, G.; Schildbach, T.,(Entscheidungstheorie 1990), S. 4.

763 Vgl. Sieben, G.; Schildbach, T., (Entscheidungstheorie 1990), S. 4.

764 Vgl. Busse von Colbe, W.; Laßmann, G.,(Betriebswirtschaftstheorie 1991). S. 1 f.
} 

betriebswirtschaftlicher Entscheidungstheorie als Ausgangspunkt der Analyse

Es wurde bereits ausgeführt, daß das Simulationsmodell im Spiel zum Gegenstand der vorgesehenen Geschäftsmaßnahmen wird, wobei das Spielverhalten durch die Handlungen der Spieler hervorgerufen wird. ${ }^{765}$ Aufgrund der »Quasirealität« des Planspiels ${ }^{766}$ handelt es sich hier um $» Q u a s i h a n d l u n g e n ~ «,{ }^{767}$ die durch Entscheidungen ausgelöst werden. Somit hat die Entscheidung eine dem Handeln vorgelagerte Stellung, die Kamlah folgendermaßen zum Ausdruck bringt:

„Unser Handeln wird durch Reden geleitet, durch gemeinsames Bereden oder durch inneres Reden, oft durch beides zugleich. An der Schwelle zwischen Begehren und Handeln steht der „Beschluß“, wenn mehrere gemeinsam handeln, der ,Entschlu $\beta^{\prime \prime}$, wenn ich allein handle. Unter mehreren beredeten Möglichkeiten erwählt der Entschluß eine und schließt die anderen aus. Das noch unentschlossene Bereden von Möglichkeiten heißt umgangssprachlich „,beraten“ bzw., wenn der einzelne es „für sich“ vollzieht, „überlegen“. Wer sich aber zum Handeln entschlossen hat und befragt wird, der spricht nun von dem, was er ,beabsichtigt“ oder was er „,will“. .768

Diese Definition läßt bereits deutlich werden, daß im Entscheidungsprozeß Daten ganz besonderer Art, die insbesondere einen Einfluß auf die bei der Betrachtung des Entscheidungsprozesses auftretenden organisatorischen Probleme haben, von dem einzelnen Entscheidenden ausgehen. Daher sind zur Klärung der mit dem Entscheidungsprozeß zusammenhängenden Problematik auch all die Einflüsse, welche vom Entscheidenden ausgehen, respektive auf ihn einwirken, von beson-

\footnotetext{
765 Vgl. Abschnitt 2.1.2.2: Das Unternehmensplanspiel: Ein Entscheidungsspiel.

766 Vgl. Abschnitt 2.2.1.1: Einsatzgebiete von Unternehmensplanspielen.

767 Vgl. Abschnitt 5.1: Das Unternehmensplanspiel: Ein komplexes Lehr-Lern-Arrangement.

768 Kamlah, W., (Anthropologie 1972), S. 32 f.
} 
derer Bedeutung. ${ }^{769}$ Es bedingt sich somit zunächst die Relevanz der empirischrealistischen betriebswirtschaftlichen Entscheidungstheorie.

Weiterhin bedingt sich aber auch die Relevanz der normativen Entscheidungstheorie, da in der Ökonomie weitestgehend davon ausgegangen wird, daß sich ein Individuum in vollem Bewußtsein über alle Alternativen und unter Maximierung entscheidet. So führt bspw. Boulding folgendes aus:

„Economic behavior is conceived as a process of „maximization." Economic man is supposed to be capable of at least three processes involving an image[770]. In the first place he is supposed to be conscious of the alternatives which lie before him. “ [...], „In addition to the image of the alternatives economic man is also supposed to be able to give value-ordering to all relevant alternatives, that is, to all parts of this image. [...] His final task after his imagination has performed these labors of Hercules as a simple one. All he needs to do is to scan all possible combinations which are open to him and all his alternative acts, rank them in order on the parade ground of value, and pick out the top of the class. This process of selection of the best alternative is what is known as maximization and a great deal of economic theory is built around it."771

Im Unternehmensplanspiel ist es nun so, daß der Teilnehmer an einer Ausbildungsmaßnahme offensichtlich nicht unter vollkommener Rationalität entscheidet. ${ }^{772}$ Es ist daher an dieser Stelle sinnvoll, eine Betrachtung der grundsätzlichen

769 Vgl. Hauschildt, J., (Entscheidungsziele 1977), S. 119.

770 Unter „Image“ versteht Boulding die Vorstellung, die sich ein Individuum von der 'Welt' macht. Wesentlich ist dabei die subjektive Komponente, in der Boulding auch die Grenze zwischen Vorstellung und Wissen sieht. Während das Wissen des Menschen den Anspruch von Validität und Wahrheit impliziert, ist mit der Vorstellung gemeint, was das einzelne Individuum für wahr hält das subjektive Wissen, welches in hohem Maße das Verhalten steuert. Vgl. Boulding, K.E., (Image 1997), S. 5 f.

772 Vgl. Abschnitt 6.3: Die unternehmerische Handlungskompetenz. 
Annahmen der normativen und der empirisch-realistischen betriebswirtschaftlichen Entscheidungstheorie vorzunehmen, ${ }^{773}$ wobei dies an dieser Stelle dazu dient eine Differenz zwischen den beiden Sichtweisen zu identifizieren.

Die Annahmen der normativen Entscheidungstheorie gehen davon aus, daß die Vorstellung, die sich ein Mensch von der Welt macht, den Anspruch der Validität und Wahrheit besitzt. Der Mensch geht bei seiner Entscheidungsfindung jedoch immer von einem subjektiven, vereinfachten Bild der Umwelt aus. Dies ist eine zentrale These der verhaltenswissenschaftlichen Entscheidungstheorie. ${ }^{774}$ Die Entscheidungen, die mit Hilfe des erworbenen Wissens gefällt werden, sind daher mit subjektiven Elementen durchsetzt, welche nicht einfach vernachlässigt werden können. ${ }^{775}$

Zunächst gilt dieser Zusammenhang für den Rationalitätsgedanken ${ }^{776}$ der normativen Entscheidungstheorie. Im verhaltenswissenschaftlichen Ansatz der Entscheidungstheorie weicht der Rationalitätsgedanke einer relativeren Anschauung von Rationalität. Die einseitig auf das Maximalziel ausgerichtete Beurteilung weicht im verhaltenstheoretischen Ansatz der Vorstellung, daß der Mensch überhaupt keine Maximal- bzw. Minimalziele verfolgt, sondern vielmehr satisfizierende Lösungen anstrebt. Damit ist das menschliche Handeln, insbesondere das Entscheidungshandeln, auf die Zielgröße des Anspruchsniveaus des Individuums ausgerichtet und gewährleistet, daß der Mensch, trotz äußeren Leistungsdrucks, zu interner Zufriedenheit und internem Gleichgewicht gelangen kann. ${ }^{777}$

Weiterhin gilt dieser Zusammenhang für die Handlungsmöglichkeiten, die nicht direkt entdeckt werden. ${ }^{778}$ So verweist Streit auf Boulding, der diesen Zusammenhang wie folgt ausdrückt: „Alternativen haben üblicherweise nicht die Höflich-

\footnotetext{
773 Vgl. hierzu auch die Ausführungen von Werner. Dieser betrachtet die Annahmen rationalen Entscheidungsverhaltens der normativen Entscheidungstheorie und der beschränkten Rationalität des Menschen in Zusammenhang mit der Entwicklung des Entscheidungsunterstützungssystems aMAdEUS für die Unternehmenssimulation Puma. Vgl. Werner, L., (Entscheidungsunterstützungssysteme 1992), S. 14 ff.

Vgl. Kirsch, W., (Entscheidungsprozesse -Band 1- 1970), S. 76.

775 Vgl. Streit, M.E., (Marktwirtschaft 1998), S. 151.

776 Vgl. Abschnitt 6.1.1: Der Gegenstand der normativen und deskriptiven Entscheidungstheorie.

777 Vgl. Bronner, R., (Entscheidung 1973), S. 14 und die dort angegebene Literatur.

778 Vgl. Streit, M.E., (Marktwirtschaft 1998), S. 151.
} 
keit, sich in Reih und Glied auf dem Exerzierplatz unserer Vorstellungskraft $\mathrm{zu}$ präsentieren. "779 Sie müssen eher durch eine Erkenntnisleistung ermittelt werden, die von subjektiver, oder gegebenenfalls auch von schöpferischer Natur sein kann. Dabei ist die Erkenntnisleistung ,[...] vorstellbar als Ergebnis des Bemühens, neues Wissen zu der Vorstellung oder der persönlichen Wissensstruktur in Beziehung zu setzen, die der Akteur im Hinblick auf seine ökonomische Umwelt bereits hatte". ${ }^{\text {" }} 80$ Dies führt dazu, daß alle Entscheidungen in zweifachem Sinne als subjektiv einzuschätzen sind. Sie basieren zum einen auf subjektiven Wissensgrundlagen, zum anderen auch auf subjektiven Einschätzungen der Handlungsmöglichkeiten. ${ }^{781}$

Luhmann führt in diesem Zusammenhang weitere Aspekte an, die er als typische Einwendungen gegen das Optimalprinzip klassifiziert. So ist das Entscheiden zum einen auch im Hinblick auf seine Abhängigkeit von individuellen Werten als subjektiv zu bezeichnen. Weiterhin führt Optimierung zwangsläufig zu Operationalsierung, um eindeutig bestimmbares Handeln ableiten zu können, wodurch sich ein Widerspruch ergibt, da Operationalisierung in ihren Prämissen Optimierung ausschließt. Schließlich ist das menschliche Auffassungsvermögen unter der Prämisse prinzipiellen Optimierens überfordert. ${ }^{782}$

Von einer Überforderung des menschlichen Antizipationsvermögens unter der Prämisse prinzipiellen Optimierens soll im folgenden ausgegangen werden. ${ }^{783} \mathrm{Sie}$, die menschliche Überforderung, kann nach Bronner als »duale Spannungslage« bezeichnet werden. Darunter ist zu verstehen, daß der Existenz und Bewältigung eines inhaltlich bestimmten Sachproblems die Existenz und Bewältigung von psychisch-kognitiv bestimmtem Streß gegenübersteht, wobei die Erarbeitung sachgerechter Problemlösungen geradezu als Funktion der Streßüberwindung begriffen

\footnotetext{
779 Boulding, K.E., (Image 1997), S. 84.

780 Streit, M.E., (Marktwirtschaft 1998), S. 151.

781 Vgl. Streit, M.E., (Marktwirtschaft 1998), S. 151.

782 Vgl. Luhmann, N., (Zweckbegriff 1968), S. 75 ff. und die dort angegebene Literatur.

783 Unter diesem Aspekt erlangt die klassische Entscheidungstheorie den Grad einer formalen Entscheidungslogik, wobei jedoch angemerkt werden muß, daß die klassische Entscheidungstheorie dadurch durchaus nicht ihren Wert für unterschiedliche wirtschaftswissenschaftliche Disziplinen, wie bspw. die Unternehmensforschung, verliert und darüber hinaus
} 
wird. ${ }^{784}$ Zusammenfassend kann sie hier auch als Differenz zwischen den Annahmen der normativen Entscheidungstheorie und den Annahmen der deskriptiven Entscheidungstheorie angesehen werden.

Dabei wird der Tatsache, daß der Umgang mit einem Unternehmensplanspiel für die Planspielteilnehmer ein Problem darstellt didaktisch entsprochen indem die Komplexität des Unternehmensplanspiels an das Vorwissen der Teilnehmer angepaßt wird. Hier wird dieses Vorwissen als Spiegel des Erfahrungshintergrundes eines Individdums angenommen, worauf im folgenden einzugehen ist.

Der Erfahrungshintergrund eines Individuums

\subsection{1}

Merkmale des Erfahrungshintergrundes eines Individuums

Verschiedene Menschen haben keinesfalls identische oder strukturell ähnliche Gedächtnisse. Individuen sind im Gegenteil geprägt von der erlebten Geschichte, von den Erfahrungen, die sie jeweils gemacht haben. Geschichte und Erfahrungen stehen in unmittelbarem Zusammenhang mit der Persönlichkeit und dem Selbst eines Menschen. ${ }^{785}$ Daher kommt den Erfahrungen, die ein Individuum gemacht hat, eine besondere Bedeutung zu. ${ }^{786}$ Denn: „Indem uns Gutes und Schlimmes widerfährt, indem unser Handeln gelingt und mißlingt, machen wir „Erfahrungen“, lernen wir für unser künftiges Verhalten."

So leitet der Mensch aus seinen Überzeugungen oder naiven Theorien darüber, wie die Welt funktioniert, Erklärungen für Ereignisse um sich herum ab. Darüber hinaus sind seine Annahmen über die Welt zu einem gewissen Umfang in der Lage, Ereignisse zu beeinflussen. Je enger die Ereignisse mit der eigenen Person und

auch Ansätze zur Erklärung tatsächlichen individuellen Entscheidungsverhaltens liefert. Vgl. Werner, L., (Entscheidungsunterstützungssysteme 1992), S. 16.

784 Vgl. Bronner, R., (Entscheidung 1973), S. 15. In Analogie dazu ist die Aufgabe der Wirtschaftspädagogik zu sehen. So konnte ausgeführt werden, daß die Wirtschaftspädagogik dazu aufgefordert ist, Lehr-Lern-Techniken zu entwickeln, die helfen, die Differenz zwischen Qualifikationsanforderungen und den subjektiven Bedingungen der Lernenden zu überwinden. Vgl. Abschnitt 5.1: Das Unternehmensplanspiel: Ein komplexes Lehr-LernArrangement.

785 Vgl. Lehner, F., (Memory 2000), S. 85.

786 Vgl. Spinath, B., (Theorien 1998), S. 4.

787 Kamlah, W., (Anthropologie 1972), S. 33. 
dem eigenen Handeln in Zusammenhang stehen, um so wahrscheinlicher ist dies der Fall. So wird beispielsweise eine Person, die davon überzeugt ist, eine bestimmte Position nicht zu erreichen, weniger bemüht sein, diese zu erreichen als eine zweite mit einer optimistischeren Sicht. Naive Theorien schaffen sich somit alltägliche Realitäten, die sie bestätigen. ${ }^{788}$

In diesem Zusammenhang wird wichtig, daß der Mensch Schwierigkeiten hat, sich andere Wege des Denkens zu erschließen. Die durch ihn gelernten Assoziationen und Denkgewohnheiten, "HABITS OF THOUGHT«, sind oft fest verankert. Dies führt zu Schwierigkeiten im Rahmen des Problemlösens macht den erfolgreichen Umgang mit neuen Situationen und Problemen vielleicht sogar unmöglich. Der Mensch zieht es vor, an Lösungswegen festzuhalten, die einmal zu einer erfolgreichen Lösung geführt haben und neigt dazu, diese immer wieder anzuwenden, selbst wenn es Möglichkeiten gibt, ein Problem schneller und einfacher zu lösen. Im Deutschen wird in diesem Zusammenhang von Einstellungen gesprochen, im Englischen von einem »MENTAL SET«, wobei beide Begriffe synonym verwendet werden.

Neben dieser mangelnden Bereitschaft des Menschen, sich neuen Wegen des Problemlösens gegenüber offen zu zeigen, bereitet das sogenannte Prinzip der Geschlossenheit dem Menschen Schwierigkeiten beim Problemlösen. So suchen Menschen bei Dingen, die von ihnen wahrgenommen werden, nach Vollständigkeit. Dies geschieht in der Form, daß sie, dem eigenen Erfahrungshintergrund gemäß, sinnvoll erscheinen, was wiederum dazu führt, daß Menschen bei unterbrochenen Reizen die Ganzheit nach ihren Vorstellungen herbeiführen. Weitere Probleme schafft die funktionale Gebundenheit des menschlichen Denkens, ${ }^{789}$ sowie die Schwierigkeit des Menschen, Problemstellungen in einem anderen als dem bisher betrachteten Kontext zu untersuchen. ${ }^{790}$

Menschen verfügen jedoch über Organisationen zum Zwecke des Problemlösens, von welchen angenommen wird, daß sie zum großen Teil ein Resultat von Lern-

788 Vgl. Spinath, B., (Theorien 1998), S. 4.

789 Vgl. Banyard, P.; Hayes, N.,(Denken 1995), S. 145. 
und Erziehungsprozessen sind. Durch derartige Erziehungsprozesse sind sie demnach auch wieder zu verändern. So formuliert Dörner: „Wenn jedoch Problemlöse- und Denkorganisationen mehr oder minder instabile Gebilde sind, so muß man annehmen, daß es irgendwelche elementaren Informationsverarbeitungsprozesse gibt [...]."791 Dörner spricht hier von Atomen der Informationsverarbeitungsprozesse. Aus ihnen ist die Problemlöseorganisation, die zu einem bestimmten Zeitpunkt in einem Individuum gegenwärtig ist, vergleichbar zusammengesetzt, wie bspw. ein kompliziertes Eiweißmolekül aus einer großen Ansammlung von Atomen. Gleichzeitig verweist jedoch auch Dörner auf die Probleme von Individuen, ihre überkommenen Denkschemata zu hinterfragen und zu entwickeln, sowie auf Theorien, aus welchen sich schließen läßt, daß Problemlöseprozeduren keinen hohen Grad an Individualität aufweisen. Er wirft in diesem Zusammenhang die Frage auf, ,[...] ob die Grenzen der freien Variierbarkeit der Informationsverarbeitungsatome nicht doch sehr eng gesteckt sind.“792

\subsubsection{Vorwissen: Spiegel des Erfahrungshintergrundes eines Indivi- duums}

In Zusammenhang mit dem Wissen, welches eine Person in eine vorgegebene Situation mitbringt, wird allgemein von Vorwissen gesprochen, welches hier daher als Spiegel des Erfahrungshintergrundes eines Individuums angenommen werden kann. Bei Prozessen, die mit der Bearbeitung komplexer Probleme in computersimulierter Form zusammenhängen, orientiert sich ein Proband sehr stark an bereits vorliegenden Erfahrungen bzw. Vorwissen, das er nutzbar machen kann. ${ }^{793}$ So haben theoretische Analysen der Rolle des Vorwissens beim Problemlösen ergeben, daß das Vorwissen die entscheidende Determinante jedes weiteren Wissenserwerbs darstellt. ${ }^{794}$ Beim Aufbau einer internen Repräsentation des Ausgangszustandes und des erwünschten Zielzustandes versucht der Problemlöser, eine Integration mit bereits vorhandenem Wissen herzustellen. Die Problembearbeitung insgesamt kann damit abhängig sein von Art und Umfang des

\footnotetext{
790 Vgl. Banyard, P.; Hayes, N.,(Denken 1995), S. 143 ff.

791 Dörner, D., (Organisation 1974), S. 11.

792 Dörner, D., (Organisation 1974), S. $11 \mathrm{f}$.

793 Vgl. Hesse, F.W., (Analyse 1985), S. 139 f.

794 Vgl. Strohschneider, S., (Wissenserwerb 1990), S. 91.
} 
Vorwissens, das dabei herangezogen wird. Fehlt bspw. ein für die Problembearbeitung relevantes Vorwissen, so muß der Problemlöser seine Problembearbeitung entsprechend anders organisieren. ${ }^{795}$ Viele Studien innerhalb der Problemlöseforschung weisen auf diesen Zusammenhang hin. Dabei wird die Bedeutung des Vorwissens in Verbindung mit dem Aufbau des Problemraums, das heißt der internen Problemrepräsentation, der Zielbildung, der Suche nach Lösungsstrategien sowie der Problemlösegüte gesehen. ${ }^{796}$

Es ist daher notwendig, bei der Klassifikation von Problemen die mit dem Problem korrespondierenden mentalen Prozesse der Probanden zu berücksichtigen. ${ }^{797}$ Es ist daher erforderlich, durch eine klare Diagnose der Fähigkeiten des einzelnen festzustellen, wo er seine persönlichen Stärken und Schwächen im Umgang mit komplexen Systemen hat. Durch eine solche Diagnose wird es möglich, den einzelnen seinen Fähigkeiten entsprechend zu plazieren und ihn gezielt zu trainieren. ${ }^{798}$ Dies kann durch Methoden zur Erhebung von Vorwissen gelingen, auf die daher im folgenden eingegangen werden soll.

\subsubsection{Methoden zur Erhebung von Vorwissen}

Um Aufschluß über das Vorwissen von Personen zu erlangen, muß es in irgendeiner Form erhoben werden. Dazu existieren unterschiedliche Methoden.

Eine Methode zur Erhebung von Vorwissen stellt zunächst der Intelligenztest dar. Fraglich ist jedoch dessen Eignung in Zusammenhang mit der Erhebung von Vorwissen bezüglich eines komplexen Systems. So wird an Aufgaben aus Intelligenztests kritisiert, daß sie fast ausschließlich als gut definierte Probleme einzu-

\footnotetext{
795 Vgl. Hesse, F.W., (Analyse 1985), S. 139 f.

796 Vgl. Weber, S., (Vorwissen 1994), S. 16. Für das handlungsorientierte Lernen ist die Bedeutung des Vorwissens bzw. der Vorerfahrungen in Analogie zur Rolle des Vorwissens beim Problemlösen zu sehen. Hier erfolgen Zielbildung, Handlung und Kontrolle auf Grundlage des subjektiv wahrgenommenen Handlungsfeldes. Vgl. dies., (Vorwissen 1994), S. 16 und die dort angegebene Literatur.

797 Vgl. Süss, H.-M., (Intelligenz 1996), S. 7.

798 Vgl. Reichert, U.; Stäudel, T.,(Diagnostik 1991), S. 102 f.
} 
ordnen sind. 799 Der Ausgangspunkt, d.h. die Lösung, ist in Intelligenztests meist aus Antwortalternativen auszuwählen, womit sie nicht in jedem Fall als repräsentativ für alltägliche Probleme angesehen werden können. So werden schlecht definierte Probleme, bei denen z.B. eine teilweise intransparente Problemstellung vorliegt, die durch aktive Datensuche erst transparent zu machen ist, in Intelligenztests nicht berücksichtigt. Dies gilt weiterhin auch für dynamische Probleme, bei denen sich die Situationsmerkmale über die Zeit hinweg ändern und nicht nur zu einem, sondern zu aufeinanderfolgenden Zeitpunkten Lösungen zu generieren und möglicherweise auch wieder zu revidieren sind. Schließlich werden selten komplexe und vernetzte Probleme berücksichtigt, die nicht allein eine Lösung erfordern, sondern nur durch ein Muster von Lösungsvorschlägen angemessen bearbeitet werden können. ${ }^{800}$

Weiterhin wird an testtheoretischen Ansätzen kritisiert, daß sie weitestgehend von der Annahme quantitativer Unterschiede ausgehen. Daher sind sie strukturellen Konzepten wie Produktionssystemen oder semantischen Netzwerken kaum kongruent. Individuelle Wissensunterschiede entsprechen in einem Produktionssystem einem Unterschied in der Menge und der Art der verfügbaren Produktionen. In semantischen Netzwerken lassen sich Wissensunterschiede in einer unterschiedlichen Anzahl von Knoten und Verbindungen darstellen. Gerade diese Ansätze verdeutlichen, wie schwierig es ist, vom Reaktionsverhalten eines Menschen auf dessen individuellen Wissensbesitz zu schließen. Dies ist wohl auch als wesentlicher Grund zu werten, daß sowohl die Diskussion von Produktionssystemen als auch von semantischen Netzwerken die Entwicklung von Diagnoseinstrumenten im Wissensbereich kaum beeinflußt hat. ${ }^{801}$

Diese Zusammenhänge wurden durch verschiedene Untersuchungen aufgezeigt, die hier zusammenfassend referiert werden sollen.

799 Vgl. Putz-Osterloh, W., (Problemlöseforschung 1989), S. 87. Die Einordnung erfolgt in Anlehnung an allgemein akzeptierte Klassifikationsschemata. Vgl. zu solchen Klassifikationsschemata bspw. Dörner, D., (Problemlösen 1976), S. 11 ff. Vgl. auch Abschnitt 2.3.2: Einfache und komplexe Probleme.

800 Vgl. Putz-Osterloh, W., (Problemlöseforschung 1989), S. 87.

801 Vgl. Kluwe, R.H.; Spada, H., (Wissen 1981), S. 318 f. 
Die Untersuchung von Intelligenztestleistungen bildet einen Schwerpunkt der BAYREUTHER ARBEITSGRUPPE um Putz-Osterloh. 802 Ausgangspunkt der Untersuchungen von Putz-Osterloh bildeten Untersuchungen zum Umgang von Personen mit komplexen Systemen sowie Untersuchungen zum Vorgehen von Personen bei der Lösung von Aufgaben aus dem Intelligenztest „ADVANCED PROGRESSIVE MATRICE“ [,APM“] von Raven. 803

In einer weiteren Untersuchung analysierte Putz-Osterloh die Ergebnisse von Versuchspersonen im Intelligenztest „APM“ im Vergleich zu den Ergebnissen mit dem betriebswirtschaftlichen System SCHNEIDERWERKSTATT. ${ }^{804}$ Sie ging davon aus, daß es zwischen den Ergebnissen einer Person in einem Intelligenztest und denen in einer komplexen Anforderungssituation einen Zusammenhang gibt. ${ }^{805}$ Diese Annahme bestätigte sich nicht. So konnte zwischen der Leistung in einem Intelligenztest und der Problemlöseleistung kein systematischer Zusammenhang gefunden werden, obwohl in beiden Problembereichen Beziehungen zwischen Variablen richtig zu analysieren und Regeln für diese Beziehungen aufzustellen waren. ${ }^{806}$ Es konnte daher gefolgert werden, daß komplexe Systeme zusätzliche Anforderungen stellen, die in Intelligenztests nicht erfaßt werden, bzw. dort nicht eingesetzt zu werden brauchen. Diese Anforderungen stellen sich wie folgt dar:807

Eine aktive Beschaffung von Informationen, die durch direkte Fragen sowie die Erprobung variationsreicher Maßnahmen realisiert werden kann.

Eine Analyse von Nebenwirkungen, die durch eine Analyse netzwerkartiger Strukturen und nicht durch ,eingleisige“ Analysen erfolgt.

Eine systematische Planung und Kontrolle des eigenen Vorgehens, bei der erkannt wird, wann genügend Informationen für ein zielgerichtetes Handeln vorliegen sowie überprüft wird, ob die geplanten Maßnahmen auch wirklich realisiert wurden.

\footnotetext{
802 Vgl. Abschnitt 2.3.1: Komplexes Problemlösen - Ein Aufriß.

803 Vgl. Putz-Osterloh, W., (Problemlöseprozesse 1981).

804 Vgl. Putz-Osterloh, W., (Beziehung 1981), S. 84 ff.

805 Vgl. Putz-Osterloh, W., (Beziehung 1981), S. 84.

806 Vgl. Putz-Osterloh, W., (Beziehung 1981), S. 90 u. S. 96 ff.
} 
Hesse formuliert weiterhin die folgenden Anforderungen: ${ }^{808}$

$>$ Die Analyse von Variablen, die untereinander verknüpft sind und sich gegenseitig beeinflussen können.

$>$ Die Steuerung von Variablen mit Eigendynamik, die sich auch ohne äußere Einwirkungen weiterentwickeln.

Die Auswahl von Maßnahmen zur Steuerung eines Systems, das die Versuchspersonen in der Regel nur zum Teil überschauen.

$>$ Das Finden einer Lösung nicht durch eine Antwort bzw. Maßnahme, sondern durch die Bearbeitung einer Problemsituation über mehrere Zeittakte, wobei sich der Problemlöser jeweils auf eine veränderte Situation einzustellen hat.

Der Intelligenztest von Raven und das System SCHNEIDERWERSTATT waren auch Grundlage einer späteren Untersuchung, die von Putz-Osterloh \& Lüer durchgeführt wurde. Hier sollte exemplarisch gezeigt werden, welche Arten intelligenten Verhaltens ein Individuum in Relation zu welchen Problemanforderungen zeigt. Darüber hinaus sollte gezeigt werden, wo ein Verhalten, welches durch gängige Intelligenztestverfahren erhoben werden kann, innerhalb dieser Varianten menschlicher Intelligenz eingeordnet werden kann. ${ }^{809}$ Ein Teil dieser Prozesse läßt sich als erfolgreicher Umgang mit Intransparenz beschreiben. Sie stellt das Hauptmerkmal komplexer Problemstellungen dar. Im Gegensatz zu transparenten Problemen ${ }^{810}$ gelten intransparente Probleme als Probleme, deren Variablen erst durch aktives Eingreifen des Problemlösers für ihn erkennbar und analysierbar werden, da ihm zu Beginn nicht genügend Informationen über die Variablen vorliegen. ${ }^{811}$

Zusammenfassend läßt sich daher festhalten, daß Erfolge beim Lösen komplexer, dynamischer Probleme aus Ergebnissen herkömmlicher Intelligenztests erst dann

807 Vgl. Putz-Osterloh, W., (Beziehung 1981), S. 97.

808 Vgl. Hesse, F.W., (Effekte 1982), S. 64.

809 Putz-Osterloh, W.; Lüer, G., (Vorhersagbarkeit 1981), S. 310.

810 Als transparent sehen Putz-Osterloh \& Lüer in Anlehnung an Dörner Probleme an, bei denen der Problemlöser alle für die Problemstellung relevanten Variablen überblicken kann und aktiv keine weiteren Informationen über diese Variablen beschaffen muß. Vgl. PutzOsterloh, W.; Lüer, G., (Vorhersagbarkeit 1981), S. 312 und Dörner, D., (Problemlösen 1976), S. $18 \mathrm{f}$.

811 Vgl. Putz-Osterloh, W.; Lüer, G., (Vorhersagbarkeit 1981), S. 312. 
vorhersagbar werden, wenn bestimmte Transparenzbedingungen bei der Problemstellung eingehalten werden, ${ }^{812}$ wozu vor allem ein Informationsangebot über die relevanten Variablen und das Relationsgefüge, durch welches sie untereinander verknüpft sind, gehört. ${ }^{813}$

Ein Instrumentarium in Zusammenhang mit der Erhebung von Vorwissen in intransparenten Problembereichen zu entwickeln, machte sich Weber zur Aufgabe. Sie verfolgt in ihrer Arbeit das Ziel ,[...] ein Instrumentarium zu entwickeln, mit dessen Hilfe es möglich ist, Vorwissensstrukturen von Schülern unter dem Aspekt der Vernetztheit, Komplexität und Prozeßorientierung zu erheben, zu beschreiben und zu bewerten“814. Dazu wählte sie zum einen das Verfahren der Netzwerkbildung aus. ${ }^{815}$ Weiterhin entwickelte sie zur Ergänzung der Netzwerkbildung ein anderes Verfahren, welches von ihr als Fenster-Technik bezeichnet wird.

Eine Netzwerkbildung kann dabei in der von Weber wie folgt vorgenommenen Weise erfolgen. ${ }^{816}$ In einer Vorstudie fand zunächst eine Entwicklung der Netzwerkbildung statt, die auf einer Erhebung an zwei niedersächsischen kaufmännischen Schulen beruhte. Dabei wurde davon ausgegangen, daß Versuchspersonen persönliches Vorwissen, hier verstanden als ökonomisches Zusammenhangswissen, am besten unter Heranziehung selbst gewählter Konzepte darstellen können. Zunächst wurden die Schüler dazu aufgefordert, Begriffe zu nennen, die ihnen in Zusammenhang mit Betrieben sowie Tätigkeiten in Betrieben einfallen, was dazu diente, einen übersichtlichen Begriffs-Pool ${ }^{817}$ für die Netzwerkbildung abzuleiten. Die Netzwerkbildung erfolgte dann in einer zweiten Vorstudie, in der Relationen erhoben werden konnten, indem die Schüler dazu aufgefordert wurden, einen effizient arbeitenden Betrieb durch ein Netzwerk darzustellen. ${ }^{818}$ Die in den Vorstudien gewonnen Ergebnisse wurden dann dazu genutzt, das Instrumentarium der

\footnotetext{
812 Vgl. Putz-Osterloh, W.; Lüer, G., (Vorhersagbarkeit 1981), S. 330.

813 Vgl. Putz-Osterloh, W.; Lüer, G., (Vorhersagbarkeit 1981), S. 330.

814 Vgl. Weber, S., (Vorwissen 1994), S. 6 f.

815 Vgl. Weber, S., (Vorwissen 1994), S. $31 \mathrm{ff}$.

816 Vgl. Weber, S., (Vorwissen 1994), S. 31 ff.

817 Dazu wurden die gewonnenen Daten mit einer kategorialen Inhaltsanalyse aggregiert und auf 65 Begriffe oder Konzepte zurückgeführt. Vgl. Weber, S., (Vorwissen 1994), S. 32 ff.

818 Die in diesem Zusammenhang erhobenen Relationen wurden auf 22 Relationen aggregiert. Vgl. Weber, S., (Vorwissen 1994), S. 38.
} 
Netzwerkbildung zu entwickeln. Dieses Instrumentarium beinhaltet die bereits erläuterte Generierung eines Begriffs- und Relations-Pools sowie einer damit verbundenen Fragestellung, anhand der das Zusammenhangswissen expliziert werden soll. ${ }^{819}$

Das Verfahren der Fenster-Technik wurde von Weber in Anlehnung an die Computertechnik entwickelt und ergänzt die Konzepte der Netzwerkbildung. Die Fenster-Technik wird hierbei dazu genutzt, herauszufinden, welche Inhalte sich für Schüler hinter den Konzepten der Netzwerkbildung verbergen. Assoziieren sie damit bspw. Worthülsen, sind es für sie Bilder oder stellen sie Teilnetze dar? Um diese Frage zu klären, wurden die Schüler aufgefordert sich zu ausgewählten ökonomischen Begriffen zu verhalten. Dazu wurde ihnen ein von Weber entwickeltes Arbeitsblatt, ${ }^{820}$ auf welchem in unterschiedlichen »Fenstern« Einträge vorgenommen werden konnten, zur Verfügung gestellt. Dabei sollten die Schüler in den unterschiedlichen »Fenstern« Auskunft darüber geben, was sie unter den ausgewählten Begriffen verstehen. ${ }^{821}$

Neben der Relevanz von Vorwissen für den erfolgreichen Umgang mit einer Problemstellung spielt auch der Wissenserwerb während der Auseinandersetzung mit einem komplexen System eine Rolle, zu dem ebenfalls Erhebungsmethoden existieren.

\subsubsection{Methoden zur Erhebung des Wissenserwerbs}

Die Überprüfung und Formulierung von Hypothesen stellt einen zentralen Aspekt in Zusammenhang mit der menschlichen Denkleistung dar. Nach Aussage von Spada, Reimann \& Häußler wurde diesem Aspekt in der Psychologie bisher nur wenig Aufmerksamkeit geschenkt. Sie entwickelten daher ein Verfahren zur Hypothesenentwicklung und -überprüfung, welches Ergebnisse über Wissen und Wissenserwerb von Probanden liefert.

\footnotetext{
819 Vgl. Weber, S., (Vorwissen 1994), S. 41 ff.

820 Vgl. Weber, S., (Vorwissen 1994), S. 242 ff.

821 Vgl. Weber, S., (Vorwissen 1994), S. 51 ff.
} 
Dieses Verfahren gelangte im Bereich geometrischer Optik zur Anwendung und wurde von Spada, Reimann \& Häußler als das Verfahren der WEIV-Sequenzen bezeichnet. ${ }^{822}$ Neben der Verwendung im Bereich geometrischer Optik läßt sich diese Methode jedoch auch generell zur Datenerhebung von Prozessen des Wissenserwerbs und der Wissensanwendung nutzen. ${ }^{823}$ In dem von den Autoren diskutierten Untersuchungsparadigma geht es um Prozesse der Hypothesenbildung und Hypothesenmodifikation anhand von Versuchsdaten. Wesentlich ist hierbei eine Informationsaufbereitung, die es dem Individuum ermöglicht eine sequentielle und von ihm selbst gesteuerte Informationsaufnahme durchzuführen. Die Versuchsperson wählt aus dem dargebotenen Informationsangebot aus [Wahl eines Informationsangebots]. Anschließend erläutert sie die auf Grundlage des aktuellen Wissens getroffene Auswahl in bezug auf den erwarteten Informationsinhalt gegenüber dem Versuchsleiter [Erwartung eines Ergebnisses]. Im nächsten Schritt erfolgt das Ergebnisfeedback, indem die Information zur Verfügung gestellt wird [Darbietung der Information]. Im letzten Schritt erfolgt die Ableitung von Hypothesenkonsequenzen, wodurch die Informationsverarbeitung angestrebt wird [Verarbeitung der Information]. Damit dient der gesamte Prozeß der Formulierung von Hypothesen und der Überprüfung ihrer Gültigkeit. Hypothesenformulierung und Hypothesentestung erfolgen hier Schritt für Schritt. ${ }^{824}$

Als weiteres Verfahren zur Erhebung des Wissenserwerbs sowie des Wissensstandes am Ende eines Spieldurchlaufs stellen Vance \& Gray ein Modell zur Evaluation von Fähigkeiten im Bereich „Business Gaming“ vor. ${ }^{825}$ Um die Ge-

822 Vgl. Spada, H.; Reimann, P.; Häusler, B.,(Hypothesenerarbeitung 1983), S. 142 ff. Im Versuchsaufbau werden unterschiedliche physikalische Phänomene angesprochen. Die Versuchsperson ist jeweils dazu aufgefordert, ein Experiment auszuwählen, dessen Wahl zu erläutern, das angenommene Ergebnis einzuzeichnen, es zu beschreiben und zu begründen, sowie außerdem die Ergebnisse mit der vorgenommenen Hypothese zu vergleichen. Vgl. dies., (Hypothesenerarbeitung 1983), S. 143.

823 Vgl. Opwis, K., (Modelle 1985), S. 27.

824 Vgl. Spada, H.; Reimann, P.; Häusler, B.,(Hypothesenerarbeitung 1983), S. 142 ff.

825 Vgl. Vance, S.C.; Gray, C.F., (Performance 1967), S. 29 ff. Das Verfahren von Vance \& Gray wurde für ein spezifisches management game entwickelt. Die Autoren verweisen jedoch auf dessen leichte Übertragbarkeit oder Möglichkeit der Modifikation im Hinblick auf andere business games. „The particular performance evaluation model used in this research was designed for the Management Decision Simulation game. The criteria used to evaluate performance are similar to those used by stock market analysts - growth, profit, dividends, etc.; therefore it would be a simple matter to develop similar models for other business games.“ dies., (Performance 1967), S. 29. 
samtheit der Spiel-Performanz eines Teilnehmers auszudrücken, definieren sie sechs Variablen, die in die Betrachtung einfließen. Diese Variablen stellen bspw. die Inventory-Performanz, die Cash-Performanz und die Profit-Performanz dar. Aus der Summe ergibt sich schließlich die totale Spiel-Performanz T des Spielers. [Vgl. Abbildung 6-1].

\begin{tabular}{|l|l|}
\hline M & Share of the market performance \\
\hline I & Inventory performance \\
\hline C & Cash performance \\
\hline P & Profit performance \\
\hline E & Owners' equity performance \\
\hline D & Dividend performance \\
\hline $\begin{array}{l}\text { T }= \\
\text { M }+ \text { I }+ \text { C + P + E + D }\end{array}$ & Total game performance \\
\hline
\end{tabular}

Abbildung 6-1: Variablen zur Errechnung der Spiel-Performanz eines Teilnehmers ${ }^{826}$

Der Spieler erhält dann regelmäßig, jeweils nach einem Viertel des Spiels, ein Performanz-Ergebnis. Dadurch ist es ihm möglich, sich bezüglich der definierten Variablen ein Bild von seiner Qualifikation zu machen, den Lernfortschritt zu beobachten und gegebenenfalls Handlungsregulationen vorzunehmen. 827

Die Notwendigkeit die Komplexität an das Vorwissen der Planspielteilnehmer anzupassen, erwuchs nun aus der Tatsache, daß der Umgang von Personen mit Unternehmensplanspielen für dieselben ein Problem darstellt. Dabei ist es jedoch so, daß Personen existieren, die mit einem komplexen System qualitativ besser umgehen können als andere. Diese wurden in der Expertiseforschung als Experten bezeichnet, wobei bereits festgehalten werden konnte welche Qualitäten das Wissen von betriebswirtschaftlichen Experten aufweist. An dieser Stelle ist nun davon auszugehen, daß Experten über ein umfangreicheres Wissen in den relevanten Dimensionen verfügen als Novizen, womit Experten den objektiven Anforderungen des Unternehmensplanspiels in einem größeren Umfang entsprechen können als dies Novizen tun. 
In Zusammenhang mit dem betriebswirtschaftlichen Rationalitätsgedanken stellt sich hier nun zum einen die Frage, was in der Betriebswirtschaftslehre unter unternehmerischer Handlungskompetenz verstanden werden kann zum anderen, welche Rolle die unterschiedlichen Qualitäten von Wissen in diesem Zusammenhang spielen.

\subsection{Die unternehmerische Handlungskompetenz}

Im Planspiel wird der Lernbegriff zum Begriff des Lernhandelns. ${ }^{828}$ Dabei werden einem Planspielteilnehmer die Ergebnisse seines Handelns in Form von Ergebnisberichten zur Verfügung gestellt, ${ }^{829}$ wodurch er eine Rückmeldung über die Qualität seines Handelns bekommt; denn im Lichte eines Ergebnisses erfahren Handlungen eine gewisse Objektivierung. ${ }^{830} \mathrm{Er}$ ist dann aufgefordert, die Ergebnisse zu reflektieren, ${ }^{831} \mathrm{um}$ in einer weiteren Entscheidungsrunde erneut $\mathrm{zu}$ handeln. ${ }^{832}$ Dabei unternimmt der Planspielteilnehmer den Versuch, sein bereits vorhandenes Wissen zu dem zu lernenden Sachverhalt in Beziehung zu setzen ${ }^{833}$ und dadurch einen Wissenserwerbsprozeß zu vollziehen. ${ }^{834}$

Die Ergebnisse stellen damit ein Reflektionsinstrument für den Teilnehmer dar gleichzeitig sind sie aber auch Ausdruck individueller sowie gruppenbezogener Handlungskompetenz. Allgemein kann Handlungskompetenz dabei als die Fähigkeit angesehen werden, schematisches Handlungswissen anzuwenden und damit etwas zu »Können«. ${ }^{835}$ In einem weiteren Begriffsverständnis definiert Volpert die dem Individuum eigene Handlungskompetenz ,[...] als die dem Individuum zur Verfügung stehenden Regel- und Elementesysteme zur Erzeugung realisierbarer Pläne“. 836

\footnotetext{
828 Vgl. Abschnitt 5.1: Das Unternehmensplanspiel: Ein komplexes Lehr-Lern-Arrangement.

829 Vgl. Abschnitt 3.2.2: Ablauf von Unternehmensplanspielen.

830 Vgl. Aebli, H., (Denken 1981), S. 84.

831 Vgl. Abschnitt 6.6.1.3: Metakognition: Die Steuerung des eigenen Denkens.

832 Vgl. Abschnitt 2.1.2.2: Das Unternehmensplanspiel: Ein Entscheidungsspiel.

833 Vgl. Abschnitt 6.2.2: Vorwissen: Spiegel des Erfahrungshintergrundes eines Individuums.

834 Vgl. Abschnitt 6.6.1.1: Interpretation kognitiver betriebswirtschaftlicher Lernprozesse durch die $\mathrm{ACT}^{*}$-Theorie.

835 Vgl. Aebli, H., (Denken 1980), S. 83 ff.

836 Volpert, W., (Zusammenhang 1979), S. 27.
} 
In der Betriebswirtschaftslehre wird die Handlungskompetenz des Individuums als eine spezielle Schlüsselqualifikation angesehen, ${ }^{837}$ wobei der Begriff der Schlüsselqualifikationen seinen Ursprung in der Arbeitsmarktforschung hat ${ }^{838}$ und später auf die Berufsbildung übertragen wurde. ${ }^{839}$ Unter der Annahme eines epistemologischen Subjektmodells ${ }^{840}$ sowie der Handlungsorientierung des Unternehmensplanspiels und dem damit in Zusammenhang stehenden Handlungsbegriff ${ }^{841}$ kann Handlungskompetenz an dieser Stelle als die Fähigkeit verstanden werden, „[...] ein Verhalten zu zeigen, das vom Handlungssubjekt bewußt, zielorientiert und gegenstandsbezogen und unter Berücksichtigung des situativen und historischen Kontextes sowie der geltenden Regeln und Normen geplant, durchgeführt und kontrolliert wird“842.

In einer von Bloech, Kauer \& Orth durchgeführten Untersuchung bezüglich des Entscheidungsverhaltens von kaufmännischen Auszubildenden in konkreten betrieblichen Problemstellungen konnte nun festgestellt werden, daß das im Unterricht erworbene Sachwissen nicht oder nur selten angewendet wird, was als Mangel an unternehmerischer Handlungskompetenz interpretiert wurde. ${ }^{843}$ Ein Teilnehmer eines Planspiels, dem es an unternehmerischer Handlungskompetenz mangelt, sieht sich demgemäß im Umgang mit einem Unternehmensplanspiel mit einem Problem konfrontiert und kann als Problemlöser angesehen werden. Dabei erfordert eine Problemlösung, im Unterschied zu einer Aufgabe, für die eine bekannte Lösungsmethode zur Verfügung steht und lediglich reproduktives Denken erforderlich ist, die Entwicklung von etwas Neuem. ${ }^{844}$

837 Vgl. zu dieser Sichtweise in Zusammenhang mit Unternehmensplanspielen Bloech, J.; Hartung, S.; Kauer, G.; Orth, C., (Einsatz 1996), S. 16 ff.; Hartung, S., (Förderung 2000), S. 29 ff.; Orth, C., (Unternehmensplanspiele 1999), S. 57 ff.

Vgl. Mertens, D., (Schlüsselqualifikationen 1975).

839 Vgl. Beck, H., (Schlüsselqualifikationen 1995), S. 12 ff. Vgl. auch Arnold, R., (Schlüsselqualifikationen 1996); Breyde, C., (Schlüsselqualifikationen 1996); Bunk, G.P.; Kaiser, M.; Zedler, R., (Schlüsselqualifikationen 1991); Dubs, R., (Schlüsselqualifikationen 1996); Elbers, D.; Heckenauer, M.; Mönikes, W.; Pornschlegel, H.; Tillmann, H., (Schlüsselqualifikationen 1975); Goetze, W., (Qualität 1996); Kaiser, A., (Schlüsselqualifikationen 1992).

840 Vgl. Abschnitt 3.4.3.2.1: Das epistemologische Subjektmodell.

841 Vgl. Abschnitt 5.1: Das Unternehmensplanspiel: Ein komplexes Lehr-Lern-Arrangement.

842 Flothow, K., (Förderung 1992), S. $52 \mathrm{f}$.

843 Vgl. Bloech, J.; Kauer, G.; Orth, C., (Unternehmensplanspiele 1996), S. 38. Auf diese Untersuchung wurde bereits in Zusammenhang mit der Untersuchung von Hartung eingegangen, deren Überlegungen sich darauf stützten. Vgl. Abschnitt 2.2.2.3.2: Zum Zusammenhang von Komplexität und Lernerfolg. 
Die Erarbeitung sachgerechter Problemlösungen hängt dabei von der Entscheidungskompetenz des Problemlösers ab und kann für die Entscheidungskompetenz im Planspiel wie folgt angenommen werden: „Ein Auszubildender eines Planspielkurses verfügt über eine ausgeprägtere unternehmerische Entscheidungskompetenz, wenn er dauerhaft in der Lage ist, in unterschiedlichen Anforderungssituationen hinsichtlich Unternehmenslage und konjunktureller Rahmenbedingungen unternehmerische Entscheidungen zu treffen, die zu höheren Unternehmensgewinnen führen, als dies Entscheidungen anderer Personen tun. “845

Da für die weiteren Betrachtungen die Qualität des Wissens relevant ist, kann in diesem Zusammenhang die Definition des betriebswirtschaftlichen Experten als einer Person, die sowohl über ein umfangreiches bereichsspezifisches Wissen als auch über generalisierbares Systemwissen sowie weiterhin heuristisches Wissen verfügt, ${ }^{846}$ herangezogen und die Entscheidungskompetenz wie folgt in Wissensgrößen ausgedrückt werden: Ein Auszubildender eines Planspielkurses verfügt über eine ausgeprägtere unternehmerische Entscheidungskompetenz, wenn er dauerhaft in der Lage ist, in unterschiedlichen Anforderungssituationen hinsichtlich Unternehmenslage und konjunktureller Rahmenbedingungen unter Anwendung seines bereichsspezifischen Wissens, des vorhandenen generalisierbaren Systemwissens sowie des heuristischen Wissens unternehmerische Entscheidungen zu treffen, die zu höheren Unternehmensgewinnen führen als dies Entscheidungen anderer Personen tun.

In dieser Definition kommt nun zum Ausdruck, was in Kapitel drei bereits ausgeführt werden konnte. ${ }^{847}$ Dies ist die Tatsache, daß die Qualität des Wissens Einfluß auf den Umgang mit komplexen Systemen hat, wobei Expertentum zu qualitativ besseren Entscheidungen führt als Novizentum. Sie können auch als optimalere Entscheidungen bezeichnet werden. Da das Vorwissen in der vorliegenden Arbeit als Spiegel des Erfahrungshintergrundes eines Individuums angenommen

\footnotetext{
845 Bloech, J.; Kauer, G.; Orth, C.,(Unternehmensplanspiele 1996), S. 40.

846 Vgl. Abschnitt 3.3.3: Untersuchungsdesigns zum Umgang von Personen mit komplexen betriebswirtschaftlichen Systemen.

847 Vgl. Abschnitt 3.3: Umgang von Personen mit komplexen Systemen.
} 
wurde, ${ }^{848}$ kann davon ausgegangen werden, daß die Unterschiede bezüglich des Erfahrungshintergrundes von Experten und Novizen offensichtlich Ausdruck eines Entwicklungsprozesses sind, der unter der Annahme eines Zeitpfades vom Experten bereits abgeschlossen ist, während er vom Novizen noch zu vollziehen ist. Die Qualität des Expertenwissens kann demnach Aufschluß darüber geben, welche Größen im Lernprozeß des Novizen eine Rolle spielen.

In diesem Zusammenhang wird hier nun davon ausgegangen, daß sich bei der Konzeption einer didaktischen Maßnahme auch die Frage nach den objektiven Anforderungen eines Unternehmensplanspiels stellt. So können aus der Beschreibung der Aufgabenumwelt zwar keine Rückschlüsse darüber gezogen werden, wie der Problemraum des Probanden aufgebaut ist, ${ }^{849}$ es können jedoch Aussagen darüber gemacht werden wie er aufgebaut sein müßte, damit der Umgang mit komplexen Systemen besser gelingt.

Es ist hier die objektive Komplexität eines Unternehmensplanspiels von Interesse, wobei Komplexität in Kapitel vier bereits als attributive Eigenschaft von Systemen angenommen werden konnte; und Komplexitätsdimensionen bestimmt sowie ein Komplexitätsmaß abgeleitet wurden. ${ }^{850}$ Im Umgang mit einem Unternehmensplanspiel stehen diese Komplexitätsdimensionen nun in Zusammenhang mit dem Lernprozeß, der sich über Entscheidungshandeln vollzieht. Da Komplexität als attributive Eigenschaft von Systemen angenommen wurde, stellt sie in $\mathrm{Zu}$ sammenhang mit dem zu erlernenden Wissens offensichtlich eine Dimension des generellen Systemwissens, welches über Entscheidungshandeln erworben wird, dar. Dabei wird dieses Systemwissen in konstruktiver, ganzheitlicher Weise erworben, wonach hier vom konstruktiven Systemansatz auszugehen ist, der das Gesamtsystem systematisch in seine Einzelteile zerlegt, wieder zusammensetzt und weiter zerlegt bis jede Unklarheit ausgeräumt ist. ${ }^{851}$ Das impliziert auch, daß

848 Vgl. Abschnitt 6.2.2: Vorwissen: Spiegel des Erfahrungshintergrundes eines Individuums.

849 Vgl. Süss, H.-M., (Intelligenz 1996), S. 7.

850 Vgl. Kapitel 4: Das Unternehmensplanspiel als Modell über einen komplexen Gegenstandsbereich.

851 In diesem Sinne ist der Systemansatz als Aufbautechnik zu verstehen, für den die Begriffe der Gestaltung, der Reflektik und der Konstruktion charakteristisch sind. Dabei meint Gestaltung die Einwirkung auf ein System, die Reflektik die Gestaltungsverantwortung, die Reflexion sowohl nach innen als auch nach außen erfordert sowie Konstruktion die Schaffung von etwas Funktionsfähigem. Vgl. Müller-Merbach, H., (Arten 1992), S. 861 ff. An 
sie, die Komplexität, objektive Charakteristika aufweist, die durch den Entscheider subjektiv wahrgenommen werden. Sollen die Anforderungen von Komplexität nun näher beleuchtet werden, so muß auf der einen Seite offensichtlich ihrem objektiven Charakter auf der anderen ihrer subjektiven Wahrnehmung entsprochen werden.

Dazu wird Komplexität im folgenden als attributive Eigenschaft von Entscheidungen angenommen; wobei zum einen von der objektiven Komplexität einer Entscheidung und zum anderen von der subjektiven Komplexität einer Entscheidung auszugehen ist; und sowohl subjektive als auch objektive Komplexitätsdimensionen, die in Zusammenhang mit dem Lernprozeß im Unternehmensplanspiel stehen, abgeleitet werden können.

\section{Komplexität als attributive Eigenschaft von Entscheidungen}

\subsubsection{Die objektive Komplexität einer Entscheidungssituation}

Für Kloidt, Dubberke \& Göldner ist das Entscheiden, wie auch die damit verbundenen Vorüberlegungen, zunächst insoweit komplex, als es sich hier um Aufgaben handelt, bezüglich derer der Entscheidungsträger die Möglichkeit hat, diese in Teilaufgaben zu zerlegen. ${ }^{852}$ Weiterhin hat ein Entscheidungsträger die Möglichkeit, auf das Wahrnehmen von Komplexität durch Informationssuche, deren Verarbeitung, durch Verhandlungen mit Markt- und systeminternen Partnern, durch Organisation des Entscheidungsprozesses mit Hilfe von Prozedurregelungen, die mit der Zielbildungsaktivität verknüpft sind, zu reagieren. Dabei kann die Komplexität einer realen Entscheidungssituation bspw. wie die von Dörner, Kreuzig, Reither \& Stäudel in ihrer Lohhausen-Studie beschriebene Entscheidungssituation eines Bauunternehmers aussehen:

diesem Systemansatz richtet auch die Wirtschaftsinformatik die ganzheitliche Gestaltung von Informationssystemen aus. Vgl. Biethahn, J.; Muksch, H.; Ruf, W., (Informationsmanagement 2000), S. 84 ff. Vgl. zur Vorgehensweise bei der Gestaltung eines ganzheitIchen Informationssystems Biethahn, J.; Mucksch, H.; Ruf, W., (Informationsmanagement 1991), S. $18 \mathrm{ff}$.

Vgl. Kloidt, H.; Dubberke, H.-A.; Göldner, J.,(Problematik 1959), S. 19. 
„Ein Bauunternehmer verhandelt mit einem Kunden. Dieser verlangt von ihm für die Fertigstellung eines Rohbaus eine bindende Terminzusage. Er verlangt diese Zusage sehr schnell, da er andernfalls zu einem anderen Unternehmer gehen will. Der Unternehmer muß das Risiko von Gewinn und Verlust gegeneinander abwägen. Das ist aber nicht einfach. Es geht gegen den Winter und es ist unklar, ob und wie lange der Bau wegen Frost eingestellt werden muß. Mit welchem Krankenstand seiner Leute muß er rechnen? Einzelne Gewerke müssen an Subunternehmer vergeben werden. Haben diese in der entsprechenden Zeit die entsprechende Kapazität? Ist mit einer Erhöhung der Energiepreise zu rechnen? In welcher Höhe könnte diese liegen? - jenseits dieser denkbaren und in ihrer Wahrscheinlichkeit zumindest grob abschätzbaren Ereignisse können noch solche auftreten, an die im Augenblick gar nicht gedacht wird. - Angesichts aller dieser Unwägbarkeiten muß der Leiter der Unternehmung zu einem Entschluß kommen." ${ }^{853}$

Bei der Komplexität im Unternehmensplanspiel erfolgt hingegen durch die Modellbildung eine Abstraktion über die realen Gegebenheiten, ${ }^{854}$ so daß in diesem Zusammenhang nicht von einer realen Komplexität auszugehen ist, sondern die in Kapitel vier als Abbildungskomplexität ${ }^{855}$ bezeichnete Komplexität in Zusammenhang mit dem Lernprozeß steht. Von Löfgren wird diese Art von Komplexität als Beschreibungskomplexität bezeichnet: „Associated with the learning process is the complexity of describing, the d-complexity. It is measured by the difficulty associated with extracting the description of a system $[\ldots]^{\text {“. } .856}$

Weiterhin kann im Hinblick auf die spezifische Simulationstechnik beim Planspiel nach Simulationsmodell und Entscheidungsspiel unterschieden werden, da

853 Dörner, D.; Kreuzig, H.W.; Reither, F.; Stäudel, T. (Hrsg.),(Lohhausen 1983), S. 19.

854 Vgl. Abschnitt 2.1.2.1: Das Unternehmensplanspiel: Ein Simulationsmodell.

855 Vgl. Abschnitt 4.3.1.2: Komplexität im Unternehmensplanspiel.

856 Löfgren, L., (Complexity 1977), S. 198. 
das Modell im Spiel zum Gegenstand der vorgesehenen Geschäftsmaßnahmen wird. ${ }^{857}$ Dabei ist es nicht zwingend der Fall, daß Simulationsmodell und Entscheidungsspiel den gleichen Umfang haben. Der Anteil des Steuerungsmodells am Gesamtmodell macht vielmehr, je nach Spielform, einen unterschiedlichen Umfang aus. Bleicher unterscheidet hierbei drei Ausprägungen. Zum einen kann das Steuerungsmodell im Rahmen des Gesamtmodells den größten Raum einnehmen. Zum zweiten können Steuerungsmodell und Simulationsmodell in etwa den gleichen Umfang haben. Drittens schließlich kann das Simulationsmodell den größten Raum einnehmen. ${ }^{858}$

Dörner, Kreuzig, Reither \& Stäudel sprechen nun in ihrer Lohhausen-Studie von einem partiell beeinflußbaren Realitätsausschnitt und unterscheiden die Variablen nach partiell beeinflußbaren und partiell unbeeinflußbaren Variablen. ${ }^{859}$ Somit sind die an den Wirkungszusammenhängen in einem Planspiel beteiligten Variablen zum einen dem Steuerungsmodell, zum anderen dem Simulationsmodell zuzurechnen. Es erscheint daher naheliegend, in bezug auf den Lernprozeß eine Unterscheidung zwischen systemtheoretischer und entscheidungstheoretischer Komplexität vorzunehmen, ${ }^{860}$ da die Teilnehmer an einem Planspiel mit einer durch die direkt beeinflußbaren Variablen hervorgerufenen Entscheidungskomplexität konfrontiert sind. $\mathrm{Zu}$ beachten ist jedoch, daß auch die nichtbeeinflußbaren Variablen Einfluß auf die Entscheidung nehmen. Dies konnte in der Betrachtung der Entscheidungskomplexität im Planspiel EpUS bereits aufgezeigt werden. ${ }^{861}$

857 Vgl. Abschnitt 2.1.2.1: Das Unternehmensplanspiel: Ein Simulationsmodell und Abschnitt 2.1.2.2: Das Unternehmensplanspiel: Ein Entscheidungsspiel.

858 Vgl. Bleicher, K., (Unternehmungsspiele 1969), Sp. 1689.

859 Vgl. Dörner, D.; Kreuzig, H.W.; Reither, F.; Stäudel, T. (Hrsg.),(Lohhausen 1983), S. $26 \mathrm{ff}$.

860 Zu einer Unterscheidung von systemtheoretischer und entscheidungstheoretischer Komplexität vgl. Hauschildt, J., (Komplexität 1990), S. 132. Zu einer Unterscheidung zwischen systemtheoretischer und entscheidungstheoretischer Komplexität in Zusammenhang mit Unternehmensplanspielen vgl. Orth, C., (Unternehmensplanspiele 1999), S. 66 ff.

861 Vgl. Abschnitt 3.4.5.2: Darstellung der Entscheidungssituation im Planspiel EpUS. Bei der stochstischen Simulation wird auch Ungewissheit als Modellbestandteil zugelassen. Um Modellkomplexität zu reduzieren wird jedoch oft darauf verzichtet, obgleich sie wesentichen Einfluß auf die Entscheidungen haben kann. Vgl. Nissen, V.; Biethahn, J., (Beispiel 1999), 108. Sie gehört aber in keinem Fall zu den beeinflußbaren Variablen. 
In diesem Zusammenhang vertritt Hauschildt die Sichtweise, daß aus entscheidungstheoretischer Perspektive Komplexität nicht die Eigenschaft eines Systems, sondern eines Problems darstellt ${ }^{862}$ und daher gleichsam von Problemkomplexität gesprochen werden kann. So führt dieser aus: „Die entscheidungstheoretische Betrachtungsweise sieht Komplexität als die Eigenschaft eines Problems, spricht also genaugenommen von Problemkomplexität. Sie unternimmt den Versuch, die Komplexität von außen, objektbezogen zu bestimmen, also nicht die Perspektive des handelnden Entscheidungsträgers einzunehmen.“ Der Terminus Problemkomplexität beinhaltet jedoch bereits den subjektiven Bezug, da Probleme keine objektiven Realitäten darstellen, die unabhängig von den Subjekten bestehen. Vielmehr hängt es von einer Person ab, ob eine Situation als Problem bezeichnet werden kann. ${ }^{863}$

Hier soll Komplexität daher, in bezug auf die durch die Teilnehmer direkt beeinflußbaren Variablen, als Kontrollkomplexität angesehen werden. ${ }^{864} \mathrm{Sie}$, die Kontrollkomplexität, stellt demgemäß die objektive Komplexität einer Entscheidungssituation im Unternehmensplanspiel dar; und steht der subjektiv wahrgenommenen Komplexität auf seiten des Individuums gegenüber, auf die im folgenden eingegangen werden soll.

\subsubsection{Die subjektive Komplexität einer Entscheidungssituation}

Die Komplexität vom Objekt her abzuleiten ist ein häufig angewandtes Verfahren, wobei jedoch die Wahrnehmung von Komplexität durch den Entscheidungsträger nicht berücksichtigt wird. ${ }^{865}$ Ein System ist aber immer als komplex im Hinblick auf ein Gegenüber zu betrachten, ${ }^{866}$ was es notwendig erscheinen läßt, die objektive Komplexität einer Situation von der durch einen Entscheidungsträger subjektiv wahrgenommenen Komplexität abzugrenzen. Diese Relativität und Subjektbe-

862 Vgl. Hauschildt, J., (Komplexität 1990), S. 132.

863 Fisch, R.; Wolf, M.F., (Handhabung 1990), S. 12.

864 Der Terminus Kontrollkomplexität findet sich bei Funke. Soll er hier jedoch als die durch einen Teilnehmer des Planspiels beeinflußbaren Variablen verstanden werden, so bezeichnet Funke diese als Kontrollvariablen. Kontrollkomplexität drückt bei Funke hingegen das Verhältnis zwischen Störungs- und Kontrollvariablen aus. Vgl. Funke, J., (Diagnose 1984), S. 163.

865 Kirsch, W., (Handhabung 1988). 
zogenheit der Komplexität betont auch Dörner, da je nach Wissensstand etwas komplex oder nicht komplex erscheint. Der Umfang der Komplexität steht somit in Relation zum Handlungsrepertoire, über welches die Teilnehmer verfügen, ${ }^{867}$ womit die Komplexität, die durch einen Entscheidungsträger subjektiv wahrgenommen wird, von der Transparenz abhängt, mit der ein System wahrgenommen wird. ${ }^{868}$ Diese Komplexität wird hier als Kognitive Komplexität bezeichnet.

In einem interaktiven Ansatz wird nun die Wechselbeziehung zwischen der Kontrollkomplexität, im weiteren Sinne auch der Abbildungskomplexität, und dem Grad der Kognitiven Komplexität eines Individuums wichtig.

Es konnte dabei bereits festgehalten werden, daß sich zwischen den Annahmen der normativen Entscheidungstheorie und den Annahmen der deskriptiven Entscheidungstheorie eine Differenz identifizieren läßt. Unter dem Aspekt einer Überforderung des menschlichen Auffassungsvermögens bei kontinuierlicher Optimierung konnte diese nach Bronner auch als »duale Spannungslage« bezeichnet werden. Darunter wurde verstanden, daß die Existenz und Bewältigung eines inhaltlich bestimmten Sachproblems der Existenz und Bewältigung von psychischkognitiv bestimmten Streß gegenübersteht, wobei die Erarbeitung sachgerechter Problemlösungen geradezu als Funktion der Streßüberwindung begriffen wird. ${ }^{869}$

Da Komplexität nun als attributive Eigenschaft von Entscheidungen angenommen wurde, ist weiterhin davon auszugehen, daß auch zwischen objektiver und subjektiver Komplexität, respektive zwischen der Kontrollkomplexität und der Kognitiven Komplexität, eine Differenz besteht. Sie, die Differenz, wirkt auf die »duale Spannungslage« und führt damit zu einer Erhöhung des psychisch-

\footnotetext{
866 Vgl. bspw. Dörner, D., (Logik 1999), S. 61 f.; Witte, E., (Informations-Verhalten 1972), S. 27.

867 Kastner, M., (Denken 1995), S. 31.

868 Vgl. Kirsch, W., (Handhabung 1988), S. 161.

869 Vgl. Abschnitt 6.1.3: Die operative Differenz zwischen normativer und deskriptiver betriebswirtschaftlicher Entscheidungstheorie als Ausgangspunkt der Analyse.
} 
kognitiven Stresses, der im weiteren als Problemkomplexität bezeichnet werden soll. ${ }^{870}$ Lernprozessbezogene Komplexität im Unternehmensplanspiel

\title{
6.5.1
}

\author{
Dimensionen der Kontrollkomplexität im Unternehmensplan- \\ spiel
}

In den vorangegangenen Ausführungen ließen sich verschiedene Komplexitätsdimensionen entwickeln, die in Zusammenhang mit dem Lernprozeß stehen. Diese werden hier noch einmal im Überblick dargestellt und in einen Beziehungszusammenhang mit den in Kapitel vier entwickelten Komplexitätsdimensionen des offenen Systems gestellt. ${ }^{871}$

Dies geschieht in Anlehnung an den von Kieras \& Polson beschriebenen Zusammenhang: „To describe user complexity fully and usefully, it is essential to be able to describe the device in a way that can be related to the knowledge required to operate it“. .872 Dabei wird 'knowledge' in diesem Zusammenhang als generalisierbares Systemwissen verstanden, wozu Komplexität in ihrer Eigenschaft als attributive Eigenschaft von Systemen zu zählen ist. ${ }^{873}$

Die zusammenfassende Darstellung der Komplexitätsdimensionen, die in $\mathrm{Zu}$ sammenhang mit dem Lernprozeß stehen, ergibt sich zunächst wie folgt:

In Zusammenhang mit dem Lernprozeß steht zunächst die in Kapitel vier angeführte Abbildungskomplexität. ${ }^{874}$ Sie kann daher hier als erste Komplexitätsdimension angeführt werden. Weiterhin wurde im Hinblick auf die Unterscheidung zwischen Simulationsmodell und Entscheidungsspiel eine Unterscheidung zwischen systemtheoretischer und entscheidungstheoretischer Komplexität vorge-

\footnotetext{
870 Es soll angemerkt werden, daß hier keine Aussage darüber gemacht werden kann, ob hier nur eine Verstärkung des psychisch-kognitiven Stresses vorliegt oder die Komplexität möglicherweise dessen Auslöser ist.

871 Vgl. Abschnitt 4.3.1: Differenzierung und Komplexität im Unternehmensplanspiel.

872 Kieras, D.; Polson, P.G., (Approach 1985), S. 365.

873 Vgl. Abschnitt 4.2.2: Komplexität als attributive Eigenschaft von Systemen sowie Abschnitt 6.3: Die unternehmerische Handlungskompetenz.
} 
nommen. Dabei kann die entscheidungstheoretische Komplexität als Entscheidungskomplexität bezeichnet werden. Diese ist dann wiederum nach beeinflußbaren und nicht-beeinflußbaren Variablen zu unterscheiden, wobei die Entscheidungskomplexität, welche die beeinflußbaren Variablen umfaßt, als Kontrollkomplexität bezeichnet wird. ${ }^{875}$ Die Kognitive Komplexität meint schließlich die subjektive Wahrnehmung von Komplexität auf Seiten des Individuums und beruht auf komplexitätsabhängigen Faktoren der Aufgabenschwierigkeit. ${ }^{876}$

Es lassen sich daher die folgenden Dimensionen von Komplexität feststellen:

\begin{tabular}{|c|c|}
\hline KOMPLEXITÄTSDIMENSION I & Abbildungskomplexität \\
\hline KOMPLEXITÄTSDIMENSION II & Entscheidungskomplexität \\
\hline N III & Kontrollkomplexität \\
\hline OMPLEXITÄTSDIMENSIO & Kognitive Komplexität \\
\hline
\end{tabular}

Die in Kapitel vier abgeleiteten Komplexitätsgrade und Komplexitätsdimensionen eines offenen Systems stehen nun in Zusammenhang mit der Komplexitätsdimension Kontrollkomplexität, wobei stets auf die beeinflußbaren Variablen abgestellt wird. Sie seien hier noch einmal angeführt:877

\begin{tabular}{|c|c|}
\hline KOMPLEXITÄTSDIMENSION II & Komplexitätsgrad K II \\
\hline KOMPLEXITÄTSDIMENSION III & Komplexitätsgrad K III \\
\hline KOMPLEXITÄTSDIMENSION IV & Komplexitätsgrad K IV \\
\hline KOMPLEXITÄTSDIMENSION V & Komplexitätsgrad $K V$ \\
\hline KOMPLEXITÄTSDIMENSION VI & Komplexitätsdimension K VI \\
\hline KOMPLEXITÄTSDIMENSION VII & Komplexitätsdimension K VII \\
\hline KOMPLEXITÄTSDIMENSION VIII & Komplexitätsdimension K VIII \\
\hline
\end{tabular}

\footnotetext{
874 Vgl. Abschnitt 6.4.1: Die objektive Komplexität einer Entscheidungssituation.

875 Vgl. Abschnitt 6.4.1: Die objektive Komplexität einer Entscheidungssituation.

876 Vgl. Abschnitt 6.4.2: Die subjektive Komplexität einer Entscheidungssituation.

877 Vgl. Abschnitt 4.3.1.2: Komplexität im Unternehmensplanspiel und Abschnitt 4.3.2: Entwicklung eines Komplexitätsmaßes für das offene System der Unternehmung.
} 
Es wurde nun davon ausgegangen, daß objektive und subjektive Komplexität in einer Wechselbeziehung stehen. Da die objektive Komplexität hier die Kontrollkomplexität darstellt, steht sie in Wechselbeziehung mit der subjektiven Wahrnehmung der Kontrollkomplexität, also der Kognitiven Komplexität, auf seiten eines Teilnehmers an einem Unternehmensplanspiel. Die unter der Komplexitätsdimension Kontrollkomplexität angenommenen Komplexitätsgrade und Komplexitätsdimensionen KI - KVIII stellen daher die potentiellen Wirkfaktoren auf die Kognitive Komplexität eines Teilnehmers dar. Wird in diesem Zusammenhang die Kontrollkomplexität, mit den Komplexitätsgraden und Komplexitätsdimensionen KI - KVIII, in Beziehung zu der Kognitiven Komplexität auf seiten des Teilnehmers gestellt, so läßt sich eine potentielle Differenz zwischen objektiver und subjektiver Komplexität auf seiten des Teilnehmers als Problemkomplexität identifizieren. [Vgl. Abbildung 6-2].

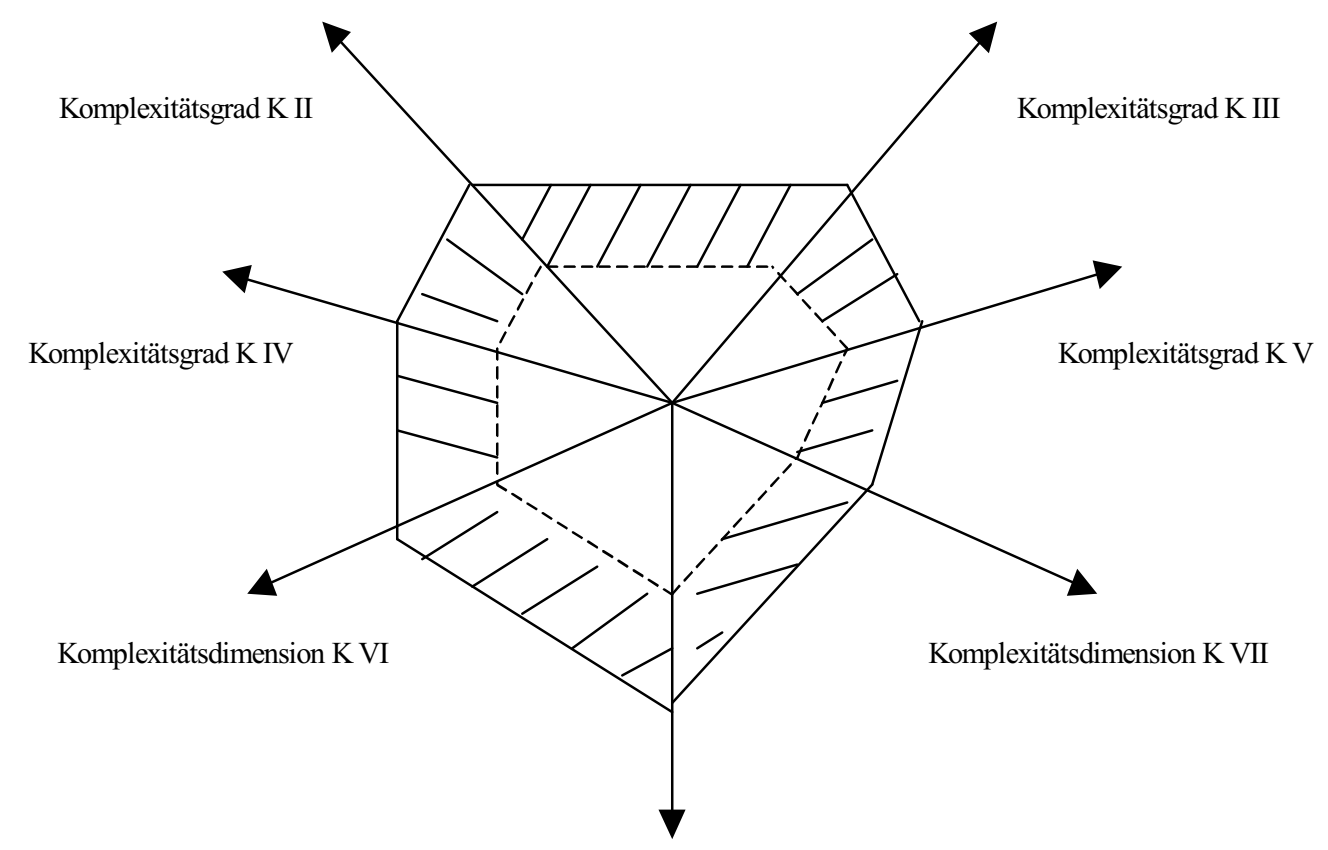

Komplexitätsdimension K VIII

Abbildung 6-2: Die Kontrollkomplexität eines offenen Systems und ihr Zusammenhang mit der Kognitiven Komplexität eines Planspielteilnehmers mit

= Kontrollkomplexität: objektive Komplexität des Unternehmensplanspielmodells in bezug auf die beeinflußbaren Variablen 
$=$ Kognitive Komplexität: in bezug auf die beeinflußbaren Variablen subjektiv wahrgenommene Komplexität eines Teilnehmers

$=$ Der Grad an Problemkomplexität eines Teilnehmers

Wird diese Problemkomplexität nun in Zusammenhang mit Personenmerkmalen gestellt, so kann sie als Systemschwierigkeit identifiziert werden, worauf im folgenden eingegangen werden soll.

Die Systemschwierigkeit: Ausdruck der Problemkomplexität in Wissensgrößen

Im vorangehenden Abschnitt wurde davon ausgegangen, daß auf seiten des Teilnehmers an einem Unternehmensplanspiel eine potentielle Differenz zwischen objektiver und subjektiver Komplexität, respektive Kontrollkomplexität und Kognitiver Komplexität existiert. Diese wurde als Problemkomplexität bezeichnet. ${ }^{878}$ Weiterhin wurde Komplexität als attributive Eigenschaft von Entscheidungen angenommen. ${ }^{879}$ Es kann daher davon ausgegangen werden, daß sich der Umfang an Problemkomplexität auf seiten des Individuums auf sein Entscheidungsverhalten auswirkt und es erscheint daher naheliegend, Problemmerkmale und Personenmerkmale gegenüberzustellen.

Solch eine Gegenüberstellung findet sich bei Hussy. Er stellt Problemmerkmale versus Personmerkmale unter dem Terminus Problemschwierigkeit gegenüber. Dabei sieht er als Problemmerkmale die Anzahl der Variablen, deren Vernetztheit sowie deren Transparenz an und faßt diese unter dem Terminus Problemkomplexität zusammen. Diese kann, nach Fisch \& Wolf, unter Anwendung der systemtheoretischen Komplexitätsdimensionen von Luhmann $^{880}$, auch weiter gefaßt werden. Sie definieren wie folgt: „Die Komplexität eines Problems kann definiert werden erstens durch die Anzahl seiner Elemente, zweitens durch die Menge der Beziehungen zwischen diesen Elementen (Relationen) und drittens durch die Ver-

878 Vgl. Abschnitt 6.5.1: Dimensionen der Kontrollkomplexität im Unternehmensplanspiel.

879 Vgl. Abschnitt 6.4: Komplexität als attributive Eigenschaft von Entscheidungen. 
schiedenartigkeit dieser Beziehungen, wobei oft nur die Relation »Abhängigkeit» berücksichtigt wird. Als vierter Aspekt gilt vielfach die Zeitdimension, wobei die Veränderungen der Komplexitätskonstituenten pro Zeiteinheit berücksichtigt werden $[\ldots]^{“}{ }^{881}$ In einem weiteren Problemzusammenhang nennen sie noch die Komponenten der Anzahl und der Verschiedenartigkeit der Beziehungen zwischen einem Problem und anderen Problemen. ${ }^{882}$

Sowohl Hussy als auch Fisch \& Wolf gehen damit von einer objektiven Komplexität aus, die sie als Problemkomplexität klassifizieren. Hier wird hingegen weiter davon ausgegangen, daß Probleme erst in bezug auf ein Gegenüber erwachsen. Es wird daher weiterhin von dem Terminus Kontrollkomplexität, mit den identifizierten Komplexitätsgraden und Komplexitätsdimensionen, Gebrauch gemacht. Ebenso wird infolgedessen an dieser Stelle auch nicht der Terminus Problemmerkmale gebraucht, sondern der Terminus Systemmerkmale sowie weiterhin der Begriff Systemschwierigkeit.
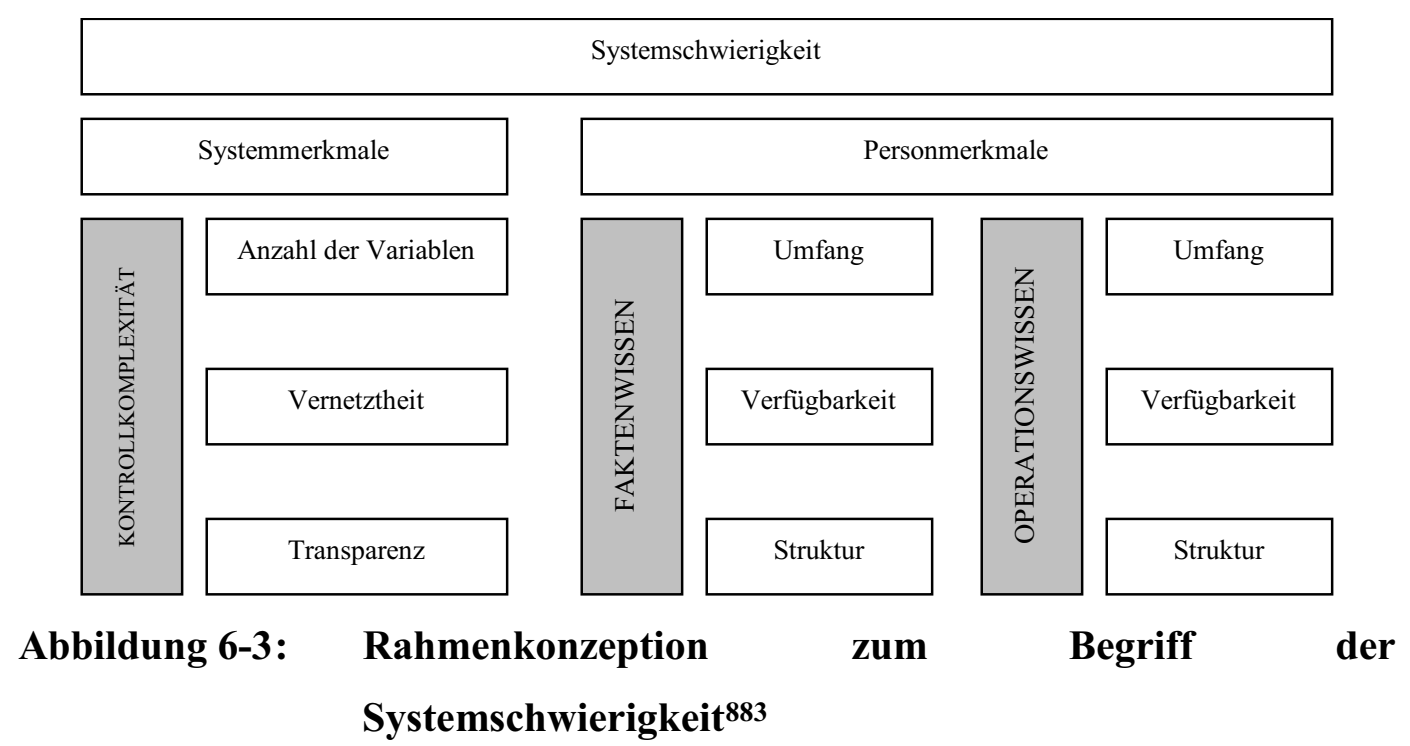

Als Personenmerkmale klassifiziert Hussy Umfang, Verfügbarkeit und Struktur des Faktenwissens sowie Umfang, Verfügbarkeit und Struktur des Operationswissens. [Vgl. unter Berücksichtigung der Termini Kontrollkomplexität, Systemmerkmale und Systemschwierigkeit Abbildung 6-3].

\footnotetext{
880 Vgl. Luhmann, N., (Komplexität 1980), Sp. 1064 f.

881 Fisch, R.; Wolf, M.F., (Handhabung 1990), S. 13.

882 Fisch, R.; Wolf, M.F., (Handhabung 1990), S. 13.
} 
Damit stehen der Kontrollkomplexität, die durch das Individuum zu kontrollieren ist, die Personmerkmale Faktenwissen und Handlungswissen gegenüber, die unter dem Begriff der Systemschwierigkeit subsumiert werden können. Wird nun davon ausgegangen, daß eine Bewältigung der Kontrollkomplexität durch den Einsatz von Wissensgrößen auf Seiten des Individuums erfolgt, so ist weiterhin davon auszugehen, daß die Problemkomplexität, die als Differenz zwischen Kontrollkomplexität und Kognitiver Komplexität angenommen wurde, auch als Wissensdefizit auf seiten des Individuums ausgedrückt werden kann.

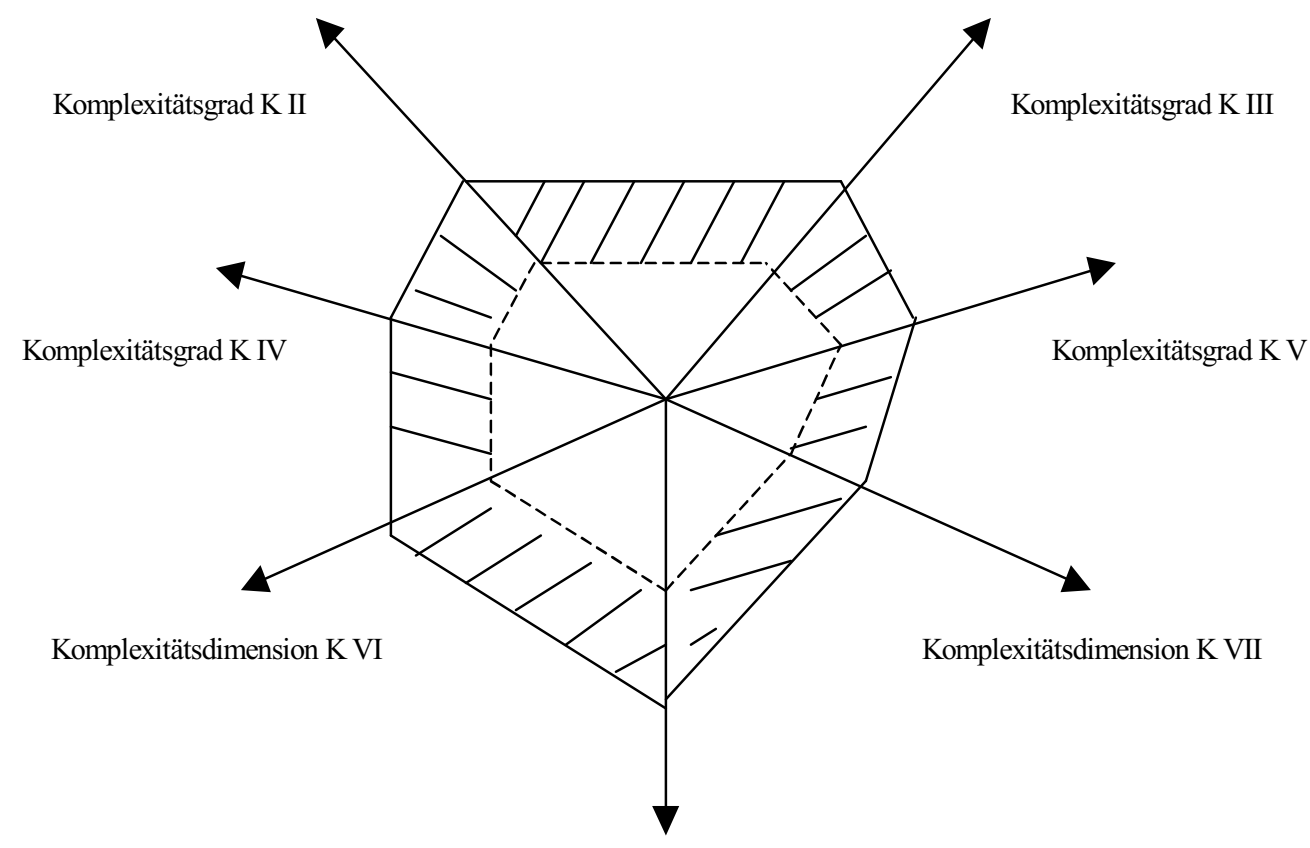

Komplexitätsdimension K VIII

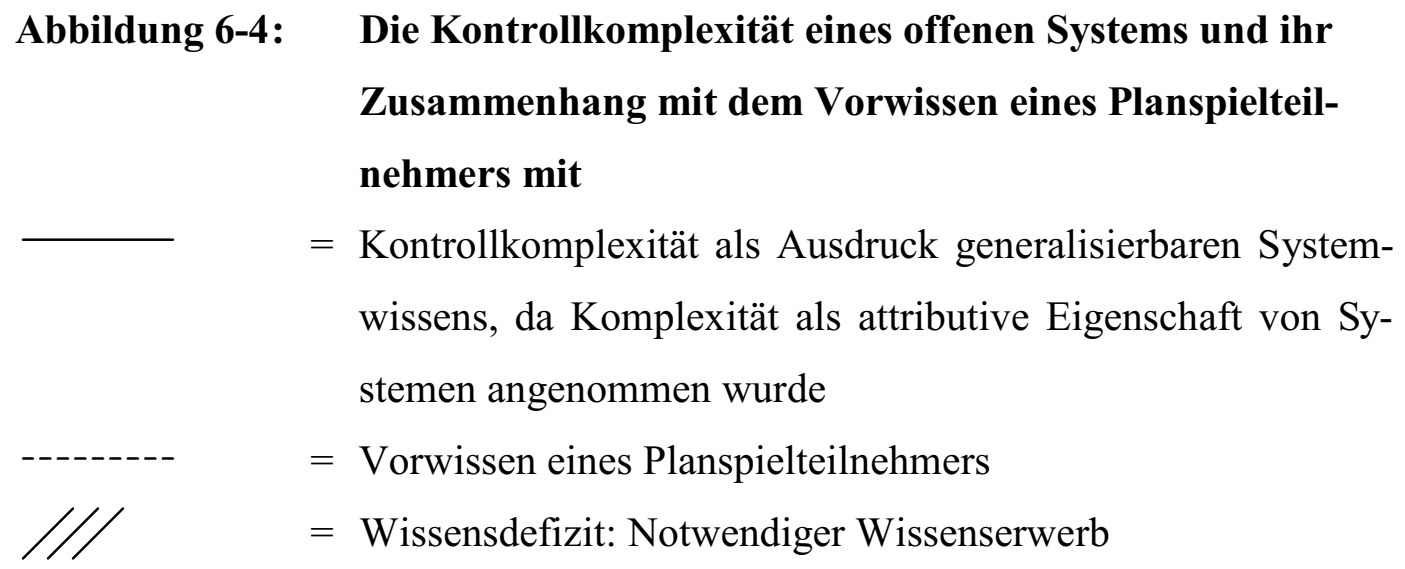


Wird dieser Sachverhalt wiederum in einem Polarkoordinatensystem dargestellt, gelingt es das Wissensdefizit formal zu bestimmen. Dabei umfaßt der Begriff Systemschwierigkeit die System- und Personmerkmale, die, unter der Annahme einer potentiellen Differenz, den Umfang an notwendigem Wissenserwerb auf seiten des Individuums ausdrücken und als Wissensdefizit bezeichnet werden können. Dabei wird unter Berücksichtigung der Notwendigkeit einer Anpassung der Planspielkomplexität an das Vorwissen der Teilnehmer ${ }^{884}$ der Begriff Vorwis$\operatorname{sen}^{885}$ verwendet. [Vgl. Abbildung 6-4].

Unter Berücksichtigung der von Hussy klassifizierten Personenmerkmale kann das Vorwissen nun wiederum als Fakten- und Operationswissen angenommen werden. Weiterhin kann unter dem Aspekt, daß Komplexität als attributive Eigenschaft eines Systems und damit als Merkmal generellen Systemwissens angenommen wurde, der Schluß gezogen werden, daß generelles Systemwissen generelles Fakten- und Operationswissen beinhaltet und die Aufstellung der folgenden Hypothese ermöglicht:

»Das generelle Systemwissen, mit den generellen Komponenten Fakten- und Operationswissen, stellt die metakognitive Komponente zu bereichsspezifischem Fakten- und Operationswissen dar.«

Für die weiteren Betrachtungen konnte in diesem Zusammenhang bereits festgestellt werden, daß ein betriebswirtschaftlicher Experte als eine Person anzusehen ist, die sowohl über ein umfangreiches bereichsspezifisches Wissen, als auch über generalisierbares Systemwissen sowie heuristisches Wissen verfügt. ${ }^{886}$ Weiterhin konnten diese Wissensarten als Bestandteile unternehmerischer Handlungskompetenz angenommen werden, womit die Relevanz dieses Aspektes für die Betriebswirtschaftslehre bereits aufgezeigt wurde. ${ }^{887}$ Findet außerdem die aufgestellte Hypothese in der Definition eines betriebswirtschaftlichen Experten Be-

884 Vgl. Abschnitt 2.2.2.3.2: Zum Zusammenhang von Komplexität und Lernerfolg.

885 Vgl. Abschnitt 6.2.2: Vorwissen: Spiegel des Erfahrungshintergrundes eines Individuums.

886 Vgl. Abschnitt 3.3.3: Untersuchungsdesigns zum Umgang von Personen mit komplexen betriebswirtschaftlichen Systemen.

887 Vgl. Abschnitt 6.3: Die unternehmerische Handlungskompetenz. 
rücksichtigung, so kann den weiteren Betrachtungen die folgende Definition zugrunde gelegt werden:

Bei einem betriebswirtschaftlichen Experten handelt es sich um eine Person, die sowohl über ein umfangreiches bereichsspezifisches Wissen, über damit in Zusammenhang stehendes metakognitives Systemwissen sowie weiterhin heuristisches Wissen verfügt, wobei diese auch an dieser Stelle als Bestandteil unternehmerischer Entscheidungskompetenz angenommen werden.

Weiterhin kann davon ausgegangen werden, daß diese Wissensarten auf seiten eines betriebswirtschaftlichen Experten in Form eines mentalen Modells repräsentiert werden, da diese als hybride Systeme angenommen werden können ${ }^{888}$. In diesem Zusammenhang ist nun auf die in Kapitel drei ausgeführten ExpertenNovizen-Vergleiche ${ }^{889}$ zurückzukommen. So ist davon auszugehen, daß ein betriebswirtschaftlicher Novize, ausgehend vom seinem Entwicklungsstand, welcher hier durch sein Vorwissen in den relevanten Dimensionen repräsentiert wird, sein mentales Modell im Umgang mit einem Unternehmensplanspiel wiederum in den relevanten Dimensionen weiterentwickelt. Diese Wissensgrößen, die des betriebswirtschaftlichen Experten, stellen folglich die Zielgröße im betriebswirtschaftlichen Lernprozeß dar.

In diesem Zusammenhang ist es nun wichtig die in Kapitel fünf angeführten Modelle der Psychologie heranzuziehen. Sie müssen hier in bezug zum betriebswirtschaftlichen Lernprozeß im Unternehmensplanspiel gestellt werden, um Aufschluß über die Informationsverarbeitung beim Menschen im Hinblick auf die für einen betriebswirtschaftlichen Experten identifizierten Wissensgrößen zu erlansteme. 
gen. Im folgenden wird dazu zunächst die ACT*-Theorie von Anderson ${ }^{890}$ wieder aufgegriffen.

6.6

Betriebswirtschaftliche Lernprozesse im Unternehmensplanspiel

6.6.1

Zur Relevanz der ACT*-Theorie in der Betriebswirtschaftslehre

6.6.1.1

Interpretation kognitiver betriebswirtschaftlicher Lernprozesse durch die $\mathrm{ACT}^{*}$-Theorie

In Zusammenhang mit Lernprozessen im Unternehmensplanspiel erfolgte durch Orth bereits eine Interpretation betriebswirtschaftlicher Lernprozesse mit der ACT*-Theorie. Auf diese Arbeit wird hier bezug genommen. Das betriebswirtschaftliche Wissen kann zunächst, unter Interpretation durch die ACT*-Theorie von Anderson, nach deklarativem und prozeduralem Wissen unterschieden werden. 891

Gemäß den Ausführungen von Orth kann davon ausgegangen werden, daß ein Planspielteilnehmer bereits vor Beginn des Planspielseminars über deklarative und prozedurale Wissensbestände verfügt. Dabei kann deklaratives betriebswirtschaftliches Wissen bspw. Wissen über Kosten und Produktionsfaktoren, oder auch über unternehmerische Teilbereiche und dazwischen existierende Interdependenzen sein. Prozedurales betriebswirtschaftliches Wissen beinhaltet bspw. Wissen über Zielbildung sowie Problemerkenntnis und -analyse, weiterhin Alternativensuche und Prognose als auch schließlich Bewertung und Entscheidung. Es kann auch als Wissen über Methoden und Techniken interpretiert werden.

Deklarativer Wissenserwerb erfolgt nun in der Informationsphase des Planspiels. Hier wird das Faktenwissen über den im Modell abgebildeten Realitätsausschnitt

890 Vgl. Abschnitt 5.5.3: Anderson's ACT*-Theorie. Zur Diskussion der in Kapitel fünf ausgeführten Produktionssysteme von Dörner, Newell und Anderson vgl. weiterhin Abschnitt 5.6: Diskussion und Gegenüberstellung der Produktionssysteme.

891 Vgl. Orth, C., (Unternehmensplanspiele 1999), S. 49 ff und die dort angegebene Literatur. 
erworben sowie weiterhin im Gedächtnis gespeichert. Dabei ist von einem lediglich schematischen und lückenhaften Wissenserwerb auszugehen, der auf den durch die Planspielunterlagen gewährten Informationen beruht. Im weiteren Spielverlauf ist jedoch von einer zunehmenden Differenzierung und Erweiterung des deklarativen Wissens auszugehen, da in den Rückkoppelungs- und Analysephasen weitere Informationen in den Spielablauf eingegeben werden sowie weiterhin die Möglichkeit besteht, Fragen an den Spielleiter zu richten.

Der prozedurale Wissenserwerb erfolgt durch Anwendung des vorhandenen, prozeduralen Wissens auf das bereits verfügbare, deklarative Wissen. Auch hier bietet der Rückkoppelungscharakter des Planspiels die Möglichkeit zu einer Ausdifferenzierung des vorhandenen Wissens. Dabei wird der Wissenserwerb durch die von Anderson beschriebenen drei Phasen des Wissenserwerbs ${ }^{892}$ angenommen. Durch das Regelkreisprinzip im Planspiel ist es dem Teilnehmer möglich, gemäß dem Prinzip der Wissenskompilierung ein prozedurales Bereichswissen aufzubauen. So erfolgt durch die wiederholte Anwendung allgemeiner Produktionen eine zunehmend automatisierte und dadurch beschleunigte Anwendung des Wissens. Der in der Phase der Wissenskompilierung aufgebaute Wissensbestand kann in der Phase der Wissensoptimierung einer Generalisation zugeführt werden. So werden hier die bereits vorhandenen Produktionen zunehmend angepaßt und neue, allgemeingültigere Regeln entworfen.

Insgesamt wird im Planspiel von einer zunehmenden Qualität des prozeduralen Wissens ausgegangen, die, entsprechend der zeitlich ausgedehnten sowie dem Regelkreisprinzip unterliegenden Planspielsituation, sukzessive aufgebaut wird. Das führt dazu, daß die Planspieler stetig ihre Entscheidungskompetenz ausbauen und zunehmend weniger Zeit benötigen, um die von ihnen erwarteten Entscheidungen zu treffen. 


\subsubsection{Kognition, Emotion und Motivation}

Neben kognitiven Faktoren nehmen, wie bereits ausgeführt werden konnte, auch die Faktoren der Motivation und der Emotion Einfluß auf den Umgang mit komplexen Systemen. ${ }^{893}$ Daher ist in diesem Zusammenhang der Aspekt zu beleuchten, ob Informationsverarbeitungsmodelle hinreichend aussagekräftig bezüglich der tatsächlichen Informationsverarbeitung beim Menschen sind; wobei in diesem Zusammenhang auch die Aussage von Lantermann zu sehen ist, der folgendes einwendet: „Psychologische Handlungstheorien scheinen mir zum gegenwärtigen Zeitpunkt weniger den Status psychologischer Theorien zu haben als vielmehr den einer normativen Setzung: sie enthalten, ein wenig überpointiert formuliert, eher Regeln, deren Befolgen, rationales, bewußtes zielgerichtetes Handeln zu garantieren scheint.“"894

So ist dagegen bspw. einzuwenden, daß aufgabenbezogene Prozesse beim Auftreten von Emotionen, die einen mittleren oder hohen Intensitätsgrad aufweisen, entweder gänzlich beendet oder nur wenig effektiv weiterverfolgt werden. Demgegenüber rückt die Bearbeitung emotionaler Inhalte in den Vordergrund. Es wird ein neues Ziel generiert, das in der Bewältigung der aufgetretenen Emotion besteht. Erst wenn das emotionsbezogene Ziel erreicht wurde, wird das aufgabenbezogene Ziel weiterverfolgt. ${ }^{895}$ Nun wurden die Aspekte der Kognitions- und Emotionspsychologie in der Psychologie lange Zeit unabhängig voneinander betrachtet und erforscht. Hier hat jedoch eine Veränderung stattgefunden, so daß in neueren Forschungen berücksichtigt wird, daß sich Emotion und Kognition ${ }^{896}$ und nicht zuletzt auch Motivation ${ }^{897}$ wechselseitig beeinflussen.

Vgl. Abschnitt 5.5.3.2: Prozeß des Wissenserwerbs in ACT*.

Vgl. Abschnitt 3.4.3.1: Der Einfluß von Personenmerkmalen auf den Umgang mit komplexen Systemen.

Lantermann, E.D.,(Prozesse 1983), S. 248.

Vgl. Lantermann, E.D., (Prozesse 1983), S. 262 ff.

Vgl. Spies, K.; Hesse, F.W., (Interaktion 1986). Die Überlegungen von Wiener lassen sich bereits auf diesen Zusammenhang zurückführen. So konnte in Kapitel zwei ausgeführt werden, daß es gelang, eine verbesserte Flubzeugabwehr zu entwickeln, weil Menschen unter Streß die Neigung haben, zu in der Ausbildung erlernten Verhaltensmustern zurückzukdren. Vgl. Abschnitt 2.1.2.3: Interpretation der Simulationstechnik beim Unternehmensplanspiel.

Vgl. Becker, D.; Oldenbürger, H.-A.; Piehl, J.,(Motivation 1987), S. 433. 
Hartung diskutiert in diesem Zusammenhang die Ansätze von Dörner ${ }^{898}$ und von Anderson. ${ }^{899}$ Bezüglich des Kognitivismus führt sie aus, daß die Ansätze die Aspekte der Emotion und Motivation vernachlässigen ${ }^{900}$ und nimmt in Zusammenhang mit Unternehmensplanspielen ein Modell von Becker, Oldenbürger \& Piehl als Grundlage zur Konzeption von Unterricht an. ${ }^{901}$ So stellen Becker, Oldenbürger \& Piehl die Merkmale Emotion, Motivation und Kognition in ihrem Modell in einen gemeinsamen Bezugsrahmen, wobei die genannten Faktoren, sowie darüber hinaus auch Wahrnehmung und Verhalten, als verschiedene Arten des Person-Umwelt-Bezuges angenommen werden. [Vgl. Abbildung 6-5]. Davon kann auch an dieser Stelle ausgegangen werden.

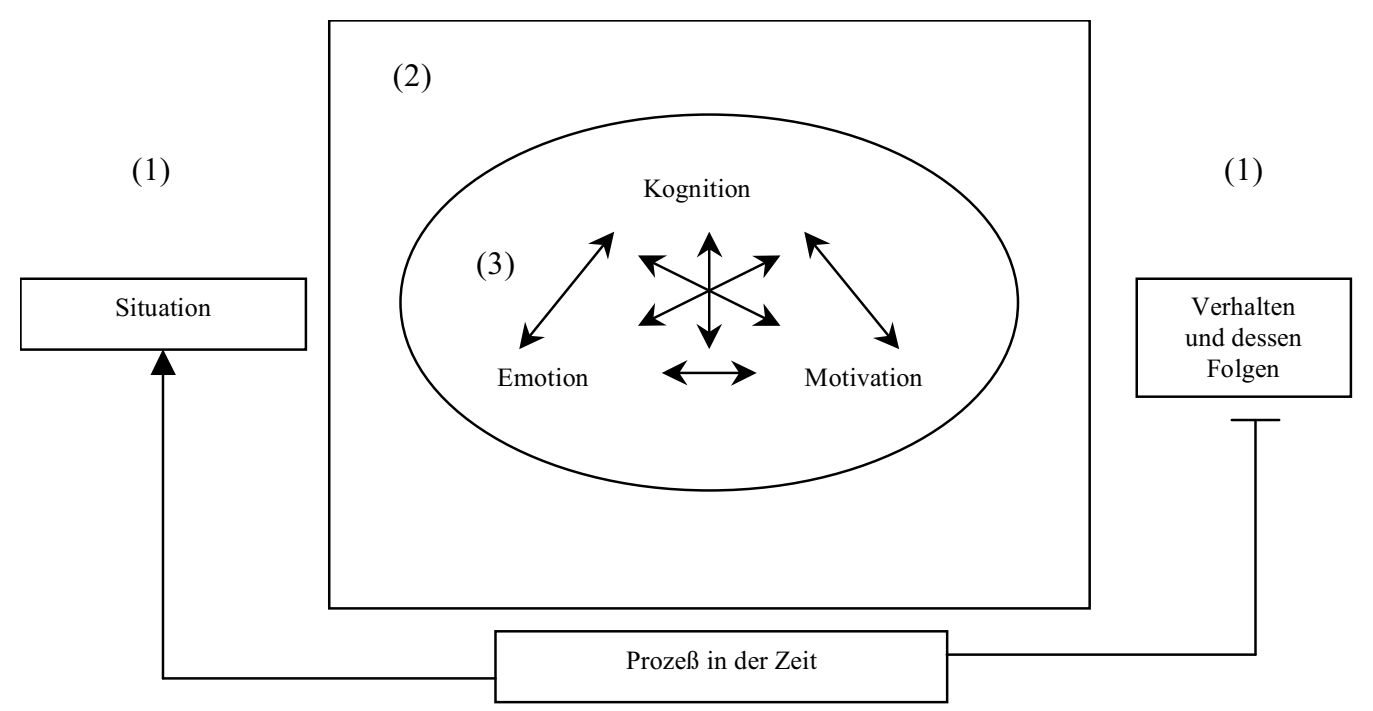

\section{Abbildung 6-5: Struktur des psychophysischen Systems im Person- Umwelt -Bezug mit ${ }^{902}$ \\ (1): physikalisch-soziale Außenwelt (Umgebung) \\ (2): psychophysischer Organismus (Person) \\ (3): innerpsychische Welt(en)}

Weiterhin leitet Hartung, unter dem Aspekt, daß den Ansätzen die Operatordok$\operatorname{trin}^{903}$ zugrunde liegt, nach der Denken als Verkettung einer Menge elementarer

\footnotetext{
898 Vgl. Abschnitt 5.5.1: Dörner's Theorie der Absichtsbehandlung.

899 Vgl. Abschnitt 5.5.3: Anderson's ACT*-Theorie.

900 Vgl. Hartung, S., (Förderung 2000), S. 20.

901 Vgl. Hartung, S., (Förderung 2000), S. 25 ff.

902 Becker, D.; Oldenbürger, H.-A.; Piehl, J.,(Motivation 1987), S. 433.
} 
Operatoren definiert wird, die Schlußfolgerung ab, daß, ,[...] das Problem der Intentionalität [einer problemlösenden Handlung] weiterhin ein Problem für Erklärungsmuster der Alltagspsychologie“ darstellt. ${ }^{904}$ Das Prinzip der Zieldominanz stellt in Anderson's Theorie jedoch das vorrangige Kriterium der Produktionsauswahl dar, so daß eine Produktion nur dann zur Anwendung gelangt, wenn in der Bedingungskomponente ein Ziel genannt wird, das dem aktuellen Ziel einer Person entspricht. ${ }^{905}$ Daher liefert sie auch unter Berücksichtigung des epistemologischen Subjektmodells, das die Annahme der Intentionalität impliziert, ${ }^{906}$ einen geeigneten Rahmen, um Aufschluß über Prozesse der menschlichen Informationsverarbeitung zu gewinnen. Das konnte in Zusammenhang mit der Darstellung der Interpretation betriebswirtschaftlicher Lernprozesse von Orth bereits aufgezeigt werden. ${ }^{907}$ Es soll daher hier der Integrationsversuch bezüglich des Aspektes der Emotion durch Spies \& Hesse ausgeführt werden. ${ }^{908}$ Auf eine Interpretation des betriebswirtschaftlichen Lernprozesses im Unternehmensplanspiel wird dabei verzichtet, da die Benennung konkreter emotionaler Verfassungen und ihr Einfluß auf den Lernprozeß im Unternehmensplanspiel noch aussteht.

Zunächst ist festzustellen, daß in der Theorie von Anderson die als wesentlich gekennzeichneten Aspekte der Motivation und Emotion nicht berücksichtigt werden. Hesse führt jedoch, im Hinblick auf den Aspekt der Emotion, aus, daß ACT* diesen Anspruch auch nicht erhebt. Er wirft aber die Frage auf, inwieweit ACT* nicht zu kognitivistisch ist und verweist in diesem Zusammenhang auf die hinreichend zuverlässigen Ergebnisse von Bower \& Cohen, ${ }^{909}$ wonach in Abhängigkeit von emotionalen Verfassungen der Zugriff zum Inhalt des Gedächtnisses unterschiedlich sein kann und diese darüber hinaus auch direkt beobachtbare Verhaltensweisen verändern. Dieser Aspekt konnte weiter oben schon ausgeführt werden. Somit erlangen die durch Anderson angenommenen Aktivationsprozesse, unter Einbeziehung emotionaler Aspekte, eine Veränderung. Diese sind dann nur

903 Wie ausgeführt, gilt das auch für Newell's SOAR-Architektur. Vgl. Abschnitt 5.6: Diskussion und Gegenüberstellung der Produktionssysteme.

904 Hartung, S., (Förderung 2000), S. 20.

905 Vgl. Spies, K.; Hesse, F.W., (Interaktion 1986), S. 86.

906 Vgl. Abschnitt 3.4.3.2.1: Das epistemologische Subjektmodell.

907 Vgl. Abschnitt 6.6.1.1: Interpretation kognitiver betriebswirtschaftlicher Lernprozesse durch die $\mathrm{ACT}^{*}$-Theorie.

908 Vgl. Spies, K.; Hesse, F.W., (Interaktion 1986).

909 Vgl. Bower, G.H.; Cohen, P.R., (Influences 1982). 
durch zusätzliche Annahmen zu erklären. Diese Erklärung bietet Anderson nicht an. ${ }^{910}$ Hesse vermutet jedoch, daß Interaktionen innerhalb der ACT*-Konzeption mit wenigen Zusatzannahmen darstellbar sind ${ }^{911}$ und unternahm mit Spies bereits den Versuch einer Integration von Emotion in die Zusammenhänge der $\mathrm{ACT}^{*}$ Theorie. ${ }^{912}$

Spies \& Hesse verfolgen dabei die Möglichkeit einer Erweiterung des prozeduralen Gedächtnisses um emotionsbezogene Produktionen, wobei sie unter emotionsbezogenen Produktionen derartige Produktionen verstehen, ,[...] die in einer ihrer Bedingungskomponenten emotionale Aspekte enthalten“. Damit geben emotionsbezogene Produktionen an, wie zu handeln ist, wenn bestimmte emotionale Zustände auftreten.

Sie gehen weiterhin davon aus, daß die in $\mathrm{ACT}^{*}$ bezüglich der kognitiven Produktionen angenommenen Kontrollprinzipien ${ }^{913}$ auch die Auswahl emotionsbezogener Produktionen steuern können. ${ }^{914}$ Dabei beziehen sie sich auf das in

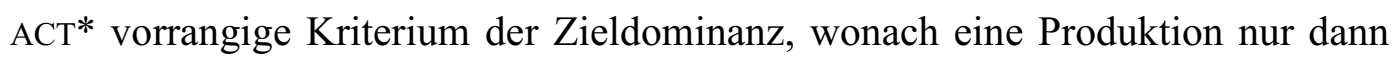
zur Anwendung gelangen kann, wenn in der Bedingung ein Ziel genannt wird, welches auch dem aktuellen Ziel einer Person entspricht. Sie folgern daraus, daß eine emotionsbezogene Produktion nur dann zur Anwendung gelangen kann, wenn das Ziel auch emotionsbezogene Aspekte aufweist. Spies \& Hesse verweisen in diesem Zusammenhang auf ein bereits von Anderson angeführtes Beispiel: „IF I suddenly feel extreme pain Then set my goal to escape the pain“915

Es wird damit davon ausgegangen, daß ein aufgabenbezogenes Ziel zurückgedrängt wird, wenn emotionale Verfassungen auftreten. In diesem Zusammenhang wird zunächst das emotionsbezogene Ziel verfolgt und das aufgabenbezogene Ziel erlangt erst dann wieder an Relevanz, wenn das emotionsbezogene Ziel als erledigt gilt. Es ist jedoch nicht so, daß aufgabenbezogene Prozesse zwangsläufig in

\footnotetext{
910 Vgl. Hesse, F.W., (Review 1985), S. 236.

911 Vgl. Hesse, F.W., (Review 1985), S. 236.

912 Vgl. Spies, K.; Hesse, F.W., (Interaktion 1986).

913 Vgl. Abschnitt 5.5.3.1: Die Architektur von ACT*.

914 Vgl. Spies, K.; Hesse, F.W., (Interaktion 1986), S. 86.
} 
den Hintergrund gedrängt werden, wenn emotionale Verfassungen auftreten. Im Gegenteil ist es sogar häufig der Fall, daß die Bewältigung aufgabenbezogener und emotionsbezogener Prozesse parallel zueinander erfolgt.

Insgesamt kann festgehalten werden, daß zur Aktivation emotionsbezogener Bedingungsmuster und zur Auswahl von emotionsbezogenen Produktionen dieselben Annahmen gelten können, die Anderson in Zusammenhang mit kognitiven Produktionen aufstellt. 916

\subsubsection{Metakognition: Die Steuerung des eigenen Denkens}

Ein zentrales Charakteristikum des epistemologischen Subjektmodells war weiterhin die Annahme der Selbstreflexivität. ${ }^{917}$ In diesem Zusammenhang wird im Unternehmensplanspiel davon ausgegangen, daß eine Förderung der Selbstreflexivität sich auf den Lernerfolg des Individuums auswirkt. So setzte Hartung ${ }^{918}$ in ihrer Untersuchung Instrumente ein, welche die Selbstreflexivität des Individuums im Unternehmensplanspiel fördern sollten. Die Förderung der Selbstreflexivität wurde dabei durch den gekreuzten Einsatz von Fragebögen in der Planspielphase unterstützt. Dies geschah zum einen im Hinblick auf die zu treffenden Entscheidungen, zum anderen durch Aufforderung der Teilnehmer, ihre Emotionen und Motivationen in der aktuellen Anforderungssituation zu reflektieren. Es wurde dabei davon ausgegangen, daß ein solches Vorgehen dazu führt, Rückschlüsse über die Wahrnehmung des Planspiel-Einsatzes auf seiten der Teilnehmer zu gewinnen. Weiterhin wurde davon ausgegangen, daß sich die Förderung auf den Lernerfolg auswirkt. Diese Annahme bestätigte sich. So konnte eine Planspielgruppe, die einen Fragebogen zum situativen Erleben der Planspielsituation bekam, wesentlich bessere Ergebnisse erzielen als eine Gruppe ohne diesen Fragebogen. Diese Gruppe war zwar im Posttest besser, war jedoch nicht in der Lage, ihre Leistungen nennenswert zu steigern. ${ }^{919}$

\footnotetext{
915 Vgl. Spies, K.; Hesse, F.W., (Interaktion 1986), S. 86.

916 Vgl. Spies, K.; Hesse, F.W., (Interaktion 1986), S 86 f.

917 Vgl. Abschnitt 3.4.3.2: Der Spieler als Handlungssubjekt im Unternehmensplanspiel.

918 Vgl. zu den bereits ausgeführten Aspekten dieser Untersuchung auch Abschnitt 2.2.2.3.2:

Zum Zusammenhang von Komplexität und Lernerfolg.
} 
Es ist nun davon auszugehen, daß auf seiten des Lernenden stets schon ein gewisses Maß an Selbstreflexivität vorhanden ist. ${ }^{920}$ So besitzen Menschen die Fähigkeit zur Selbstreflexivität, sie wissen einiges über sich selbst und sind in der Lage, sich selbst zu beeinflussen. ${ }^{921}$ Ansätze, die direkt an der Selbstreflexivität des Lernenden anknüpfen, waren Entwicklungsgegenstand der Metakognitionsforschung, die eine Teilklasse ,[...] kognitionspsychologischer Theorien [darstellt], in denen kognitive Operationen oder Prozesse oder Resultate früherer kognitiver Prozesse („Wissen“) selbst entweder Gegenstand oder Inhalt des Wissens oder Objekt kognitiver Operationen sind“922. Die Metakognitionsforschung umfaßt dabei zwei Brennpunkte, die unter den Begriffen Kompetenz und Performanz prägnanter gefaßt werden können. Performanz bezeichnet dabei all jene Prozesse, die als Realisation von Kompetenz gelten können. Es handelt sich hier also um feststellbares kognitives Verhalten des Organismus, oder, falls dieses nicht beobachtet werden konnte, kognitive Produkte, die auf die Einwirkung bestimmter mentaler Prozesse schließen lassen. ${ }^{923}$ Kompetenz bezeichnet hingegen die Ausstattung des Organismus an Fähigkeiten, wozu Fischer \& Mandl auch deklaratives und prozedurales Wissen im Sinne Dörner's zählen.

Werden Kompetenz und Performanz in Form von Modellen ausgedrückt, so gelten dafür folgende Voraussetzungen: ,Werden metakognitive Objekte stärker als Wissen oder Wissensbestandteile akzentuiert, spricht man von Kompetenzmodellen; wird hingegen ihr Einsatz als Objekt metakognitiver Steuerung im konkreten Denkvollzug betont, geht es um Exekutiv- oder Performanzmodelle."924

Das kognitive Handeln einer Person wird nun von der Selbstreflexivität mehr oder weniger differenziert begleitet. ${ }^{925}$ So wird von allgemeinem und bereichsspezifi-

\footnotetext{
919 Vgl. Hartung, S., (Förderung 2000), S. 284 u. S. 287.

920 Diese Begabung ist einzigartig für den Menschen. So führt Boulding aus: „The image of man is also characterized by a much greater degree of self-consciousness and of selfawareness than that of the lower animals. We not only know, but we know that we know. This reflective character of the human image is unique, and is what leads to philosophy." Boulding, K.E., (Image 1997), S. 25.

922 Kötter, L.; Mandl, H., (Ansätze 1983), S. 30.

923 Vgl. Fischer, P.M.; Mandl, H.,(Förderung 1983), S. 266.

924 Kötter, L.; Mandl, H., (Ansätze 1983), S. 30.

925 Vgl. Kötter, L.; Mandl, H.,(Ansätze 1983), S. 30.
} 
schem Veränderungswissen das Wissen über das eigene Problemlöseverhalten und seinen Erfolg unterschieden.926 So kann Wissen, unter Berücksichtigung metakognitiver Mechanismen sowie selbstreflexiver Prozesse, genutzt werden. Dabei dient dies dem Ziel, bewußt neue Lösungsstrategien zu entwerfen.927

Werden die ausgeführten Ansätze nun in Bezug zur weiter oben ausgeführten Hypothese gesetzt, daß »generelles Systemwissen, mit den generellen Komponenten Fakten- und Operationswissen, die metakognitive Komponente zu bereichsspezifischem Fakten- und Operationswissen darstellt«, so führt die Argumentation an dieser Stelle der Arbeit zu der Erkenntnis, daß die implizite Annahme, wonach die angeführten Produktionssysteme auch unter dem Aspekt einer Betrachtung generellen Systemwissens aussagekräftig seien, hier nicht ausreichend ist. Es muß hingegen festgestellt werden, daß in diesem Zusammenhang offensichtlich ein komplexeres Modell menschlicher Informationsverarbeitung erforderlich ist, welches als ein Produktionssystem denkbar sein könnte, welches in der Lage ist, metakognitives Wissen zu nutzen sowie weiterhin die Aspekte der Motivation und Emotion zu berücksichtigen.

In diesem Zusammenhang kann hier noch eine zweite Hypothese aufgestellt werden. So konnten Bloech, Kauer \& Orth in ihren Untersuchungen eine unzureichende Anwendung des betriebswirtschaftlichen Sachwissens im Unternehmensplanspiel analysieren, was als Mangel an unternehmerischer Handlungskompetenz interpretiert wurde. 928 Der Grund dafür kann nun unter Berücksichtigung der aufgestellten Hypothese in Ermangelung eines mit dem betriebswirtschaftlichen Sachwissen in Zusammenhang stehenden generellen Systemwissens vermutet werden; was gleichsam ein paradoxer Schluß ist, da dieses Wissen ja gerade durch den Einsatz von Unternehmensplanspielen vermittelt werden soll. Die entsprechende Hypothese lautet also wie folgt:

»Die unzureichende Anwendung betriebswirtschaftlichen

Sachwissens im Unternehmensplanspiel, ist auf einen

\footnotetext{
926 Vgl. Putz-Osterloh, W., (Wissen 1988), S. 251.

927 Vgl. Putz-Osterloh, W., (Wissen 1988), S. 251.

928 Vgl. Abschnitt 6.3: Die unternehmerische Handlungskompetenz.
} 
Mangel an damit in Zusammenhang stehendem, generellem Systemwissen zurückzuführen.«

Unter Berücksichtigung der Interpretation von Bloech, Kauer \& Orth kann sie auch wie folgt formuliert werden:

»Die unzureichende Anwendung betriebswirtschaftlichen Sachwissens im Unternehmensplanspiel ist auf einen Mangel an damit in Zusammenhang stehendem, generellem Systemwissen zurückzuführen; und äußert sich als mangelnde unternehmerische Handlungskompetenz.«

Für das hypothetisch angenommene betriebswirtschaftliche mentale Modell eines Experten kann damit insgesamt festgehalten werden, daß es offensichtlich ein Produktionssystem beinhalten muß, welches in der Lage ist, metakognitives Wissen zu nutzen sowie weiterhin die Aspekte der Motivation und Emotion zu berücksichtigen. 929

Wird nun von Personen unterschiedlichen Erfahrungshintergrundes ausgegangen, so sind deren mentale Modelle unterschiedlich entwickelt; und weisen somit in bezug auf die erforderlichen Wissensqualitäten im Umgang mit einem Unternehmensplanspiel unterschiedliche Entwicklungszustände auf. Für einen Planspielteilnehmer, der sich beim Umgang mit einem Unternehmensplanspiel mit einem Problem konfrontiert sieht, ist davon auszugehen, daß das mentale Modell in bezug auf die Anforderungssituation nicht hinreichend ausgebildet ist. In diesem Zusammenhang wurde das Vorwissen in der vorliegenden Arbeit als Spiegel des Erfahrungshintergrundes eines Individuums angenommen, womit davon ausgegangen werden konnte, daß die Unterschiede bezüglich des Erfahrungshintergrundes von Experten und Novizen Ausdruck eines Entwicklungsprozesses sind, der unter der Annahme eines Zeitpfades vom Experten bereits abgeschlossen ist, während er vom Novizen noch zu vollziehen ist. Demnach kann die Qualität des

929 Vgl. zu metakognitiven Ansätzen bspw. Lantermann, E.D., (Prozesse 1983); Brown, A.L., (Development 1975); Brown, A.L., (Schooling 1975). 
Expertenwissens Aufschluß darüber geben, welche Wissensgrößen im Lernprozeß des Novizen eine Rolle spielen.

In diesem Zusammenhang wurde bereits mehrfach darauf eingegangen, daß die Wirtschaftspädagogik dazu aufgefordert ist, die Komplexität des Lernstoffes an das Vorwissen der Teilnehmer anzupassen, ${ }^{930}$ wodurch die Differenz zwischen den Qualifikationsanforderungen, welche das Lernobjekt stellt, und den subjektiven Bedingungen der Lernenden, von Wolff als »pädagogische Lücke« bezeichnet, zum Ausdruck kommt. ${ }^{931}$ Durch die Annahme eines unterschiedlichen Erfahrungshintergrundes unterschiedlich qualifizierter Personen, der offensichtlich Ausdruck eines Entwicklungsprozesses ist, stellt sich nun weiter die Frage wie der Entwicklungsprozeß der Planspielteilnehmer didaktisch unterstützt werden kann.

Dazu sind an dieser Stelle zunächst die Pole, die Experten und Novizen, des Entwicklungsprozesses bezüglich der Qualität ihres Wissens näher zu charakterisieren. Dabei wird hier als erstes auf die Novizen eingegangen, wodurch die didaktische Maßnahme die Komplexität des Unternehmensplanspiels an das Vorwissen der Planspielteilnehmer anzupassen, widergespiegelt wird. Für diese, die Novizen, kann davon ausgegangen werden, daß ihr Vorgehen im Umgang mit einem Unternehmensplanspiel von Fehlern geprägt ist; ${ }^{932}$ weshalb hier ein Klassifikationsansatz menschlicher Fehler von Reason ausgeführt wird.

\subsubsection{Kognitive Grenzen: Der Klassifikationsansatz menschlicher Fehler von Reason}

In Kapitel drei wurde der Versuch unternommen, Ergebnisse der Problemlöseforschung zu Fehlleistungen des Individuums im Umgang mit komplexen Systemen in einen gemeinsamen Bezugsrahmen mit Unternehmensplanspielen zu stellen. ${ }^{933}$ Diese Betrachtung erfolgte zum einen in Zusammenhang mit der in Kapitel zwei dargestellten Diskussion bezüglich der wünschenswerten Zusam-

\footnotetext{
930 Vgl. bspw. Abschnitt 2.2.2.3: Zum Zusammenhang von Komplexität, Lernzielen und Lernerfolg im Unternehmensplanspiel.

931 Vgl. Abschnitt 5.1: Das Unternehmensplanspiel: Ein komplexes Lehr-Lern-Arrangement.

932 Vgl. Abschnitt 3.4: Das S-H-E-L-L - Modell.

933 Vgl. Kapitel 3: Das S-H-E-L-L - Modell: Ein Mensch-Maschine-System.
} 
menarbeit zwischen Betriebswirtschaftslehre und Psychologie, ${ }^{934}$ vordergründig jedoch in Zusammenhang mit der unzureichenden Anwendung betriebswirtschaftlichen Sachwissens in Unternehmensplanspielen. ${ }^{935}$

Die Notwendigkeit einer Anpassung der Planspielkomplexität an das Vorwissen der Teilnehmer hatte hier bereits den problematischen Charakter von Unternehmensplanspielen bezüglich des Umgangs mit diesen aufgezeigt. Wird weiterhin die unzureichende Anwendung des Sachwissens im Unternehmensplanspiel berücksichtigt, so kann an dieser Stelle noch einmal festgehalten werden, daß ein Unternehmensplanspiel nicht den Charakter einer Aufgabe, sondern den eines Problems besitzt. ${ }^{936}$ Es wird daher erforderlich, zwischen Problemlöseprozeß und Entscheidungsprozeß zu differenzieren. Dies ist möglich, wenn das Suchverhalten, das der Entscheidung vorgelagert ist, als Problemlösung bezeichnet wird. ${ }^{937}$ Oder, um den zeitlich ausgedehnten Prozeß expliziter herauszustellen, mit den Worten von Duncker: ,[...] die Endform einer Lösung [wird] typisch auf dem Wege über vermittelnde Prozeßphasen erreicht, deren jede nach rückwärts Lösungscharakter, nach vorwärts Problemcharakter besitzt."938 Die Entscheidung stellt daher einen sich wiederholenden Akt im Problemlöseprozeß und zugleich auch dessen Abschluß dar. Davon wird im folgenden ausgegangen, wobei der Problemlöseprozeß selbst als Anwendung und Erwerb von Wissen vorstellbar ist, ${ }^{939}$ der seinen Ausgangspunkt bei der individuell empfundenen Systemschwierigkeit findet. 940

Der Klassifikationsansatz von Reason wurde in diesem Zusammenhang ausgewählt, da er Theorien menschlicher Fehler integriert und zugleich einen Regel-

\footnotetext{
934 Vgl. Abschnitt 2.3.3: Einsatz von Simulationsmodellen im Forschungsfeld des Komplexen Problemlösens.

935 Vgl. Abschnitt 6.3: Die unternehmerische Handlungskompetenz.

936 Werner spricht in Zusammenhang mit dem Planspiel Puma von »echten« Problemen, wdchen ein Planspielteilnehmer gegenübersteht. Vgl. Werner, L., (Entscheidungsunterstützungssysteme 1992), S. 186 ff.

937 Vgl. Kirsch, W., (Entscheidungsprozesse -Band 1- 1970), S. 74.

938 Duncker, K., (Psychologie 1963), S. 10.

939 Vgl. Abschnitt 5.2: Komplexes Problemlösen und Wissen.

940 Vgl. Abschnitt 6.5.2: Die Systemschwierigkeit: Ausdruck der Problemkomplexität in Wissensgrößen.
} 
kreis zur Systemkontrolle darstellt, der in Zusammenhang mit verschiedenen Wissensebenen, als Orte möglicher Fehlerquellen, steht.

Die Formulierung geeigneter Kategorien zur Klassifikation menschlichen Fehlverhaltens macht einen wesentlichen Anteil der Forschung im Bereich menschlichen Fehlverhaltens aus. ${ }^{941}$ Die Klassifikation GEMS, ein Akronym für „GENERIC ERROR-MODELLING SYSTEM“, von Reason verfolgt das Anliegen, einen einfachen, allgemeinen Rahmen zu bieten, in dem prinzipielle kognitive Grenzen und Richtungen, die vorhersehbare Varianten menschlicher Fehler verursachen, Berücksichtigung finden. ${ }^{942}$ Reason geht zunächst davon aus, daß zwei existierende Theorien menschlicher Fehler zu integrieren sind:943

1. SLIPS werden von Reason wie folgt angenommen: „Departures of action from intention, or execution failures [...].“ Bei den slips handelt es sich somit vor allem um versehentlich falsch ausgeführte Operationen, da sie nicht der ursprünglichen Absicht entsprechen. ${ }^{944}$ Norman definiert: „A slip is a form of human error defined to be the performance of an action that was not what was intended."

2. MISTAKES: Fehlern liegen hingegen falsche Absichten und Ziele zugrunde. ${ }^{946}$ Sie werden von Reason wie folgt angenommen: „Errors in which the actions may run according to plan, but where the plan is inadequate to achieve its desired outcome.“

Vgl. Kluwe, R.H., (Analysen 1991), S. 276.

942 Vgl. Reason, J., (System 1987), S. 63.

943 Vgl. Reason, J., (System 1987), S. 63 und die dort angegebene Literatur.

944 Vgl. Kluwe, R.H., (Analysen 1991), S. 276.

945 Norman, D.A., (Categorization 1981), S. 1. Diese Art von Fehlern beschreibt Brauchle wie folgt: „Eine sehr interessante Ausstrahlung des Unbewußten sind ,[...] die sogenannten Fehlleistungen. Man versteht darunter fehlgegangene, am Ziel der Absicht vorbeigegangene Leistungen. Man will etwas sagen und sagt doch etwas anderes, man will etwas behalten und verliert, vergißt es doch, man will richtig schreiben und muß feststellen, daß die Feder etwas anderes hingesetzt hat, als unserer Absicht entsprach. Aber diese falschen Leistungen sind nur falsch vom Standpunkt der ursprünglichen Absicht aus; sie sind richtig vom Standpunkt einer zweiten Absicht, die sich mehr oder weniger verborgen hält. Eine gestörte Absicht und eine störende Absicht vereinigen sich in der Hervorbringung der Fehlleistung." Brauchle, A., (Macht 1975), S. 35 f. 
Weiterhin nimmt in Reason's Klassifikation menschlicher Irrtümer eine Klassifikation von Rasmussen eine zentrale Stellung ein. ${ }^{947}$ So kann sich nach Rasmussen die Kontroll- und Steuerungstätigkeit eines Operateurs auf einer von drei Ebenen bewegen, die sich hinsichtlich ihrer kognitiven Anforderungen unterscheiden:948 [Vgl. Abbildung 6-6].

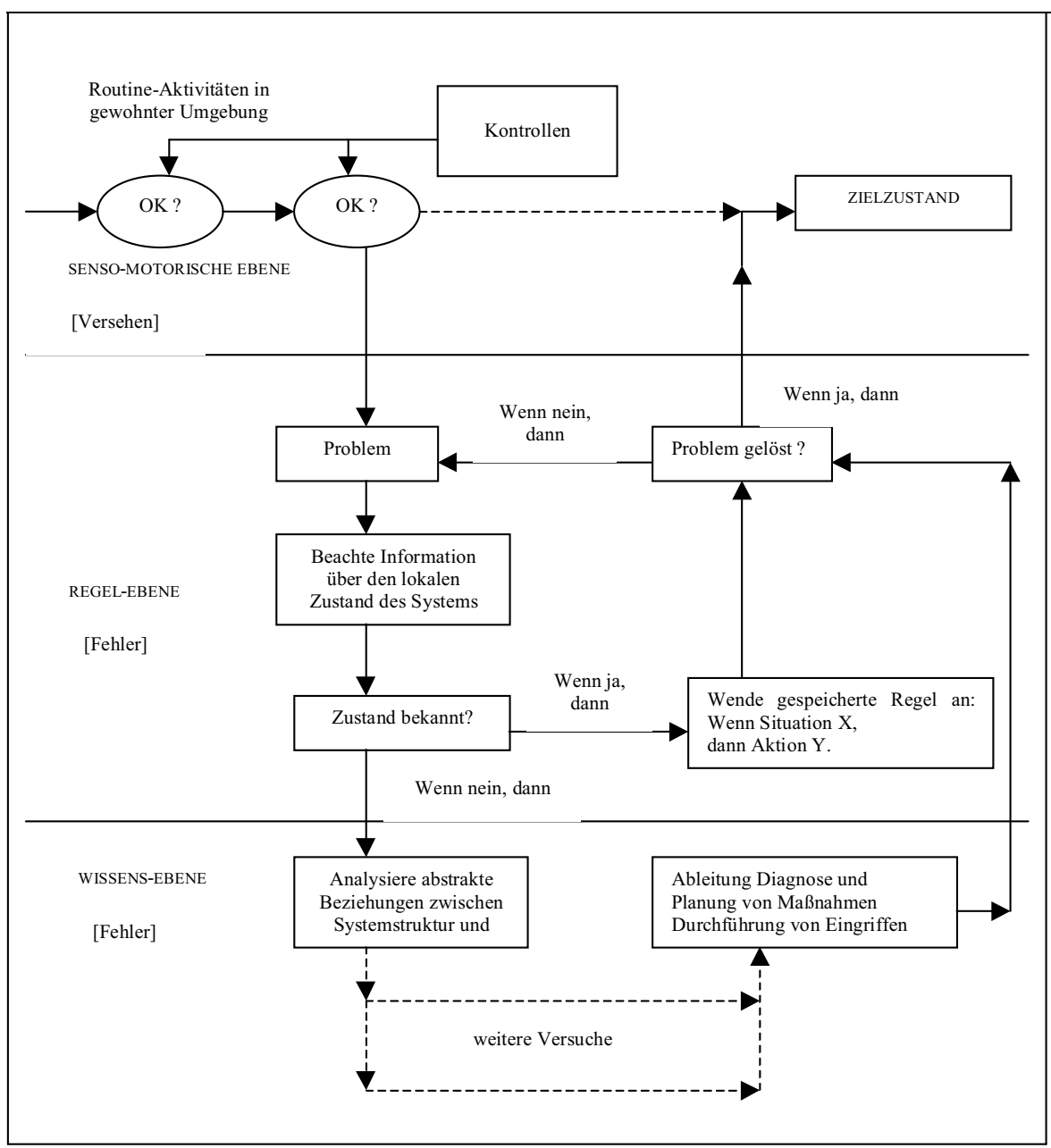

\section{Abbildung 6-6: $\quad$ Ebenen der Kontroll- und Steuerungstätigkeit ${ }^{949}$}

Das SKILL-BASED BEHAVIOUR bezeichnet ein Verhalten auf der Ebene routinierter Kontroll- und Steuerungstätigkeit, welches stark sensomotorisch basiert ist. Gemäß Rasmussen unterliegt ein solches Verhalten nur gelegentlich einer bewußten Kontrolle. Er führt aus: „The skill-based behaviour represents sensory-motor per-

947 Vgl. Reason, J., (System 1987), S. 64.

948 Vgl. Kluwe, R.H., (Analysen 1991), S. 276, zitiert nach Rasmussen, J., (Skills 1983), S. $258 \mathrm{ff}$.

949 Vgl. Kluwe, R.H., (Analysen 1991), S. 278 nach Reason, J., (System 1987), S. 66. 
formance during acts or activities which, following a statement of an intention, take place without conscious control as smooth, automated, and highly integrated patterns of behaviour. Only occasionally is performance based on simple feedback control, where motor output is a response to the observation of an error signal representing the difference between the actual state and the intended state in a time-space environment, and where the control signal is derived at a specific point in time. " $" 950$

Abweichungen im Systembetrieb werden daher vorwiegend auf der Ebene des RULE-BASED BEHAVIOUR beobachtet und korrigiert. Weiterhin werden dort gespeicherte Kontroll- und Steuerungsregeln aufgerufen, die zur Korrektur der festgestellten Abweichungen dienen. Dabei erfolgt der Abruf von gespeicherten Regeln auf der Grundlage von im Vorfeld gemachten Erfahrungen. „The point here is that performance is goal-oriented but structured by „feedforward control“ through a stored rule. Very often, the goal is not even explicitly formulated but is found implicitly in the situation releasing the stored rules. The control is teleological in the sense that the rule or control is selected from previous successful experiences. “951 Als KNOWLEDGE-BASED BEHAVIOUR bezeichnet Rasmussen schließlich das Verhalten auf der Ebene, wo weder Routineaktivitäten noch der Abruf verfügbarer Kontroll- und Steuerungsregeln genügen. Es liegen ungewöhnliche Systemzustände vor. Dies führt dazu, daß es notwendig ist, einen sinnvollen Plan zu entwickeln. Dies geschieht in der Weise, daß unterschiedliche Pläne entworfen werden, ihre Wirkung im Hinblick auf den Grad der Zielerreichung erprobt wird, was schließlich zur Auswahl eines Planes führt. ${ }^{952}$

Auf unterschiedliche Anforderungen muß ein Individuum nun unterschiedlich reagieren. Dazu muß es von einer Ebene auf die andere wechseln. Reason interpretiert die Interaktion der drei Ebenen, sprich den Wechsel zwischen ihnen, wie folgt: 953

\footnotetext{
950 Rasmussen, J., (Skills 1983), S. 258.

951 Rasmussen, J., (Skills 1983), S. 259.

952 Vgl. Rasmussen, J., (Skills 1983), S. 259.

953 Reason, J., (System 1987), S. 68 f.
} 
- Der Wechsel von der SENSO-MOTORISCHEN EBENE auf die REGEL-EBENE:

Wird eine Problemlösung angestrebt, wird stets zunächst die Regel-Ebene betrachtet; womit die Regel-Ebene in dem Moment relevant wird, wenn eine Überprüfung erreichter Ergebnisse oder angestrebter Ziele ein Problem identifiziert. Eine Problemlösung, die auf der Regel-Ebene vorangetrieben wird, zielt dabei auf die Entwicklung hochroutinierter Aktivitäten in ähnlichen Umgebungen. Dabei muß bei schweren Problemen der Zirkel stets mehrfach durchlaufen werden, bevor er zu hochroutinierten Aktivitäten führt.

- Der Wechsel von der REGEL-EBENE auf die WISSENS-EBENE:

In Zusammenhang mit der einfachen Logik von GEMS erfolgt der Wechsel von der Regel-Ebene auf die Wissens-Ebene, wenn der Problemlöser realisiert, daß sein Repertoire an regelbasierten Lösungen nicht ausreicht, um eine Lösung herbeizuführen und infolgedessen neue Regeln ausgebildet werden müssen.

- Der Wechsel von der WISSENS-EBENE auf die REGEL-EBENE:

Aktivitäten auf der Wissens-Ebene können klassifiziert werden als die Suche nach einer adäquaten Problemlösung. Diese Lösung konstituiert möglicherweise einen neuen Handlungsplan, der ein neues Set von regelbasierten Routinen enthält. Dieser Zusammenhang wird in der Abbildung durch die Frage verdeutlicht, ob das Problem als gelöst gilt.

Es kann festgehalten werden, daß der vorliegende Klassifikationsansatz lediglich in stark vereinfachter Weise die wesentlich komplexere Form der Informationsverarbeitung in der Theorie von Anderson widerspiegelt. Demgegenüber weist er jedoch eine reflexive Komponente auf, da Problemlösungen nach einem Regelkreisprinzip vorangetrieben werden und der Problemlöser in bewußter Weise vorzugehen hat. So wird durch die Fragestellungen impliziert, daß der Problemlöser Probleme bewußt als solche identifiziert, sowie weiterhin Problemlösungen in bewußter Weise vorantreibt. Er hat auch ein Bewußtsein darüber, wo er sich im System befindet und reflektiert die Ergebnisse im Hinblick auf einen definierten Zielzustand. 
Als zweiter Pol ist nun der Expertenpol näher zu charakterisieren. Dies geschieht durch die Berücksichtigung von Expertensystemen, da durch die Annahme eines Zeitpfades davon ausgegangen werden kann, daß die Wissensqualitäten des Experten eine Zielgröße im Entwicklungsprozeß des Novizen darstellen. Da die Wissensakquisition bei Experten hier die Möglichkeit bietet, Wissen auf Computern darzustellen, soll im folgenden auf Expertensysteme eingegangen werden. ${ }^{954}$

\subsubsection{Darstellung von Expertenwissen in Expertensystemen}

In einem Teilbereich der Künstlichen Intelligenz, welche einen Forschungsbereich der Informatik darstellt, ${ }^{955}$ werden sogenannte Expertensysteme oder auch Wissensbasierte Systeme ${ }^{956}$ konzipiert, die in der Lage sind, die Fähigkeiten zum Problemlösen von Experten zu simulieren. ${ }^{957}$ Sie können in diesem Zusammenhang wie folgt definiert werden: „Expertensysteme sind Programme, mit denen das Spezialwissen und die Schlußfolgerungsfähigkeit qualifizierter Fachleute auf eng begrenzten Aufgabengebieten nachgebildet werden soll. Dabei gehen wir vorläufig von der Vorstellung aus, daß Experten ihre Problemlösungen aus Einzelkenntnissen zusammensetzen, die sie selektieren und in passender Anordnung verwenden. Expertensysteme benötigen daher detaillierte Einzelkenntnisse über das Aufgabengebiet und Strategien, wie dieses Wissen zur Problemlösung benutzt werden soll." ${ }^{9} 958$

954 Dabei erfolgt die Darstellung von Wissen in Expertensystemen unter der Berücksichtigung der vorliegenden Repräsentationsformen, wobei bspw. auch hybride Systeme angenommen werden können. Vgl. Kretschmar, T., (Datentypen 1991), S. 173 ff.

Dabei verstehen sich die einzelnen Forschungsbereiche der Künstlichen Intelligenz als ergänzende Komponenten im Bereich der Informatik. Vgl. Johannes, P., (Expertensysteme 1990), S. 9. Der Begriff der Künstlichen Intelligenz (KI) stellt die Übersetzung des engIschen Begriffs „Artifical Intelligence (AI)“ dar. Die Anfänge dieses Forschungsbereiches sind in der Mitte der fünfziger Jahre zu finden, wobei es das vordergründige Ziel dieses Forschungsgebietes war, beim Computer eine Verhaltensweise zu erzeugen, die beim Menschen als intelligent zu bezeichnen wäre. Vgl. Borkowski, V.; Geis, W., (Intelligenz 1990), S. 252. Namentlich gehen die Anfänge der KI aufJ. von Neumann sowie insbesondere auf A. Turing zurück. Vgl. Kurbel, K., (Entwicklung 1992), S. 2. Zu weiterführenden Aus@gen zur Entwicklung der KI vgl. ders. (Entwicklung 1992), S. 2 ff. In der Literatur setzt sich zunehmend der Begriff des Wissensbasierten Sytems durch, da die Entwicklung dahin geht, neben Expertenwissen auch anderes Wissen auf Computern darzustellen. Vgl. Spang, S., (Expertensysteme 1991), S. 1. Demnach sind Expertensysteme ein spezieller Ausdruck von Wissensbasierten Systemen. Vgl. Neumann, B., (Expertensysteme 1986), S. 11.

957 Vgl. Borkowski, V.; Geis, W., (Expertensysteme 1990), S. 173.

958 Puppe, F., (Einführung 1991), S. 2. 
Ursprünglich zur Nachahmung des menschlichen Problemlösungsverhaltens entwickelt, ist diese sehr allgemeingültige Sichtweise inzwischen einer differenzierteren Sichtweise gewichen, wonach Expertensysteme nach zugrunde gelegtem Forschungsinteresse unterschiedlich definiert werden können. So existiert inzwischen eine Vielzahl unterschiedlich orientierter Ansätze, wobei im Bereich betrieblicher Expertensysteme die leistungsorientierten, die strukturorientierten und die funktionsorientierten Ansätze Relevanz besitzen. Die strukturorintierten Ansätze beschreiben nun den Aufbau von Expertensystemen, ${ }^{959}$ der sich in eine Serie funktionaler Komponenten unterteilt, in denen das Wissen von Experten beschrieben wird: 960

Die Kernkomponenten stellen zunächst die Wissensbasis sowie die Inferenzkomponente dar. Darüber hinaus sind zu den funktionalen Komponenten eines Expertensystems die Dialogkomponente, die Erklärungskomponente und die Wissensakquisitionskomponente zu rechnen: [Vgl. Abbildung 6-7].

Die Wissensbasis stellt den Teil eines Expertensystems dar, in dem die problemspezifischen Fakten und Regeln einer Wissensdomäne dargestellt werden. ${ }^{961}$ Dieses Wissen wird in einem Expertensystem strikt von der Ablaufsteuerung getrennt, wonach Wissen, welches die Verarbeitung von Wissen anbelangt, in der Inferenzmaschine dargestellt wird. ${ }^{962}$ Dabei ist diese Trennung von Wissen und Problemlösungsstrategie zunächst das grundlegende Organisationsprinzip von Expertensystemen.963 Die Dialogkomponente stellt weiterhin die Schnittstelle zum Benutzer eines Expertensystems dar, wobei die Akzeptanz eines Expertensystems sehr stark von der Benutzerfreundlichkeit der Dialogkomponente abhängt. ${ }^{964}$ Durch die Erklärungkomponente ist es einem Anwender möglich, den Schlußfol-

\footnotetext{
959 Vgl. Wandel, H.-U., (Expertensysteme 1992), S. 17 ff. und die dort angegebene Literatur.

960 Bezüglich des Aufbaus von Expertensystemen besteht in der Literatur weitgehend Eingkeit. Vgl. hier bspw. Bendig, R.; Frick, D.; Gabriel, R.; Meier, G.; ReinersmannRescheleit; Timm, H., (Expertensysteme 1987), S. 14 ff.; Wandel, H.-U., (Expertensysteme 1992), S. 18 ff.; Borkowski, V.; Geis, W., (Expertensysteme 1990), S. 174 ff.; Frank, U., (Expertensysteme 1988), S. 26 ff.

961 Vgl. Weidenhaupt, T.M.,(Grundlagen 1991), S. 16.

962 Vgl. Weidenhaupt, T.M.,(Grundlagen 1991), S. 16.

963 Vgl. Puppe, F., (Einführung 1991), S. 2.

964 Vgl. Bendig, R.; Frick, D.; Gabriel, R.; Meier, G.; Reinersmann-Rescheleit; Timm, H., (Expertensysteme 1987), S. $50 \mathrm{ff}$.
} 
gerungen eines Expertensystems zu folgen. ${ }^{965}$ Durch die Anwendung von Methoden der Wissensakquisition wird schließlich das Wissen, welches Eingang in das Expertensystem findet, erfaßt. ${ }^{966}$ Dabei sollte die Wissensakquisition für ein Expertensystem als Komponente der gesamtbetrieblichen Informationsbereitsstellungsaufgaben verstanden werden und nicht als isolierte Insellösungen. 967

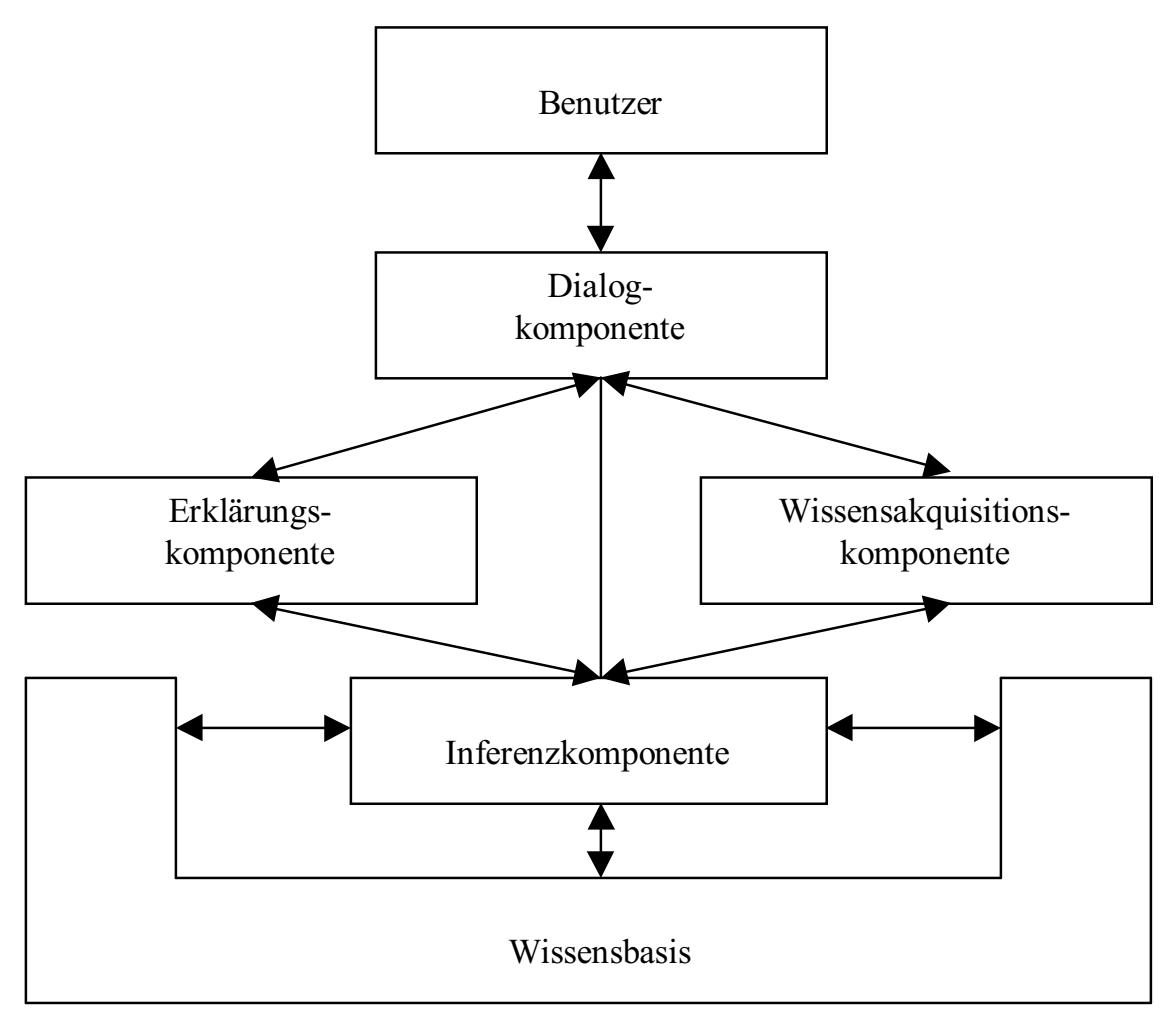

\section{Abbildung 6-7: $\quad$ Architektur eines Expertensystems ${ }^{968}$}

Über diese Grundkonzeption eines Expertensystems hinaus, wäre es nun auch wünschenswert, wenn das Expertensystem lernfähig wäre. Als problematisch erweist sich in diesem Zusammenhang jedoch, daß Expertensysteme ihrer Natur nach statisch sind, da sie der Lernfähigkeit von Experten nicht im ausreichendem Maße entsprechen. So führt Biethahn aus, daß Experten lernfähig sind; und dies auch von Expertensystemen zu erwarten sein sollte. Dabei verfügen Expertensysteme für die Aufnahme neuen Wissens in der Tat über eine sogenannte Wissenserwerbskomponente. Diese kann jedoch in der Regel nur vom Wissensinge-

966 Vgl. Bendig, R.; Frick, D.; Gabriel, R.; Meier, G.; Reinersmann-Rescheleit; Timm, H., (Expertensysteme 1987), S. 60 ff.

967 Vgl. Biethahn, J., (Arten 1991), S. 189. 
nieur benutzt werden, wobei dies in der Weise geschieht, daß dieser nur unter ausreichendem Vorwissen über das Expertensystem und den Wissensbereichs Wissen in die Wissensbasis oder Wissensbank einfließen lassen kann. Für den Experten ist die Schnittstelle selbst hingegen noch nicht hinreichend geeignet. Somit kann der Wissenszugang in Expertensystemen im Allgemeinen nur periodisch durch einen Wissensingenieur und nicht über den Normalnutzer erfolgen, wodurch die Expertensysteme einen weitgehend statischen Charakter aufweisen. ${ }^{969}$ So kann die Praxis auch sehr wenige Erfolge nachweisen, die sich auf die Implementierung einer Lernkomponente beziehen. ${ }^{970}$

Wird nun davon ausgegangen, daß Expertenwissen eine Zielgröße im betriebswirtschaftlichen Lernprozeß am Unternehmensplanspiel darstellt, so stellt sich an dieser Stelle die Frage, inwieweit Expertensysteme die Möglichkeit bieten zur Entscheidungsunterstützung im Unternehmensplanspiel eingesetzt zu werden. Es erweist sich in diesem Zusammenhang als problematisch, daß eine solche Maßnahme nicht dem didaktischen Erfordernis entspräche, die Komplexität des Unternehmensplanspiels an das Vorwissen der Planspielteilnehmer anzupassen. Im Hinblick auf den Einsatz von Expertensystemen in der Lehre bedarf es unter Berücksichtigung des Zeitaspektes offensichtlich einer anderen Kategorie von Expertensystemen. Vor den Erkenntnissen der vorliegenden Arbeit soll daher die Bedeutung des Faktors Zeit im System beleuchtet werden.

\subsubsection{Entscheidungsunterstützung im Unternehmensplanspiel}

\subsubsection{System, Handlung und Zeit}

Die Zeit hat in bezug auf das wirtschaftliche Handeln und Denken eine zentrale Bedeutung. „Alles Wirtschaften als ein ökonomisch determiniertes Tätigsein vollzieht sich im Zeitablauf.“971 Trotzdem wird der Zeitabhängigkeit des Handelns in

\footnotetext{
968 Raulefs, P., (Expertensysteme 1982), S. 63.

969 Vgl. Biethahn, J., (Grenzen 1991), S. 293.

970 Vgl. Bendig, R.; Frick, D.; Gabriel, R.; Meier, G.; Reinersmann-Rescheleit; Timm, H., (Expertensysteme 1987), S. 67.

971 Kern, W., (Zeit 1992), S. 41.
} 
der Betriebswirtschaftslehre nur unzureichende Aufmerksamkeit entgegengebracht. Analysen erfolgen statisch, komparativ-statisch oder unter ceteris-paribusBedingungen, die die Annahme eines stabilen Zustandes zulassen.972

Das Unternehmensplanspiel ist nun seinem Wesen nach statisch; im Spiel wird es jedoch zum Gegenstand der vorgesehenen Geschäftsmaßnahmen und erhält hier einen dynamischen Charakter. So unterliegt das Simulationsmodell Veränderungen im Zeitablauf, die durch das Entscheidungshandeln der Planspielteilnehmer hervorgerufen werden. ${ }^{973}$ Parallel dazu erweitert sich das mentale Modell des Planspielteilnehmers. Vor der Annahme eines Zeitpfades erweitert der Planspielteilnehmer seinen Erfahrungshintergrund, wofür in der vorliegenden Arbeit die Unterscheidung nach Experten und Novizen Pate stehen kann. Sie beschreiben hier zwei Pole eines in der Zeit sich erstreckenden Entwicklungsprozesses. ${ }^{974}$ Dabei konnte unter Berücksichtigung eines Zeitpfades folgendes angenommen werden: Da das Vorwissen in der vorliegenden Arbeit als Spiegel des Erfahrungshintergrundes eines Individuums angenommen wurde, konnte davon ausgegangen werden, daß die Unterschiede bezüglich des Erfahrungshintergrundes von Experten und Novizen Ausdruck eines Entwicklungsprozesses sind, der unter der Annahme eines Zeitpfades vom Experten bereits abgeschlossen ist, während er vom Novizen noch zu vollziehen ist. Die Qualität des Expertenwissens kann demnach Aufschluß darüber geben, welche Größen im Lernprozeß des Novizen eine Rolle spielen.975 Weiterhin konnte davon ausgegangen werden, daß der Novize die Qualitäten seines Wissens durch Reflektion seiner Fehler entwickelt; 976 der Experte diese Wissensqualitäten hingegen bereits erworben hat und für Expertensysteme zur Verfügung stellt ${ }^{977}$.

In diesem Zusammenhang ist nun schließlich auf das Wesen der Komplexität bezug zu nehmen. So konnte in Kapitel zwei folgendes ausgeführt werden: Oft wird die Welt mit den Begriffen Ordnung und Unordnung beschrieben; wobei die le-

\footnotetext{
972 Vgl. Kern, W., (Zeit 1992), S. 41 f.

973 Vgl. Abschnitt 2.1.2.1: Das Unternehmensplanspiel: Ein Simulationsmodell sowie Abschnitt 2.1.2.2: Das Unternehmensplanspiel: Ein Entscheidungsspiel.

974 Vgl. Abschnitt 3.3: Umgang von Personen mit komplexen Systemen.

975 Vgl. Abschnitt 6.3: Die unternehmerische Handlungskompetenz.

976 Vgl. Abschnitt 6.6.2: Kognitive Grenzen: Der Klassifikationsansatz menschlicher Fehler von Reason.
} 
digliche Nutzung dieser, zwei gegensätzliche Pole beschreibenden Begriffe jedoch wenig interessant ist. So ist Unordnung nicht klar zu umschreiben und ist das, was sie selbst offenbart. Ordnung ist hingegen schnell zu beschreiben, wird rasch trivial und in der Folge schnell uninteressant. Daher kann das Folgende angenommen werden: „Es muß etwas Drittes geben, das weder totale Unordnung noch vollkommene Ordnung ist, etwas, das nicht trivial, sondern kompliziert ist, ohne ungeordnet zu sein: Komplexität.“ Dabei meint Komplexität stets etwas Drittes, das sich zwischen zwei entgegengesetzten Polen befindet. Etwas Drittes, daß Nørretranders als den Kontinent der Komplexität bezeichnet; und worüber es keinen gesicherten Begriff gibt. 978

Die zweite Polarisierung in der vorliegenden Arbeit stellt weiterhin die nach normativer und deskriptiver Entscheidungstheorie dar. Liegt das Wesen der Komplexität nun stets zwischen zwei Polen, so handelt es sich bei der Komplexität, die zwischen Experten und Novizen liegt um Erfahrungskomplexität; bei der Komplexität, die zwischen der deskriptiven und der normativen Entscheidung liegt hingegen um Sachkomplexität, wonach der Teilnehmer an einem Unternehmensplanspiel offensichtlich zwei Probleme bewältigen muß: Zum einen ein Erfahrungsproblem und zum anderen ein Sachproblem, die beide durch Komplexität gekennzeichnet sind.

Dabei ist in Zusammenhang mit der Zeit zunächst folgendes wichtig: „Handlungen sind keine Prozesse, sondern Ereignisse. Sie können nicht analog zu Bewegungen (Körperbewegungen!) begriffen werden, sie sind vielmehr Entscheidungspunkte, an denen sich Beziehungsnetze restrukturieren, und Prozesse ergeben sich erst durch die Verknüpfung einer Mehrzahl von Handlungen. Mit dem Begriff der Bewegung ist immer eine Selbständerung gemeint. Ereignisse aber, und das gilt auch für Handlungen, ändern nicht sich selbst, sondern anderes. “979

Das Entscheidungshandeln der Planspielteilnehmer im Umgang mit einem Unternehmensplanspiel verändert demgemäß zwar das System, es verändert jedoch 
nicht zwangsläufig auch die Planspielteilnehmer selbst. Daher ist davon auszugehen, daß das Entscheidungshandeln im Unternehmensplanspiel der Erschließung des Sachproblems dient, der Erschließung des Erfahrungsproblems hingegen nicht. In diesem Zusammenhang soll hier die folgende Hypothese aufgestellt werden:
»Die Entscheidungshandlung im Unternehmensplanspiel gelingt nur unzureichend, weil das Erfahrungsproblem nicht erschlossen wird.«

Dabei ist unter Berücksichtigung des Zeitaspektes weiterhin folgendes wichtig: Im System besitzt jedes Ereignis, welches ein Element mit temporalisierter Komplexität darstellt, eine ihm eigene und nicht zu wiederholende Vergangenheitsund Zukunftsperspektive. Als gegenwärtig-passierend kann ein Ereignis nur begriffen werden, wenn seine unmittelbare Vergangenheit sowie seine unmittelbare Zukunft auszugsweise mit in die Betrachtung einbezogen wird, wobei dies in jedem Fall für Handlungen gilt. ${ }^{980}$ Es können zwar auch Handlungen nach ihrer Dauer untersucht werden, da alles, was in der Zeit existiert, eine wie immer minimale zeitliche Extension aufweist, aber diese Extension der Handlung hat im Handlungssystem nur die Funktion eines Differenzpunktes zwischen Zukunft und Vergangenheit. Sie wird ausschließlich unter diesem Gesichtspunkt in Betracht gezogen, wobei jede Handlung, dauert sie für Umschaltvorgänge im System zu lange, in Teilhandlungen aufgesplittet werden kann, womit eine Annäherung an Punktualität erfolgt. Sie, die Zeit, ist außerdem in Handlungszusammenhängen schon längst konstituiert, wenn sie in Zusammenhang mit Zweck und Mittel gestellt wird, welche hier dann eine Art Prüf- und Kontrollsinn erfüllen. ${ }^{981}$

Dies ist im Unternehmensplanspiel der Fall. So werden die zur Entscheidungsfindung aufgewandten Mittel in Zusammenhang mit dem zum Betrachtungszeitpunkt erreichten Ergebnis in Beziehung zu dem übergeordneten Ziel gesetzt, wodurch Zeit konstituiert wird. Die Konstitution von Zeit verhindert aber die Möglichkeit zur Reflexion. Das fortgesetzte Wiederholen der gleichen Entscheidungssituation 
durch den Planspielteilnehmer suggeriert daher offensichtlich nur die Möglichkeit zur Reflexion. Vielmehr ist es so, daß nach Durchführung einer Entscheidungsrunde das System an Struktur gewonnen hat, welche in der folgenden Entscheidungsrunde durch erneutes Treffen einer Entscheidung fortgeschrieben wird. Die vorangegangene Struktur ist insoweit festgeschrieben und irreversibel. Die Handlung des Planspielteilnehmers in Form der Quasi-Handlung des Simulationsprogrammes stellt daher ein Ereignis im Simulationsprozeß dar, welches zwischen vergangenem und zukünftigen trennt. Insofern findet der Planspielteilnehmer in der nun folgenden Entscheidungsrunde zwar die gleichen Entscheidungsparameter vor, nicht jedoch ein in gleicher Weise strukturiertes System. Er ist aufgefordert durch eine erneute Entscheidung das System fortzuschreiben; hat jedoch nicht immer die Möglichkeit die vorangegangene Entscheidungsrunde isoliert $\mathrm{zu}$ reflektieren, was gewährleisten würde, daß Fehler in der Entscheidung auch ihren Konsequenzen zugerechnet werden könnten. ${ }^{982}$ So konnte ausgeführt werden, daß die Reflektion der Planspielergebnisse zumeist zum Ende eines Planspieleinsatzes erfolgen. Auch in der Untersuchung von Hartung hat sich jedoch bereits gezeigt, daß durch den Einsatz von Fragebögen in der Planspielphase, die ein Reflektionsinstrument darstellen, eine Verbesserung der Planspielergebnisse erzielt werden kann. ${ }^{983}$

In diesem Zusammenhang wird hier nun noch eine letzte Hypothese aufgestellt:984

$»$ Die Erschließung von Wissen zu einem Sachproblem ist durch Instruktion möglich. Die Erschließung von Wissen zu einem Erfahrungsproblem ist hingegen nur durch Konstruktion möglich; wobei davon ausgegangen wird, daß sich in einer didaktischen Konzeption zu einem Unter-

\footnotetext{
981 Vgl. Luhmann, N., (Temporalstrukturen 1979), S. 38 f.

982 Vgl. Abschnitt 3.2.2: Ablauf von Unternehmensplanspielen.

983 Vgl. zu dieser Untersuchung Abschnitt 2.2.2.3.2: Zum Zusammenhang von Komplexität und Lernerfolg sowie zum Einsatz der Fragebögen Abschnitt 6.6.1.3: Metakognition: Die Steuerung des eigenen Denkens.

984 Vgl. in diesem Zusammenhang auch die Ausführungen bei Gruber, Mandl \& Renkl zur Entstehung von Trägem Wissen sowie zur Notwendigkeit ein Gleichgewicht zwischen Konstruktion und Instruktion umzusetzen. Gruber, H.; Mandl, H.; Renkl, A., (Kluft 2000), S. 139 ff. sowie S. 143 ff.
} 
nehmensplanspiel Phasen der Instruktion und der Konstruktion abwechseln müssen, um einen Lernfortschritt zu gewährleisten.«

Unter Berücksichtigung des Zeitaspektes ist es nun erforderlich zur Entscheidungsunterstützung Systeme einzusetzen, die in der Lage sind, den Faktor Zeit zu berücksichtigen. In diesem Zusammenhang wird im folgenden auf Entscheidungsunterstützungssysteme eingegangen, die beim Planspieleinsatz zur Entscheidungsunterstützung eingesetzt werden.

\subsubsection{Entscheidungsunterstützungssysteme}

Neben Expertensystemen ${ }^{985}$ existieren konventionelle Formen der Computerunterstützung. Dies sind Executive-Informationssysteme, Managementinformationssysteme und Entscheidungsunterstützungssysteme, die sich nach dem »Grad der Programmiertheit« der Entscheidungen, die durch das System zu stützen oder zu treffen sind, von Expertensystemen abgrenzen lassen. Dabei stellen ExecutiveInformationssysteme und Managementinformationssysteme lediglich Informationen zur Verfügung, während Entscheidungsunterstützungssysteme zusätzlich auch den Problemlösungsprozeß unterstützen. Von Expertensystemen unterscheiden sich demnach alle drei Formen der Computerunterstützung darin, daß sie keine selbständigen Aussagen darüber vornehmen können, wie Wissen, welches in der Wissensbasis dargestellt wird, weiterverarbeitet werden kann. 986

$\mathrm{Da}$ in der Entscheidungsunterstützung bei Unternehmensplanspielen Entscheidungsunterstützungssysteme zur Anwendung gelangen, soll darauf im folgenden näher eingegangen werden. Entscheidungsunterstützungssysteme oder auch Decision Support Systems (DSS) können wie folgt definiert werden: „DSS entscheiden nicht, sie nehmen dem Entscheidungsträger keine Entscheidung ab, sondern dienen der Entscheidungsberatung, d.h. der Entscheidungsvorbereitung und -findung, der Analyse der Vor- und Nachteile von offenstehenden Alternativen, der Überprüfung der Machbarkeit bzw. Widersprüchlichkeit von Entscheidungsvor-

\footnotetext{
985 Vgl. Abschnitt 6.6.3: Darstellung von Expertenwissen in Expertensystemen.

986 Vgl. Wandel, H.-U., (Expertensysteme 1992), S. 19 ff. und die dort angegebene Literatur.
} 
schlägen, und schließlich dienen sie der Dokumentation und der Erklärung oder

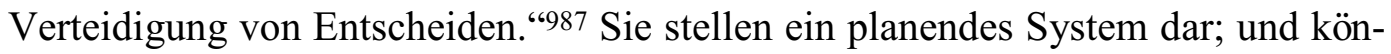
nen darüber hinaus Entscheidungsvorschläge zur zielgerichteten Veränderung entstandener Probleme ableiten, die zum einen auf den Resultaten der Simulation und zum anderen auf den im System enthaltenen Regeln zur Selektion einer Entscheidungsalternative basieren. ${ }^{988}$ In diesem Sinne sind sie,,[...] hochorganisierte Informationssysteme, welche speziell zum Zwecke der Entscheidungsunterstützung in einem klar abgegrenzten Entscheidungsumfeld aufgebaut wurden und sich kontinuierlich mit diesem weiterentwickeln“. 989

Um all dies zu gewährleisten bestehen sie, die Entscheidungsunterstützungssysteme, typischerweise aus Menschen mit verschiedenen Funktionen im Entscheidungsprozeß, einem spezifischen und abgrenzbaren Entscheidungskontext, einem Zielsystem, einer Wissensbasis sowie einer Arbeitsumgebung; und können entweder passive oder aktive Entscheidungsunterstützung leisten. Passive Entscheidungsunterstützung meint dabei, daß das Entscheidungsunterstützungssystem nicht in der Lage ist Entscheidungsvorschläge oder Lösungen zu generieren, aktive hingegen wenn es dies kann.990

Ein Entscheidungsunterstützungssystem, welches zum Planspiel PUMA entwickelt wurde, stellt nun bspw. das von Werner entwickelte Entscheidungsunterstützungssystem aMAdEUS, ein Akronym für Marketing-Entscheidungs-Unterstützungssystem, dar. ${ }^{991}$ Hier stehen den Teilnehmern des Unternehmensplanspiels PUMA verschiedene Entscheidungshilfen und Unterstützungsfunktionen zur Verfügung, die von diesen selbständig abgerufen werden können. So besteht zum einen die Möglichkeit, gezielt Informationen abzufragen zum anderen aber auch die Möglichkeit, gezielt Regelwissen an ausgewählten Problemstellungen zu trainieren. 992 Demgemäß wird einem Planspielteilnehmer durch die Möglichkeit von Informationsabfragen die Gelegenheit gegeben, erkannte Wissensdefizite durch selbst abge-

\footnotetext{
987 Hättenschwiler, P., (Konzepte 1999), S. 1.

988 Vgl. Gmilkowsky, P.; Roth, F., (Simulation 1999), S. 225.

989 Vgl. Hättenschwiler, P., (Konzepte 1999), S. 1.

990 Vgl. Hättenschwiler, P., (Konzepte 1999), S. 1.

991 Vgl. Werner, L., (Entscheidungsunterstützungssysteme 1992), S. 201 ff.
} 
rufene Informationen auszugleichen. Weiterhin können Defizite im Bereich des Regelwissens gezielt trainiert werden.

Außerdem findet der Umstand Berücksichtigung, daß der ausgewählte Bereich Marketing ein Subsystem des Gesamtsystems Unternehmung darstellt und nicht isoliert betrachtet werden kann. Er ist vielmehr als integrativer Bestandteil des Gesamtsystems Unternehmung anzusehen. Das impliziert wiederum, daß zunächst die Gesamtsituation des Unternehmens unter Berücksichtigung der Gewinn- und Verlustrechnung, der Bilanz und des Finanzberichtes zu analysieren ist, bevor ausgewählte Problemstellungen analysiert werden können. ${ }^{993}$

In Zusammenhang mit den Erkenntnissen der vorliegenden Untersuchung, ist ein solches Vorgehen als Berücksichtigung generellen Systemwissens zu interpretieren. Einem Planspielteilnehmer wird dadurch die Möglichkeit gegeben, das System der Unternehmung unter ganzheitlichen und spezifischen Gesichtspunkten $\mathrm{zu}$ analysieren und $\mathrm{zu}$ verstehen. Hier ist gleichsam auch ein zentraler Anknüpfungspunkt für weitere Forschungsaktivitäten zu sehen. So wird davon ausgegangen, daß der Lerner, unter verstärkter Berücksichtigung generellen Systemwissens, dessen betriebswirtschaftliche Relevanz bereits aufgezeigt werden konnte ${ }^{994}$ und hypothetisch als metakognitive Komponente bereichsspezifischen Wissens angenommen wurde, ${ }^{995}$ Aktivitäten des geleiteten Problemlösens vollziehen kann und dadurch seine Handlungskompetenz verbessert. In diesem Zusammenhang kann weiterhin davon ausgegangen werden, daß der Lerner seine Handlungskompetenz ebenfalls verbessert, wenn dem Erfahrungsproblem entsprochen wird. Dabei ist das Wissen, was in diesem Zusammenhang zu erwerben ist ebenfalls metakognitiver Natur. Es ist als Wissen über sich selbst anzunehmen und wird durch Reflexion erworben.

Im folgenden wird nun wiederum auf den Gegenstand Unternehmensgründung bezug genommen. Es wird dessen Stellung in der Lehre aufgezeigt und die kriti-

\footnotetext{
992 Vgl. Werner, L., (Entscheidungsunterstützungssysteme 1992), S. 210 ff.

993 Vgl. Werner, L., (Entscheidungsunterstützungssysteme 1992), S. $211 \mathrm{ff}$.

994 Vgl. Abschnitt 4.3: Das Unternehmensplanspiel als Modell über das komplexe System der Unternehmung.
} 
schen Faktoren Finanzierung und Information unter Berücksichtigung der TopDown-Zerlegung eines offenen Systems ${ }^{996}$ dynamisch betrachtet.

\section{7}

\section{Entrepreneurship Education}

\subsubsection{Unternehmensgründung als Gegenstand der Lehre}

Es wurde bereits darauf hingewiesen, daß sich die Forschung der 90er Jahre mit der Notwendigkeit beschäftigt, den Forschungsgegenstand der Unternehmensgründung vermehrt an Hochschulen zu etablieren.997 Zweck einer solchen Integration ist die Ausbildung von Personen, welche die Absicht haben, ein Unternehmen zu gründen. ${ }^{998}$ Dabei sollte dieses Ziel nicht nur auf Absolventen der Wiso-Fakultäten abzielen, sondern bspw. auch auf Absolventen der Ingenieurwissenschaften, Informatik und Naturwissenschaften. ${ }^{999}$ In diesem Zusammenhang stellt sich daher die Frage, ob und in welcher Form Entrepreneurship ein Gegenstand der Lehre sein kann. ${ }^{1000}$

Ein Vorbild dafür kann offensichtlich der anglo-amerikanische Sprachraum bieten. Dies bedingt sich einmal dadurch, daß Entrepreneurship international schon seit längerem Gegenstand der Lehre ist. ${ }^{1001}$ Die der Lehre zugrunde liegende anglo-amerikanische Forschung kann dabei in fünf Hauptkategorien unterteilt werden. Die erste, welche nicht in einem wirklich engen Zusammenhang mit der Theorie steht, fokussiert die Definition des Begriffes Entrepreneur. Eine zweite Kategorie stellt die Erforschung von Eigenschaften, wie z.B. die Untersuchung psychologischer Eigenschaften von Personen, die als Entrepreneurs bezeichnet werden können, dar. Eine weitere ist die Erforschung von Erfolgsfaktoren, d. h.

\footnotetext{
995 Vgl. Abschnitt 6.5.2: Die Systemschwierigkeit: Ausdruck der Problemkomplexität in Wissensgrößen.

996 Vgl. Abschnitt 4.3.1: Differenzierung und Komplexität im Unternehmensplanspiel.

997 Vgl. Abschnitt 4.4.1: Chronologische Entwicklung der Unternehmensgründungsforschung.

998 Vgl. Ripsas, S., (Entrepreneurship 1997), S. 231.

999 Vgl. Klandt, H., (Entrepreneurship 1999), S. 246.

1000 Es sei darauf hingewiesen, daß der englischsprachige Begriffskern „Entrepreneurship Education“" synonym zum deutschen Begriffskern Unternehmerausbildung verwandt werden kann. Davon soll auch hier ausgegangen werden. Vgl. dazu die Ausführungen bei Klandt, H., (Entrepreneurship 1999), S. $241 \mathrm{ff.}$

1001 Vgl. Klandt, H., (Entrepreneurship 1999), S. 241.
} 
die Erforschung von Gründen, die den Erfolg von neu gegründeten und existierenden Unternehmen erklären. Eine vierte Kategorie ist die Untersuchung der Struktur und des Aufbaus neuer Unternehmen. Schließlich sind noch Studien zu nennen, welche die Effekte von Umweltfaktoren auf unternehmerisches Handeln untersuchen. 1002

In diesem Zusammenhang weist Klandt zum anderen darauf hin, daß die in den englisch- und deutschsprachigen Veröffentlichungen vorzufindenden Inhalte, trotz abweichender Begriffsdefinitionen, eine weitgehende Deckung aufweisen. ${ }^{1003}$

Daraus wäre zu folgern, daß auch in der deutschen Gründungsforschung ein ausreichendes $\mathrm{Ma}$ an Forschung geleistet wurde, um die Basis für eine Gründungslehre darzustellen. Dies zeigt auch die gegenwärtig zu verzeichnende Entwicklung, da Gründung zunehmend zum Gegenstand der Lehre wird. So verweist Klandt, selbst Inhaber des Stiftungslehrstuhls für Allgemeine Betriebswirtschaftslehre, insbesondere Gründungsmanagement und Entrepreneurship, darauf, daß sich in der Zukunft mehr als 20 Lehrstühle mit dem Ausbildungs- und Forschungsansatz des Entrepreneurship etablieren werden. ${ }^{1004}$ In diesem Zusammenhang ist die Persönlichkeit des Unternehmers von Interesse, worauf im folgenden eingegangen werden soll.

\subsubsection{Der Begriff des Unternehmensgründers}

Die Person des Unternehmensgründers hat eine tragende Funktion im Gründungsgeschehen. So wird der Erfolg der Unternehmensgründung außer durch einen ausgefeilten Geschäftsplan ${ }^{1005}$ auch durch die Person des Unternehmensgründers wesentlich bestimmt. ${ }^{1006}$

Von Schumpeter wurde der Unternehmer als eine Person definiert, welche die bestehende Ökonomie durch Einführung neuer Produkte und Services, durch neue

\footnotetext{
1002 Vgl. Bull, I.; Willard, G.E., (Theory 1993), S. 184.

1003 Vgl. Klandt, H., (Entrepreneurship 1999), S. 241.

1004 Klandt, H., (Entrepreneurship 1999), S. 241.

1005 Vgl. Abschnitt 6.8.1: Der Geschäftsplan als Instrument der Gründungsplanung.

1006 Vgl. Klandt, H., (Person 1980), S. 321 f.
} 
Organisationsformen oder neue Materialien zerstört. ${ }^{1007}$ Die große Mehrheit neuer Unternehmen werden jedoch in bestehende Märkte hinein initiiert, was Bygrave dazu veranlaßte, eine umfassendere Definition des Entrepreneur's zu formulieren. Sie umfaßt jeden, der eine Existenz gründet. Ein Entrepreneur ist jemand, der eine Gelegenheit sieht und eine Organisation konzipiert, um diese umzusetzen. ${ }^{1008}$

Das durch den Unternehmenssgründer zu überblickende System ist dabei zumeist wenig arbeitsteilig. Dem Gründer oder dem Gründungsteam obliegt es, unterschiedlichste Anforderungen gleichzeitig zu bewältigen. Er steht besonders in der Gründungs- und Frühentwicklungsphase im Vordergrund und verbindet in einer Person die Funktion des strategischen Planers mit der des Durchsetzers sowie die des Vollziehenden. Durch diese Personalunion sind die Personenmerkmale des Gründers mit den Eigenschaften -den Stärken und Schwächen- der Gründungsunternehmung gleichzusetzen. ${ }^{1009}$

Daher fungiert der Unternehmensgründer gleichsam als Kontrolleur des Systems und hat in diesem Zusammenhang vielfältige Planungsaufgaben zu erfüllen. Zur Erstellung der grundlegenden Konzeptionen und Planungen dient dabei zunächst der Geschäftsplan, auf den im folgenden eingegangen wird.

\subsection{Liquiditätsplanung einer Unternehmensgründung}

\subsubsection{Der Geschäftsplan als Instrument der Gründungsplanung}

Im Vergleich zur etablierten Unternehmung ist ein Merkmal der Gründungsunternehmung, daß sie keine Vergangenheitsdaten aufzuweisen hat, welche die Basis darstellen, um eine Prognose der zukünftigen Entwicklung der Unternehmung vorzunehmen. Eine solche Prognose ist jedoch zwingend erforderlich, da der In-

1007 Vgl. Schumpeter, J.A., unter Beteiligung von Preiswerk, S., (Kapitalismus 1946), S. 214 f.

1008 Vgl. Bygrave, W.T., (Process 1997), S. 2. Der Begriff Entrepreneur betont stärker das Gründen neuer Einheiten, als den, eine Unternehmung zu übernehmen oder fortzuführen. Vgl. Klandt, H., (Aktivität 1984), S. 14. 
teressentenkreis einer solchen Prognose sehr groß ist. So hat daran zunächst einmal der Unternehmungsgründer selbst sowie beteiligte Personen, insbesondere aber auch die Geldgeber, ein gesteigertes Interesse. Die Basis einer solchen Prognose stellt der Unternehmungsgründer selbst ${ }^{1010}$ sowie weiterhin die ausformulierte Geschäftsidee dar, auf die im folgenden eingegangen werden soll.1011

Um eine Existenz zu gründen, ist zunächst eine Geschäftsidee erforderlich, welche später, im Rahmen einer Unternehmensgründungsplanung, zu einem tragfähigen Konzept ausgebaut werden muß. ${ }^{1012}$ Dabei ist eine Vielzahl von Entscheidungen zu berücksichtigen, die ein hohes Maß an Komplexität aufweisen. Denn vergleichsweise sind in bestehenden Unternehmen in ähnlich kurzer Zeit, wie sie die Gründungsphase gewöhnlich mit sechs bis zwölf Monaten umfaßt, nur in Extremsituationen zugleich Entscheidungen zu treffen über Produkt/Markt-Kombination, Standort und Lage, Rechtsform, Finanzierung der Gesamtunternehmung, Absatzorganisation/Vertriebskanäle, Personalorganisation Beschaffungskanäle/Einkaufsorganisation, Produktion/Dienstleistungserstellung, die gleichsam nur einige der wichtigsten darstellen. ${ }^{1013}$

In jüngerer Zeit wird für eine solche Unternehmensgründungsplanung der Geschäftsplan, der auch als Business-Plan, Unternehmensplan, Gründungsplan, Geschäftskonzept und ähnliches bezeichnet wird, als ein geeignetes Instrument angesehen. ${ }^{1014}$ Hier sollen die Begriffe Businessplan und Geschäftsplan verwendet und im folgenden synonym verstanden werden, da Businessplan wörtlich übersetzt soviel wie Geschäftsplan heißt. ${ }^{1015}$ Definiert werden kann der Business- oder Geschäftsplan, Steuck verwendet bspw. die Bezeichnung Businessplan, dabei wie folgt: „Der Businessplan ist ein Dokument, das die wichtigsten Eckdaten und

1009 Vgl. Szyperski, N.; Nathusius, K., (Probleme 1999), S. 35 f. Vgl. auch Klandt, H., (Aktivität 1984); Klandt, H., (Person 1990), S. 29; Mitton, D.G., (Entrepreneur 1989), S. 919.

1010 Vgl. Abschnitt 6.7.2: Der Begriff des Unternehmensgründers.

1011 Vgl. Klandt, H., (Person 1980), S. 321.

1012 Vgl. BMWi (Hrsg.), (Starthilfe 1999), S. 18.

1013 Vgl. Rentrop, N., (Strategien 1985), S. 16.

1014 Vgl. BMWi (Hrsg.), (Starthilfe 1999), S. 18; Klandt, H.; Finke-Schürmann, T., (Existenzgründung 1998), S. 15. In den USA gehört die Erstellung und Vorlage eines BusinessPlans hingegen schon lange zur Notwendigkeit, um externen Zielgruppen die eigene Geschäftsidee nahezubringen und von der Tragfähigkeit des Unternehmenskonzeptes zu überzeugen. Vgl. dies., (Existenzgründung 1998), S. 15. 
Planvorgaben für ein geschäftliches Vorhaben -häufig eine Unternehmensgründung- umfaßt und an einen bestimmten Adressatenkreis -häufig potentielle Kapitalgeber- gerichtet ist.“"1016

Steuck nimmt damit bezug auf eine der verschiedenen externen Funktionen des Businessplans, welchen die Erstellung des Geschäftsplans, neben internen Funktionen, dient. ${ }^{1017}$ Diese externe Funktion, die der Kapitalbeschaffung dient, stellt gleichsam die zentrale externe Funktion des Geschäftsplans dar. So macht die Erstellung des Geschäftsplans, und damit die Ausformulierung der unternehmerischen Idee, das Konzept für externe Kapitalgeber transparent. Dabei legen Banken Wert auf einen in sich schlüssigen Business-Plan, der ihnen ein stimmiges und realistisches Geschäftskonzept, einen mit dem Produkt verbundenen Kundennutzen, realistische Marktchancen und plausible Planungsannahmen, offenlegt. Darüber hinaus liegt ein besonderer Fokus auf dem Management, da der Erfolg oder Mißerfolg einer Gründung stark mit der Persönlichkeit des Gründers in Verbindung steht. ${ }^{1018}$

Die interne Funktion dient dem Unternehmensgründer zur Vergegenwärtigung und Benennung seiner Ideen, deren Formulierung und stetigen Soll-IstVergleichen. Demgemäß dient er nach Steuck zum einen der Verständnis- und Erkenntnissteigerung sowie daneben auch dem Zwecke der Kanalisierung der Geschäftsidee, des Risikomanagements und der Steuerung und Kommunikation. ${ }^{1019}$ Gerade in diesem Zusammenhang ist es auch wichtig, daß er nicht nur in der Gründungsphase, sondern auch in den darauffolgenden Phasen zur Anwendung gelangt ${ }^{1020}$ und zu ständigen Soll-Ist-Vergleich dient. ${ }^{1021}$

Als problematisch ist in diesem Zusammenhang anzusehen, daß die zu planenden Vorhaben und die Prozesse der Planung sich sehr unterschiedlich darstellen und

1015 Vgl. Benzel, W.; Wolz, E., (Businessplan 2000), S. 10.

1016 Steuck, J.W., (Businessplan 1999), S. 12.

1017 Vgl. Struck, U., (Geschäftspläne 1990), S. 1. Zu internen Aufgaben und externen Zielgruppen des Business-Plans vgl. weiterhin Klandt, H., (Gründungsmanagement 1999).

1018 Vgl. Wupperfeld, U., (Business-Plan 1999), S. 8 f.

1019 Steuck, J.W., (Businessplan 1999), S. 19.

1020 Vgl. Benzel, W.; Wolz, E., (Businessplan 2000), u. a. S. 10 u. S. 18. 
es daher nicht möglich ist, einen allgemeingültigen Plan oder Planungsprozeß zu entwerfen und vorzugeben. Diesen Schluß läßt bereits die Vielzahl möglicher Gründungen zu. ${ }^{1022}$ Es ist jedoch möglich, Planungsprozesse zu typologisieren. So entwirft bspw. Steuck, gestützt durch praktische Beispiele, drei typische Planungssituationen, welche er als Einzelgründung, komplexe Hightech-Neugründung und Diversifizierung im Großunternehmen bezeichnet. ${ }^{1023}$

In diesem Zusammenhang soll an dieser Stelle nun der Versuch unternommen werden, mit Hilfe des in Kapitel vier entwickelten Modells eine dynamische Finanzmittelplanung formal darzustellen. ${ }^{1024}$ Dazu wird zunächst auf die Möglichkeit der Dekomposition einer Planungsaufgabe eingegangen.

\subsubsection{Dekomposition einer Planungsaufgabe}

Durch den Planungsprozeß werden die unternehmerischen Entscheidungen vorbereitet. ${ }^{1025}$ Dabei ist die Qualität der Planung von entscheidender Bedeutung, da die Realitätsnähe der Planung und der Grad der Zielerreichung davon abhängen. ${ }^{1026}$ Der Planungsbegriff kann grundlegend definiert werden als ,,[...] ein geordneter, informationsverarbeitender Prozeß zur Erstellung eines Entwurfs, welcher Größen für das Erreichen von Zielen vorausschauend festlegt.“1027 Analog dazu läßt sich die Definition eines Planes festlegen: „Ein Plan ist als Ergebnis eines geordneten, informationsverarbeitenden Prozesses ein Entwurf, welcher Größen für das Erreichen von Zielen vorausschauend festlegt.“"1028

Der Planer ist dabei in erster Linie mit zwei Schwierigkeiten konfrontiert: Das sind zum einen die vielfach unvollständig und unvollkommen zur Verfügung ste-

\footnotetext{
1021 Vgl. Benzel, W.; Wolz, E., (Businessplan 2000), S. 14 ff.

1022 Vgl. Abschnitt 4.4.2.3: Formen der Unternehmensgründung.

1023 Steuck, J.W., (Businessplan 1999), S. 19.

1024 Eine Betrachtung unter statischen Gesichtspunkten, die durch das statische Wesen des Simulationsmodells impliziert wurde, konnte in Kapitel vier bereits vorgenommen werden. Vgl. Abschnitt 4.5: Insolvenz als zentraler Faktor des Scheiterns junger Unternehmen. Vgl. Abschnitt 3.4.6.1.1: Allgemeiner Überblick über das Unternehmensplanspiel MARKUS.

1026 Vgl. Hax, K., (Entwicklung 1959), S. 23.

1027 Schweitzer, M., (Planung 1989), S. 11.

1028 Schweitzer, M., (Planung 1989), S. 11.
} 
henden Informationen, zum anderen die Planung unter Ungewißheit der zukünftigen Situation. ${ }^{1029}$

Informationsdefizite stellen im Gründungsprozeß nun die zweithäufigste Konkursursache nach den finanziellen Mängeln dar. Dabei betrifft der Informationsbedarf alle wesentlichen Teilbereiche der Betriebswirtschaft und muß zum Teil durch eigene Befragungen in Zusammenhang mit Faktoren, wie bspw. der Kundenfrequenz oder der Verkehrsanbindung, selbst erhoben werden. ${ }^{1030}$ Es ist daher $\mathrm{zu}$ gewährleisten, daß ein ausreichendes Maß an Informationen zur Verfügung steht, womit das folgende »Prinzip« gilt: „Die Aussage: „Wie die Planung - so die Gründung!“ kann wie folgt ergänzt werden: „Wie die Information - so die Planung!"“1031

In Zusammenhang mit Planungsaufgaben können verschiedene Möglichkeiten zur Dekomposition angenommen werden. So formuliert Hauer unter anderem die folgenden: 1032

1. Zum einen ist es möglich, die Planungsaufgabe durch vertikale Dekomposition in verschiedene hierarchisch gestaffelte Teilaufgaben zu zerlegen. Dabei ist es erforderlich, daß ein System aus einer endlichen Anzahl von mindestens zwei Einheiten besteht oder sich in eine endliche Anzahl von mindestens zwei Einheiten dekomponieren läßt, um es als hierarchisch bezeichnen zu können. Weiterhin ist es erforderlich, daß die Einheiten im Hinblick auf einen konkretisierungsbedürftigen Aspekt in einer Beziehung der Über- oder Unterordnung stehen.

2. Durch horizontale Dekomposition, als zweite Problemzerlegungsform, kann dagegen die Gesamtaufgabe in mehrere gleichgeordnete Teilaufgaben zerlegt werden. Kennzeichnend hierfür ist das Fehlen einer hierarchischen Über- bzw. Unterordnung. Interdependenzen zwischen den Teilaufgaben können durch

1029 Vgl. Hax, K., (Entwicklung 1959), S. 23.

1030 Vgl. Schoeffling, H., (Businessplan 2001), S. 32.

1031 Schoeffling, H., (Businessplan 2001), S. 32. Schoeffling führt verschiedene generelle, regionale als auch internetbasierte Informationsquellen an. Vgl. ders., (Businessplan 2001), S. $32 \mathrm{ff}$. 
iterative Anpassungen der Koppelungsfaktoren in den Teilplänen berücksichtigt werden.

Das Beispiel von Chronos \& Tempus zeigt, im Hinblick auf den Faktor Zeit, auf, daß Chronos durch Aufteilung des Konstruktionsprozesses erheblich weniger Zeit zur Produktion seiner Uhren benötigt als Tempus. Die Dekomposition einer Planungsaufgabe kann daher am konkreten Beispiel als planvolles Handeln identifiziert werden.

„Zwei Uhrmacher, Chronos und Tempus, erzeugen mit gleicher Präzision Uhren aus tausend Teilen in zweierlei Weise: Während Tempus seine Uhren sequentiell produziert und dadurch bei einer Störung jedesmal von vorne beginnen muß, teilt Chronos den Konstruktionsprozeß in Teilschritte auf. In jedem Teilschritt werden 10 Einheiten zusammengebaut. Auf der ersten Ebene entsteht so aus 10 Einzelteilen ein Bauteil. Auf der zweiten Ebene ergeben 10 Bauteile eine Komponente. Auf der dritten und letzten Ebene schließlich werden 10 Komponenten zur fertigen Uhr zusammengesetzt. Wenn Chronos gestört wird, verliert er also nur jeweils das gerade in Bearbeitung befindliche Teil der jeweiligen Ebene. Stört man ihn gerade, wenn er aus den 10 Komponenten die Uhr bildet, muß er diesen Schritt nochmals wiederholen. Die Komponenten, die aus je 100 Einzelteilen bestehen, bleiben ihm jedoch erhalten. Die Wahrscheinlichkeit für die beiden Uhrmacher, eine Uhr fertigzustellen bei gegebener Störwahrscheinlichkeit $\mathrm{p}$ beträgt für Tempus $(1-\mathrm{p})^{1000}$, für Chronos $(1-p)^{10}$. Nimmt man eine Störwahrscheinlichkeit von $\mathrm{p}=0,01$ an, braucht auf lange Sicht Tempus 20000 mal mehr Zeit als Chronos." $" 1033$ 
3. Durch Anwendung einer horizontal-vertikalen Dekomposition, einer Kombination aus vertikaler und horizontaler Dekomposition, können schließlich Teilaufgaben gebildet werden, die sowohl eine Über- und Unterordnung als auch eine Gleichordnung aufweisen können.

6.8.3

Dekomposition einer Finanzmittelplanung im Hinblick auf den Aspekt der Beteiligungsfinanzierung

Zunächst ist davon auszugehen, daß eine Finanzmittelplanung über einen sich in der Zeit erstreckenden Prozeß, wie die Gründung ihn darstellt, ${ }^{1034}$ zunächst einmalig im Rahmen der Erstellung des Geschäftsplans ${ }^{1035}$ vorgenommen wird. Diese Planung erfährt jedoch im Zeitverlauf Aktualisierungen und Anpassungen, so daß der Unternehmungsgründer sowohl dazu aufgefordert ist, die finanzielle Situation der gegründeten Unternehmung stets im Auge zu behalten, als auch stets über Möglichkeiten der Finanzierung informiert zu sein. Weiterhin ist der Unternehmungsgründer dazu aufgefordert, den Plan stets im Auge zu behalten, ihn zu reflektieren und gegebenenfalls $\mathrm{zu}$ modifizieren. Da Informationsdefizite die zweithäufigste Ursache für das Scheitern von Unternehmensgründungen darstellen, ${ }^{1036}$ ist in diesem Zusammenhang davon auszugehen, daß der Unternehmungsgründer sich dabei mit einem Problem konfrontiert sieht. Er ist daher an dieser Stelle als Problemlöser anzusehen, der Instrumente zur Problemlösung benötigt.

In diesem Zusammenhang ist von der in Kapitel vier entwickelten Top-DownZerlegung eines offenen Systems auszugehen, die aus den konstituierenden Merkmalen der Allgemeinen Systemtheorie sowie weiterhin aus den daraus entwickelten Möglichkeiten zur Dekomposition eines Systems hergeleitet wurde. Werden in diesem Zusammenhang die Möglichkeiten zur Dekomposition einer Planungsaufgabe betrachtet, ${ }^{1037}$ so ist davon auszugehen, daß die entwickelte Top-DownZerlegung eines offenen Systems einen geeigneten Rahmen darstellt, um Pla-

1034 Vgl. Abschnitt 4.4.2.2: Der Prozeß der Unternehmensgründung.

1035 Vgl. Abschnitt 6.8.1: Der Geschäftsplan als Instrument der Gründungsplanung.

1036 Vgl. Abschnitt 6.8.2: Dekomposition einer Planungsaufgabe.

1037 Vgl. Abschnitt 6.8.2: Dekomposition einer Planungsaufgabe. 
nungsaspekte formal zu erfassen. Dies konnte in Kapitel vier, unter bezug auf die an dieser Stelle vorgenommenen Ausführungen, bereits aufgezeigt werden. An dieser Stelle ist nun davon auszugehen, daß sich das vorliegende Modell auch unter dynamischen Gesichtspunkten für Planungszwecke nutzen läßt. Diese Betrachtung erfolgt dabei unter dem Aspekt, daß das Simulationsmodell im Spiel zum Gegenstand der vorgesehenen Geschäftsmaßnahmen wird. ${ }^{1038}$ Weiterhin kann, unter dem Aspekt der notwendigen Reflexion des eigenen Handelns durch den Unternehmungsgründer, das formale Regelkreisprinzip des Klassifikationsansatzes menschlicher Fehler von Reason ${ }^{1039}$ implizit als Möglichkeit zur Problemlösung angenommen werden.

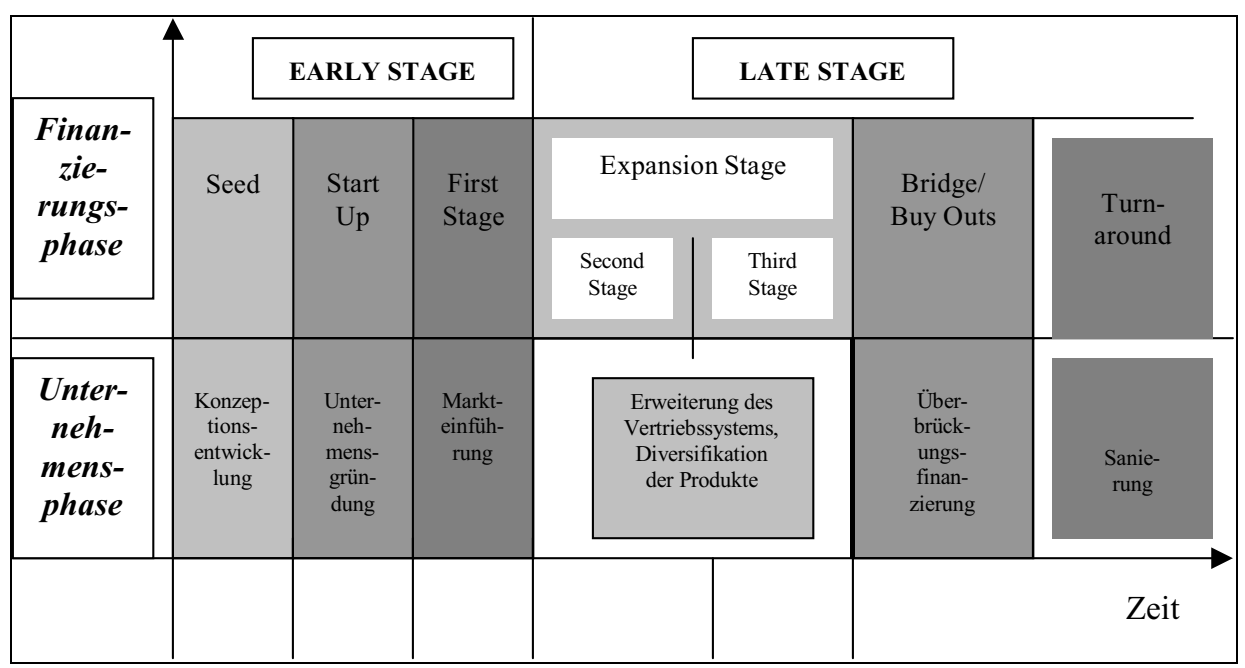

\section{Abbildung 6-8: $\quad$ Beteiligungsfinanzierung in den Lebensphasen eines}

\section{Unternehmens ${ }^{1040}$}

In den weiteren Ausführungen wird dabei von einer zeitraumbezogenen Betrachtung der Möglichkeiten zur Beteiligungsfinanzierung von Betsch, Groh \& Schmidt ausgegangen und die Frühphasenfinanzierung mit den drei Sub-Phasen des Seed-, Start Up- und First Stage - Financing herangezogen: ${ }^{1041}$ [Vgl. Abbildung 6-8].

Die Frühphasen-Finanzierung beinhaltet zum einen die Finanzierung der Konzeptentwicklung auf der Basis einer Idee vor Unternehmensgründung. Zum ande-

\footnotetext{
1038 Vgl. Abschnitt 2.1.2.2: Das Unternehmensplanspiel: Ein Entscheidungsspiel.

1039 Vgl. Abschnitt 6.6.2: Kognitive Grenzen: Der Klassifikationsansatz menschlicher Fehler von Reason.

1040 Betsch, O.; Groh, A.P.; Schmidt, K., (Unternehmen 2000), S. 20.

1041 Vgl. Betsch, O.; Groh, A.P.; Schmidt, K.,(Unternehmen 2000).
} 
ren beinhaltet sie die Finanzierung bis zum Start der Produktion und der Vermarktung. Diese Frühphasen-Finanzierung beinhaltet weiterhin eine Reihe von Subphasen, die im zeitlichen Prozeß einer Unternehmensgründung durchlaufen werden: 1042

1. DAS SEED FINANCING: In der Phase der Grundlagenentwicklung und der Erstellung von Prototypen erfolgt die Finanzierung überwiegend aus eigenen Mitteln und aus öffentlichen Fördermitteln. Der Einsatz von Venture Capital ist hier schwierig, weil die Marktchancen des Produktes oft noch nicht beurteilt werden können. Dennoch sind Venture Capital-Finanzierungen auch in dieser Phase keine Seltenheit und beinhalten die finanzielle Unterstützung zur Entwicklung der Idee bis hin zu ersten verwertbaren Resultaten, auf deren Basis ein Geschäftskonzept für ein zu gründendes Unternehmen erstellt werden kann. [Vgl. Abbildung 6-9].

Zur Gewinnung von Eigenkapital können möglicherweise Verwandte und Freunde hilfreich einspringen, das Eigenkapitalhilfe-Programm der Bundesregierung kann in Anspruch genommen werden, oder Partner gewonnen werden, die dann allerdings auf einem Mitspracherecht bestehen. Weiterhin besteht auch die Möglichkeit, sich öffentlich geförderte oder private Beteiligungsgesellschaften zu suchen. Als öffentliche Fördermittel wurden für diese Phase beispielhaft das ERPEigenkapital-Programm, das DtA-Startgeld sowie die Beratungsförderung angenommen. Dabei sind diese Förderungen, obgleich es sich um öffentliche Fördermittel handelt, grundsätzlich bei der Hausbank zu beantragen. ${ }^{1043}$ In den Abbildungen werden die Finanzierungsmöglichkeiten jeweils als Umweltparameter begriffen, die potentiell durch den Gründer in Anspruch genommen werden können.

1042 Vgl. auch die Betrachtungen in Abschnitt 4.5: Insolvenz als zentraler Faktor des Scheiterns junger Unternehmen. Die Annahmen der Elemente zur Beteiligungsfinanzierung stellen potentielle Annahmen zur Beteiligungsfinanzierung dar. 


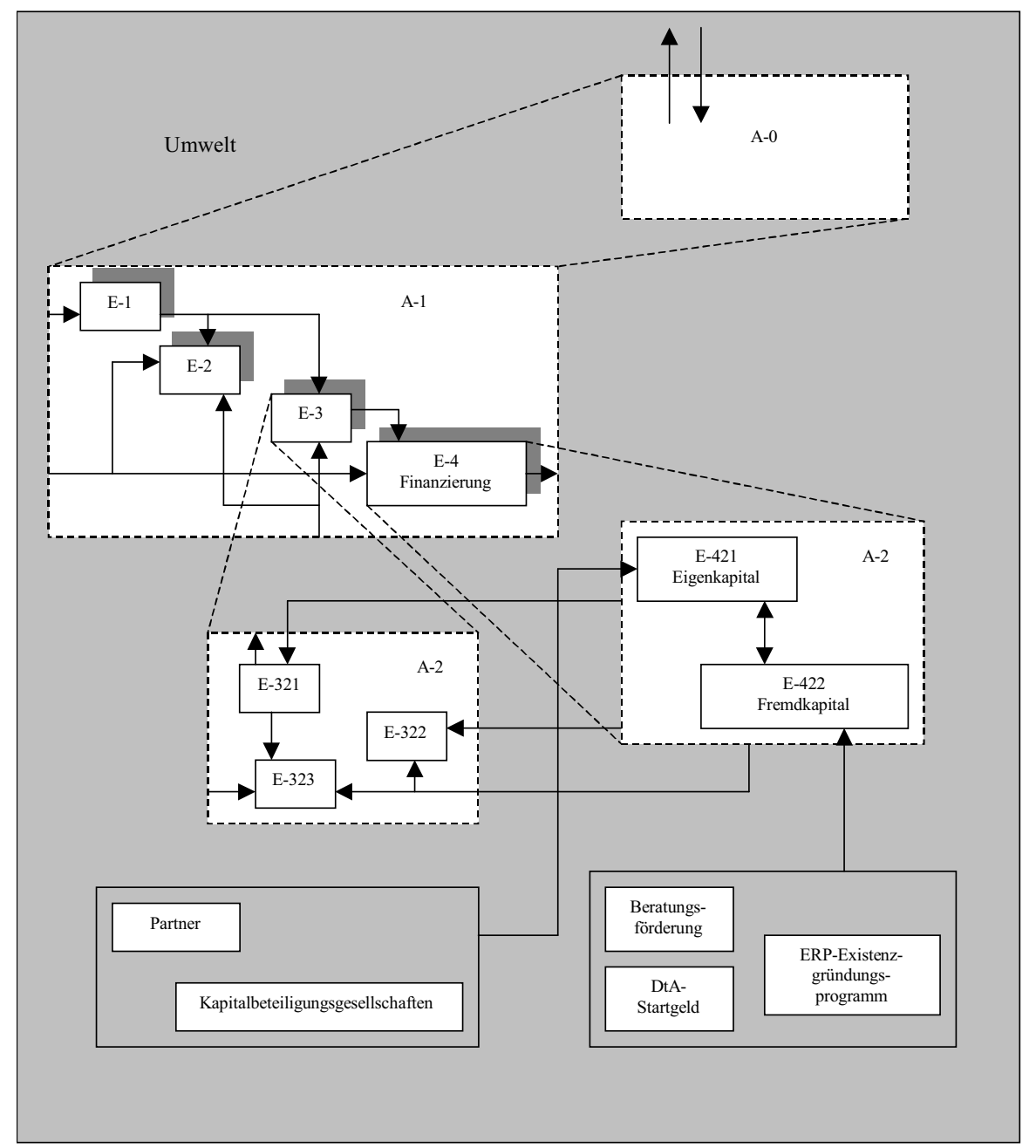

\section{Abbildung 6-9: Möglichkeiten der Beteiligungsfinanzierung in der Phase des Seed Financing1044}

2. START UP FINANCING: Das neue Produkt wird in dieser Phase zur Produktionsreife weiterentwickelt, und es kommt zum Abschluß der Produktionsvorbereitung. Zur späteren Markteinführung des Produktes erfolgt die Ausarbeitung eines Marketingkonzeptes. Dabei muß der wachsende Kapitalbedarf beinahe vollständig durch Eigenkapital gedeckt werden, da die anfallenden Verluste eine Aufnahme von Bankkrediten erschweren. Somit stellt die Start-up-Beteiligung vielfach den Beginn einer Venture Capital-Finanzierung dar, da in neuen Unternehmen die Produktionsvorbereitung meist zeitgleich mit der Unternehmensgründung erfolgt. [Vgl. Abbildung 6-10]. 


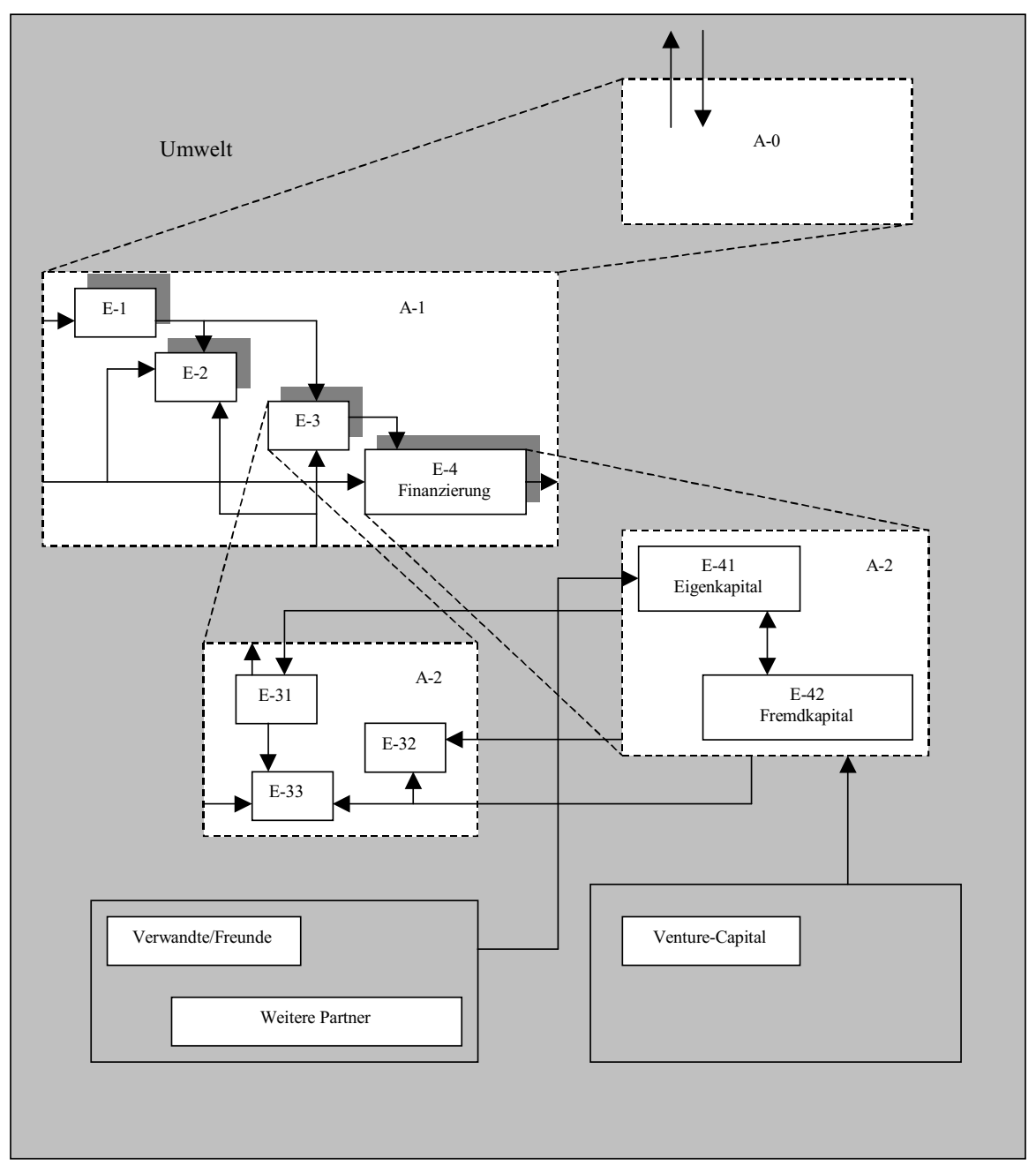

Abbildung 6-10: Möglichkeiten zur Beteiligungsfinanzierung in der Phase des Start Up Financing

3. FIRST STAGE FINANCING: In dieser Phase wird mit der Produktion begonnen und das Produkt auf dem Markt eingeführt. Dies beinhaltet gleichzeitig den Aufbau des Vertriebsnetzes, der Produktionsanlagen sowie der ersten Marketingaktivitäten. Dabei beinhaltet die First-Stage-Finanzierung nun die höchsten Fremdmittel des Innovationsprozesses. Die First-Stage-Finanzierung endet mit dem Übergang in das Wachstumsstadium eines Unternehmens. Hier verbessern die ersten Gewinne die Kreditwürdigkeit des Unternehmens, so daß die Aufnahme von Bankkrediten möglich wird. [Vgl. Abbildung 6-11]. 


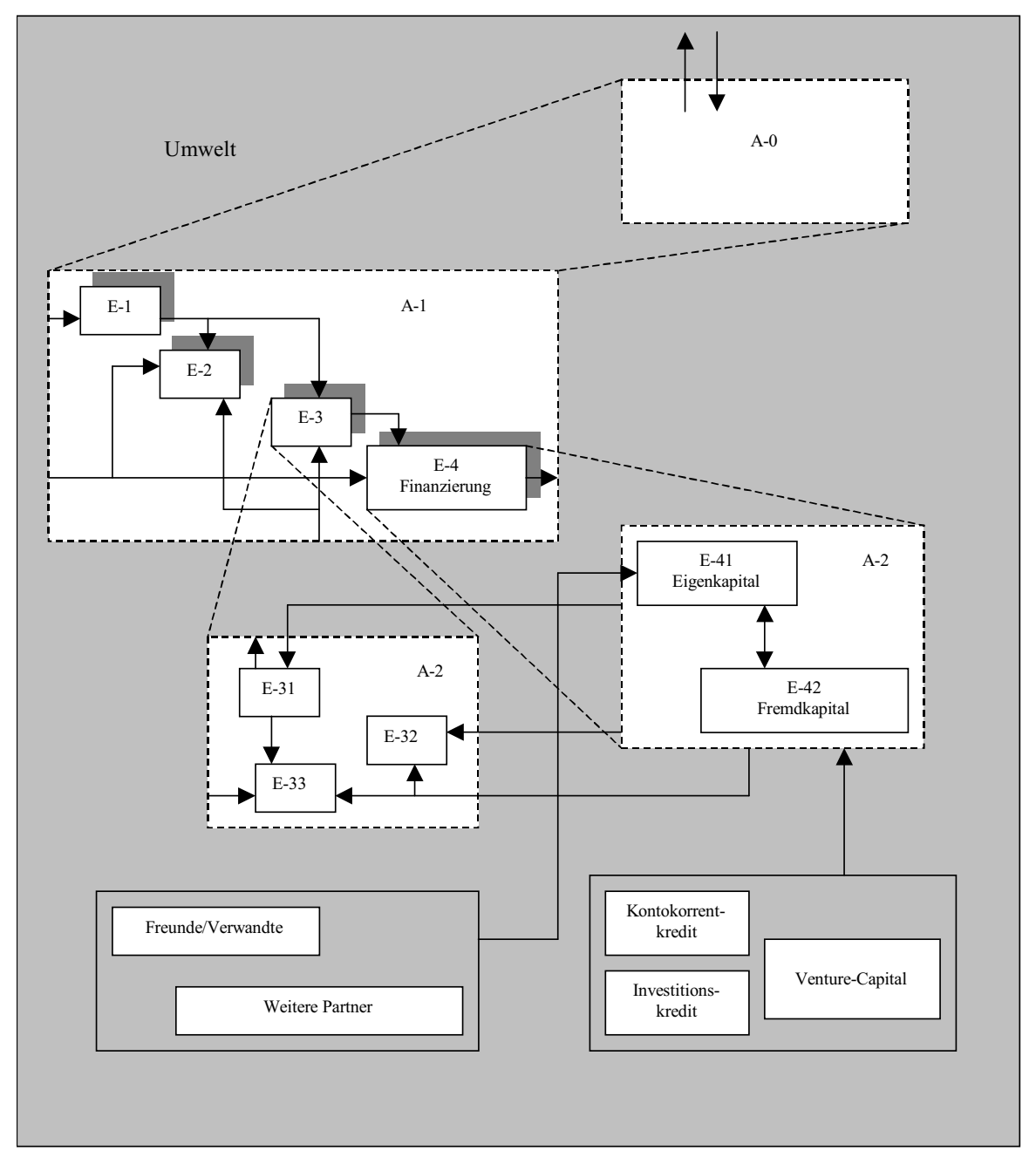

\section{Abbildung 6-11: Möglichkeiten zur Beteiligungsfinanzierung in der Phase des First Stage Financing}

Schließlich kann von einer Betrachtung im Zeitverlauf ausgegangen werden.[Vgl. Abbildung 6-12]. 


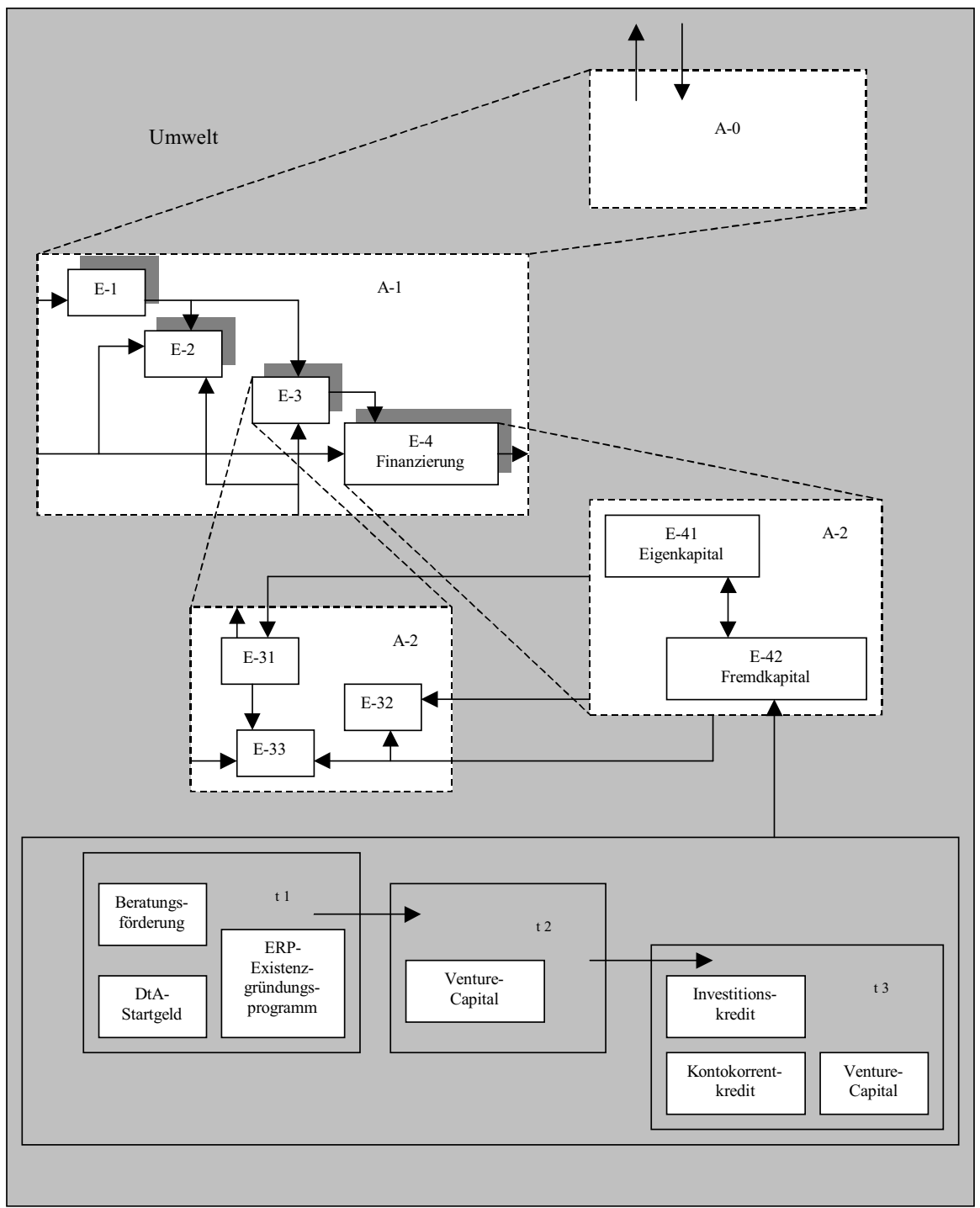

Abbildung 6-12: Möglichkeiten zur Beteiligungsfinanzierung im Zeitverlauf 
Die Überlegungen zu dieser Arbeit gingen vom Gegenstandsbereich der Unternehmensgründung aus. Aus der chronologischen Entwicklung des Forschungsstandes konnte in diesem Zusammenhang abgeleitet werden, daß ein hoher Anteil der wissenschaftstheoretischen Diskussionsbeiträge deskriptiv bleibt und die wenigen modellgestützten Untersuchungen Lücken aufweisen, die der Komplexität der Problemstellung nicht gerecht werden. In Zusammenhang mit dem von Dietz, unter Berücksichtigung chaostheoretischer Aspekte, entwickelten Modells ${ }^{1045}$ wurde dann eine modelltheoretische Auseinandersetzung mit dem Bereich der Unternehmensgründung angeregt, wozu die Möglichkeit der Modellbildung in Form von Unternehmensplanspielen ausgewählt wurde. In diesem Zusammenhang konnte dem Aspekt der Komplexität sowohl im Bereich der Unternehmensgründung als auch im Bereich von Unternehmensplanspielmodellen eine zentrale Rolle zugewiesen werden.

In einem Planspiel ist Komplexität dabei vor allem im Hinblick auf das vordergründig verfolgte Ziel zu sehen, komplexes Denken zu schulen. In diesem Zusammenhang konnte unter einer allgemeinen Betrachtung des Komplexitätsbegriffes sowie der generellen Relevanz des Verständnisses von Komplexität und deren Bewältigung, der Schluß gezogen werden, daß Komplexität im Unternehmensplanspiel offensichtlich einen mehrdimensionalen Bezugsrahmen aufweist. Ausgehend von der Feststellung, daß in der Literatur kein allgemeingültiger Index dazu existiert, der die Komplexität eines Unternehmensplanspielmodells entsprechend determiniert, wurde dann zunächst die Frage aufgeworfen, worin das Prinzip der Komplexität in Zusammenhang mit Unternehmensplanspielen überhaupt besteht.

Um in dieser Frage zu einer Annäherung zu gelangen, war auf die spezifische Simulationstechnik beim Planspiel Bezug zu nehmen, bei der nach Simulationsmodell und Entscheidungsspiel unterschieden werden kann. Durch diese Unterscheidung gelingt es, den Komplexitätsbegriff im Unternehmensplanspiel zunächst in zweifacher Weise zu verankern: So wurde Komplexität in Zusammen- 
hang mit der Modellbildung als attributive Eigenschaft von Systemen interpretiert. In Zusammenhang mit der Funktion als Lehr-Lern-Instrument wurde Komplexität als attributive Eigenschaft von Entscheidungen angenommen. Diese Sichtweise ermöglichte es wiederum, die Betrachtung weiterer Dimensionen von Komplexität im Unternehmensplanspiel in zwei Untersuchungsbereichen fortzuführen: Im Bereich der Modellbildung wurde die Untersuchung objektiver Eigenschaften von Komplexität unter Berücksichtigung der Systemtheorie möglich. Im Bereich der Lehr-Lern-Forschung wurde unter Berücksichtigung kognitionspsychologischer Forschung die Untersuchung subjektiver Wahrnehmungen von Komplexität möglich. Dabei konnten die gewonnenen Erkenntnisse weiterhin in einen gemeinsamen Bezugsrahmen gestellt werden.

In Zusammenhang mit der Modellbildung konnte in Kapitel vier die Allgemeine Systemtheorie herangezogen werden. Dabei wurden zunächst die Merkmale ausgeführt, unter denen sie zu ihrem universellen Anspruch gelangt. Da diese gleichsam den Hintergrund für die durch Luhmann vollzogenen Annahmen einer Theorie der Systemdifferenzierung und einer Theorie der Systemkomplexität darstellen, ${ }^{1046}$ konnte die Argumentation unter Berücksichtigung dieser Aspekte fortgesetzt werden. In der Anwendung der erläuterten Gesichtspunkte auf das offene System der Unternehmung wurde so die Identifikation von verschiedenen Komplexitätsgraden sowie weiterhin eine Top-down-Zerlegung eines offenen Systems möglich. Unter Ergänzung der Komplexitätsgrade durch Komplexitätsdimensionen von Ehlken ${ }^{1047}$ gelang es dann weiterhin, ein Komplexitätsmaß für ein Unternehmensplanspielmodell abzuleiten, welches ein offenes System abbildet. Außerdem konnte schließlich der Faktor Insolvenz als kritische Variable im Gründungsgeschehen identifiziert und eine Anwendung der in diesem Kapitel entwickelten Top-down-Zerlegung eines offenen Systems vorgenommen werden.

In Kapitel sechs wurde hingegen, ausgehend von einer zwischen der normativen und deskriptiven Entscheidungstheorie aufgezeigten Differenz, eine Komplexitätsbetrachtung als attributive Eigenschaft von Entscheidungen durchgeführt. In diesem Zusammenhang ließ sich die potentiell angenommene Differenz zwischen 
objektiver und subjektiver Komplexität als potentielle Problemkomplexität identifizieren. Durch eine Gegenüberstellung der Problemkomplexität sowie weiterer Komplexitätsdimensionen, die in den Ausführungen abgeleitet wurden, mit den in Kapitel vier analysierten Komplexitätsgraden und Komplexitätsdimensionen gelang es dann, das Ausmaß an Problemkomplexität formal zu bestimmen. Weiterhin erfolgte eine Gegenüberstellung der Problemkomplexität mit Personmerkmalen wodurch die Systemschwierigkeit abgeleitet und als Ausdruck eines potentiellen Wissensdefizits auf Seiten des Individuums formal darstellbar wurde.

Schließlich wurde die folgende Hypothese aufgestellt:

»Das generelle Systemwissen, mit den generellen Komponenten Fakten- und Operationswissen, stellt die metakognitive Komponente zu bereichsspezifischem Fakten- und Operationswissen dar.«

Die weiteren Betrachtungen dienten der Identifikation relevanter Ansätze der kognitionspsychologischen Forschung, die in Kapitel fünf ausgeführt wurden, für den betriebswirtschaftlichen Lernprozeß, unter kognitiven, emotionalen und metakognitiven Gesichtspunkten. Diesen Ansätzen wurde die aufgestellte Hypothese gegenübergestellt und festgestellt, daß unter Berücksichtigung generellen Systemwissens offensichtlich ein komplexeres Modell menschlicher Informationsverarbeitung erforderlich ist. Dabei ist ein solches Modell als ein Produktionssystem denkbar, das in der Lage ist, metakognitives Wissen $\mathrm{zu}$ nutzen und die Aspekte der Motivation und Emotion zu integrieren.

In diesem Zusammenhang konnte dann noch eine zweite Hypothese aufgestellt werden. So konnten Bloech, Kauer \& Orth in ihrer Untersuchung eine unzureichende Anwendung des betriebswirtschaftlichen Sachwissens im Unternehmensplanspiel nachweisen, was als Mangel an unternehmerischer Handlungskompetenz interpretiert wurde. 1048 Der Grund dafür könnte unter Berücksichtigung der aufgestellten Hypothese im Mangel an generellem Systemwissen vermutet wer- 
den, welches mit dem betriebswirtschaftlichen Sachwissen in Zusammenhang steht. Dies ist gleichsam ein paradoxer Schluß, da dieses Wissen ja gerade durch den Einsatz von Unternehmensplanspielen vermittelt werden soll. Die ursprüngliche Hypothese lautete zunächst wie folgt:

»Die unzureichende Anwendung betriebswirtschaftlichen Sachwissens im Unternehmensplanspiel ist auf einen Mangel an damit in Zusammenhang stehendem, generellem Systemwissen zurückzuführen.«

Unter Berücksichtigung der Interpretation dieses Sachverhalts als mangelnder unternehmerischer Handlungskompetenz durch Bloech, Kauer \& Orth konnte diese zusätzlich wie folgt erweitert werden:

»Die unzureichende Anwendung betriebswirtschaftlichen Sachwissens im Unternehmensplanspiel ist auf einen Mangel an damit in Zusammenhang stehendem, generellem Systemwissen zurückzuführen; « und äußert sich als mangelnde unternehmerische Handlungskompetenz.

Im Hinblick auf anknüpfende Forschungsaktivitäten ergibt sich die Möglichkeit, die aufgestellten Hypothesen durch entsprechend zu konzipierende Instrumentarien zu untersuchen. Bei einer Bestätigung der Hypothese könnten sich didaktische Implikationen ergeben, da generelles Systemwissen demnach auch in betriebswirtschaftlichen Seminaren und Vorlesungen zu vermitteln wäre bzw. als Entscheidungsunterstützung im Planspiel.

Im weiteren Verlauf wurde dann an die Ausführungen in Kapitel drei angeknüpft, welches eine Gegenüberstellung betriebswirtschaftlicher und psychologischer Forschung zum Ziel hatte. Diese erwies sich als notwendig, um dem in Kapitel zwei aufgezeigten Bedarf nach einer Zusammenarbeit zwischen der Betriebswirtschaftslehre und der Psychologie zu entsprechen, diente vordergründig jedoch der in Kapitel sechs weiter entwickelten Ausgangsfrage, warum die Teilnehmer an 
einem Unternehmensplanspiel ihr Sachwissen nur unzureichend oder gar nicht anwenden. ${ }^{1049}$

Für die Beantwortung dieser Frage erwies es sich als problematisch, daß aus der spezifischen Simulationstechnik beim Planspiel unterschiedliche Modellkonstruktionen entwickelt und die vorliegenden psychologischen Untersuchungen bisher an unterschiedlichen Versuchsanordnungen vorgenommen wurden. Durch Berücksichtigung des in der Luft- und Raumfahrttechnik entwickelten S-H-E-L-L - Modells gelang hier jedoch eine Gegenüberstellung der beiden Bereiche. So werden in diesem Modell die grundlegenden Elemente von Systemen berücksichtigt, die im Hinblick auf grundlegende Merkmale von Unternehmensplanspielmodellen zu interpretieren und in einen Beziehungszusammenhang mit Erkenntnissen der Psychologie zu Fehlleistungen im Umgang mit komplexen Systemen zu stellen waren.

In diesem Zusammenhang konnte unter Berücksichtigung der erfolgten Interpretation der grundlegenden Systemelemente eine Abgrenzung des Unternehmensplanspielsystems erfolgen. Weiterhin ließ sich festhalten, daß die Zuordnung von Fehlleistungen nicht in allen Bezugspunkten gelingt. Hier ergibt sich daher ein weiterer Anknüpfungspunkt für künftige Forschungsaktivitäten. Weiterhin kann durch die Betrachtungen in Kapitel drei gefolgert werden, daß eine vertiefte $\mathrm{Zu}$ sammenarbeit zwischen Psychologie und Betriebswirtschaftslehre sicherlich für die Zukunft wichtige Erkenntnisse liefern kann.

An die Betrachtungen in Kapitel drei anzuknüpfen stellte der Gedanke dar, daß davon auszugehen ist, daß die mangelnde unternehmerische Handlungskompetenz Fehlleistungen im Umgang mit komplexen Systemen nach sich zieht. Ging es im Kontext von Kapitel drei jedoch um die konkreten Ausprägungen von Fehlern, so stellte sich in Kapitel sechs nun die Frage nach deren Ursachen und ihrer Überwindung. So konnte es in Kapitel sechs durch die Annahme eines Zeitpfades gelingen, den Novizen und den Experten als zwei Pole eines sich in der Zeit erstrekkenden Entwicklungsprozesses anzunehmen, den der Experte bereits abgeschlossen hat während der Novize ihn sich durch Reflexion seiner Fehler erschließt.

1049 Vgl. Bloech, J.; Kauer, G.; Orth, C., (Unternehmensplanspiel 1996), S. 38. 
Dies, die Reflexion des Novizen, zu beschreiben, wurde der Klassifikationsansatz menschlicher Fehler von Reason ausgewählt, der in vereinfachter Form die als betriebswirtschaftlich relevant erachteten und vermuteten kognitiven Aspekte berücksichtigt. ${ }^{1050}$ Die Tatsache, daß Experten diese Entwicklung bereits zurückgelegt haben, fand hingegen dadurch Berücksichtigung, daß das Expertenwissen die Möglichkeit bietet es in Expertensystemen darzustellen.

Da der Einsatz von Expertensystemen in der Entscheidungsunterstützung im Unternehmensplanspiel aufgrund ihres statischen Charakters jedoch als problematisch anzusehen ist, wurde schließlich die Bedeutung des Faktors Zeit in Zusammenhang mit der Handlung im System beleuchtet. Dabei konnte abgeleitet werden, daß der Unternehmensplanspieler zusätzlich zu seinem Sachproblem auch mit einem Erfahrungsproblem konfrontiert ist, wobei offensichtlich von folgendem auszugehen ist:

»Die Entscheidungshandlung im Unternehmensplanspiel gelingt nur unzureichend, weil das Erfahrungsproblem nicht erschlossen wird.«

Wobei der Bewältigung sowohl des Sachproblems als auch des Erfahrungsproblems didaktisch offensichtlich wie folgt entsprochen werden könnte:

»Die Erschließung von Wissen zu einem Sachproblem ist durch Instruktion möglich. Die Erschließung von Wissen zu einem Erfahrungsproblem ist hingegen nur durch Konstruktion möglich; wobei davon ausgegangen wird, daß sich in einer didaktischen Konzeption zu einem Unternehmensplanspiel Phasen der Instruktion und der Konstruktion abwechseln müssen, um einen Lernfortschritt zu gewährleisten.«

Dazu können, wie es auch bereits geschieht, Entscheidungsunterstützungssysteme im Lernprozeß eingesetzt werden. Hier wurde beispielhaft das mit dem Planspiel 
PUMA in Zusammenhang stehende Entscheidungsunterstützungssystem aMAdEUS ausgeführt. ${ }^{1051}$ Allgemein legen die Erkenntnisse der vorliegenden Untersuchung den Schluß nahe, daß ein zentraler Anknüpfungspunkt für weitere Forschungsaktivitäten in der Entscheidungsunterstützung unter Berücksichtigung generellen Systemwissens zu sehen ist.

In Zusammenhang mit dem Gegenstandsbereich Unternehmensgründung konnte dieser Aspekt, unter Berücksichtigung der Top-Down-Zerlegung eines offenen Systems, bereits unter statischen Gesichtspunkten aufgezeigt werden. An dieser Stelle erfolgte dann eine weitere Betrachtung der kritischen Faktoren Finanzierung und Information unter dynamischen Gesichtspunkten. Dabei wurde der Klassifikationsansatz menschlicher Fehler von Reason den weiteren Ausführungen lediglich implizit zugrunde gelegt.

1051 Vgl. Werner, L., (Entscheidungsunterstützungssysteme 1992), S. 201 ff. 


\section{Abkürzungsverzeichnis für Zeitschriftentitel}

BFuP

BWP

CATCA

CEP

DB

DBW

DUZ

ET \& P

HBR

IEEE TransInfTheory

Int. J. General Systems

Int. J. Man-Machine Studies

Int. J. Th. Phys.

Io

OR

Psychol. Res.

QZ

RKW

SMC

WiSt

$\mathrm{WiSu}$

WP

ZBW

ZfB
Betriebswirtschaftliche Forschung und Praxis

Berufsbildung in Wissenschaft und Praxis

Canadian Air Traffic Control Association

Control Engineering Practice

Der Betrieb

Die Betriebswirtschaft

Deutsche Universitätszeitung

Entrepreneurship Theory \& Practice: the Official Journal of the United States Association for Small Business and Entrepreneurship

Harvard Business Review

IEEE transactions on information theory:

a journal devoted to the theoretica

International Journal of General Systems

International Journal of Man-Machine Studies

International Journal of Theoretical Physics

Industrielle Organisation

Operations Research

Psychological Research

Qualität und Zuverlässigkeit: QZ; Zeitschrift für industrielle Qualitätssicherung

Rationalisierungs-Kuratorium der Deutschen Wirtschaft

IEEE Transactions on Systems, Man, And Cybernetics

Wirtschaftswissenschaftliches Studium

Das Wirtschaftsstudium

Kölner Zeitschrift für „Wirtschaft und Pädagogik“

Zeitschrift für Berufs- und Wirtschaftspädagogik Zeitschrift für Betriebswirtschaft 
$\mathrm{ZfbF}$

ZfhF

zfo

Z.f.Päd.

$\mathrm{ZP}$

Z. Psychol.
Schmalenbachs Zeitschrift für betriebswirtschaftliche Forschung

Zeitschrift für handelswissenschaftliche Forschung

Zeitschrift Führung und Organisation

Zeitschrift für Pädagogik

Zeitschrift für Planung

Zeitschrift für Psychologie mit Zeitschrift

für angewandte Psychologie

\section{Literaturverzeichnis}

Achtenhagen, F., (Lösen 1990): Zum Lösen komplexer Probleme, in: Unterrichtswissenschaft, Heft 4, 18. Jg., 1990, S. 290-294.

Achtenhagen, F., (Überlegungen 1990): Einige Überlegungen zur Bedeutung der Problemlöseforschung für die Didaktik des Wirtschaftslehreunterrichts, in: Feger, H. (Hrsg.), Wissenschaft und Verantwortung: Festschrift für Karl Josef Klauer zum sechzigsten Geburtstag, Göttingen 1990, S. 109129.

Achtenhagen, F.; Meyer, H.L., (Curriculumrevision 1971): Curriculumrevision, 3. Aufl., München 1971.

Achtenhagen, F.; Tramm, T.; Preiss, P.; Seemann-Weimar, H.; John, E.G.; Schunck, A., (Lernhandeln 1992): Lernhandeln in komplexen Situationen, Neue Konzepte der betriebswirtschaftlichen Ausbildung, Wiesbaden 1992.

Ackoff, R.L.; Emery, F.E., (Systeme 1975): Zielbewußte Systeme: Anwendung der Systemforschung auf gesellschaftliche Vorgänge, Frankfurt, New York 1975.

Adelson, B., (Problem 1981): Problem Solving and the Development of Abstract Categories in Programming Languages, in: Memory \& Cognition, No. 4, Vol. 9, 1981, S. 422-433.

Adelson, B., (Difficulty 1984): When Novices surpass Experts: The Difficulty of a Task may increase with Expertise, in: Journal of Experimental Psychology: Learning, Memory and Cognition, 10, 1984, S. 483-495. 
Aebli, H., (Denken 1980): Denken: Das Ordnen des Tuns. Band I: Kognitive Aspekte der Handlungstheorie, 1. Aufl., Stuttgart 1980.

Aebli, H., (Denken 1981): Denken: Das Ordnen des Tuns. Band 2: Denkprozesse, Stuttgart 1981.

Albach, H., (Entscheidungsprozeß 1961): Entscheidungsprozeß und Informationsfluß in der Unternehmensorganisation, in: Schnaufer, E.; Agthe, K. (Hrsg.), Organisation, Berlin, Baden-Baden 1961, S. 355-402.

Albach, H., (Aufgaben 1967): Stand und Aufgaben der Betriebswirtschaftslehre heute, in: ZfbF, Heft 7, 19. Jg., 1967, S. 446-469.

Albach, H., (Unternehmensspiele 1974): Unternehmensspiele als Mittel der Führungskräfteschulung, in: Fasshauer, R.; Wurzbacher, W. (Hrsg.), Unternehmensspiele - Stand und Entwicklungstendenzen, IBM-Beiträge zur Datenverarbeitung, Methoden und Techniken 4, Stuttgart 1974, S. 29-38.

Allwood, C.M., (Novices 1986): Novices on the Computer: a Review of the Literature, in: Int. J. Man-Machine Studies, Vol. 25, 1986, S. 633-658.

Anderson, J.R., (Language 1976): Language, Memory and Thought, Hillsday, N.J. 1976.

Anderson, J.R., (Acquisition 1982): Acquisition of Cognitive Skill, in: Psychological Review, No. 4, Vol. 89, 1982, S. 369-406.

Anderson, J.R., (Architecture 1983): The Architecture of Cognition, Cambridge 1983.

Anderson, J.R., (Theory 1989): A Theory of the Origins of Human Knowledge, in: Artifical Intelligence, No. 1-3, Vol. 40, 1989, S. 313-351.

Anderson, J.R., (Character 1990): The Adaptive Character of Thought, Hillsdale, N.J. 1990.

Anderson, J.R., (Psychologie 1996): Kognitive Psychologie: eine Einführung, 2. Aufl., Heidelberg 1996.

Anderson, J.R.; Bower, G.H., (Human 1973): Human Associative Memory, New York 1973.

Anderson, J.R.; Thompson, R., (Analogy 1989): Use of Analogy in a Production System Architecture, in: Vosniadou, S.; Ortony, A., (Eds.), Similarity and Analogical Reasoning, Cambridge 1989, S. 267-297. 
Arbeitskreis Gamer, (Unternehmungsspiele 1963): Unternehmungsspiele und ihre Bedeutung für die betriebswirtschaftliche Ausbildung an Hochschulen, in: ZfhF (N.F.), Heft 4, 15. Jg., 1963, S. 149-190.

Arnold, R., (Schlüsselqualifikationen 1996): Schlüsselqualifikationen. „Fachwissen in der Krise“ oder Erziehung zur Inkompetenz?, in: Seyd, W.; Witt, R. (Hrsg.), Situation, Handlung, Persönlichkeit. Kategorien wirtschaftspädagogischen Denkens. Festschrift für Lothar Reetz, Hamburg 1996, S. 57-70.

Ashby, W.R., (Variety 1958): Requisite Variety and its Implications for the Control of Complex Systems, in: Cybernetica, No. 2, Vol. 1, 1958, S. 83-99.

Bachmann, W., (Grundlagen 1988): Theoretische Grundlagen einer handlungstheoretischen Wirtschaftsdidaktik, Bergisch Gladbach 1988.

Baetge, J.; Biethahn, J.; Bokranz, R., (Information 1997): Management Information zum OPEX II-Unternehmungsspiel von J. Biethahn, 12. Aufl., Münster 1997.

Bainbridge, L., (Equations 1987): Mathematical Equations or Processing Routines, in: Rasmussen, J.; Duncan, K.; Leplat, J. (Eds.), New Technology and Human Error, Chichester 1987, S. 271-286.

Baldegger, U., (Motivation 1988): Die Motivation der Gründer gewerblicher Produktionsunternehmen, St. Gallen, Zürich 1988.

Banyard, P.; Hayes, N., (Denken 1995): Denken und Problemlösen, in: Gerstenmaier, J. (Hrsg.), Einführung in die Kognitionspsychologie, München, Basel 1995, S. 121-152.

Barth, S., (Existenzgründer 1995): Existenzgründer in den neuen Bundesländern: psychologische Dimensionen und wirtschaftlicher Erfolg, Wiesbaden 1995.

Beck, H., (Schlüsselqualifikationen 1995): Schlüsselqualifikationen. Bildung im Wandel, 2. Aufl., Darmstadt 1995.

Becker, D.; Oldenbürger, H.-A.; Piehl, J., (Motivation 1987): Motivation und Emotion, in: Lüer, G. (Hrsg.), Allgemeine experimentelle Psychologie: eine Einführung in die methodischen Grundlagen mit praktischen Übungen für das experimentelle Praktikum, Stuttgart 1987, S. 431-470.

Beer, S., (Kybernetik 1962): Kybernetik und Management, Hamburg 1962. 
Bellinger, B., (Gründung 1975): Gründung, in: Handwörterbuch der Betriebswirtschaft, 4. Aufl, Bd. 2, 1975, Stuttgart 1975.

Bendig, R.; Frick, D.; Gabriel, R.; Meier, G.; Reinersmann-Rescheleit, M.; Timm, H., (Expertensysteme 1987): Expertensysteme: Systeme zur Lösung betriebswirtschaftlicher Problemstellungen, Teil 1, Diskussionsbeitrag des Fachbereichs Wirtschaftswissenschaft der UniversitätGesamthochschule-Duisburg Nr. 104, Duisburg 1987.

Benzel, W.; Wolz, E., (Businessplan 2000): Businessplan für Existenzgründer: Geschäftspläne erstellen und erfolgreich umsetzen; mit zahlreichen Beispielen, Regensburg, Düsseldorf, Berlin 2000.

Berndts, P.; Harmsen, D.-M., (Technologieorientierte 1985): Technologieorientierte Unternehmensgründungen in Zusammenarbeit mit staatlichen Forschungseinrichtungen, Studie im Auftrag des BMFT, TechnologieTransfer, Bd. 8, Köln 1985.

Berry, D.C.; Broadbent, D.E., (Relationship 1984): On the Relationship between Task Performance and Associated Verbalizable Knowledge, in: The Quarterly Journal of Experimental Psychology, Vol. 36 A, 1984, S. 209231.

Berry, D.C.; Broadbent, D.E., (Combination 1987): The Combination of Explicit and Implcit Learning Processes in Task Control, in: Psychol. Res., Vol. 49, 1987, S. 7-15.

Bertalanffy, L. v., (Biologie 1942): Theoretische Biologie. Zweiter Band: Stoffwechsel, Wachstum, Berlin-Zehlendorf 1942.

Bertalanffy, L. v., (Systemlehre 1949): Zu einer allgemeinen Systemlehre, in: Biologia Generalis, Heft 1, Bd. 19, Wien 1949, S. 114-129.

Bertalanffy, L. v., (Systemtheorie 1957): Allgemeine Systemtheorie, Wege zu einer neuen Mathesis Universalis, in: DUZ, Heft 5/6, 12. Jg., 1957, S. 812.

Bertalanffy, L. v., (General 1962): General System Theory - A Critical Review, in: General Systems, Bd. 7, 1962.

Bertalanffy, L. v., (System 1988): General System Theory - Foundations, Development, Applications, Revised Edition, Tenth Printing, New York 1988. 
Betsch, O.; Groh, A.P.; Schmidt, K., (Unternehmen 2000): Gründungs- und Wachstumsfinanzierung innovativer Unternehmen, München, Wien 2000.

Bierwagen, T., (Fluglotsen 1999): Kognitive Prozesse von Fluglotsen bei der Konflikterkennung: Modellierung und Implementierung, Sinzheim 1999.

Biethahn, J., (Ansatz 1991): Ein Ansatz zur systematischen Wissensverarbeitung auf der Basis der Erfahrungen beim Einsatz von XPS-Shells, in: Biethahn, J.; Bloech, J.; Bogaschewsky, R.; Hoppe, U. (Hrsg.), Wissensbasierte Systeme in der Wirtschaft: Anwendungen und Tools, Wiesbaden 1991, S. 183-190.

Biethahn, J., (Grenzen 1991): Aktuelle Grenzen von Expertensystemen und Entwicklungstrends, in: Biethahn, J.; Hoppe, U. (Hrsg.), Entwicklung von Expertensystemen: eine Einführung, Wiesbaden 1991, S. 281-300.

Biethahn, J.; Mucksch, H.; Ruf, W., (Informationsmanagement 1991): Ganzheitliches Informationsmanagement, Band 2: Daten- und Entwicklungsmanagement, München, Wien 1991.

Biethahn, J.; Muksch, H.; Ruf, W., (Informationsmanagement 2000): Ganzheitliches Informationsmanagement, Band 1: Grundlagen, 5., unwesentlich veränderte Aufl., München, Wien 2000.

Bleicher, K., (Unternehmungsspiel 1960): Das Unternehmungsspiel - eine neue Lehrmethode, in: Fortschrittliche Betriebsführung, Heft 2, 9. Jg., 1960, S. $36-40$.

Bleicher, K., (Entscheidungssimulation 1962): Entscheidungssimulation und Unternehmungsspiele, in: ZfB, Heft 1, 32. Jg., 1962, S. 15-30.

Bleicher, K., (Planspiel UB-5 1962): Planspiel UB-5 zum Treffen unternehmerischer Entscheidungen, Baden-Baden 1962.

Bleicher, K., (Planspiel UB-10 1962): Planspiel UB-10 für unternehmerische Entscheidungen, Baden-Baden 1962.

Bleicher, K., (Unternehmungsspiele 1962): Unternehmungsspiele. Simulationsmodelle für unternehmerische Entscheidungen, Baden-Baden 1962.

Bleicher, K., (Entscheidungsprozesse 1965): Entscheidungsprozesse an Unternehmungsspielen. Unternehmungsspiele als Darstellungsmittel der Unternehmungspolitik, Baden-Baden, Bad Homburg vor der Höhe 1965. 
Bleicher, K., (Unternehmungsspiele 1969): Unternehmungsspiele, in: Grochla, E. (Hrsg.), Handwörterbuch der Organisation, Stuttgart 1969, Sp. 16851694.

Bleicher, K., (Entwicklung -Heft 1- 1970): Die Entwicklung eines systemorientierten Organisations- und Führungsmodells der Unternehmung, in: zfo, Heft 1, 39. Jg., 1970, S. 3-8.

Bleicher, K., (Entscheidungsprozesse 1974): Entscheidungsprozesse an Unternehmungsspielen. Band I: Die Darstellung von Unternehmungspolitik und -planung an Idealmodellen, 3. Aufl., Baden-Baden, Bad Homburg vor der Höhe 1974.

Bliss, C., (Management 2000): Management von Komplexität: Ein integrierter, systemtheoretischer Ansatz zur Komplexitätsreduktion, Wiesbaden 2000.

Bloech, J., (Modellierung 1989): Modellierung komplexer betrieblicher Situationen und ihre didaktische Vermittlung mit Hilfe neuer Informations- und Kommunikationstechniken, in: Bundesminister für Bildung und Wissenschaft (Hrsg.), Neue Informationstechniken in kaufmännischen Modellversuchen: Ergebnisse schulischer Modellversuche und Möglichkeiten ihrer Umsetzung in die kaufmännische Ausbildung; Dokumentation einer Fachtagung in Göttingen, Bad Honnef 1989, S. 102-119.

Bloech, J.; Döhne, S.R.; Hartung, S.; Orth, C., (Einsatz 1998): Einsatz eines Planspiels in der kaufmännischen Weiterbildung-Verschiedene Arten der Komplexitätssteigerung im Planspiel-, Arbeitsbericht Nr. 1/1998 des Instituts für Betriebswirtschaftliche Produktions- und Investitionsforschung der Georg-August-Universität Göttingen, Göttingen 1998.

Bloech, J.; Hartung, S.; Kauer, G.; Orth, C., (Einsatz 1996): Einsatz eines Planspiels in der kaufmännischen Weiterbildung -Untersuchungen zur Förderung unternehmerischer Handlungskompetenz-, Arbeitsbericht Nr. 2/1996 des Instituts für Betriebswirtschaftliche Produktions- und Investitionsforschung der Georg-August-Universität Göttingen, Göttingen 1996.

Bloech, J.; Hartung, S.; Orth, C., (Unternehmensplanspiele 1998): Unternehmensplanspiele in der kaufmännischen Ausbildung - Anpassung der Komplexität an den Lernfortschritt, in: Beck, K.; Dubs, R. (Hrsg.), Kompetenzentwicklung in der Berufserziehung: kognitive, motivationale und moralische Dimensionen kaufmännischer Qualifizierungsprozesse, 
in: Zeitschrift für Berufs- und Wirtschaftspädagogik, Beiheft 14, Stuttgart 1998, S. 38-56.

Bloech, J.; Hartung, S.; Orth, C., (Einsatz 1999): Einsatz eines Planspiels in der kaufmännischen Weiterbildung, in: Wirtschaft und Erziehung, Heft 11, 51. Jg., 1999, S. 389-391.

Bloech, J.; Hartung, S.; Orth, C., (Förderung 1999): Förderung der unternehmerischen Handlungskompetenz durch Unternehmensplanspiele, in: Schelten, A.; Sloane, P.F.E.; Straka, G.A. (Hrsg.), Berufs- und Wirtschaftspädagogik im Spiegel der Forschung, Forschungsberichte des DGfE-Kongresses 1998, Opladen 1999, S. 121-131.

Bloech, J.; Kauer, G.; Orth, C., (Unternehmensplanspiele 1996): Unternehmensplanspiele in der kaufmännischen Ausbildung - Untersuchungen zum Wissenserwerb, in: Zeitschrift für Berufs- und Wirtschaftspädagogik, Beiheft 13, 1996, S. 37-52.

Bloech, J.; Rüscher, H., (BAP 1987): Unternehmensplanspiel BAP: Computersimulation mit Lotus - 123. Modellbeschreibung und Entscheidungsunterlagen, Göttingen 1987.

Bloech, J.; Rüscher, H., (EPUS 1992): Unternehmensplanspiel EPUS - eine experimentell ausgerichtete Unternehmenssimulation, in: Biethahn, J.; Hummeltenberg, W.; Schmidt, B. (Hrsg.), Simulation als betriebliche Entscheidungshilfe, Bd. 3, Berlin, Heidelberg, New York 1992, S. 268282.

Bloech, J.; Rüscher, H., (PENTA 1992): Die Unternehmenssimulation PENTA, in: Lücke, W.; Schulz, K. (Hrsg.), Umweltschutz und Investitionen, Wiesbaden 1992, S. 65-93.

Bloech, J. in Zusammenarbeit mit Rüscher, H.; Rill, W., (EpUS 1991): Modellbeschreibung und Bedienungsanweisungen zur Unternehmenssimulation EpUS: Ein-Platz-Unternehmens-Simulation, Spieler-Handbuch, Göttingen 1991.

Bloech, J. in Zusammenarbeit mit Rüscher, H.; Schubert, U., (Penta 1991): Modellbeschreibung zur Unternehmenssimulation PENTA. Planspiel Entsorgungs- Und Abfallwirtschaft, Göttingen 1991. 
Blohm, H., (Planspiele 1966): Differenziertere Planspiele, in: Der Volkswirt, Wirtschafts- und Finanz-Zeitung, Nr. 44., 20. Jg., 4. Quartal 1966, S. $2155-2156$.

BMWi (Hrsg.), (Starthilfe 1999): Starthilfe. Der erfolgreiche Weg in die Selbständigkeit, Köln 1999.

BMWi (Hrsg.), (Unternehmensnachfolge 1999): Unternehmensnachfolge. Der richtige Zeitpunkt - optimale Nachfolgeplanung, 4., überarb. Aufl., Köln 1999.

Böhret, C.; Wordelmann, P., (Planspiel 1975): Das Planspiel als Methode der Fortbildung. Zur allgemeinen und speziellen Verwendung der Simulationsmethode in der öffentlichen Verwaltung, Bonn 1975.

Boos, M., (Entscheidungsfindung 1996): Entscheidungsfindung in Gruppen: eine Prozeßanalyse, Bern, Göttingen, Toronto, Seattle 1996.

Borkowski, V.; Geis, V., (Expertensysteme 1990): Expertensysteme, Grundlagen, in: Mertens, P. (Haupthrsg.), Lexikon der Wirtschaftsinformatik, 2., vollst. neu bearb. u. erw. Aufl., Berlin, Heidelberg u.a. 1990, S. $173-$ 176.

Borkowski, V.; Geis, V., (Intelligenz 1990): Künstliche Intelligenz, in: Mertens, P. (Haupthrsg.), Lexikon der Wirtschaftsinformatik, 2., vollst. neu bearb. u. erw. Aufl., Berlin, Heidelberg u.a. 1990, S. 252-253.

Bossel, H., (Simulation 1992): Simulation dynamischer Systeme: Grundwissen, Methoden, Programme, 2., verb. Aufl., Braunschweig, Wiesbaden 1992.

Boulding, K.E., (Systems 1956): General Systems Theory-The Skeleton of Science, in: Management Science, No. 3, Vol. 2, 1956, S. 197-208.

Boulding, K.E., (Systemtheorie 1975): Die allgemeine Systemtheorie als Skelett der Wissenschaft, in: Baetge, J., (Hrsg.), Grundlagen der Wirtschaftsund Sozialkybernetik, Opladen 1975, S. 16-31.

Boulding, K.E., (Image 1997): The Image. Knowledge in Life and Society, Ann Arbor 1997.

Bower, G.H.; Cohen, P.R., (Influences 1982): Emotional Influences in Memory and Thinking: Data and Theory, in: Clark, M.S.; Fiske, S.T. (Eds.), Affect and Cognition, Hillsdale, N.J. 1982, S. 291-333.

Bramsemann, R., (Controlling 1990): Handbuch Controlling: Methoden und Techniken, 2., überarb. und erw. Aufl., München, Wien 1990. 
Brauchle, A., (Macht 1975): Von der Macht des Unbewußten: Tiefenpsychologie, 6. Aufl., Stuttgart 1975.

Breyde, C., (Schlüsselqualifikationen 1996): Die Schlüsselqualifikationen als endlose Debatte oder der Wunsch nach Klarheit über einen zu Recht vielschichtigen Begriff, in: Seyd, W.; Witt, R., (Hrsg.), SituationHandlung-Persönlichkeit. Kategorien wirtschaftspädagogischen Denkens. Festschrift für Lothar Reetz, Hamburg 1996, S. 237-244.

Broadbent, D.E.; FitzGerald, P.; Broadbent, M.H.P., (Knowledge 1986): Implicit and Explicit Knowledge in the Control of Complex Systems, in: British Journal of Psychology, Vol. 77, 1986, S. 33-50.

Bronner, R., (Entscheidung 1973): Entscheidung unter Zeitdruck. Eine Experimentaluntersuchung zur empirischen Theorie der Unternehmung, Tübingen 1973.

Bronner, R., (Komplexität 1992): Komplexität, in: Frese, E. (Hrsg.), Handwörterbuch der Organisation, 3. Aufl., Stuttgart 1992, Sp. 1121-1130.

Brown, A.L., (Development 1975): The Development of Memory: Knowing, Knowing about Knowing, and Knowing how to Know, in: Reese, H.W. (Ed.), Advances in Child Development and Behaviour, New York 1975, 10, S. 103-152.

Brown, A.L., (Schooling 1975): Development, Schooling and the Acquisition of Knowledge about Knowledge, in: Anderson, R.C.; Spiro, R.J.; Montague, W.E. (Eds.), Schooling and the Acquisition of Knowledge, Hillsdale, N.J., 1977, S. 241-257.

Brüderl, J.; Preisendörfer, P.; Ziegler, R., (Erfolg 1996): Der Erfolg neugegründeter Betriebe - Eine empirische Studie zu den Chancen und Risiken von Unternehmensgründungen, Berlin 1996.

Bull, I.; Willard, G.E., (Theory 1993): Towards a Theory of Entrepreneurship, in: Journal of Business Venturing, Vol. 8., 1993, S. 183-195.

Bunk, G.P.; Kaiser, M.; Zedler, R., (Schlüsselqualifikationen 1991): Schlüsselqualifikationen - Intention, Modifikation und Realisation in der beruflichen Aus- und Weiterbildung, in: Mitteilungen aus der Arbeitsmarktund Berufsforschung, Heft 2, 24. Jg., 1991, S. 365-374. 
Busse von Colbe, W.; Laßmann, G., (Betriebswirtschaftstheorie 1991): Betriebswirtschaftstheorie. Band 1: Grundlagen, Produktions- und Kostentheorie, 5., durchges. Aufl., Berlin, Heidelberg u.a. 1991.

Bygrave, W.D., (Process 1997): The Entrepreneurial Process, in: Bygrave, W.D. (Ed.), The Portable MBA in Entrepreneurship, $2^{\text {nd }}$ Ed., 1997.

Casti, J., (Connectivity 1979): Connectivity, Complexity, and Catastrophe in large-scale Systems, New York 1979.

Ceci, S.J.; Liker, J.K., (Study 1986): A Day at the Races: A Study of IQ, Expertise and Cognitive Complexity, in: Journal of Experimental Psychology: General, Vol. 115, 1986, S. 255-266.

Ceppi, C., (Erfahrungen 1970): Erfahrungen mit Management Games, in: io, Heft 7, 39. Jg., 1970, S. 305-308.

Chaitin, G.J., (Information 1982): Gödel's Theorem and Information, in: Int. J. Th. Phys., No. 12, Vol. 21, 1982, S. 941-954.

Chase, W.G.; Simon, H.A., (Perception 1973): Perception in Chess, in: Cognitive Psychology, No. 1, Vol. 4, 1973, S. 55-81.

Chi, M.T.H.; Feltovich, P.J.; Glaser, R., (Categorization 1981): Categorization and Representation of Physics Problems by Experts and Novices, in: Cognitive Science, 5, 1981, S. 121-152.

Cohen, K.J.; u.a., (Management Game 1960): The Carnegie Tech Management Game, in: The Journal of Business, No. 4, Vol. 33, 1960, S. 303-321.

Dahremöller, A., (Existenzgründungsstatistik 1987): Existenzgründungsstatistik - Nutzung amtlicher Datenquellen zur Erfassung des Gründungsgeschehens, Stuttgart 1987.

deKleer, J.; Brown, J.S., (Assumptions 1983): Assumptions and Ambiguities in Mechanistic Mental Models, in: Gentner, D.; Stevens, A.L. (Eds.), Mental Models, Hillsdale, N.J. 1983, S. 155-190.

Dietz, J.-W., (Gründung 1989): Gründung innovativer Unternehmen, Wiesbaden 1989.

Dietz, J.-W.; Roski, R., (Management 1989): Management von Innovationen, Ventures und Diskontinuitäten, in: Lücke, W.; Dietz, J.-W. (Hrsg.), Innovation und Controlling, Wiesbaden 1989, S. 285-332. 
Dill, W.R.; Doppelt, N., (Acquisition 1963): The Acquisition of Experience in a Complex Management Game, in: Management Science, No. 1, Vol. 10, 1963, S. 30-46.

Dörner, D., (Organisation 1974): Die kognitive Organisation beim Problemlösen. Versuche zu einer kybernetischen Theorie der elementaren Informationsverarbeitungsprozesse beim Denken, Bern, Stuttgart, Wien 1974.

Dörner, D., (Problemlösen 1976): Problemlösen als Informationsverarbeitung, 3. Aufl., Stuttgart, Berlin, Köln, Mainz 1976.

Dörner, D., (Struktur 1977): Kognitive Struktur und Information, in: Reulecke, W. (Hrsg.), Strukturelles Lernen, Hamburg 1977, S. 38-49.

Dörner, D., (Schwierigkeiten 1981): Über die Schwierigkeiten menschlichen Umgangs mit Komplexität, in: Psychologische Rundschau, 32. Jg., 1981, S. 163-179.

Dörner, D., (Probleme 1982): Wie man viele Probleme zugleich löst - oder auch nicht!, in: Sprache \& Kognition, Heft 2, 1. Jg., 1982, S. 55-66.

Dörner, D., (Informationsverarbeitung 1985): Informationsverarbeitung, in: Herrmann, T.; Lantermann, E.-D. (Hrsg.), Persönlichkeitspsychologie: ein Handbuch in Schlüsselbegriffen, München, Wien, Baltimore 1985, S. 371-380.

Dörner, D., (Difficulties 1987): On the Difficulties People have in Dealing with Complexity, in: Rasmussen, J.; Duncan, K.; Leplat, J. (Eds.), New Technology and Human Error, Chichester 1987, S. 97-109.

Dörner, D., (Wissen 1988): Wissen und Verhaltensregulation: Versuch einer Integration, in: Mandl, H.; Spada, H. (Hrsg.), Wissenspsychologie, München, Weinheim 1988, S. 264-279.

Dörner, D., (Logik 1999): Die Logik des Mißlingens: Strategisches Denken in komplexen Situationen, Reinbek bei Hamburg 1999.

Dörner, D.; Drewes, U.; Reither, F., (Problemlösen 1975): Über das Problemlösen in sehr komplexen Realitätsbereichen, in: Tack, W.H. (Ed.), Bericht über den 29. Kongreß der DGfP in Salzburg 1974, Band 1, Göttingen 1975, S. 339-340.

Dörner, D.; Kreuzig, H.W.; Reither, F.; Stäudel, T. (Hrsg.), (Lohhausen 1983): Lohhausen: vom Umgang mit Unbestimmtheit und Komplexität, Bern, Stuttgart, Wien 1983. 
Dörner, D.; Schaub, H., (Spiel 1986): Spiel und Wirklichkeit: Über die Verwendung und den Nutzen computersimulierter Planspiele, in: WP, Köln 1986, S. 55-78.

Dubs, R., (Schlüsselqualifikationen 1996): Schlüsselqualifikationen - werden wir erneut um eine Illusion ärmer?, in: Gonon, P. (Hrsg.), Schlüsselqualifikationen kontrovers, Aarau 1996, S. 49-57.

Duden, (Etymologie 1963): Etymologie, Herkunftswörterbuch der deutschen Sprache, Mannheim 1963.

Dulisch, F., (Lernen 1986): Lernen als Form menschlichen Handelns. Eine handlungstheoretisch orientierte Analyse von Lernprozessen unter besonderer Berücksichtigung des Selbststeuerungsaspektes, Bergisch Gladbach 1986.

Duncker, K., (Psychologie 1963): Zur Psychologie des produktiven Denkens, 2., unveränd. Neudruck der 1935 erschienenen 1. Aufl., Berlin, Göttingen, Heidelberg 1963.

Dworatscheck, S., (Management-Informations-Systeme 1971): ManagementInformations-Systeme, Berlin 1971.

Edwards, E., (Machine 1972): Man and Machine: Systems for Safety, in: Technical Symposium. British Air Line Pilots Association, No. 13, Harlington 1972.

Ehlken, J., (Modelleinsatz 1999): Modelleinsatz bei der Bewertung und Auswahl von Forschungs- und Entwicklungsprojekten - Darstellung der Modelle, Analyse von Problemen und Identifikation von Lösungsansätzen, Göttingen, London 1999.

Eisele, W., (Gründung 1993): Gründung, in: Wittmann, W.; u.a. (Hrsg.), Handwörterbuch der Betriebswirtschaft, Teilband 1, 5., völlig neu gestaltete Aufl., Stuttgart 1993, Sp. 1550-1562.

Elbers, D.; Heckenauer, M.; Mönikes, W.; Pornschlegel, H.; Tillmann, H., (Schlüsselqualifikationen 1975): Schlüsselqualifikationen - ein Schlüssel für die Berufsbildungsforschung?, in: BWP, Heft 4, 4. Jg., 1975, S. 2629. 
Eyferth, K.; Schömann, M.; Widowski, D., (Umgang 1986): Der Umgang von Psychologen mit Komplexität, in: Sprache \& Kognition, Heft 1, 5. Jg., 1986, S. 11-26.

Fabel, M., (Fortentwicklungsperspektiven 1993): Fortentwicklungsperspektiven für Unternehmungsplanspiele, in: ZP, Heft 1, 4. Jg., 1993, S. 49-64.

Faßbender, S., (Management 1973): Wie lehrt und lernt man Management? Ziele und Methoden der Weiterbildung von Führungskräften, Frankfurt am Main 1973.

Fassheber, P.; Terjung, B., (Symlog-Teamdiagnostik 1986): SymlogTeamdiagnostik als Organisationsentwicklung. Teilprojekt: Organisationsentwicklung, Göttingen 1986.

Fassheber, P.; Terjung, B., (Symlog-Rating-Daten 1989): Symlog-Rating-Daten und ihre Beziehung zu Leistung und Verhalten außerhalb der Gruppensituation. Teilprojekt: Kleingruppenforschung, 2. Aufl., Göttingen 1989.

Fiedler, P.A., (Funktion 1976): Zur Funktion der Verstärkung in Problemlösungsprozessen: Experimentelle Untersuchung zur Einflußnahme verschiedener Rückmeldebedingungen auf das kognitivsoziale Verhalten bei der Lösung komplexer Probleme, Münster 1976.

Fisch, R.; Wolf, M. F., (Handhabung 1990): Die Handhabung von Komplexität beim Problemlösen und Entscheiden, in: Fisch, R.; Boos, M. (Hrsg.), Vom Umgang mit Komplexität in Organisationen: Konzepte, Fallbeispiele, Strategien, Konstanz 1990, S. 11-39.

Fischer, P.M.; Mandl, H., (Förderung 1983): Förderung von Lernkompetenz und Lernregulation. Zentrale Komponenten der Steuerung und Regulation von Lernprozessen, in: Kötter, L.; Mandl, H. (Hrsg.), Kognitive Prozesse und Unterricht. Jahrbuch für Empirische Erziehungswissenschaft 1983, 1. Aufl., Düsseldorf 1983, S. 263-317.

Fiske, S.T.; Kinder, D.R.; Larter, W.M., (Novice 1983): The Novice and the Expert: Knowledge-Based Strategies in Political Cognition, in: Journal of Experimental Social Psychology, Vol. 19, 1983, S. 381-400.

Flechtner, H.J., (Grundbegriffe 1969): Grundbegriffe der Kybernetik: Eine Einführung, 4. Aufl., Stuttgart 1969. 
Flothow, K., (Förderung 1992): Förderung von Handlungskompetenz in der beruflichen Erstausbildung durch Lern- und Arbeitstechniken, Bergisch Gladbach 1992.

Foerster, H. v., (Constructing 1973): On Constructing a Reality, in: Preiser, W.F.E. (Hrsg.), Environmental Design Research, Bd. 2, Stroudsbourg Pa. 1973, S. 35-46.

Frank, U., (Expertensysteme 1988): Expertensysteme: Neue Automatisierungspotentiale im Büro- und Verwaltungsbereich?, Wiesbaden 1988.

Freudenreich, D., unter Mitarbeit von Großhans, F., (Planspiel 1979): Das Planspiel in der sozialen und pädagogischen Praxis: Beispiele für den Umgang mit Organisationen, Gruppen und Personen, München 1979.

Friedrich, H.; Tschersig, R., (Planspiel 1978): Das Planspiel als Hochschullehrveranstaltung: Beispiel »Konzertierte Aktion«, Hamburg 1978.

Fuchs, H., (Systemtheorie 1969): Systemtheorie, in: Grochla, E. (Hrsg.), Handwörterbuch der Organisation, Stuttgart 1969, Sp. 1618-1630.

Fuchs, H., (Systemtheorie 1973): Systemtheorie und Organisation. Die Theorie offener Systeme als Grundlage zur Erforschung und Gestaltung betrieblicher Systeme, Wiesbaden 1973.

Fuchs-Wegner, G., (,Systemanalyse“ 1974): „Systemanalyse“: Eine Forschungsund Gestaltungsstrategie, in: ZfbF, Sonderheft 3, 1974, S. 69-82.

Funke, J., (Mondlandung 1981): Mondlandung - ein neuer Aufgabentyp zur Erforschung komplexen Problemlösens, Trierer Psychologische Berichte 8, Heft 9, 1981.

Funke, J., (Diagnose 1984): Diagnose der westdeutschen Problemlöseforschung in Form einiger Thesen. Mit einigen Anmerkungen von D. Dörner, in: Sprache \& Kognition, Heft 3, 3. Jg., 1984, S. 159-172.

Funke, J., (Problemlösen 1984): Komplexes Problemlösen: kritische Bestandsaufnahme und weiterführende Perspektiven, Trier 1984.

Funke, J., (Problemlösen 1985): Problemlösen in komplexen computersimulierten Realitätsbereichen, in: Sprache \& Kognition, Heft 3, 4. Jg., 1985, S. 113-129.

Funke, J., (Steuerung 1985): Steuerung dynamischer Systeme durch Aufbau und Anwendung subjektiver Kausalmodelle, in: Z. Psychol., Heft 4, 193. Jg., 1985, S. 443-465. 
Funke, J., (Systemmerkmale 1990): Systemmerkmale als Determinanten des Umgangs mit dynamischen Systemen, in: Sprache \& Kognition, Heft 3, 9. Jg., 1990, S. 143-154.

Fürstenau, B., (Problemlösen 1994): Komplexes Problemlösen im betriebswirtschaftlichen Unterricht. Mit einem Geleitwort von Frank Achtenhagen, Wiesbaden 1994.

Gediga, G.; Schöttke, H.; Tücke, M., (Problemlösen 1982): Problemlösen in einer komplexen Situation. Ein experimenteller Beitrag im Umgang mit einem schwer durchschaubaren Problem. Psychologischer Forschungsbericht Nr. 27 aus dem Fachbereich 8 der Universität Osnabrück, Osnabrück 1982.

Geisler, M.A., (Simulation 1962): Appraisal of Laboratory Simulation Experiences, in: Management Science, No. 3, Vol. 8, 1962, S. 239-245.

Gell-Mann, M., (Quark 1998): Das Quark und der Jaguar: Vom Einfachen zum Komplexen. Die Suche nach einer neuen Erklärung der Welt, 2. Aufl, München 1998.

Gerjets, P.; Westermann, R., (Theorien 1997): Theorien der kognitiven Psychologie und das Problem der Intentionalität, in: Kluwe, R.H. (Hrsg.), Strukturen und Prozesse intelligenter Systeme, Wiesbaden 1997, S. 255279.

Geuting, M., (Planspiel 1992): Planspiel und soziale Simulation im Bildungsbereich. Studien zur Pädagogik, Andragogik und Gerontagogik, Bd. 10, Frankfurt am Main u.a. 1992.

Gmilkowsky, P.; Roth, F., (Simulation 1999): Simulation und Wissensverarbeitung als integrale Bestandteile eines Systems zur Fertigungsdisposition, in: Biethahn, J.; Hummeltenberg, W.; Schmidt, B.; Stähly, P.; Witte, T. (Hrsg.), Simulation als betriebliche Entscheidungshilfe. State of the Art und neuere Entwicklungen, Heidelberg 1999, S. 219-254.

Gödel, K., (Sätze 1931): Über formal unentscheidbare Sätze der Principia Mathematica und verwandter Systeme, in: Monatshefte für Mathematik und Physik, 38. Jg., 1931, S. 173-198. 
Goertzen, H., (Simultanplanung 1990): Simultanplanung von Produktion und Beschaffung bei substitutionalen Produktionsfaktoren - Eine Entscheidungshilfe im Planspiel, Göttingen 1990.

Goetze, W., (Qualität 1996): Von SQ (Schlüsselqualifikationen) zu QS (Qualitätssicherung): Sollten wir mehr über Qualität von Bildungsangeboten reden - und weniger über Schlüsselqualifikationen?, in: Gonon, P. (Hrsg.), Schlüsselqualifikationen kontrovers, Aarau 1996, S. 58-66.

Golas, B., (Complexity 1981): Complexity and Learning in Business Policy Games, in: Simulation \& Games, No. 3, Vol. 12, 1981, S. 295-306.

Gomez, P.; Probst, G., (Praxis 1995): Die Praxis des ganzheitlichen Problemlösens: vernetzt denken, unternehmerisch handeln, persönlich überzeugen, Bern, Stuttgart, Wien 1995.

Gorelik, G., (Bodganov's 1987): Bodganov's Tektologia, General Systems Theory, and Cybernetics, in: Cybernetics \& Systems, No. 2, Vol. 18, 1987, S. $157-175$.

Götze, U.; Rudolph, F., (Instrumente 1994): Instrumente der strategischen Planung, in: Bloech, J.; Götze, U.; Huch, B.; Lücke, W.; Rudolph, F. (Hrsg.), Strategische Planung. Instrumente, Vorgehensweisen und Informationssysteme, Heidelberg 1994, S. 1-56.

Graf, H., (Möglichkeiten 1984): Möglichkeiten der Nutzung computergestützter Instrumente in der Gründungsplanung, in: Nathusius, K.; Klandt, H.; Kirschbaum, G. (Hrsg.), Unternehmungsgründung: Konfrontation von Forschung und Praxis: Festschrift gewidmet Herrn Prof. Dr. Norbert Szyperski aus Anlaß des 10jährigen Bestehens der Projektgruppe Gründungsforschung am Planungsseminar der Universität zu Köln. Mit einem Geleitwort von Erwin Grochla, Bergisch Gladbach 1984, S. 241-260.

Graf, J., (Prinzip 1992): Das Prinzip der Komplexität, in: Graf, J. (Hrsg.), Planspiele: simulierte Realitäten für den Chef von morgen; mit PlanspielMarktübersicht, Speyer 1992, S. 11-18.

Greeno, J.G., (Hobbits 1974): Hobbits and Orcs: Acquisition of a Sequential Concept, in: Cognitive Psychology, No. 2, Vol. 6, 1974, S. 270-292.

Gregor, C., (Franchising 1990): Franchising, in: Dieterle, W.K.M.; Winckler, E.M. (Hrsg.), Unternehmensgründung: Handbuch des Gründungsmanagement, München 1990, S. 549-557. 
Greulich, W. (Hrsg.), (Lexikon 1999): Lexikon der Physik: in sechs Bänden, Bd. 2, Heidelberg 1999.

Grochla, E., (Automation 1966): Automation und Organisation: Die technische Entwicklung und ihre betriebswirtschaftlich-organisatorischen Konsequenzen, Wiesbaden 1966.

Grochla, E., (Systemtheorie 1970): Systemtheorie und Organisationstheorie, in: ZfB, Heft 1, 40. Jg., 1970, S. 1-16.

Grochla, E., (Systemtheorie 1972): Systemtheorie und Organisationstheorie, in: Bleicher, K. (Hrsg.), Organisation als System, Wiesbaden 1972, S. 123137.

Groeben, N., (Handlungsperspektive 1981): Die Handlungsperspektive als Theorierahmen für Forschung im pädagogischen Feld, in: Hofer, M. (Hrsg.), Informationsverarbeitung und Entscheidungsverhalten von Lehrern, München, Wien, Baltimore 1981, S. 20-44.

Groeben, N.; Scheele, B., (Argumente 1977): Argumente für eine Psychologie des reflexiven Subjekts: Paradigmawechsel vom behavioralen zum epistemologischen Menschenbild, Darmstadt 1977.

Grossmann, C., (Komplexitätsbewältigung 1992): Komplexitätsbewältigung im Management: Anleitungen, integrierte Methodik und Anwendungsbeispiele, St. Gallen 1992.

Gruber, H.; Mandl, H.; Renkl, A., (Schule 2000): Was lernen wir in Schule und Hochschule: Träges Wissen?, in: Mandl, H.; Gerstenmaier, J. (Hrsg.), Die Kluft zwischen Wissen und Handeln. Empirische und theoretische Lösungsansätze, Göttingen, Bern, Toronto, Seattle 2000, S. 139-156.

Gruber, H.; Ziegler, A., (Expertise 1996): Expertise als Domäne psychologischer Forschung, in: Gruber, H.; Ziegler, A. (Hrsg.), Expertiseforschung. Theoretische und methodische Grundlagen, Opladen 1996, S. 716.

Guyote, M.J.; Sternberg, R.S., (Theory 1981): A Transitive-Chain Theory of Syllogistic Reasoning, in: Cognitive Psychology, No. 4, Vol. 13, 1981, S. 461-525. 
Hardiman, P.T.; Dufresne, R.; Mestre, J.P., (Relation 1989): The Relation between Problem Categorization and Problem Solving among Experts and Novices, in: Memory \& Cognition, No. 5, Vol. 17, 1989, S. 627-638.

Hartmann, R., (Betrieb 1967): Der Betrieb als kybernetisches System, in: BFuP, Heft 2, 19. Jg., 1967, S. 65-79.

Hartung, S., (Förderung 2000): Förderung der Lerneffizienz beim Einsatz von Unternehmensplanspielen in der kaufmännischen Fortbildung berufstätiger Erwachsener, Göttingen 2000.

Hasselmann, D., (Einsatz 1990): Der Einsatz computersimulierter, komplexer Probleme in betrieblichen Assessmentcenter-Veranstaltungen, in: Frey, D. (Hrsg.), Bericht über den 37. Kongreß der DGfP in Kiel 1990, Bd. 1, Göttingen 1990, S. 64-65.

Hasselmann, D., (Problemstellungen 1993): Computersimulierte komplexe Problemstellungen in der Management-Diagnostik: die Theorie zum Konzept Herausforderung Komplexität, 1. Aufl., Hamburg 1993.

Hättenschwiler, P., (Konzepte 1999): Neue Konzepte der Entscheidungsunterstützung. Interne Publikation des Instituts für Informatik der Universität Freiburg Nr. 99-4, Freiburg 1999.

Hauer, G., (Entscheidungsrechnung 1994): Hierarchische kennzahlenorientierte Entscheidungsrechnung: ein Beitrag zum Investitions- und Kostenmanagement, München 1994.

Hauschildt, J., (Entscheidungsziele 1977): Entscheidungsziele. Zielbildung in innovativen Entscheidungsprozessen: Theoretische Ansätze und empirische Prüfung, Tübingen 1977.

Hauschildt, J., (Komplexität 1990): Komplexität, Zielbildung und Effizienz von Entscheidungen in Organisationen, in: Fisch, R.; Boos, M. (Hrsg.), Vom Umgang mit Komplexität in Organisationen: Konzepte, Fallbeispiele, Strategien, Konstanz 1990, S. 131-147.

Hawkins, F.H., (Human 1984): Human Factors Education in European Air Transport Operations, in: Nijoff, M. (Ed.), Breakdown in Human Adaptation to Stress: Towards a Multidisciplinary Approach, for the Commission of the European Communities, Vol. 1, The Hague 1984. 
Hax, K., (Entwicklung 1959): Die Entwicklung der deutschen Betriebswirtschaftslehre nach dem zweiten Weltkrieg, in: The Annals of the School of Business Administration, Kobe University, No. 3, 1959, S. 13-25.

Hebig, M., (Existenzgründungsberatung 1994): Existenzgründungsberatung: steuerliche, rechtliche und wirtschaftliche Gestaltungshinweise zur Unternehmensgründung, 3., überarb. und erw. Aufl., Bielefeld 1994.

Heidack, C., (Planspiel-Praxis 1980): Planspiel-Praxis. Schwerpunkte der Methodik und Didaktik der Planspiel-Praxis unter besonderer Berücksichtigung des Verhaltenstrainings, Speyer 1980.

Heinen, E., (Wissenschaftsprogramm 1969): Zum Wissenschaftsprogramm der entscheidungsorientierten Betriebswirtschaftslehre, in: ZfB, Heft 4, 39. Jg., 1969, S. 207-220.

Heinen, E., (Ansatz 1971): Der entscheidungsorientierte Ansatz der Betriebswirtschaftslehre, in: ZfB, Heft 7, 41. Jg., 1971, S. 429-444.

Hertlein, A., (Gründung 1965): Gründung der Unternehmung, in: Beckerath, E. v.; u.a. (Hrsg.), Handwörterbuch der Sozialwissenschaften. Zugleich Neuauflage des Handwörterbuchs der Staatswissenschaften, Bd. 4, Göttingen 1965, Sp. 673-675.

Hesse, F.W., (Effekte 1982): Effekte des semantischen Kontexts auf die Bearbeitung komplexer Probleme, in: Zeitschrift für experimentelle und angewandte Psychologie, Heft 1, 29. Jg., 1982, S. 62-91.

Hesse, F.W., (Analyse 1985): Vergleichende Analyse kognitiver Prozesse bei semantisch unterschiedlichen Problemeinbettungen, in: Sprache \& Kognition, Heft 3, 4. Jg., 1985, S. 139-153.

Hesse, F.W., (Review 1985): Review: John R. Anderson (1983), The Architecture of Cognition, in: Sprache \& Kognition, Heft 4, 4. Jg., 1985, S. 231-237.

Hesse, F.W.; Spies, K.; Lüer, G., (Einfluß 1983): Einfluß motivationaler Faktoren auf das Problemlöseverhalten im Umgang mit komplexen Problemen, in: Zeitschrift für experimentelle und angewandte Psychologie, Heft 3, 30. Jg., 1983, S. 400-424.

Hesselbach, J., (Verhaltensforschung 1970): Verhaltensforschung bei unternehmerischen Entscheidungen, in: ZfB, Heft 10, 40. Jg., 1970, S. 647-664.

Heylighen, F., (Growth 2001): The Growth of Structural and Functional Complexity during Evolution, [Online in Internet], 
URL:http://pespmc1.vub.ac.be/papers/ComplexityGrowth.html, ] [Stand 01.08.2001].

Hill, G.E., (Opportunities 1994): Market Opportunities and Marketing, in: Bygrave, W.D. (Ed.), The Portable MBA in Entrepreneurship, New York 1994, S. 67-112.

Högsdal, B., (Planspiele 1996): Planspiele. Einsatz von Planspielen in der Ausund Weiterbildung. Praxiserfahrungen und bewährte Methoden, Bonn 1996.

Hopfenbeck, W., (Unternehmen 1989): Allgemeine Betriebswirtschafts- und Managementlehre: das Unternehmen im Spannungsfeld zwischen ökonomischen, sozialen und ökologischen Interessen, 1. Aufl., Landsberg am Lech 1989.

Hopkin, V.D., (Human 1995): Human Factors in Air Traffic Control, London u.a. 1995.

Horvarth, P., (Controlling 1994): Controlling, 5. Aufl., München 1994.

Huch, B., (Rechnungswesen 1975): Das Rechnungswesen als Grundlage der Unternehmenspolitik, Würzburg, Wien 1975.

Huck, K., (Analyse 1975): Die Analyse des Problemlöseverhaltens in Gruppen, München 1975.

Hussy, W., (Denkpsychologie1984): Denkpsychologie: ein Lehrbuch. Band 1: Geschichte, Begriffs- und Problemlöseforschung, Intelligenz, Stuttgart 1984.

Innerhofer, P., (Regelmodell 1974): Ein Regelmodell zur Analyse und Intervention in Familie und Schule: Abänderung und Erweiterung des S-R-KModells, in: Zeitschrift für Klinische Psychologie, Heft 1, 3. Jg., 1974, S. $1-29$.

Isaac, A.R.; Ruitenberg, B., (Air 1999): Air Traffic Control: Human Performance Factors, Aldershot 1999.

Jäger, W., (Gründung 1976): Gründung, in: Büschgen, H.E. (Hrsg.), Handwörterbuch der Finanzwirtschaft, Stuttgart 1976, Sp. 787-794. 
Jeffries, R.; Polson, P.G.; Razran, L.; Atwood, M.E., (Process 1977): A Process Model for Missionaries-Cannibals and other River-Crossing Problems, in: Cognitive Psychology, No. 4, Vol. 9, 1977, S. 412-440.

Johannes, P., (Expertensysteme 1990): Expertensysteme: Entscheidungskriterien für Manager, München 1990.

Johnson, R.A.; Kast, F.E.; Rosenzweig, J.E., (Theory 1967): The Theory and Management of Systems, 2. Aufl., New York, San Francisco, Toronto, London 1967.

Johnson-Laird, P.N., (Models 1983): Mental Models. Towards a Cognitive Science of Language, Inference, and Consciousness, Cambridge 1983.

Johnson-Laird, P.N.; Steedman, M., (Psychology 1978): The Psychology of Syllogisms, in: Cognitive Psychology, No.1, Vol. 10, 1978, S. 64-99.

Kabuschat, H., (Scheinfirmenarbeit 1958): Produktivitätssteigerung durch neuartige Berufsausbildung: Eine Darstellung der Methode der Scheinfirmenarbeit für Lehrbetriebe, Ausbilder und Pädagogen, Hamburg 1958.

Kaiser, A., (Schlüsselqualifikationen 1992): Schlüsselqualifikationen in der Arbeitnehmerweiterbildung. Landesarbeitsgemeinschaft für katholische Erwachsenenbildung in Nordrhein-Westphalen e.V., Neuwied 1992.

Kaiser, F.-J.; Kaminski, H., (Methodik 1994): Methodik des Ökonomie-Unterrichts, Grundlagen eines handlungsorientierten Lernkonzepts mit Beispielen, Bad Heilbronn/Obb. 1994.

Kaminski, G., (Überlegungen 1981): Überlegungen zur Funktion von Handlungstheorien, in: Lenk, H. (Hrsg.), Handlungstheorien interdisziplinär III: Verhaltenswissenschaftliche und psychologische Handlungstheorien, 1. Halbband, München 1981, S. 93-121.

Kamlah, W., (Anthropologie 1972): Philosophische Anthropologie. Sprachkritische Grundlegung und Ethik, Zürich 1972.

Kappel, R.; Schwarz, I.A., (Systemforschung 1981): Systemforschung 19701980; Entwicklungen in der Bundesrepublik Deutschland: Materialien zu einem Förderungsschwerpunkt der Stiftung Volkswagenwerk. Mit einem Beitrag von Anatol Rapoport, Göttingen 1981. 
Karczewski, S., (Entwicklung 1991): Die Entwicklung einer modularen Gesamtarchitektur für die Softwarekomponenten von Planspielen, Wiesbaden 1991.

Kastner, M., (Denken 1995): Systemisches Denken, in: Geilhardt, T.; Mühlbradt, T. (Hrsg.), Planspiele im Personal- und Organisationsmanagement, Göttingen 1995, S. 27-43.

Keese, C., (Firmen 2000): Wer Firmen fusioniert, sollte von der Evolution lernen

- Im Rausch der Größe ignorieren viele Unternehmen die menschliche Biologie: Unsere Fähigkeit zur Kommunikation mit anderen Menschen ist begrenzt, in: Financial Times Deutschland vom 25. September 2000, S. 30 .

Kern, W., (Research 1987): Operations Research: Einführung und Überblick, 6., völlig überarb. u. erw. Aufl., Stuttgart 1987.

Kern, W., (Zeit 1992): Die Zeit als Dimension betriebswirtschaftlichen Denkens und Handelns. Statik und Dynamik; Planung und Unsicherheit; Modellbildung; Rechnungslegung; Zeitmanagement, in: DBW, Heft 1, 52. Jg. 1992, S. 41-58.

Kieras, D.; Polson, P.G., (Approach 1985): An Approach to the Formal Analysis of User Complexity, in: Int. J. Man-Machine Studies, 22. Jg., 1985, S. 365-394.

Kirsch, W., (Entscheidungsprozesse -Band 1- 1970): Entscheidungsprozesse. Verhaltenswissenschaftliche Ansätze der Entscheidungstheorie, Bd. 1, Wiesbaden 1970.

Kirsch, W., (Handhabung 1988): Die Handhabung von Entscheidungsproblemen. Einführung in die Theorie der Entscheidungsprozesse, 3. Aufl., München 1988.

Kirschbaum, G.; Naujoks, W., (Erfolgreich 1986): Erfolgreich in die berufliche Selbständigkeit, Tips und Ratschläge für Existenzgründer, 2. Aufl., Freiburg im Breisgau 1986.

Kistner, K.-P.; Südfeld, E. u.a., (Statistische 1987): Statistische Erfassung von Unternehmensgründungen: Umfang, Ursachen, Wirkungen, in: Statistisches Bundesamt (Hrsg.), Schriftenreihe Forum der Bundesstatistik, Bd. 8, Stuttgart, Mainz 1988. 
Klandt, H., (Person 1980): Die Person des Unternehmungsgründers als Determinante des Gründungserfolgs, in: BfuP, Heft 4, 32. Jg., 1980, S. 321-335.

Klandt, H., (Aktivität 1984): Aktivität und Erfolg des Unternehmungsgründers: eine empirische Analyse unter Einbeziehung des mikrosozialen Umfeldes, 1. Aufl., Bergisch Gladbach 1984.

Klandt, H., (Person 1990): Zur Person des Unternehmensgründers, in: Dieterle, W.K.M.; Winckler, E.M. (Hrsg.), Unternehmensgründung: Handbuch des Gründungsmanagement, München 1990, S. 29-43.

Klandt, H., (Entrepreneurship 1999): Entrepreneurship: Unternehmerausbildung an deutschen Hochschulen, in: BFuP, Heft 3, 51. Jg., 1999, S. 241-255.

Klandt, H., (Gründungsmanagement 1999): Gründungsmanagement: Der integrierte Unternehmensplan, München, Wien 1999.

Klandt, H.; Finke-Schürmann, T., (Existenzgründung 1998): Existenzgründung für Hochschulabsolventen - So erstellen Sie einen überzeugenden Business Plan, Frankfurt am Main 1998.

Klandt, H.; Münch, G., (Gründungsforschung 1990): Gründungsforschung im deutschsprachigen Raum - Ergebnisse einer empirischen Untersuchung, in: Szyperski, N.; Roth, P. (Hrsg.), Innovative Unternehmensgründung als Aufgabe, Stuttgart 1990, S. 171-186.

Klix, F., (Information 1976): Information und Verhalten. Kybernetische Aspekte der organismischen Informationsverarbeitung. Einführung in naturwissenschaftliche Grundlagen der Allgemeinen Psychologie, Berlin 1976.

Kloidt, H.; Dubberke, H.-A.; Göldner, J., (Problematik 1959): Zur Problematik des Entscheidungsprozesses, in: Kosiol, E. (Hrsg.), Organisation des Entscheidungsprozesses, Berlin 1959, S. 9-22.

Klotzbücher, R., (Planspielentwicklung 1996): Objektorientierte Planspielentwicklung: Konzept für den Versicherungssektor, Wiesbaden 1996.

Kluwe, R.H., (Kontrolle 1990): Kontrolle und Steuerung komplexer Systeme durch Menschen: Anmerkungen zum Stand der kognitionspsychologischen Forschung, in: Grawe, K.; Hänni, R.; Semmer, N.; Tschan, F. (Hrsg.), Über die richtige Art, Psychologie zu betreiben, Göttingen 1990, S. 239-254.

Kluwe, R.H., (Analysen 1991): Kognitionspsychologische Analysen der Unzulänglichkeiten von Menschen beim Umgang mit risikoreichen Systemen, 
in: Frey, D. unter Mitarbeit von Köhnken, G. (Hrsg.), Bericht über den 37. Kongreß der DGfP in Kiel 1990, Bd. 2, Göttingen 1991, S. 275-283.

Kluwe, R.H., (Problem 1991): Zum Problem der Wissensvoraussetzung für Prozeß- und Systemsteuerung, in: Z. Psychol., Heft 11, 199. Jg., 1991, S. 311-324.

Kluwe, R.H., (Gedächtnis 1992): Gedächtnis und Wissen, in: Spada, H. (Hrsg.), Lehrbuch allgemeine Psychologie, 2., korrigierte Aufl., Bern, Göttingen, Toronto, Seattle 1992, S. 115-187.

Kluwe, R.H.; Haider, H., (Modelle 1990): Modelle zur internen Repräsentation komplexer technischer Systeme, in: Sprache \& Kognition, Heft 4, 9. Jg., 1990, S. 173-192.

Kluwe, R.H.; Misiak, C.; Haider, H., (Ergebnisse 1989): Erste Ergebnisse zu einem Modell der Steuerung eines komplexen Systems, in: Dörner, D.; Michaelis, W. (Hrsg.), Idola fori et idola theatri. Festschrift aus Anlaß der Emeritierung von Prof. Dr. phil. et Dr. med. Hermann Wegener, Göttingen 1989, S. 101-119.

Kluwe, R.H.; Misiak, C.; Schmidle, R., (Wissenserwerb 1985): Wissenserwerb beim Umgang mit umfangreichen Systemen: Lernvorgänge als Ausbildung subjektiver Ordnungsstrukturen, in: Albert, D. (Hrsg.), Bericht über den 34. Kongreß der DGfP in Wien 1984, Bd. 1: Grundlagenforschung, Göttingen 1985, S. 255-257.

Kluwe, R.H.; Schilde, A.; Fischer, C.; Oellerer, N., (Problemlöseleistungen 1991): Problemlöseleistungen beim Umgang mit komplexen Systemen und Intelligenz, in: Diagnostica, Heft 4, 37. Jg., 1991, S. 291-313.

Kluwe, R.H.; Spada, H., (Wissen 1981): Wissen und seine Veränderung: Einige psychologische Beschreibungsansätze, in: Foppa, K.; Groner, R., (Hrsg.), Kognitive Strukturen und ihre Entwicklung, Bern, Stuttgart, Wien 1981, S. 284-327.

Koller, H., (Planspieltechnik 1969): Simulation und Planspieltechnik: Berechnungsexperimente in der Betriebswirtschaft, Wiesbaden 1969.

Koller, H., (Planspieltechnik 1974): Planspieltechnik, in: Fasshauer, R.; Wurzbacher, W. (Hrsg.), Unternehmensspiele - Stand und Entwicklungstendenzen. IBM-Beiträge zur Datenverarbeitung, Methoden und Techniken 4, Stuttgart 1974, S. 5-13. 
Kosiol, E.; Szyperski, N.; Chmielewicz, K., (Standort 1972): Zum Standort der Systemforschung im Rahmen der Wissenschaften (einschließlich ihrer Beziehungen zur Organisations-, Automations- und Unternehmensforschung), in: Bleicher, K., (Hrsg.), Organisation als System, Wiesbaden 1972, S. 65-97.

Kötter, L.; Mandl, H., (Ansätze 1983): Kognitionstheoretische Ansätze und Forderungen gegenwärtiger Unterrichtsforschung, in: Kötter, L.; Mandl, H. (Hrsg.), Kognitive Prozesse und Unterricht. Jahrbuch für Empirische Erziehungswissenschaft 1983, 1. Aufl., Düsseldorf 1983, S. 11-43.

Kratky, K.W., (Denken 1989): Vom linearen zum systemischen Denken, in: Kratky, K.W.; Bonet, E.M. (Hrsg.), Systemtheorie und Reduktionismus, Wiener Studien zur Wissenschaftstheorie, 1. Aufl., Bd. 3, Wien 1989, S. 11-32.

Kreikebaum, H., (Unternehmensplanung 1993): Strategische Unternehmensplanung, 5., überarb. Aufl., Stuttgart u.a. 1993.

Kretschmar, T., (Datentypen 1991): Abstrakte Datentypen zur flexiblen Wissensrepräsentation und -verarbeitung, in: Biethahn, J.; Bloech, J.; Bogaschewsky, R.; Hoppe, U. (Hrsg.), Wissensbasierte Systeme in der Wirtschaft: Anwendungen und Tools, Wiesbaden 1991, S. 171-181.

Kulicke, M., (Technologieorientierte 1987): Technologieorientierte Unternehmen in der Bundesrepublik Deutschland. Eine empirische Untersuchung der Strukturbildungs- und Wachstumsphase von Neugründungen, Frankfurt am Main, Bern, New York 1987.

Kurbel, K., (Entwicklung 1992): Entwicklung und Einsatz von Expertensystemen: Eine anwendungsorientierte Einführung in wissensbasierte Systeme, 2., verb. Aufl., Berlin, Heidelberg, New York, Tokyo 1992.

Lachman, R.; Lachman, J.L.; Butterfield, E.C., (Psychology 1979): Cognitive Psychology and Information Processing: An Introduction, Hillsdale, N.J. 1979.

Laird, J.E.; Newell, A.; Rosenbloom, P.S., (SOAR 1987): SOAR: An Architecture for General Intelligence, in: Artifical Intelligence, No. 1, Vol. 33, 1987, S. 1-64. 
Langenscheidt-Redaktion (Hrsg.), (Lateinisch-Deutsch 1973): LateinischDeutsch, Deutsch-Lateinisch. Ein Langenscheidt-Wörterbuch, Berlin, München 1973.

Langenscheidt-Redaktion (Hrsg.), (Griechisch 1992): Langenscheidts Eurowörterbuch Griechisch. Griechisch-Deutsch, Deutsch-Griechisch, Berlin, München 1992.

Lantermann, E.D., (Prozesse 1983): Kognitive und emotionale Prozesse beim Handeln, in: Mandl, H.; Huber, G.L. (Hrsg.), Emotion und Kognition, München, Wien, Baltimore 1983, S. 248-281.

Larkin, J.; McDermott, J.; Simon, D.P.; Simon, H.A., (Expert 1980): Expert and Novice Performance in Solving Physics Problems, in: Science: American Association for the Advancement of Science, No. 4450, Vol. 208, 1980, S. 1335-1342.

Lass, U.; Lüer, G., (Problemlöseforschung 1990): Psychologische Problemlöseforschung, in: Unterrichtswissenschaft, Heft 4, 18. Jg., 1990, S. 295-312.

Laszlo, E., (Systemtheorie 1998): Systemtheorie als Weltanschauung: eine ganzheitliche Vision für unsere Zeit, München 1998.

Lehner, F., (Memory 2000): Organisational Memory. Konzepte und Systeme für das organisatorische Lernen und das Wissensmanagement, München, Wien 2000.

Levitt, T., (Imitation 1966): Innovative Imitation, in: HBR, No. 5, Vol. 44, 1966, S. 63-70.

Lewis, C., (Skill 1981): Skill in Algebra, in: Anderson, J.R. (Ed.), Cognitive Skills and their Acquisition, Hillsdale, N.J. 1981, S. 85-110.

Lewis, M.W.; Anderson, J.R., (Discrimination 1985): Discrimination of Operator Schemata in Problem Solving: Learning from Examples, in: Cognitive Psychology, No. 1, 17. Jg., 1985, S. 26-65.

Löfgren, L., (Complexity 1977): Complexity of Descriptions of Systems: A Foundational Study, in: Int. J. General Systems, No. 4, Vol. 3, 1977, S. 197-214.

Lücke, W. (Hrsg.), unter Mitarbeit von Bloech, J.; u.a., (Investitionslexikon 1991): Investitionslexikon, 2., völlig neubearb. Aufl., München 1991. 
Lüer, G., (Denkabläufe 1973): Gesetzmäßige Denkabläufe beim Problemlösen. Ein empirischer Beitrag für eine psychologische Theorie der Entwicklung des Denkens, Weinheim, Basel 1973.

Lüer, G.; Lass, U., (Einleitung 1997): Einleitung, in: Lüer, G.; Lass, U. (Hrsg.), Erinnern und Behalten: Wege zur Erforschung des menschlichen Gedächtnisses, Göttingen 1997, S. 1-3.

Luhmann, N., (Funktionen 1964): Funktionen und Folgen formaler Organisation, Berlin 1964.

Luhmann, N., (Theorie 1966): Theorie der Verwaltungswissenschaft: Bestandsaufnahme und Entwurf, Köln 1966.

Luhmann, N., (Zweckbegriff 1968): Zweckbegriff und Systemrationalität. Über die Funktion von Zwecken in sozialen Systemen, Tübingen 1968.

Luhmann, N., (Temporastrukturen 1979), Temporalstruturen des Handlungssystems. Zum Zusammenhang von Handlungs- und Systemtheorie, in: Schluchter, W. (Hrsg.), Verhalten, Handeln und System. Talcott Parsons' Beitrag zur Entwicklung der Sozialwissenschaften, Frankfurt am Main 1979, S. 32-67.

Luhmann, N., (Komplexität 1980), Komplexität, in: Grochla, E. (Hrsg.), Handwörterbuch der Organisation, 2., völlig neu gestaltete Aufl., Stuttgart 1980, Sp. 1064-1070.

Luhmann, N., (Systeme 1996): Soziale Systeme: Grundriß einer allgemeinen Theorie, 6. Aufl., Frankfurt am Main 1996.

Malik, F., (Strategie 1989): Strategie des Managements komplexer Systeme: Ein Beitrag zur Management-Kybernetik evolutionärer Systeme, 3. Aufl., Bern, Stuttgart 1989.

Mandl, H.; Friedrich, H.F.; Hron, A., (Psychologie 1986): Psychologie des Wissenserwerbs, in: Weidenmann, B.; Krapp, A.; Hofer, M.; Huber, G.L.; Mandl, H. (Hrsg.), Pädagogische Psychologie, München 1986, S. 143-218.

Mandl, H.; Friedrich, H.F.; Hron, A., (Ansätze 1988): Theoretische Ansätze zum Wissenserwerb, in: Mandl, H.; Spada, H. (Hrsg.), Wissenspsychologie, München, Weinheim 1988, S. 123-160. 
Mandl, H.; Spada, H., (Wissenspsychologie 1988): Wissenspsychologie: Einführung, in: Mandl, H.; Spada, H. (Hrsg.), Wissenspsychologie, München, Weinheim 1988, S. 1-16.

Manstedten, B.C., (Entwicklung 1997): Entwicklung von Organisationsstrukturen in der Gründungs- und Frühentwicklungsphase von Unternehmungen, Förderkreis Gründungs-Forschung, Dortmund 1997.

Manstetten, R., (Kommunikation 1982): Kommunikation und Interaktion im Unterricht: eine fachdidaktische Analyse, Düsseldorf 1982.

Marca, D.A.; McGowan, C.L., (SADT 1988): SADT: Structured Analysis and Design Technique, with a Foreword by Douglas T. Ross, New York, St. Louis u.a. 1988.

Maser, S., (Grundlagen 1972): Grundlagen der allgemeinen Kommunikationstheorie: Eine Einführung in ihre Grundbegriffe und Methoden (mit Übungen), Stuttgart 1972.

May, E., (Existenzgründungen 1981): Erfolgreiche Existenzgründungen und öffentliche Förderung: eine vergleichende empirische Analyse geförderter und nichtgeförderter Gründungsunternehmen, Göttingen 1981.

Mayrhofer, M., (Analyse 1985): Analyse und graphische Darstellung eines Qualitätssicherungssystems, in: QZ, Heft 4, 30. Jg., 1985, S. 111-114.

McFarland, A., (Power 1969): The Power of Leadership in pluralist Systems, Stanford 1969.

Meffert, H., (Systemtheorie 1971): Systemtheorie aus betriebswirtschaftlicher Sicht, in: Schenk, K.E. (Hrsg.), Systemanalyse in den Wirtschafts- und Sozialwissenschaften, Berlin 1971, S. 174-206.

Mertens, D., (Schlüsselqualifikationen 1975): Schlüsselqualifikationen und Berufsbildung - Versuch einer Erwiderung, in: BWP, Heft 5, 4. Jg., 1975, S. 24-25.

Meyer, R., (Entscheidungstheorie 2000): Entscheidungstheorie. Ein Lehr- und Arbeitsbuch, 2., durchgesehene Aufl., Wiesbaden 2000.

Miller, G.A., (Number 1956): The Magic Number Seven, Plus or Minus Two: Some limits on our Capacity for Processing Information, in: Psychological Review, No. 2, Vol. 63, 1956, S. 81-97. 
Minsky, M., (Framework 1975): A Framework for Representing Knowledge, in: Winston, P.H. (Ed.), The Psychology of Computer Vision, New York 1975, S. 211-280.

Mitton, D.G., (Entrepreneur 1989): The Compleat Entrepreneur, in: ET \& P, Vol. 14, 1989, S. 9-19.

Morin, E., (Méthode 1977): La Méthode, Bd. 1, Paris 1977.

Müller, K., (Systemtheorie 1996): Allgemeine Systemtheorie. Geschichte, Methodologie und sozialwissenschaftliche Heuristik eines Wissenschaftsprogramms, Opladen 1996.

Müller-Merbach, H., (Arten 1992): Vier Arten von Systemansätzen, dargestellt in Lehrgesprächen, in: ZfB, Heft 8, 62. Jg., 1992, S. 853-876.

Nathusius, K.; Klandt, H.; Kirschbaum, G. (Hrsg.), (Unternehmensgründung 1984): Unternehmensgründung - Konfrontation von Forschung und Praxis. Festschrift für Norbert Szyperski, mit einem Geleitwort von Erwin Grochla, Bergisch Gladbach 1984.

Neumann, B., (Expertensysteme 1986): Was sind Expertensysteme?, in: Krallmann, H. (Hrsg.), Expertensysteme im Unternehmen. MöglichkeitenGrenzen-Anwendungsbeispiele, 2., unveränderte Aufl., Berlin 1986, S. 11-12.

Newell, A., (Theories 1994): Unified Theories of Cognition, third printing, Cambridge 1994.

Newell, A.; Rosenbloom, P.S.; Laird, J.E., (Architectures 1991): Symbolic Architectures for Cognition, in: Posner, M.I. (Ed.), Foundations of Cognitive Science, Third printing, Cambridge 1991, S. 93-131.

Newell, A.; Shaw, J.C.; Simon, H.A., (Elements 1958): Elements of a Theory of Human Problem Solving, in: Psychological Review, No. 3, Vol. 65, 1958, S. 151-166.

Nissen, V.; Biethahn, J., (Beispiel 1999): Ein Beispiel zur stochastischen Optimierung mittels Simulation und einem Genetischen Algorithmus, in: Biethahn, J.; Hummeltenberg, W.; Schmidt, B.; Stähly, P.; Witte, T. (Hrsg.), Simulation als betriebliche Entscheidungshilfe. State of the Art und neuere Entwicklungen, Heidelberg 1999, S. 108-125. 
Noelle-Neumann, E.; Schulz, W.; Wilke, J. (Hrsg.), (Publizistik 1994): Das Fischer-Lexikon Publizistik - Massenkommunikation, akt., vollst. überarb. Neuausgabe, Frankfurt am Main 1994.

Norman, D.A., (Categorization 1981): Categorization of Action Slips, in: Psychological Review, No. 1, Vol. 88, 1981, S. 1-15.

Norman, D.A., (Observations 1983): Some Observations on Mental Models, in: Gentner, D.; Stevens, A.L. (Eds.), Mental Models, Hillsdale, N.J. 1983, S. 7-14.

Norman, D.A., (Engineering 1986): Cognitive Engineering, in: Norman, D.A.; Draper, S.W. (Eds.), User Centered System Design. New Perspectives on Human-Computer Interaction, Hillsdale, N.J. 1986, S. 31-61.

Nørretranders, T., (Welt 1997): Spüre die Welt: Die Wissenschaft des Bewußtseins, Reinbek bei Hamburg 1997.

O'Connor, J., (Thinking 1997): Thinking Past the Obvious: What is a System? [Online in Internet], http://www.radix.net/ crbnblu/assoc/oconnor/chapt1.html, 06.11.1997].

Oerter, R., (Informationsverarbeitung 1978): Informationsverarbeitung im Rahmen eines Modells kognitiver Sozialisation, in: Mandl, H.; Huber, G.L. (Hrsg.), Kognitive Komplexität: Bedeutung - Weiterentwicklung - Anwendung, Göttingen 1978, S. 141-155.

Opwis, K., (Modelle 1985): Mentale Modelle dynamischer Systeme: Analyse und Weiterführung methodischer Grundlagen von psychologischen Experimenten zum Umgang von Personen mit Systemen, Forschungsberichte des Psychologischen Instituts der Albert-Ludwigs-Universität Freiburg im Breisgau, Nr. 30, Freiburg 1985.

Opwis, K., (Modelle 1987): Mentale Modelle dynamischer Systeme, in: Amelang, M. (Hrsg.), Bericht über den 35. Kongreß der DGfP in Heidelberg 1986, Bd. 2, Göttingen 1987, S. 265-270.

Opwis, K., (Produktionssysteme 1988): Produktionssysteme, in: Mandl, H.; Spada, H. (Hrsg.), Wissenspsychologie, München, Weinheim 1988, S. 74-98. 
Opwis, K., (Modellierung 1992): Kognitive Modellierung: zur Verwendung wissensbasierter Systeme in der psychologischen Theoriebildung, 1. Aufl., Bern, Göttingen, Toronto, Seattle 1992.

Opwis, K.; Spada, H.; Schwiersch, M., (Erwerb 1985): Erwerb und Anwendung von Wissen über ein ökologisches System. Forschungsbericht Nr. 23 des Psychologischen Instituts der Universität Freiburg, Freiburg 1985.

Orth, C., (Unternehmensplanspiele 1999): Unternehmensplanspiele in der betriebswirtschaftlichen Aus- und Weiterbildung - Entwicklung eines Planspiels mit variabler Modellkomplexität, Lohmar, Köln 1999.

Oswald, M.; Gadenne, V., (Wissen 1984): Wissen, Können und künstliche Intelligenz: Eine Analyse der Konzeption des deklarativen und prozeduralen Wissens, in: Sprache \& Kognition, Heft 3, 3. Jg., 1984, S. 173-184.

Paar, I., (Handlungsziel 1993): Handlungsziel, Wissensaufbau und Wissensnutzung in sportlicher Wettkampfsituation: Experten-Novizen-Vergleich zur Erkennung und Beantwortung gegnerischer Verteidigungskonstellation beim Basketball, Saarbrücken 1993.

Penndorf, B., (Geschichte 1966): Geschichte der Buchhaltung in Deutschland, veranstalteter reprographischer Nachdruck der 1. Aufl. von 1913, Frankfurt am Main 1966.

Petersen, U., (Finanzmittelplanung 1990): Finanzmittelplanung, in: Dieterle, W.K.M.; Winckler, E.M. (Hrsg.), Unternehmensgründung: Handbuch des Gründungsmanagement, München 1990, S. 275-292.

Petzing, F., (Untersuchung 1993): Untersuchung des Wissenserwerbs bei einem Unternehmensplanspiel mit Verfahren des Operations Research, Heidelberg 1993.

Pfohl, H.-C., (Entscheidungsfindung 1977): Problemorientierte Entscheidungsfindung in Organisationen, 1. Aufl., Berlin, New York 1977.

Popper, K.R., (Logik 1994): Logik der Forschung, 10., verb. u. vermehrte Aufl., Tübingen 1994.

Pörner, R., (Management 1989): Strategisches Management für innovative technologieorientierte Gründerunternehmen, Frankfurt am Main, Bern, New York, Paris 1989. 
Porr, B., (Definitionen 2001): Allgemeine Definitionen, [Online in Internet], URL: http://www.neurop.ruhr-unibochum.de/ porr/luhmann3/node3.html, [Stand 30. 07. 2001].

Posch, P.; Schneider, W.; Mann, W.E., (Unterrichtsplanung 1977): Unterrichtsplanung, Wien 1977.

Prehm, H.-J., (ZP-Stichwort 1991): ZP-Stichwort: Unternehmensspiele, in: ZP, Heft 3, 2. Jg., 1991, S. 291-294.

Prehm, H.-J., (MARKUS 1995): Marketing-Unternehmensspiel MARKUS: Modelldarstellung und Instrumente zur Entscheidungsvorbereitung, Wiesbaden 1995.

Prehm, H.-J.; Ehlken, J., (ISOS 1995): Unternehmensspiel ISOS - Simulation internationaler Standorte und Märkte, Göttingen 1995.

Puppe, F., (Einführung 1991): Einführung in Expertensysteme, 2. Aufl., Berlin, Heidelberg, New York u.a. 1991.

Pürer, H., (Einführung 1993): Einführung in die Publizistikwissenschaft. Systematik, Fragestellungen, Theorieansätze, Forschungstechniken, 5., überarb. Aufl., München 1993.

Putz-Osterloh, W., (Beziehung 1981): Über die Beziehung zwischen Testintelligenz und Problemlöseerfolg, in: Z. Psychol., Heft 1, 189. Jg., 1981, S. 80-100.

Putz-Osterloh, W., (Problemlöseprozesse 1981): Problemlöseprozesse und Intelligenztestleistung, Bern 1981.

Putz-Osterloh, W., (Experten 1987): Gibt es Experten für komplexe Probleme?, in: Z. Psychol., Heft 1, 195. Jg., 1987, S. 63-84.

Putz-Osterloh, W., (Wissen 1988): Wissen und Problemlösen, in: Mandl, H.; Spada, H. (Hrsg.), Wissenspsychologie, München, Weinheim 1988, S. $247-263$

Putz-Osterloh, W., (Problemlöseforschung 1989): Problemlöseforschung und Intelligenzdiagnostik: Ein Anwendungsbeispiel, in: Dörner, D.; Michaelis, W. (Hrsg.), Idola fori et idola theatri. Festschrift aus Anlaß der Emeritierung von Prof. Dr. phil. et Dr. med. Hermann Wegener, Göttingen 1989, S. 87-100.

Putz-Osterloh, W.; Lüer, G., (Vorhersagbarkeit 1981): Über die Vorhersagbarkeit komplexer Problemlöseleistungen durch Ergebnisse in einem Intelli- 
genztest, in: Zeitschrift für experimentelle und angewandte Psychologie, Heft 2, 28. Jg., 1981, S. 309-334.

Rabe, M., (Einführung 1998): Einführung, in: Kuhn, A.; Rabe, M. (Hrsg.), Simulation in Produktion und Logistik: Fallbeispielsammlung, Berlin, Heidelberg, New York u.a. 1998, S. 1-10.

Ramnarayan, S.; Strohschneider, S.; Schaub, H., (Trappings 1997): Trappings of Expertise and the Pursuit of Failure, in: Simulation \& Gaming, No. 1, Vol. 28, 1997, S. 28-43.

Rasmussen, J., (Skills 1983): Skills, Rules, and Knowledge; Signals, Signs, and Symbols, and Other Distinctions in Human Performance Models, in: SMC, No. 3, Vol. 13, 1983, S. 257-266.

Raulefs, P., (Expertensysteme 1982): Expertensysteme, in: Bibel, W.; Siekmann, J.H. (Hrsg.), Künstliche Intelligenz: Frühjahrsschule Teisendorf, 15.-24. März 1982, Informatik Fachberichte, hrsg. v. W. Brauer, Bd. 59, Berlin, Heidelberg, New York 1982, S. 62-98.

Reason, J., (System 1987): Generic Error-Modelling System (GEMS): A Cognitive Framework for Locating Common Human Error Forms, in: Rasmussen, J.; Duncan, K.; Leplat, J. (Eds.), New Technology and Human Error, Chichester 1987, S. 63-83.

Reichert, U.; Dörner, D., (Heurismen 1988): Heurismen beim Umgang mit einem «einfachen» dynamischen System, in: Sprache \& Kognition, Heft 1, 7. Jg., 1988, S. 12-24.

Reichert, U.; Stäudel, T., (Diagnostik 1991): Computergestützte Diagnostik der Fähigkeiten für den Umgang mit komplexen und vernetzten Systemen, in: Schuler, H.; Funke, U. (Hrsg.), Eignungsdiagnostik in Forschung und Praxis: Psychologische Information für Auswahl, Beratung und Förderung von Mitarbeitern, Bd. 10, Stuttgart 1991, S. 102-105.

Rentrop, N., (Strategien 1985): Ausgewählte Strategien im Gründungsprozeß Die Strategie der innovativen Imitation und das Konzept der kritischen Erfolgsfaktoren als strategische Ansätze zur Verbesserung der Qualität von Unternehmensgründungen, Bergisch Gladbach 1985. 
Rentrop, N., (Imitation 1990): Die Strategie der innovativen Imitation, in: Dieterle, W.K.M.; Winckler, E.M. (Hrsg.), Unternehmensgründung: Handbuch des Gründungsmanagement, München 1990, S. 119-128.

Ripsas, S., (Entrepreneurship 1997): Entrepreneurship als ökonomischer Prozeß: Perspektiven zur Förderung unternehmerischen Handelns. Mit einem Geleitwort von Dietrich Winterhager, Wiesbaden 1997.

Robinson, J., (Research 1962): A Research Design for Comparing Simulation with Case Studies and Problem Papers in Teaching Political Sciences, in: Dill, W.R.; Jackson, J.R.; Sweeney, J.W. (Eds.), Proceedings of the Conference on Business Games as Teaching Devices, School of Business Administration, Tulane University, 1962, S. 123-129.

Rohn, W.E., (Führungsentscheidungen 1964): Führungsentscheidungen im Unternehmensplanspiel, Essen 1964.

Rohn, W.E., (Methodik 1980): Methodik und Didaktik des Planspiels, Köln 1980.

Rohn, W.E., (Planspiel-Übersicht 1988): Deutsche Planspiel-Übersicht 1988, 4. Aufl., Wuppertal 1988.

Rohn, W.E., (Management-Lernen 1991): Management-Lernen durch Planspiele. Eine Bilanz: 30 Jahre Unternehmens-Planspiel in Deutschland, in: Personalführung, Heft 10, 1991, S. 764-773.

Rosen, R., (Complexity 1977): Complexity as a System Property, in: Int. J. General Systems, No. 4, Vol. 3, 1977, S. 227-232.

Rosenbloom, P.S.; Laird, J.E.; Newell, A.; McCarl, R., (Analysis 1991): A Preliminary Analysis of the Soar Architecture as a Basis for General Intelligence, in: Artifical Intelligence, No. 1-3, Vol. 47, 1991, S. 289-325.

Ross, D.T., (Applications 1985): Applications and Extensions of SADT, in: IEEE Computer, No. 4, Vol. 18, 1985, S. 25-34.

Rubinstein, S.L., (Grundlagen 1973): Grundlagen der Allgemeinen Psychologie, 8. Aufl., Berlin 1973.

Rumelhart, D.E., (Schemata 1980): Schemata: The Building Blocks of Cognition, in: Spiro, R.J.; Bruce, B.C.; Brewer, W.F. (Eds.), Theoretical Issues in Reading Comprehension: Perspectives from Cognitive Psychology, Linguistics, Artifical Intelligence, and Education, Hillsdale, N.J. 1980, S. 33-58. 
Ryle, G., (Begriff 1969): Der Begriff des Geistes, Stuttgart 1969.

Schank, R.C.; Abelson, R.P., (Scripts 1977): Scripts, Plans, Goals and Understanding. An Inquiry into Human Knowledge Structures, Hillsdale, N.J. 1977.

Schanz, G., (Pluralismus 1973): Pluralismus in der Betriebswirtschaftslehre: Bemerkungen zu gegenwärtigen Forschungsprogrammen, in: ZfbF, Heft 2, 25. Jg., 1973, S. 131-154.

Schanz, G., (Wissenschaftspraxis 1975): Traditionelle Wissenschaftspraxis und systemtheoretisch-kybernetische Ansätze, in: Jehle, E. (Hrsg.), Systemforschung in der Betriebswirtschaftslehre, Stuttgart 1975, S. 1-22.

Schaub, H., (Modellierung 1993): Modellierung der Handlungsorganisation, Bern, Göttingen, Toronto, Seattle 1993.

Schaub, H., (Fehler 2001): "Exception Error": Über Fehler und deren Ursachen beim Handeln in Unbestimmtheit und Komplexität, [Online in Internet], URL: $\quad \underline{\text { http://www.uni-bamberg.de/ ba2dp1/lit.html\#Schaub, [Stand }}$ 14.08.2001].

Schoeffling, H., (Businessplan 2001): So erstellen Sie einen Businessplan Handbuch für Existenzgründer, Brühl 2001.

Schroder, H.M., (Bedeutsamkeit 1978): Die Bedeutsamkeit von Komplexität, in: Mandl, H.; Huber, G.L. (Hrsg.), Kognitive Komplexität: BedeutungWeiterentwicklung-Anwendung, Göttingen, Toronto, Zürich 1978, S. 3550.

Schumpeter, J.A., (Unternehmer 1928): Der Unternehmer, in: Elster, L. (Hrsg.), Handwörterbuch der Staatswissenschaften, Bd. 8, 4. Aufl., 1928.

Schumpeter, J.A., unter Beteiligung von Preiswerk, S., (Kapitalismus 1946): Kapitalismus, Sozialismus und Demokratie, Bern 1946.

Schweitzer, M., (Planung 1989): Planung und Kontrolle, in: Bea, F.X.; Dichtl, E.; Schweitzer, M. (Hrsg.), Allgemeine Betriebswirtschaftslehre, Bd. 1: Grundfragen, 4. Aufl., Stuttgart 1989, S. 11-48.

Sellien, R.; Sellien, H. (Hrsg.), (Wirtschaftslexikon 1988): Gabler Wirtschaftslexikon, 12., vollständig neu bearb. U. erw. Aufl., Bd. 4 und 5, Wiesbaden 1988. 
Sembill, D., (Problemlösefähigkeit 1992): Problemlösefähigkeit, Handlungskompetenz und Emotionale Befindlichkeit - Zielgrößen Forschenden Lernens, Göttingen, Toronto, Zürich 1992.

Sieben, G.; Schildbach, T., (Entscheidungstheorie 1990): Betriebswirtschaftliche Entscheidungstheorie, 3., überarb. und erw. Aufl., Düsseldorf 1990.

Simon, H.A., (Architecture 1962): The Architecture of Complexity, in: Proceedings of the American Philosophical Society, No. 6, Vol. 106, 1962, S. 467-482.

Simon, H.A., (Equivalence 1975): The Functional Equivalence of Problem Solving Skills, in: Cognitive Psychology, 1975.

Skinner, B.F., (Freiheit 1973): Jenseits von Freiheit und Würde, Reinbek bei Hamburg 1973.

Sonderegger, W., (Entwurf 1982): Entwurf von computerisierten Volkswirtschafts-Planspielen in verschiedenen Schwierigkeitsgraden, Bamberg 1982.

Spada, H.; Reimann, P.; Häusler, B., (Hypothesenerarbeitung 1983): Hypothesenerarbeitung und Wissensaufbau beim Schüler, in: Kötter, L.; Mandl, H. (Hrsg.), Kognitive Prozesse und Unterricht. Jahrbuch für empirische Erziehungswissenschaft 1983, 1. Aufl., Düsseldorf 1983, S. 139-167.

Spang, S., (Expertensysteme 1991): Einführung: Expertensysteme - Verständnis einer neuen Technologie, in: Spang, S.; Kraemer, W. (Hrsg.), Expertensysteme: Entscheidungsgrundlage für das Management, Wiesbaden 1991, S. 1-5.

Spies, K.; Hesse, F.W., (Interaktion 1986): Interaktion von Emotion und Kognition, in: Psychologische Rundschau, 37. Jg., 1986, S. 75-90.

Spinath, B., (Theorien 1998): Implizite Theorien über die Veränderbarkeit von Intelligenz und Begabung als Bedingung von Motivation und Leistung, Bielefeld 1998.

Stachowiak, H., (Denken 1965): Denken und Erkennen im kybernetischen Modell, Wien 1965.

Stahl, S.R., (Beschränkungen 1999): Kulturelle Beschränkungen im Transformationsprozeß: Eine sozialpsychologisch-kognitive Theorie institutionellen Wandels, Jena 1999. 
Steiner, G., (Repräsentationen 1988): Analoge Repräsentationen, in: Mandl, H.; Spada, H. (Hrsg.), Wissenspsychologie, München, Weinheim 1988, S. 99-119.

Sternberg, R.J., (Reasoning 1982): Reasoning, Problem Solving, and Intelligence, in: Sternberg, R.J., (Ed.), Handbook of Human Intelligence, Cambridge 1982, S. 225-307.

Steuck, J.W., (Businessplan 1999): Businessplan, Berlin 1999.

Streit, M.E., (Marktwirtschaft 1998): Die Marktwirtschaft, ein offenes und komplexes System, in: Wobus, A.M.; Wobus, U.; Parthier, B. (Hrsg.), Vom Einfachen zur Ganzheitlichkeit: das Problem der Komplexität auf organismischer und soziokultureller Ebene / Gaterslebener Begegnung 1997. Gemeinsam veranstaltet vom Institut für Pflanzengenetik und Kulturpflanzenforschung und von der Deutschen Akademie der Naturforscher Leopoldina am 23. und 24. Mai 1997, Heidelberg, Leipzig 1998.

Strohner, H., (Systeme 1995): Kognitive Systeme: Eine Einführung in die Kognitionswissenschaft, Opladen 1995.

Strohschneider, S., (Wissenserwerb 1990): Wissenserwerb und Handlungsregulation, Wiesbaden 1990.

Strohschneider, S.; Schaub, H., (Manager 1991): Können Manager wirklich so gut managen? Über die Effekte unterschiedlichen heuristischen Wissens beim Umgang mit komplexen Problemen, in: Z. Psychol., Suppl. 11, 1991, S. 325-340.

Struck, U., (Geschäftspläne 1990): Geschäftspläne. Voraussetzungen für eine erfolgreiche Kapitalbeschaffung, Stuttgart 1990.

Süllwold, F., (Bedingungen 1960): Bedingungen und Gesetzmäßigkeiten des Problemlösungsverhaltens, in: Thomas, H. (Hrsg.), Bericht über den 22. Kongreß der DGfP in Heidelberg 1959, Göttingen 1960, S. 96-115.

Süss, H.-M., (Intelligenz 1996): Intelligenz, Wissen und Problemlösen: kognitive Voraussetzungen für erfolgreiches Handeln bei computersimulierten Problemen, Göttingen, Bern, Toronto, Seattle 1996.

Szyperski, N., (Orientierung 1971): Zur wissenschaftsprogrammatischen und forschungsstrategischen Orientierung der Betriebswirtschaftslehre, in: ZfbF, 23. Jg., 1971, S. 261-282. 
Szyperski, N.; Kirschbaum, G., (GRÜMOD 1979): GRÜMOD: Ein computergestütztes Simulationsmodell zur Unternehmensgründung, unveröffentlichtes Rezensionsexemplar des Arbeitsbericht Nr. 26 des Seminars für Allgemeine Betriebswirtschaftslehre und betriebswirtschaftliche Planung der Universität zu Köln, Köln 1979.

Szyperski, N.; Klandt, H., (Research 1981): The empirical Research on Entrepreneurship in the Federal Republic of Germany, in: Vesper, K.H. (Ed.), Frontiers of Entrepreneurship, Proceedings of the 1981 Babson College Entrepreneurship Research Conference, $3^{\text {rd }}$ unrevised Printing, Wilmington 1981, S. 158-178.

Szyperski, N.; Kirschbaum, G., (Unternehmensfluktuation 1981): Unternehmensfluktuation in Nordrhein-Westfalen - Eine empirische Untersuchung zur Entwicklung von Gründungen und Liquidationen im Zeitraum von 1973 bis 1979, Göttingen 1981.

Szyperski, N.; Nathusius, K., (Information 1975): Information und Wirtschaft. Der informationstechnische Einfluß unterschiedlicher Wirtschaftssysteme, Frankfurt, New York 1975.

Szyperski, N.; Nathusius, K., (Probleme 1999): Probleme der Unternehmensgründung: eine betriebswirtschaftliche Analyse unternehmerischer Startbedingungen, 2. Aufl., Lohmar, Köln 1999.

Tack, W.H., (Ziele 1987): Ziele und Methoden der Wissensrepräsentation, in: Sprache \& Kognition, Heft 3, 6. Jg., 1987, S. 150-163.

Taddicken, U., (Simulationsspiele 1977): Simulationsspiele im Wirtschaftslehreunterricht: Ein Beitrag zur Didaktik der Wirtschafts- und Gesellschaftslehre, Bad Homburg vor der Höhe, Berlin, Zürich 1977.

Tergan, S.-O., (Modelle 1986): Modelle der Wissensrepräsentation als Grundlage qualitativer Wissensdiagnostik, Opladen 1986.

Tergan, S.-O., (Grundlagen-Teil I-1989): Psychologische Grundlagen der Erfassung individueller Wissensrepräsentationen. Teil I: Grundlagen der Wissensmodellierung, in: Sprache \& Kognition, Heft 3, 8. Jg., 1989, S. 152165.

Thom, P., (Structural 1975): Structural Stability and Morphogenesis, Reading 1975. 
Tramm, T., (Übungsfirma 1984): Übungsfirma als Lernumwelt. Konzeption und erste Ergebnisse eines Forschungsprojekts zur Analyse des Lernpotentials schulischer Übungsfirmen, in: Kell, A.; Lipsmeier, A. (Hrsg.), Berufliches Lernen ohne berufliche Arbeit? Beiheft 5 der Zeitschrift für Berufs- und Wirtschaftspädagogik, Stuttgart 1984, S. 60-74.

Ueckert, H., (Computer-Simulation 1983): Computer-Simulation, in: Bredenkamp, J.; Feger, H. (Hrsg.), Enzyklopädie der Psychologie. Themenbereich B: Methodologie und Methoden. Serie I: Forschungsmethoden der Psychologie. Band 5: Hypothesenprüfung, Göttingen 1983, S. 530-616.

Ulrich, H., (Unternehmung 1970): Die Unternehmung als produktives soziales System. Grundlagen der allgemeinen Unternehmungslehre, 2., überarb. Aufl., Bern, Stuttgart 1970.

Ulrich, T.A.; Cole, G.S., (Entrepreneurs 1987): Toward More Effective Training of Future Entrepreneurs, in: Journal of Small Business Management, No. 4, Vol. 25, 1987, S. 32-39.

Vance, S.C.; Gray, C.F., (Performance 1967): Use of a Performance Evaluation Model for Research in Business Gaming, in: Academy of Management Journal, No. 10, Vol. 9, 1967, S. 27-37.

Vogt, H.M., (Persönlichkeitsmerkmale 1998): Persönlichkeitsmerkmale und komplexes Problemlösen: der Zusammenhang von handlungstheoretischen Persönlichkeitskonstrukten mit Verhaltensweisen und Steuerungsleistungen bei dem computersimulierten komplexen Szenario UTOPIA, München, Mering 1998.

Vogt, R., (Systemwissenschaften 1983): Die Systemwissenschaften: Grundlagen und wissenschaftstheoretische Einordnung, Frankfurt am Main 1983.

Volpert, W., (Zusammenhang 1979): Der Zusammenhang zwischen Arbeit und Persönlichkeit aus handlungstheoretischer Sicht, in: Groskurth, P. (Hrsg.), Arbeit und Persönlichkeit: Berufliche Sozialisation in der arbeitsteiligen Gesellschaft, Reinbek bei Hamburg 1979, S. 21-46.

Voss, J.F., (Lösen 1990): Das Lösen schlecht strukturierter Probleme - ein Überblick, in: Unterrichtswissenschaft, Heft 4, 18. Jg., 1990, S. 313-337. 
Voss, J.F.; Fincher-Kiefer, R.H.; Greene, T.R.; Post, T.A., (Performance 1986): Individual Differences in Performance: The Contrastive Approach to Knowledge, in: Sternberg, R.J. (Ed.), Advances in the Psychology of Human Intelligence, Vol. 3, Hillsdale, N.J., 1986, S. 297-334.

Wagenaar, W.A.; Sagaria, S.D., (Misperception 1975): Misperception of Exponential Growth, Perception \& Psychophysics, No.6, Vol. 18, 1975, S. 416-422.

Wandel, H.-U., (Expertensysteme 1992): Expertensysteme in der strategischen Planung, Göttingen 1992.

Watzlawick, P., (Wirklichkeit 1995): Wie wirklich ist die Wirklichkeit?: WahnTäuschung-Verstehen, 20. Aufl., München 1995.

Weber, J., (Einführung 1990): Einführung in das Controlling, 2., überarb. Aufl., Stuttgart 1990.

Weber, S., (Vorwissen 1994): Vorwissen in der betriebswirtschaftlichen Ausbildung: eine struktur- und inhaltsanalytische Studie. Mit einem Geleitwort von Frank Achtenhagen, Wiesbaden 1994.

Weidenhaupt, T.M., (Grundlagen 1991): Grundlagen von Expertensystemen, in: Biethahn, J.; Hoppe, U. (Hrsg.), Entwicklung von Expertensystemen: eine Einführung, Wiesbaden 1991, S. 9-31.

Weiser, M.; Shertz, J., (Problem 1983): Programming Problem Represantation in Novice and Expert Programmers, in: International Journal of ManMachine Studies, 19, 1983, S. 391-398.

Welge, M.K. unter Beteiligung von Rüth, D., (Unternehmungsführung 1985): Unternehmungsführung, Stuttgart 1985.

Wender, K.-F., (Netzwerke 1988): Semantische Netzwerke als Bestandteil gedächtnispsychologischer Theorien, in: Mandl, H.; Spada, H. (Hrsg.), Wissenspsychologie, München, Weinheim 1988, S. 55-73.

Wender, K.-F., (Methoden 1992): Ausgewählte Methoden, in: Spada, H., (Hrsg.), Lehrbuch allgemeine Psychologie, 2., korrigierte Aufl., Bern, Göttingen, Toronto, Seattle 1992, S. 561-595.

Werbik, H., (Handlungstheorien 1978): Handlungstheorien, Stuttgart 1978. 
Werner, L., (Entscheidungsunterstützungssysteme1992): Entscheidungsunterstützungssysteme: ein problem- und benutzerorientiertes ManagementInstrument, Heidelberg 1992.

Whitfield, D., (Study 1979): A Preliminary Study of the Air Traffic Controller's 'Picture', in: CATCA Journal, No. 1, Vol. 11, 1979, S. 19-28.

Whitfield, D.; Jackson, A., (Air 1982): The Air Traffic Controller's Picture as an Example of a Mental Model, in: Johannsen, G.; Rijnsdorp, J.E. (Eds.), Proceedings of the IFAC Conference on Analysis, Design and Evaluation of Man-Machine Systems, London 1982, S. 37-44.

Wiener, N., (Mathematik 1962): Mathematik-mein Leben, 1. Aufl., Düsseldorf, Wien 1962.

Wiener, N., (Kybernetik 1963): Kybernetik: Regelung und Nachrichtenübertragung im Lebewesen und in der Maschine, 1. deutsche Aufl. der 2., revidierten und ergänzten Aufl., Düsseldorf, Wien 1963.

Wild, J., (Grundlagen 1982): Grundlagen der Unternehmensplanung, 4. Aufl., Opladen 1982.

Wilson, J.R.; Rutherford, A., (Models 1989): Mental Models: Theory and Application in Human Factors, in: Human Factors, Vol. 31, 1989, S. 617634.

Winkel, A.; Schuster, H.; Trommsdorf, V., (Insolvenzgründe 1986): Insolvenzgründe junger technologieorientierter Unternehmen. Untersuchung der Forschungsstelle für den Handel Berlin (FfH) e.V. und des Marketinglehrstuhls der Technischen Universität Berlin, Berlin 1986.

Winograd, T., (Representations 1975): Frame Representations and the Declarative/Procedural Controversy, in: Bobrow, D.G.; Collins, A. (Eds.), Representation and Understanding. Studies in Cognitive Science, New York 1975 , S. $185-210$.

Witte, E., (Informations-Verhalten 1972): Das Informations-Verhalten in Entscheidungsprozessen, in: Witte, E. (Hrsg.), Das Informationsverhalten in Entscheidungsprozessen, Tübingen 1972, S. 1-88.

Witte, E., (Unternehmungsspiel 1974): Das Unternehmungsspiel in Lehre und Forschung, in: Fasshauer, R.; Wurzbacher, W. (Hrsg.), Unternehmensspiele - Stand und Entwicklungstendenzen. IBM-Beiträge zur Datenverarbeitung, Methoden und Techniken 4, Stuttgart 1974, S. 14-20. 
Wolf, M., (Leistungsfähigkeit 1979): Die Leistungsfähigkeit des systemorientierten Ansatzes für die Modellbildung, in: Bea, F.X.; Bohnet, A.; Klimesch, H. (Hrsg.), Systemmodelle: Anwendungsmöglichkeiten des systemtheoretischen Ansatzes, München, Wien 1979, S. 11-37.

Wolff, K., (Umgang 1994): Umgang mit Komplexität in Lernsituationen: Reduktion und Transformation als integrale Bestandteile eines auf Ganzheitlichkeit angelegten Konzeptes zur Komplexitätsbewältigung, Köln 1994.

Woll, A., (Volkswirtschaftslehre 1971): Allgemeine Volkswirtschaftslehre, 4. Aufl., München 1971.

Wupperfeld, U., (Business-Plan 1999): Der Business-Plan für den erfolgreichen Start: Anforderungen, Aufbau, Gestaltung, Präsentation, Landsberg am Lech 1999.

Yates, F.E., (Complexity 1978): Complexity and the Limits to Knowledge, in: American Journal of Physiology, No. 5, Vol. 4, 1978, S. R 201-R 204.

Zayer, H., (Prinzipien 1975): Prinzipien und Anwendung der Simulation in der Psychologie, in: Hawel, W. (Hrsg.), Datenverarbeitung in der Psychologie, Stuttgart 1975, S. 17-35. 
Name:

Regina Ahlbrecht

Anschrift:

Mauerstraße 24/13, 37073 Göttingen

geboren:

22. Juli 1967 in Hameln

Staatsangehörigkeit: deutsch

Schulbildung:

1974-1978 Grundschule Hehlen

1978-1980 Orientierungsstufe Bodenwerder

1980-1988 Schillergymnasium Hameln

Praktika

06/88-10/88 Hotel Mirabeau in Zermatt/Schweiz

01/89-06/89

BHW Bausparkasse Hameln

Studium

$10 / 89-11 / 97$

Studium der Betriebswirtschaftslehre

an der Georg-August-Universität

Göttingen

Promotion

03/98-07/02

Doktorandin bei Prof. Dr. Dr. h.c. J. Jürgen Bloech, Institut für Betriebswirtschaftliche Produktions- und Investitionsforschung der GeorgAugust-Universität Göttingen, Abteilung Unternehmensplanung

Wissenschaftliche

Tätigkeit

08/97-04/00 Wissenschaftliche Hilfskraft mit Examen im Arbeitskreis für Doktorandinnen an der Georg-AugustUniversität Göttingen zur wissenschaftlichen Qualifizierung speziell von Frauen 
Ich versichere, daß ich die Arbeit selbständig und ohne Benutzung anderer als der angegebenen Hilfsmittel angefertigt habe. Alle Stellen, die wörtlich oder sinngemäß aus Veröffentlichungen oder anderen Quellen entnommen sind, sind als solche kenntlich gemacht.

Regina Ahlbrecht 